\title{
DESIGN AND TESTING OF A NOVEL COMBINED ADHESION AND LOCOMOTION METHOD FOR WALL CLIMBING VEHICLES
}

\author{
A Thesis \\ presented to \\ the Faculty of California Polytechnic State University, \\ San Luis Obispo
}

\author{
In Partial Fulfillment \\ of the Requirements for the Degree \\ Master of Science in Mechanical Engineering
}

by

James Roy Stefani

June 2016 
(C) 2016

James Roy Stefani

ALL RIGHTS RESERVED 


\section{COMMITTEE MEMBERSHIP}

TITLE:

AUTHOR:

DATE SUBMITTED:

COMMITTEE CHAIR:

COMMITTEE MEMBER:

COMMITTEE MEMBER:
Design and Testing of a Novel Combined Adhesion and Locomotion Method for Wall Climbing Vehicles

James Roy Stefani

June 2016
Thomas Mackin, Ph.D.

Professor of Mechanical Engineering

John Ridgely, Ph.D.

Professor of Mechanical Engineering

Joseph Mello, Ph.D.

Professor of Mechanical Engineering 


\begin{abstract}
Design and Testing of a Novel Combined Adhesion and Locomotion Method for Wall Climbing Vehicles James Roy Stefani

The objective of this project was to design, construct and test a wall climbing vehicle which uses a novel vacuum tread system for both adhesion and locomotion. The design and manufacturing of this proof of concept vehicle is detailed with particular emphasis on the design decisions that proved most impactful to the performance of both the vehicle and the tread system. Adhesion performance was characterized by a series of tests that validate the concept, but also identify improvements and design recommendations for future embodiments of the adhesion/locomotion system.
\end{abstract}

Keywords: Wall-Climbing, vacuum, sliding tread adhesion, robot, vehicle, timing belt. 


\section{ACKNOWLEDGMENTS}

There are numerous people who made this project possible that I would like to thank. Firstly, I would like to thank Dr. Mackin. Without him there would have been no project, or funding, and I would have most likely lost my mind without his support. So, thanks Dr. Mackin, it was an honor and a pleasure to work with you and without your help I doubt this project would have gotten off the ground.

I would like to thank the two partner with whom I worked with on this project, Erik van Broekhoven and Lynda Tesillo. Erik, I had a blast working with you on that proof of concept model with you and I hope you did too. Lynda, you were with me through thick and thin, and the mutual support we provided each other through these trying times was invaluable. I truly appreciate the friendship that we have and look forward to continuing that friendship for years to come.

To all of the different Cal Poly shop technicians, thank you all so much for the help making this thing. In particular I would like to thank Brett Johnson and Grant Pocklington for doing what I could not, which was weld. I would also like to thank Larry Coolidge for helping me get my damn wheels printed and reprinted and reprinted again.

To everyone in the grad lab, and there were far too many of you to thank individually, thank you all for being my friends and companions through the journey that was graduate school. The support and comradery that we shared was priceless and I will consider each of you a lifelong friend.

I would also like to thank Patrick Hutchinson for keeping me from going off the deep end during this endeavor. You have become one of the best friends that I have ever had. I could not have finished this project without you keeping me grounded, coherent and distracting me when it was appropriate.

Finally, I would like to thank my parents, Dave and Danielle Stefani, because without them I would truly not have been here or gotten this far. You guys have only ever shown me love, support and respect and for that I will forever love you. Oh, and my sister Katie too, I guess. 


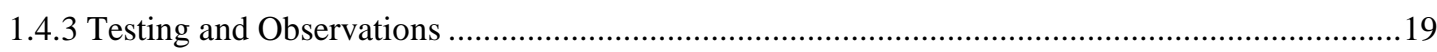

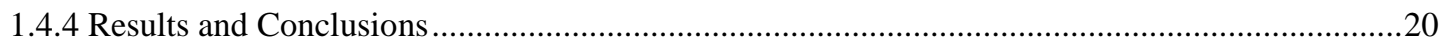

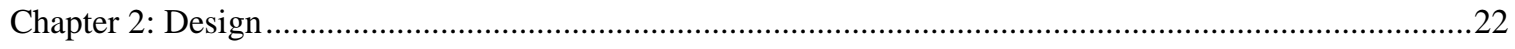

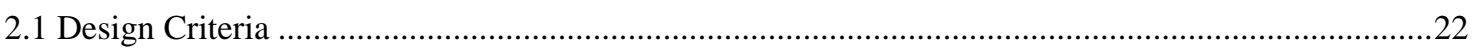

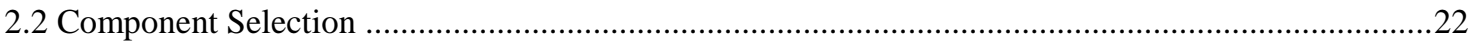

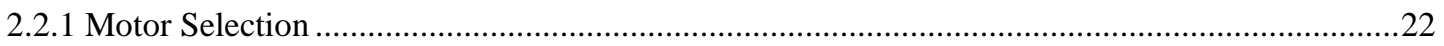

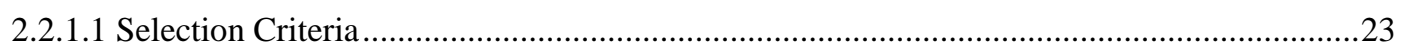

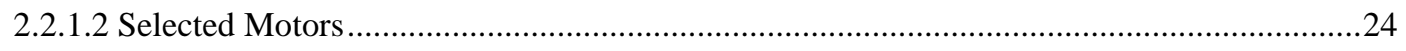

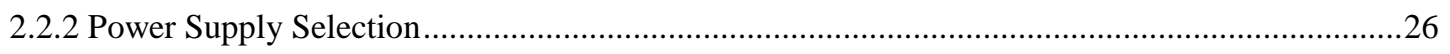

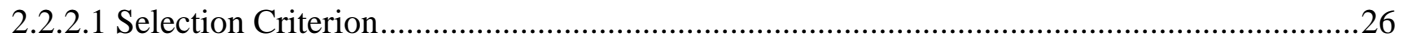

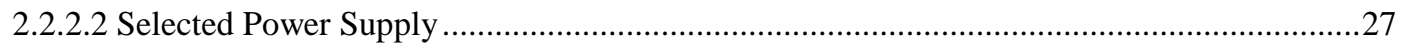

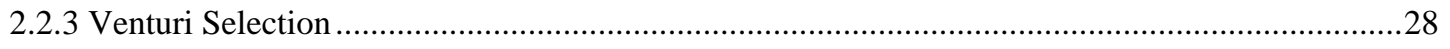

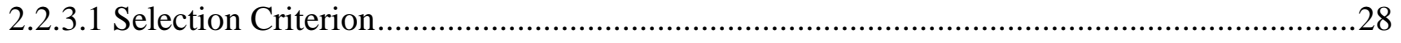

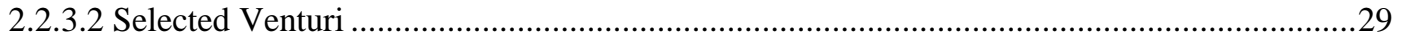

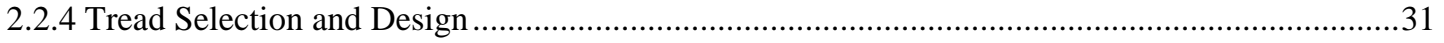

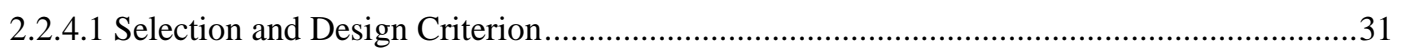

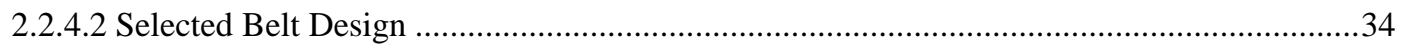

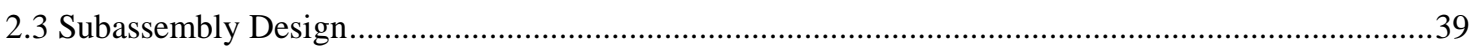

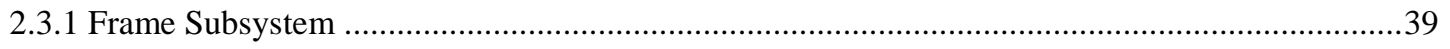

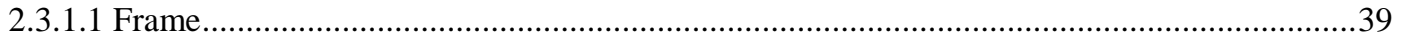

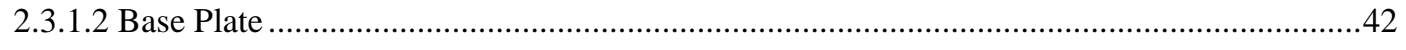

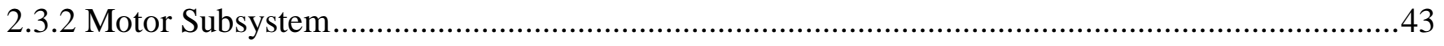

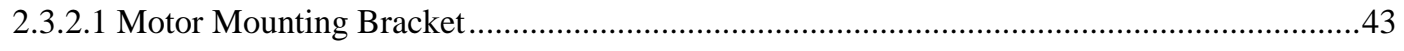

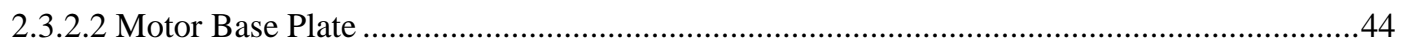

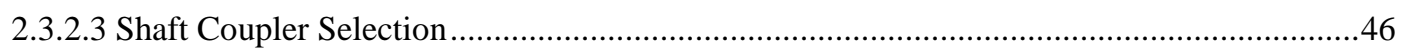




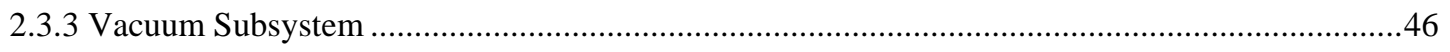

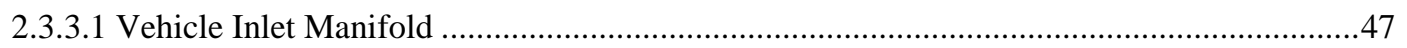

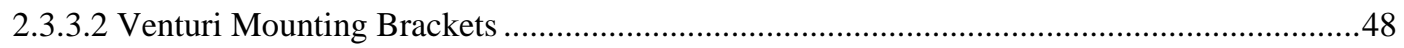

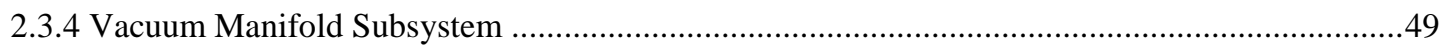

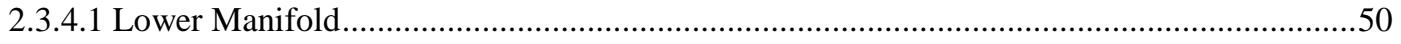

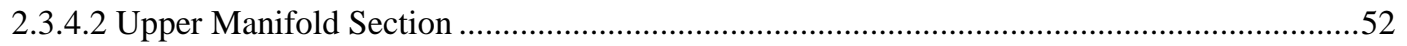

2.3.4.3 Manifold Gasket ..............................................................................................................

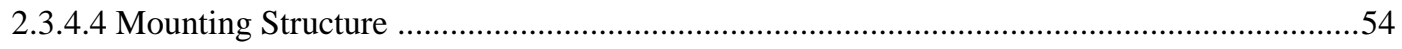

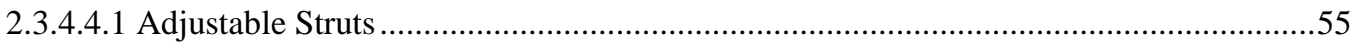

2.3.4.4.2 Threaded Uprights …………...............................................................................56

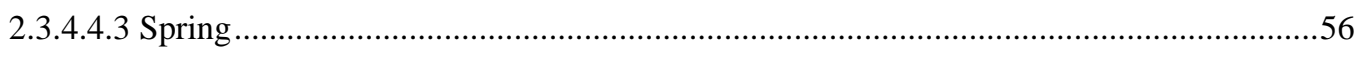

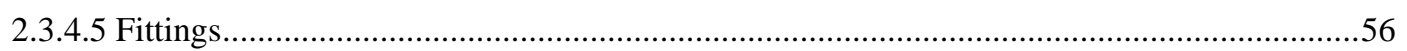

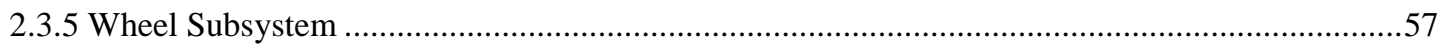

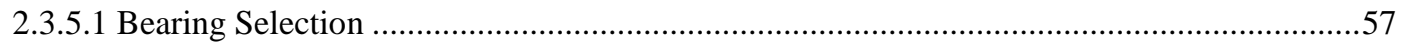

2.3.5.1.1 Flanged Bearing ………....................................................................................

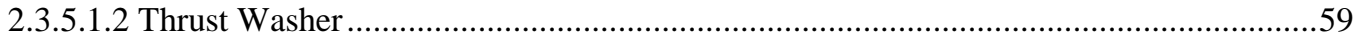

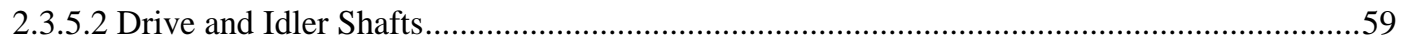

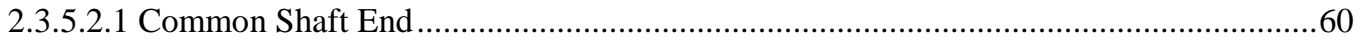

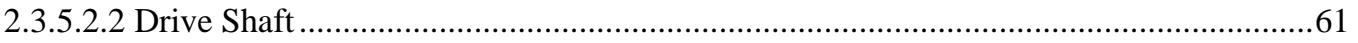

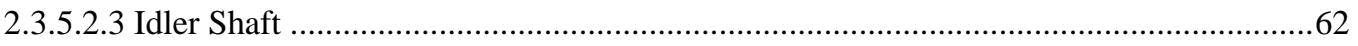

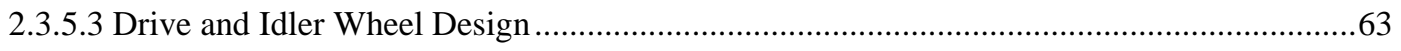

2.3.5.3.1 Common Drive and Idler Wheel Design Elements ......................................................63

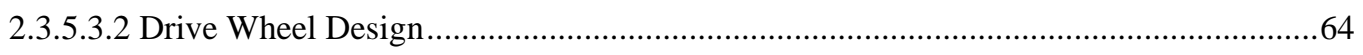

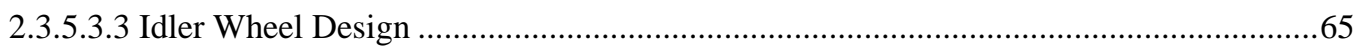

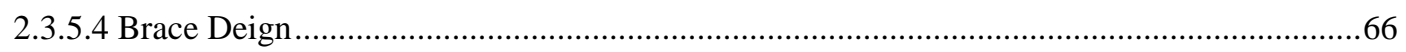

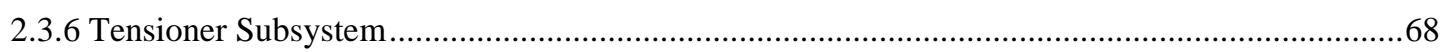

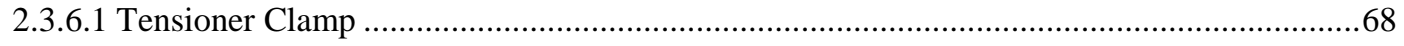

2.3.6.1.1 Tensioner Clamp - Interior .......................................................................................69

2.3.6.1.1 Tensioner Clamp - Exterior ...................................................................................

2.3.6.2 Tensioner Wheel Assembly .........................................................................................

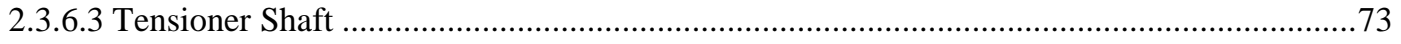

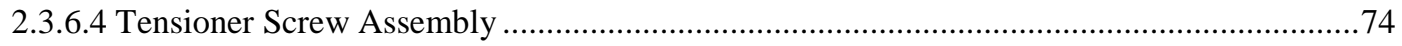

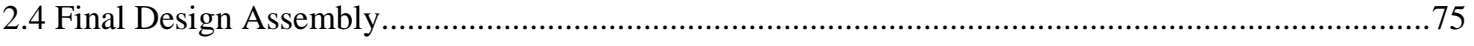

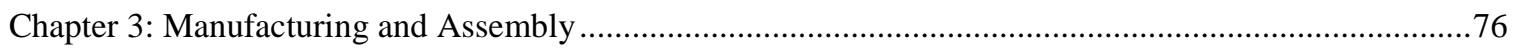




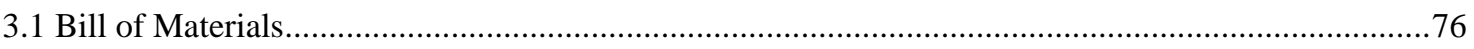

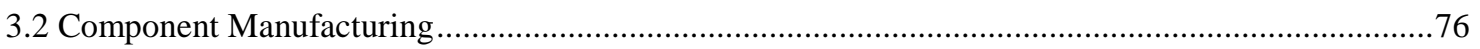

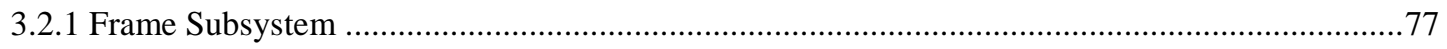

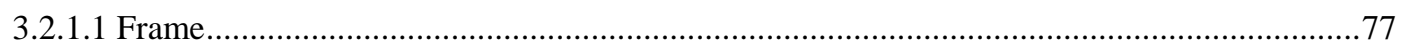

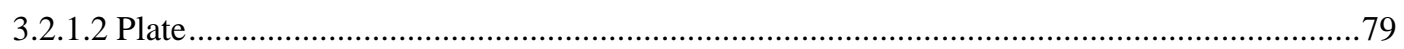

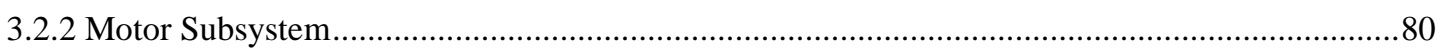

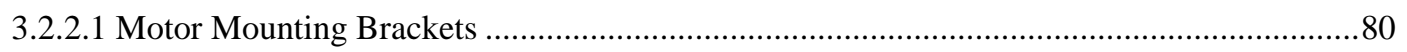

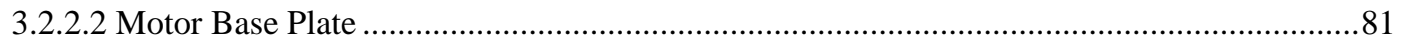

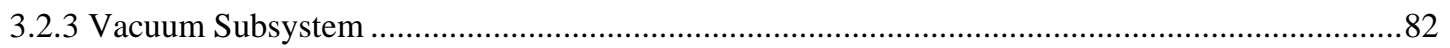

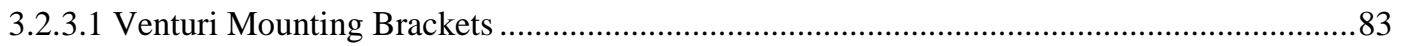

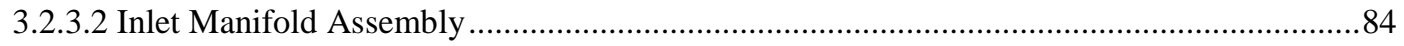

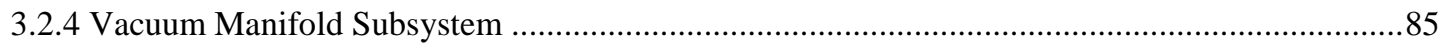

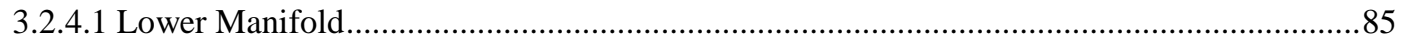

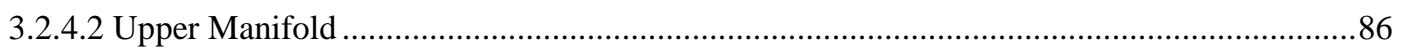

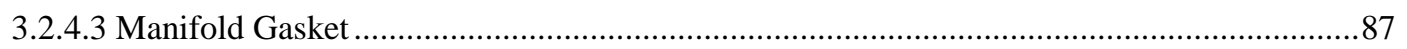

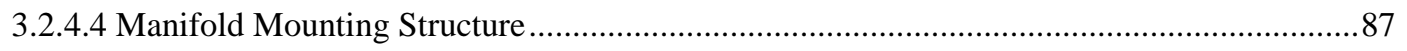

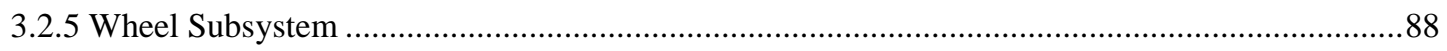



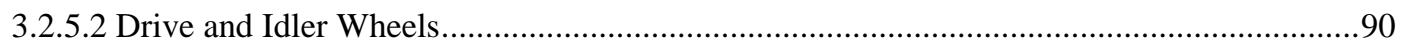

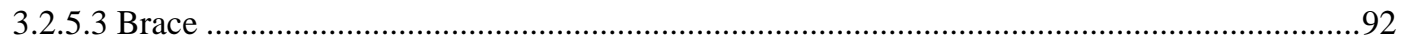

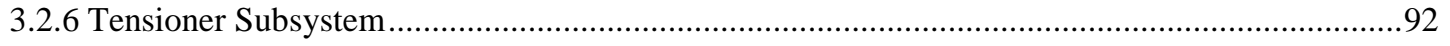

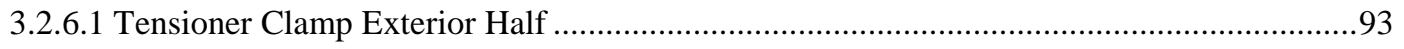

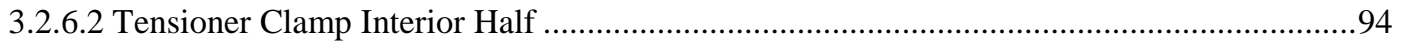

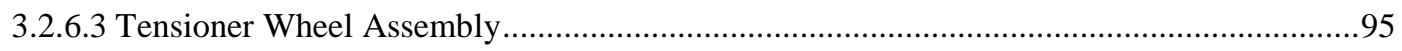

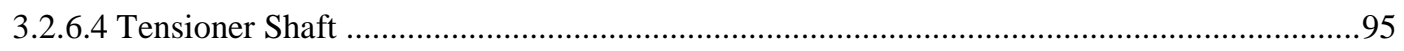

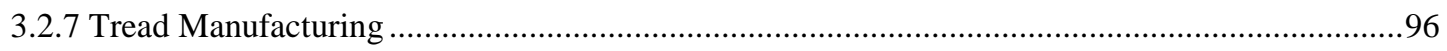

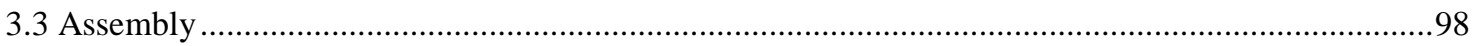

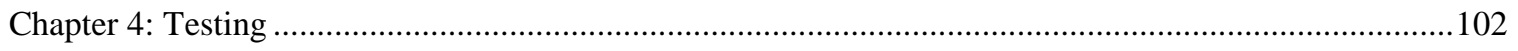

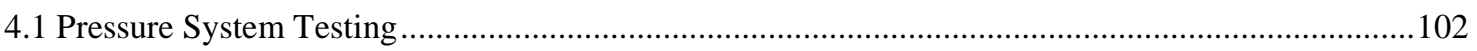

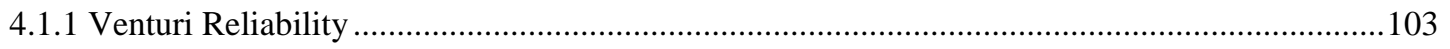




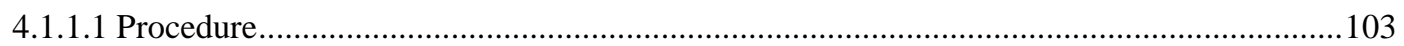

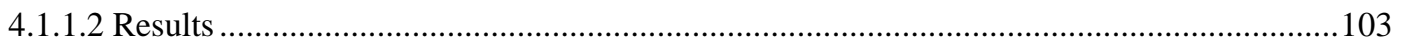

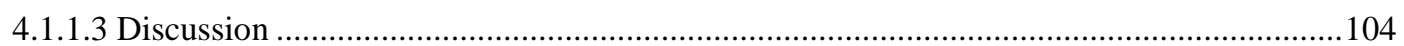

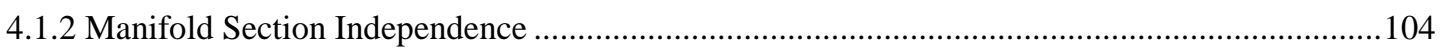

4.1.2.1 Procedure

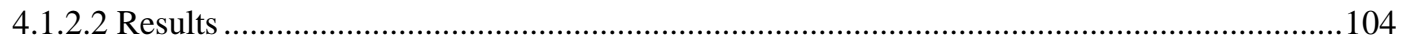

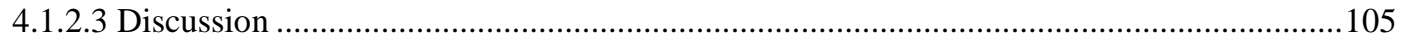

4.1.3 Tread Installation Effect and Fixed Manifold Testing..................................................................105

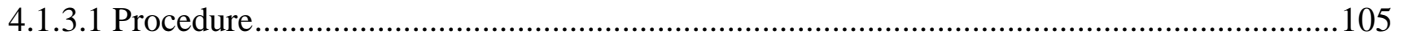

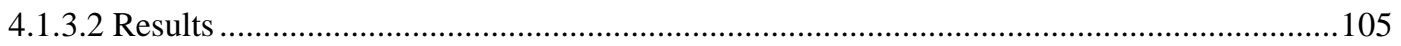

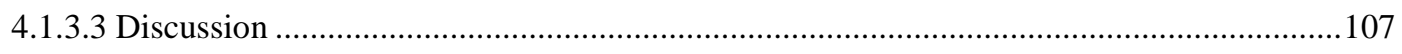

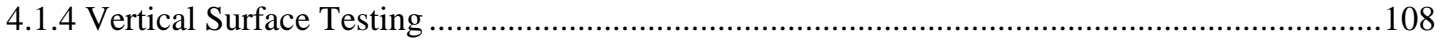

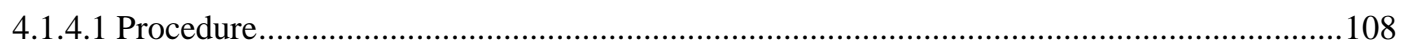

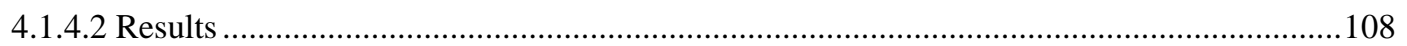

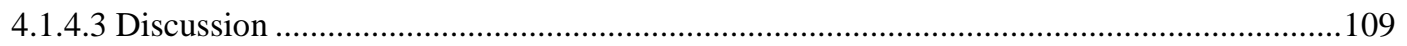

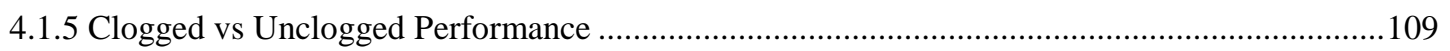

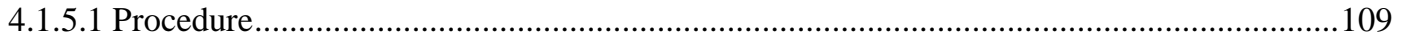

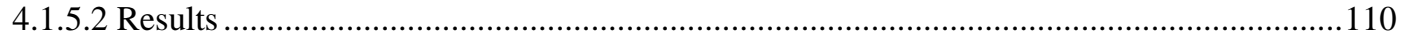

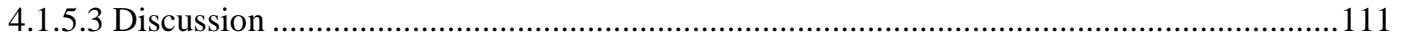

4.1.6 Split Manifold Testing .................................................................................................... 111

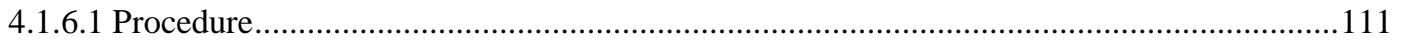

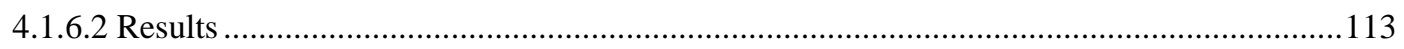

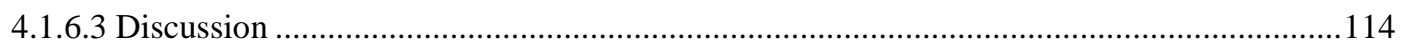

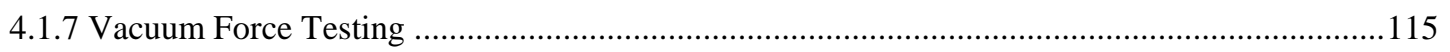

4.1.7.1 Procedure

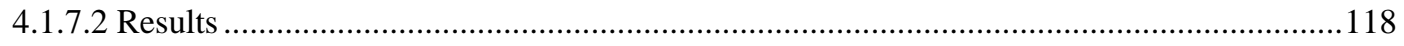

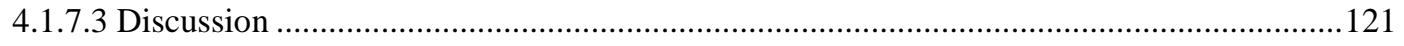

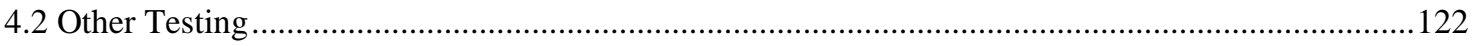

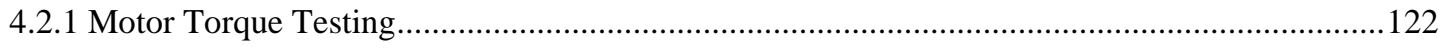

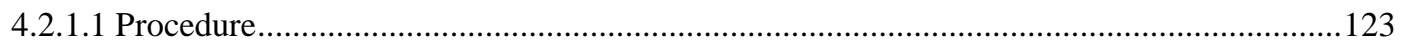

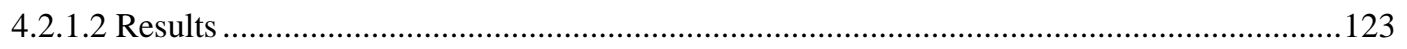

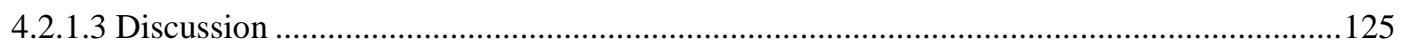

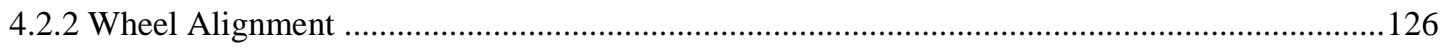


4.2.2.1 Procedure

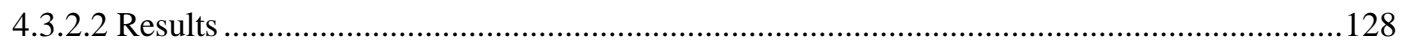

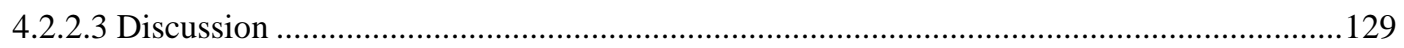

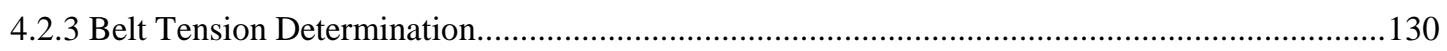

4.2.3.1 Procedure

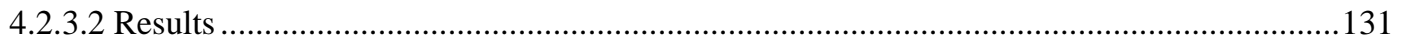

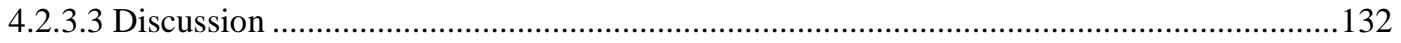

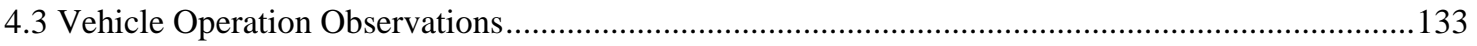

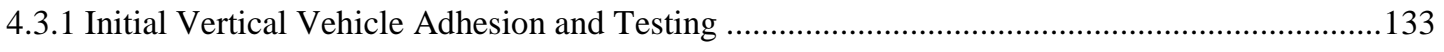

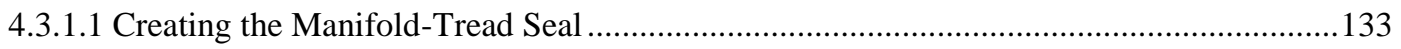

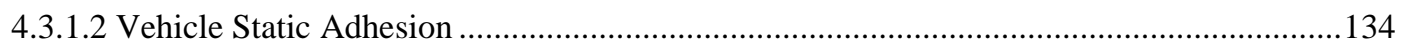

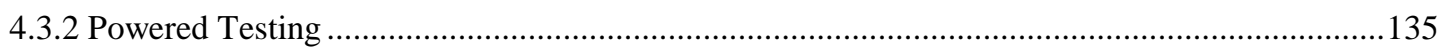

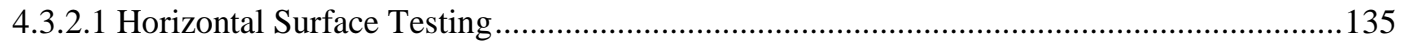

4.3.2.2 Initial Vertical Surface Testing ……................................................................................ 135

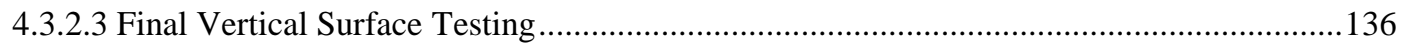

Chapter 5: Recommendations for Future Work and Conclusion.................................................................138

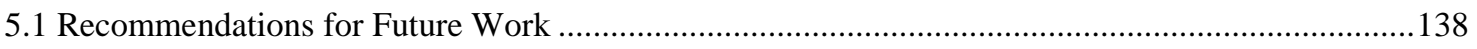

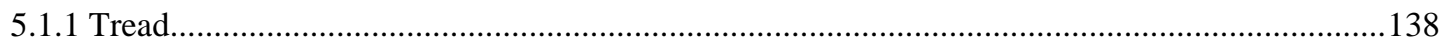

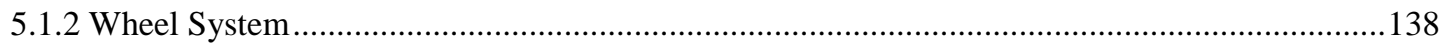

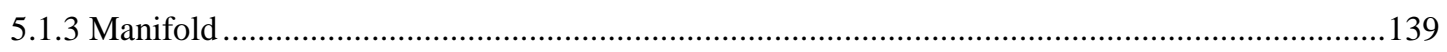

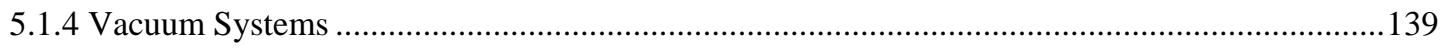

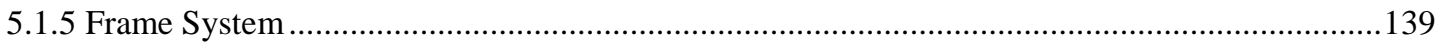

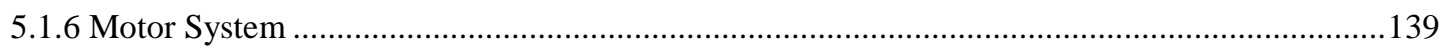

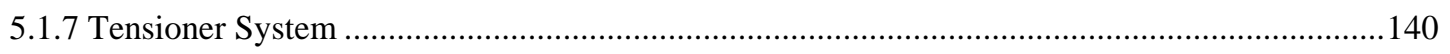

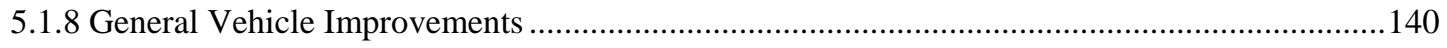

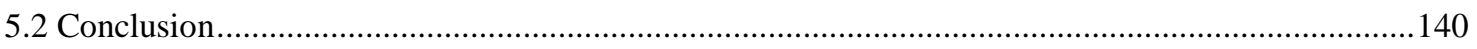

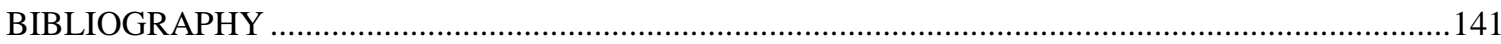




\section{APPENDICES}

Appendix A - Simulations and Calculations

A.1 Symbolic Calculation of Motor Torque..................................................................... 144

A.1.1 Calculation Description............................................................................ 144

A.1.2 Assumptions .................................................................................................... 144

A.1.3 Free Body and Mass Acceleration Diagrams ............................................................. 145

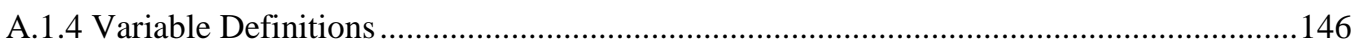

A.1.5 Symbolic Manipulation ....................................................................................... 147

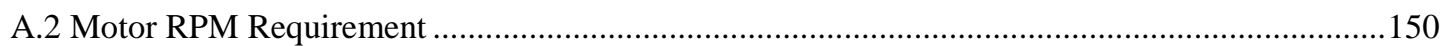

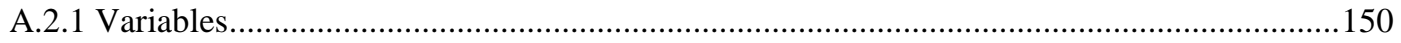

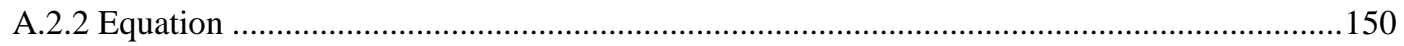

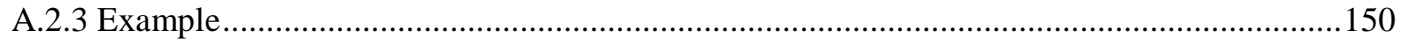

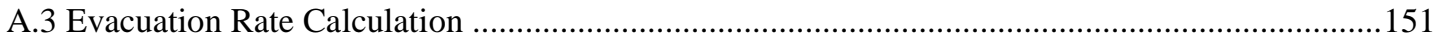

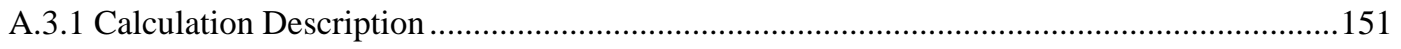

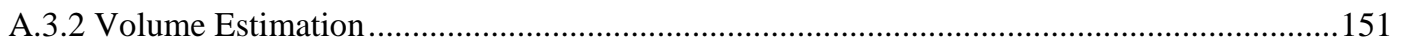

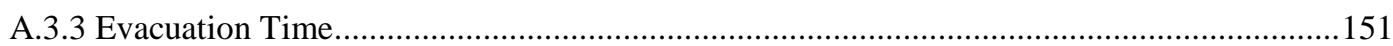

A.4 Minimum Belt Tension Requirement Calculation .............................................................. 153

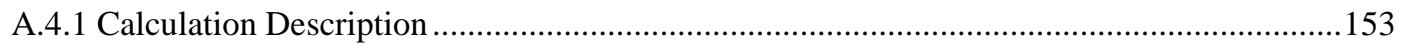

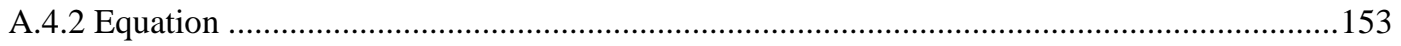

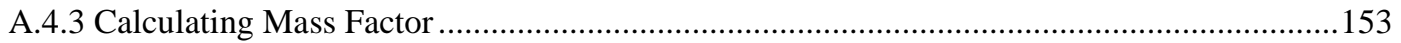

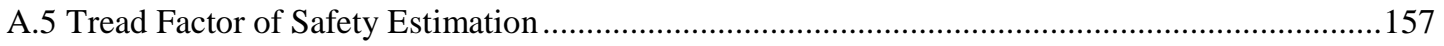

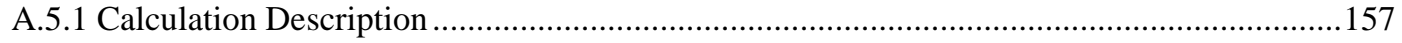

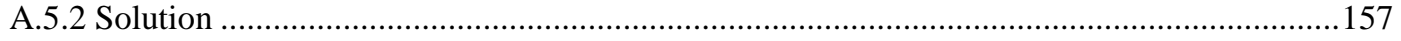

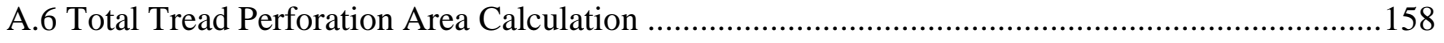

A.6.1 Calculation Description .................................................................................... 158

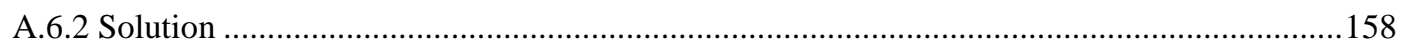

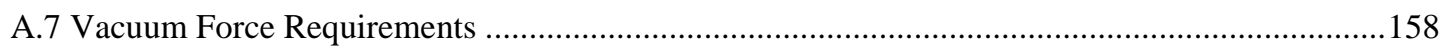

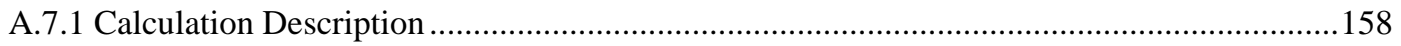

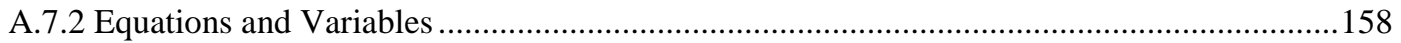

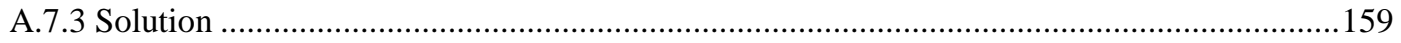

A.8 Final Tread Hole Pattern and Manifold Slot Length Calculation ...........................................160

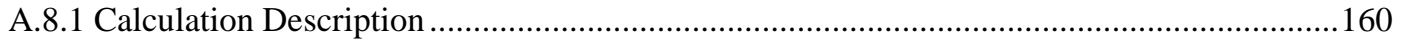




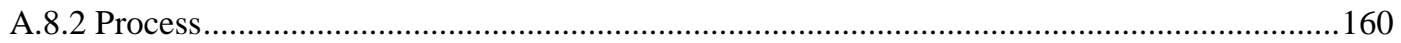

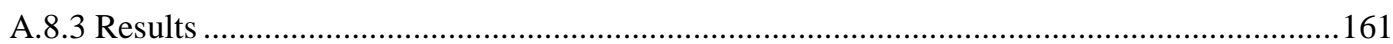

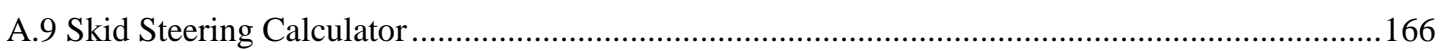

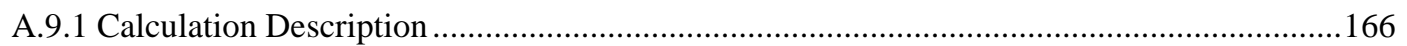

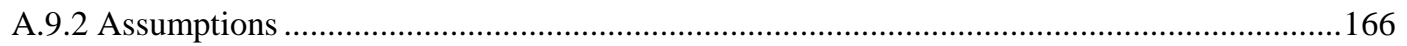

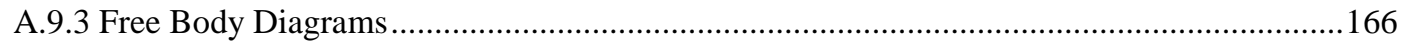

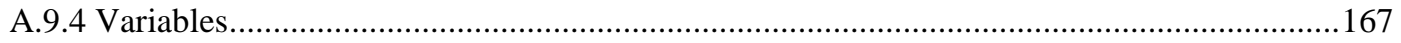

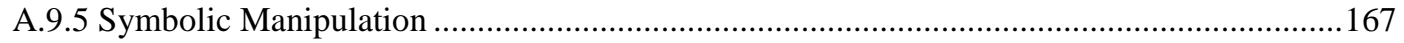

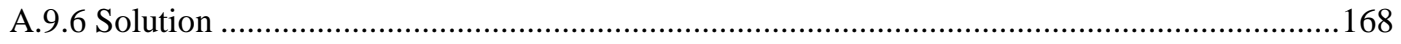

A.10 Allowable Friction Between The Tread and The Manifold .......................................................169

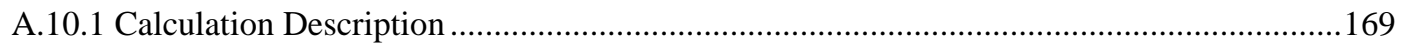

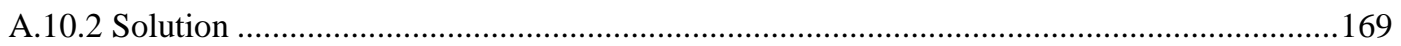

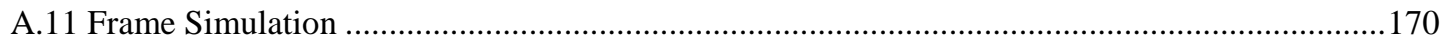

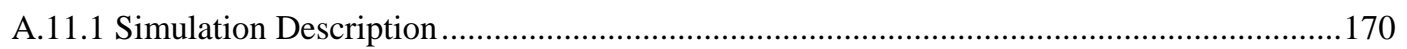

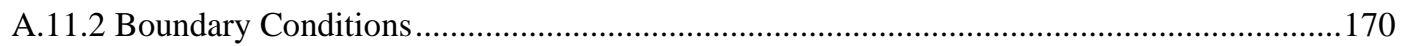

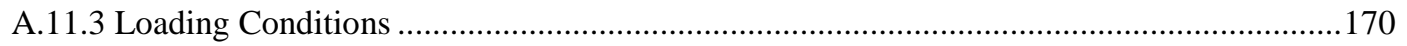

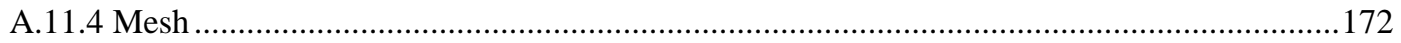

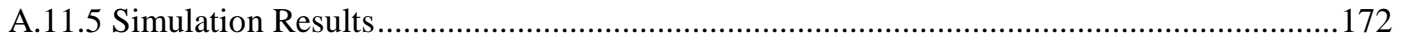

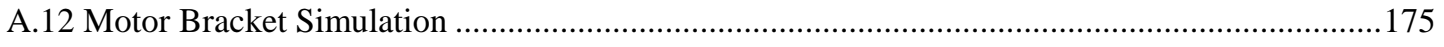

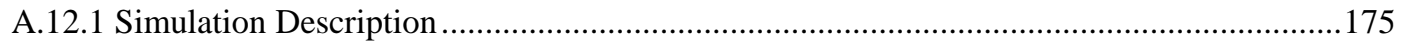

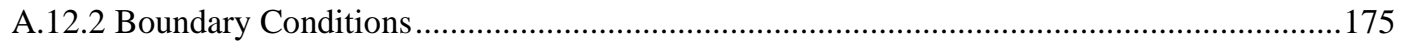

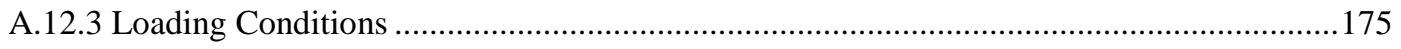

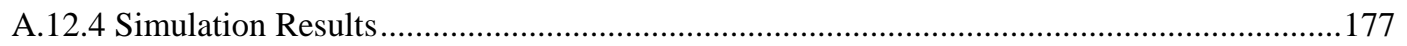

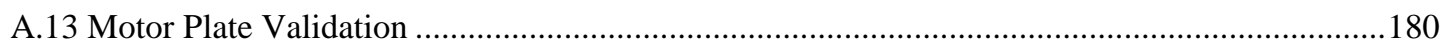

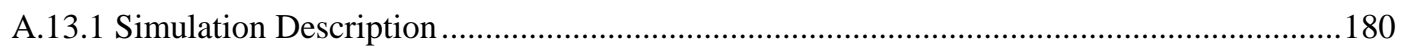

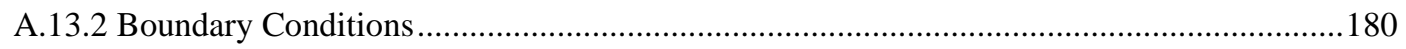

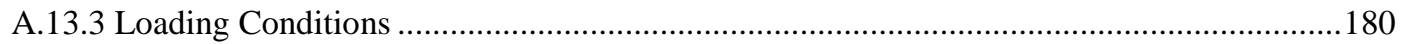

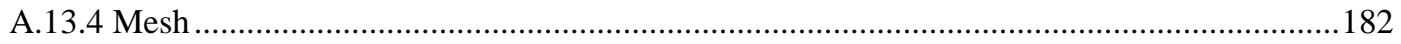

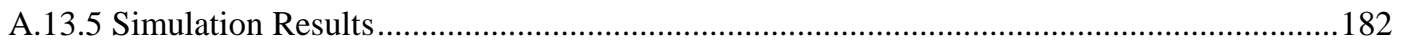

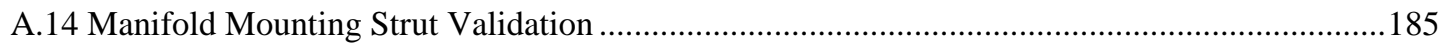

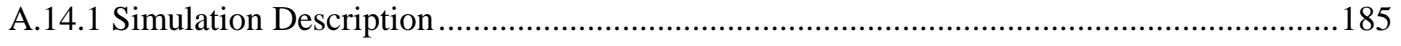

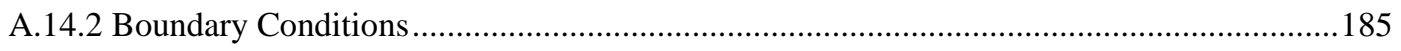

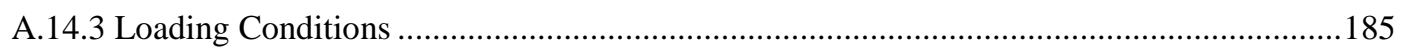

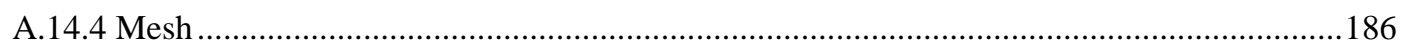

xiii 


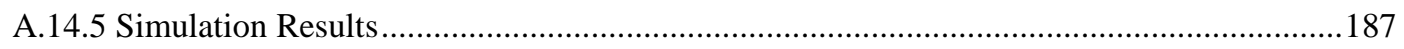

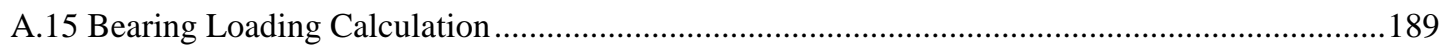

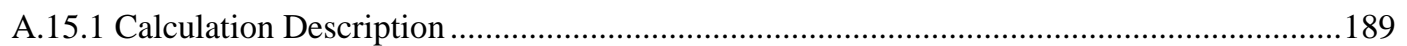

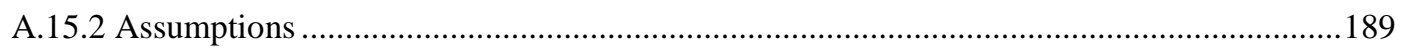

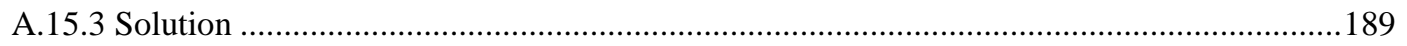

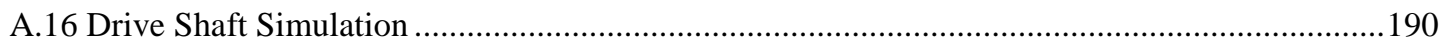

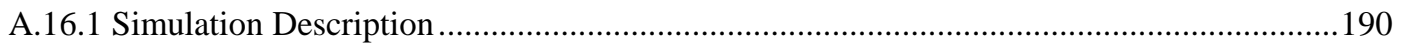

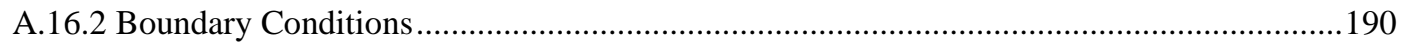

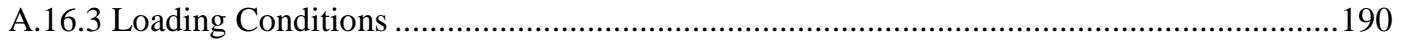

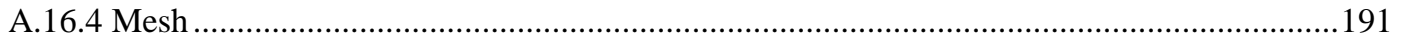

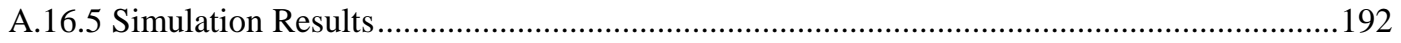

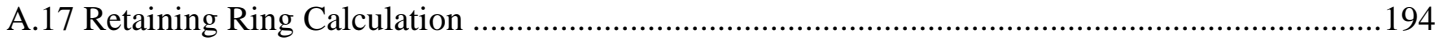

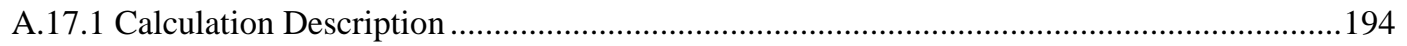

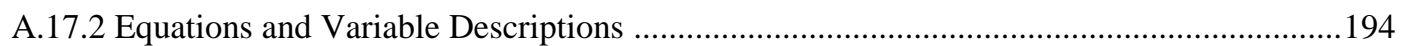

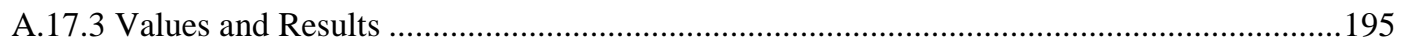

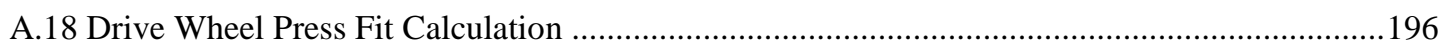

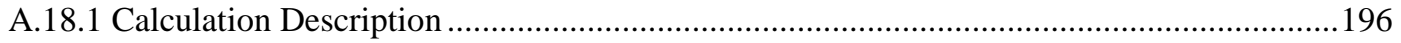

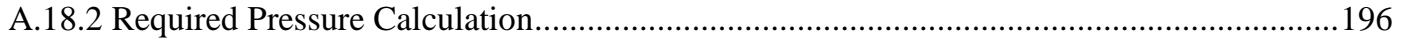

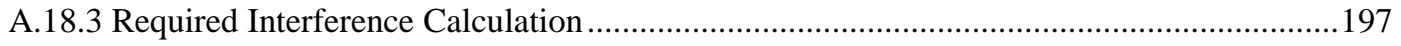

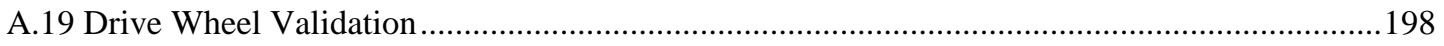

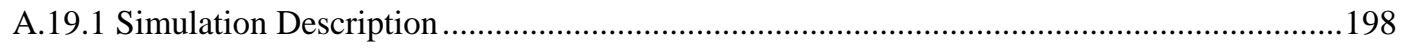

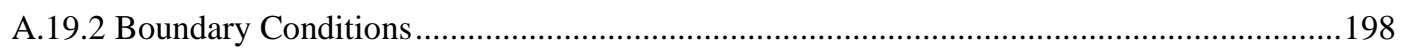

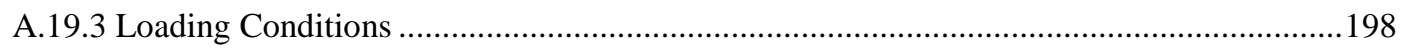

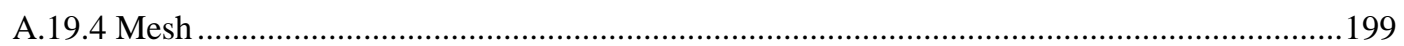

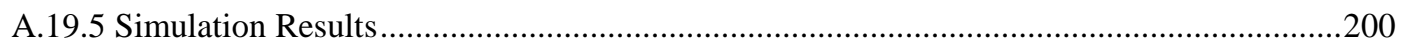

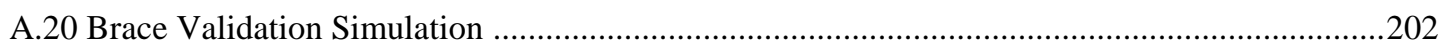

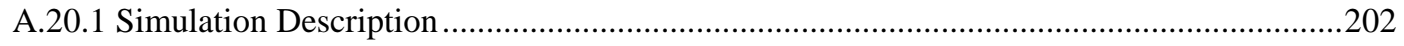

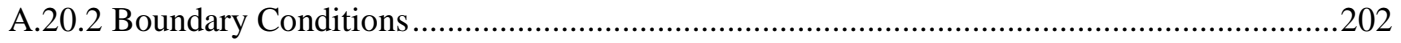

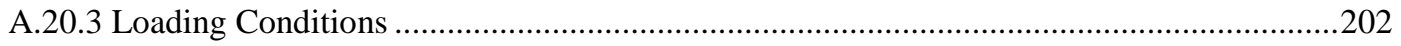

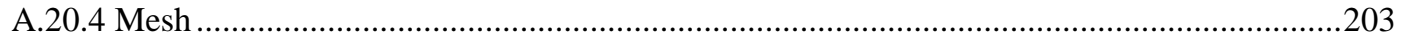

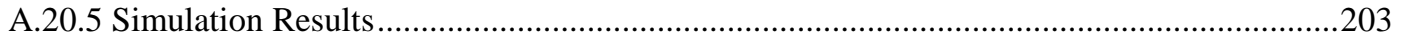

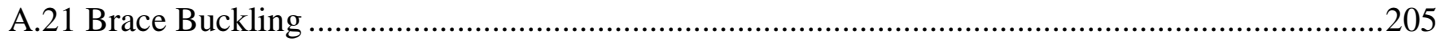

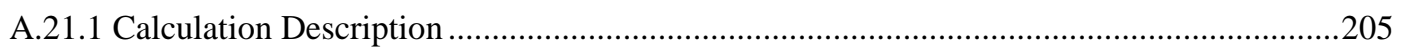




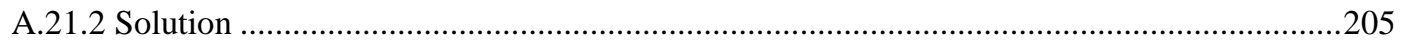

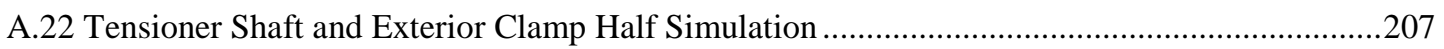

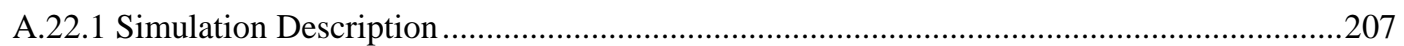

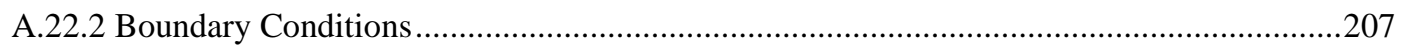

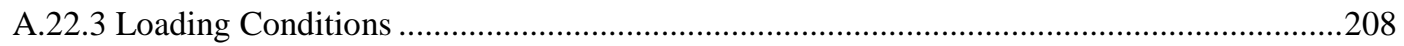

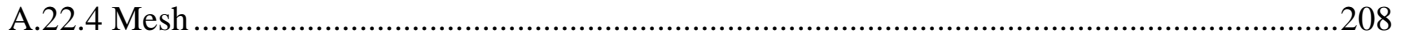

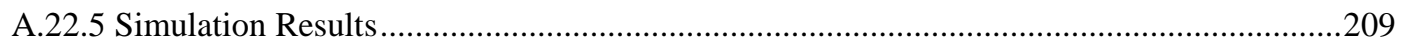

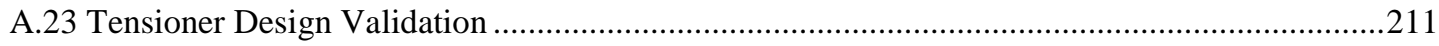

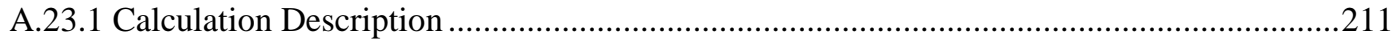

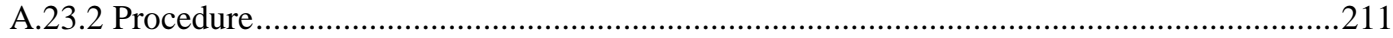

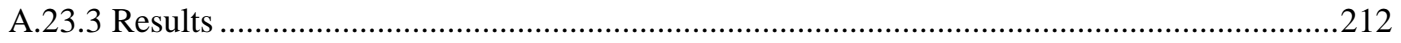

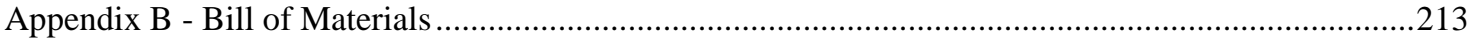

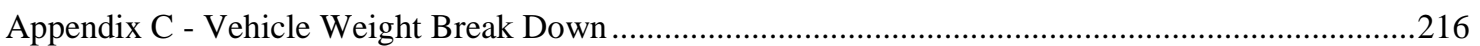

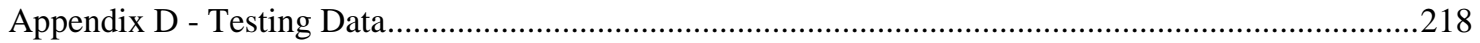




\section{LIST OF TABLES}

Table

Table 1: Pull-off force on different surface types.

Table 2: Observations made about the conceptual model during testing. ..............................................19

Table 3: The criteria used to design and test the climbing robot system. ...........................................22

Table 4: Maximum allowable electrical characteristics of the motors. Values taken from the

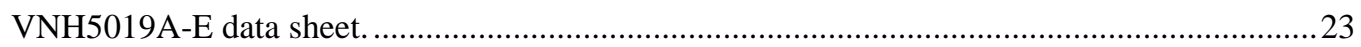

Table 5: Physical requirements used to select the drive motors for the climbing robot. ............................24

Table 6: Specifications for the two motors which best satisfied the projects requirements. ......................24

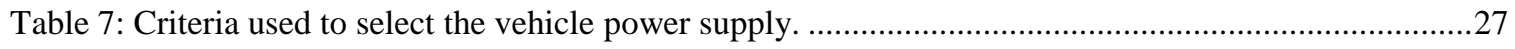

Table 8: Parameters used to select the venturi models used in the robot. ................................................29

Table 9: Evacuation rate, evacuation time for the estimated volume of a single vacuum section and the cost of each of the different venturi models in Vaccon's miniature venturi line.

Table 10: Minimum tread tension for the robots treads to function properly and the factor of safety estimated for the tread.

Table 11: Expected required vacuum forces for both vertical operation and operation upside-down and the tread perforation area which is required to achieve the greater of the two force values. .......33

Table 12: Maximum friction force allowable between the tread and the manifold and the resulting maximum allowable coefficient of static friction between the tread and the manifold.

Table 13: Hole pattern dimensions of the final tread design and the projected area of the wall exposed to the treads.

Table 14: Predicted and actual pitch length of the final tread design, as well as the number of teeth on the final tread design.

Table 15: Decision matrix used to determine the frame material used in the project...... .40

Table 16: Tubing selected to make up the vehicle frame. .40

Table 17: Final dimensions, weight and the simulation results for the frame. . .41

Table 18: Final dimensions and weight of the base plate.

Table 19: Final dimensions, weight and simulation results of the motor mounting bracket. .44

Table 20: Final dimensions, weight and simulation results for the motor plates. .45

Table 21: Parameters associated with the selected coupler. .46

Table 22: Information on the fittings used in the inlet manifold. .......................................................47

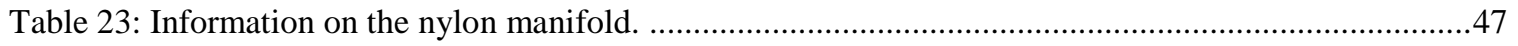

Table 24: Final dimensions and weight of the venturi mounting bracket..............................................48

Table 25: Major dimensions and weight of the lower manifold. .......................................................51

Table 26: Dimensions and weight of the final design of the upper manifold.......................................53

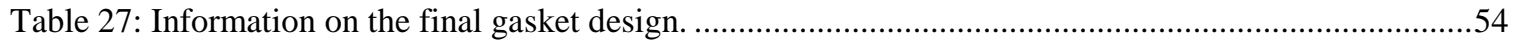

Table 28: Information on the final design of the mounting struts. .....................................................55

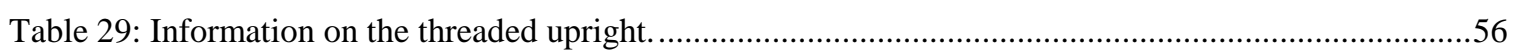

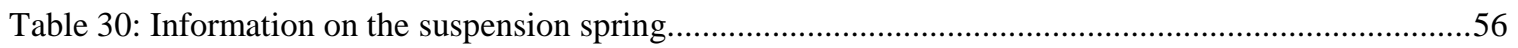


Table 31: Information on the selected flange bearing and the retaining compound used.

Table 32: Information on the chosen thrust washer.

Table 33: Dimensions of the common shaft end and the results of the retaining ring calculations. .61

Table 34: Final dimension, weight and simulation results for the drive shaft. ......................................62

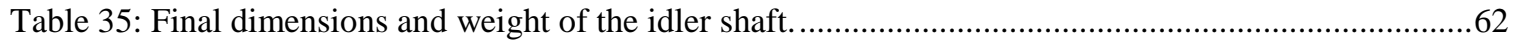

Table 36: Final dimensions, weight and simulation results for the drive wheel.....................................65

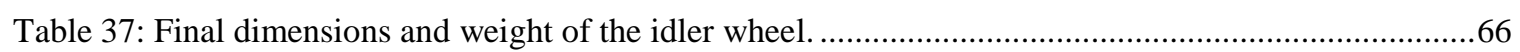

Table 38: Final dimensions, weight and simulation results for the brace.............................................67

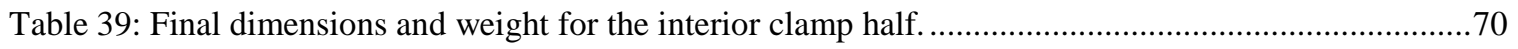

Table 40: Final dimensions, weight and simulation results for the exterior clamp half. ..........................71

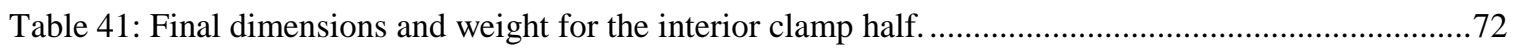

Table 42: Final dimensions, weight and simulation results for the interior clamp half.............................73

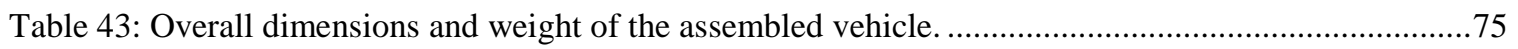

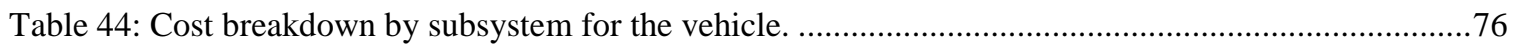

Table 45: Average measured ventuir vacuum pressure versus predicted vacuum pressure. ....................103

Table 46: Right side venturi section independence results...................................................... 104

Table 47: Left side venturi section independence results. ............................................................. 105

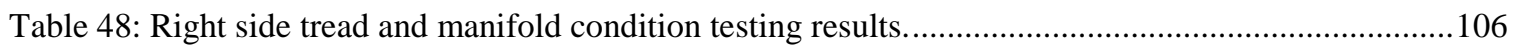

Table 49: Left side tread and manifold condition testing results.................................................... 106

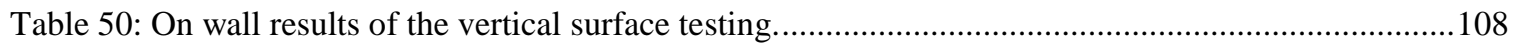

Table 51: Off wall results of the vertical surface testing.............................................................109

Table 52: Cumulative results of the clogged versus unclogged testing for the right manifold. ................110

Table 53: Cumulative results of the clogged versus unclogged testing for the left manifold....................110

Table 54: Performance of the split right manifold versus the whole right manifold for the section independence test.

Table 55: Performance of the split right manifold versus the whole right manifold for the vehicle tread

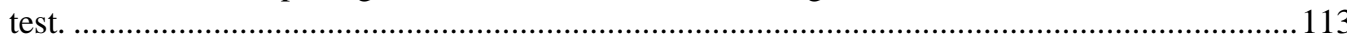

Table 56: Performance of the split right manifold versus the whole right manifold for the fixed manifold test.

Table 57: Performance of the split right manifold versus the whole right manifold for the fixed manifold test.

Table 58: Performance of the split right manifold versus the whole right manifold for the vertical

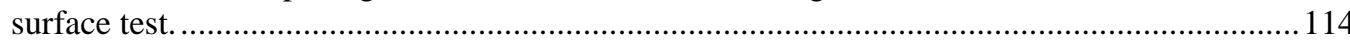

Table 59: Results of the applied force test for the right manifold. Applied load was 14 lbf. ...................114

Table 60: Results of the applied force test for the left manifold. Applied load was 14 lbf. .....................114

Table 61: Holes engaged during the pull force testing. ..............................................................118

Table 62: Average pull off force and manifold pressure for the horizontal and vertical tests..................118

Table 63: Predicted versus actual pull off force for the horizontal test with the mass included in the predicted value. 
Table 64: Predicted versus actual pull off force for the vertical test.

Table 65: Pressure in the right and left manifold sections at different pull off force values.

Table 66: Predicted versus actual pull off force for the horizontal test without the mass included in the predicted value.

Table 67: Current values measured during different test cases and the corresponding torque values.........124

Table 68: Calculated values for different motor torque contributors. ................................................125

Table 69: Calculated versus measured values of the motor torque contribution of different elements of the design.

Table 70: Angular and parallel misalignment between wheels on the right side of the vehicle................128

Table 71: Angular and parallel misalignment between wheels on the left side of the vehicle. .................128

Table 72: Angular misalignment between wheels on the right side of the vehicle a flat surface..............128

Table 73: Angular misalignment between wheels on the left side of the vehicle a flat surface. ................129

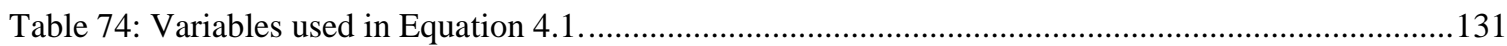

Table 75: Predicted tension in the left tread and the values used to calculate it..................................132

Table 76: Predicted tension in the right tread and the values used to calculate it. .................................132

Table 77: A comparison between the measured and desired tread tension values. ...............................132

Table 78: Variable symbols, units and descriptions for the symbolic motor torque calculator. .................146

Table 79: Final input variables used in the motor torque calculator...................................................148

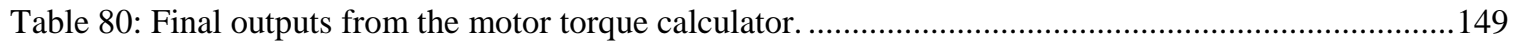

Table 81: Variable symbols, units and descriptions for the motor RPM requirement calculation. ............150

Table 82: Results of the motor RPM requirements calculation for different motor and wheel

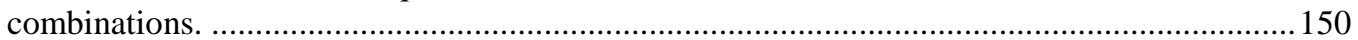

Table 83: Variable symbols, units and descriptions for the evacuation rate calculation. ..........................151

Table 84: The evacuation rate and time to evacuate for each of the venturi models considered for the project.

Table 85: Variable symbols, units and descriptions for the minimum belt tension requirement

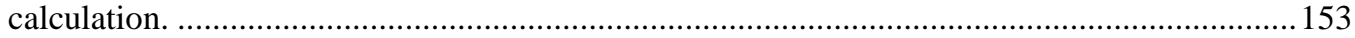

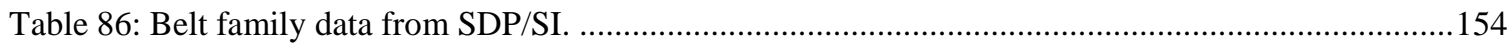

Table 87: The estimated mass factor per belt width and estimated mass factor for the H-Type belt used for the tread.

Table 88: The input variables used to estimate the minimum belt tension required and the results of the calculation.

Table 89: Variables and their values used to calculate the maximum allowable tread tension. ................157

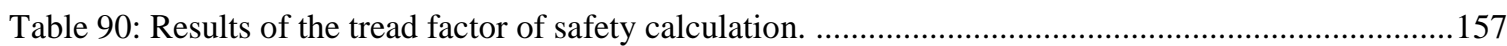

Table 91: Variable symbols, units and descriptions for the vacuum force requirements calculation.

Table 92: Input values for the vacuum force requirements calculation and the results. ..........................159

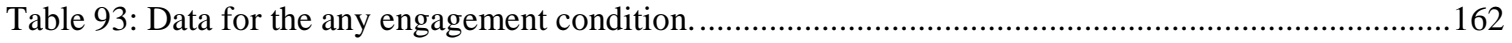

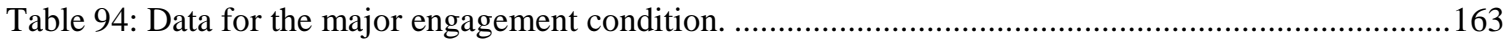

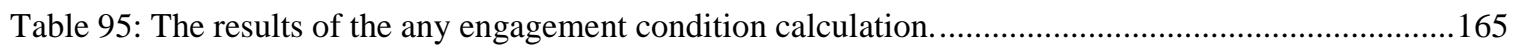


Table 96: The results of the major engagement condition calculation.

Table 97: Variable symbols, units and description for the skid steering calculation.

Table 98: The input variables for the skid steering calculation and the results. .168

Table 99: The variable symbols, units and description for the allowable tread/manifold friction calculation.

Table 100: The results of the allowable tread/manifold friction calculation.

Table 101: Loading conditions of the Frame.

Table 102: Results of the motor bracket simulation. 172

Table 103: Results of the motor bracket simulation. 177

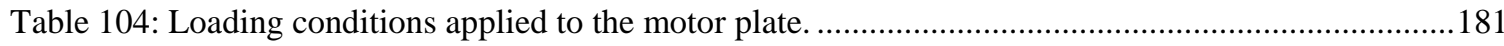

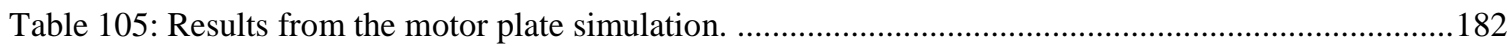

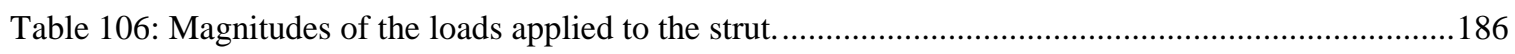

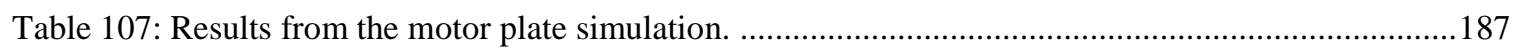

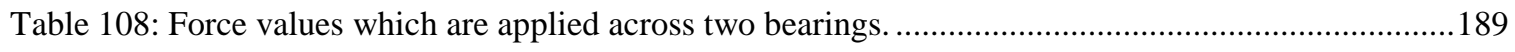

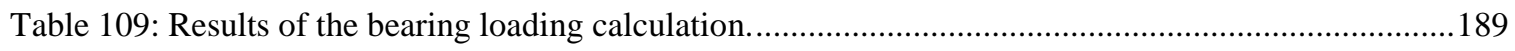

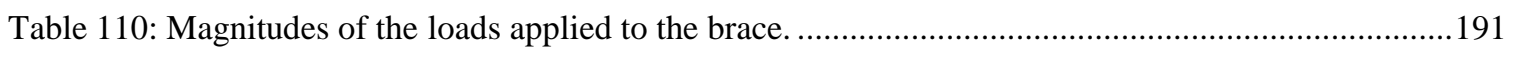

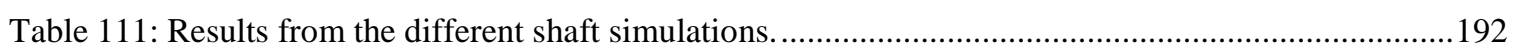

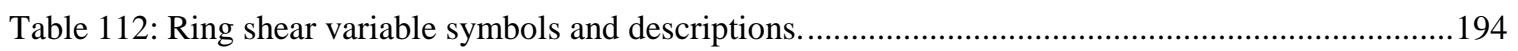

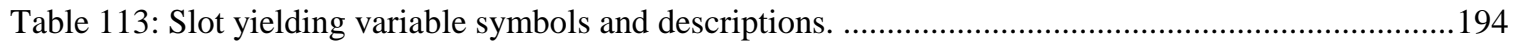

Table 114: Input variable values for the drive and idler shaft retaining rings and the results of the calculation.

Table 115: Input variable values for the tensioner shaft retaining rings and the results of the calculation..195

Table 116: The input variable symbols, descriptions and values used to calculate the required press fit pressure.

Table 117: The output variable symbols, descriptions and values from the required press fit pressure calculation.

Table 118: Input variable symbols, descriptions and values used to calculate the required interference and the resulting interference.

Table 119: Magnitudes of the loads applied to the drive wheel.

Table 120: Results from the drive wheel simulation.

Table 121: Magnitudes of the loads applied to the brace. .............................................................202

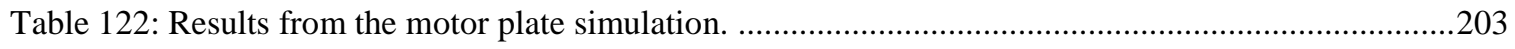

Table 123: Values used to calculate the buckling condition and factor of safety for the brace.................205

Table 124: Results from the tensioner shaft and exterior clamp half simulation....................................209

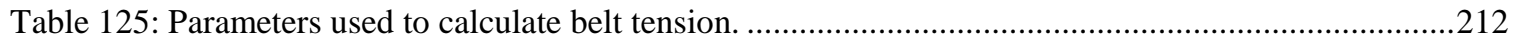

Table 126: Results of the tensioner design validation calculation...................................................212

Table 127: Frame raw material supplier, quantity and cost breakdown. ............................................213

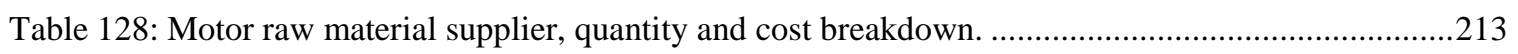

Table 129: Vacuum raw material supplier, quantity and cost breakdown........................................213 
Table 130: Manifold raw material supplier, quantity and cost breakdown. ........................................214

Table 131: Wheel raw material supplier, quantity and cost breakdown...........................................214

Table 132: Tensioner raw material supplier, quantity and cost breakdown. .........................................214

Table 133: Tread raw material supplier, quantity and cost breakdown. ..........................................214

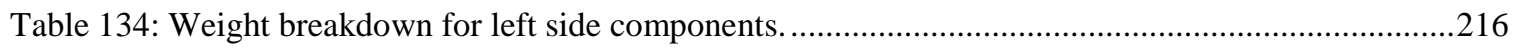

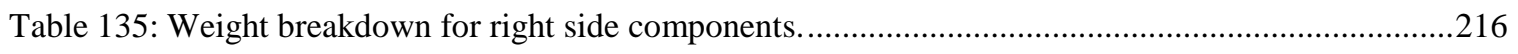

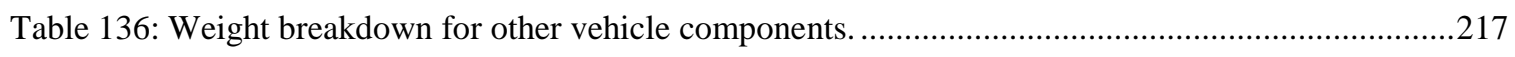

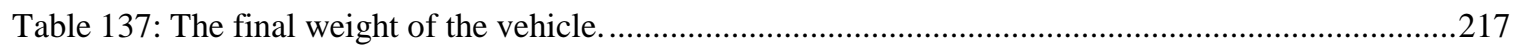

Table 138: Data for Fixed Right Manifold Unclogged Test..........................................................218

Table 139: Data for Unfixed Right Manifold Unclogged Test.......................................................218

Table 140: Data for the right manifold independence unclogged test. ...............................................219

Table 141: Data for the right manifold off vehicle unclogged test....................................................219

Table 142: Data for Fixed Right Manifold Clogged Test......................................................................220

Table 143: Data for Unfixed Right Manifold Clogged Test............................................................220

Table 144: Data for the right manifold independence clogged test. ................................................221

Table 145: Data for the right manifold off vehicle clogged test......................................................221

Table 146: Data for Fixed Left Manifold Unclogged Test...............................................................222

Table 147: Data for Unfixed Left Manifold Unclogged Test. ...............................................................223

Table 148: Data for the left manifold independence unclogged test. .................................................223

Table 149: Data for the left manifold off vehicle unclogged test. ..................................................224

Table 150: Data for Fixed Left Manifold clogged Test....................................................................2224

Table 151: Data for Unfixed Left Manifold clogged Test..................................................................225

Table 152: Data for the left manifold independence clogged test. ......................................................225

Table 153: Data for the left manifold off vehicle unclogged test. ....................................................226

Table 154: Vertical surface test and following table top test data test set 1 ......................................226

Table 155: Vertical surface test and following table top test data test set 2.....................................227

Table 156: Vertical surface test and following table top test data test set 3.......................................227

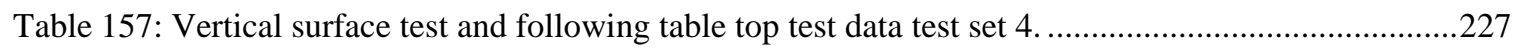

Table 158: Vertical surface test and following table top test data test set 5.....................................227

Table 159: Split Manifold Testing. All Pressure Value in inHg. .....................................................228

Table 160: The split manifold vertical surface test data with and without applied load. .........................228

Table 161: Fixed Split Manifold Influence Testing. All Pressure Values in inHg.................................2229

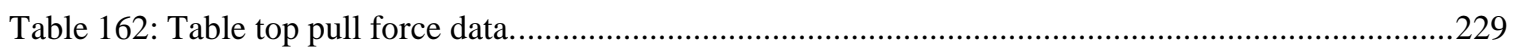

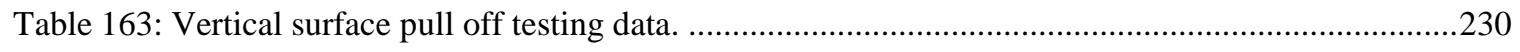

Table 164: Holes engaged with manifold sections during pull force testing.......................................230 


\section{LIST OF FIGURES}

Figure $\quad$ Page

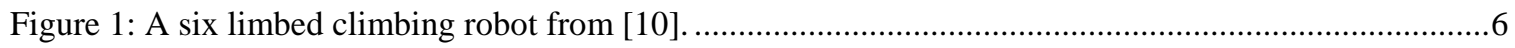

Figure 2: The Alicia II robot from [11] uses wheels to locomote. ..............................................................

Figure 3: An example of a treaded wall climbing robot from [8]...........................................................

Figure 4: An example of a slibing frame climbing robot [12] ...............................................................

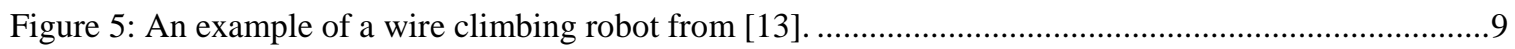

Figure 6: (Left) The Sadie robot, which combines a sliding frame with magnets [7]. (Right) The Tripillar robot which combines a tread system with magnets [14] ............................................ 10

Figure 7: An example of a climbing robot which uses suction cups [20]...............................................10

Figure 8: (Left) The ICM Climber which employs an active vacuum adhesion system [21]. (Right) The Alicia VTX robot which employs a vortex system [22] .........................................................11

Figure 9: (Left) The RiSE robot which uses small claws to adhere to surfaces [16]. (Right) The Treebot which uses gripper to climb small trees [17] .....................................................................12

Figure 10: A climbing robot which employs an electroadhesive tread [18] ...............................................13

Figure 11: A climbing robot which uses passive suction cups attached to a continuous tread [20].............14

Figure 12: The Tripillar robot, which uses a continuous tread with embedded magnets [14].....................15

Figure 13: A climbing robot which uses active suction cups attached to a continuous tread [8].................16

Figure 14: Sample of the images used to determine exposed tread hole area. .............................................17

Figure 15: Solidworks model of proof of concept model. .......................................................................

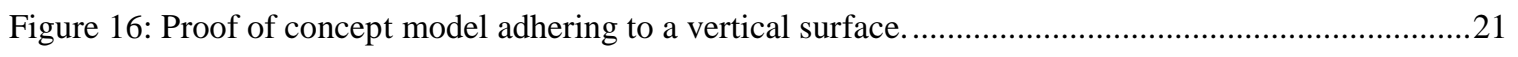

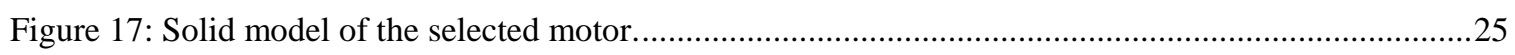

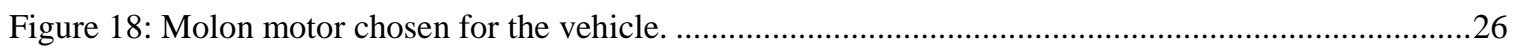

Figure 19: Agilent N5747A power supply used to power the vehicle .........................................................27

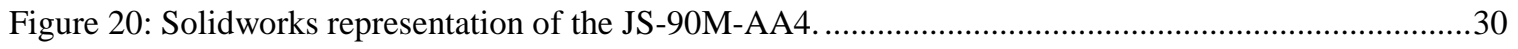

Figure 21: A table containing the dimensions of the different belt patterns taken from F.N. Sheppard's design catalog. The highlighted belt sections are those which were considered for the tread

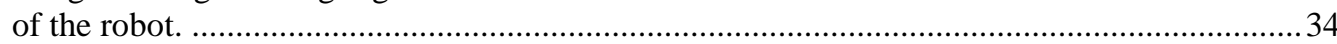

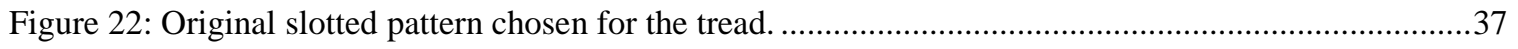

Figure 23: Solidworks model of the final tread hole pattern. ....................................................................

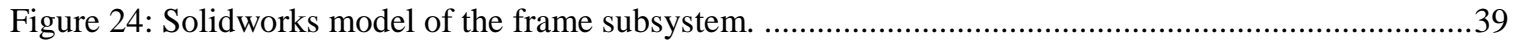

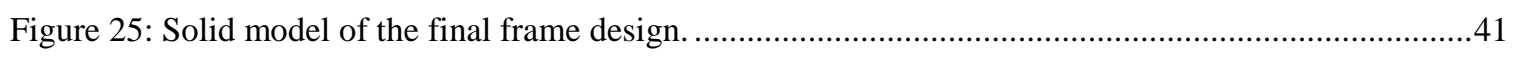

Figure 26: Solid model of the final base plate design. ............................................................................42

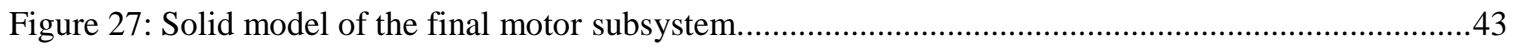

Figure 28: Solidworks model of the final motor mounting bracket design. ...............................................44

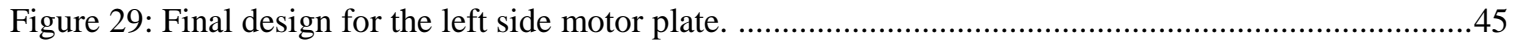

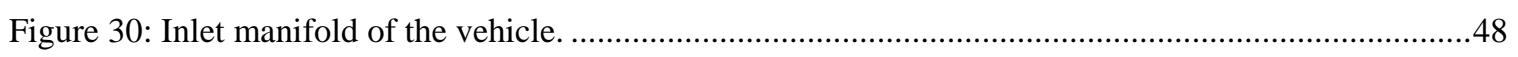

Figure 31: Solid model of the venturi mounting bracket........................................................................49 
Figure 32: Solid model of the vacuum manifold.

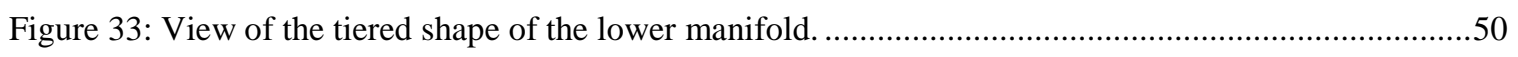

Figure 34: Solid model of the final lower manifold design. ................................................................52

Figure 35: Solid model of the final upper manifold design. ......................................................53

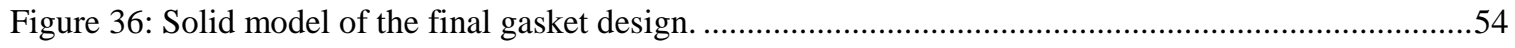

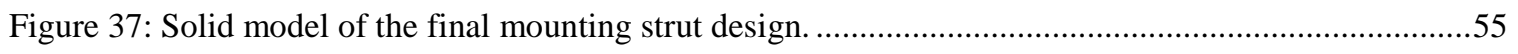

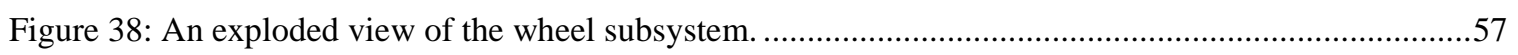

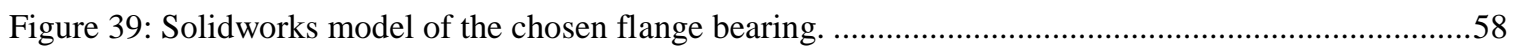

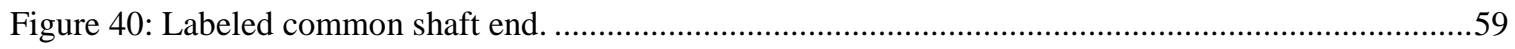

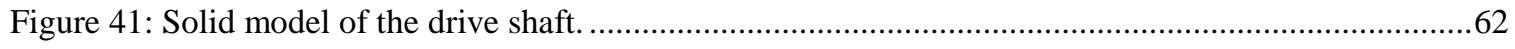

Figure 42: Solid model of the idler wheel shaft. ..............................................................................63

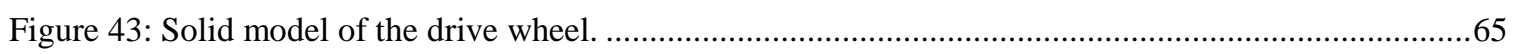

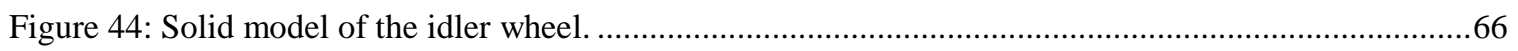

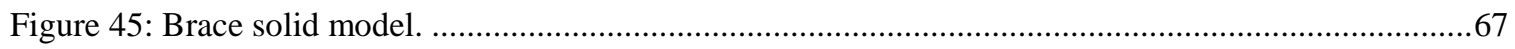

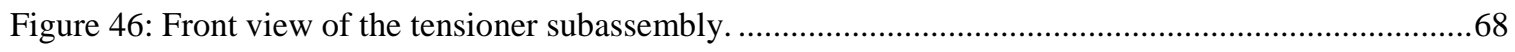

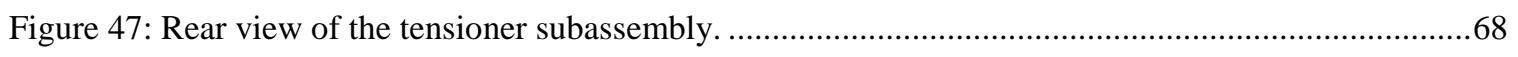

Figure 48: Solid model of the interior half of the tensioner clamp....................................................69

Figure 49: Solid model of the exterior half of the tensioner clamp. .....................................................

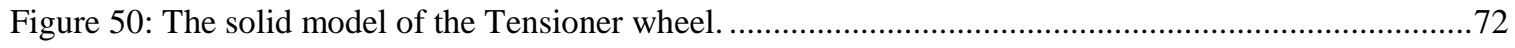

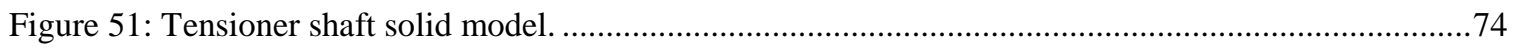

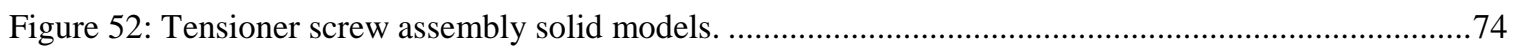

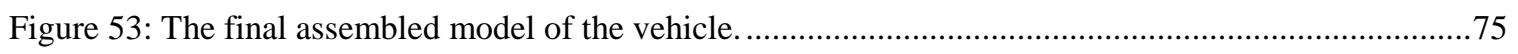

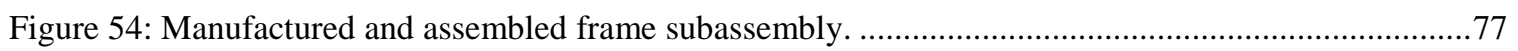

Figure 55: Misalignment of the frame at the right corner. The corner was raised by $1 / 32^{\text {nd }}$ of an inch.......78

Figure 56: Angular misalignment of the idler strut on the left side of the vehicle. ................................78

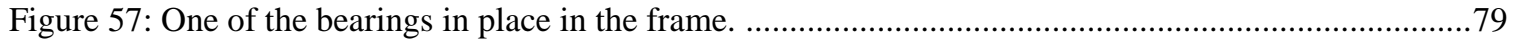

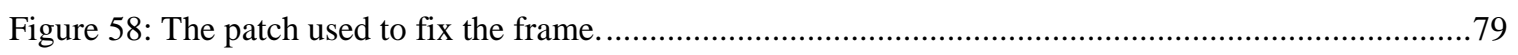

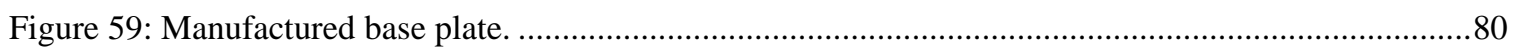

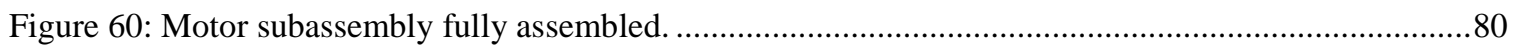

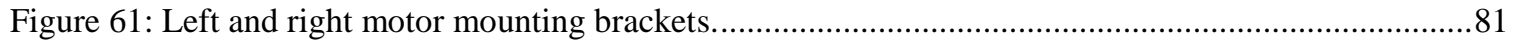

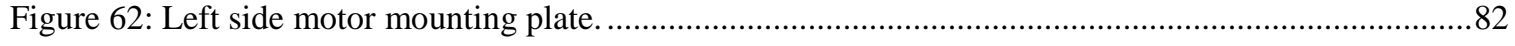

Figure 63: Right side motor mounting plate. Note the slots cut so the motor could be adjusted. ................82

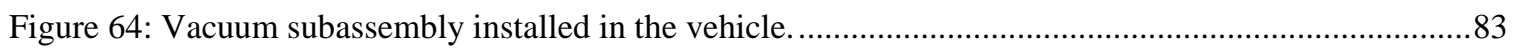

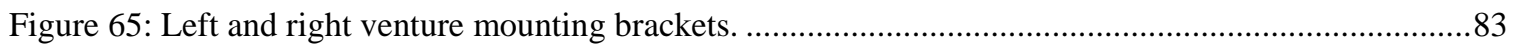

Figure 66: Venturi mounting bracket with the venturis installed. .................................................84

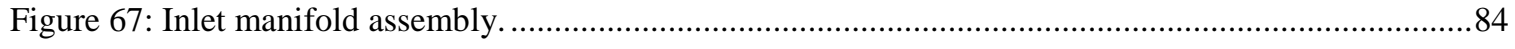

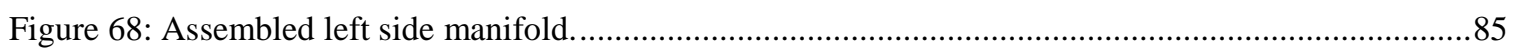




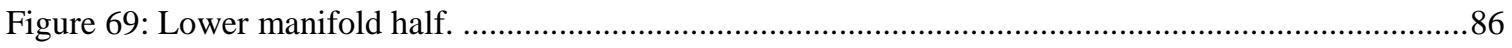

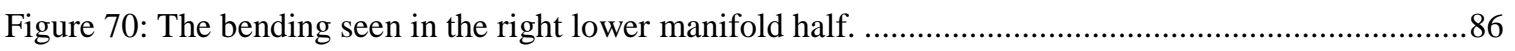

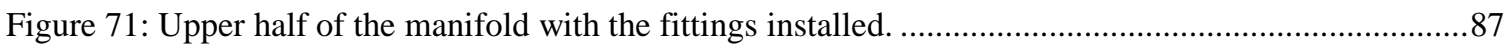

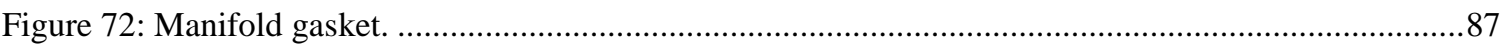

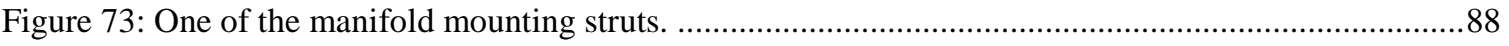

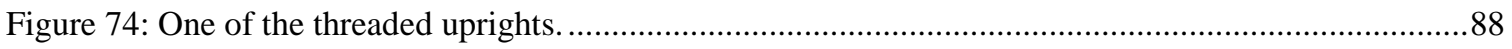

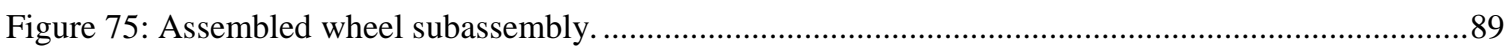

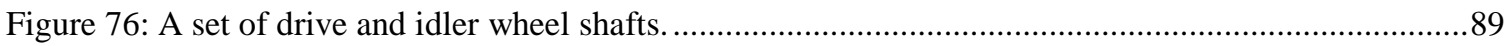

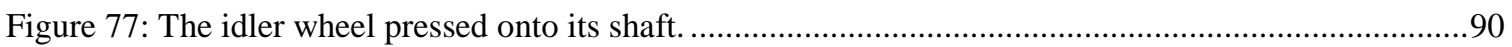

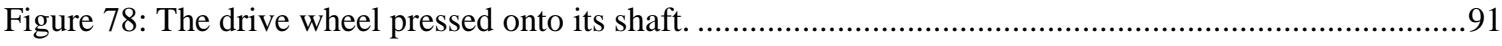

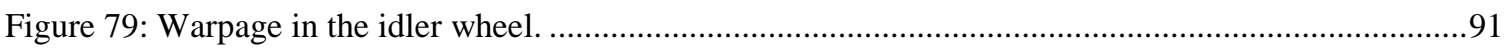

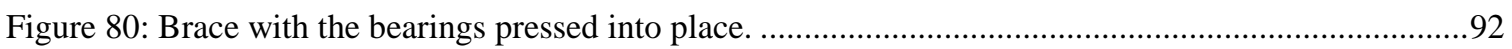

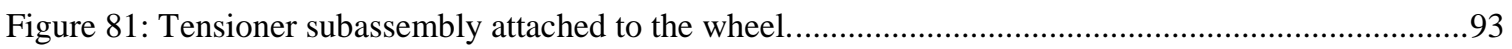

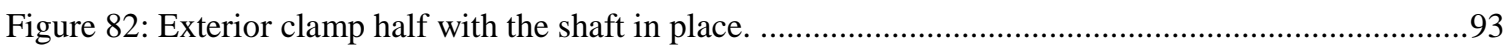

Figure 83: Spacer used to correct the misalignment of the left side tensioner wheel...............................94

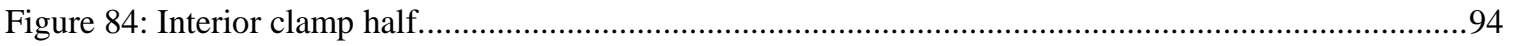

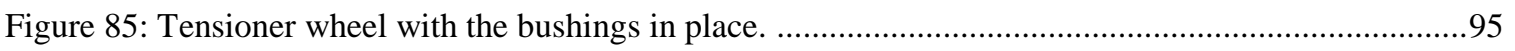

Figure 86: Tensioner shaft after it was welded to the exterior clamp half............................................96

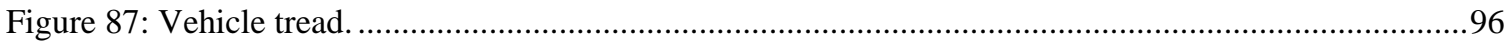

Figure 88: Low friction fabric before the application of the silicone sealant. .....................................97

Figure 89: Low friction fabric after the application of the silicone sealant.......................................97

Figure 90: Tread backing before sealant was applied. ..................................................................98

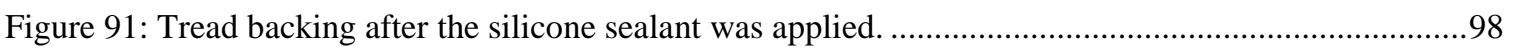

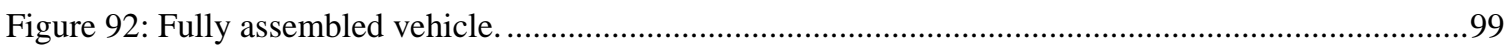

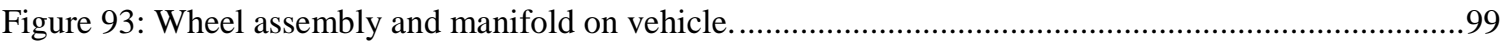

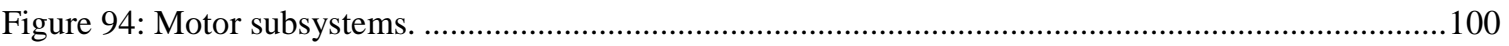

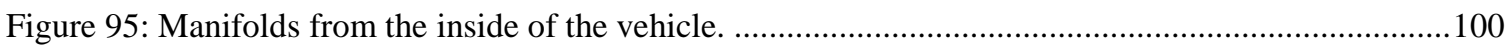

Figure 96: Vacuum system installed in the vehicle. ........................................................................ 101

Figure 97: Vacuum gauge used during the pressure system testing. ................................................. 102

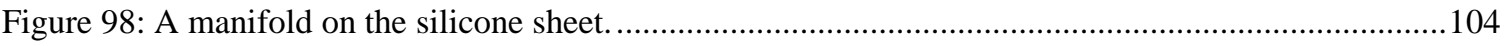

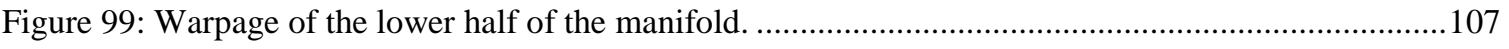

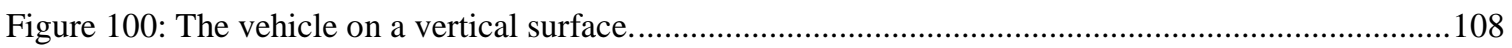

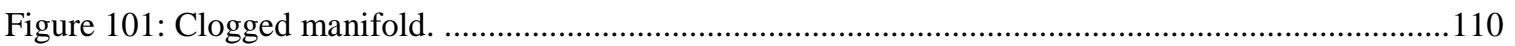

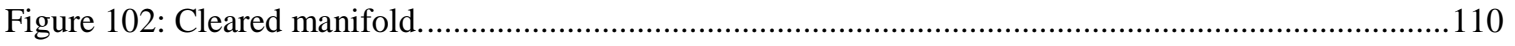

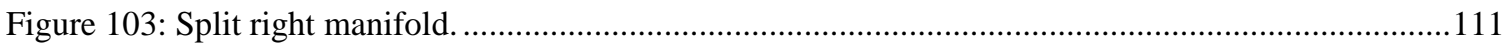

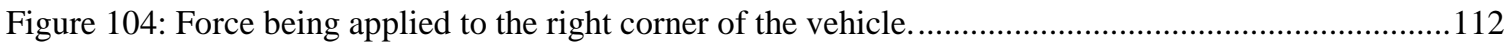


Figure 105: (Left) the right tread after it was pressed onto the wall. (Right) the right tread before it was pressed onto the wall.

Figure 106: A section of the manifold and the tread holes which it engages. ...........................................116

Figure 107: The zip tie suspension system used to perform the pull tests................................................116

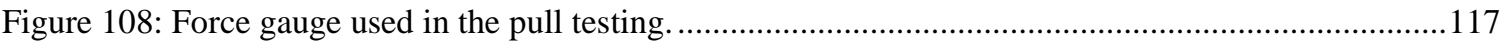

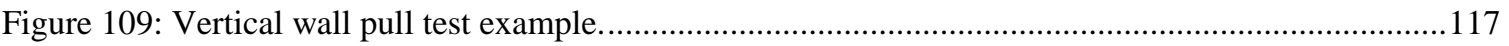

Figure 110: Right side manifold pressure as load was applied.............................................................120

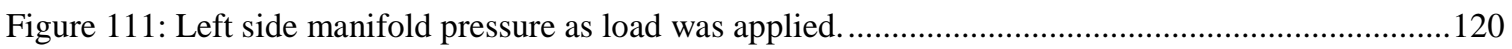

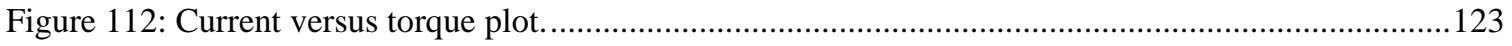

Figure 113: Torque versus current plot showing each test cases torque and current value. ........................124

Figure 114: Straight edge going between the drive and tensioner wheels on the right side of the vehicle. 126

Figure 115: Angular misalignment of the tensioner wheel on the right side of the vehicle. .......................127

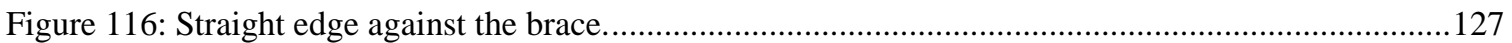

Figure 117: Angular misalignment between the brace and the tensioner wheel on the right side of the vehicle

Figure 118: The force gauge used to determine the tread tension. ......................................................130

Figure 119: The tension in the left tread being measured......................................................................131

Figure 120: A tread being adhered to the wall with a manifold. .................................................................133

Figure 121: The vehicle being adhered to a vertical surface. .................................................................134

Figure 122: Interior of a manifold after it had become clogged...........................................................136

Figure 123: Grease spikes which were squeezed into the tread holes during operation..............................136

Figure 124: Low friction fabric after the silicone sealant had been applied................................................137

Figure 125: Free body and mass acceleration diagrams for the body of the vehicle..................................145

Figure 126: Free body and mass acceleration diagrams for the drive wheel.............................................145

Figure 127: Free body and mass acceleration diagrams for the manifold. ................................................146

Figure 128: Free body and mass acceleration diagrams for the tread.....................................................146

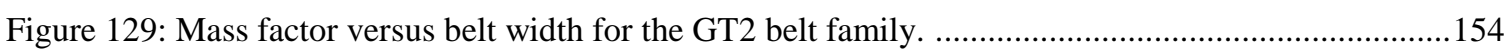

Figure 130: Mass Factor versus belt width for the trapezoidal tooth family. ...............................................155

Figure 131: Mass factor per belt width versus pitch length for the GT2 belt family.................................155

Figure 132: Mass factor per belt width versus pitch length for the trapezoidal tooth belt family...............156

Figure 133: A top view of the model used to determine the tread hole engagement..................................160

Figure 134: Tread holes engaged with the manifold sections with the manifold at zero displacement.......161

Figure 135: Tread holes engaged with the manifold sections with the manifold at a tenth of an inch of displacement.

Figure 136: (Left) A hole which would be disregarded in the major engagement condition. (Right) A hole which would be considered engaged for the major engagement condition.......................162

Figure 137: A plot of the total engaged holes versus the manifold displacement for the any engagement condition. 
Figure 138: A plot of the total engaged holes versus the manifold displacement for the major engagement condition.

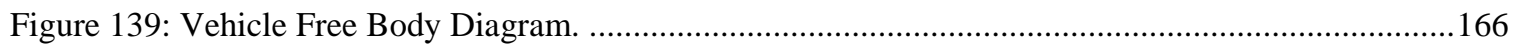

Figure 140: Wheel System Free Body Diagram.....................................................................................167

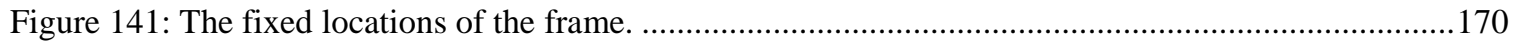

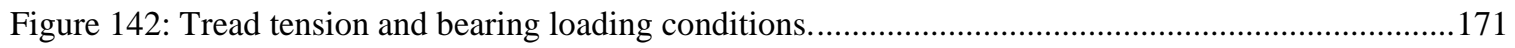

Figure 143: Torque produced by the tensioner assembly on the frame................................................171

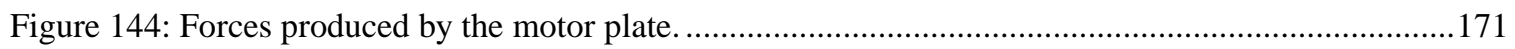

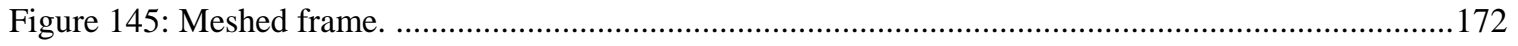

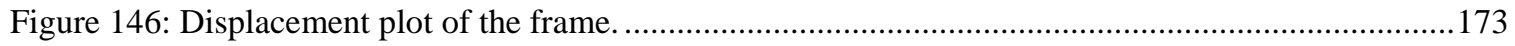

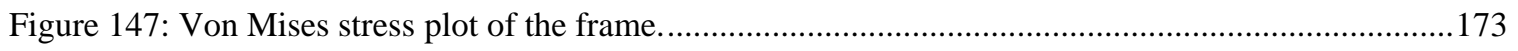

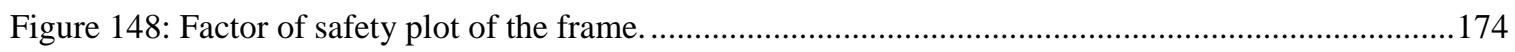

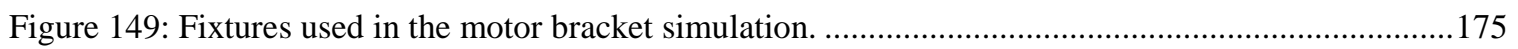

Figure 150: The loading on the motor bracket due to the mass of the motor. .............................................176

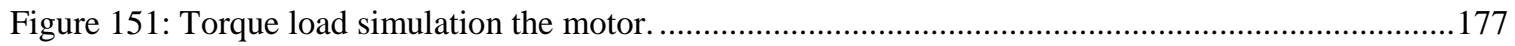

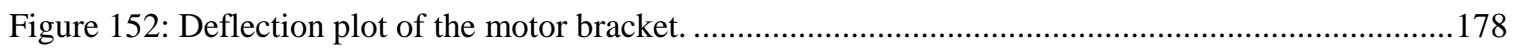

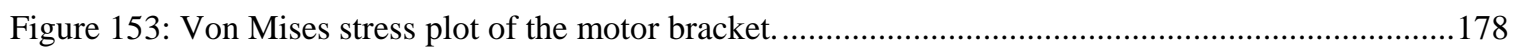

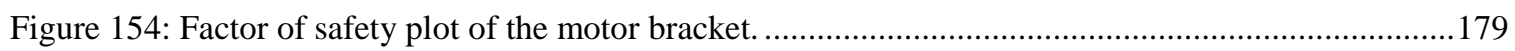

Figure 155: Reactions at the fixed holes of the motor bracket. ………..................................................179

Figure 156: Fixtures used in the motor plate simulation, Highlighted in blue. ……..................................180

Figure 157: An image of the rear hole showing the loading conditions..............................................181

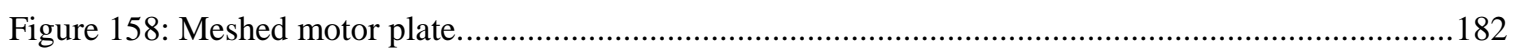

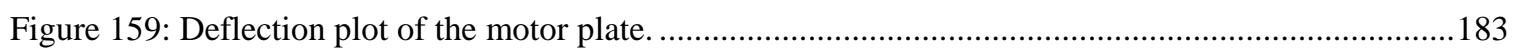

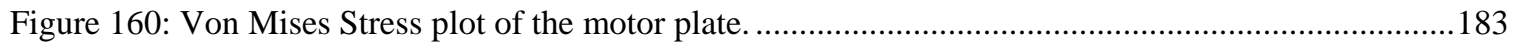

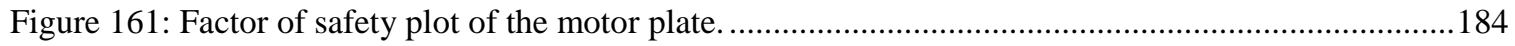

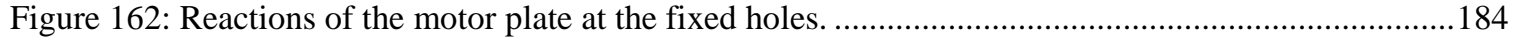

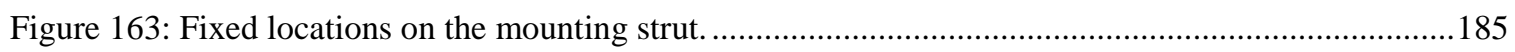

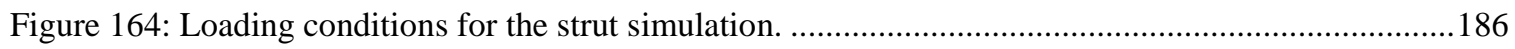

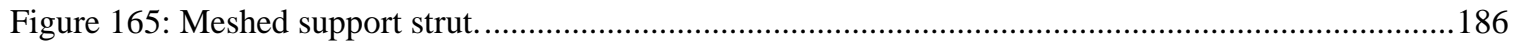

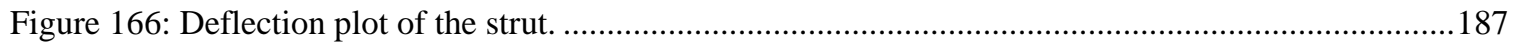

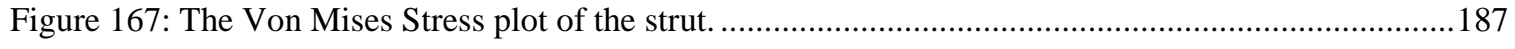

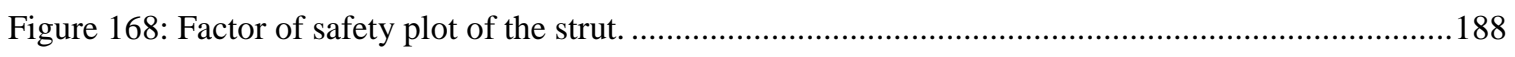

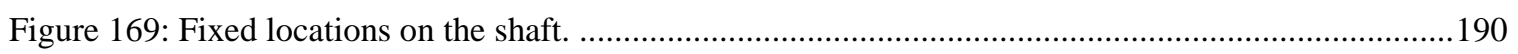

Figure 170: Locations of the loads applied to the brace..........................................................................191

Figure 171: Meshed drive shaft...................................................................................................191

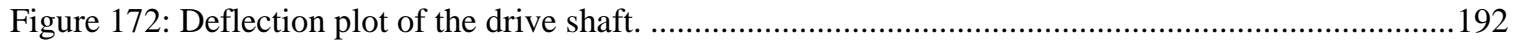

Figure 173: The Von Mises Stress plot of the drive shaft. .....................................................................193 


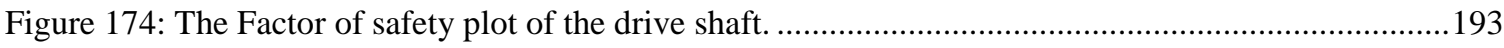

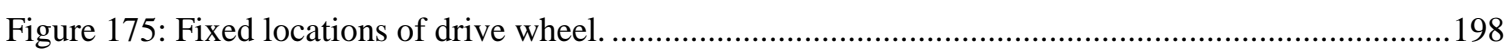

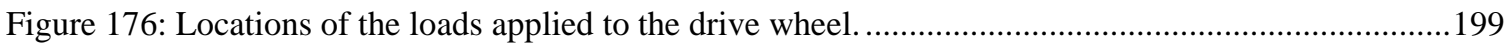

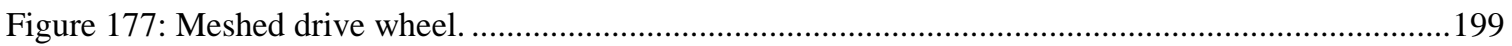

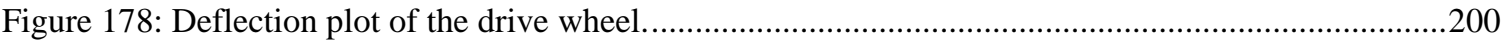

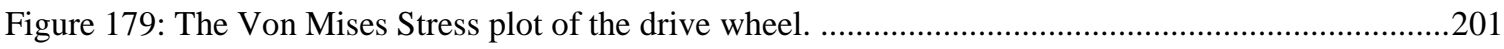

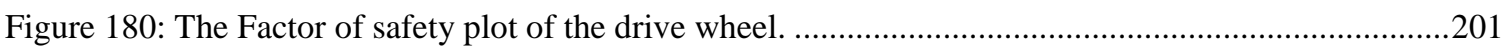

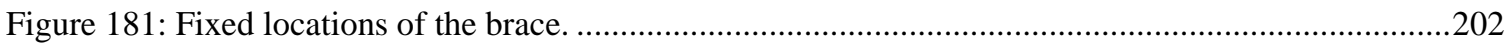

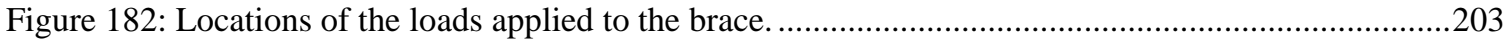

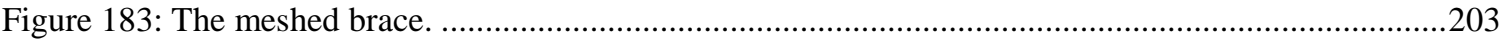

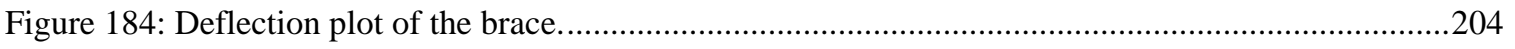

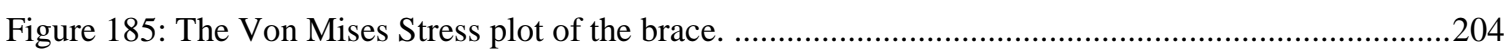

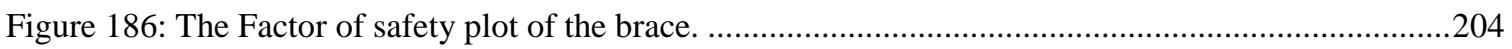

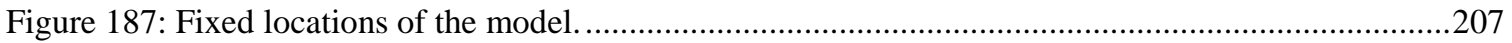

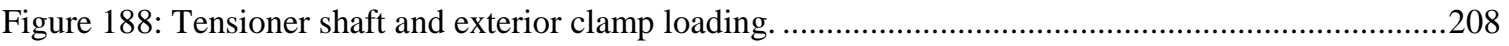

Figure 189: Meshed tensioner shaft and exterior clamp half..............................................................208

Figure 190: Deflection plot of the tensioner shaft and exterior clamp half. ..............................................209

Figure 191: The Von Mises Stress plot of the tensioner shaft and exterior clamp half...............................209

Figure 192: The Factor of safety plot of the tensioner shaft and exterior clamp half................................210

Figure 193: The slack condition of the belt. Belt length 33.83 in. .........................................................211

Figure 194: The assumed maximum tensioner height. Belt length 34.01 in. ...........................................212 


\section{Chapter 1: Introductory Material}

\subsection{Problem Statement}

Climbing robots represent an important development for many different technical tasks which occur on vertical surfaces, ceilings or in areas which would be hazardous or inaccessible to people. These applications include, among others, inspection of structures, maintenance, construction and data acquisition. Climbing robots can be useful in several different ways. They can be more cost effective, accurate and efficient than human technicians because they can be run continuously and autonomously. They can access areas which would be difficult or impossible for a human technician, such as the interiors of ship hulls. They can also remove the need to have human technicians placed in dangerous or hazardous environments, such as nuclear reactors or chemical storage tanks. This makes them an attractive tool for many different industrial and scientific operations.

This is not to say that climbing robots do not have their draw backs. Many of the designs which have been developed can only be applied to a single application or subset of applications. The environments they can operate in are often limited by their adhesion and locomotion methods, as well as by their overall dimensions and load-bearing capacity. This ultimately means that, for any given application, a climbing robot must be specially designed, which reduces its cost effectiveness.

The purpose of this project is to introduce a novel combined adhesion and locomotion system, i.e. a tank tread which acts as a gasket for an active vacuum system, which has the potential to be applied to many different operating environments. The system also has the potential to be scaled easily, which means that it could be used for robots of many sizes and with a variety of carrying capacities.

\subsection{Project Overview}

As stated above, the purpose of this project is to produce and demonstrate a novel combined adhesion and locomotion system for climbing robots. This includes the design, manufacture and testing of the proposed system. 
The proposed system uses two treads, similar to a tank, to locomote. Perforations are cut through the tread to create chambers through which a vacuum can be drawn, adhering the robot to a surface. These chambers are located along a central channel in the tread, surrounded by timing belt teeth to allow power engagement. The tread system is composed of three distinct layers, a timing belt made of stiff neoprene which interfaces with the drive and idler wheels, a soft neoprene layer used to create a seal between the vacuum chambers and the tread, and a layer of low friction fabric which covers the central channel.

A vacuum manifold with four separate vacuum chambers rides in the central channel of the tread and distributes vacuum to the holes cut into the tread. Each vacuum chamber is connected to a venturi vacuum generator, which produces the vacuum that adheres the robot to a climbing surface. A total of eight venturis are used in the system and all of the venturis are fed by a common positive air pressure source.

Three different wheels are used in the tread system. A drive wheel is used to transfer power from a gear motor to the tread. An idler wheel at the back of the robot is used to facilitate the smooth motion of the tread as it moves and to provide support to the tread system. A tensioning idler is used to tension the belt to ensure that no slipping occurs during operation and to allow the belt to be easily inserted and removed from the robot.

A full description of the design process used to create the robot, how the robot was manufactured and the testing procedures used to validate the robot's design can be found later in the report.

\subsection{Background and Survey of Existing Systems}

\subsubsection{Common Applications for Climbing Robots}

There are a wide variety of applications which currently use climbing robot, ranging from relatively mundane to extremely critical. Many of the industrial climbing robots are designed to perform cleaning tasks on vertical surfaces or in hazardous environments. The NERO II, for instance, is used to clean the interiors of nuclear reservoirs [1], while other robots, such as the SURF, are used to clean the exteriors of high rises without the use of scaffolding [4]. Still others are used to inspect and clean ships, such as the OCTOPUS, and airplanes [6]. 
Because climbing robots equipped with sensors produce repeatable and unbiased results with a high degree of accuracy, they are often used in inspection applications. Climbing robots are used to inspect bridges for corrosion and condition, both in concrete [5] and steel [7] bridges. They are also used to inspect oil tanks and pipes in chemical systems [6,7], which tend to be both difficult to access and are hazardous to humans. One of the most critical and potentially hazardous inspection task climbing robots perform is in nuclear storage areas $[1,6]$.

Climbing robots can also be used to perform tasks which are not necessarily obvious. Welding is one such application which climbing robots are relatively well suited for because they can move at a constant rate and lay consistent welds over large seams, which can be difficult for human welders. For instance, engineers at the University of Canterbury have developed the TigBot, a robot which performs TIG welding on stainless steel structures [9]. Another interesting application is the use of climbing robots in search and rescue operation conducted by fire departments [6]. These robots are often used to scout potentially hazardous or difficult to reach areas, which allows fire fighters to both determine if the area is safe to enter and to locate any trapped or incapacitated people. Similarly, climbing robots have been developed to aid in military reconnaissance, allow soldiers to scout buildings before entering them [3].

Clearly, there are a wide range of applications which climbing robots are being applied to. Additionally, as climbing robot design becomes more sophisticated it will become more feasible to use climbing robots in a wider range of applications.

\subsubsection{Major Design Principles of Climbing Robots in Non-Research Applications}

To be effective at their designated tasks, climbing robots must meet certain design requirements. These requirements can be broken down into five major categories which can be applied to almost every climbing robot in some way or another [7]. This section contains a brief description of each of these categories based upon the list put forth by [7].

\subsubsection{Velocity}

The robot must be able to achieve a speed at which it can complete its required task in a reasonable amount of time, while remaining accurate and safe. This requirement depends upon several factors, such as the 
dimensions of its work space, the maximum speed at which its chosen adhesion method can handle before the robot falls and the rate at which its work must be done. For instance, an inspection robot working on a bridge may be required to traverse relatively large distances between inspection areas, which means it need to move quickly between these points to remain efficient. Once it reaches an inspection point, it may need to move slowly for its inspection equipment to operate.

\subsubsection{Mobility}

The robot must be able to maneuver around its work environment efficiently and precisely, as well as be able to maneuver its tools or inspection equipment into the required location. This means that the robot must be able to position itself accurately on its work surface, maintain a desired trajectory, and move smoothly if its sensors or tools require smooth motion to operate. It must also be able to navigate over any obstacles it may encounter.

\subsubsection{Payload}

The robot must able to carry the tools and equipment which are required to complete its task. These can range from small and light weight systems, such as cameras, or larger and heavier systems, such as inspection equipment or tools. They must be able to accommodate these systems both in terms of carrying capacity, i.e. the weight they can hold while maintaining surface adhesion and locomotion, and of dimensional requirements, i.e. the space required to house the system.

\subsubsection{Safety and Reliability}

The robot system must be safe to use for both the human operator and bystanders, as wells as for the robot itself. It must also be robust enough to be usable, i.e. it must be able complete its desired task without frequent break downs and maintenance. They should have a high factor of safety for their given adhesion method and should also be capable of detecting and recovering from potentially hazardous conditions.

\subsubsection{Usability}

This is something of a catch all category dealing with the general usability of the robot, which can include everything from the weight of the system to the power requirements to how easy it is to operate. For the 
system to be practical it should be at least as effective of the currently employed methods used to complete the desired task. This effectiveness can be defined in multiple ways, such as how effective the system is, if it is more powerful than the current method and if it is less dangerous than the current method. The system should be adaptable to multiple applications, i.e. it should be able to handle different payloads. It should also be easy to repair, maintain and trouble shoot, both in the field and when being serviced. It should not be too bulky or unwieldy so that its operators can easily handle it. There are a myriad of other factors which fall into this category and they are often specific to the required task or task which the robot is required to perform.

\subsubsection{Existing Methods for Locomotion and Adhesion}

A myriad of different climbing robots have been designed and implemented, many of which employ similar adhesion and locomotion in different combinations. The most common types of these adhesion and locomotion systems are discussed below [7]. A more comprehensive survey can be found in [7].

\subsubsection{Locomotion methods}

This section gives a brief description of the different types of locomotion methods used in wall climbing robots. It also discusses some of the advantages and disadvantages of each method.

\subsection{Arms and Legs}

The use of arms or legs is relatively common in wall climbing robots. These systems use multiple, often multi-degree of freedom, limbs to move. Generally, each of these limbs has an adhesive component on its tip which holds the robot to the wall. Because these limbs can be moved independently of one another and the robot can test the adhesion of the limb, limbed robots are quite adept at traversing relatively rough terrain and obstacles. But, because of the complex nature of the limbs, it is often difficult to control limbed robots and to operate them smoothly. Limbs also tend to be relatively slow when compared to other locomotion methods. This is because, generally, only a single limb can be moved at a given time to ensure the robot maintains contact with the wall. An example of a limbed robot can be found in Figure 1. 


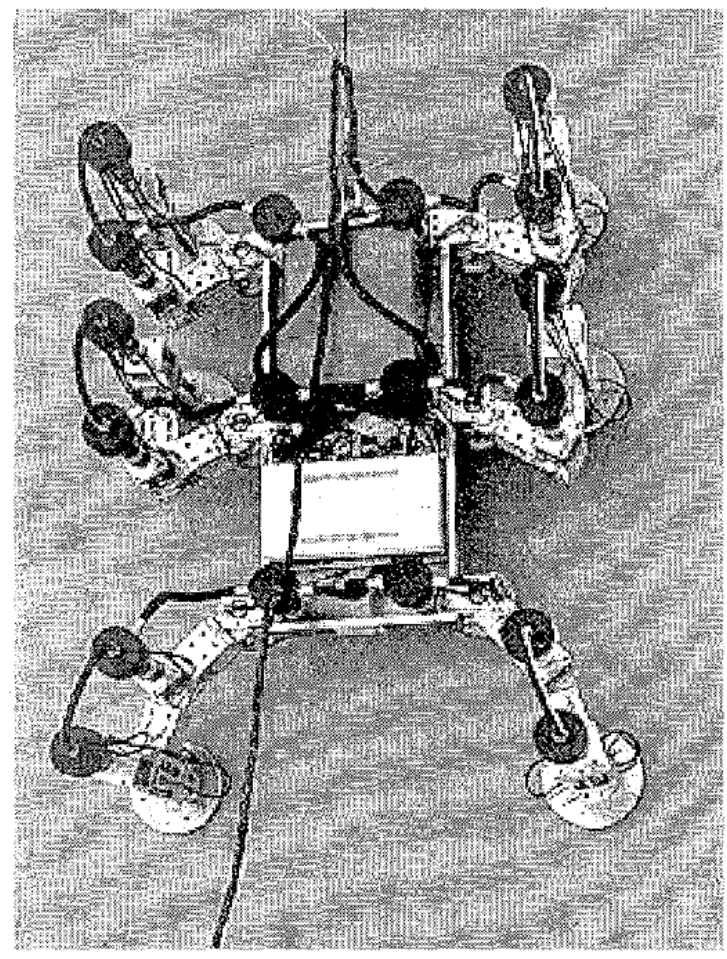

Figure 1: A six limbed climbing robot from [10].

\subsection{Wheels and Tread}

Wheels or treads are relatively common forms of locomotion for climbing robots, especially when the robot operates on a relatively smooth surface. Wheels and treads are relatively fast and provide smooth and continuous locomotion, which is desirable in many applications. They are also relatively simple to control and are also mechanically simple. The main disadvantage of wheeled or tracked robots is their inability to traverse steps and obstacles, which limits the application that they can be applied to. An example of a wheeled climbing robot can be found in Figure 2, while an example of a treaded climbing robot can be found in Figure 3. 


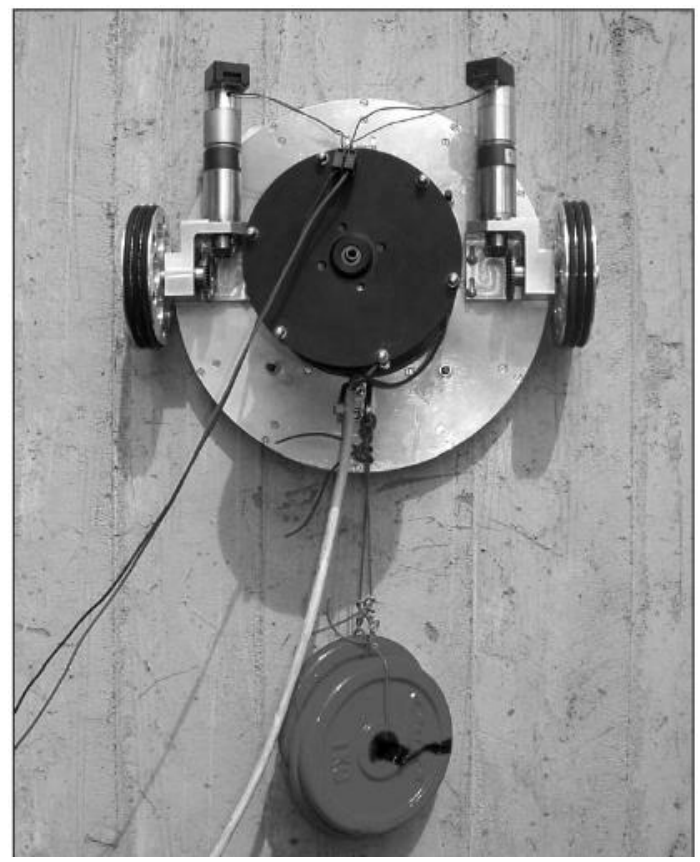

Figure 2: The Alicia II robot from [11] uses wheels to locomote.

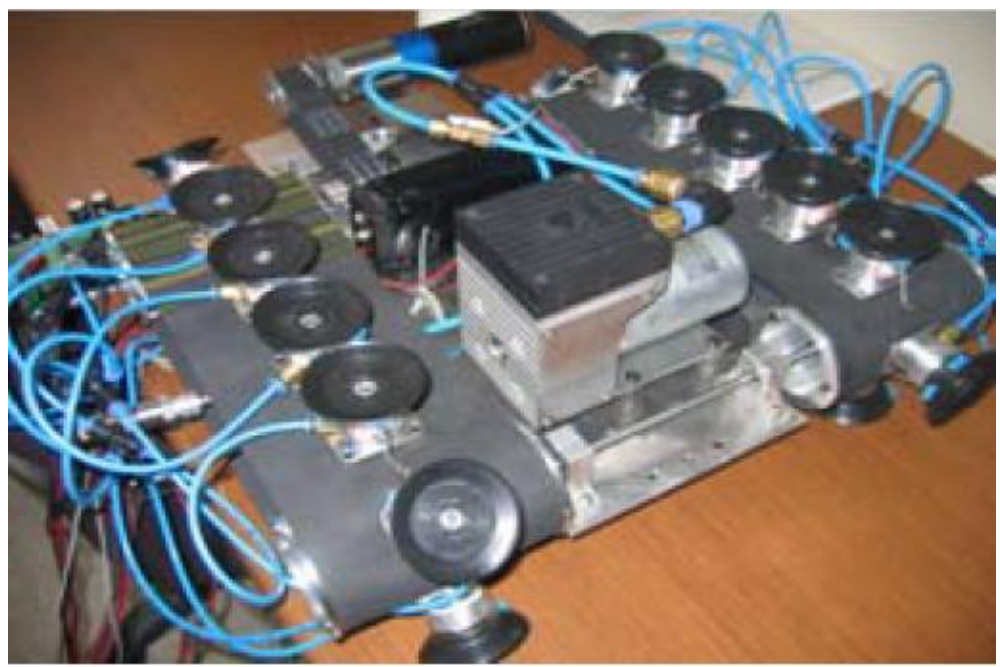

Figure 3: An example of a treaded wall climbing robot from [8].

\subsection{Sliding Frame}

A fairly common form of wall climbing robot locomotion is the sliding frame. These systems generally employ two frames which can translate or rotate relative to one another, with each frame containing its own adhesion system. To move, the robot fixes one frame to the surface and then moves the second frame forwards or backwards. The second frame attaches itself to the surface and then the process repeats with the first frame moving. Sliding frames tend to be reliable because of their ability to test their footing. They are 
also easy to control because of their relatively simple mechanical structure. However, like limbed robots, they tend to be slow and move in a discontinuous manner. They also tend to be bulky when compared to other designs. An example of a sliding frame climbing robot can be seen in Figure 4.

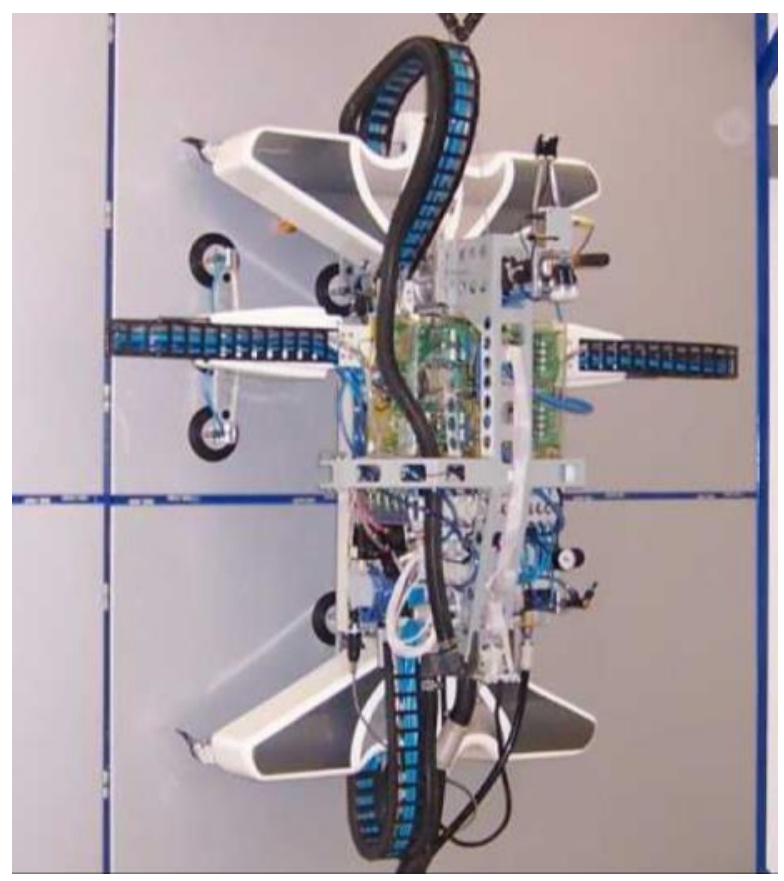

Figure 4: An example of a slibing frame climbing robot [12].

\subsection{Rails and Wires}

When the path of a climbing robot is well defined, wires or rails can be used as a method for locomotion. The robot travels along the guiding structure and uses its adhesion method only to fix itself in position on its work surface. Because the load is carried by the wire or rail, the adhesion method can be relatively weak and the robot itself can be quite heavy when compared to the other locomotion methods. Though the mechanisms used to move the robot along the rail or wire can be extremely simple and fast, the robot is restricted to use the given path, which means they must be highly specialized. An example of a wire driven climbing robot can be found in Figure 5. 


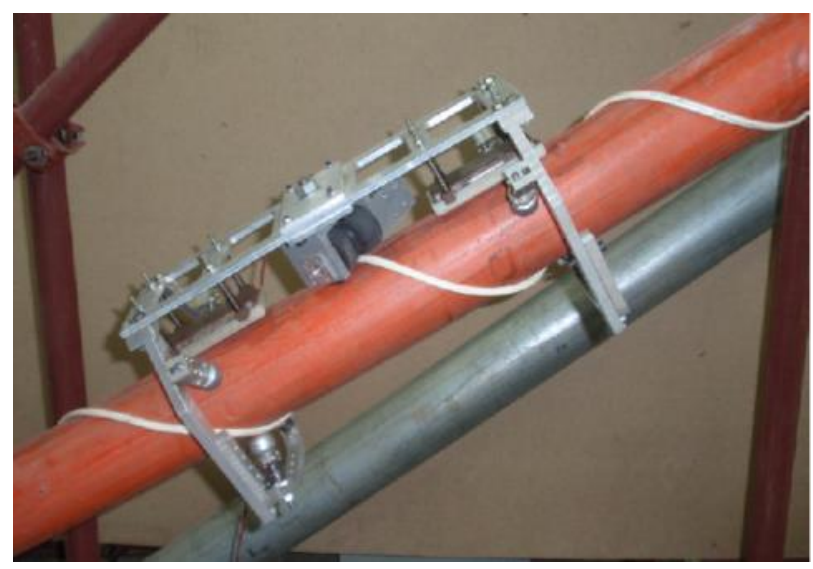

Figure 5: An example of a wire climbing robot from [13].

\subsubsection{Adhesion Methods}

This section contains a brief description of the most commonly used adhesion methods in climbing robots. It also discusses the advantages and disadvantages of each method.

\subsection{Magnetic}

Magnets, both permanent and electromagnets, are commonly used as an adhesion method when a climbing robot works on ferromagnetic surfaces. A significant distinction can be made between the behavior, in application, of permanent magnets and electromagnets. The force generated by electromagnets can be controlled relatively easily by varying the current passing through the coils, which means that they can be positioned near the working surface without having to worry about generating too much attractive force or friction. However, electromagnets require power to operate, which reduces the electrical efficiency of the system as a whole. Permanent magnets can generate large attractive forces with a relatively small footprint and require no power, but they must be carefully placed. This is to ensure that they do not generate excessive force, which can damage the robot or cause excess friction. Because magnets can only work on ferrous surfaces, climbing robot design which employ them are limited in the types of applications in which they can be employed. Two examples of climbing robots which employ the magnet adhesion method are show in Figure 6. 

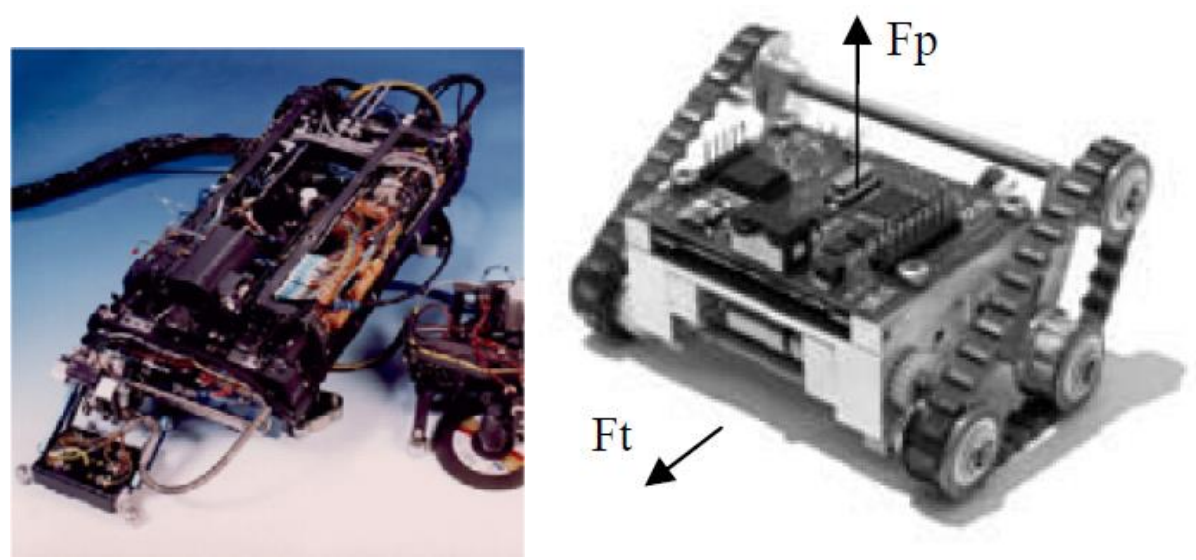

Figure 6: (Left) The Sadie robot, which combines a sliding frame with magnets [7]. (Right) The Tripillar robot which combines a tread system with magnets [14].

\subsection{Pneumatic}

Perhaps the most common adhesion methods used in climbing robots is the used of pneumatic adhesion. Pneumatic adhesion can be broken down into two major categories, active pneumatics and passive pneumatics. Suction cups are the most common, if not the only, form of passive pneumatics used in climbing robots. Suction cups have two major disadvantages associated with their use in climbing robots. The first is that they require a smooth surface to maintain negative pressure, which can be foiled by rough surfaces or particulates on a smooth surface. The second is that the cups must be reapplied to the surface relatively frequently because if the cups remain static for too long negative pressure is lost due to small leaks. This makes climbing robots which employ suction cups difficult or impossible to employ in most applications. An example of a robot which uses suction cups for adhesion can be found in Figure 7.

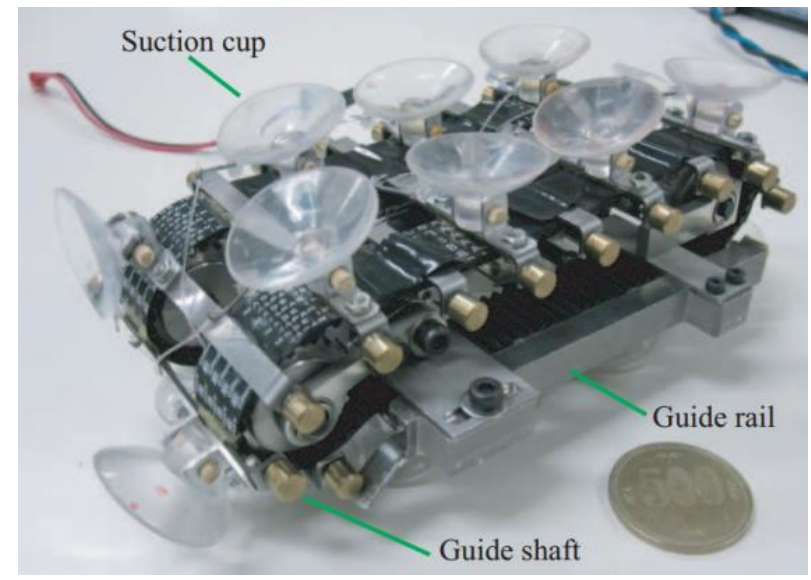

Figure 7: An example of a climbing robot which uses suction cups [20]. 
Active pneumatics can be broken down into two different major categories, true active vacuum adhesion and thrust or vortex adhesion. Active vacuum adhesion uses vacuum generators, generally pumps, to pull a vacuum in sealed, or semi-sealed, chambers to adhere the robot to work surface. Active vacuum adhesion is by far the most commonly employed of the pneumatic adhesion techniques, as it can be applied to a variety of surface types and the force generated can be controlled by the system using valves. The main disadvantage of active vacuum systems is the requirement of either on board or remote vacuum generators, which can add weight to the system or limit its operating range. Active vacuum systems also require a near perfect seal with the work surface so that the system does not lose suction, which limits the surfaces they can be used on as well as the size obstacles these robots can safely navigate. Vortex adhesion is an uncommon form of pneumatic adhesion which is still being researched. Robots which employ this method do so by creating a vortex with a high-speed fan. The main advantage of the vortex system is it does not require a seal to maintain adhesion like the other more common pneumatic methods. Thrust systems maintain adhesion by creating a thrust force, usually with fans, directed away from the wall, thereby pressing the climbing robot against it. Like vortex systems, thrust systems do not require a seal, but they tend to be unstable and difficult to control which makes them impractical. An example of a climbing robot which employs an active vacuum system and one which employs a vortex system can be found in Figure 8 .

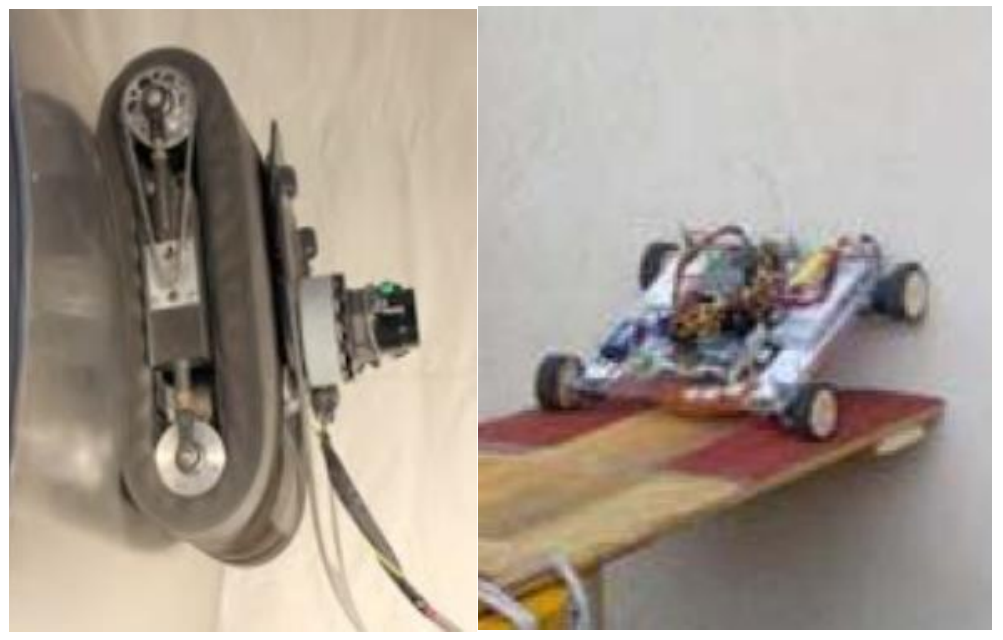

Figure 8: (Left) The ICM Climber which employs an active vacuum adhesion system [21]. (Right) The Alicia VTX robot which employs a vortex system [22]. 


\subsection{Mechanical}

Mechanical means of adhesion are quite diverse, though most can be broken down into two major categories, claws or grippers. Claws are used on surfaces which are rough enough for the claws to find purchase, while grippers are used to grasp portions of a structure. Once adhesion has been achieved in a mechanical system, there is often very little power required to maintain adhesion onto a surface. In fact, gripers can be designed in such a way that the system can lose power completely and still maintain adhesion. This makes mechanical adhesion methods relatively safe. However, mechanical adhesion tends to force the climbing robot to move slowly and carefully to ensure that adhesion has been achieved, while at the same time making it difficult to maneuver the robot. Figure 9 shows an example of the claw adhesion method and the gripper adhesion method. An example of gripper and claw adhesion systems can be seen in Figure 9.

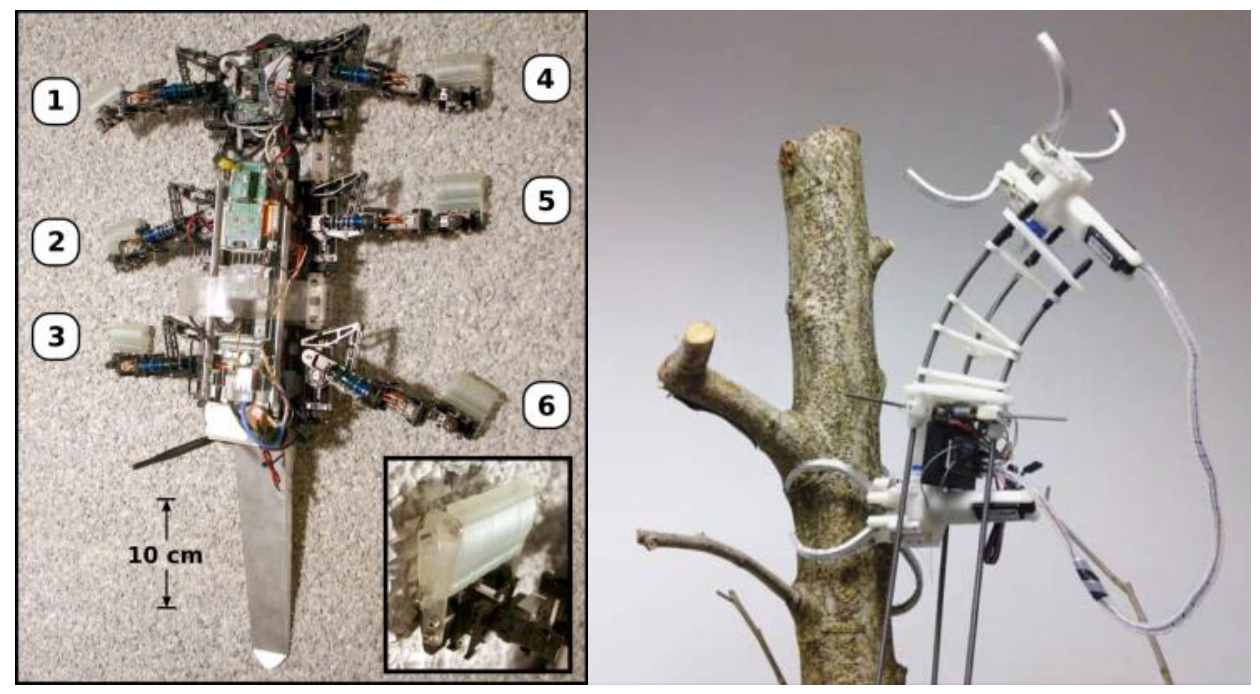

Figure 9: (Left) The RiSE robot which uses small claws to adhere to surfaces [16]. (Right) The Treebot which uses gripper to climb small trees [17].

\subsection{Electrostatic}

Although few robots employing this adhesion method exist, initial research shows that electrostatic adhesion offers a promising alternative to the more common adhesion methods used in industrial applications. In this adhesion method, pads comprised of electrodes and insulating substrate generate Van der Waals forces between the work surface and the robot. Because of the nature of the electrostatic force, a wide variety of surface, such as concrete, wood and glass, can be adhered to by robots employing this 
adhesion method. It has also been shown that a low power to force ratio can be achieved, which means that it is also a relatively energy efficient method for adhesion. Currently, payloads for robots employing electrostatic adhesion are relatively low, but as the concept is developed further, it could very easily begin to compete with the currently employed adhesion methods. An example of a climbing robot which employs electrostatic adhesion is shown in Figure 10.

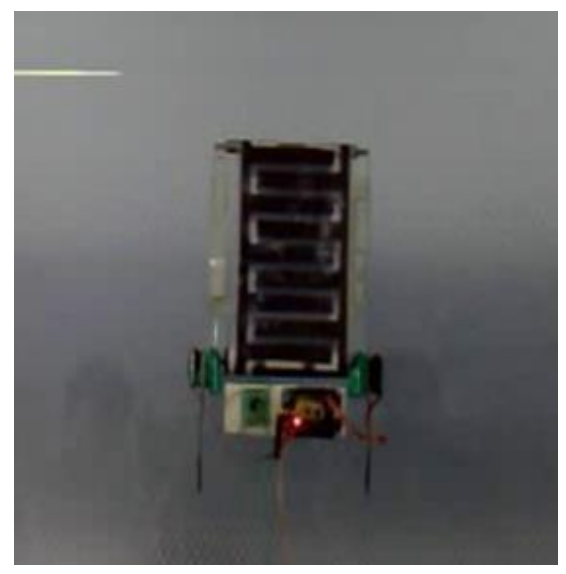

Figure 10: A climbing robot which employs an electroadhesive tread [18].

\subsection{Chemical}

Chemical adhesion is one of the least common adhesion methods and has, as of now, been confined to the laboratory. This category includes tapes, tacky elastomers and thermal glues. Each of these methods offers a low power adhesion solution, which is desirable. However, each method has its drawbacks. The glue and tape methods require frequent replacement, which can be cumbersome and difficult. The tack elastomer method requires relatively large forces to remove from the work surface once placed. Further research and development is required before any of these methods are capable of being applied in practical applications.

\subsubsection{Designs Similar to the Proposed System}

This section contains a brief description of climbing robot designs which are similar to the proposed system. It addresses why each design is similar to the proposed design and what differences exist between the proposed design and the existing design, focusing on the adhesion and locomotion method. It does not comment why one design might be superior to another. 


\subsubsection{Treaded Robot with Passive Vacuum Suction Cups}

This design uses passive suction cups attached to treads to achieve both locomotion and adhesion. The system is relatively small and only weighs 0.3 kilograms. It uses a single DC motor to drive both belts, which means that the robot cannot be turned.

Once pressed onto a wall, which attaches the first set of suction cups, the robot attaches the suction cups to the wall using a torsion spring, which pushes the suction cups as they travel beneath the drive wheels. Once attached, pins on the suction cups mechanisms move into a guide rail, which distributes the attachment load evenly across all of the attached cups. To detach the cups, a wire is connected between the suction cups release tab and the neighboring suction cup such that the tab is pulled as the neighboring wheel moves around the back idler wheel. This means that the robot can only move forward. A more detailed description of the robot and its operating principle can be found in [20] and Figure 11 shows the completed robot.

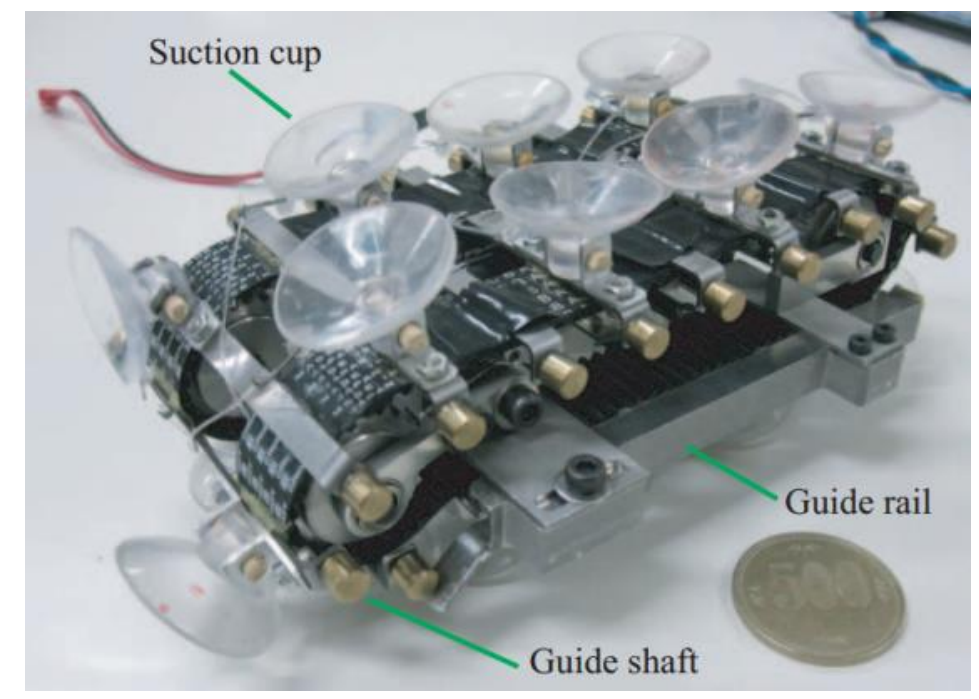

Figure 11: A climbing robot which uses passive suction cups attached to a continuous tread [20].

This design is similar to the proposed design in that it uses a combined adhesion and locomotion method. Like the proposed design it uses a pneumatic adhesion method and a tread for locomotion. However, the proposed design uses an active pneumatic system while this design uses passive suction cups. The proposed design will also be capable of turning 360 degrees, turning each tread independently and turning the treads both forwards and backwards. 


\subsubsection{The Tripillar}

This Tripillar uses magnets embedded in a continuous tread to locomote on and adhere to ferrous surfaces. The system weighs 214 grams and uses power from a battery pack. Two DC motors are used to drive the treads, which allows the system to skid steer. It also contains a Bluetooth module to allow the system to be controlled remotely. Two IR sensors are used to align the robot with the surface it is going to climb.

The tread wraps around three wheels, which allows the system to transition between surfaces that are perpendicular to one another. A longitudinal cable is wired into the tread to ensure that it remains rigid when moving. The magnets are molded into the tread and the poles of the magnets are alternated. The operating principle is relatively simple; the magnets adhere to the ferrous surface which produces sufficient friction so that the robot can move without the tread slipping when it is driven by the motors. A full description of the Tripillar can be found in [14] and Figure 12 shows an image of the Tripillar system.

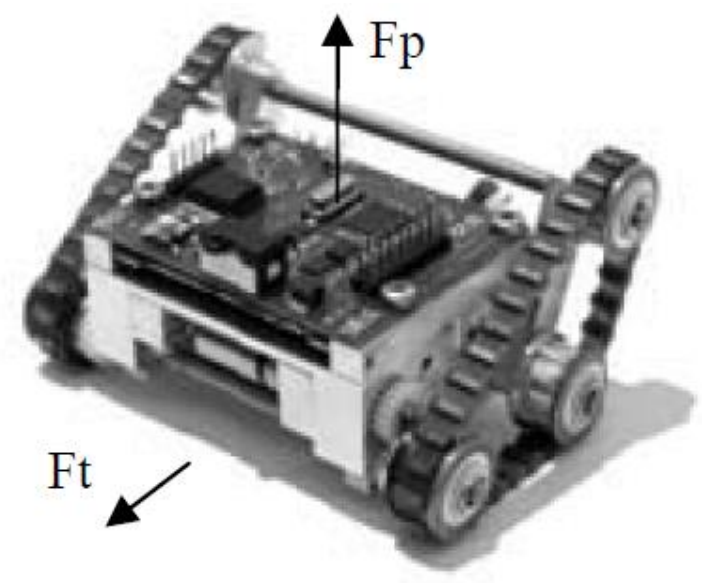

Figure 12: The Tripillar robot, which uses a continuous tread with embedded magnets [14].

Like the design discussed above, this design is similar to the proposed design in that it uses a combined adhesion and locomotion method. Like the proposed design, this system uses a continuous tread to locomote. Both the Tripillar and the proposed design are capable of skid steering. The main difference is that the proposed design uses a pneumatic adhesion method, while the Tripillar uses magnetic adhesion. This limits the Tripillar to operating on ferrous surfaces, but means that it does not require a tether, which the proposed design does require. 


\subsubsection{Treaded Robot with Active Vacuum Suction Cups}

This design uses active suctions cups attached to a continuous tread for adhesion and locomotion. The system weighs about 14 kilo grams. It uses a single DC motor to drive the tread which means it is incapable of turning. The system contains an internal power supply and vacuum generator, which means it is not tethered.

The tread is a timing belt, which is driven by two sets of pulleys. Each of the pulley sets is composed of two pulleys which are separated to allow the suction cup assemblies to travel between them. The suction cup assemblies are bolted to the tread, with the cups on the outside of the tread and trigger mechanism on the insides. Each assembly is connected to a rotating manifold which connected to the vacuum generator. When an assembly is not in contact with the wall, the cup is cut off from the vacuum supply. As the cup comes into contact with the wall, the trigger mechanism enters a guide channel which depresses the trigger and exposes the cup to the vacuum. A full description of the system can be found in [8] and Figure 13 contains an image of the system.

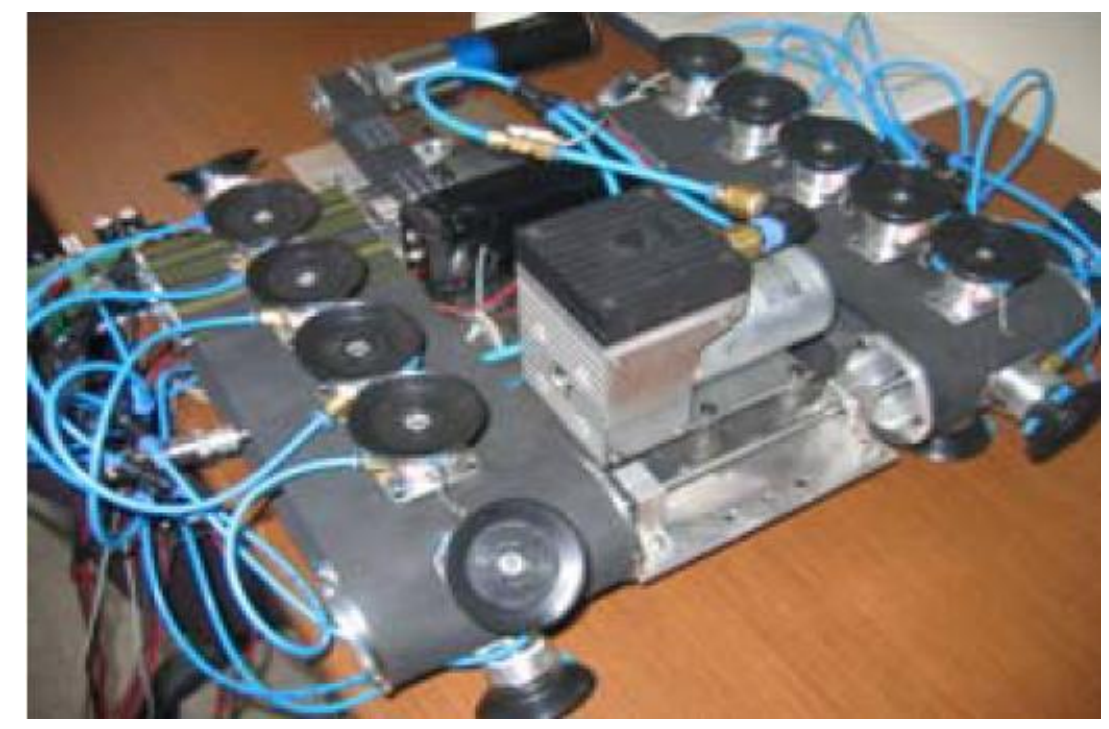

Figure 13: A climbing robot which uses active suction cups attached to a continuous tread [8].

This climbing robots design is the most similar to the proposed design that could be found. Both designs use active vacuum adhesion which is incorporated into a continuous tread. Several differences separate the proposed design from this design. The most obvious of these differences is that the proposed design uses its treads perforations as suction chambers, while this design uses suction cups. Additionally, the proposed 
design uses venturis and a positive pressure air supply to generate vacuum, while this design uses an electric vacuum generator. The proposed design is also capable of skid steering, which this design is not.

\subsection{Proof of Concept Model}

Before this thesis was started, the author and a graduate student from the Netherlands, Erik van Broekhoven, designed and built a proof of concept model. The purpose of this model was to demonstrate that a perforated tread was capable of being used as an adhesion and locomotion methods for wall climbing robot. It was also meant to determine any problems associated with the design, so that that information could be used in this project.

\subsubsection{Design}

The project began with a literature study to determine whether or not the proposed concept, i.e. the perforated tread acting as a suction cup, had been designed by another party. It was found that the concept was novel and so the project moved forward.

A model of the tread and manifolds was created to determine an optimized pattern for both parts which would allow the vacuum system to provide consistent force between the wall and the vehicle. This optimization was done using a Matlab script. The script analyzed images of different tread and manifold hole patterns overlaid with each other to determine the area exposed to vacuum as the manifold was moved across the tread. An image of the types of images used in the program can be found in Figure 14.

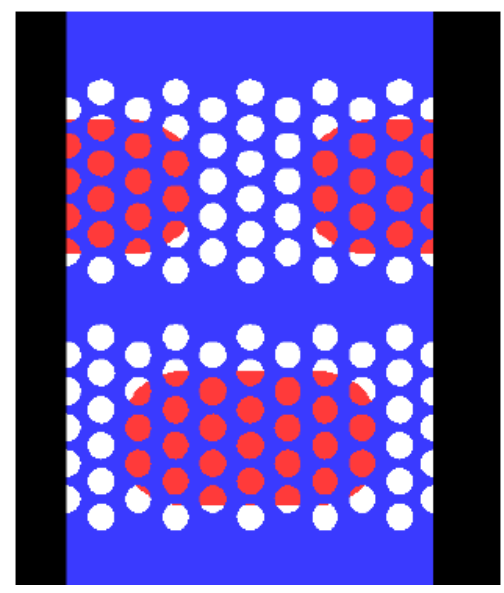

Figure 14: Sample of the images used to determine exposed tread hole area. 
Once the hole patterns of the tread and manifold were finalized, the material of the tread and manifold were chosen. The material for the tread was chosen to be fabric reinforced neoprene because it was compliant in the direction of the thickness of the tread but stiff longitudinally and laterally. The manifolds material was chosen to be acrylic with a thin sheet of Teflon were the manifold contacted the tread to reduce friction.

To shorten the time to manufacture the vehicle a radio controlled tank chassis was purchased from Taigen Tanks. The tank offered many components which could be used directly in the project such as its motor system, its drive and idler wheels, its frame and its tensioning system. The model of the vehicle was based on the dimensions of this chassis and components were added as necessary to complete the design. An image of the final model can be found in Figure 15.

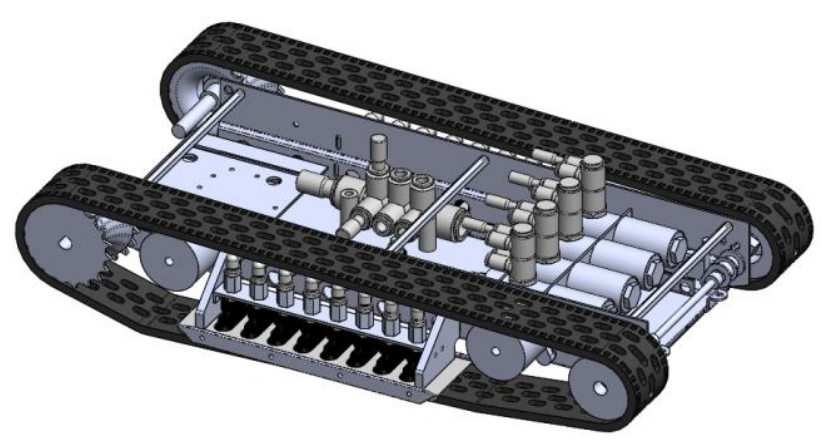

Figure 15: Solidworks model of proof of concept model.

\subsubsection{Manufacturing}

The manufacturing of the vehicle itself was relatively straight forward. The tank chassis was stripped down to its sheet metal frame and the superfluous components were removed. Struts were added to in interior of the frame to stiffen it and holes were drilled in its base to mount the venturi system. Lipped delrin wheels were machined to replace the idler and tensioner wheels that came with the tank to help retrain the tread during operation.

The tread and manifold plates were laser cut as their geometries were complicated. The treads were then stitched together using Kevlar thread to form continuous belts. Silicone paste was then used to seal the stitched area. Guide rails were then attached to the manifold plates to guide the tread as it passed under the manifold plates. 


\subsubsection{Testing and Observations}

The first test which was conducted was a pull off force measurement test performed on different surfaces. The test involved adhering the vehicle to different surfaces then pulling the vehicle off the surface using a force scale. The results of this test can be found in Table 1 .

Table 1: Pull-off force on different surface types.

\begin{tabular}{|l|r|}
\hline \multicolumn{1}{|c|}{ Material } & Force [N] \\
\hline Acrylic & $35-40$ \\
\hline Wall & $75-80$ \\
\hline Wood & $45-50$ \\
\hline Painted door & $55-60$ \\
\hline Painted steel closet & $55-60$ \\
\hline Glass & $80-85$ \\
\hline
\end{tabular}

The second test which was conducted was to see if the vehicle was able to drive under its own power. This test was unsuccessful as the friction between the tread and manifold was too great for the stock motors on the tank chassis to handle. As an alternative, the drive wheels were rotated by hand and forward motion was achieved without the vehicle dropping from the wall, though it was extremely difficult to move.

A myriad of observations were made about the vehicles performance during testing. These ranged from problems in the tread system to difficulties with the vehicles frame. Table 2 lists the significant observations which were made during the testing of the vehicle.

Table 2: Observations made about the conceptual model during testing.

\begin{tabular}{|l|l|}
\hline \multicolumn{1}{|c|}{ Observation } & \multicolumn{1}{c|}{ Description } \\
\hline Motor Torque & $\begin{array}{l}\text { The motor torque was insufficient to drive the vehicle up the wall. } \\
\text { The cause of this problem was a combination of friction between the } \\
\text { manifold and the tread and the weight of the vehicle. }\end{array}$ \\
\hline Tread/Manifold Friction & $\begin{array}{l}\text { The amount of friction between the tread and the manifold was far } \\
\text { more significant than initially predicted. }\end{array}$ \\
\hline $\begin{array}{l}\text { Deflection of the drive } \\
\text { and Tensioner shafts }\end{array}$ & $\begin{array}{l}\text { Significant deflections were observed in the drive and idler wheel } \\
\text { shafts when the belt was tensioned. This cause the tread to slip when } \\
\text { the wheels of the vehicle were turned. }\end{array}$ \\
\hline Frame Deflections & $\begin{array}{l}\text { Deflections in the frame were observed after the belt was tensioned. } \\
\text { Tread Stiffness Problems } \\
\text { tread when the wheels were turned. This included deformation at the } \\
\text { hole where the drive wheel engaged with the tread, warping of the } \\
\text { vacuum through holes and buckling when the tread was pushed } \\
\text { under the manifold. }\end{array}$ \\
\hline Venturi clogging & $\begin{array}{l}\text { Several instances of venture clogging were observed during the } \\
\text { testing. }\end{array}$ \\
\hline Adhesion Failures & $\begin{array}{l}\text { Several instances of adhesion failure between the wall and the } \\
\text { vehicle were observed. Each instance of failure was the result of a } \\
\text { single failed vacuum section. }\end{array}$ \\
\hline
\end{tabular}




\begin{tabular}{|l|l|}
\hline Low Adhesion Force & $\begin{array}{l}\text { The adhesion force between the wall and vehicle was lower than } \\
\text { expected. }\end{array}$ \\
\hline Peel Off & $\begin{array}{l}\text { The motors at the front of the vehicle created a moment which } \\
\text { caused the treads to peel off the wall. }\end{array}$ \\
\hline
\end{tabular}

\subsubsection{Results and Conclusions}

Despite the fact that the vehicle could not locomote under its own power, the ultimate results of the proof of concept model were promising and provided information which was applied to the next iteration of the design. A list of improvements to future designs that was developed during the course of the proof of concept phase of the project follows.

- Create a detailed model of the vehicle to determine the motor torque required and ensure a sufficient factor of safety is used when selecting the motors.

- Use professionally manufactured belts as treads to ensure the tread is stiff enough to handle the loads it will be subjected to.

- Use a brace between the drive ad idler wheels and stiff shafts to minimize deflections in the wheel system.

- Use material combinations for the tread and manifolds which have a low coefficient of friction.

- Have a means of measuring the vacuum pressure in the manifolds directly to make characterizing the vehicles adhesion performance easier.

- Try to ensure that the center of gravity of the vehicle is near the center of the manifolds to reduce peel off forces.

- Ensure that the frame is stiff enough to handle the tension and operation loads it will be subjected to.

- Ensure that the tread is unable to slip off of the wheels during operation.

- $\quad$ Ensure the wheels are well aligned.

The most significant outcome during the design of the project was that a rubber tread could be used to seal a vacuum system and hold the vehicle to a wall. Figure 16 shows the first proof of concept model adhering to a wall. 


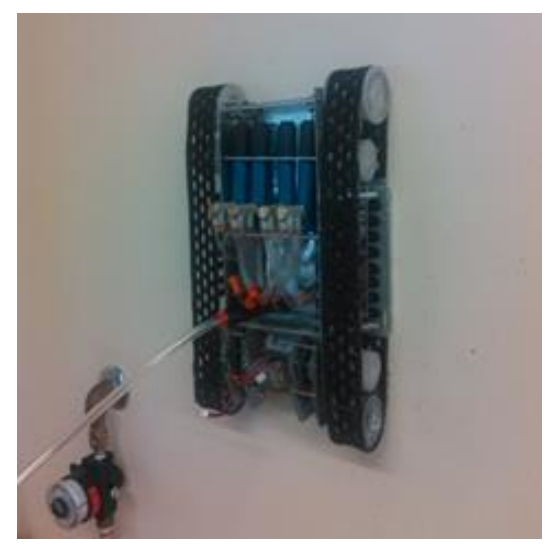

Figure 16: Proof of concept model adhering to a vertical surface. 


\section{Chapter 2: Design}

\subsection{Design Criteria}

This section details the criteria that were used to guide the design of the climbing robot. These criteria are described in detail in Table 3.

Table 3: The criteria used to design and test the climbing robot system.

\begin{tabular}{|l|r|l|}
\hline \multicolumn{1}{|c|}{ Criteria } & Numerical Value & \multicolumn{1}{|c|}{ Description } \\
\hline Maximum System Weight & $15 \mathrm{lbf}$ & $\begin{array}{l}\text { The system should be a reasonable weight so that } \\
\text { the user can easily and safely carry it and apply it } \\
\text { to surfaces. }\end{array}$ \\
\hline Minimum Payload Capacity & $1.5 \mathrm{lbf}$ & $\begin{array}{l}\text { The minimum payload which the system should } \\
\text { be able to carry. }\end{array}$ \\
\hline Minimum Drive Speed & $0.5 \mathrm{ft} / \mathrm{s}$ & $\begin{array}{l}\text { This is the minimum drive speed which the robot } \\
\text { should be able to achieve during forward motion. }\end{array}$ \\
\hline $\begin{array}{l}\text { Maximum Required Input Air } \\
\text { Pressure }\end{array}$ & $100 \mathrm{psi}$ & $\begin{array}{l}\text { The maximum pressure which the input to the } \\
\text { system should require. Set by the maximum } \\
\text { possible pressure output from testing station in } \\
\text { Mustang 60. }\end{array}$ \\
\hline $\begin{array}{l}\text { Minimum Pull Off Force Factor } \\
\text { of Safety }\end{array}$ & 2.0 & $\begin{array}{l}\text { This is the minimum force which should be } \\
\text { required to remove the robot from a surface when } \\
\text { it has payload. }\end{array}$ \\
\hline $\begin{array}{l}\text { Minimum Number of Failed } \\
\text { Vacuum Sections for Continued } \\
\text { Adhesion }\end{array}$ & 2 & $\begin{array}{l}\text { This is the minimum number of vacuum sections } \\
\text { which can be exposed to the atmosphere or have } \\
\text { failed and still allow the robot to maintain } \\
\text { adhesion. }\end{array}$ \\
\hline Minimum Surface Roughness & 0.005 in. & $\begin{array}{l}\text { The minimum surface roughness which the robot } \\
\text { should be able to operate on. }\end{array}$ \\
\hline Maximum Voltage Input & $120 \mathrm{VAC}$ & $\begin{array}{l}\text { The system should be able to be powered using a } \\
\text { standard wall outlet. }\end{array}$ \\
\hline
\end{tabular}

\subsection{Component Selection}

This section details the selection of the major system components. These components drove much of the rest of the systems design. These components include the selection of the motors, the power supply, the venturis and the belt system.

\subsubsection{Motor Selection}

The motors were a critical portion of the design. This was because they ultimately control both the total weight that the system can carry and the speed at which the system can travel. The inability of the proof of concept model to locomote under its own power also demonstrated the importance of the using sufficiently powerful motors in the new system. 


\subsubsection{Selection Criteria}

It was decided early in the project that the motors would be direct current (DC). This was because the controller which was to be created for the robot would be simpler to design and program if the motors it was driving were DC. The controller, more specifically the motor driver chips, also determined the maximum allowable input voltage and current for the motors. The allowable electrical parameters of the motors can be found in Table 4. These parameters were used as a guide when selecting the motors which would be used to drive the motors.

Table 4: Maximum allowable electrical characteristics of the motors. Values taken from the VNH5019A-E data sheet.

\begin{tabular}{|l|r|}
\hline \multicolumn{1}{|c|}{ Parameter } & Value \\
\hline Current Type & Direct \\
\hline Maximum Voltage & 40 Volts \\
\hline Maximum Current & 30 Amps \\
\hline Maximum Power & 1.2 Kilowatts \\
\hline
\end{tabular}

The physical requirements of the motors were determined next. The output torque and speed of the motor shaft were the most important physical parameters of the motors. To calculate the required torque output, a simple conceptual model of the system was created. This model was based on the predicted physical parameters of the final system, using the proof of concept model as a guide. The model was then symbolically analyzed to determine the required torque for the motor. The results of this analysis were used to create a calculator which would take in known or projected system parameters and output the motor torque. This calculator was used throughout the design process to ensure that the selected motors would be able to meet the torque requirements of the final design. The symbolic calculations used to create the calculator can be found in Appendix A, as well as an outline of the calculator and sample results.

The speed requirements of the motors were calculated next. This was a relatively simple calculation. It involved back calculating the motors RPM from the desired minimum speed of the robot and the projected drive wheel radius, and assumed that the speed of the robot could be calculated as if the drive wheels were placed directly on a surface rather than on treads. Again, this calculation was updated as the design of the robot was iterated. The calculation used to determine the required output RPM of the motor can be found in Appendix A. 
The other physical parameters of the motor were also considered. This includes the weight of the motor, the motors size and how easily the motor could be mounted. To ensure that the motors did not expend a large amount of their output power lifting themselves, it was determined that they should not be a large fraction of the systems overall weight. With this in mind, was decided that the motors should each contribute less than $10 \%$ of the overall mass of the system. To allow for as much room as possible for the other components in the robot, a compact overall package for the motors was desired. Based on preliminary sizing of the system, motors which fit with in a 4"x3"x3" space would be considered ideal, though this was a flexible requirement and motors which fell outside this space by +0.5 " on any of the parameters would be considered. A list of all of the physical requirements for the motors can be found in Table 5 .

Table 5: Physical requirements used to select the drive motors for the climbing robot.

\begin{tabular}{|l|r|}
\hline \multicolumn{1}{|c|}{ Physical Parameter } & Value \\
\hline Required Output Torque From Calculator & $26.7 \mathrm{in}-\mathrm{lb}$ \\
\hline Output Angular Velocity & $40 \mathrm{RPM}$ \\
\hline Maximum Motor Weight & $1.75 \mathrm{lb}$ \\
\hline Desired Motor Dimensions & 4 "x3"x3" \\
\hline
\end{tabular}

\subsubsection{Selected Motors}

Using the above parameters, a search for DC gear motors was conducted. This search turned up two different motors which satisfied most of the conditions required for the project. These two motors, one from Molon motors and the other from Maxon motors, were compared. Table 6 contains a comparison of the relevant specifications for each of the motors which were considered.

Table 6: Specifications for the two motors which best satisfied the projects requirements.

\begin{tabular}{|l|r|r|}
\hline \multicolumn{1}{|c|}{ Parameter } & Molon Motor & \multicolumn{1}{c|}{ Maxon Motor } \\
\hline Maximum Torque & $50 \mathrm{in}-\mathrm{lb}$ & $60 \mathrm{in}-\mathrm{lb}$ \\
\hline Torque Factor of Safety & 1.87 & 2.25 \\
\hline Max Angular Velocity & $25 \mathrm{RPM}$ & $50 \mathrm{RPM}$ \\
\hline Required Voltage & $24 \mathrm{VDC}$ & $24 \mathrm{VDC}$ \\
\hline Max Current & $1.08 \mathrm{~A}$ & $2.73 \mathrm{~A}$ \\
\hline Dimensions & $4.17 ” \times 2.75 ” \times 3 "$ & $1.12 \mathrm{lb}$ \\
\hline Weight & $1.2 \mathrm{lb}$ & 1.26 " dia. x 4.65” long \\
\hline Cost per Motor & $\$ 53.16$ & $\$ 744.88$ \\
\hline
\end{tabular}

While the Maxon motor better satisfied the speed and torque requirements above, the Molon motor was chosen for several reasons. The first and most significant was cost. The Maxon motor was almost fifteen times the cost of the Molon motor, which put it far outside the budget of the project. Also, two of the 
Maxon motors required a relatively large current which would make them less safe to use. The length of the Maxon motors also made it difficult to fit them easily in the space available.

The Molon motors, while not able to match the Maxon motor in output speed or torque, do satisfy most of the projects requirements. The only area which they do not satisfy is desired output speed, but this is acceptable because with the finalized drive wheel size they can move the robot at approximately .29 feet per second, which is reasonable. The Molon motors torque output also has a low factor of safety when compared with the initial torque requirement predictions, but the final weight of the system and final wheel size reduce the projected torque requirement significantly, making them more than powerful enough. They have a relatively low current draw, meaning they will not over tax the controller or the power supply when running near their maximum current draw. Their compactness also means that they were easy to integrate into the space that was available for them. Images of the Molon motors selected for the project can be seen in Figure 17 and Figure 18.

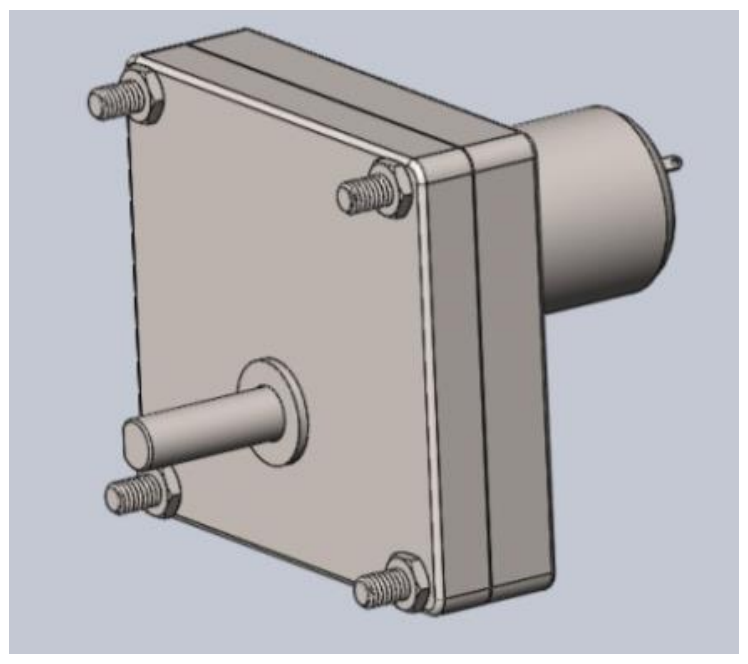

Figure 17: Solid model of the selected motor. 


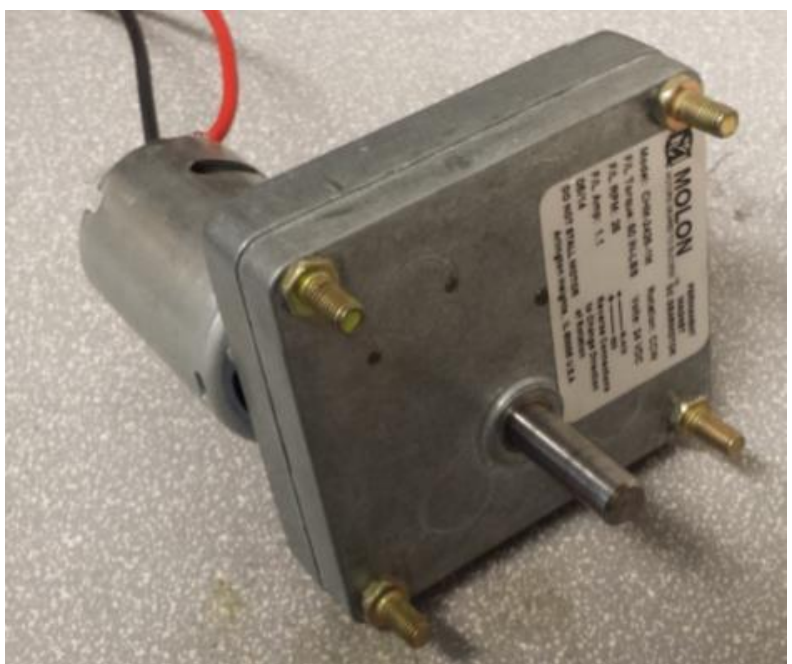

Figure 18: Molon motor chosen for the vehicle.

\subsubsection{Power Supply Selection}

The power supply was an important factor to consider in the design of the vehicle, as it provided the vehicle with electrical power for the controller, the motors and the sensors. Three parameters were considered in the selection process for the power supply used. These were the weight contributed to the vehicle by the power supply and its cables, the power requirements of the motors and the controller and the amount of space the power supply occupied in the vehicle.

\subsubsection{Selection Criterion}

The criteria used to select the power supply used in the project were relatively strait forward. The first and most critical of these criterion was the ability of the power supply to meet the electrical power requirements of the vehicles. This requirement was determined by summing the current draw of the motors and the controllers and by finding the maximum voltage that the motors could safely operate at. The weight requirement for the power supply was based on its total contribution to the overall weight of the vehicle. It was decided that the power supply and its cabling should not weigh more than $10 \%$ of the total weight of the vehicle. To maximize the space inside the vehicle, a limit was placed on the foot print dimensions. Table 7 contains the criterion used to select the power supply. 
Table 7: Criteria used to select the vehicle power supply.

\begin{tabular}{|c|c|}
\hline Criteria & Value \\
\hline Minimum Voltage Output & $24 \mathrm{~V}$ \\
\hline Minimum Current Output & $2.75 \mathrm{Amps}$ \\
\hline Minimum Power Output & 66 Watts \\
\hline Maximum Allowable Weight & $1.5 \mathrm{lbf}$ \\
\hline Maximum Foot Print Dimensions & 3 in. $x 4$ in. \\
\hline Maximum Height & 3 in. \\
\hline
\end{tabular}

\subsubsection{Selected Power Supply}

Three different types of power supplies were proposed for the system. These were a battery pack, an on vehicle AC/DC converter and an off vehicle AC/DC converter. The battery pack option was quickly dismissed because while a battery pack would eliminate the need of an electrical tether for the vehicle, they are extremely heavy and has large foot prints at the required voltage and power needs of the vehicle.

Ultimately, the power supply type chosen was the off vehicle AC/DC converter because it offered several advantages over the on vehicle AC/DC converter. The most significant of these advantages was the fact that the off vehicle converter contributed almost no weight to the vehicle, with only the weight of the lines between the converter and the vehicle contributing to the vehicles overall weight. The off vehicle converter also did not require any space in the interior of the vehicle, which would make it easier to work inside the robot after assembly. The off vehicle converter was also much safer, as an on vehicle converter would require an $\mathrm{AC}$ line to be run to the vehicle. This meant there would be significant risk of electrocution if the line or the converter were grounded to the vehicle. Figure 19 shows the off vehicle AC/DC converter that was used as the projects power supply.

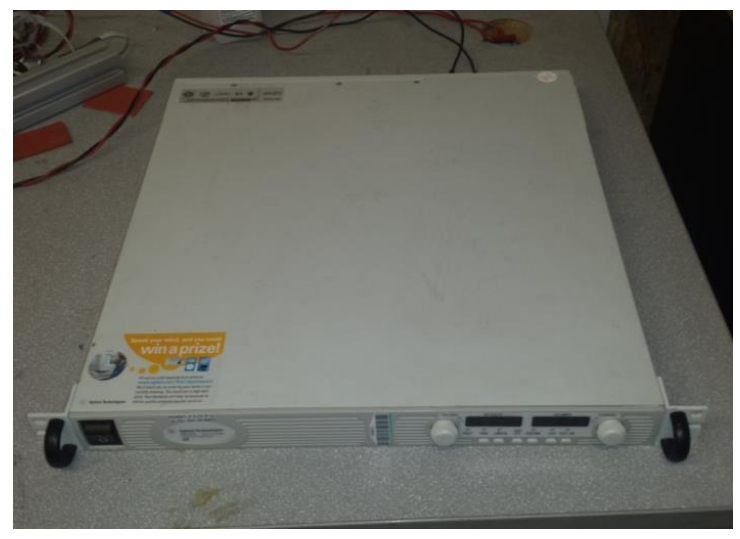

Figure 19: Agilent N5747A power supply used to power the vehicle. 


\subsubsection{Venturi Selection}

The selection of the venturis used in the robot was a critical portion of the design because they generate the vacuum used to adhere the system to surfaces. Three major parameters were used in determining which venturis would be employed in the design. These were the maximum vacuum which could be generated by the venturis, the required input pressure for the venturis and the rate at which the vacuum can evacuate the required volume. Other considerations which were taken into account, but were less critical, were the dimensions and weight of the venturis. Because the proof of concept model used venturis from Vaccon and they proved to be of high quality, it was decided that the venturis for this project would also be purchased from Vaccon.

\subsubsection{Selection Criterion}

To minimize the required area exposed to the vacuum, and thereby reduce the size of the tread, to ensure safe adhesion venturis which could generate high vacuum were desired. It was found that the maximum pressure achievable by Vaccon's venturis was 13.75 psi. This maximum pressure was shared by all of their miniature and midsized venturis. This was also true of the required input pressure for each of the venture models. They all required an 80 psi input to achieve maximum suction.

The weight and the dimensions of the miniature venturis were also all standard, with no variation between models. The exception to this was in the different silencers which could be used with the venturis. Three different silencers were available, a closed end silencer, a straight-through silencer and hybrid silencer. The closed end and straight-through silencers were similar in their overall weight, but the closed end silencer was shorter by about .4 inches. The hybrid silencer was quite a bit larger than the other two options both in increase in length and weight of the venture system. To keep the length of the venturis to a minimum, the closed end silencers were selected.

This left the evacuation rate to determine which should be used in the project. To determine the required evacuation rate, the volume that the venturis were first estimated. This calculation was based on the estimated volumes of the manifold chamber, the connecting tubing and the tread perforation volumes. The estimated volume was determined to be $9.4 \times 10^{\wedge}-4 \mathrm{ft}^{\wedge} 3$. The desired time to evacuation was determined based on the rate that the pressure sensors measuring the vacuum could operate at. The time to evacuation 
was selected as $0.01 \mathrm{sec}$, which allowed the pressure sensors to take around 5 measurements during the evacuation. The required evacuation rate was then calculated and found to be about $10 \sec$ per $\mathrm{ft}^{\wedge} 3$. All of the calculations related to estimating the evacuation rate can be found in Appendix A. Table 8 contains the requirements for determining which model of venturi would be used.

Table 8: Parameters used to select the venturi models used in the robot.

\begin{tabular}{|l|r|}
\hline \multicolumn{1}{|c|}{ Parameter } & Value \\
\hline Input Pressure & $80 \mathrm{psi}$ \\
\hline Maximum Vacuum & $13.75 \mathrm{psi}$ \\
\hline Evacuation Rate & $10 \mathrm{~s} / \mathrm{ft}^{\wedge} 3$ \\
\hline Length (Including Silencer) & $4.20 \mathrm{in}$ \\
\hline Diameter & $0.75 \mathrm{in}$ \\
\hline
\end{tabular}

\subsubsection{Selected Venturi}

Because most of the parameters for each of the venturi models were similar, the selection process was relatively quick. The decision was made by comparing the evacuation rate between each of the miniature venturi models offered by Vaccon. Cost was also factored into the decision, as well as the fact that venturis from the proof of concept model were available.

Using the estimated volume of a single section of the vacuum system and the evacuation rates provided by Vaccon, the time to evacuation was calculated for each of the miniature venturi models. The costs of each of the venturi models with the closed end silencer were also gathered. This information can be found in Table 9.

Table 9: Evacuation rate, evacuation time for the estimated volume of a single vacuum section and the cost of each of the different venturi models in Vaccon's miniature venturi line.

\begin{tabular}{|l|r|r|r|r|}
\hline \multicolumn{1}{|c|}{ Parameter } & \multicolumn{1}{c|}{ JS-60M } & \multicolumn{1}{c|}{ JS-90M } & \multicolumn{1}{c|}{ JS-100M } & \multicolumn{1}{|c|}{ JS-150M } \\
\hline Evacuation Rate & $28.24 \mathrm{~s} / \mathrm{ft}^{\wedge} 3$ & $10.05 \mathrm{~s} / \mathrm{ft}^{\wedge} 3$ & $8.99 \mathrm{~s} / \mathrm{ft}^{\wedge} 3$ & $4.46 \mathrm{~s} / \mathrm{ft}^{\wedge} 3$ \\
\hline Evacuation Time & $0.027 \mathrm{~s}$ & $0.009 \mathrm{~s}$ & $0.008 \mathrm{~s}$ & $0.004 \mathrm{~s}$ \\
\hline Cost & $\$ 65.00$ & $\$ 71.50$ & $\$ 78.25$ & $\$ 84.75$ \\
\hline
\end{tabular}

The venturi model which was ultimately selected was the JS-90M, for several different reasons. The JS$90 \mathrm{M}$ allowed for a sufficient number of readings during the re-evacuation process. The shorter evacuation time also allows the robot to recover more quickly when sections of the vacuum system are exposed to the atmosphere and also allows the vacuum system to deal with minor leaks which may occur during operation.

The cost of each of the different models were all similar, but the JS-90M falls at the lower end of the cost spectrum. This, coupled with the fact that four of the JS-90M models were available from the proof of 
concept model meant that it was the most cost effective option. An image of the JS-90M model can be found in Figure 20, which shows both the venturi's main body and the closed end silencer.

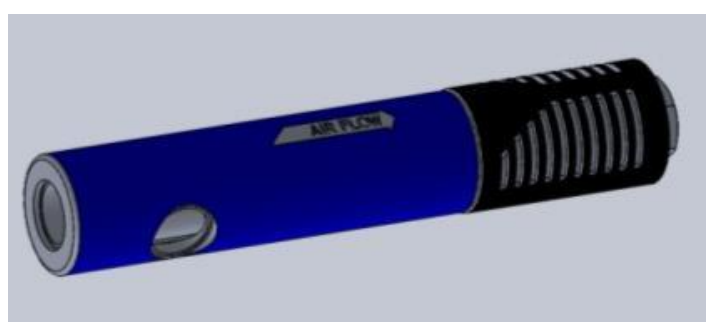

Figure 20: Solidworks representation of the JS-90M-AA4. 


\subsubsection{Tread Selection and Design}

The treads which drive the robot and act as the gasket for the vacuum system are perhaps the most critical portion of the design. The design of the new tread system was begun by looking at the problems associated with the proof of concept models tread. With this insight, criteria were set forth for the design of the new tread, both in terms of the materials used in the tread and the shape that the tread would take.

The proof of concept model suffered from several different problems associated with its treads, which this design seeks to address. The first of these problems was buckling of the tread when under vacuum, which was caused by high friction between the tread and the manifold, as well as the low stiffness of the tread material. The second problem which the proof of concept models tread had was a large amount of deflection in the tread when it was under tension and when driven. The perforations in the tread which interacted with the drive wheels warped significantly, which caused damage to the edges of the belt. The third problem with proof of concept tread was that it was not initially continuous and had to be stitched to attach the free ends. The stitched area suffered from small tears each time the belt was tensioned, which would eventually cause failure. This tearing also meant that the stitched area was difficult to seal effectively and that leaks caused by the tears would eventually become great enough to cause the vacuum system to fail. The stitched area was also raised on both sides of the tread, which cause the manifold to be pushed away from the tread as it moved over the stitched area dethatching the robot from the wall.

\subsubsection{Selection and Design Criterion}

It was decided early in the projected that using timing belts as the basis of the treads would offer several advantages over other potential designs, such as the sprocket driven belt in the proof of concept model. Timing belts are generally stiff and can be tensioned without causing any deflection or stretching in the belt. This stiffness also means that they are less likely to buckle during operation, which is especially important for the robot to function properly. The teeth of timing belts also do not suffer from the deflections seen in the proof of concept models drive holes at even relatively high torques. Teeth also help to ensure that no slipping around the drive pulley, even at high speed or torques.

Timing belts also allow for the tread to be flat against the wall for its entire length between the drive wheels and the back idlers. This was not possible in the proof of concept model because the drive sprocket and 
back idler would contact the wall before the tread. Having the tread leaving the wheels parallel to the wall allows for the drive and idler wheels to take a portion of the reaction loads from the wall that would otherwise be carried by the manifold. It also means that the manifold does not need to force the tread flat against the wall, which the manifold in the proof of concept model had to do.

Another advantage of using timing belts is the fact that several companies exist which produce custom belts. By using an outside manufacturer, belts of greater complexity could be produced for much less effort and time than if they were machined on site. A survey of different custom belt companies was conducted and it was found that F.N. Sheppard had the widest variety of customization options available. These options included the removal of teeth in a given area of the belt, the application of low friction material on belt surfaces, the application of backing material on the outside of the belt and perforations of the belt. Each of these capabilities was important for the design of the tread. Their website included a design catalog which contains a large amount of information which was used to design the belts used in the robot.

Several different belt materials were available from F.N. Sheppard. These included urethane, various neoprene rubbers and silicone. The most common materials used in the construction of their belts were urethane with a durometer of 90 shore-A and neoprene with a durometer of 70 shore-A. Each of these materials could be reinforced with different types of internal fibers, the urethane with steel or Kevlar and the neoprene with fiberglass. These reinforcement fibers allow for the belts to be tensioned without stretching. F.N. Sheppard also offered a variety of different tooth patterns, each with different dimensions. These patterns were common between the different available belt materials.

To determine which belt material and dimensions should be used in the project, two different factors were considered. The tension in the belt was perhaps the most critical because if the belt were too lose it could jump teeth on the wheels or buckle, while if it were too tight the shafts, bearings or the belt itself might fail. Using an equation found in a timing belt catalog, the minimum required tension of the belt was estimated. This equation uses the design torque acting on the belt, the diameter of the drive pulley, the mass per unit length and the speed of the drive pulley. The calculation used to determine this estimate and the belt factor of safety can be found in Appendix A. Table 10 contains the estimated values for the minimum required belt tension. 
Table 10: Minimum tread tension for the robots treads to function properly and the factor of safety estimated for the tread.

\begin{tabular}{|l|r|}
\hline \multicolumn{1}{|c|}{ Parameter } & Value \\
\hline Minimum Belt Tension & $55 \mathrm{lb}$ \\
\hline Factor of Safety & 16 \\
\hline
\end{tabular}

The next important criterion for the design of the tread was the area of the perforations that the vacuum would be pulled through. Using the projected weight of the system and the desired factor of safety, a required vacuum force was calculated. This calculation was performed assuming for two cases, when the robot is attached to a vertical surface and when the robot is attached to the ceiling. Using this vacuum force and the vacuum pressure which could be generated by the venturis, the perforation area was calculated.

Table 11 contains the values for the required vacuum and for the required area of the perforations, and the calculations used to obtain these values can be found in Appendix A.

Table 11: Expected required vacuum forces for both vertical operation and operation upside-down and the tread perforation area which is required to achieve the greater of the two force values.

\begin{tabular}{|l|r|}
\hline \multicolumn{1}{|c|}{ Parameter } & Value \\
\hline Vacuum Force - Vertical Surface & $33 \mathrm{lb}$ \\
\hline Vacuum Force - Ceiling & $33 \mathrm{lb}$ \\
\hline Perforation Area & $2.4 \mathrm{in}^{\wedge} 2$ \\
\hline
\end{tabular}

Another important factor which was considered was the friction between the manifold and the tread. One of the main reasons which the proof of concept model could not drive itself and the models treads buckled was due to the friction between the treads and the manifolds. In the model, Teflon was added to the bottoms of the manifold plates but the rubber of the tread still caused a large amount of friction. To determine the desired coefficient of friction between the tread and manifold, a short calculation was performed. First, it was decided that the friction force between the tread and the manifold should not exceed twenty percent of the force output of the motors. From this number, the maximum allowable friction force was calculated. This force value was then used to determine the maximum allowable coefficient of friction by dividing the frictional force by the vacuum force determined above. The calculated maximum coefficient of friction value is the static coefficient of friction, because the static coefficient of friction is always greater than the dynamic coefficient of friction. Table 12 contains the maximum friction force allowed between the tread 
and the manifold, as well as the maximum static coefficient of friction allowable. Appendix A contains the calculations used to determine these two values.

Table 12: Maximum friction force allowable between the tread and the manifold and the resulting maximum allowable coefficient of static friction between the tread and the manifold.

\begin{tabular}{|l|rr|}
\hline \multicolumn{1}{|c|}{ Parameter } & Value \\
\hline Maximum Friction Force & $10.5 \mathrm{lb}$ \\
\hline Maximum Desired Coefficient of Friction & .5 \\
\hline
\end{tabular}

\subsubsection{Selected Belt Design}

The first step which was taken in the design of the belt was the determination of which of the materials offered by F.N. Sheppard, urethane or neoprene, should be used, as well as the tooth pattern which should be used. This was accomplished using both the design catalog provided by F.N. Sheppard and recommendations by one of their support staff. The design catalog contained detailed design information for both urethane reinforced with steel and urethane with Kevlar. This information included the stiffness, allowable tension, weight and tensile strength for different tooth pitches. The catalog did not contain any of this information for the neoprene timing belts, except for the durometer of the neoprene. This made the urethane belts more attractive initially, as their properties could be directly compared to the requirements above. The tooth pattern of the belt was selected next and Figure 21contains a table from the catalog which contains the dimensional information for each of the available tooth patterns. Three of these patterns were considered for the project. These were the XL, L and H patterns.

\begin{tabular}{|c|c|c|c|c|c|c|c|}
\hline & $\begin{array}{c}\text { Belf } \\
\text { Section }\end{array}$ & $\begin{array}{l}\text { Construction } \\
\text { Urethane/ } \\
\text { Neoprene }\end{array}$ & $\begin{array}{l}\text { Pitch } \\
\text { (in.) }\end{array}$ & $\begin{array}{l}\text { hb } \\
\text { (in.) }\end{array}$ & $\begin{array}{l}\text { hit } \\
\text { (in.) }\end{array}$ & $\begin{array}{l}\text { Tooth } \\
\text { Angle } \\
\text { (Degree) }\end{array}$ & $\begin{array}{l}\text { bf } \\
\text { (in.) }\end{array}$ \\
\hline & $40 \mathrm{DP}$ & Urethane & 0.0816 & 0.048 & 0.018 & 60 & - \\
\hline & MXL & Urethane \& Neoprene & 0.080 & 0.045 & 0.018 & 40 & 0.045 \\
\hline$\underbrace{}_{k \rightarrow \text { Pitch } \rightarrow 1} \sqrt{\frac{\hat{t}}{h t}}$ & $\mathrm{XL}$ & Urethane \& Neoprene & 0.200 & 0.090 & 0.050 & 50 & 0.101 \\
\hline & L & Urethane \& Neoprene & 0.375 & 0.140 & 0.075 & 40 & 0.183 \\
\hline & $\mathrm{H}$ & Urethane \& Neoprene & 0.500 & 0.160 & 0.090 & 40 & 0.241 \\
\hline & $\mathrm{XH}$ & Urethane \& Neoprene & 0.875 & 0.440 & 0.250 & 40 & 0.495 \\
\hline & $X X \mathrm{H}$ & Neoprene & 1.250 & 0.620 & 0.375 & 40 & 0.750 \\
\hline
\end{tabular}

Figure 21: A table containing the dimensions of the different belt patterns taken from F.N. Sheppard's design catalog. The highlighted belt sections are those which were considered for the tread of the robot. 
Based on the design criterion from the previous section, each of the tooth patterns which were considered would be able to handle the projected minimum tension and tooth loading, even if the width of the belts was relatively thin. Several different factors ultimately lead to the selection of the type $\mathrm{H}$ belt pattern. The first was that it was the stiffest of the options and could carry the greatest tension. This meant that the tread would be less likely to buckle when driven and would allow a wider range of tensions to be tested. The type $\mathrm{H}$ pattern also had the strongest teeth of the three options, again meaning that a wide range of tensions could be tested without fear of damaging the belt teeth. The height of the teeth in the type $\mathrm{H}$ pattern also meant that the belt would be less likely to slip off the wheels during skid steering.

Urethane reinforce with Kevlar was initially chosen as the material for the tread because, as stated above, little information was given about the neoprene belt material beyond its durometer. This choice was reconsidered and ultimately changed after consulting with F.N. Sheppard. Although a neoprene belt would be less stiff than a urethane belt, it was found that neoprene would ultimately work better for the robot for several different reasons. Firstly, after describing the parameters of the robot and the expected loading on the belt, the representative was confident that a neoprene belt would more than meet the projects requirements. The cost of the neoprene belts was significantly lower than that of a urethane belt. The production time for the neoprene was also significantly shorter than that of the urethane belt, as the neoprene belts were prefabricated and only required modification. The neoprene belt offered by the company was molded, and so did not require any welding to make it continuous, which the urethane belt would. This weld interferes with the continuity of the perforation pattern for the vacuum system, causing a dead spot at the welded section. So, based on the advice of the F.N. Sheppard representative, the neoprene reinforced with fiber glass was chosen.

The next step in designing the treads was the selection of the backing material which would be on the outside of the tread and the selection of the low friction material which the manifold would contact on the inside of the tread. The backing material was a relatively simple selection. The neoprene belt used in the proof of concept models tread, which was 70 Shore A durometer, showed that even relatively stiff neoprene could form a sufficient seal. A brief survey was conducted to determine the standard durometer of neoprene used in suction cups and it was found the between 40 and 60 Shore A durometer neoprene is commonly 
used. This information, coupled with a backing selection chart from F.N. Sheppard, lead to the selection of quarter inch thick 40 Shore A durometer neoprene being selected for the backing.

One of the services which F.N. Sheppard provided was the application of the Teflon coating on belts and the application of low friction fabrics. Initially, Teflon coating was chosen for the area were the tread and manifold would come into contact because the bottom of the manifold would also be coated in Teflon. This would have made the coefficient of friction between the tread and manifold around 0.04 , which is extremely low. After consulting with the F.N. Sheppard representative it was discovered that the Teflon coating could not be applied to the area of the belt were the manifold would be traveling. This meant that a low friction fabric would be applied to this area instead. No specific data was given for the properties of the low friction fabric, but the F.N. Sheppard representative was confident that the fabric would ensure that the friction between the manifold and tread would be lower than the maximum allowable force.

With all of the belt materials and tooth pattern chosen, the final portion of the belt system that was designed was the physical design of the belt. This included the width of the belt, the length of the belt, where the belt teeth should be removed, if an indented channel should be cut in the belt for the manifold and the perforation pattern layout. The first and easiest of these was the determination of where in the belt the teeth should be removed. Because the tread would undergo lateral forces during skid steering, it made sense that the teeth should be removed in the center of the tread. This left teeth on both sides of the tread which could then be used to align the tread with the wheels, align the manifold with the perforations in the tread and allow the wheels to carry the lateral loads of the skid steering through the teeth.

To determine the width of the area where teeth were removed, and by extension the width of the tread itself, the next physical parameter determined about the tread was the pattern of perforations. Initially, a slotted pattern similar to the proof of concept models pattern was designed. Slots were chosen in an attempt to increase the area exposed to the vacuum while also ensuring that the area of the tread exposed to the vacuum was minimized. An image of the initial slotted pattern can be found in Figure 22. After consulting with F.N. Sheppard it was discovered that using a slotted pattern would increase the cost of the belts dramatically. So the slotted perforations were abandoned in favor of circular perforations. F.N Sheppard offered several different standard circular hole patterns, with various diameters and pitches, which were 
more cost effective than the slotted pattern. To determine which pattern would provide the most consistent vacuum force at the proper magnitude, a test assembly was created in Solidwoks. Using this assembly, different potential hole patterns were tested against each other. The full details of this test assembly and the methods used to determine the final hole pattern can be found in Appendix A. The final pattern which was decided upon three rows of the quarter inch holes at a half inch pitch along the rows. The rows were staggered relative to one another to allow for the overall width of the pattern to be minimized. Table 13 contains the projected performance information gathered from the Solidworks test assembly. Figure 23 contains an image of the final perforation pattern selected for the tread and a detailed drawing of the treads perforation pattern can be found in the drawing package which accompanies this report.

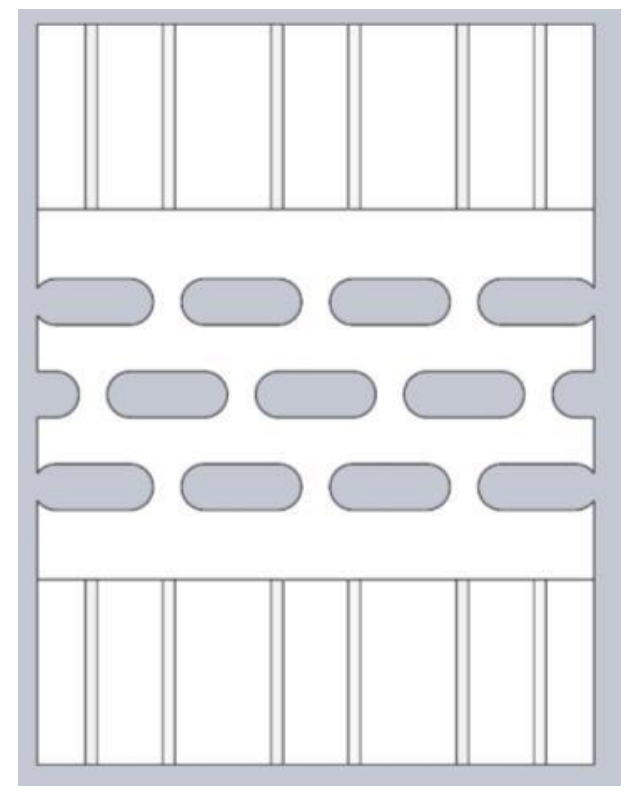

Figure 22: Original slotted pattern chosen for the tread.

Table 13: Hole pattern dimensions of the final tread design and the projected area of the wall exposed to the treads.

\begin{tabular}{|l|r|}
\hline \multicolumn{1}{|c|}{ Parameter } & \multicolumn{1}{c|}{ Value } \\
\hline Hole Diameter & 0.25 in \\
\hline Number of Rows & 3 \\
\hline Pitch in Row & 0.5 in \\
\hline Projected Average Area of Wall Exposed to the Manifold & $2.24 \mathrm{in}^{\wedge} 2$ \\
\hline Projected Vacuum Force & $30.3 \mathrm{lb}$ \\
\hline Maximum Pattern Width & $0.75 \mathrm{in}^{\wedge} 2$ \\
\hline
\end{tabular}




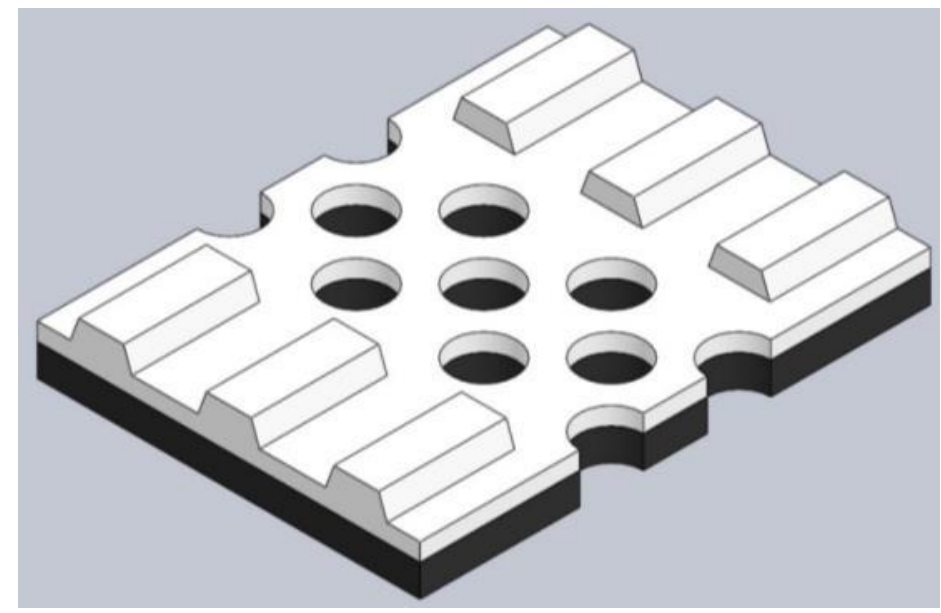

Figure 23: Solidworks model of the final tread hole pattern.

With the width of the perforation pattern determined, the width of the area where the teeth were removed from the belt could be determined. It was decided that an eighth of an inch gap should be left on either side of the tread pattern. This was to both ensure that the perforations in the tread were completely covered by the manifold, even if the manifold shifted relative to the tread, and to allow for continuous reinforcement fibers to remain in the area where teeth were removed. This meant that the section where the teeth were removed would be one inch wide. It was decided that the overall tread width would be two inches wide.

This was to ensure that the teeth of the tread and wheels would have a large are over which to contact.

The determination of the belts length was relatively easy. After a Solidworks layout of the drive and back idler wheel was created, the belt tool in Solidworks was used to determine the pitch length of the belt which would snuggly fit around the wheels. With this number in hand, the standard neoprene belt lengths produced by F.N. Sheppard were consulted. A belt that was slightly longer than the Solidmodels predicted length was then chosen to allow the tread to be placed around the wheels without difficulty. The belt length also determined the number of teeth on the belt. Table 14 contains the predicted required belt length by Solidworks, as well as the selected length of the final belt and number of teeth on the final belt.

Table 14: Predicted and actual pitch length of the final tread design, as well as the number of teeth on the final tread design.

\begin{tabular}{|l|r|}
\hline \multicolumn{1}{|c|}{ Parameter } & \multicolumn{1}{c|}{ Value } \\
\hline Solidworks Minimum Predicted Length & 33.8 in. \\
\hline Final Belt Length & 34.0 in \\
\hline Number of Teeth & 68 \\
\hline
\end{tabular}




\subsection{Subassembly Design}

This section contains descriptions of the design process of the subassemblies of the vehicle and their components. This includes the criterion used to design each component, the process used to select the final design of each component and the process used to validate the final design of the components. A complete solid model package of all components manufactured at Cal Poly, as well as their respective drawings, can be found in files which accompany this report

\subsubsection{Frame Subsystem}

The frame subassembly was key to the functionality of the vehicle as a whole. It provided attachment points for all of the other major subsystems of the design and gave the vehicle its rigidity. Therefore it neededmust be strong enough to withstand the forces of operation as well as light enough to allow for the other subsystems weight. Figure 24 shows the frame subsystem.

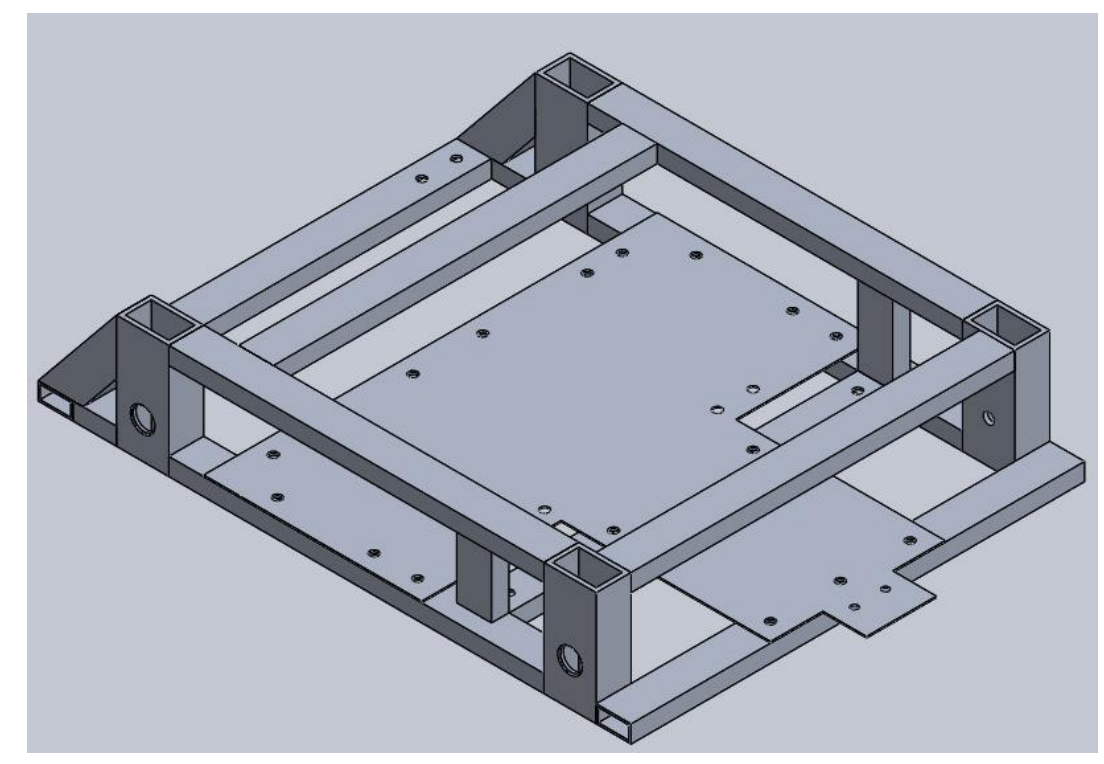

Figure 24: Solidworks model of the frame subsystem.

\subsubsection{Frame}

The frame of the vehicle was required to be both rigid and light weight, while providing sufficient space and attachment points for the other subassemblies. The first step in the design of the frame was determining what type of material the frame would be made of. Three different possibilities where conceptualized. These were: a sheet metal frame, an aluminum tubular frame and an aluminum t-slot frame. Each of these 
concepts was compared to the others to determine which would best suit the projects requirements. This comparison can be found in Table 15.

Table 15: Decision matrix used to determine the frame material used in the project.

\begin{tabular}{|c|c|c|c|c|c|}
\hline Criteria & Criteria Description & $\begin{array}{c}\text { Criteria } \\
\text { Multiplier }\end{array}$ & $\begin{array}{l}\text { Sheet } \\
\text { Metal }\end{array}$ & Tubular & T-Slot \\
\hline Weight & $\begin{array}{l}\text { The overall weight of the } \\
\text { frame. }\end{array}$ & 6 & 2 & 3 & 1 \\
\hline Manufacturability & $\begin{array}{l}\text { How easy to manufacture the } \\
\text { frame is. }\end{array}$ & 2 & 1 & 2 & 3 \\
\hline Precision & $\begin{array}{l}\text { How precisely the frame can } \\
\text { be manufactured. }\end{array}$ & 4 & 1 & 2 & 3 \\
\hline Stiffness & $\begin{array}{l}\text { How stiff the final frame } \\
\text { could be made. }\end{array}$ & 5 & 1 & 2 & 3 \\
\hline Cost & $\begin{array}{l}\text { How little the frame would } \\
\text { cost to make. }\end{array}$ & 1 & 3 & 2 & 1 \\
\hline Attachment & $\begin{array}{l}\text { How easy attachment of } \\
\text { other systems to the frame } \\
\text { would be. }\end{array}$ & 3 & 1 & 3 & 2 \\
\hline Total & & & 29 & 51 & 46 \\
\hline
\end{tabular}

As seen above the tubular frame was superior to the other two concepts. With the general material of the frame chosen, the specifics of the frame were then designed. The tubing selected to make up the frame can be found in Table 16. To ensure that the internal components of the vehicle would fit into the frame, the first thing that was designed for the frame was the internal dimensions. The height of the frame needed to accommodate the motors and the banks of venturis with enough room to make adjustments to the internal components easily. The width was chosen to accommodate both the controller and attached pressure sensors as wells at to allow for different motors to be used if the chosen motors did not perform as expected. Finally, the length was chosen based on the theoretical length of internal component layout.

Table 16: Tubing selected to make up the vehicle frame.

\begin{tabular}{|l|l|r|r|}
\hline \multicolumn{1}{|c|}{ Location Used } & Material Type & Outside Dimensions & \multicolumn{1}{c|}{ Wall Thickness } \\
\hline Wheel Uprights & 6061-T6 Aluminum & 1 ” 0.5 ” & $0.125^{\circ}$ \\
\hline $\begin{array}{l}\text { All Other Frame } \\
\text { Members }\end{array}$ & 6061-T6 Aluminum & 0.5 ” 0.25 ” & 0.0625 ” \\
\hline
\end{tabular}

An assembly of the frame was created using the above tubing dimensions and the required internal dimensions of the frame. This layout was refined to the final design in two different ways, the first being the creation of an assembly in Solidworks which allowed different components to be placed in the frame. This allowed measurements between components to be taken and adjustments to be made to ensure that there was sufficient room in the frame to accommodate all of the components and that those components 
could be easily interacted with when in the frame. This was also the point at which the locations for the bolts holes on the frame were determined.

The second refinement method employed was the use of Solidworks Simulation Tools to perform finite element analysis on the frame to ensure that it would be able to withstand the expected loading that would be applied to it during operation. This step did not result in any major changes to the design of the frame, but did serve to validate the frames final design. The methodology and the detailed results of this analysis can be found in Appendix A.

One concern that was raised during the design of the frame was the method of joining to be used to attach the frames struts together. Two methods were proposed, the first of which was the use of brackets at the frame's joints. This method was not selected as it would increase the overall weight of the frame dramatically. The second method, and the method chosen, was to weld the frames components together. This would add relatively little weight to the frame while providing solid connections between the frames struts. The main drawback to welding aluminum is degradation of strength at the welded sites, but this was accounted for in the analysis found in Appendix A. The final design of the frame can be seen in Figure 25. The final dimensions of the frame, the weight of the frame and the summary results of the finite element analysis of the frame can be found in Table 17.

Table 17: Final dimensions, weight and the simulation results for the frame.

\begin{tabular}{|l|r|}
\hline \multicolumn{1}{|c|}{ Parameter } & Value \\
\hline Dimensions (L x W x H) & 17.0 in. x 13.0 in. x 3.25 in. \\
\hline Predicted Weight & $3.44 \mathrm{lbs}$ \\
\hline Maximum Von Mises Stress & $4436.5 \mathrm{psi}$ \\
\hline Maximum Displacement & $0.002 \mathrm{in}$. \\
\hline Minimum Factor of Safety & 7.4 \\
\hline
\end{tabular}

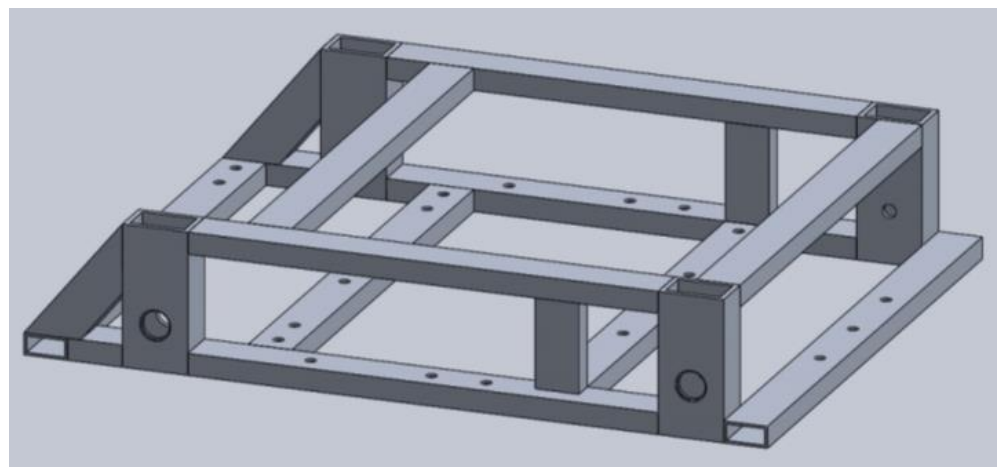

Figure 25: Solid model of the final frame design. 


\subsubsection{Base Plate}

The base plate provides attachment points and support for some of the interior components of the vehicle.

These included the controller, pressure sensors and vacuum components. The base plate was designed after the frame design was finalized, which meant that most of the dimensional constraints of the plate were already set. This also dictated the hole pattern that was required for the plate. This meant that the only major design decisions that were made for the base plate was the material used in its construction and the thickness of the plate. To keep the overall weight of the plate lower while keeping the plate relatively stiff, 6061-T6 aluminum was chosen as the material for the design. The plate was assumed to carry low loads when the vehicle was assembled. This meant that the weight of the plate was the primary driver in the selection of the thickness of the plate. The final design of the Base Plate can be seen in Figure 26. The final dimensions of the plate, the weight of the plate and the summary results of the finite element analysis of the plate can be found in Table 18 .

Table 18: Final dimensions and weight of the base plate.

\begin{tabular}{|l|r|}
\hline \multicolumn{1}{|c|}{ Parameter } & Value \\
\hline Length & $13.5{ }^{\prime \prime}$ \\
\hline Width & $13.0^{\prime \prime}$ \\
\hline Thickness & $0.4{ }^{\prime \prime}$ \\
\hline Weight & $0.44 \mathrm{lbs}$ \\
\hline
\end{tabular}

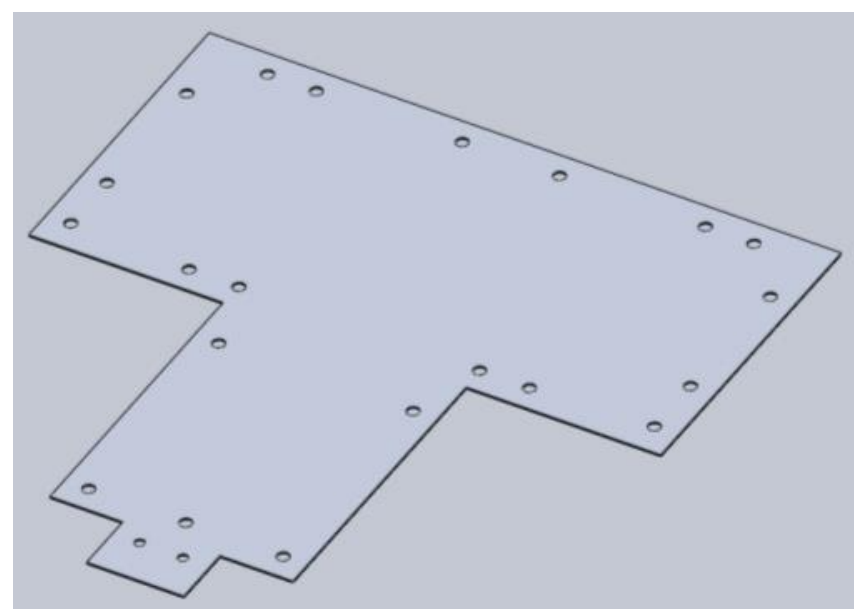

Figure 26: Solid model of the final base plate design. 


\subsubsection{Motor Subsystem}

The motor subassembly served several different functions for the vehicle. These functions are the alignment of the motors shafts with the drive wheel shafts, the attachment of the motor to the vehicle and the coupling of the motor shafts to the drive wheel shafts. There are three components which make up the motor assembly excluding the motor itself. These components are the motor mounting bracket, the motor base plate and the shaft coupler. An image of the motor subsystem including the motor can be found in

Figure 27.

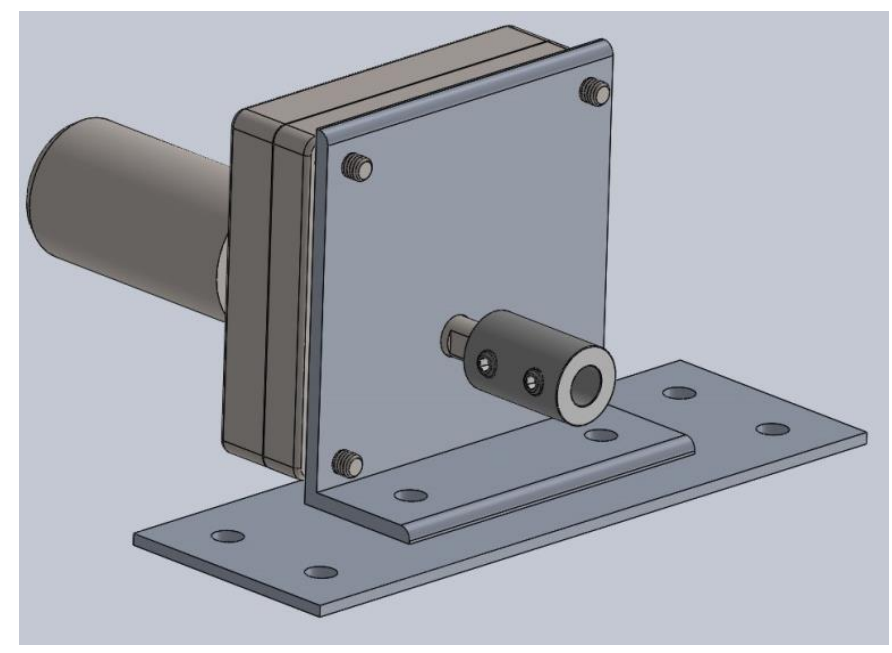

Figure 27: Solid model of the final motor subsystem.

\subsubsection{Motor Mounting Bracket}

The motor mounting bracket attaches directly to the motor. There were several factors that were taken into consideration when designing the bracket such as the stiffness of the bracket, the weight of the bracket, the ability of the bracket to withstand the operating loads applied to it by the motor and the precision required in the alignment of the motor and drive shafts.

Because 6061-T6 aluminum is lightweight, stiff and easy to machine it was chosen as the material for the bracket. An L-Shape was chosen for general shape of the bracket as this would allow for convenient mounting of both the motor to the bracket and the bracket to the mounting plate, as well as allowing easy access to the shafts after assembly. 
With the shape and material of the bracket selected, the dimensions of the bracket were then determined. A hole pattern corresponding to the mounting bolts and shaft of the motor was laid out on the upright portion of the bracket. This pattern dictated the height of upright leg as well as the brackets width. The final step in the design of the mounting bracket was performing a simulation to verify that the final design would be able to handle the forces applied to it during the operation of the motor. The methodology and the detailed results of this simulation can be found in Appendix A. Figure 28 contains an image of the final design of the motor mounting bracket. The final weight, dimensions and verification results of the mounting bracket can be found in Table 19 .

Table 19: Final dimensions, weight and simulation results of the motor mounting bracket.

\begin{tabular}{|l|r|}
\hline \multicolumn{1}{|c|}{ Parameter } & \multicolumn{1}{|c|}{ Value } \\
\hline Width & $3.00 "$ \\
\hline Thickness & $0.125^{\prime \prime}$ \\
\hline Motor Leg Length & $3.0{ }^{\prime \prime}$ \\
\hline Base Leg Length & 1.125 \\
\hline Weight & $0.14 \mathrm{lbs}$ \\
\hline Maximum Von Mises Stress & $6390 \mathrm{psi}$ \\
\hline Maximum Displacement & $0.0035 \mathrm{in}$. \\
\hline Minimum Factor of Safety & 6.2 \\
\hline
\end{tabular}

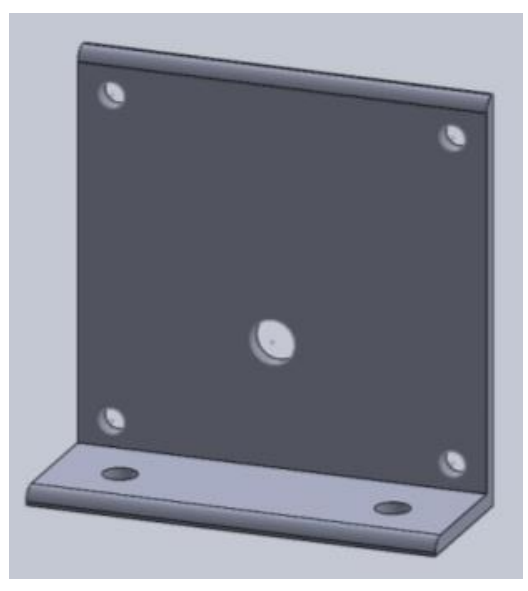

Figure 28: Solidworks model of the final motor mounting bracket design.

\subsubsection{Motor Base Plate}

The motor base plate acts as the attachment point between the motor mounting bracket and the vehicles frame. It also serves to align the motor shafts and the drive wheel shafts along the length of the vehicle. The main considerations for the plate were its mass, its stiffness and the precision of the shaft alignment. 
The length of the attachment plate as well as the bolt hole positioning for the frame to plate attachment points were dictated by the frame. The width of the plate was chosen to allow two bolts to be used to attach the plate to the frame without the bolts interfering.

Because the motors chosen did not perfectly mirror one another when flipped, two versions of the motor base plate were designed. The only difference between these two designs was the position of the bracket to plate bolt holes along the length of the plate, which were positioned in such a way that the motor shafts and drive shafts on both sides of the vehicle were aligned. The holes for attaching the motor bracket to the plate were positioned along the width of the plate to ensure that there was not interference between the motor shafts and the drive shafts when the motors were installed.

The thickness of the plate was determined by simulating the pate under operating conditions to ensure that a small amount of deflection occurred in the plate. This also served as the verification of 6061-T6 aluminum as the material choice and as the verification of the final design. The methodology and the detailed results of this simulation can be found in Appendix A. The final design of the Motor Base Plate can be seen in Figure 29. The final dimensions of the plate, the weight of the plate and the summary results of the simulation of the plate can be found in Table 20.

Table 20: Final dimensions, weight and simulation results for the motor plates.

\begin{tabular}{|l|r|}
\hline \multicolumn{1}{|c|}{ Parameter } & Value \\
\hline Dimensions (L x W x T) & 5.75 in x 2.00 in x 0.094 in \\
\hline Weight & $.1025 \mathrm{lbs}$ \\
\hline Maximum Von Mises Stress & $11,525 \mathrm{psi}$ \\
\hline Maximum Displacement & $0.005 \mathrm{in}$. \\
\hline Minimum Factor of Safety & 3.5 \\
\hline
\end{tabular}

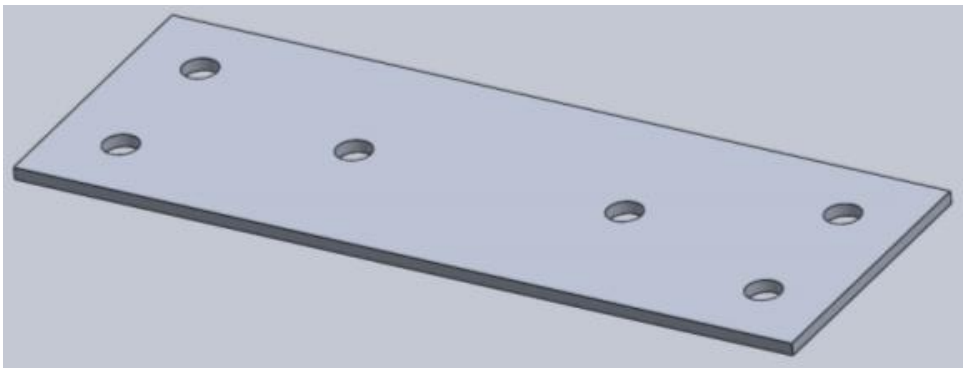

Figure 29: Final design for the left side motor plate. 


\subsubsection{Shaft Coupler Selection}

A shaft coupler was required to connect the motor's shaft with the drive wheel shaft. Several major factors contributed to the selection of the coupler for the final design, which were the size of the coupler, the diameter of the shafts being coupled, the shaft type being coupled and the loading the coupler would be likely to experience.

The length and outer diameter of the coupler was a concern, because there was limited space in the area designated for the coupler. Additionally, a smaller coupler was desired to minimize mass and the moment of inertia that was driven by the motor.

The output shafts of the chosen motors were $5 / 16$ " in diameter and were D-shaped. In an effort to simplify the selection process of the couplers, the portion of the drive shaft that coupled to the motors was designed to be $5 / 16$ " in diameter and D-shaped. Because the shafts were D-shaped, a coupler which used set screws was ideal, as set screws would provide good grip on the flats of the shafts and be easy to tighten and loosen when the shaft coupler was installed.

The coupler used in the final design was a black-oxide steel set screw rigid shaft coupler from Mcmaster Carr. This coupler met all of above criterion and was cost effective. The detail of the coupler can be found in Table 21.

Table 21: Parameters associated with the selected coupler.

\begin{tabular}{|l|r|}
\hline \multicolumn{1}{|c|}{ Parameter } & Value \\
\hline Outer Diameter & $5 / 8 "$ \\
\hline Length & $1 "$ \\
\hline Max Torque & 86 in.-lbs \\
\hline Factor of Safety & 1.7 \\
\hline
\end{tabular}

\subsubsection{Vacuum Subsystem}

The vacuum system is used to generate the vacuum used to adhere the vehicle to different surfaces. In addition to the venturis and the manifolds which are discussed in the separate sections, several different components make up the vacuum system of the vehicle. These are the vehicle inlet manifold and the brackets which hold the venturis. 


\subsubsection{Vehicle Inlet Manifold}

The purpose of the vehicle inlet manifold is to take in positive air pressure and distribute it to the inlets of the venturis. It consists of four different parts: a straight push-to-connect fitting, two 4 outlet swivel pushto-connect manifolds, a nylon manifold and a hex head plug. All of these components were required to handle the $80 \mathrm{psi}$ that the venturis required to operate.

The push-to-connect fittings were chosen to make assembly and disassembly of the vehicle easier. The straight push-to-connect fitting is used to connect the inlet of the manifold with the incoming positive pressure supply line. The two 4 port push-to-connect manifolds were chosen because each would be connected to four of the venturis. The details of both of the push-to-connect chosen can be found in Table 22.

Table 22: Information on the fittings used in the inlet manifold.

\begin{tabular}{|l|r|r|}
\hline \multicolumn{1}{|c|}{ Parameters } & $\begin{array}{c}\text { Four port push-to-connect } \\
\text { manifold }\end{array}$ & \multicolumn{2}{c|}{$\begin{array}{c}\text { Single Port push-to-connect straight } \\
\text { adaptor }\end{array}$} \\
\hline Number of Ports & 4 & 1 \\
\hline Tubing OD & $1 / 4 "$ & $3 / 8 "$ \\
\hline Thread Type & $1 / 8$ "NPT Male & $1 / 4$ " NPT Male \\
\hline Maximum Pressure & $145 \mathrm{psi}$ & $290 \mathrm{psi}$ \\
\hline
\end{tabular}

The nylon manifold served to securely connect the main air supply line to the outputs for the venturi.

Several material options were available such as aluminum and steel, but nylon was chosen to reduce weight. The manifold had two outlet ports and two inlet ports, and was chosen such that the ports matched the threads for the push-to-connect fittings. The hex head plug was used to block the unused inlet of the nylon manifold. The details about the nylon manifold can be found in Table 23, and an assembled view of the inlet manifold can be found in Figure 30.

Table 23: Information on the nylon manifold.

\begin{tabular}{|l|r|}
\hline \multicolumn{1}{|c|}{ Parameter } & Value \\
\hline Dimensions & 1.75 " x 1" x 1" \\
\hline Max Allowable Pressure & $200 \mathrm{psi}$ \\
\hline Inlet Pipe Size & $1 / 4$ " NPT \\
\hline Outlet Pipe Size & $1 / 8$ " NPT \\
\hline
\end{tabular}




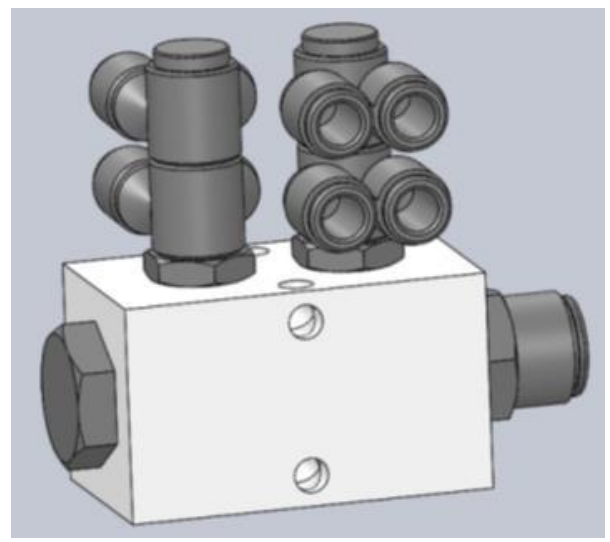

Figure 30: Inlet manifold of the vehicle.

\subsubsection{Venturi Mounting Brackets}

The purpose the venturi mounting bracket was to hold the venturis rigidly, while still allowing access to the inputs and outputs of the venturis. Each bracket was designed to hold four venturis which would feed into the same manifold. The bracket consisted of a sheet of 6061-T6 aluminum bent into an L-shape. The long leg of the bracket was used as the mounting point for the venturis, with a square pattern of tightly grouped holes which allowed the bracket to be relatively small while allowing the venturis to be easily manipulated and attached. The short leg had two mounting holes so that he brackets could be attached to the frame.

Table 24 contains details about the brackets. Figure 31 shows and image of the venturi mounting bracket without the venturis installed.

Table 24: Final dimensions and weight of the venturi mounting bracket.

\begin{tabular}{|l|r|}
\hline \multicolumn{1}{|c|}{ Parameter } & Value \\
\hline Width & $1.725^{\prime \prime}$ \\
\hline Plate Thickness & $0.0625^{\prime \prime}$ \\
\hline Base Leg Length & $1.0{ }^{\prime \prime}$ \\
\hline Venturi Leg Length & $2.0625^{\prime}$ \\
\hline Weight & $0.03 \mathrm{lbs}$ \\
\hline
\end{tabular}




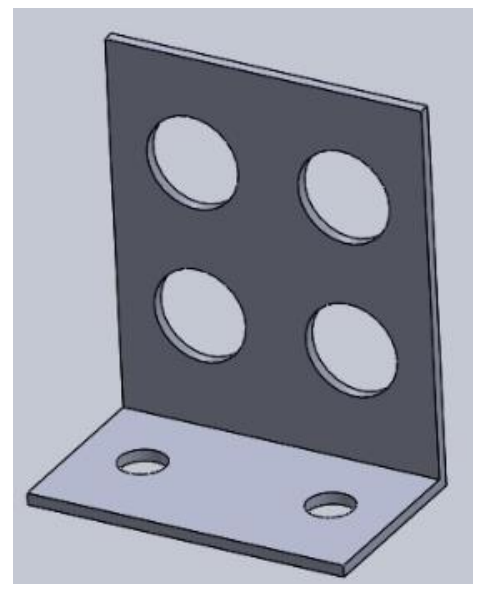

Figure 31: Solid model of the venturi mounting bracket.

\subsubsection{Vacuum Manifold Subsystem}

The vacuum manifold subsystem's purpose was to distribute vacuum across the tread to adhere the vehicle to a surface. This subsystem also included the means by which the manifold is held firmly to the tread during operation and the means by which the manifold is attached to the vehicle body. An image of the manifold subsystem can be found in Figure 32.

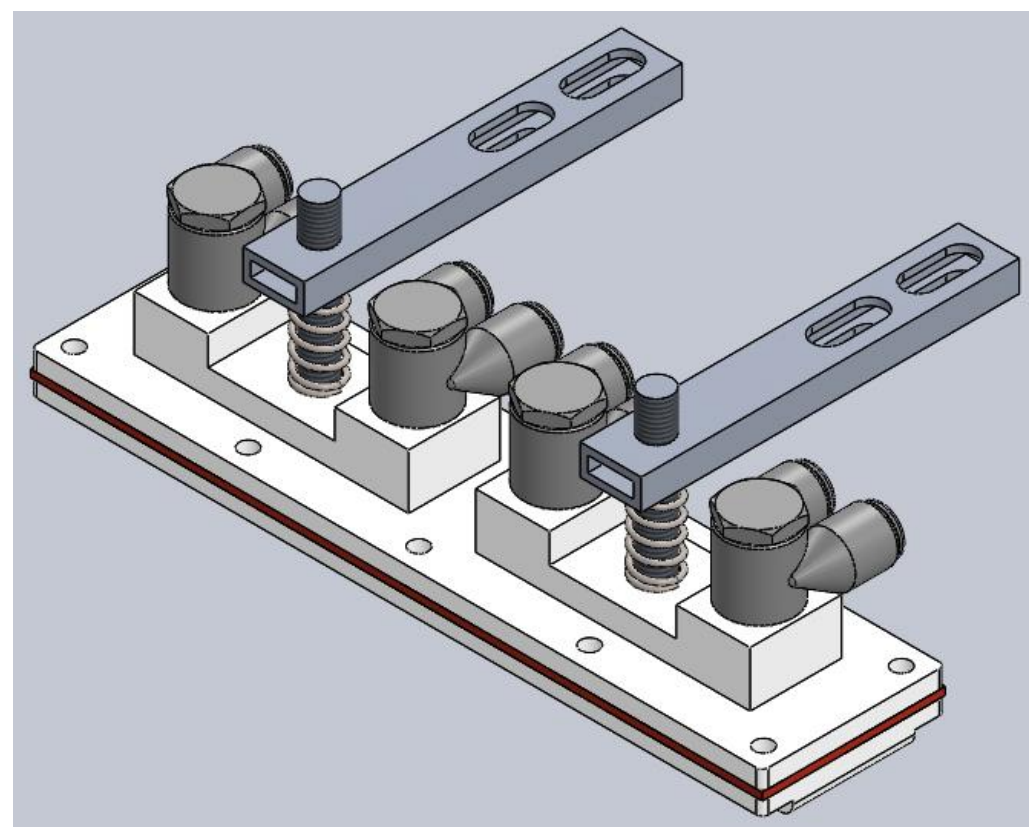

Figure 32: Solid model of the vacuum manifold. 


\subsubsection{Lower Manifold}

The lower manifold was the portion of the manifold subsystem which directly contacts the tread. The lower manifold contains four chambers which are connected to one of the four venturis on a given side. Slots are cut at the bottom of each chamber to allow for the vacuum to pass between the manifold and the wall.

The first step in designing the lower manifold was choosing its material. The chosen material needed to be lightweight and have a low coefficient of friction when in contact with the tread. Initially, Teflon was chosen as it is extremely lightweight and slick, but it proved extremely difficult to machine and was prone to warpage. This led to Delrin being chosen because it is lightweight, easy to machine and is generally stronger that Teflon.

The next step in designing the lower manifold was to determine the basic shape and dimensions that it would need to take. The length of the lower manifold was chosen so that the manifold would both cover as much of the tread as possible while also not interfering with the tensioning system or the drive wheel. Because the lower manifold needed to be in contact with the tread at all times, a two tiered shape was chosen and can be seen in Figure 33. The lower tier was designed to ride in the channel cut into the treads teeth. This meant that the width of the lower tier was smaller than that of the width of the channel in an attempt to prevent any contact between the teeth of the tread and the lower manifold. The thickness of the lower tier was chosen to give the higher tier clearance above the tread's teeth, as the upper tier is flared over the tread teeth. The upper tier is flared to allow for tapped holes to be placed along its outer edge without interfering with the gasket placed between the upper and lower manifold. The thickness of the upper tier was chosen to allow for good thread engagement of the bolts which hold the upper and lower manifold together.

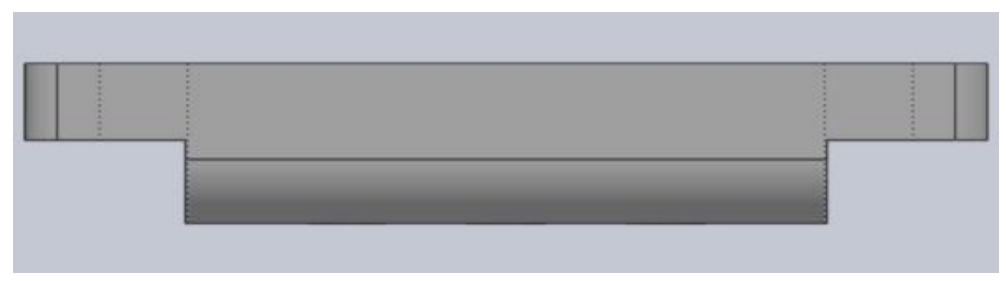

Figure 33: View of the tiered shape of the lower manifold. 
The next step in design of the manifold was the layout of the vacuum chambers and their dimensions. It was important that each of the vacuum chambers remained independent of one another to prevent the failure of one chamber from affecting the others. Because the diameter of the holes in the tread was known, the minimum distance between chambers was calculated to ensure that no hole could be engaged by more than one chamber at a time. This value would also ensure a hole could not be in contact with the atmosphere and engaged with the first or last chamber in the manifold. A margin of error was included into this distance for added insurance and this value was used to section the lower manifold to determine the length of the chambers. The width of the chambers was chosen such that a wall thickness of a tenth of an inch was between both sides of the chambers and the outside atmosphere. The depth of the chambers was chosen to ensure that the material between the slots in the manifold would not crack or warp when in use.

The final portion of the design was the design of the slotted pattern in the bottoms of the vacuum chambers. Because there were three rows of holes in the tread three slots along the width of the vacuum chambers.

These slots were laid out along the width such that their center lines corresponded to the center lines of the rows of holes on the tread. The width of the slots was chosen to be smaller than the diameter of the holes in the tread. This was to minimize the amount of the tread exposed to the vacuum in the manifold in an effort to reduce tread-manifold friction. The length of the slots was chosen such that any given time the number of holes exposed to the vacuum remained relatively constant and that enough holes were exposed to the vacuum that the minimum force between the wall and the vehicle was always met. Details on the method used to determine the length of the slots can be found in Appendix A. The final design of the lower manifold can be found in Figure 34. Table 25 contains details about the lower manifold such as the weight, final dimensions and calculation results.

Table 25: Major dimensions and weight of the lower manifold.

\begin{tabular}{|l|r|}
\hline \multicolumn{1}{|c|}{ Parameter } & Value \\
\hline Over All Dimensions (L x W x H) & 6 in x 1.5 in x .25 in \\
\hline Chamber Dimensions (L x W x D) & 1 in x .75 in x .15 in \\
\hline Slot Dimensions (L x W) & 0.825 in x .125 in \\
\hline Weight & $0.06 \mathrm{lb}$ \\
\hline
\end{tabular}




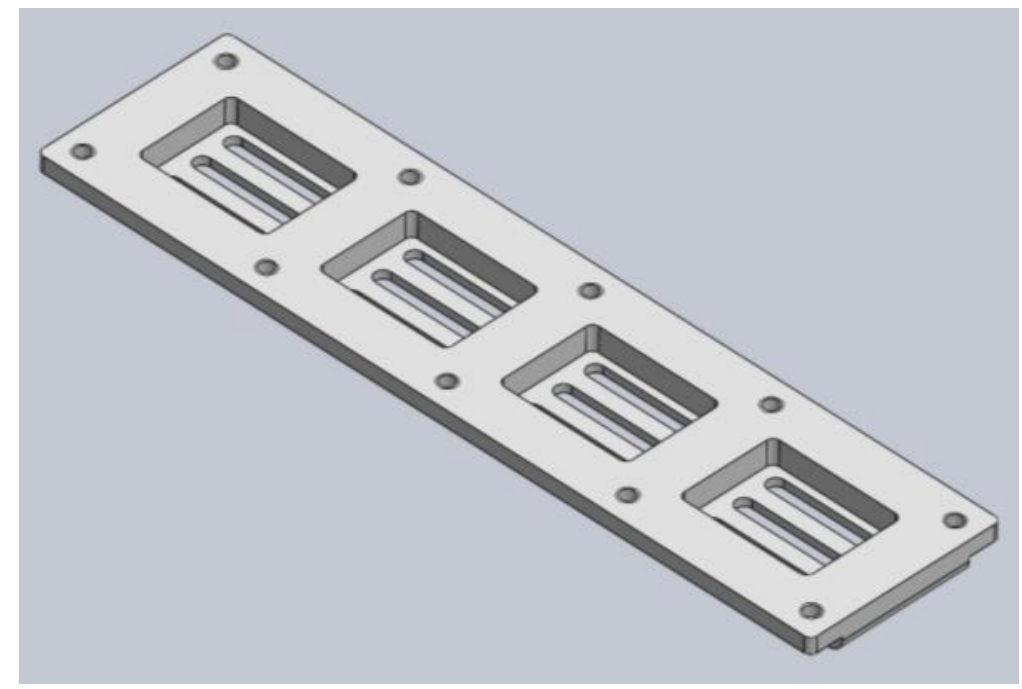

Figure 34: Solid model of the final lower manifold design.

\subsubsection{Upper Manifold Section}

The upper manifold sits on top of the lower manifold. The purpose of the upper manifold was to act as a connection between the lower manifold and the vacuum system, provide attachment points for the mounting structure and to help create a seal with the lower manifold and the gasket.

The material chosen for the upper manifold was chosen based on two criterions. These were the mass of the final product and the ease with which the material could be machined. The two materials considered were aluminum and Delrin. Delrin was chosen because it weighs about half as much as aluminum does and is also easier to machine than aluminum.

The upper manifold had a three tiered design. The first and lowest tier provided a series of through holes corresponding to the threaded holes on the lower manifolds upper tier which allows the two halves of the manifold to be joined. The second tier provides a threaded hole for the uprights used to attach the manifold to the mounting structure. The third tier had NPT threaded holes to allow for the fittings that connect the manifold and the vacuum system to be attached.

The width and length of the first tier are the same as the upper tier of the lower manifold. This made aligning the holes between the upper and lower manifold easier and also simplified the design of the gasket. The thickness of the first tier was to reduce the amount of material required in the upper manifold and to reduce the length of the bolts required to hold the upper and lower manifold together. 
The second tier was raised off the surface of the first tier such that a sufficiently deep hole could be drilled and tapped to accommodate the upright which connected the manifold to the mounting structure. The length of the second tier was chosen such that neither the upright nor the adjustment nut for the upright would interfere with the third tier or the manifold fittings. The width of the second tier was chosen to match that of the third tier to simplify machining.

The third tier was broken up into four separate platforms, with each platform corresponding to a vacuum chamber in the lower manifold. Each platform has a tapped NPT through hole at its center. The third tier's height was chosen to allow for a fully tapped NPT hole to be placed in on each of the four surfaces. The height was also chosen to ensure that the manifold fittings connection points would stand above the surface of the base plate. The width and length of the platforms was chosen to allow for NPT threads to be drilled and tapped without risking cracking any of the platforms. The final design of the upper manifold can be found in Figure 35. Table 26 contains details about the upper manifold such as the weight, final dimensions and calculation results.

Table 26: Dimensions and weight of the final design of the upper manifold.

\begin{tabular}{|l|r|}
\hline \multicolumn{1}{|c|}{ Parameter } & \multicolumn{1}{|c|}{ Value } \\
\hline Over All Dimensions (L x W x H) & 6 in x 1.5 in x 0.6 in \\
\hline First Tier Thickness & 0.15 in \\
\hline Second Tier Dim. (L x W x H) & 5.05 in x 0.75 in x 0.21 in \\
\hline Third Tier Dim. (L x W x H) & .6 in x .75 in x 0.45 in \\
\hline Through Hole Diameter & .1495 \\
\hline Upright Hole Call Out & $1 / 4-20$ \\
\hline Fitting Hole Callout & $1 / 8 \mathrm{NPT}$ \\
\hline Weight & $.1 \mathrm{lb}$ \\
\hline
\end{tabular}

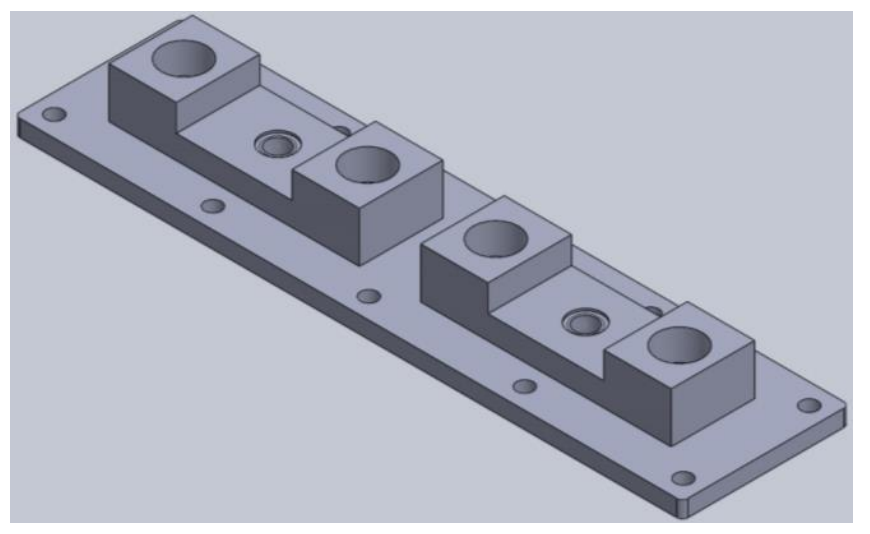

Figure 35: Solid model of the final upper manifold design. 


\subsubsection{Manifold Gasket}

The manifold gasket was designed to help create a seal between the upper and lower manifold halves. Its dimensions were based on the length and the width of the lower manifolds upper tier. Holes positioned at each of the vacuum chamber locations on the lower manifold were cut slightly smaller than the camber dimensions to allow for some overhang into the chambers.

Fiberglass reinforced silicone rubber was chosen as the material for the gasket because silicone provides a good seal and the fiberglass ensures that the sheet does not get squeezed out of place during assembly. The thickness of the sheet was chosen to reduce the amount of additional height added to the manifold by the gasket. The final design of the manifold gasket can be found in Figure 36. Table 27 contains details about the manifold gasket such as the weight, final dimensions and calculation results.

Table 27: Information on the final gasket design.

\begin{tabular}{|l|r|}
\hline \multicolumn{1}{|c|}{ Parameter } & \multicolumn{1}{|c|}{ Value } \\
\hline Dimensions (L x W x T) & 6 in x 1.5 in x 0.0625 in \\
\hline Hardness & $40 \mathrm{D}$ \\
\hline Weight & $.02 \mathrm{lb}$ \\
\hline
\end{tabular}

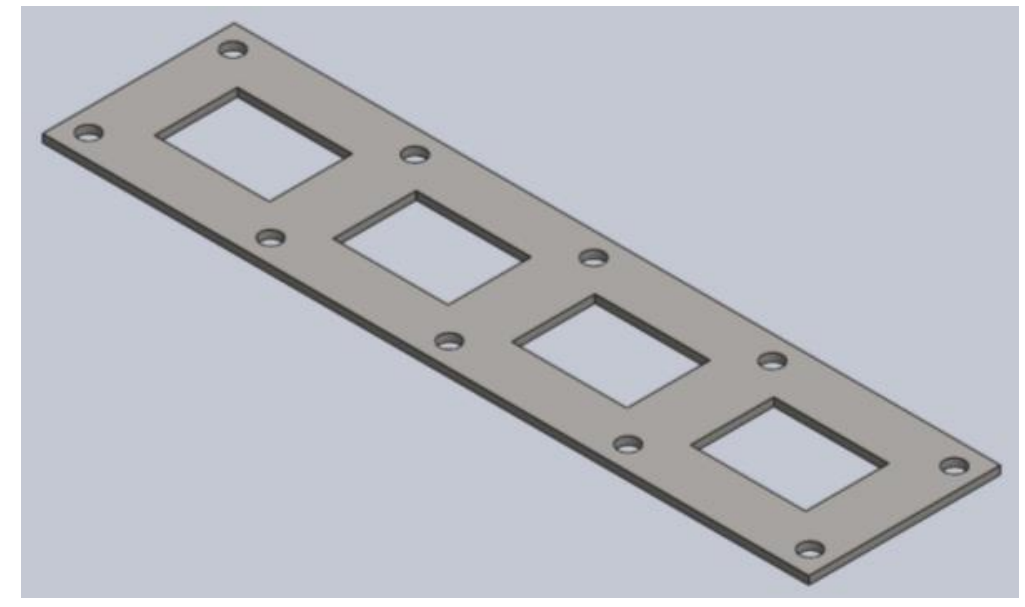

Figure 36: Solid model of the final gasket design.

\subsubsection{Mounting Structure}

The purpose of the mounting structure was to connect the manifold to frame of the vehicle. This structure consisted of two parts, the adjustable struts and the threaded uprights. The adjustable struts were attached directly to the frame of the vehicle and positioned the manifold relative to the channel in the tread. The 
threaded uprights connected the manifold to the struts and position the manifold vertically relative to the tread.

\subsection{Adjustable Struts}

The adjustable struts consisted of two rectangular tubes. These tubes had a trough hole which the threaded uprights could be placed in to at one end and a pair of slots at the other. The purpose of the slots was to allow the struts to be connected to the frame and be adjusted to align the manifold with the channel in the tread. The position of the upright hole relative to the slots was determined using a Solidworks assembly of the vehicle.

A stock aluminum rectangular tube was chosen as the base material for the struts for ease of manufacturing and to reduce weight. The design and material selection were validated by performing a simulation using the expected loading on the struts. The methodology and detailed results of this simulation can be found in Appendix A. The final design of the adjustable struts can be found in Figure 37. Table 28 contains details about the adjustable struts such as the weight, final dimensions and simulation results.

Table 28: Information on the final design of the mounting struts.

\begin{tabular}{|l|r|}
\hline \multicolumn{1}{|c|}{ Parameter } & \multicolumn{1}{|c|}{ Value } \\
\hline Overall Dimensions (L x W x H) & 3.1 in x 0.5 in x 0.25 in. \\
\hline Wall Thickness & 0.0625 in. \\
\hline Hole Diameter & .25 in. \\
\hline Slot Dimensions (L x W) & .6 in. x 0.25 in. \\
\hline Weight & .007 lbs \\
\hline Maximum Von Mises Stress & $11,550 \mathrm{psi}$ \\
\hline Maximum Displacement & 0.004 in. \\
\hline Minimum Factor of Safety & 3.5 \\
\hline
\end{tabular}

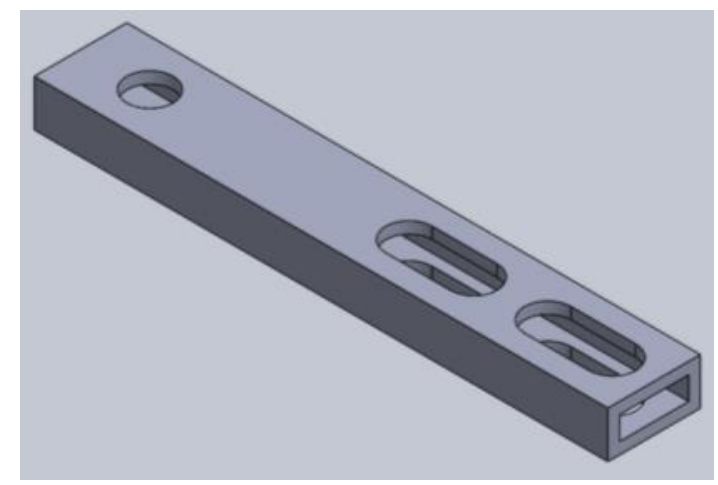

Figure 37: Solid model of the final mounting strut design. 


\subsection{Threaded Uprights}

The threaded uprights were aluminum 1/4-20 threaded rods. These rods were cut a length which would allow the manifold to be pressed firmly against the tread, while remaining short enough to not interfere with the assembly of the vehicle. Table 29 contains details about the threaded uprights such as their weight, final dimensions and simulation results.

Table 29: Information on the threaded upright.

\begin{tabular}{|c|c|}
\hline Parameter & Value \\
\hline Length & $1.5 \mathrm{in}$. \\
\hline Thread Type & $1 / 4-20$ \\
\hline Weight & $0.01 \mathrm{lbf}$ \\
\hline
\end{tabular}

\subsection{Spring}

A spring was placed around the threaded uprights and between the adjustable struts and the upper manifold. This was done to give the manifold subsystem the ability to recover more effectively from losses in vacuum pressure and to give it more compliance to prevent any shocks the vehicle might encounter during operation from causing damage to the manifold or the mounting struts. The spring which was chosen offered a good amount of compressible length combined with a high spring constant. Table 30 contains the details on the spring which was chosen.

Table 30: Information on the suspension spring.

\begin{tabular}{|l|r|}
\hline \multicolumn{1}{|c|}{ Parameter } & \multicolumn{1}{c|}{ Value } \\
\hline Uncompressed Length & $0.75 \mathrm{in}$. \\
\hline Maximum Load & $11.10 \mathrm{lbf}$ \\
\hline Spring Rate & $24.3 \mathrm{lbf} / \mathrm{in}$. \\
\hline
\end{tabular}

\subsubsection{Fittings}

The fittings that were chosen to connect the manifold to the vacuum system were push-to-connect double ninety degree swiveling elbows. These fittings were chosen because they met the vacuum requirements of the system; they rotated which allowed for easy assembly and were push-to-connect which also made assembly easy. A two port version was chosen so that an inlet line to the manifold and a line for the pressure sensors could be run from the same fitting. 


\subsubsection{Wheel Subsystem}

The purpose of the wheel subsystem was to translate motor torque to the tread and to allow the tread to smoothly translate. Two distinct wheel assemblies where used in this system, the drive wheel assembly and the idler wheel assembly. The similarities and differences of these two assemblies will be discussed in this section. Four major components make up the wheel subassembly. These are the bearings, the drive and idler shafts, the drive and idler wheels and the brace. An image of the wheel subsystem can be found in Figure 38.

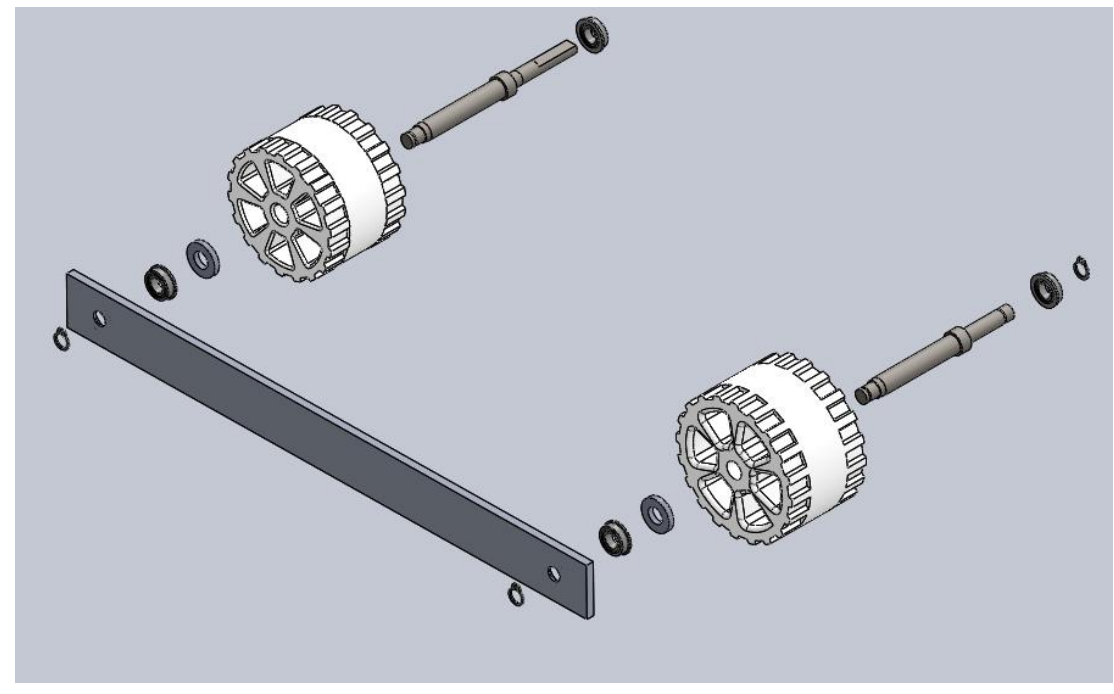

Figure 38: An exploded view of the wheel subsystem.

\subsubsection{Bearing Selection}

The bearings served an important purpose in the wheel subsystem, which was to carry the loading of the shafts while reducing friction. Two different types of bearings were chosen for the system. These were flanged roller bearings capable of taking thrust loads and low friction thrust bearing in the shape of a washer.

\subsection{Flanged Bearing}

The flanged roller bearings were selected for several different reasons. The first and most significant of these reasons was their ability to carry the predicted loads that would be applied to them during operation. This loading was calculated using the predicted belt tension that the system would require to operate. This calculation can be found in Appendix A. The second was its dimensions and shape. The bearing was 
chosen because its shaft diameter was $8 \mathrm{~mm}$ which is close to the diameter of the output shaft of the motor. This simplified the dimensions of the drive shafts and the selection of the coupler between the drive shaft and the motor. The flange on the front of the bearing allowed the bearing to be located precisely, which was required for proper belt alignment.

The bearings mounting holes in the frame and the wheel brace were designed so that the bearings could be easily and securely mounted. An inner hole was sized to snuggly fit the body of the bearing while still allowing the bearing to be pressed into place by hand. A circular indent centered on this hole was cut to a depth and diameter that would allow the flange of the bearing to be recessed making the bearings surface flush with the surface of the frame up rights or of the brace. A retaining compound was used to secure the bearings once they were installed. Figure 39 shows an image of the selected bearing. Table 31 contains details about the chosen flange bearing such as its dimensions, the results of the loading calculation and performance specifications. Table 31 also contains information on the retaining compound used to hold the bearings in place.

Table 31: Information on the selected flange bearing and the retaining compound used.

\begin{tabular}{|l|r|}
\hline \multicolumn{1}{|c|}{ Parameter } & Value \\
\hline Inner Dimeter & $0.315 \mathrm{in}$. \\
\hline Outer Diameter & $0.707 \mathrm{in}$. \\
\hline Thickness & $0.197 \mathrm{in}$. \\
\hline Loading Capacity & $280 \mathrm{lbf}$ \\
\hline Maximum Predicted Loading & $63 \mathrm{lbf}$ \\
\hline Safety Factor & 4.4 \\
\hline Retaining Compound & Loctite 609 \\
\hline Primer & 7649 Primer for Loctite \\
\hline
\end{tabular}

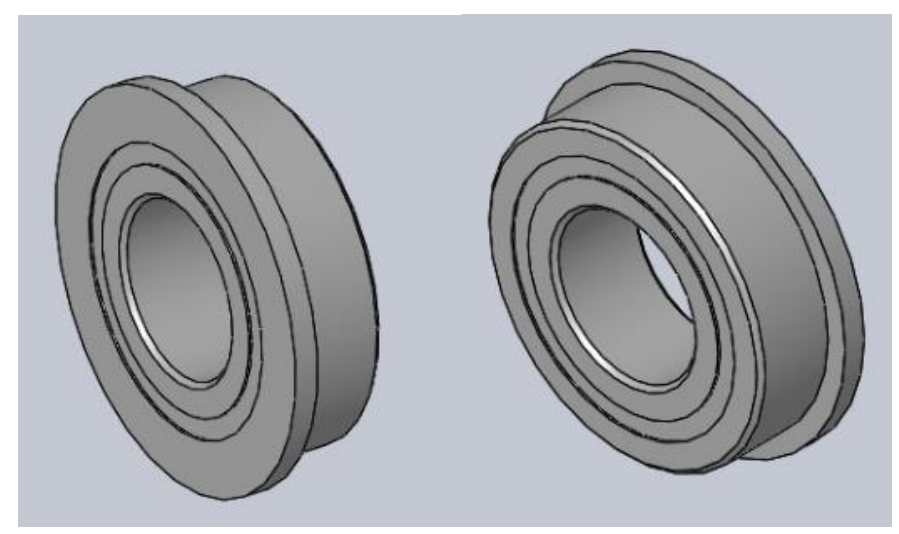

Figure 39: Solidworks model of the chosen flange bearing. 


\subsection{Thrust Washer}

The second bearing that was used in the wheel subassembly was a low friction brass thrust washer. This bearing was chosen for two reasons. The first and most significant reason for its use in the design was to act as a spacer between the wheels of the vehicle and the cross brace of the wheels. This ensured that the tensioning system would not interfere with the cross brace during tensioning and it ensured that the belt would not rub against the cross brace. The second reason that the thrust washer was chosen was to prevent the shafts of the vehicle from rubbing the flange bearings in the cross brace during operation while providing low contact friction. Table 32 contains details about the washer chosen.

Table 32: Information on the chosen thrust washer.

\begin{tabular}{|l|r|}
\hline \multicolumn{1}{|c|}{ Parameter } & Value \\
\hline Inner Diameter & $0.379 \mathrm{in}$. \\
\hline Outer Diameter & $0.75 \mathrm{in}$. \\
\hline Thickness & $0.125 \mathrm{in}$. \\
\hline Load Capacity & $70.0 \mathrm{lbf}$ \\
\hline
\end{tabular}

\subsubsection{Drive and Idler Shafts}

The drive and idler shafts provided rigid attachment points for the wheels for their respective wheels. The drive shafts also transferred torque from the motors to the drive wheels. Several key factors were considered during the design of the shafts. These were the strength of the shafts, the required dimensions of the shafts set by other components, the ease with which the wheels could be attached and positioned on the shafts and the manufacturability of the shafts. As the drive and idler shafts are identical in many respects, the similar features in their designs will be discussed together. A labeled image of the common end of the shafts can be found in Figure 40. Subsections later in this section will discuss their differences.

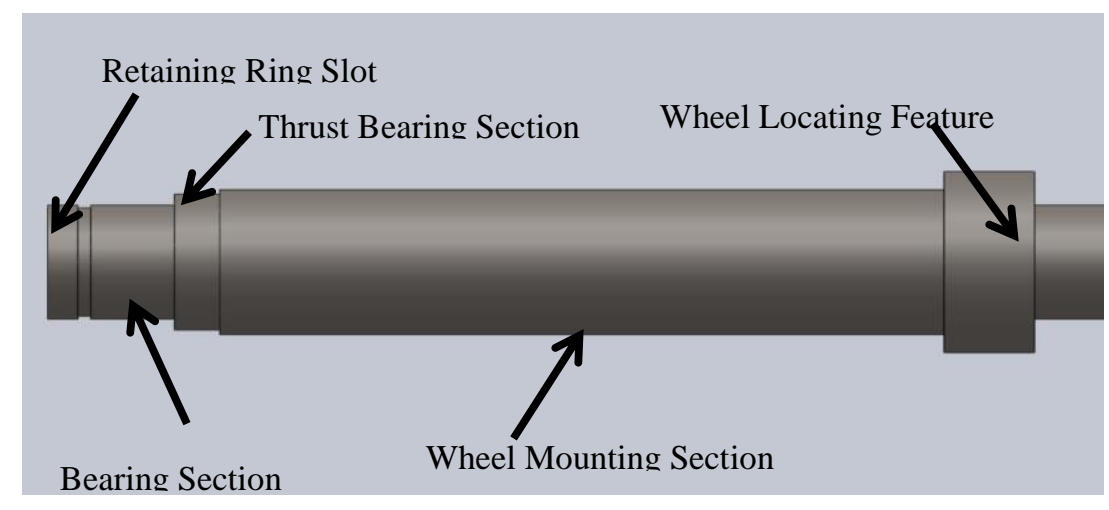

Figure 40: Labeled common shaft end. 


\subsection{Common Shaft End}

The first step taken in designing the common end of the drive and idler shafts was the determination of the dimensions of the shafts and the placement of features on the shafts. Several of these dimensions were already determined by other components in the design. The brace bearing section's diameter was determined by the inner diameter of the flanged bearings and its length was chosen to all for different retaining methods to be used to hold the cross brace in place. The diameter and length of the thrust washer section of the shafts was determined by the thickness and the inner diameter of the thrust washers. The length of the wheel mounting section of the shaft was determined by the width of the wheels. The width of the wheel locating feature was chosen to be similar to that of the thrust washers and its diameter was chosen to extend past the inner running of the flanged bearing.

With most of the common end dimensions selected, the final dimension which needed to be determined was the diameter of the wheel mounting section. This was done by creating shafts with a range of different diameters and simulating the performance of each shaft under the expected loading conditions the shafts would be subjected during operation. The primary parameter which was looked at during this study was the deflection of the shaft under load. The methodology and detailed results of these simulations can be found in Appendix A. The final diameter was chosen because it resulted in small deflections under load while allowing for well-defined steps in the shaft that would be relatively easy to machine.

The material that the shafts would be made from was determined during same simulation that the diameter of the wheel mounting section was determined. This was done by running each simulated diameter with both 6061-T6 aluminum and 1045 medium carbon steel as the material of the shaft. It quickly became apparent that the 6061-T6 aluminum would result in very shaft diameters and so the 1045 steel was chosen. The detailed results and methodology of the simulations can be found in Appendix A.

The final step in the design of the common end of the shafts was to determine the attachment method which would be used to hold the cross brace in place on the shafts. Several different options were considered, but the use of retaining rings was the ultimate choice for several reasons. Retaining rings offered good strength and were easily added to and removed from the shafts without the need to disassemble the vehicle. They also require the least amount of machining on the shafts to install. To ensure the selected ring would work 
in the vehicle, a calculation was performed to determine the rings maximum allowable thrust load. This load was compared to the expected loads that the rings would be subjected to. The details of this calculation can be found in Appendix A. The position of the slot for the retaining ring on the common end was determined by simply measuring the thickness of the cross brace when the flanged bearings were installed. The slot dimensions were based on the manufactures recommendations. Table 33 contains details about the final design of the common shaft end such as the final dimensions of the common end, the final results of the retaining ring calculations.

Table 33: Dimensions of the common shaft end and the results of the retaining ring calculations.

\begin{tabular}{|l|r|}
\hline \multicolumn{1}{|c|}{ Parameter } & Value \\
\hline Wheel Mounting Section Dimensions (L x Dia.) & 2.0 in. x 0.4 in. \\
\hline Thrust Bearing Section Dimensions (L x Dia.) & 0.125 in. x 0.375 in. \\
\hline Brace Bearing Section Dimensions (L x Dia.) & 0.231 in. x 0.315 in. \\
\hline Retaining Ring Slot Dimensions (W x Dia.) & 0.035 in. x 0.299 in. \\
\hline Wheel Locator Dimensions (L x Dia.) & 0.25 in. X 0.5 in. \\
\hline Retaining Ring Factor of Safety & 10.7 \\
\hline
\end{tabular}

\subsection{Drive Shaft}

The design of the non-common end of the drive shaft was relatively simple. It required a portion of the shaft that would fit into the flange bearing and a portion to be coupled to the drive motor.

The length of the non-common end of the drive shafts was determined by the distance from the outside edge of the frame flange bearing to the front end of the motors output shaft. This length was reduced slightly to ensure that the drive shaft would not interfere with the motor shaft and to allow for easy installation and removal of the shaft and motor.

The diameters of these two sections were easily determined as they were set by components which had already been selected. The diameter of the first three quarters of an inch of shaft after the wheel locating feature was $8 \mathrm{~mm}$ in diameter so the shaft would fit snuggly into the flange bearing. The remaining length of shaft was 5/16" in diameter so that it could be inserted into the motor to shaft coupler. An inch at the end of the shaft was D-shaped to ensure the coupler could be attached to the shaft securely. Table 34 contains the details about the final design of the non-common end of the drive shaft and of the final design of the drive shaft such as overall length and weight. Figure 41 shows the final design of the drive shaft. 
Table 34: Final dimension, weight and simulation results for the drive shaft.

\begin{tabular}{|l|r|}
\hline \multicolumn{1}{|c|}{ Parameter } & Value \\
\hline Frame Bearing Section Dimensions (L x Dia.) & 0.25 in. x 0.315 in. \\
\hline Motor Attachment Section Dimensions (L x Dia.) & 1.465 in. x 0.313 in. \\
\hline Weight & $.131 \mathrm{lb}$ \\
\hline Maximum Von Mises Stress & $17,960 \mathrm{psi}$ \\
\hline Maximum Deflection & $0.002 \mathrm{in}$. \\
\hline Minimum Factor of Safety & 4.3 \\
\hline
\end{tabular}

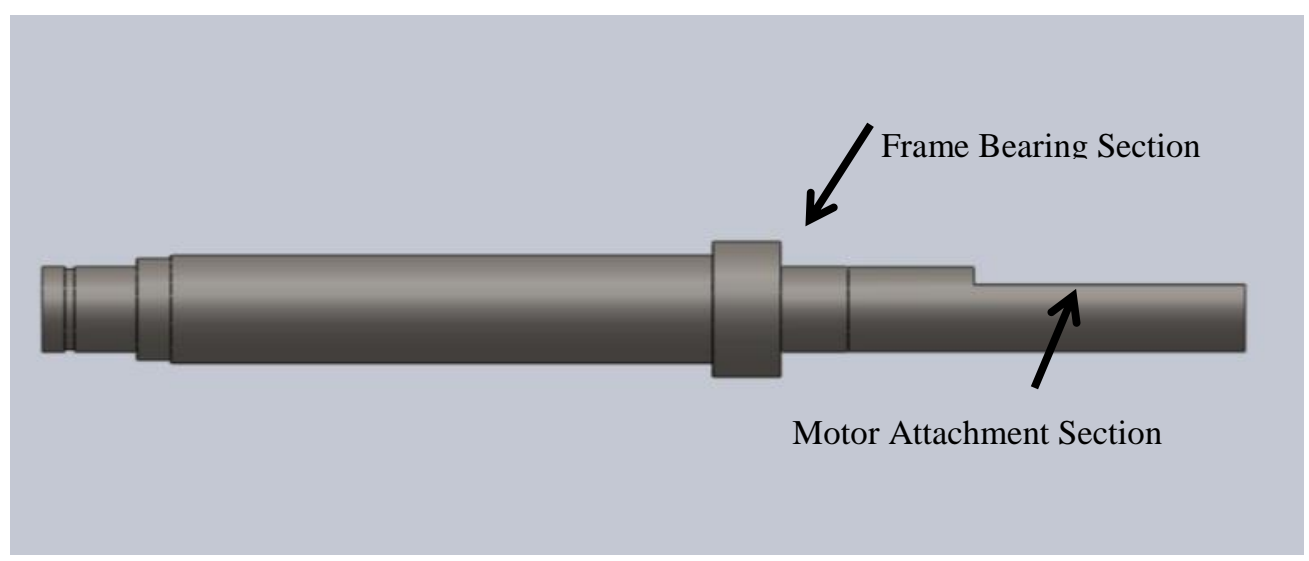

Figure 41: Solid model of the drive shaft.

\subsection{Idler Shaft}

The design of the non-common end of the idler shaft was straight forward. The length of the non-common end was chosen such that, when it was installed, the end of the shaft would protrude past the idler upright. This was to allow for a retaining ring to be installed to hold the shaft in place during operation. This retaining ring was the same ring as was used on the common end of the shafts. Finally the diameter of the shaft was chosen to be $8 \mathrm{~mm}$ so that it would fit snuggly into the flange bearing in the upright. Table 35 contains the details about the final design of the non-common end of the idler shaft and of the final design of the idler shaft such as overall length and weight. Figure 42 shows the final design of the idler shaft.

Table 35: Final dimensions and weight of the idler shaft.

\begin{tabular}{|l|r|}
\hline \multicolumn{1}{|c|}{ Parameter } & \multicolumn{2}{c|}{ Value } \\
\hline Idler End Section Length Dimensions (L x Dia.) & 1.0 in. x 0.315 in. \\
\hline Retaining Ring Slot Dimensions (W x Dia.) & 0.035 in. x 0.299 in. \\
\hline Weight & .124 \\
\hline
\end{tabular}




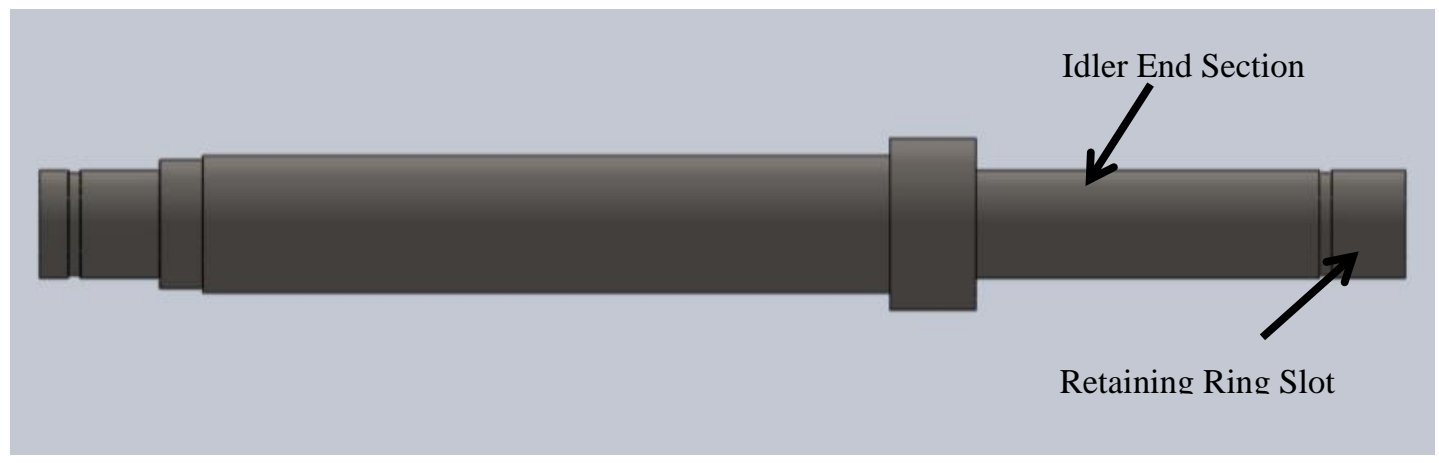

Figure 42: Solid model of the idler wheel shaft.

\subsubsection{Drive and Idler Wheel Design}

The drive and idler wheels were both critical portions of the design. They needed to be light weight, rigid, capable of handling the operating loads they would be subjected to and to be easy to manufacture. The only real difference between the drive wheels and the idler wheels is their diameter and consequently the number of teeth on each wheel.

\subsection{Common Drive and Idler Wheel Design Elements}

The first step in the design of the wheels was to determine if the wheels would be modified pulleys provided by FN Sheppard or if they would be custom designed. Ultimately it was decided that the wheels would be custom designed for several reasons. The pulleys that FN Shepard could provide were solid aluminum. This posed two major problems. The weight of these pulleys would be significant in terms of their contribution to the vehicles overall weight and to the amount of inertia which the motors would need to overcome to move them. The pulleys would also require a significant amount of machining to be usable.

Because of the complex geometries that the wheels would have, 3D printing was determined to be the most time efficient and cost effective option for the manufacturing method of the wheels. This limited the material which could be used to construct the wheels. As the 3D printer most readily available to the project printed in $\mathrm{ABS}$ plastic, $\mathrm{ABS}$ was chosen as the final material of the wheels.

The next step in the design of the wheels was to determine the dimensions of the teeth. This was relatively simply to determine because FN Sheppard provided detailed dimensions of the belt teeth found on the treads. The only change in dimension between the belt tooth geometry and the wheel tooth geometry was a 
slight reduction in the base thickness of the wheel teeth. The number of teeth on each wheel was determined by the diameter of the wheels and this number was provided by FN Sheppard.

A central band of material was designed between the toothed sections of the wheels. This band rode in the manifold channel of the tread and acted as a stop for the teeth of the tread. This assisted in the initial installation of the tread onto the wheels and prevented the belt from slipping off the wheels while the vehicle was operating. It also helped to transfer the loads on the tread during turning onto the wheels and away from the manifold.

The overall width of the wheels was chosen to exactly match the overall width of the tread. The width of the toothed sections of the wheels was chosen to be slightly larger than the width of the toothed sections on the tread. This was done to prevent rubbing between the wheels and the tread during operation. The width of the center band on the wheels was slightly smaller than that of the channel in the tread, again to reduce the amount of rubbing between the wheels and the tread.

The bodies of the wheels were designed with spokes that ran between the outer portions of the wheels, where the teeth and central band were located to the wheels hubs. These spokes were designed to reduce the weight of the wheels while still allowing them to carry the loads they would be subjected to during operation.

The wheels were designed to press fit directly onto the drive and idler shafts. Press fitting was chosen over the use of set screws, pins or adhesives to reduce the complexity of manufacturing and assembly, maintain the integrity of the wheels geometry, and to ensure that the wheels could be replaced easily. The diameter of the shaft hole was determined by performing a press fit calculation to determine the amount of interference between the wheel and the shaft was required to hold the wheels in place, the details of which can be found in Appendix A.

\subsection{Drive Wheel Design}

The diameter of the drive wheel was selected based upon the theoretical wheel radius used to calculate the motor requirements. FN Sheppard's design catalog was used to determine which of the standard pulley 
diameters was closest to the ideal diameter. This then determined both the diameter of the drive wheel and the number of teeth on the drive wheel.

The final design of the drive wheel was validated by simulating the drive wheel under expected operating conditions. The loading in used in the simulation were calculated and then applied to a model of the wheel. The detailed results, methodology and loading calculations can be found in Appendix A. Table 36 contains details on the final drive wheel design, such as weight, general dimensions and simulation results. Figure 43 shows the final design of the drive wheel.

Table 36: Final dimensions, weight and simulation results for the drive wheel.

\begin{tabular}{|l|r|}
\hline \multicolumn{1}{|c|}{ Parameter } & Value \\
\hline Outer Diameter & $2.64 \mathrm{in}$. \\
\hline Hub Diameter & $0.399 \mathrm{in}$. \\
\hline Number of Teeth & 17 \\
\hline Weight & $.26 \mathrm{bs}$ \\
\hline Maximum Von Mises Stress & $1030 \mathrm{psi}$ \\
\hline Maximum Displacement & $0.001 \mathrm{in}$. \\
\hline Minimum Factor of Safety & 4.5 \\
\hline
\end{tabular}

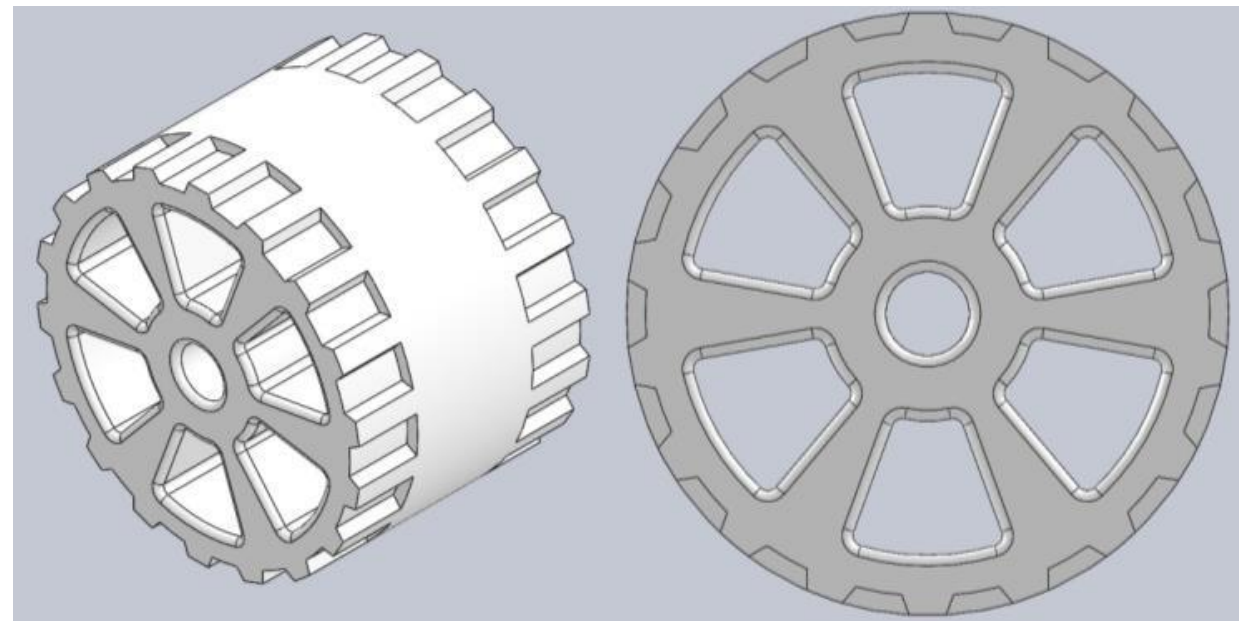

Figure 43: Solid model of the drive wheel.

\subsection{Idler Wheel Design}

The diameter of the idler wheels was chosen based on the suggestion of FN Sheppard. A minimum tensioning idler diameter was required for the tread system to function properly. This requirement was based upon the belt geometry used in the tread. The minimum diameter possible was used to reduce the weight of the idler wheel and to prevent interference between idler wheel and the tensioning system. This diameter also dictated the number of teeth on the idler. The drive wheels were subjected to more loading 
than the idler. Because the drive wheels simulation was successful, it was assumed that the idler wheels would be able to handle the loading it was subjected to and so no simulation of the idler wheel was conducted. Table 37 contains details on the final idler wheel design, such as weight, general dimensions and simulation results. Figure 44 shows the final design of the idler wheel.

Table 37: Final dimensions and weight of the idler wheel.

\begin{tabular}{|l|r|}
\hline \multicolumn{1}{|c|}{ Parameter } & \multicolumn{1}{|c|}{ Value } \\
\hline Outer Diameter & 3.31 in. \\
\hline Hub Diameter & $0.392 \mathrm{in}$. \\
\hline Number of Teeth & 20 \\
\hline Weight & $.36 \mathrm{lbs}$ \\
\hline
\end{tabular}

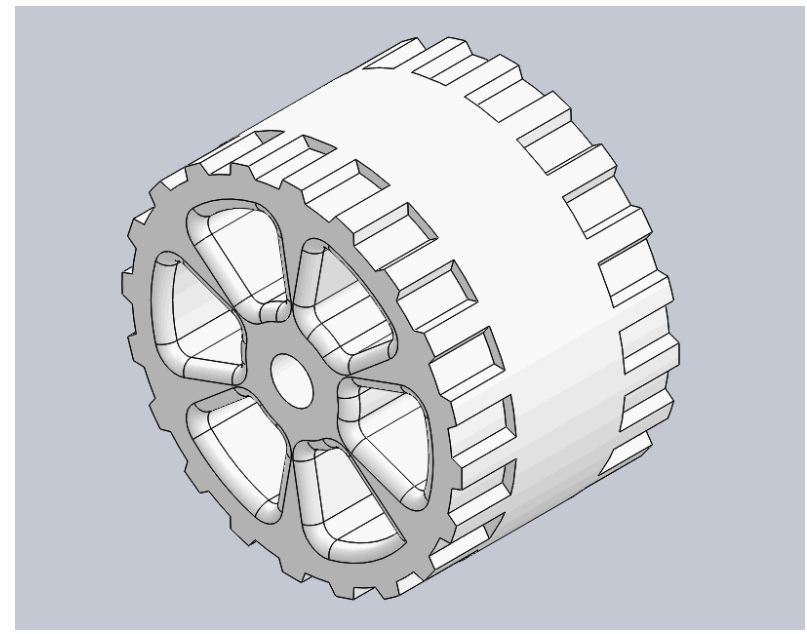

Figure 44: Solid model of the idler wheel.

\subsubsection{Brace Deign}

A major problem encountered in the proof of concept model was deflection of the drive and idler wheels of the model when the tread system was tensioned. To prevent this from happening in the new prototype, a brace between the drive and idler wheels was designed. This brace also carried a portion of the tension load in the wheel subsystem which increased the factor of safety on the flange bearings.

The design of the brace was relatively simple. The distance between the centers of the drive and idler wheel shafts was determined. This was the distance that the flange bearings in the brace would be separated by. The length of the brace was then determined by adding the radius of the bearings plus and additional quarter of an inch to both ends of the brace. The width of the brace was chosen to be the diameter of the flange of the bearing plus three quarters of an inch. 
The mounting points for the bearings in the brace were designed with a through hole and a blind hole. The through hole was sized to allow the end of the shaft to be inserted through the brace. The blind hole was designed such that the bearing could be inserted into the brace until the flange contacted the surface of the brace. The hole was chosen to be blind to leave a relatively thick portion of the brace material between the bearing and the retaining ring on the shaft. This gave the retaining rings a surface to contact without potentially damaging the bearings. This blind hole and the wall it left were used to determine the required thickness of the brace.

The design of the brace was validated by simulating the loads which would be applied to it during the operation of the vehicle. The detailed results and methodology for this simulation, as well as the loading calculations, can be found in Appendix A. Appendix A also contains a buckling calculation for the brace. Table 38 contains details on the final brace design, such as weight, general dimensions and simulation results. Figure 45 shows the final design of the brace.

Table 38: Final dimensions, weight and simulation results for the brace.

\begin{tabular}{|l|r|}
\hline \multicolumn{1}{|c|}{ Parameter } & \multicolumn{1}{|c|}{ Value } \\
\hline Brace Dimensions (L x W x T) & 14.0 in. x 1.25 in. x 0.187 in. \\
\hline Hole Separation & 12.25 in. \\
\hline Weight & $0.31 \mathrm{lbs}$ \\
\hline Maximum Von Mises Stress & $4,319 \mathrm{psi}$ \\
\hline Maximum Displacement & $0.002 \mathrm{in}$. \\
\hline Minimum Factor of Safety & 9.2 \\
\hline Buckling Factor of Safety & 114 \\
\hline
\end{tabular}

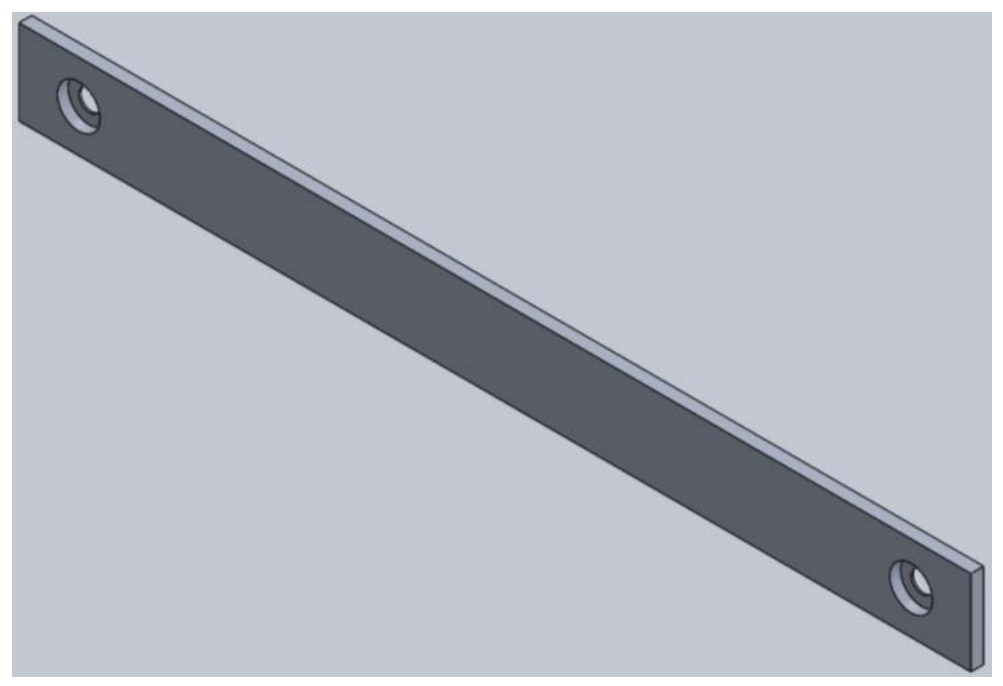

Figure 45: Brace solid model. 


\subsubsection{Tensioner Subsystem}

The purpose of the tensioner subassembly was to provide a means by which the vehicle's tread could be easily tensioned and un-tensioned while the vehicle was fully assembled. To do this, a screw driven mechanism was designed to raise and lower a toothed wheel, similar to the drive and idler wheels. When the wheel was raised into position it would engage with and begin to tension the tread. Once the desired tension in the tread was achieved, the tensioner mechanism was locked into place.

This screw driven mechanism consists of four major components. These are the tensioner wheel assembly, a clamping mechanism, a shaft and a screw assembly. The assembled mechanism can be seen in Figure 46 and Figure 47.

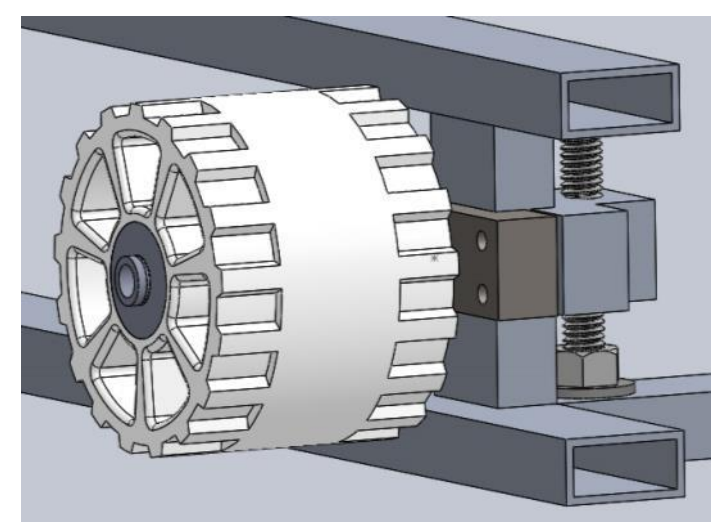

Figure 46: Front view of the tensioner subassembly.

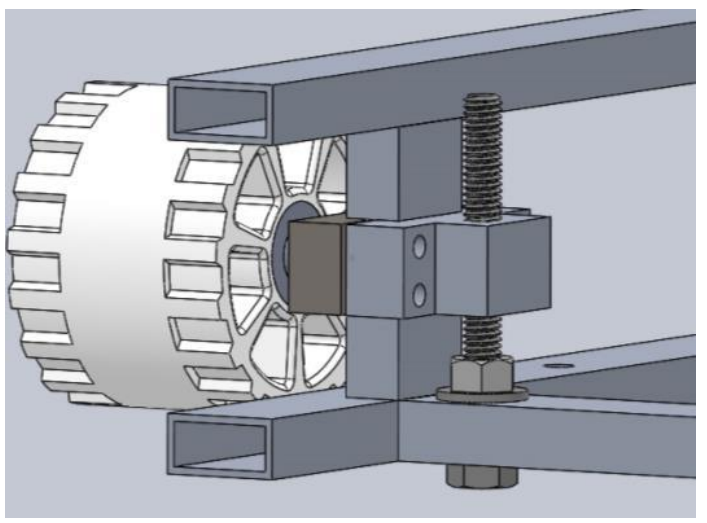

Figure 47: Rear view of the tensioner subassembly.

\subsubsection{Tensioner Clamp}

The tensioner clamp served several different purposes in the tensioner subsystem. It provided rigid support for the tensioner wheel, allowed the tensioning subsystem to be locked into place and acted as part of the 
mechanism which raised and lowered the tensioning wheel. It consisted of an interior half and an exterior half. These two halves were placed around the frames tensioner upright and were connected using bolts.

\subsection{Tensioner Clamp - Interior}

The half of the tensioner clamp which was placed on the interior of the frame was designed primarily to interact with the drive screw of the tensioner mechanism. It was also designed to fit onto the frame's tensioner upright to prevent the tensioner from slipping during the tensioning process. Figure 48 shows an image of the interior half of the tensioner clamp.

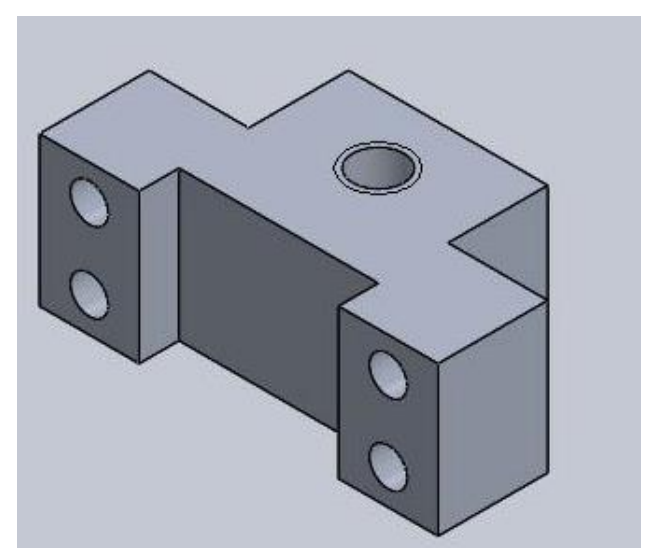

Figure 48: Solid model of the interior half of the tensioner clamp.

The overall width of the interior clamp half was chosen to accommodate the slot that allowed the clamp to be placed onto the tensioner upright and the four through holes for the bolts which hold the two clamp halves together. These through holes also determined the height of the clamp. The thickness of the clamp half was chosen to accommodate the depth of the slot and the threaded hole used for the drive screw. To reduce the length of screws required to hold the two pieces of the clamp together, the thickness of the clamp half around the bolt holes was reduced.

The width of the slot in the interior clamp half was chosen such that the clamp could slide freely on the tensioner upright, but still prevent the tensioner mechanism from shifting during vehicle operation. The depth of the slot was chosen such that the two halves of the clamp do not make contact when they are fully tightened. The slot was positioned along the center line of the part.

The tapped drive screw hole was positioned on the part such that when the clamp was in place in the vehicle the threaded hole aligned with the hole in the frame for the drive screw. The through holes for the 
clamping bolts were positioned so that the heads of the bolts would not interfere with each other or with the interior clamp half's body.

Aluminum was chosen as the material of the interior clamp half to reduce its weight contribution to the vehicle. Table 39 contains details on the final interior clamp half design, such as weight, general dimensions and calculation results.

Table 39: Final dimensions and weight for the interior clamp half.

\begin{tabular}{|l|r|}
\hline \multicolumn{1}{|c|}{ Parameter } & Value \\
\hline Interior Clamp Dimensions (L x W x H) & 1.05 in. x 2.0 in. x 0.75 in. \\
\hline Slot Dimensions (W X H x D) & 1.0 in. x 0.75 in. x 0.2 in. \\
\hline Threaded Hole Callout & $1 / 4-20$ THRU \\
\hline Attachment Through Hole Diameter & $0.19 \mathrm{in.}$ \\
\hline Weight & $0.09 \mathrm{lbf}$ \\
\hline
\end{tabular}

\subsection{Tensioner Clamp - Exterior}

The exterior half of the clamp holds and positions the tensioner shaft and design of the exterior half of the tensioner clamp was driven by the need to hold the tensioner wheel rigidly in place during operation. Like the interior half of the clamp, the exterior half used a slot to fit securely onto the tensioner upright.

The basic shape of the exterior half of the clamp was that of a rectangle. The height and width of the exterior half of the clamp were chosen to be the same as the interior half of the clamp to simplify the design. The thickness of the exterior clamp half was chosen to accommodate the depth of the slot cut into it while maintaining sufficient material for the clamping bolts and the tensioner shaft to be secured in place without risk of striping the threads. The slot cut into the exterior half of the clamp is identical to the slot cut into the interior half of the clamp.

The threaded holes used to secure the clamping bolts in place were positioned so that they corresponded to the through holes in the interior half of the clamp. The hole for the tensioner shaft was centered on the width of the width of the clamp, but offset from the center of the height of the clamp. This was done to allow the tensioner wheel to be given an offset relative to the clamp to compensate for any welds in the frame which would prevent the tensioner clam from reaching the top of the tensioner upright. Because the tensioner shaft was chosen to be steel the material that was chosen for the exterior clamp half was steel. This was done to allow the shafts to be welded into place. 
The design of the exterior half of the clamp was validated using a simulation which included the tensioner shaft and the exterior half of the clamp. The methodology and detailed results of this simulation can be found in Appendix A. Table 40 contains details on the final exterior clamp half design, such as weight, general dimensions and calculation results. Figure 49 shows the final design of the exterior clamp half.

Table 40: Final dimensions, weight and simulation results for the exterior clamp half.

\begin{tabular}{|l|r|}
\hline \multicolumn{1}{|c|}{ Parameter } & \multicolumn{1}{|c|}{ Value } \\
\hline Interior Clamp Dimensions (L x W x H) & 0.5 in. x 2.0 in. x 0.75 in. \\
\hline Slot Dimensions (W X H x D) & 1.0 in. x 0.75 in. x 0.25 in. \\
\hline Shaft Hole Diameter & $0.38 \mathrm{in}$. \\
\hline Attachment Through Hole Callout & $\# 10-24 \mathrm{THRU}$ \\
\hline Weight & $0.14 \mathrm{lbf}$ \\
\hline Maximum Von Mises Stress & $11,850 \mathrm{psi}$ \\
\hline Maximum Displacement & $0.003 \mathrm{in}$. \\
\hline Minimum Factor of Safety & 6.5 \\
\hline
\end{tabular}

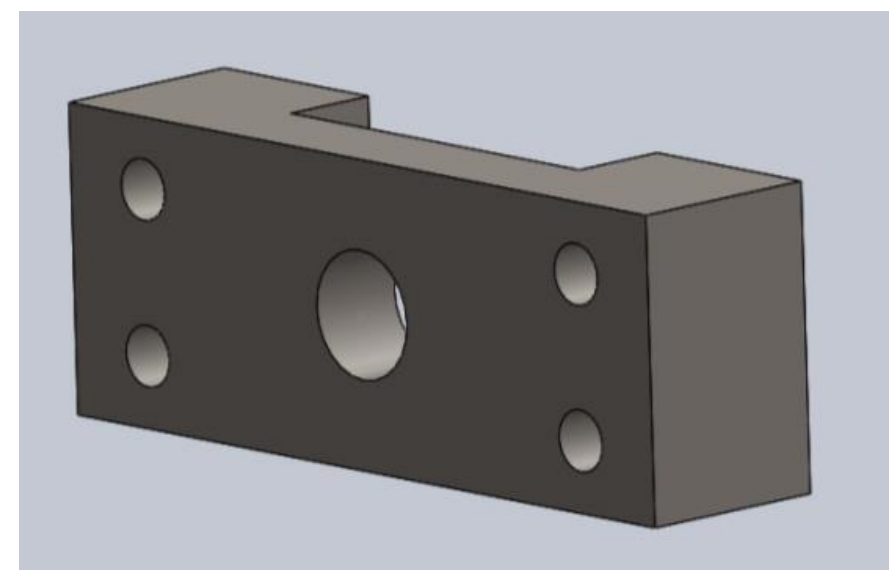

Figure 49: Solid model of the exterior half of the tensioner clamp.

\subsubsection{Tensioner Wheel Assembly}

As stated above, the tensioner wheel was very similar in design to the drive and idler wheel of the vehicles. The tooth geometry used in the tensioner wheel is the same as the tooth geometry used for the drive and idler wheels. Like the drive and idler wheels, 3D printed ABS plastic was chosen as the material that would make up the tensioner wheel. Like the drive and idler wheels, the design of the tensioner wheel consisted of an outer band which held the teeth and alignment band which was connected to a central hub by spokes.

The width of the tensioner wheel was also the same as the width of the drive and idler wheel. 
The diameter and number of teeth on the tensioner wheel were determined using the FN Sheppard design catalog and Solidwork's belt length tool. The details of this process can be found in Appendix A. The wheel diameter that was chosen provided enough potential belt extension without interfering with other subsystems.

Because the tensioner shaft was fixed, the tensioner wheel was required to rotate freely on the shaft. This was accomplished by including bushings in the design of the tensioner wheel assembly. The bushings that were chosen were selected to operate at the projected speeds and loads experienced by the tensioner wheel during vehicle operation. They also had flanges, which made them easy to insert and position in the tensioner wheel.

To accommodate the bushings, the hub of the tensioner wheel was designed differently than the hubs of the idler and drive wheels. The flange of the bushings required the diameter of the tensioner wheel hub to be increased significantly. Additionally, the diameter of the through hole of the hub was increased to accommodate the outer diameter of the bushing. A recess was added to the hub to allow the bushing to be pressed flush with the face of the tensioner wheel. Table 41 contains details on the final design of the tensioner wheel. Figure 50 shows the final design of the tensioner wheel.

Table 41: Final dimensions and weight for the interior clamp half.

\begin{tabular}{|l|r|}
\hline \multicolumn{1}{|c|}{ Parameter } & \multicolumn{1}{|c|}{ Value } \\
\hline Outer Diameter & $2.64 \mathrm{in}$. \\
\hline Hub Diameter & $0.625 \mathrm{in}$. \\
\hline Number of Teeth & 17 \\
\hline Weight & $.26 \mathrm{lbs}$ \\
\hline
\end{tabular}

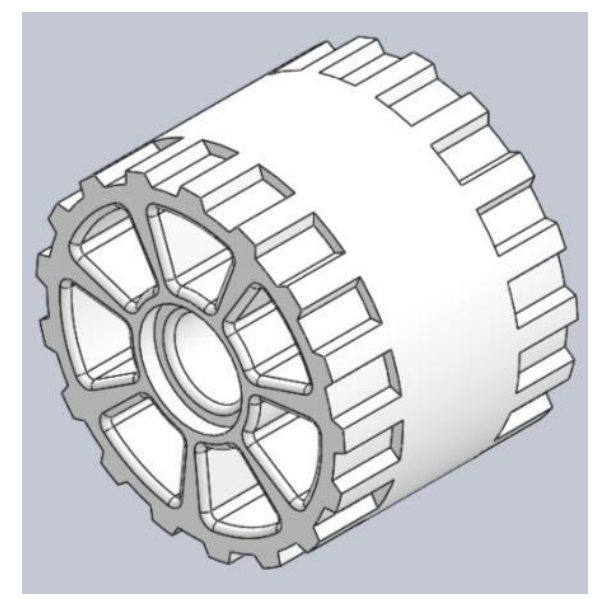

Figure 50: The solid model of the Tensioner wheel. 


\subsubsection{Tensioner Shaft}

The purpose of the tensioner shaft was to provide a rigid surface that the tensioner wheel could be both positioned on and allowed to rotate freely on. It would also need to be attached to the exterior tensioner clamp half.

The dimensions of the tensioner shaft were relatively straight forward to design. The portion of the shaft on which the tensioner wheel rested was chosen to have a length equal to the width of the tensioner wheel plus the length required to accommodate the retention ring which kept the wheel on the shaft. The diameter of this section was chosen to allow the bushings in the tensioner wheel to rotate freely on shaft. The next portion of the tensioner shaft acted as a spacer and prevented the tensioner wheel from slipping into contact with the frame and to help align the tensioner wheel with the drive and idler wheels. To make welding the tensioner shaft to the exterior clamp half easier a short section was added to the shaft that would slip into the exterior clamp half's central hole.

Like the drive and idler shafts, 1045 medium carbon steel was chosen as the material for the tensioner shaft. Steel was chosen to reduce the amount of deflection in the shaft when it was loaded, which was especially critical for the tensioner shaft because it was cantilevered from the tensioner clamp exterior half. The final design of the tensioner shaft was validated using the same simulation used to validate the design of the tensioner clamp exterior half. Again the details of this simulation can be found in Appendix A. Table 42 contains details on the final design of the tensioner shaft such as weight and major dimensions. Figure 51 shows the final design of the tensioner shaft.

Table 42: Final dimensions, weight and simulation results for the interior clamp half.

\begin{tabular}{|l|r|}
\hline \multicolumn{1}{|c|}{ Parameter } & \multicolumn{1}{|c|}{ Value } \\
\hline Wheel Mounting Section Dimensions (L x Dia.) & 2.0 in. x 0.38 in. \\
\hline Wheel Locating Section (L x Dia.) & 0.5 in. x $0.5 \mathrm{in}$ \\
\hline Mounting Section Dimension (L x Dia.) & $0.25 \mathrm{in.}$ x $0.38 \mathrm{in}$. \\
\hline Retaining Ring Slot Dimensions (W x Dia.) & 0.029 in. x 0.351 in. \\
\hline Weight & $0.1 \mathrm{lbf}$ \\
\hline Maximum Von Mises Stress & $11,850 \mathrm{psi}$ \\
\hline Maximum Displacement & $0.003 \mathrm{in}$. \\
\hline Minimum Factor of Safety & 6.5 \\
\hline
\end{tabular}




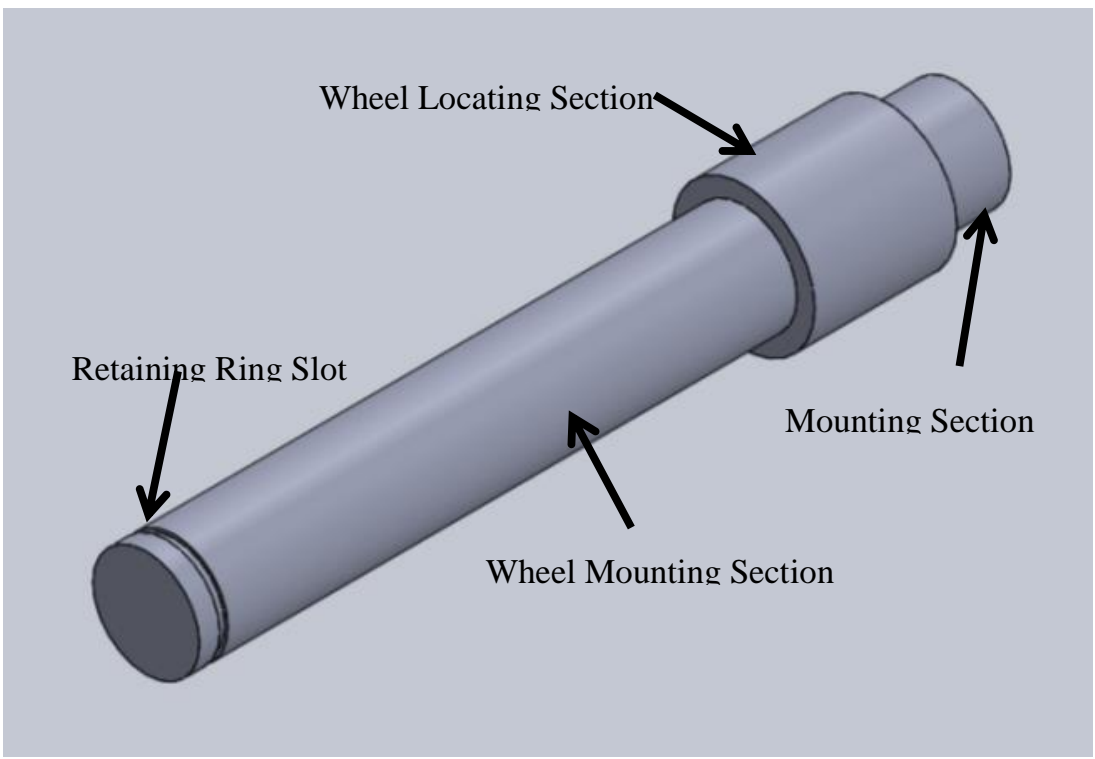

Figure 51: Tensioner shaft solid model.

\subsubsection{Tensioner Screw Assembly}

The purpose of the tensioner screw assembly was to drive the tensioner clamp up and down to tension and un-tension the treads. This assembly was made up of three components, a bolt, a lock nut and two nylon washers. Figure 52 shows the screw assembly in place in the tensioner assembly.

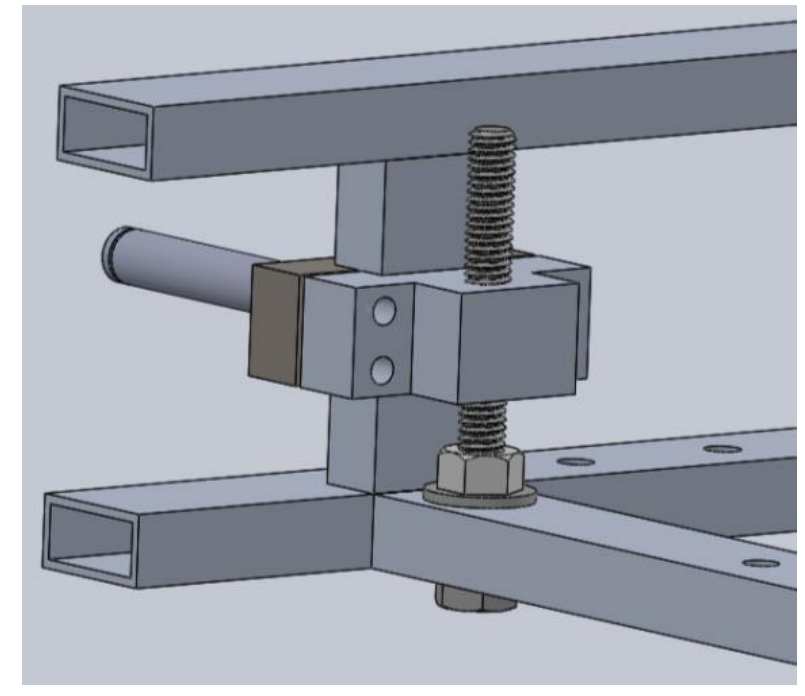

Figure 52: Tensioner screw assembly solid models.

The operation of the screw assembly was relatively simple. The bolt was placed into the hole in the frame and held in place using the lock nut. The bolt was then screwed into the interior clamp half. Because the bolt was held in place, when it was turned the interior clamp was raised or lowered. The nylon washers 
were placed between the frame and the bolt head and the frame and the lock nut to reduce friction and prevent the mechanism from binding.

\subsection{Final Design Assembly}

Figure 53 shows the final assembled Solidworks model of the vehicle and Table 43 contains details on the final assembled vehicle such as its overall dimensions and estimated final weight. This estimated weight does not include the fasteners used in the project, nor does it include the mass of the treads.

Table 43: Overall dimensions and weight of the assembled vehicle.

\begin{tabular}{|r|r|}
\hline \multicolumn{1}{|c|}{ Parameter } & \multicolumn{1}{|c|}{ Value } \\
\hline Final Assembly Dimensions (L x W x H) & $19.1 \times 18.45$ in. x 3.945 in. \\
\hline Solidworks Estimated Weight & $12.4 \mathrm{lbf}$ \\
\hline Estimated Total Weight & $13.4 \mathrm{lbf}$ \\
\hline
\end{tabular}

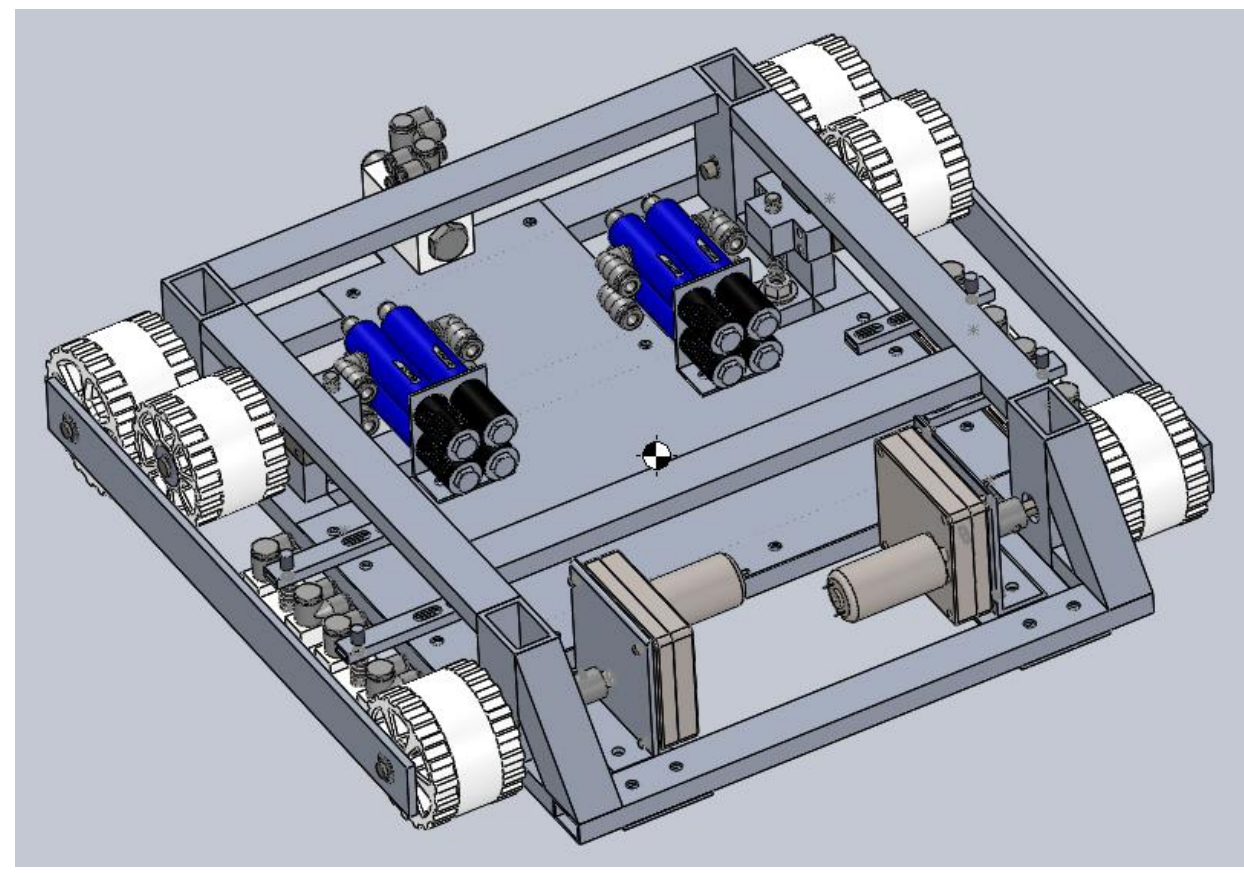

Figure 53: The final assembled model of the vehicle. 


\section{Chapter 3: Manufacturing and Assembly}

\subsection{Bill of Materials}

This section gives an overview of the total cost of the project. Table 44 gives a breakdown of the cost of the vehicles by subsystem as well as the total cost of the vehicle. A detailed bill of materials can be found in Appendix B and gives a per item cost break down for the entire vehicle, the quantities of each item used and the supplier of each item.

Table 44: Cost breakdown by subsystem for the vehicle.

\begin{tabular}{|l|r|}
\hline \multicolumn{1}{|c|}{ Vehicle System } & Cost \\
\hline Frame Subsystem & $\$ 130.22$ \\
\hline Motor Subsystem & $\$ 150.03$ \\
\hline Vacuum Subsystem & $\$ 670.83$ \\
\hline Manifold Subsystem & $\$ 226.48$ \\
\hline Wheel Subsystem & $\$ 390.74$ \\
\hline Tensioner Subsystem & $\$ 155.68$ \\
\hline Tread Subsystem & $\$ 611.16$ \\
\hline Total & $\$ 2,335.14$ \\
\hline
\end{tabular}

\subsection{Component Manufacturing}

This section give brief descriptions of the processes used to manufacture the various components of the design. Each section will deal with a different subsystem and will discuss the manufacturing method, anomalies or tolerance issues encountered in the manufacturing process and any modifications made to the designs during the manufacturing, assembly and testing processes. Each subsection will show the final product of the manufacturing process. A weight break down by part can be found in Appendix C. 


\subsubsection{Frame Subsystem}

Figure 54 shows an image of the manufactured and assembled frame subsystem.

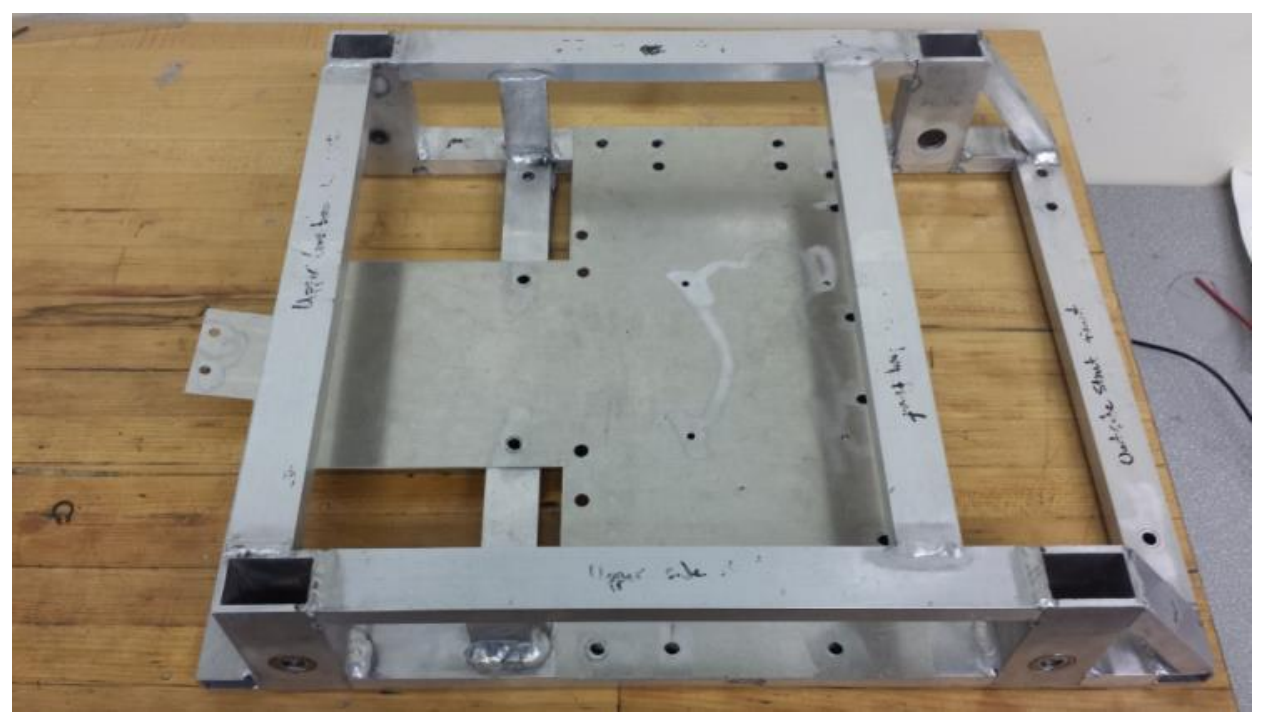

Figure 54: Manufactured and assembled frame subassembly.

\subsubsection{Frame}

The manufacturing of the frame was relatively straight forward. Sections of 6061 aluminum square tubing was rough cut on an aluminum chop saw. These tubing sections were then milled and deburred to create blank struts of the required lengths. The hole patterns were then drilled into each of the struts using a mill to ensure tight tolerances could be kept.

Once all of the individual struts were created, they were handed over to a shop tech at Cal Poly's machine shop for welding. A TIG welder was used to weld each strut into place in the frame and 4043 Aluminum filler rod was used during the welding process. Because of the complexity of the frame, no special fixtures were used during the welding process. This resulted in slight misalignments in some of the frames components, though none drastic enough to make the frame unusable. Some examples of these misalignments can be seen in Figure 55 and Figure 56 and the effects of these misalignments are discussed in Chapter 4. 


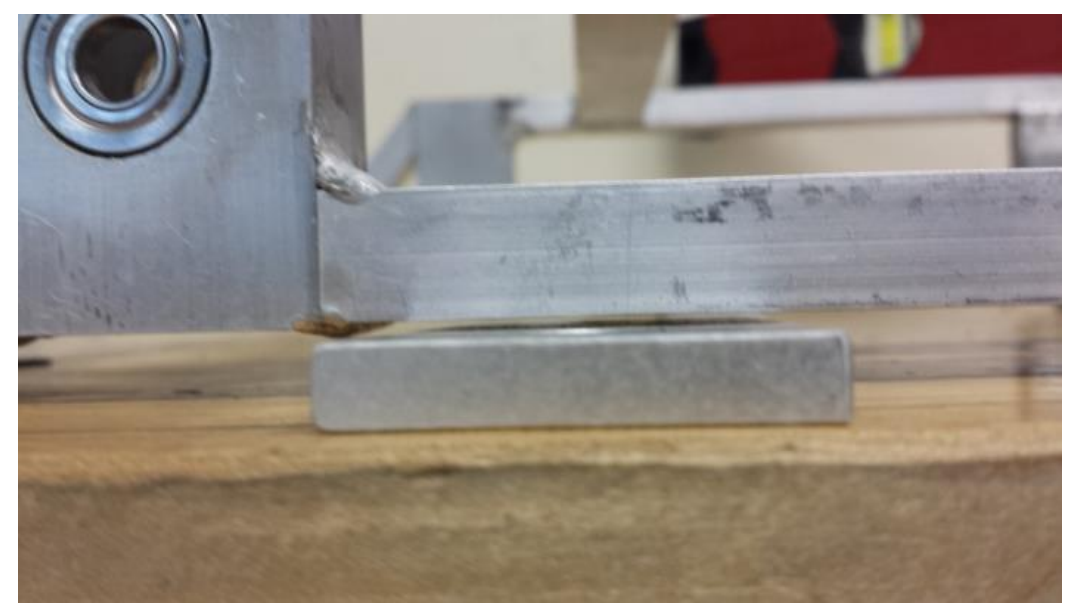

Figure 55: Misalignment of the frame at the right corner. The corner was raised by $1 / 32^{\text {nd }}$ of an inch.

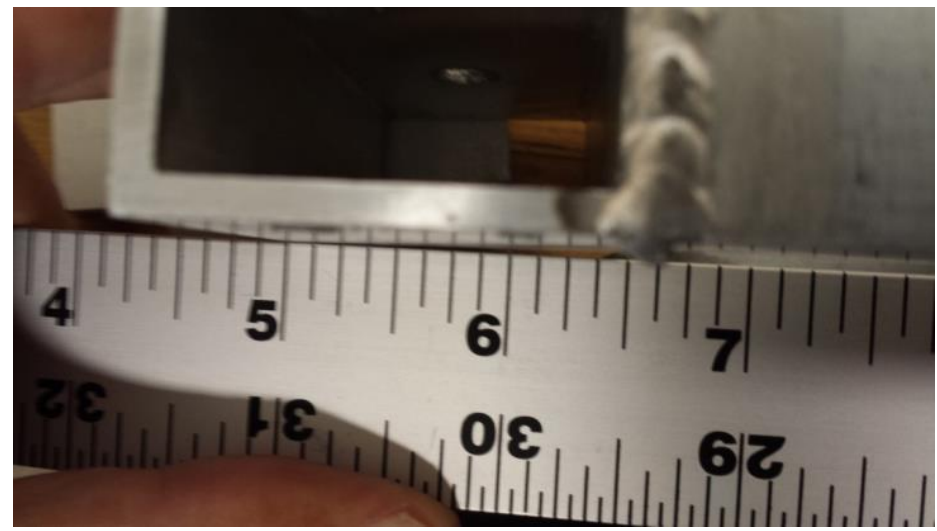

Figure 56: Angular misalignment of the idler strut on the left side of the vehicle.

Once the frame was completely welded, a dremel tool was used to smooth out some of the weld beads. This served to both improve the look of the frame and to prevent the weld beads from interfering with the other subsystems as they were attached to the frame.

Finally, the bearings were installed into the frame. The bearings and frame were thoroughly cleaned and then the retaining compounds catalyst was painted on to the outer race of the bearing and the bearing holes in the frame. The retaining compound was then applied to the bearing and the frame and the bearings were pressed into place by hand until the bearing and frame strut faces were flush. The excess retaining compound was then removed.An image of the bearing inserted in the frame can be found in Figure 57. 


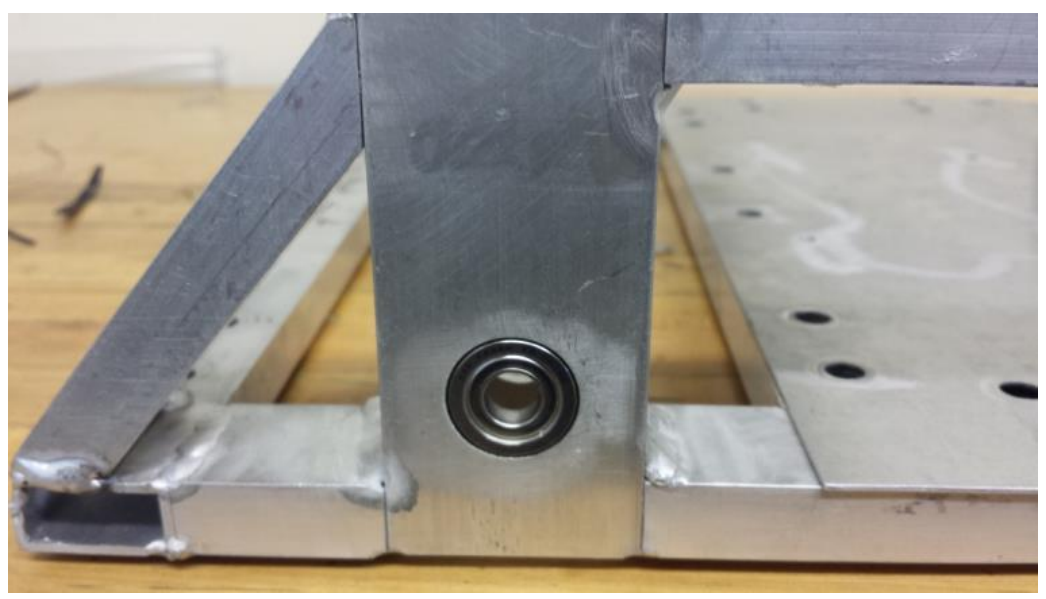

Figure 57: One of the bearings in place in the frame.

During the initial testing of the tensioner system, the tensioner on the right side of the vehicle jammed. In the process of unjamming it the frame was cracked. A patch of 6061 aluminum was welded between the struts were the crack occurred to cover the crack and provide the struts with additional support. The patched area of the frame can be seen in Figure 58



Figure 58: The patch used to fix the frame.

\subsubsection{Plate}

The base plate for the frame was manufactured from a sheet of 6061 aluminum. The basic shape and dimensions of the plate were marked out on the aluminum sheet. The sheet was then cut to shape using a vertical band saw. The sharp edges left by the cutting process were smoothed using a polishing wheel. The hole pattern was then laid out on the plate and the holes were drilled using a drill press. The holes in the 
plate were intentionally oversized to help with alignment with the frame. The final base plate can be seen in Figure 59.

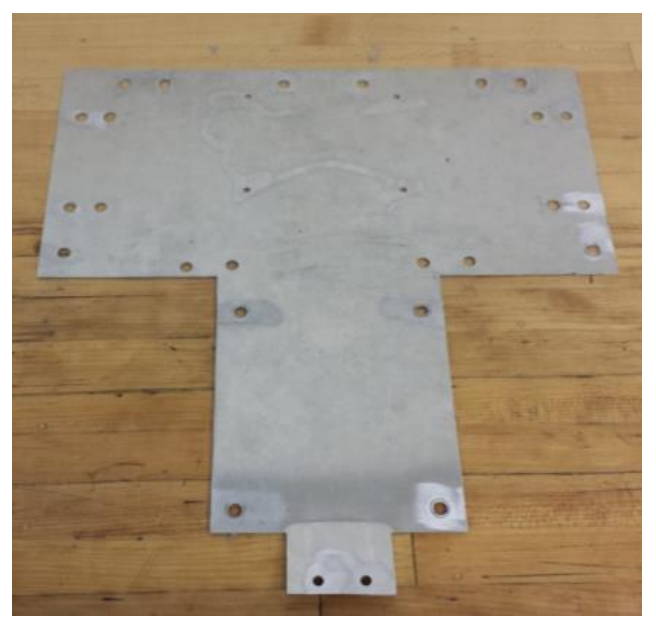

Figure 59: Manufactured base plate.

\subsubsection{Motor Subsystem}

Figure 60 shows an image of the manufactured and assembled motor subsystem. The final subsystem weight was $1.66 \mathrm{lbf}$ for both of the assembled motor subsystems.

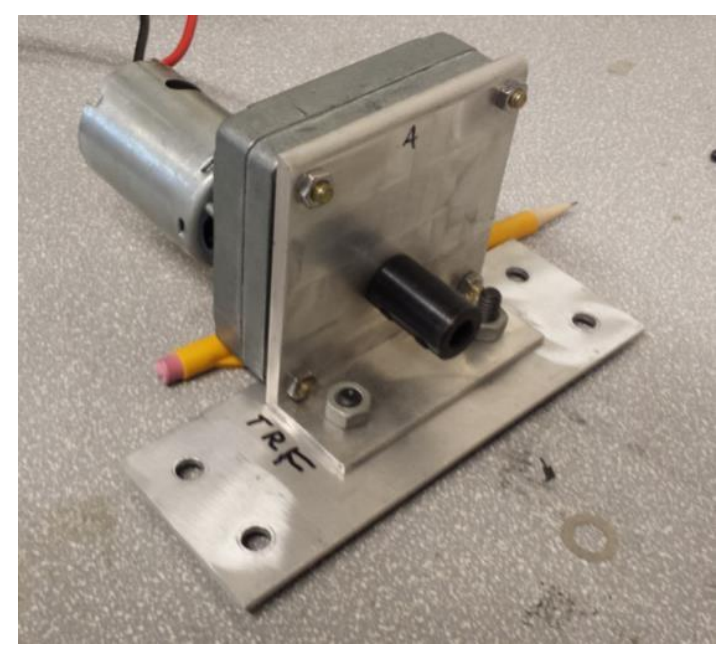

Figure 60: Motor subassembly fully assembled.

\subsubsection{Motor Mounting Brackets}

The first step in creating the motor mounting bracket was rough cutting the L-shaped stock using a band saw. These rough cuts were then milled to the final width that the brackets would have. Next, one leg of the brackets was milled to reduce its length. The face of each leg of the bracket was then milled until they were 
the desired thickness. The hole patterns for both legs were then drilled using a mill to ensure precision. The bracket was then polished on a polishing wheel to remove any burrs left by the machining process and to improve the surface finish.

The only difficulty encountered during the manufacturing of the mounting brackets was the fact that the motor's mounting screws were not positioned correctly. This resulted in motors not being able to be mounted on the bracket. This was solved by increasing the hole diameters of the motor mounting points on the brackets. Figure 61 shows both of the final mounting brackets.

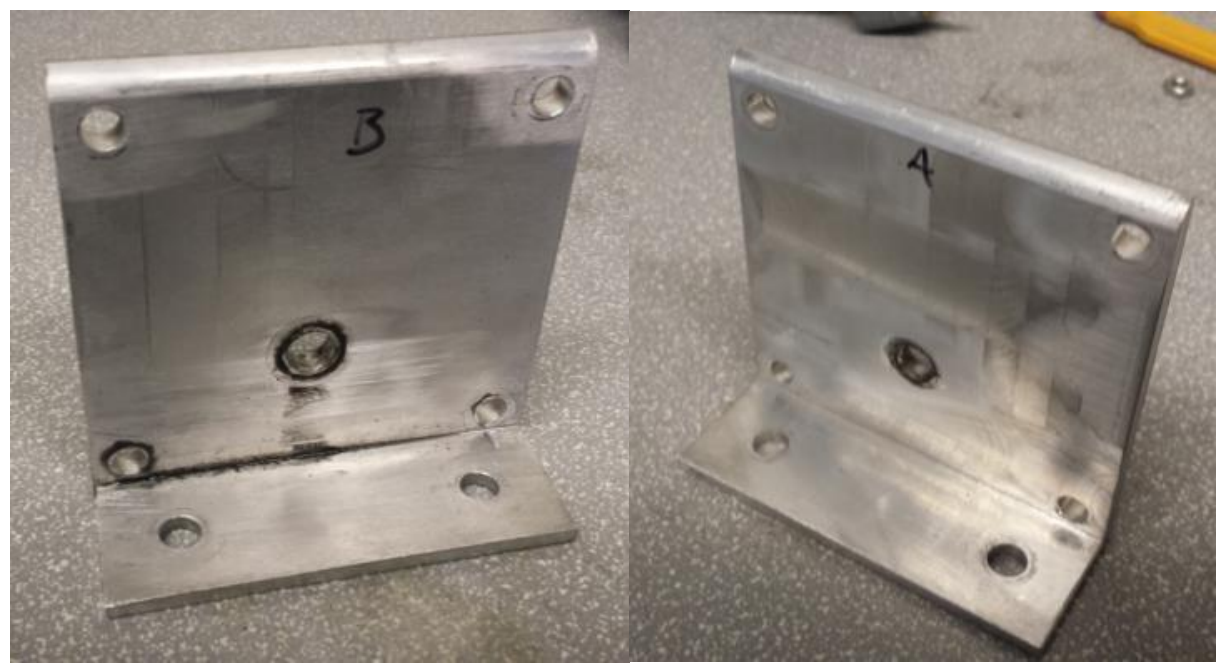

Figure 61: Left and right motor mounting brackets.

\subsubsection{Motor Base Plate}

The motor base plate was relatively simple to manufacture. The plates were rough cut from stock aluminum plates using a band saw and then milled to size. The hole patterns for the left and right side plates were then drilled into the plates using a mill to ensure the patterns precision. The plates were then deburred using a polishing wheel. Images of the final motor base plates can be seen in Figure 62 and Figure 63. 


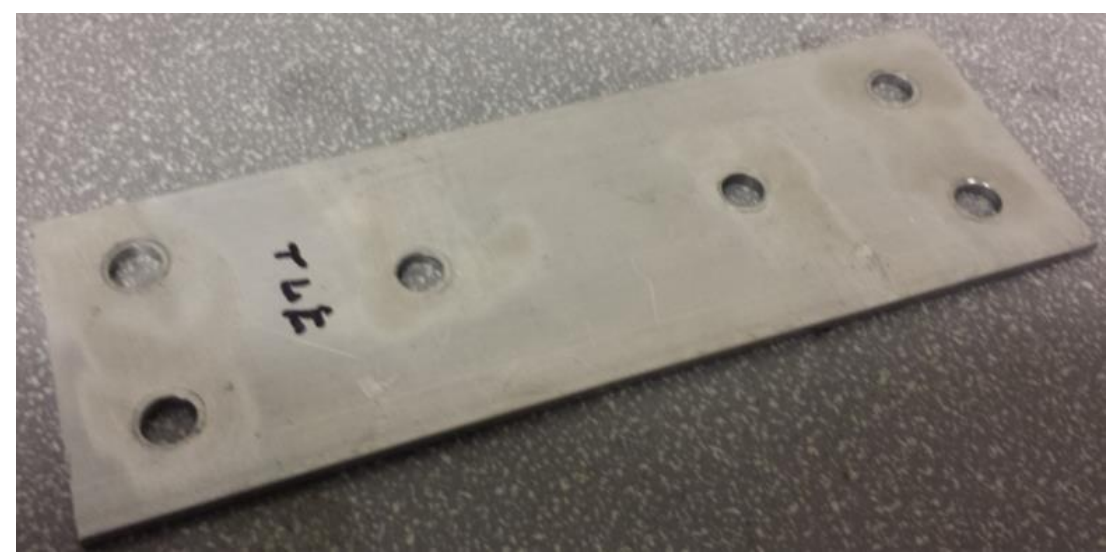

Figure 62: Left side motor mounting plate.

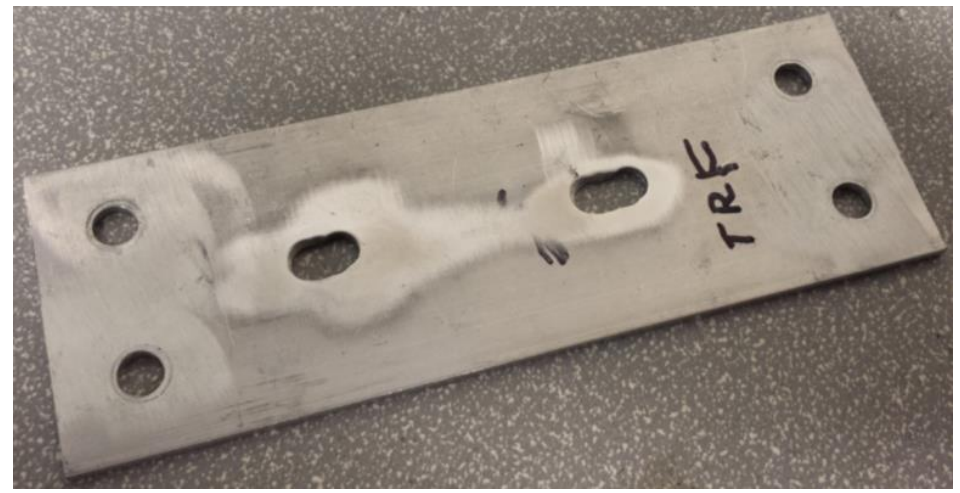

Figure 63: Right side motor mounting plate. Note the slots cut so the motor could be adjusted.

Two difficulties were encountered with the machined plates. The first was misalignment between the frame holes and the plate holes which prevented the plates from being attached to the frame. This was corrected by increasing the plate's frame mounting holes diameter until the plates could be attached to the frame.

The second problem was that while the left side plate aligned the motor shaft with the drive shaft, the right side motor shaft had significant misalignment laterally with its drive shaft. To correct this, slots were cut in the plate to allow the motor to be adjusted into the correct position.

\subsubsection{Vacuum Subsystem}

Figure 64 shows an image of the manufactured and assembled vacuum subsystem after it was installed in the frame subsystem. The final weight of the subsystem was $1.68 \mathrm{lbf}$ in total. 


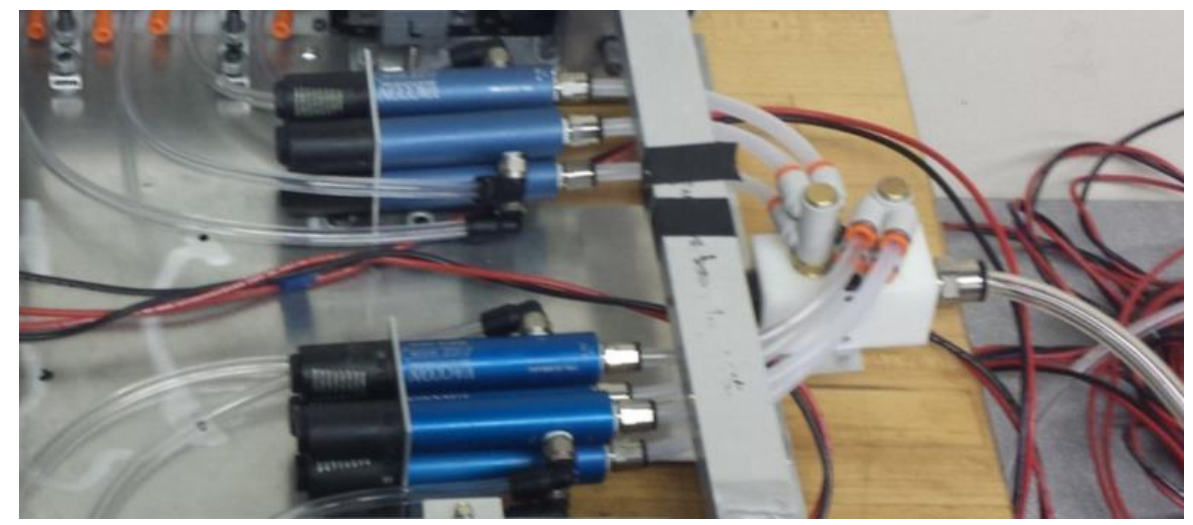

Figure 64: Vacuum subassembly installed in the vehicle.

\subsubsection{Venturi Mounting Brackets}

Stock aluminum sheeting was cut the desired size using a band saw and the edges were deburred. The hole pattern was then marked on the plate and then drilled out using a drill press. The bend point of the bracket was marked and then the bracket was bent to ninety degrees using a sheet metal bender. An image of the final venture mounting brackets can be seen in Figure 65 and the right bracket with venturis in position can be seen in Figure 66.

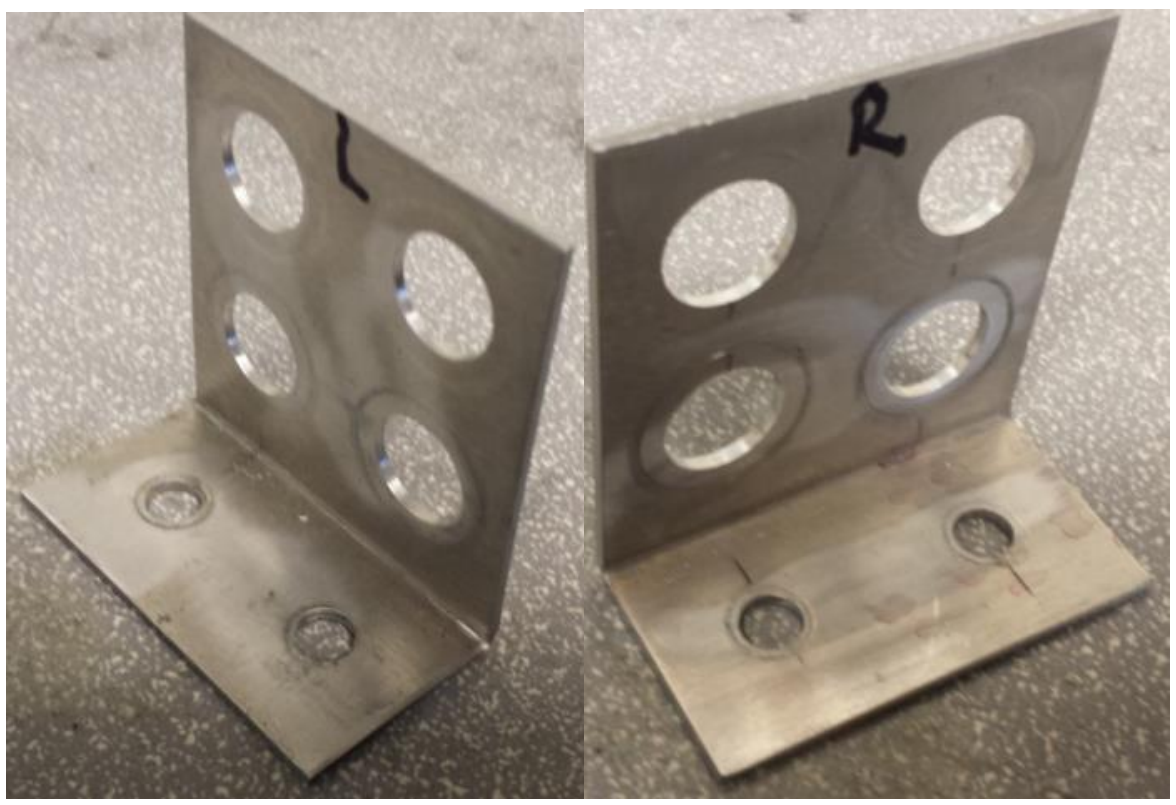

Figure 65: Left and right venture mounting brackets. 


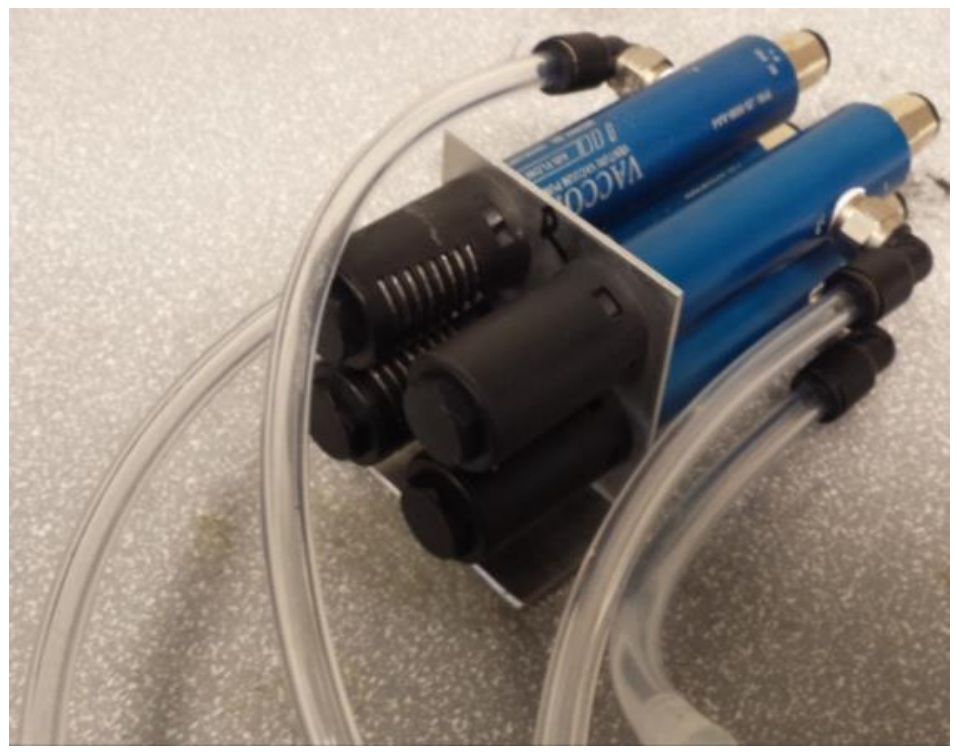

Figure 66: Venturi mounting bracket with the venturis installed.

\subsubsection{Inlet Manifold Assembly}

The inlet manifold assembly was easy to assemble. Teflon tape was added to the threads of each component and then they were screwed into the nylon block. Figure 67 shows the assembled Inlet manifold assembly.

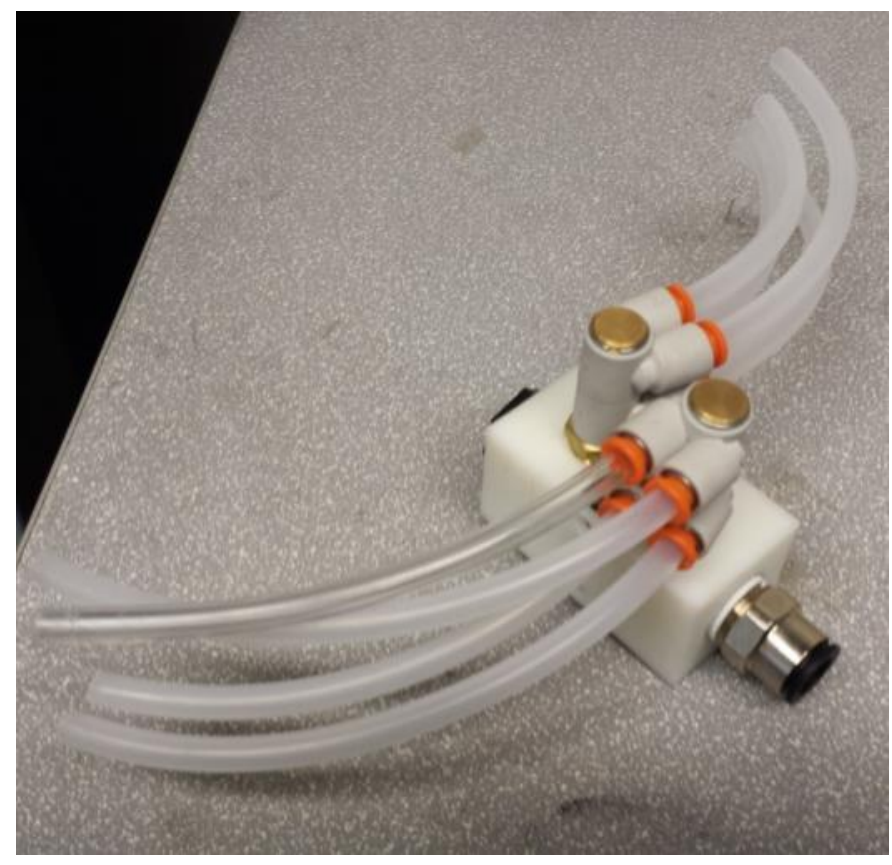

Figure 67: Inlet manifold assembly. 


\subsubsection{Vacuum Manifold Subsystem}

Figure 70 shows an image of the manufactured and assembled manifold subsystem. The weight of the manifold subsystems was $0.55 \mathrm{lbf}$ each.

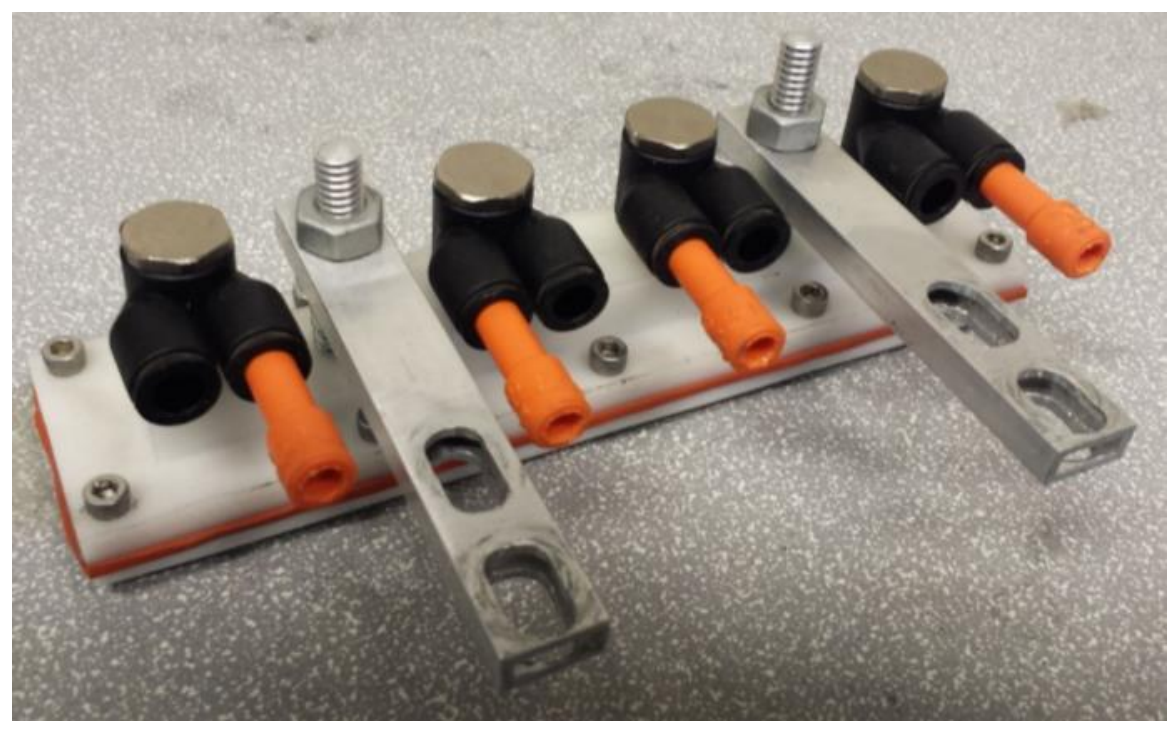

Figure 68: Assembled left side manifold.

\subsubsection{Lower Manifold}

The lower half of the manifolds was difficult and time consuming to machine. Stock delrin bars were rough cut to size using a table saw and miter saw. These bars were then machined down on a mill to the final length, width and thickness dimensions of the lower manifold half. The thickness of the outside edges was then reduce to form a tiered shape. The individual chambers and their corresponding slots were then milled into each of the lower manifold halves. Finally, the attachment hole pattern along the outside edge of the lower manifold were drilled on a mill and the tapped by hand. During the milling processes, large amounts of coolant were used to prevent heat from building up in the manifold and causing warpages. An image of the lower half of the manifold can be seen in Figure 69. 


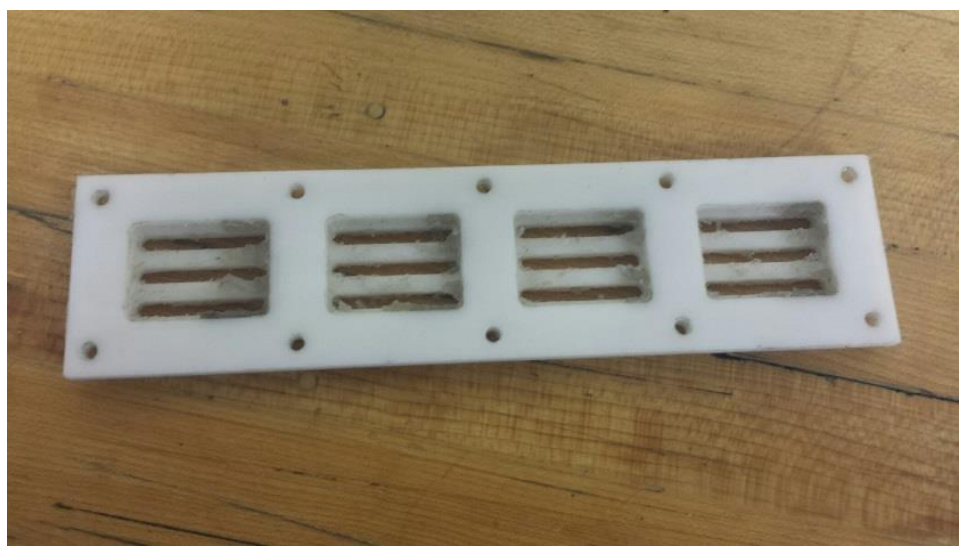

Figure 69: Lower manifold half.

Because the stock that was purchased came slightly warped and the cutting of the stock on the saws caused further warping, it was difficult to machine the lower manifolds to be perfectly flat. To account for this warp, the bars which would become the lower manifolds were machined flat before they were machined to the lower manifolds final dimensions. This significantly reduced the amount of warping in the final product, but did not eliminate it. Figure 70 shows the warpage in the final lower manifold and the effects of this warpage are discussed in Chapter 4.

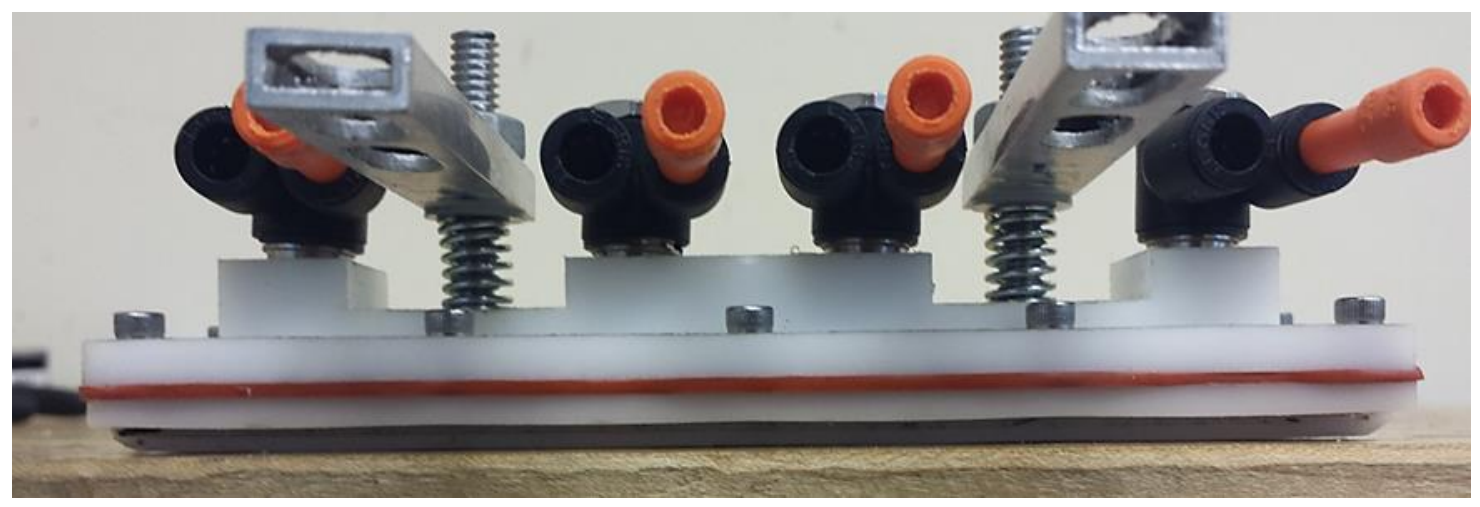

Figure 70: The bending seen in the right lower manifold half.

\subsubsection{Upper Manifold}

The process used to machine the upper half of the manifolds was identical to that of the lower manifold halves. Stock delrin bars were rough cut and the milled into the desired shape. The through holes for assembling the manifolds were drilled but not tapped and the hole for the vacuum fittings and mounting holes for the uprights were drilled then tapped. Teflon sealant tape was then applied to the vacuum fittings and they were then screwed into place. Images of the upper half of the manifold can be seen in Figure 71 . 


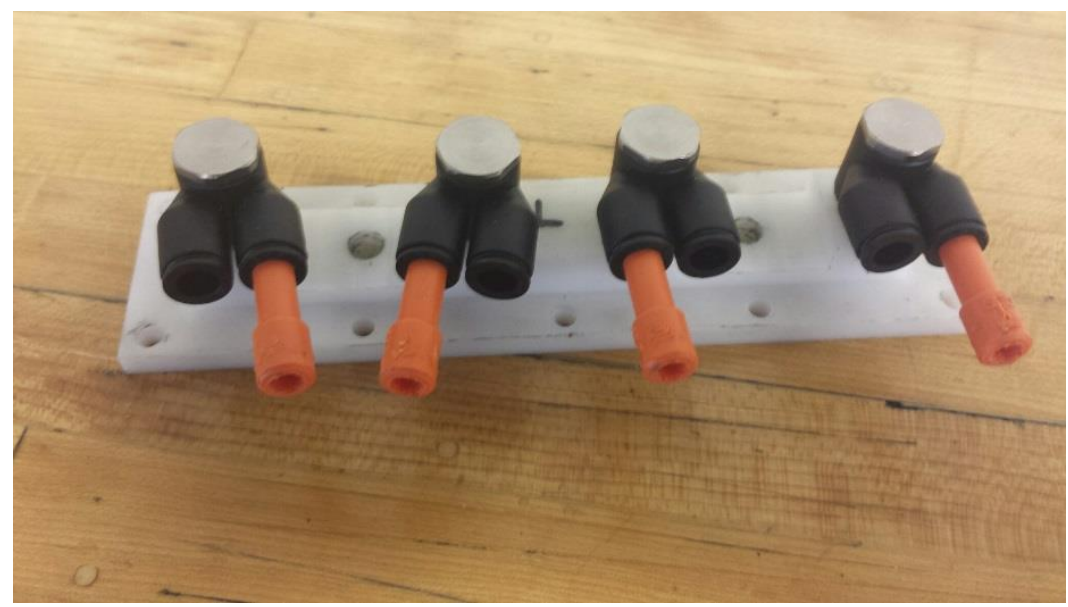

Figure 71: Upper half of the manifold with the fittings installed.

There was the slight misalignment between the attachment holes between the lower and upper halves of the manifolds. This was corrected by slightly oversizing the through holes in the upper manifold halves.

\subsubsection{Manifold Gasket}

The manifold gasket was relatively easy to manufacture. The pattern for the gasket was printed out and tapped to a sheet of silicone rubber. The pattern was then cut out by hand using a razor blade. Care was taken to clean the screw through holes to prevent binding during assembly. An image of the manifold gasket can be seen in Figure 72.

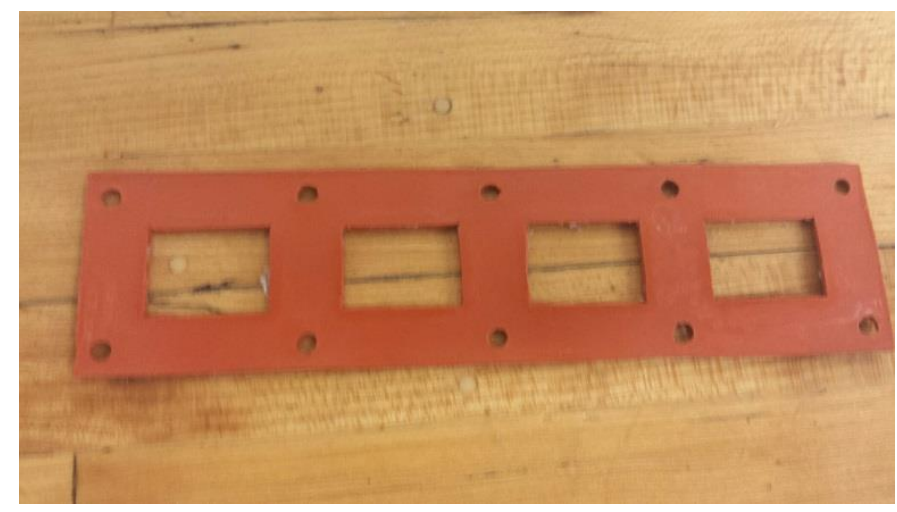

Figure 72: Manifold gasket.

\subsubsection{Manifold Mounting Structure}

The manufacturing of the manifold mounting structure occurred in two parts. The first part was the machining of the mounting struts. Stock aluminum tubing was rough cut on a band saw and then machined 
to the desired length on a mill. The hole for the threaded upright was then drilled on the mill. The slots for the frame mounting points were then cut on the mill. Each of the struts was then deburred and polished. An image of the mounting struts can be seen in Figure 73.

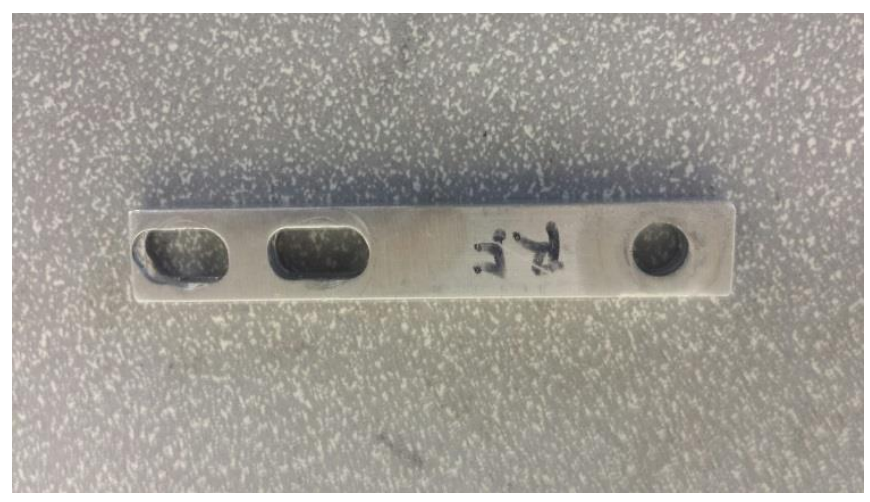

Figure 73: One of the manifold mounting struts.

The threaded uprights were extremely easy to manufacture. Stock threaded rods were rough cut to the required lengths using a band saw. The cut ends were then polished and deburred on a polishing wheel. A nut was run over each rod clean and deburr the threads. An image of the threaded uprights can be seen in Figure 74 .

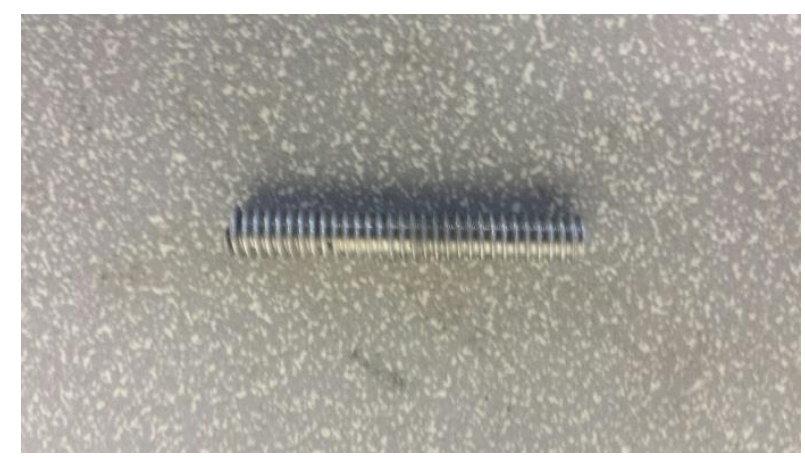

Figure 74: One of the threaded uprights.

\subsubsection{Wheel Subsystem}

Figure 75 shows an image of the manufactured and assembled wheel subsystem. The final weight of the wheel subsystems was $0.98 \mathrm{lbf}$ each. 


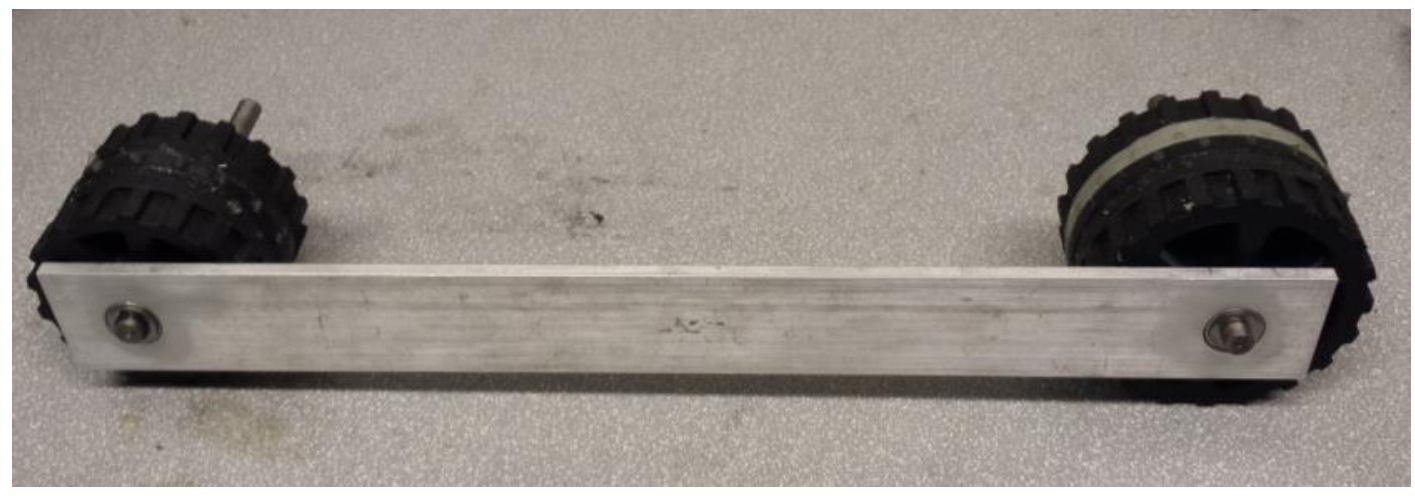

Figure 75: Assembled wheel subassembly.

\subsubsection{Drive and Idler Shafts}

The drive and idler shafts were extremely complicated and time consuming to manufacture. Steel stock was rough cut to length on a grinding saw. Each rod was then inserted into a lathe and a carbide tipped tool was used to machine common shaft end onto the end of each rod. Once the steps of the common end were machine, a parting tool was used to cut the slot to hold the retaining ring.

At this point, the dimensions of each common end were compared and the most similar shafts were paired up. The common ends of each rod were then chucked into the lathe and the unmachined ends were turned down to a diameter of $8 \mathrm{~mm}$ and cut to the correct length. The drive shaft of each pair was then turned down further to $5 / 16$ in. and a flat was ground on each shaft. A slot for the interior retaining ring was then cut into the idler shafts. Finally, the portion of each shaft that a wheel would be pressed onto was sanded until the desired diameter was achieved. An image of the drive and idler shafts can be seen in Figure 76.

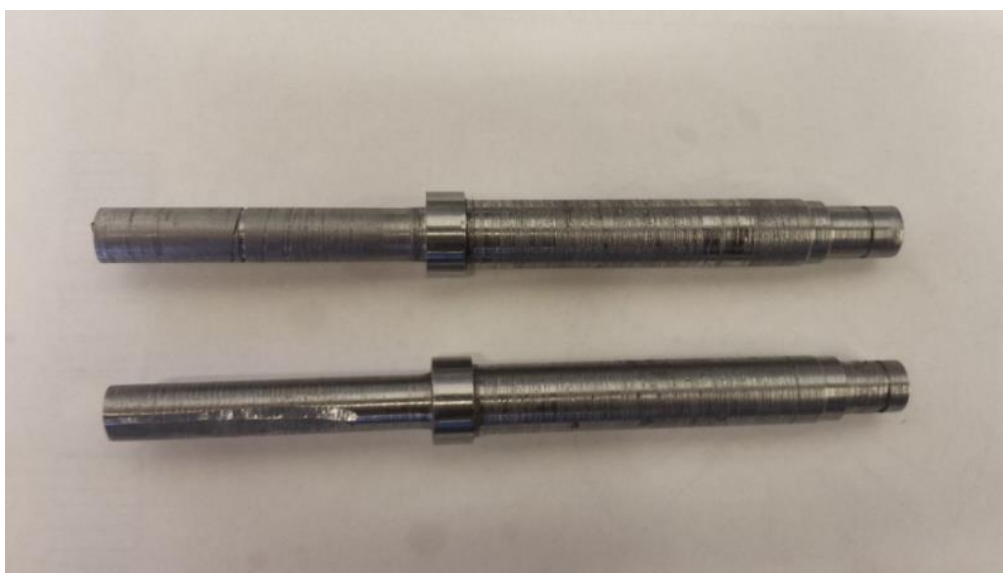

Figure 76: A set of drive and idler wheel shafts. 
Several difficulties were encountered during the manufacturing of the drive and idler shafts. The most significant of these was the lack of precision in the lathe used to manufacture the shafts. It was extremely difficult to achieve consistent diameters across the length of the shafts. This resulted in some amount of slop when the shafts were fitted into the bearings.

Another difficulty encountered during the machining of the idler and drive shafts was the jamming of a parting tool when cutting one of the idler shafts to length. This caused a significant bend in the back end of the shaft. A considerable amount of time was spent straightening out the shaft and, while the shaft was made significantly straighter, it was stilled slightly bent.

\subsubsection{Drive and Idler Wheels}

The drive and idler wheels were both $3 \mathrm{~d}$ printed. This first required the creation of .STL files from the Solidwork models of the wheels. These files were then inserted into the 3D printer software and the printer was run. Once the wheels were completed they were placed in a bath of acetic acid to remove the support material used in the printing process.

Once the wheels had dried, the interior of the hubs were sanded until their diameters fell within the desired range for press fitting onto the shafts. The wheels where then pressed onto their respective shafts using a hand press. An image of the drive and idler wheels after they were pressed onto their shafts can be seen in Figure 77 and Figure 78.

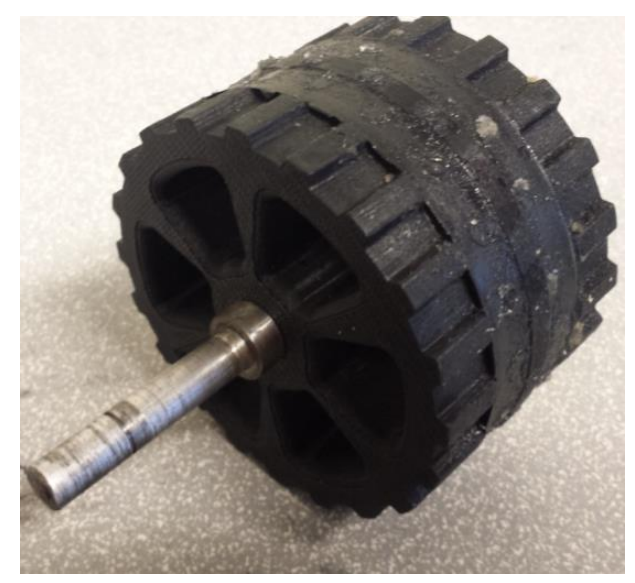

Figure 77: The idler wheel pressed onto its shaft. 


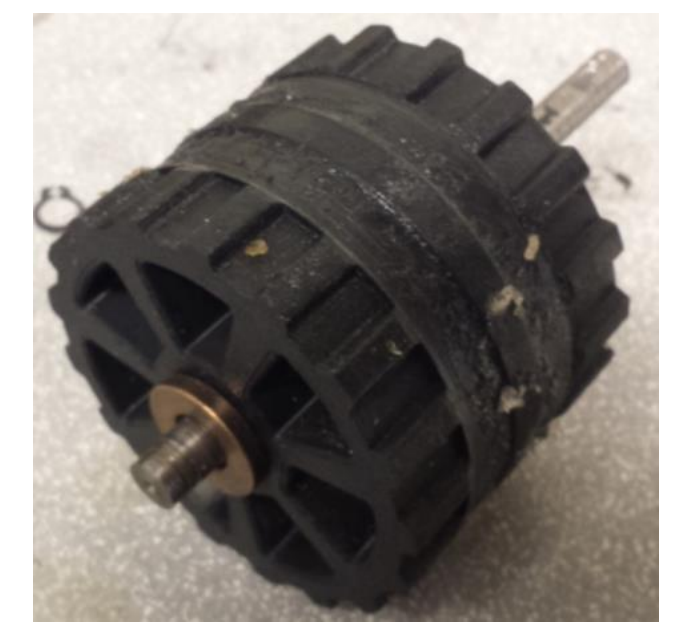

Figure 78: The drive wheel pressed onto its shaft.

The only difficulty encountered during the manufacturing of the drive and idler wheels was during a reprint one of the idler wheels. The reprinted wheel came out of the printer warped at one set of teeth. An image of this warpage can be seen in Figure 79. This was caused by the tray on which the wheel was printed shifting during the printing process due to heat expansion. This problem was corrected and a replacement wheel was produced.

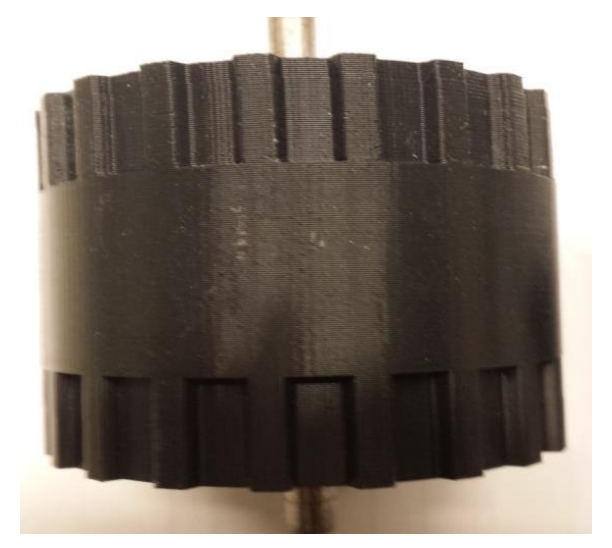

Figure 79: Warpage in the idler wheel.

In an effort to improve grease distribution on the tread, channels were machined into the central band of the drive and idler wheels. These channels were cut into the wheels by placing the wheels in a lathe and filing down the desired areas. The depth of the channels was chosen to such that the tread did not contact the bottoms of the channels but that enough material was left on the wheels that they would not break. The results of this modification to wheels are discussed further in Chapter 4. 


\subsubsection{Brace}

The manufacturing of the braces was relatively straight forward. Aluminum stock bar was rough cut to length on a band saw and the machined to length on a mill. The drilling of the mounting holes for the bearings came next and proved to be very difficult. Initially, an end mill with the same nominal diameter as the outer race of the bearing was used to create a blind hole for the bearing to be mounted in. The problem was that the actual end mill diameter was smaller than its nominal diameter, so the bearings could not be pressed in without being damaged. The solution was to drill a through hole in the brace using a drill bit, which had enough wobble to expand the hole to the desired size. A recess was then milled around the hole to accommodate the bearings flange.

The final step in manufacturing of the brace was the instillation of the bearings. The bearings and braces were thoroughly cleaned and then the retaining compounds catalyst was painted on to the outer race of the bearing and the bearing holes in the brace. The retaining compound was then applied to the bearing and the brace and the bearing was pressed into place by hand until the bearing and brace faces were flush. The excess retaining compound was then removed. An image of the braces can be seen in Figure 80.

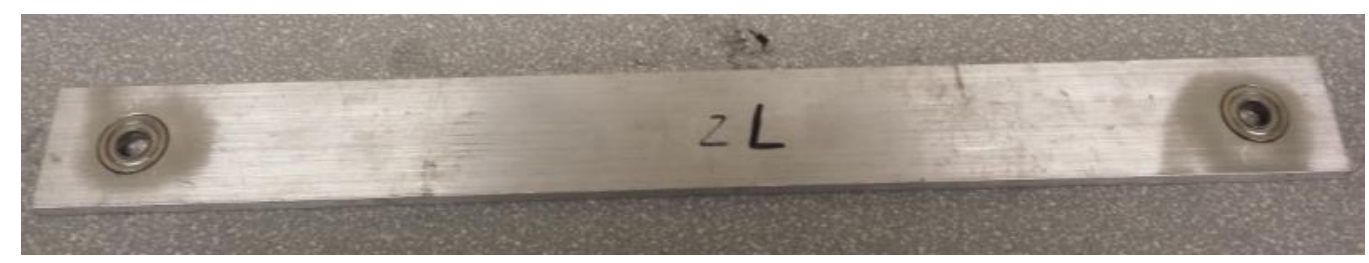

Figure 80: Brace with the bearings pressed into place.

\subsubsection{Tensioner Subsystem}

Figure 81 shows an image of the manufactured and assembled tensioner subsystem after it as assembled onto the frame. The final weight of the tensioner systems was $0.57 \mathrm{lbf}$ each. 


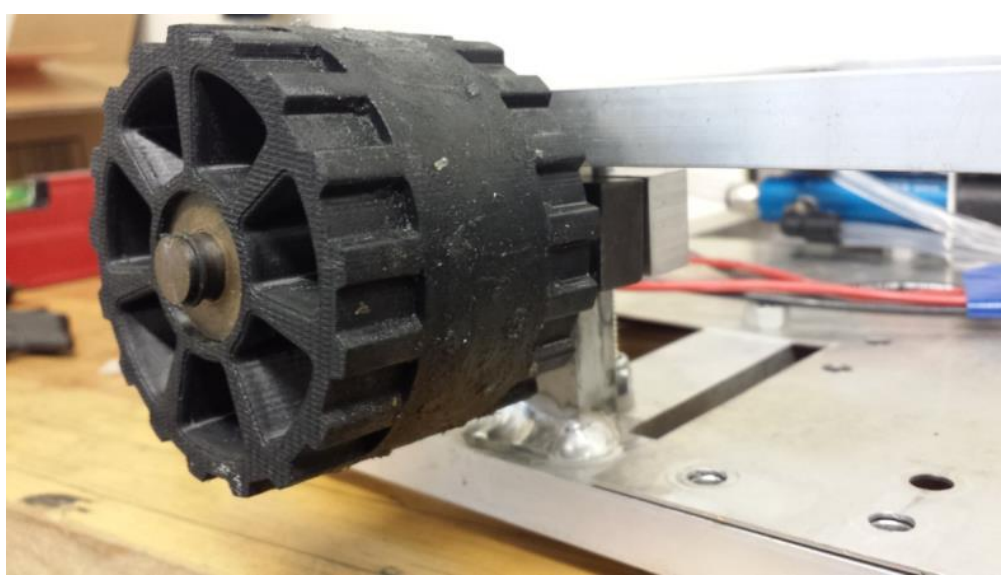

Figure 81: Tensioner subassembly attached to the wheel.

\subsubsection{Tensioner Clamp Exterior Half}

Stock steel bar was rough cut using a steel chop saw and then milled to the desired dimensions of the exterior clamp half. The slot was then milled in to the face of the clamp half and the tensioner shaft mounting hole drilled out. The holes for the attachment bolts were drilled out and tapped. The part was then deburred and polished, with special attention given to the slot. The tensioner shaft was then inserted into its mounting hole and the two parts were welded together. An image of the tensioner clamp exterior clamp half with the tensioner shaft welded in place can be seen in Figure 82.

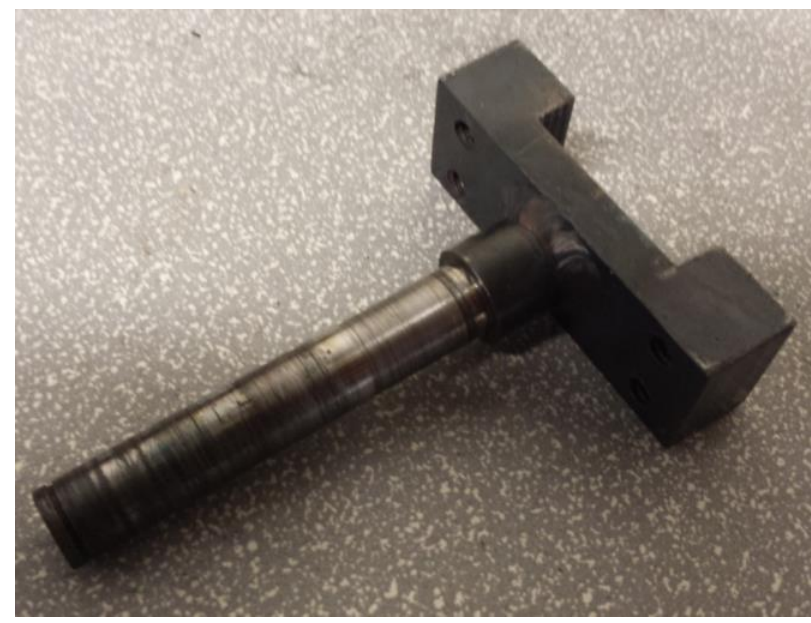

Figure 82: Exterior clamp half with the shaft in place.

The only anomalies encountered in the manufacturing of the tensioner clamp exterior half was that during assembly it was noted that the left side tensioner upright was sloped outward. This caused the tensioner wheel to be at a sever angle relative to the drive and idler wheels on that side. To correct this, two part 
putty epoxy was used to create an angled spacer on the exterior clamp half used on that side. This seemed to completely correct the issue. An image of the space on the exterior clamp half can be seen in Figure 83.

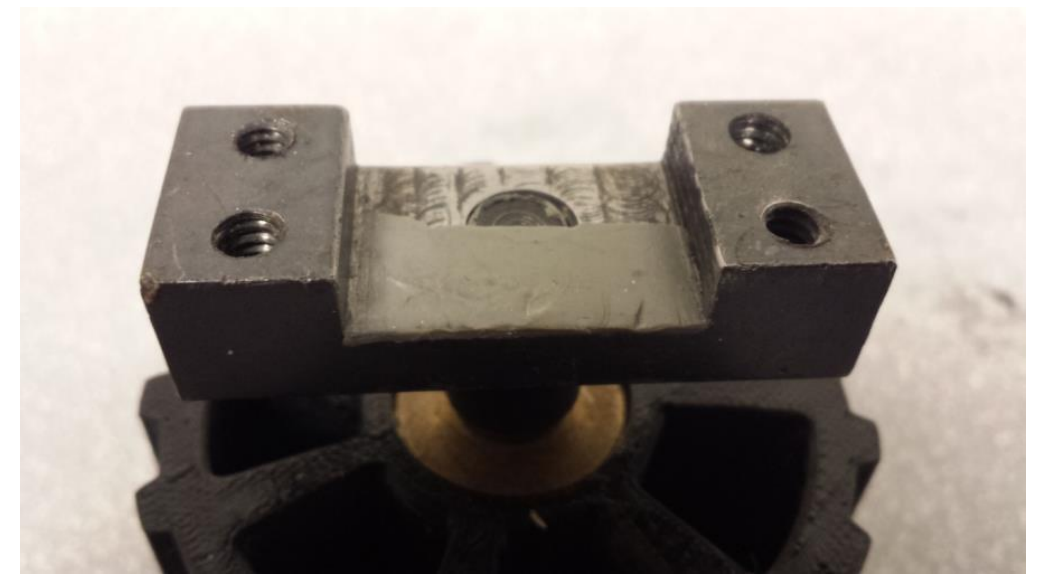

Figure 83: Spacer used to correct the misalignment of the left side tensioner wheel.

\subsubsection{Tensioner Clamp Interior Half}

Stock aluminum bar was rough cut on a band saw and then milled to the desired outer dimensions of the interior clamp half. The slot was then milled into one face of the parts and then flipped over and the exterior cuts were milled out. The through holes for the attachment bolts were then drilled out on the mill. The hole for the drive screw was then drilled and tapped. Finally the parts were deburred and polished, with special attention given to the slot. An image of the tensioner clamp interior clamp half can be seen in Figure 84.

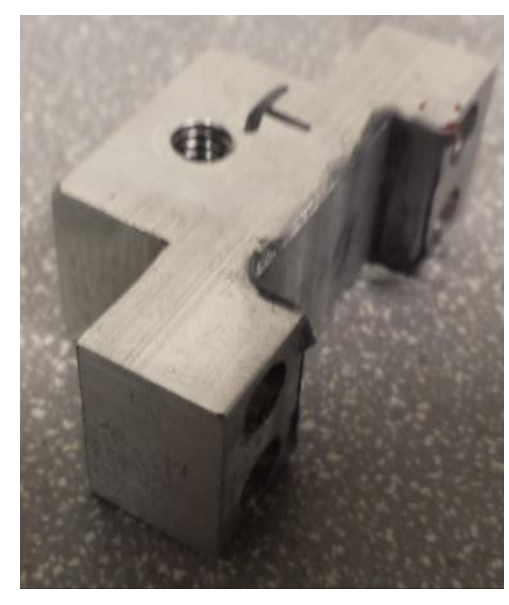

Figure 84: Interior clamp half. 


\subsubsection{Tensioner Wheel Assembly}

Like the drive and idler wheels, the tensioner wheels were 3D printed. The same over process was used, a .STL file was created and the tensioner wheels were clean and then the interior hub was sanded. Once the interior hub was smoothed, the bushings were pressed into place. To help ensure that the tensioner wheel would align properly with the drive and idler wheels, the bushings were pressed deeper than initially intended to create a recess on both faces of the tensioner wheels. Figure 85 shows the tensioner wheel with the bushings inserted.

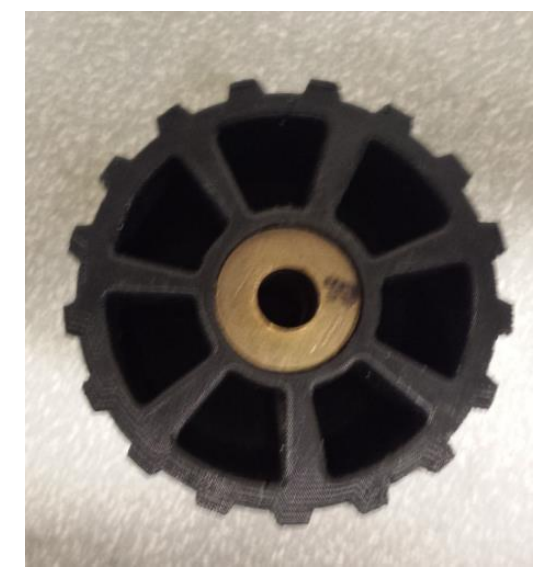

Figure 85: Tensioner wheel with the bushings in place.

\subsubsection{Tensioner Shaft}

The process of machining of the tensioner shafts was very similar to that of the process used for the drive and idler shafts. Stock steel rod was cut rough cut to length on a steel chop saw. The resulting blanks were then chucked in a lathe and the exterior portion of the shaft was turned down using a carbide tool. A slot for the retaining ring was cut near the tip of the shaft using a parting tool and then the end of the shaft was faced. The shafts were then flipped in the lathe and a parting too was used to cut the end of the shaft to length. The end of the shaft was then turned down to form the stud which would be inserted into the exterior clamp half. The shaft was then welded into place in the exterior clamp half. An image of the tensioner shaft welded in place can be seen in Figure 86. 


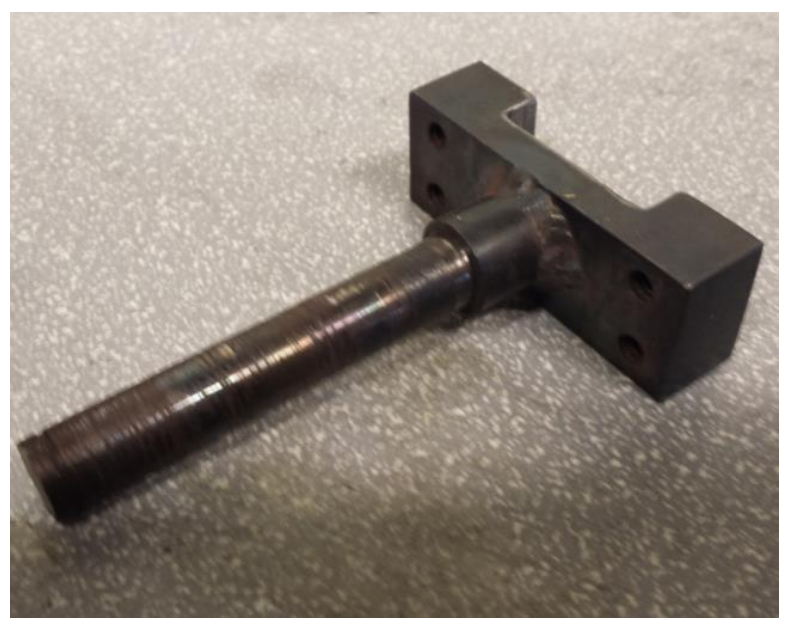

Figure 86: Tensioner shaft after it was welded to the exterior clamp half.

\subsubsection{Tread Manufacturing}

The tread was manufactured by F.N. Sheppard based on the designs sent to them. The following description of the manufacturing process was provided by an F.N. Sheppard employee. The timing belt portion of the tread was first cut to length. The teeth in the center of the belt were ground flat and the low friction fabric was glued into place. The neoprene backing was then cut to length and adhered to the timing belt and the adhesive was allowed to dry. The hole pattern was then drilled into the tread using a CNC process. Then ends of the tread were then welded together to form a continuous loop. Figure 87 contains an image of the left tread. The final weight of the treads was $0.56 \mathrm{lbf}$ each.

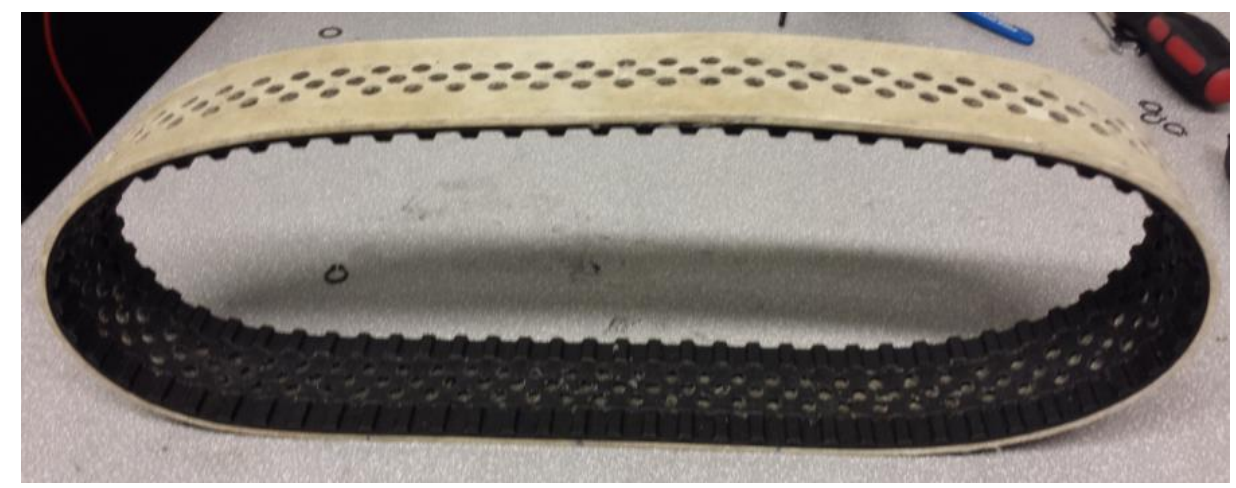

Figure 87: Vehicle tread.

Several modifications were made to the treads over the course of the project. The first of these modifications was the removal of the low friction fabric where it bunched at the teeth of the tread. The 
bunched fabric caused the surface of the channel to be uneven which in turn prevented the manifold from lying flat on the tread. Once the fabric was removed the manifold ran smoothly in the treads.

The next modification made to the treads was the application of silicone sealant over the low friction fabric in an effort to improve the seal between the manifold and the tread. The sealant was carefully aliped and smoothed to create a flat surface for the manifold to slide on and to fill in the gaps in the low friction fabric. Figure 88 shows the fabric before the sealant was applied and Figure 89 shows the fabric after the sealant was applied.Special care was taken to clear each of the tread holes so as not to clog them with the sealant. The results of the application of the sealant to the treads are discussed in Chapter 4 .

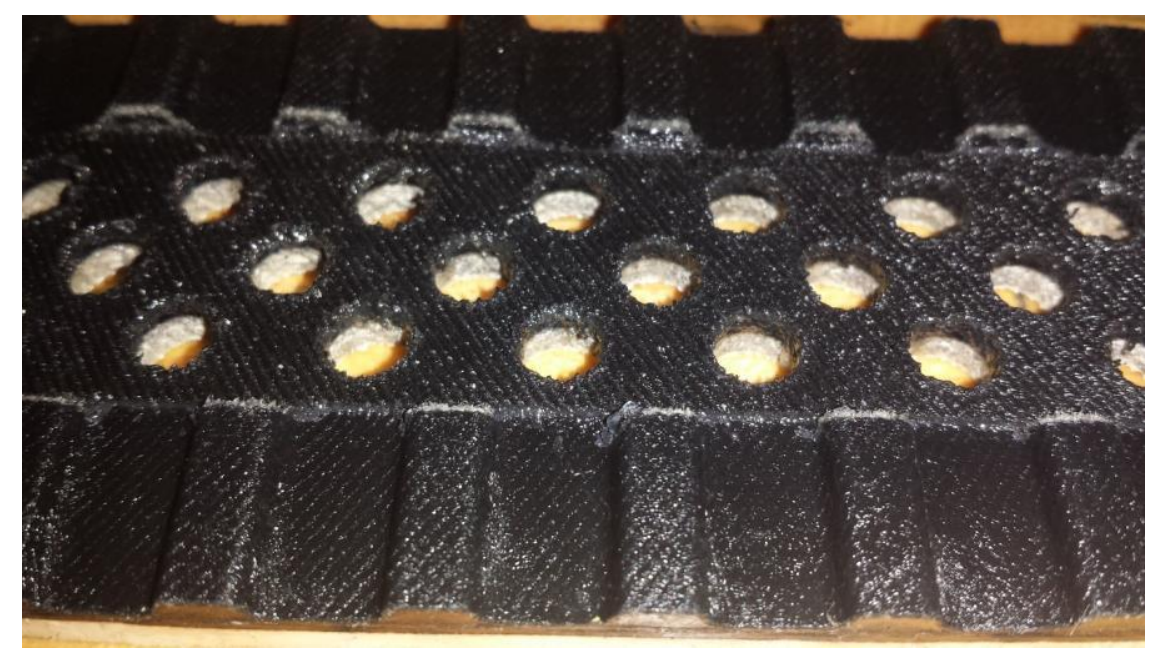

Figure 88: Low friction fabric before the application of the silicone sealant.

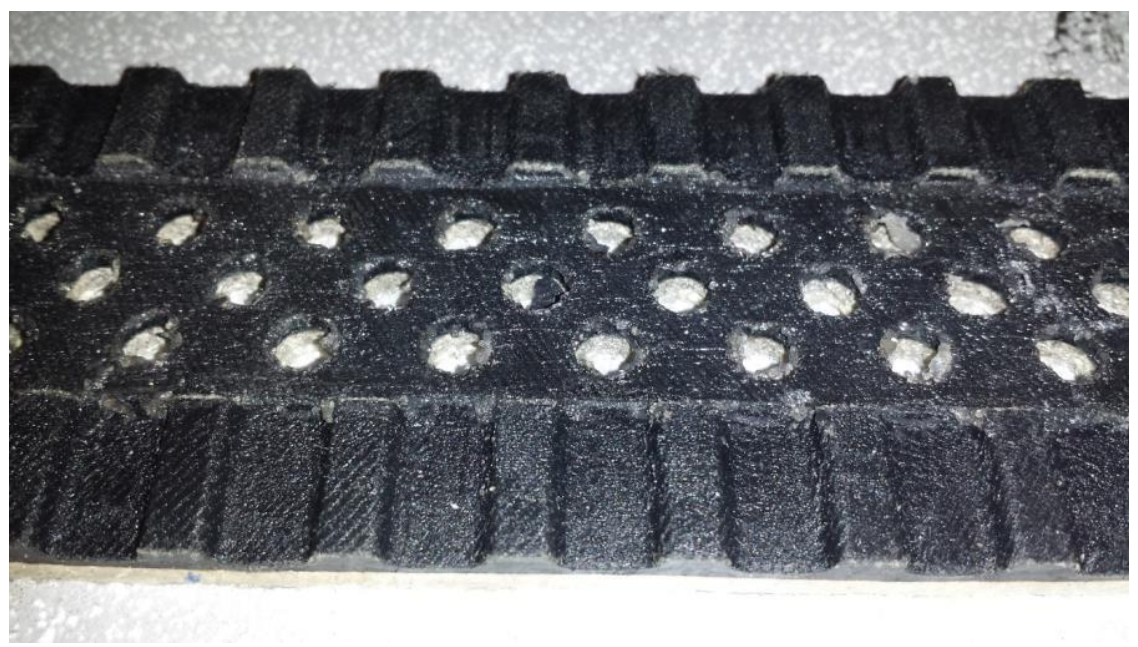

Figure 89: Low friction fabric after the application of the silicone sealant. 
Finally, the same silicone sealant that was applied to the low friction fabric on the interior of the tread was applied to the exterior of the tread in an effort to increase the coefficient of friction between the wall and the tread. The sealant was carefully applied to the outer surface of the tread and smooth. Figure 90 shows the tread backing before the sealant was applied and Figure 91 shows the tread backing after the sealant was applied. Again, each hole was cleared to prevent clogging. The results of the application of the sealant to the treads are discussed in Chapter 4 .

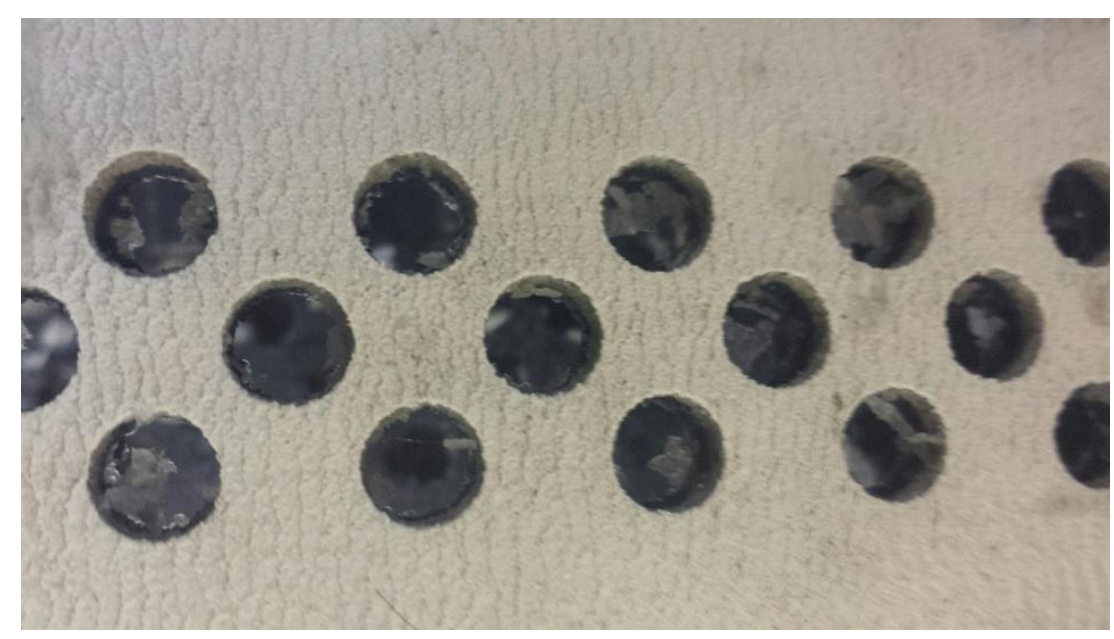

Figure 90: Tread backing before sealant was applied.

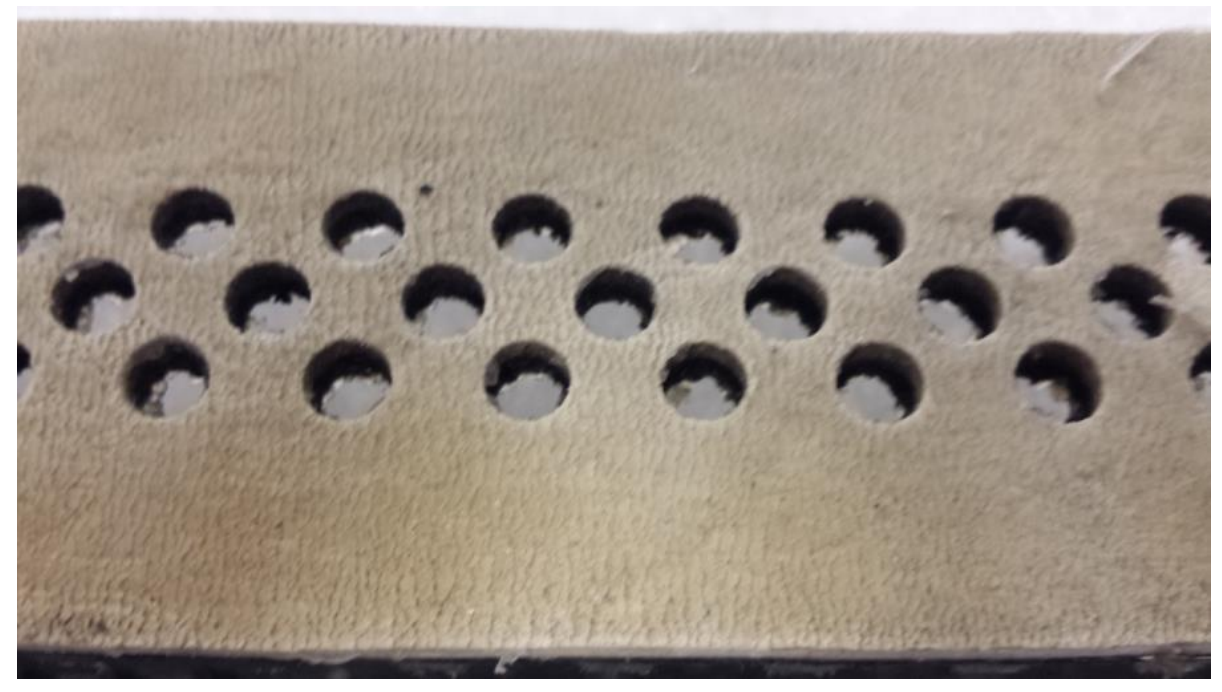

Figure 91: Tread backing after the silicone sealant was applied.

\subsection{Assembly}

This section gives brief overview of the assembled vehicle. It shows the placement of each of the subsystems within the actual vehicle. The final weight of the vehicle was 13.6 lbf. Figure 92 shows the 
entire assembled vehicle and Figure 93, Figure 94, Figure 95 and Figure 96 shows the different subsystems in place in the vehicle.

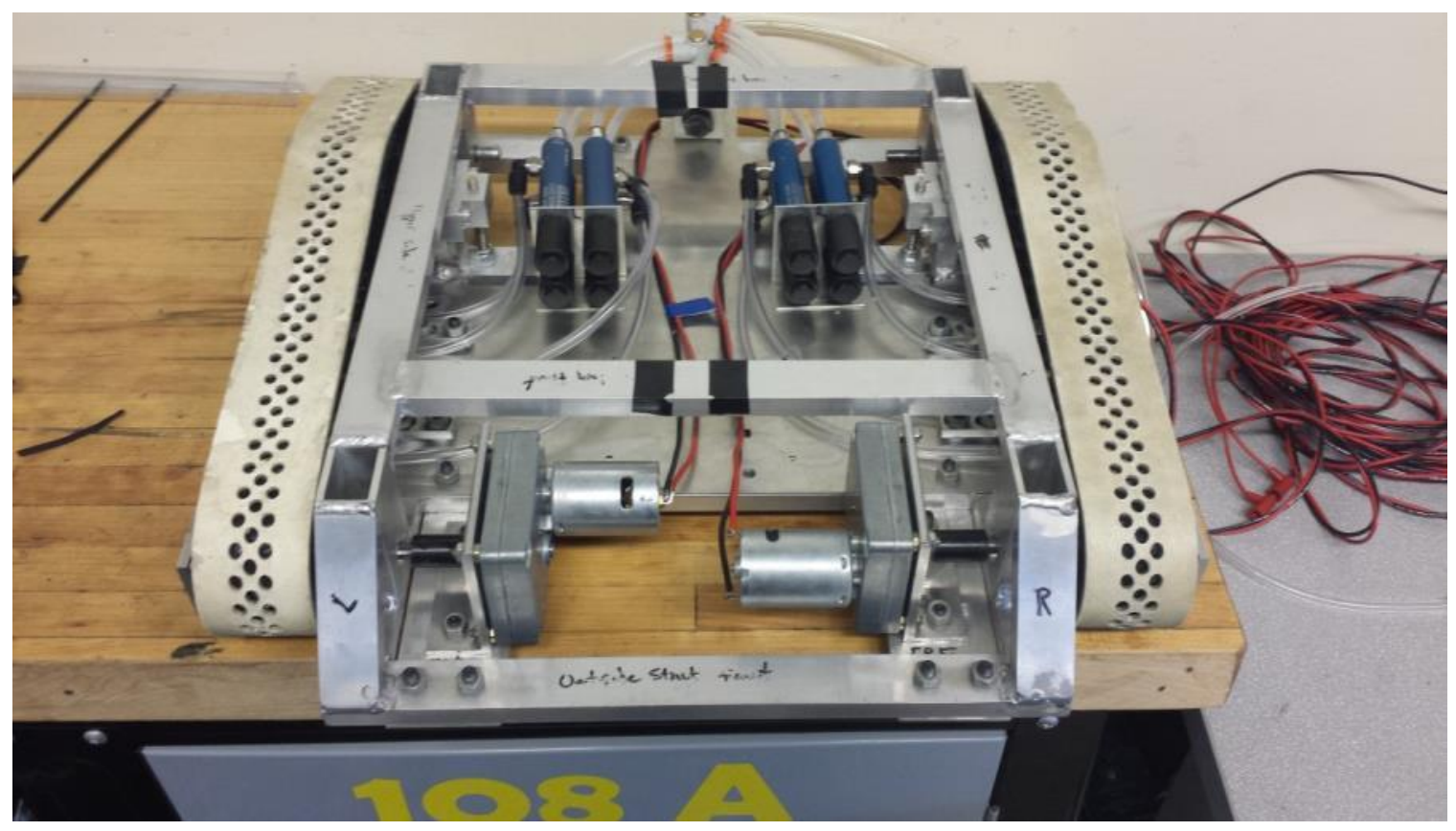

Figure 92: Fully assembled vehicle.

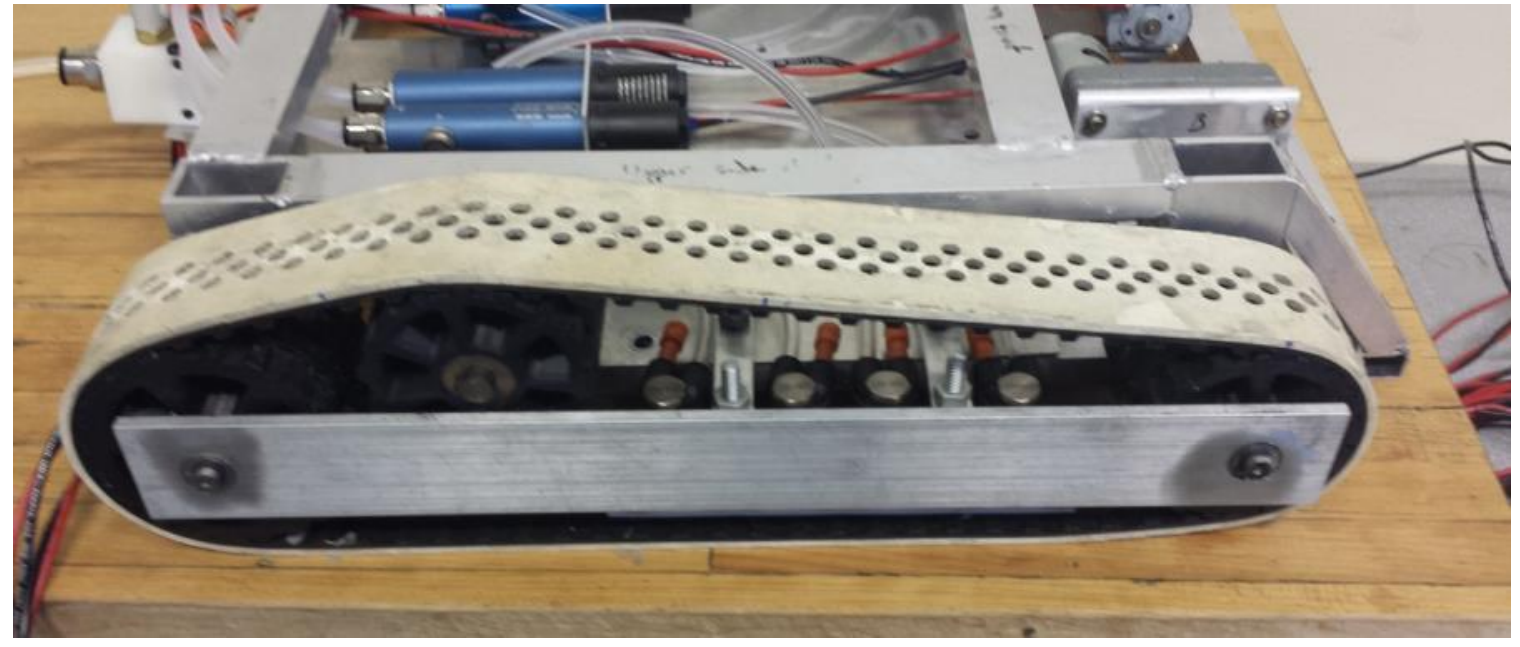

Figure 93: Wheel assembly and manifold on vehicle. 


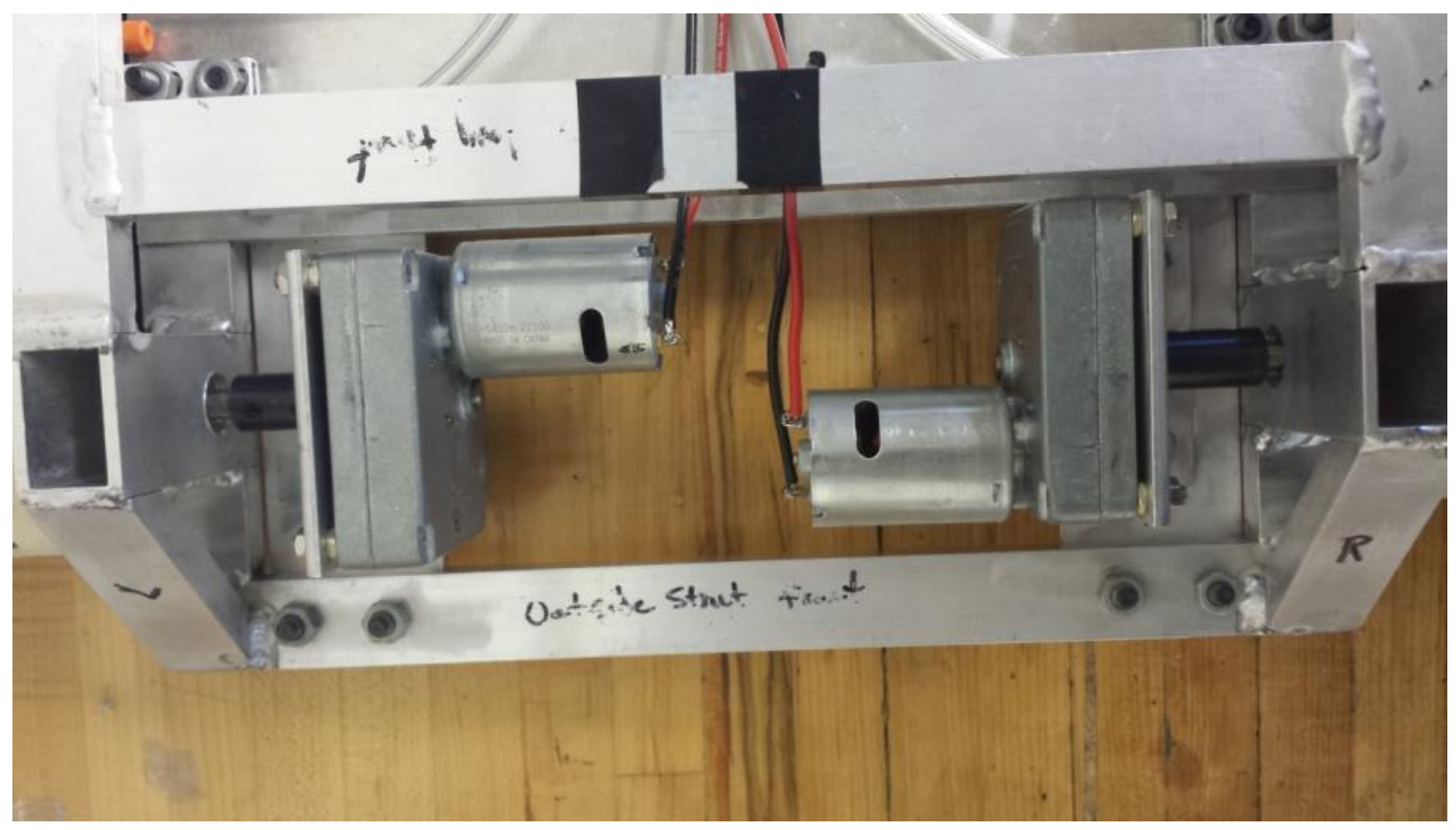

Figure 94: Motor subsystems.

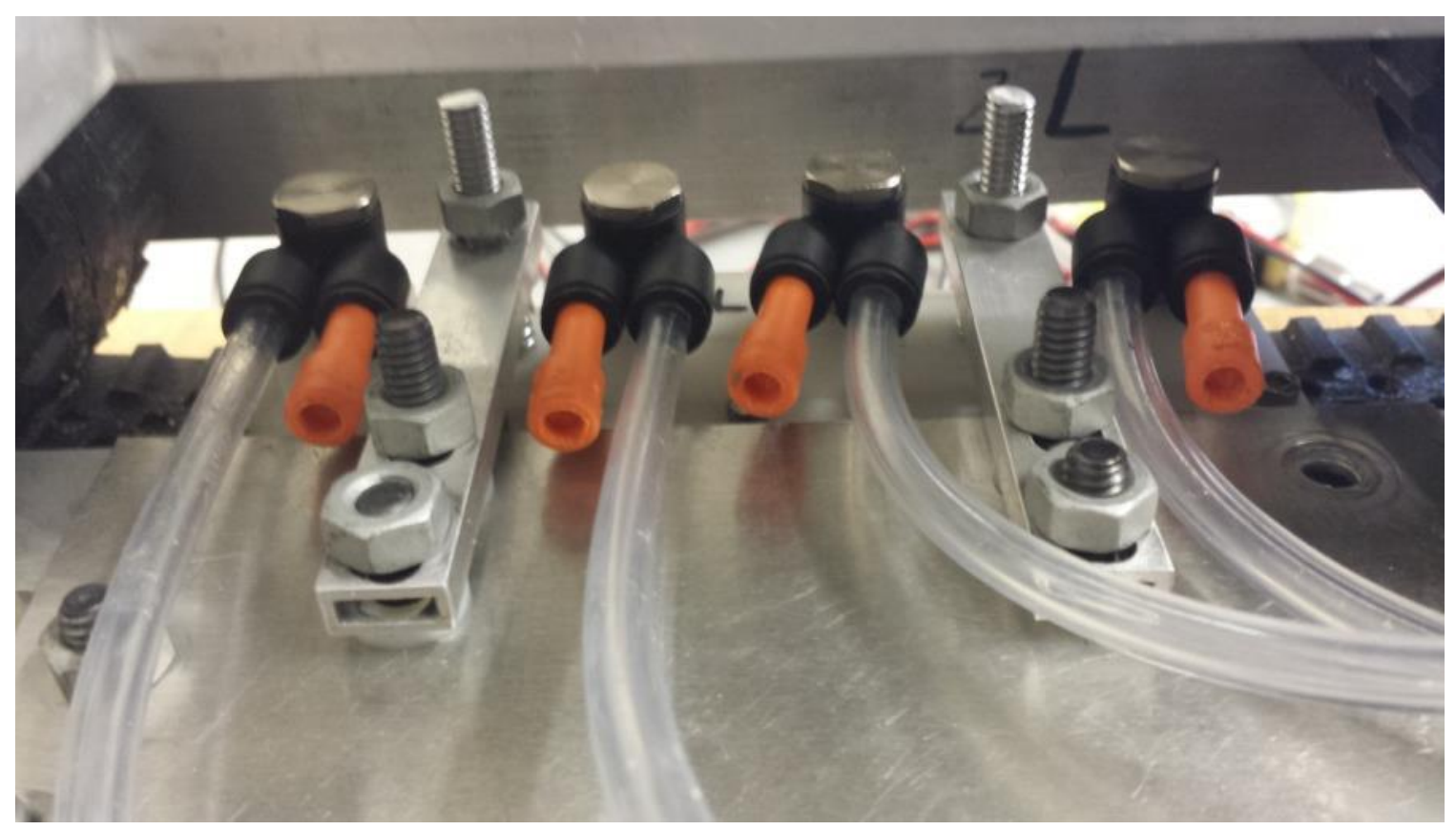

Figure 95: Manifolds from the inside of the vehicle. 


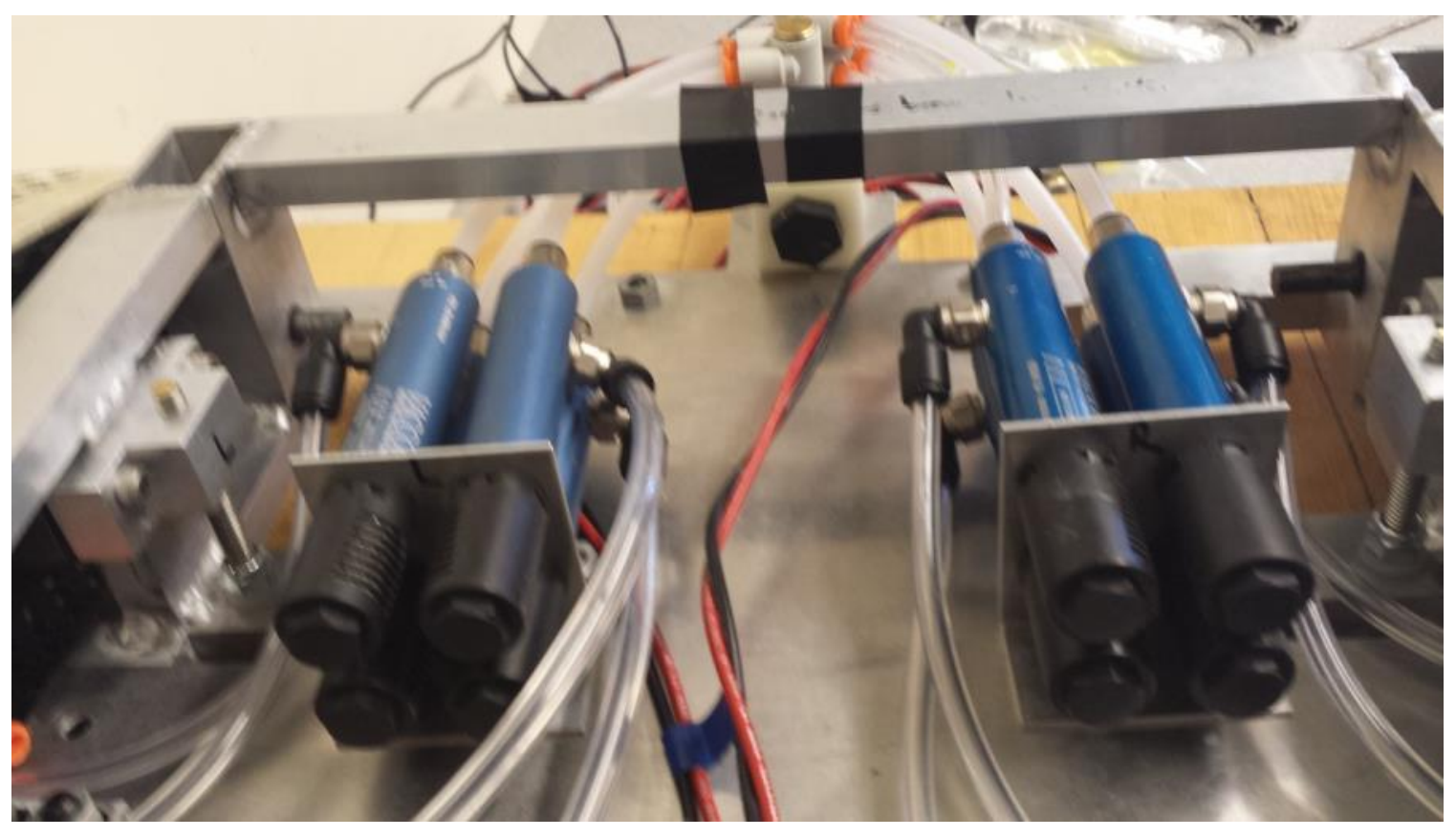

Figure 96: Vacuum system installed in the vehicle. 


\section{Chapter 4: Testing}

This chapter discusses the testing which was performed on the final vehicle. Additional test data can be found in Appendix D.

\subsection{Pressure System Testing}

This section covers a series of tests done to gain insight into the vacuum system and how it performed under different conditions. This includes the assessment of the venturi's performance, the verification that each manifold section is independent from the others and how the manifolds act under different vehicle conditions.

The basic procedure for each of the tests conducted was very similar. A vacuum gauge, seen in Figure 97, was attached to the manifold section or venturi being tested and the vacuum pressure was measured. The following subsections give more detailed descriptions of specific tests which were conducted. To eliminate variations in the tread surface from effecting the testing, each test was conducted on the same section of the left and right treads.

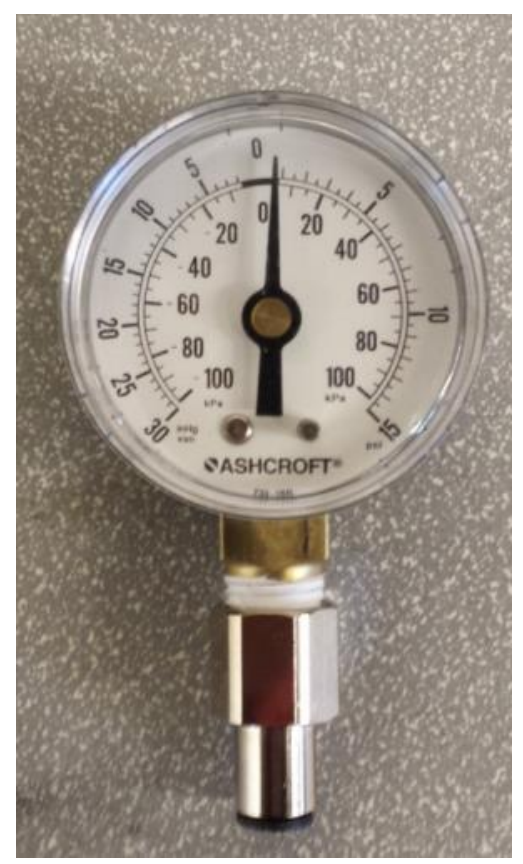

Figure 97: Vacuum gauge used during the pressure system testing.

A general procedure for the manifold testing, referred to as the general manifold testing scheme, was developed to keep the tests consistent. The scheme is as follows: 
1. The vacuum in the manifold being tested was engaged with all sections closed to the atmosphere.

2. The first manifold section to be tested was connected to the vacuum gauge.

3. The vacuum pressure was recorded.

4. The section closest to the front of the vehicle not being tested was exposed to the atmosphere by removing its plug and the pressure in the section being tested was recorded.

5. Repeat step 4 for each closed section until all sections are open except the one being tested.

6. The vacuum gauge was then moved to the next section to be tested and all open sections were closed.

7. Repeat steps 3-6 until each vacuum section has been tested.

This scheme also included the testing of each section before and after each test to determine if anything untoward occurred during the testing, such as a section becoming clogged. This was done by measuring the pressure in each section sequentially with all the other sections on the manifold closed.

\subsubsection{Venturi Reliability}

\subsubsection{Procedure}

This test was conducted by measuring the vacuum pressure that was produced by each venture and to determine if it was consistent and to determine the likelihood of clogging. Before each of the other tests in this section were run, the vacuum force produced by each venturi was measured and recorded by attaching the vacuum gauges directly to the venturi's outlet.

\subsubsection{Results}

The results of the venturi reliability testing can be found in Table 45 . No venturi clogging was seen during the pressure system testing.

Table 45: Average measured ventuir vacuum pressure versus predicted vacuum pressure.

\begin{tabular}{|l|r|r|r|}
\hline \multicolumn{1}{|c|}{ Venturi Bank } & Average Measured Pressure & Ideal Pressure & \% Difference \\
\hline Right & $13.16 \mathrm{psi}$ & $13.75 \mathrm{psi}$ & $4.3 \%$ \\
\hline Left & $13.33 \mathrm{psi}$ & $13.75 \mathrm{psi}$ & $3.1 \%$ \\
\hline Total & $13.25 \mathrm{psi}$ & $13.75 \mathrm{psi}$ & $3.6 \%$ \\
\hline
\end{tabular}




\subsubsection{Discussion}

The venturis proved to be extremely reliable. The average vacuum pressure produced by the banks is very close to the expected outputs. The discrepancies between the expected and measured values can be explained by minor losses in the fittings used on the venturis and the vacuum gauge or by losses in the tubing. No clogging of the venturis was observed during the entirety of the pressure system testing.

\subsubsection{Manifold Section Independence}

\subsubsection{Procedure}

This test was conducted to determine if the sections of each manifold were independent of each other internally and to determine the pressure losses in the manifolds to the atmosphere. To do this, each manifold was placed on a sheet of silicon rubber, as shown in Figure 98 and the vacuum was engaged. The general manifold section testing scheme was then used to test the manifolds.

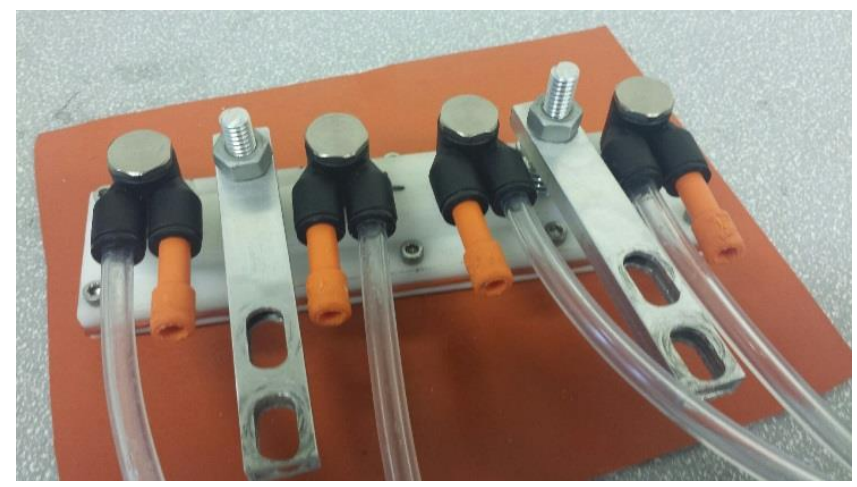

Figure 98: A manifold on the silicone sheet.

\subsubsection{Results}

The results of the manifold section independence testing can be found in Table 46 and Table 47.

Table 46: Right side venturi section independence results.

\begin{tabular}{|c|r|r|r|}
\hline Section & $\begin{array}{c}\text { Average Measured } \\
\text { Pressure }\end{array}$ & Average Venturi Pressure & \multicolumn{1}{c|}{ \% Difference } \\
\hline 1 & $12.83 \mathrm{psi}$ & $13.01 \mathrm{psi}$ & $1.4 \%$ \\
\hline 2 & $12.91 \mathrm{psi}$ & $13.50 \mathrm{psi}$ & $4.4 \%$ \\
\hline 3 & $12.85 \mathrm{psi}$ & $13.38 \mathrm{psi}$ & $4.0 \%$ \\
\hline 4 & $12.89 \mathrm{psi}$ & $13.38 \mathrm{psi}$ & $3.7 \%$ \\
\hline Average & $12.89 \mathrm{psi}$ & $13.32 \mathrm{psi}$ & $3.4 \%$ \\
\hline
\end{tabular}


Table 47: Left side venturi section independence results.

\begin{tabular}{|c|c|r|r|}
\hline Section & $\begin{array}{c}\text { Average Measured } \\
\text { Pressure }\end{array}$ & Average Venturi Pressure & \multicolumn{2}{c|}{ \% Difference } \\
\hline 1 & $13.24 \mathrm{psi}$ & $13.50 \mathrm{psi}$ & $1.9 \%$ \\
\hline 2 & $13.18 \mathrm{psi}$ & $13.26 \mathrm{psi}$ & $0.6 \%$ \\
\hline 3 & $13.01 \mathrm{psi}$ & $13.50 \mathrm{psi}$ & $3.6 \%$ \\
\hline 4 & $12.95 \mathrm{psi}$ & $13.38 \mathrm{psi}$ & $3.2 \%$ \\
\hline Average & $13.10 \mathrm{psi}$ & $13.41 \mathrm{psi}$ & $2.3 \%$ \\
\hline
\end{tabular}

\subsubsection{Discussion}

The sections in the right and left manifolds proved to be completely independent from one another. The data in Appendix D shows that even when all other sections in a given manifold are open, the measured section maintains its initial pressure. This implies that the manifold gasket provides sufficient seal to prevent all interaction between the sections and that the manifolds themselves are well sealed with few to no defects.

When the measured venturi pressure for a given section taken at the start of the test was compared to the pressure in the section only minor losses are found. These losses are likely due to minute losses in the manifold at the gasket and the inlet fitting.

\subsubsection{Tread Installation Effect and Fixed Manifold Testing}

\subsubsection{Procedure}

This test was conducted to determine if the treads being installed on the vehicle had any effect on the performance of the manifolds. To do this, each manifold was placed on its respective tread after the treads had been removed from the vehicle. The general manifold testing scheme was then used on each manifold. The treads were then placed onto the wheel system and the manifolds were placed onto the tread. The general manifold testing scheme was used on each manifold. Finally the manifolds were fixed to the vehicle and then tested using the general manifold testing scheme.

\subsubsection{Results}

Table 48 and Table 49 contain the results from the tread installation and fixed manifold testing for the right and left sides of the vehicle respectively. 
Table 48: Right side tread and manifold condition testing results.

\begin{tabular}{|c|c|c|c|c|}
\hline \multicolumn{5}{|c|}{ Tread Off Vehicle } \\
\hline $\begin{array}{c}\text { Tested } \\
\text { Section }\end{array}$ & All Sections Closed & 1 Section Open & 2 Sections Open & 3 Sections Open \\
\hline 1 & $7.37 \mathrm{psi}$ & $3.44 \mathrm{psi}$ & $1.47 \mathrm{psi}$ & $7.86 \mathrm{psi}$ \\
\hline 2 & $12.28 \mathrm{psi}$ & $12.28 \mathrm{psi}$ & $11.79 \mathrm{psi}$ & $9.82 \mathrm{psi}$ \\
\hline 3 & $12.28 \mathrm{psi}$ & $11.79 \mathrm{psi}$ & $11.05 \mathrm{psi}$ & $7.37 \mathrm{psi}$ \\
\hline 4 & $12.28 \mathrm{psi}$ & $12.28 \mathrm{psi}$ & $12.28 \mathrm{psi}$ & $10.31 \mathrm{psi}$ \\
\hline \multicolumn{5}{|c|}{ Tread On Vehicle } \\
\hline $\begin{array}{c}\text { Tested } \\
\text { Section }\end{array}$ & All Sections Closed & 1 Section Open & 2 Sections Open & 3 Sections Open \\
\hline 1 & $9.82 \mathrm{psi}$ & $7.37 \mathrm{psi}$ & $6.38 \mathrm{psi}$ & $9.82 \mathrm{psi}$ \\
\hline 2 & $12.28 \mathrm{psi}$ & $12.28 \mathrm{psi}$ & $10.80 \mathrm{psi}$ & $10.56 \mathrm{psi}$ \\
\hline 3 & $11.79 \mathrm{psi}$ & $11.79 \mathrm{psi}$ & $11.79 \mathrm{psi}$ & $9.33 \mathrm{psi}$ \\
\hline 4 & $12.28 \mathrm{psi}$ & $12.28 \mathrm{psi}$ & $12.28 \mathrm{psi}$ & $10.80 \mathrm{psi}$ \\
\hline \multicolumn{5}{|c|}{ Fixed Manifold } \\
\hline $\begin{array}{c}\text { Tested } \\
\text { Section }\end{array}$ & All Sections Closed & 1 Section Open & 2 Sections Open & 3 Sections Open \\
\hline 1 & $8.84 \mathrm{psi}$ & $3.93 \mathrm{psi}$ & $2.95 \mathrm{psi}$ & $5.65 \mathrm{psi}$ \\
\hline 2 & $12.28 \mathrm{psi}$ & $11.79 \mathrm{psi}$ & $10.80 \mathrm{psi}$ & $10.56 \mathrm{psi}$ \\
\hline 3 & $11.79 \mathrm{psi}$ & $11.79 \mathrm{psi}$ & $11.79 \mathrm{psi}$ & $10.56 \mathrm{psi}$ \\
\hline 4 & $12.28 \mathrm{psi}$ & $12.28 \mathrm{psi}$ & $12.28 \mathrm{psi}$ & $11.79 \mathrm{psi}$ \\
\hline
\end{tabular}

Table 49: Left side tread and manifold condition testing results.

\begin{tabular}{|c|c|c|c|c|}
\hline \multicolumn{5}{|c|}{ Tread Off Vehicle } \\
\hline $\begin{array}{l}\text { Tested } \\
\text { Section }\end{array}$ & All Sections Closed & 1 Section Open & 2 Sections Open & 3 Sections Open \\
\hline 1 & $10.31 \mathrm{psi}$ & $9.33 \mathrm{psi}$ & $8.84 \mathrm{psi}$ & $8.84 \mathrm{psi}$ \\
\hline 2 & $9.82 \mathrm{psi}$ & $8.59 \mathrm{psi}$ & $4.91 \mathrm{psi}$ & $1.47 \mathrm{psi}$ \\
\hline 3 & $9.33 \mathrm{psi}$ & $8.84 \mathrm{psi}$ & $4.91 \mathrm{psi}$ & $0.98 \mathrm{psi}$ \\
\hline 4 & $12.28 \mathrm{psi}$ & $12.28 \mathrm{psi}$ & $12.28 \mathrm{psi}$ & $10.80 \mathrm{psi}$ \\
\hline \multicolumn{5}{|c|}{ Tread On Vehicle } \\
\hline $\begin{array}{c}\text { Tested } \\
\text { Section }\end{array}$ & All Sections Closed & 1 Section Open & 2 Sections Open & 3 Sections Open \\
\hline 1 & $8.35 \mathrm{psi}$ & $4.91 \mathrm{psi}$ & $1.96 \mathrm{psi}$ & $6.88 \mathrm{psi}$ \\
\hline 2 & $10.31 \mathrm{psi}$ & $9.82 \mathrm{psi}$ & $4.91 \mathrm{psi}$ & $0.98 \mathrm{psi}$ \\
\hline 3 & $9.33 \mathrm{psi}$ & $9.33 \mathrm{psi}$ & $4.42 \mathrm{psi}$ & $0.49 \mathrm{psi}$ \\
\hline 4 & $11.30 \mathrm{psi}$ & $11.30 \mathrm{psi}$ & $10.80 \mathrm{psi}$ & $9.82 \mathrm{psi}$ \\
\hline \multicolumn{5}{|c|}{ Fixed Manifold } \\
\hline $\begin{array}{l}\text { Tested } \\
\text { Section }\end{array}$ & All Sections Closed & 1 Section Open & 2 Sections Open & 3 Sections Open \\
\hline 1 & $8.35 \mathrm{psi}$ & $6.88 \mathrm{psi}$ & $5.89 \mathrm{psi}$ & $10.31 \mathrm{psi}$ \\
\hline 2 & $10.80 \mathrm{psi}$ & $10.31 \mathrm{psi}$ & $7.37 \mathrm{psi}$ & $3.68 \mathrm{psi}$ \\
\hline 3 & $10.31 \mathrm{psi}$ & $9.82 \mathrm{psi}$ & $6.38 \mathrm{psi}$ & $1.96 \mathrm{psi}$ \\
\hline 4 & 11.79 psi & $11.79 \mathrm{psi}$ & $11.30 \mathrm{psi}$ & $9.82 \mathrm{psi}$ \\
\hline
\end{tabular}




\subsubsection{Discussion}

There were several different insights which were gained during this portion of the pressure testing. Each of these insights is critical to the robot's ability to function and can be used to improve the performance of future robots significantly.

The first of these insights, and perhaps the most important, is that the geometries of the manifold are critical to its ability to form a seal with the treads. In every test case pressure drops were seen in the section being measured as the sections next to it were opened. This is best seen in the raw data in Appendix D. Additionally, the vacuum pressure in the first and last sections of the manifolds is to some extent dependent on the pressure in the section that is the furthest away from it, again best illustrated in the raw data. Both of these phenomena imply that the manifolds do not lie flat against the treads. This is clearly the case as seen in Figure 99.

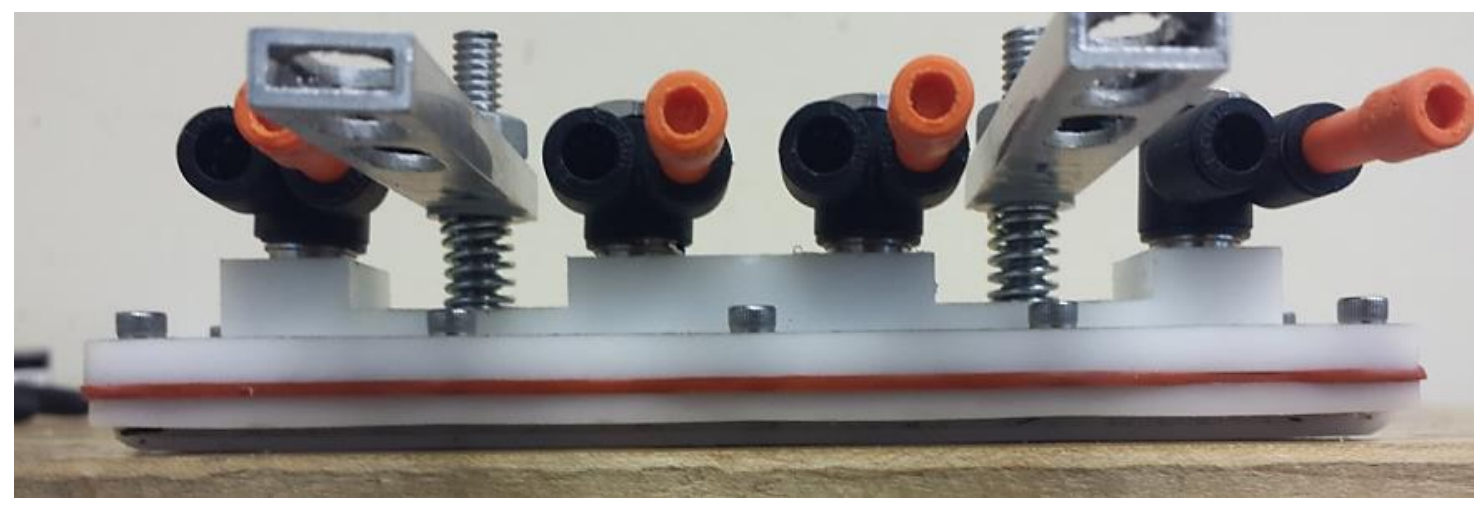

Figure 99: Warpage of the lower half of the manifold.

Another interesting insight is that the magnitudes of the forces seen when all sections are closed is very similar for each of the test cases. This indicates that neither the treads being installed on the wheels nor the manifold being fixed in place cause any impediment to the initial seal formed by the manifold. This is not the case as the manifold sections lose suction. The fixed manifold testing seems to indicate that the manifolds being fixed in place can improve a sections ability to retain its vacuum as the other sections are opened. This is most likely a consequence of both the spring suspension pushing the manifold onto the tread and the manifold's supports preventing the manifold from shifting its position on the tread as sections are opened. 


\subsubsection{Vertical Surface Testing}

\subsubsection{Procedure}

This test was conducted to determine the performance of the manifold system's when the vehicle was adhered to a vertical surface. This was done by adhering the vehicle to a wall and then measuring the pressure in each manifold section sequentially. The general manifold testing scheme was not used as the vehicle could not keep itself adhered to the wall with one manifold not producing an adhering force. Figure 100 shows the vehicle on a vertical surface.

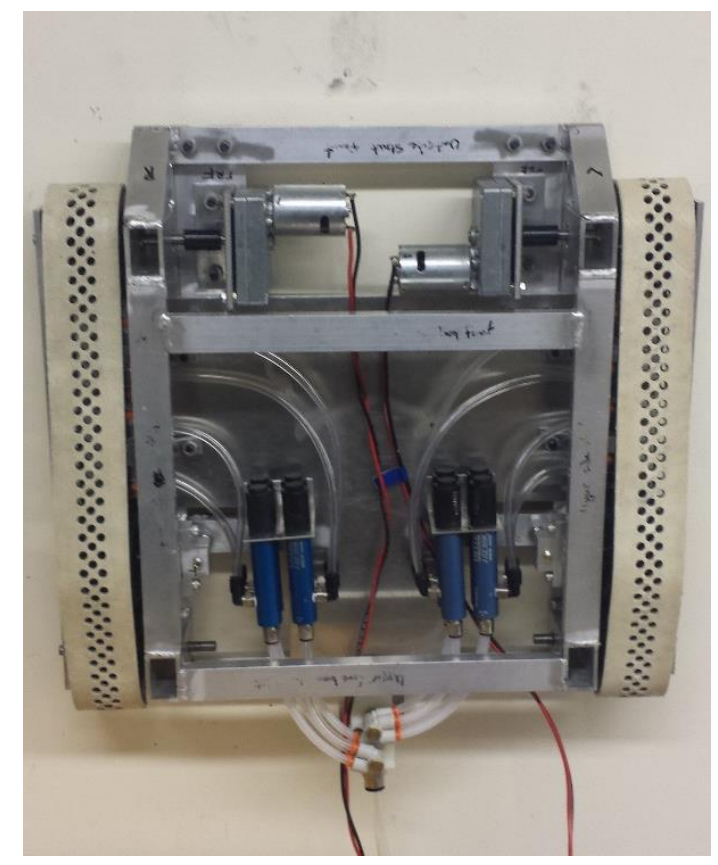

Figure 100: The vehicle on a vertical surface.

\subsubsection{Results}

The results of the vertical surface testing of the pressure system for the right and left sides of the vehicle can be found in Table 50 and Table 51 respectively.

Table 50: On wall results of the vertical surface testing.

\begin{tabular}{|c|r|r|r|r|}
\hline & \multicolumn{2}{|c|}{ Right Side } & \multicolumn{2}{c|}{ Left Side } \\
\hline Section & Average & Standard Dev. & Average & Standard Dev. \\
\hline 1 & $0.49 \mathrm{psi}$ & 0.69 & $6.38 \mathrm{psi}$ & 4.17 \\
\hline 2 & $10.61 \mathrm{psi}$ & 1.86 & $10.90 \mathrm{psi}$ & 0.73 \\
\hline 3 & $12.08 \mathrm{psi}$ & 0.27 & $10.21 \mathrm{psi}$ & 1.06 \\
\hline 4 & $12.28 \mathrm{psi}$ & 0.00 & $4.17 \mathrm{psi}$ & 3.98 \\
\hline Total & $8.86 \mathrm{psi}$ & 5.63 & $7.92 \mathrm{psi}$ & 3.19 \\
\hline
\end{tabular}


Table 51: Off wall results of the vertical surface testing.

\begin{tabular}{|c|r|r|r|r|}
\hline & \multicolumn{2}{|c|}{ Right Side } & \multicolumn{2}{c|}{ Left Side } \\
\hline Section & Average & Standard Dev. & Average & \multicolumn{1}{c|}{ Standard Dev. } \\
\hline 1 & $8.69 \mathrm{psi}$ & 4.09 & $11.15 \mathrm{psi}$ & 0.33 \\
\hline 2 & $12.57 \mathrm{psi}$ & 0.27 & $10.26 \mathrm{psi}$ & 0.96 \\
\hline 3 & $12.28 \mathrm{psi}$ & 0.00 & $10.66 \mathrm{psi}$ & 0.81 \\
\hline 4 & $9.28 \mathrm{psi}$ & 3.20 & $10.26 \mathrm{psi}$ & 1.18 \\
\hline Total & $10.71 \mathrm{psi}$ & 2.00 & $10.58 \mathrm{psi}$ & 0.42 \\
\hline
\end{tabular}

\subsubsection{Discussion}

The vertical surface testing of the vehicle revealed several different insights into the behavior of both the vehicle as whole and the manifold system itself. Firstly, the front section of the right side manifold was completely exposed to the atmosphere during each of the tests. This seems to have been the result of the vehicles frame being misaligned which cause the right corner of the vehicle to rise off of the wall. This in turn caused the tread to lift slightly which broke the seal between the wall and tread. This implies that the frames alignment is critical to the proper functioning of the manifold system.

The left hand manifolds performance during the testing was erratic, with the pressure in the front and rear sections varying dramatically between tests. This seems to have been the result of the front threaded upright consistently loosening during the testing. This allowed the manifold to shift more than it otherwise would have been able to.

\subsubsection{Clogged vs Unclogged Performance}

\subsubsection{Procedure}

There were several observed incidents during the testing of the manifolds lower chambers filling with the vacuum grease. This series of tests was done to determine if this had a direct effect on the manifolds ability to maintain a seal or would cause clogging in the vacuum system. Each tread was run continuously while grease was applied to the surface of the manifold channel until a large amount of grease had built up in the manifold. Figure 101 and Figure 102 shows the clogged and the unclogged manifolds respectively for comparison. The clogged manifolds were then run through the section independence, tread installation effect and fixed manifold testing regimes. A vertical surface test was not conducted as the clogged manifolds could not safety hold the vehicle on the wall while it was being tested. 


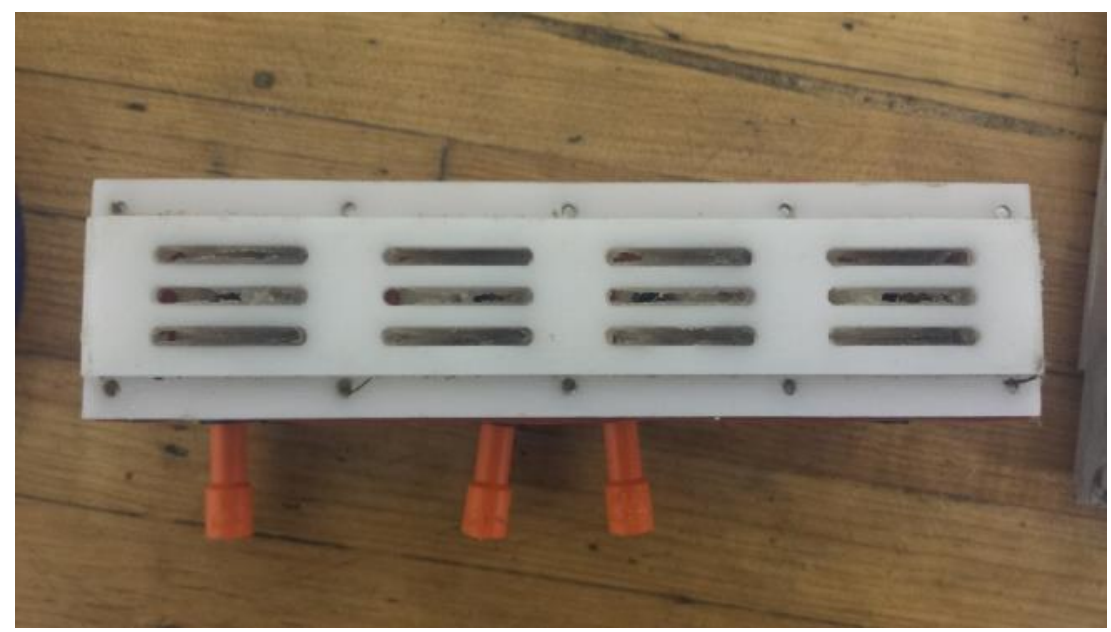

Figure 101: Clogged manifold.

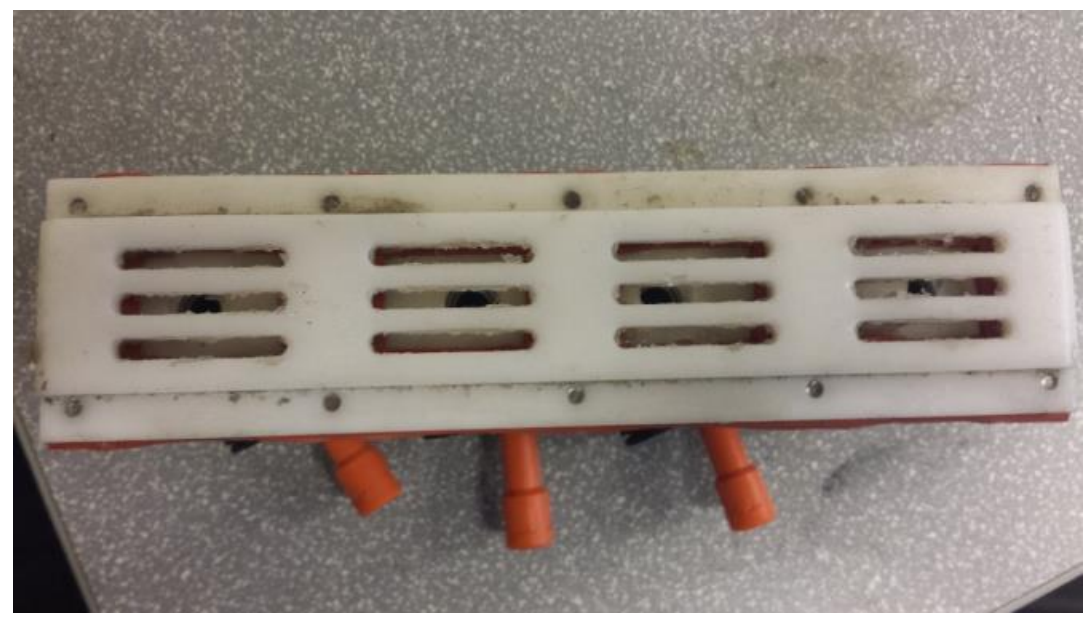

Figure 102: Cleared manifold.

\subsubsection{Results}

The results of the clogged versus unlogged testing can be found in Table 52 and Table 53.

Table 52: Cumulative results of the clogged versus unclogged testing for the right manifold.

\begin{tabular}{|c|r|r|r|}
\hline Section & \multicolumn{1}{|c|}{ Clogged } & Unclogged & \% Difference \\
\hline 1 & $8.88 \mathrm{psi}$ & $8.92 \mathrm{psi}$ & $0.46 \%$ \\
\hline 2 & $12.20 \mathrm{psi}$ & $12.28 \mathrm{psi}$ & $0.67 \%$ \\
\hline 3 & $11.87 \mathrm{psi}$ & $12.03 \mathrm{psi}$ & $1.4 \%$ \\
\hline 4 & $11.46 \mathrm{psi}$ & $12.11 \mathrm{psi}$ & $5.4 \%$ \\
\hline
\end{tabular}

Table 53: Cumulative results of the clogged versus unclogged testing for the left manifold.

\begin{tabular}{|c|r|r|r|}
\hline Section & Clogged & Unclogged & \% Difference \\
\hline 1 & $7.41 \mathrm{psi}$ & $9.29 \mathrm{psi}$ & $20 \%$ \\
\hline 2 & $11.01 \mathrm{psi}$ & $10.64 \mathrm{psi}$ & $3.5 \%$ \\
\hline 3 & $10.39 \mathrm{psi}$ & $10.03 \mathrm{psi}$ & $3.6 \%$ \\
\hline 4 & $9.78 \mathrm{psi}$ & $11.87 \mathrm{psi}$ & $17 \%$ \\
\hline
\end{tabular}




\subsubsection{Discussion}

No major discrepancies were seen between the vacuum pressures in the clogged manifold versus the manifold which had been cleared. This shows that the grease was not being pulled into the manifolds fittings and causing blockages in the section itself. The real effect that clogging had on the vehicle was the loss of vacuum force due to tread holes being cut off from the vacuum. This resulted in extremely low adhesion forces between the vehicle and the surfaces which it was adhering to.

\subsubsection{Split Manifold Testing}

\subsubsection{Procedure}

To determine if the bowing observed in the manifolds was preventing all of the manifold sections from engaging at one time, the right side manifold was cut in half as shown in Figure 103. To determine the effect that this had on the performance of the right side manifold it was tested for section independence, off wheel tread effects, fixed manifold and vertical surface testing.

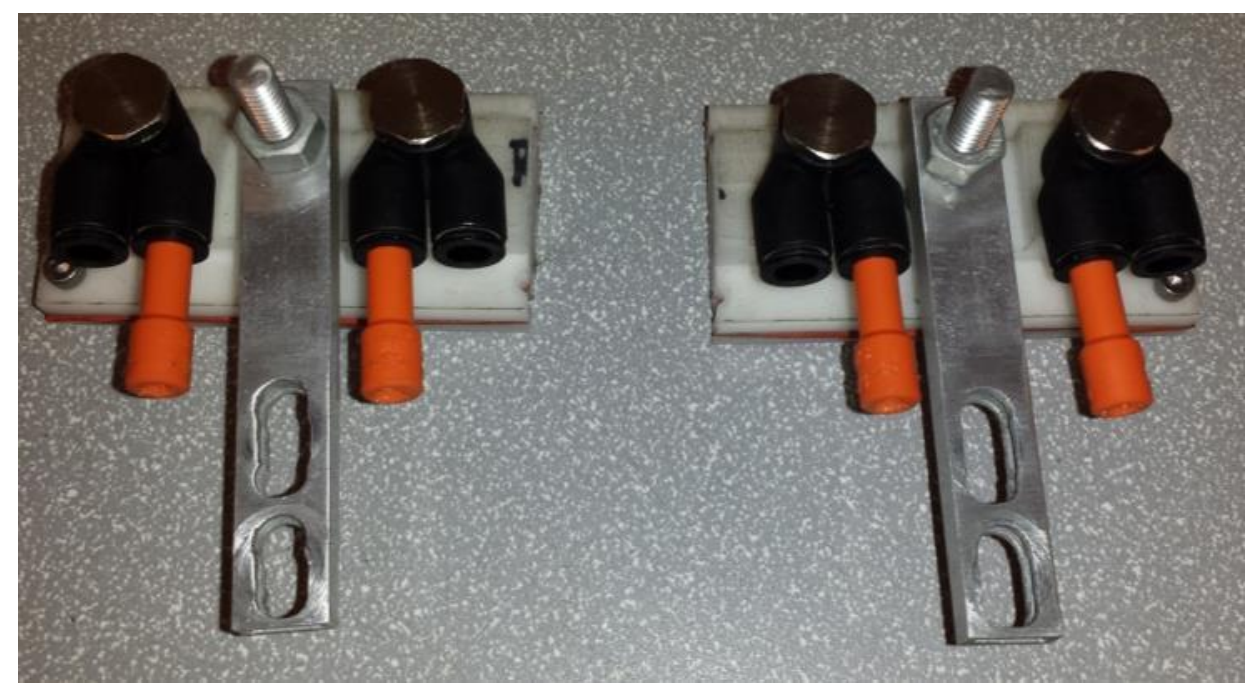

Figure 103: Split right manifold.

The results of the initial vertical surface test prompted a modified vertical surface test to be performed. In this test the vehicle was adhered to the wall as before. A known force was then applied to the right side of the frame near the drive wheel as seen in Figure 104. This forced the right hand tread to lay flat on the wall. Figure 105 shows the right hand tread before and after the tread was forced flat. The vacuum pressure was then measured as it was in the original vertical surface testing scheme. 


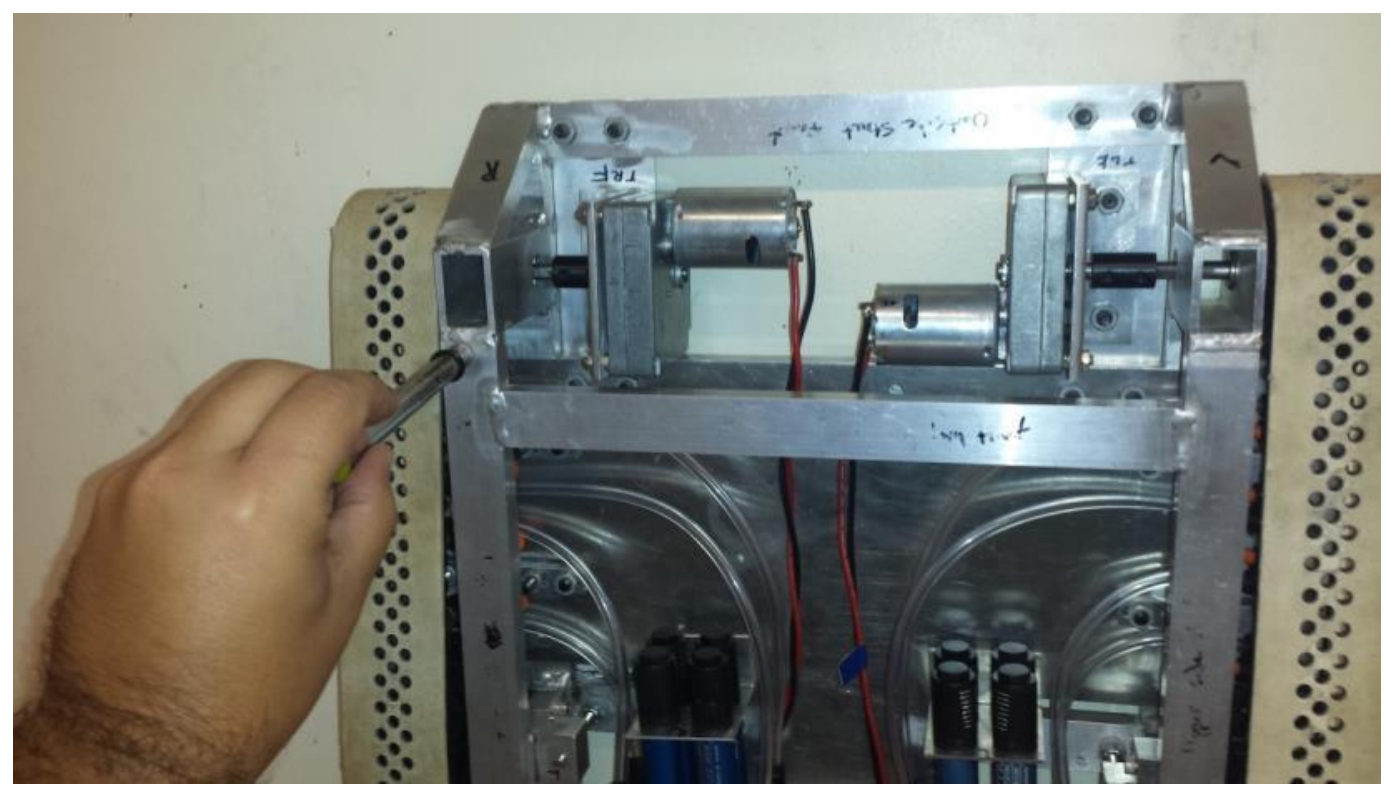

Figure 104: Force being applied to the right corner of the vehicle.

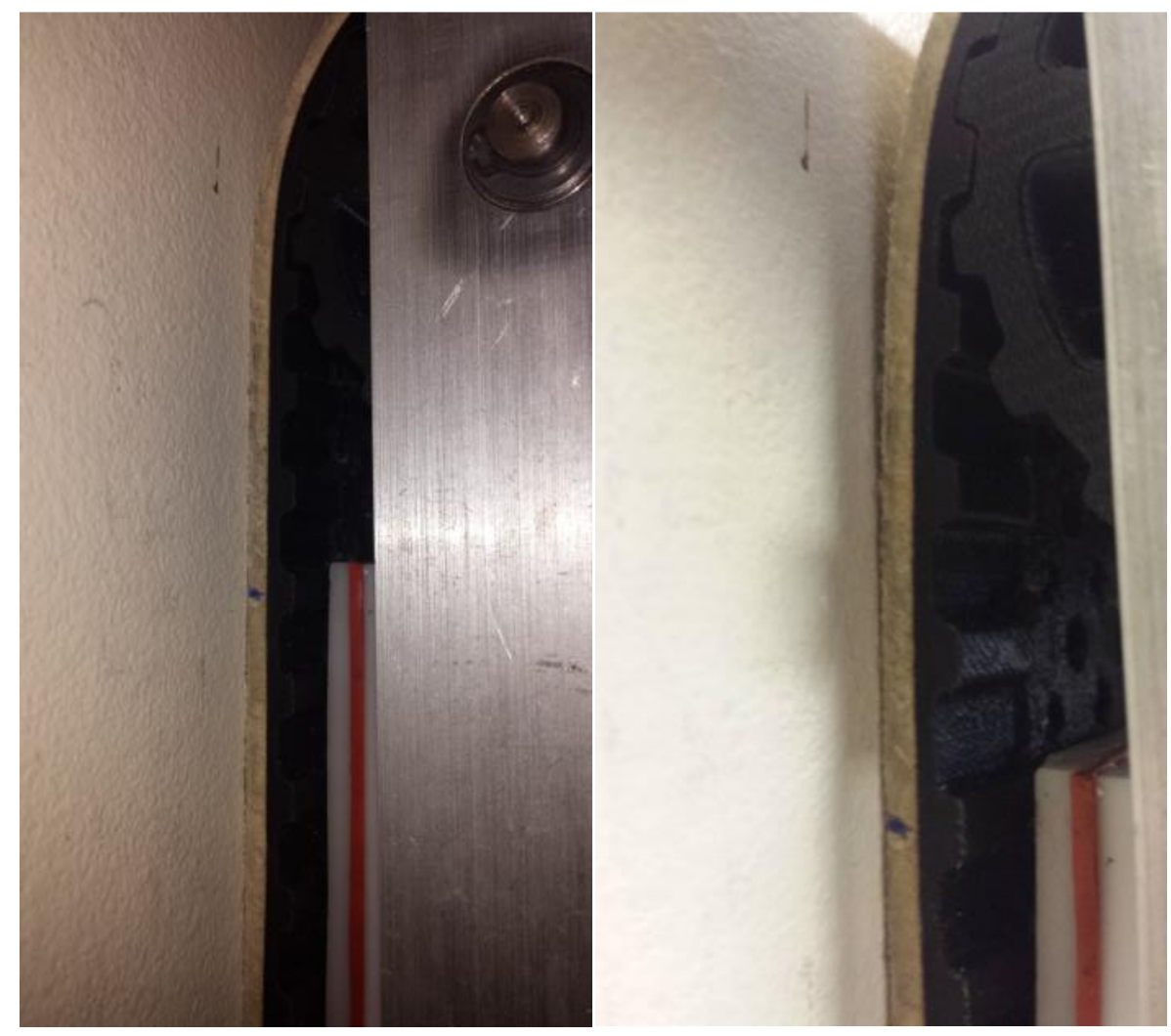

Figure 105: (Left) the right tread after it was pressed onto the wall. (Right) the right tread before it was pressed onto the wall. 


\subsubsection{Results}

The raw data from the split right manifold testing can be found in Appendix D. The results of the right split manifold section independence test and the comparison of these results to the whole right manifold section independence test can be found in Table 54. The results of the right split manifold on vehicle tread test and the comparison of these results to the whole right manifold on vehicle tread test can be found in Table 55 .

Table 56 and Table 57 gives a comparison between the fixed manifold testing results for the split right manifold and the whole right manifold.

Table 54: Performance of the split right manifold versus the whole right manifold for the section independence test.

\begin{tabular}{|c|r|r|r|}
\hline Section & $\begin{array}{c}\text { Right Manifold Pressure } \\
\text { Split }\end{array}$ & $\begin{array}{c}\text { Right Manifold Pressure } \\
\text { Whole }\end{array}$ & \% Difference \\
\hline 1 & $12.77 \mathrm{psi}$ & $12.83 \mathrm{psi}$ & $0.46 \%$ \\
\hline 2 & $13.01 \mathrm{psi}$ & $12.91 \mathrm{psi}$ & $0.77 \%$ \\
\hline 3 & $12.77 \mathrm{psi}$ & $12.85 \mathrm{psi}$ & $0.62 \%$ \\
\hline 4 & $12.77 \mathrm{psi}$ & $12.89 \mathrm{psi}$ & $0.93 \%$ \\
\hline Average & $12.83 \mathrm{psi}$ & $12.87 \mathrm{psi}$ & $0.16 \%$ \\
\hline
\end{tabular}

Table 55: Performance of the split right manifold versus the whole right manifold for the vehicle tread test.

\begin{tabular}{|c|r|r|r|}
\hline Section & Right Manifold - Split & Right Manifold - Whole & \multicolumn{1}{c|}{ \% Difference } \\
\hline 1 & $11.30 \mathrm{psi}$ & $7.37 \mathrm{psi}$ & $53 \%$ \\
\hline 2 & $11.79 \mathrm{psi}$ & $12.28 \mathrm{psi}$ & $4.0 \%$ \\
\hline 3 & $11.79 \mathrm{psi}$ & $11.79 \mathrm{psi}$ & $0.0 \%$ \\
\hline 4 & $10.31 \mathrm{psi}$ & $12.28 \mathrm{psi}$ & $16 \%$ \\
\hline Average & $11.30 \mathrm{psi}$ & $10.93 \mathrm{psi}$ & $3.4 \%$ \\
\hline
\end{tabular}

Table 56: Performance of the split right manifold versus the whole right manifold for the fixed manifold test.

\begin{tabular}{|c|r|r|r|}
\hline Section & Right Manifold - Split & Right Manifold - Whole & \% Difference \\
\hline 1 & $8.59 \mathrm{psi}$ & $9.33 \mathrm{psi}$ & $7.9 \%$ \\
\hline 2 & $12.77 \mathrm{psi}$ & $12.28 \mathrm{psi}$ & $4.0 \%$ \\
\hline 3 & $11.30 \mathrm{psi}$ & $12.28 \mathrm{psi}$ & $8.0 \%$ \\
\hline 4 & $10.80 \mathrm{psi}$ & $12.28 \mathrm{psi}$ & $12 \%$ \\
\hline Average & $10.87 \mathrm{psi}$ & $11.54 \mathrm{psi}$ & $5.9 \%$ \\
\hline
\end{tabular}

Table 57: Performance of the split right manifold versus the whole right manifold for the fixed manifold test.

\begin{tabular}{|c|r|r|r|r|}
\hline \multicolumn{5}{|c|}{ Split Right Manifold } \\
\hline $\begin{array}{c}\text { Tested } \\
\text { Section }\end{array}$ & All Sections Closed & 1 Section Open & 2 Sections Open & \multicolumn{1}{|c|}{3 Sections Open } \\
\hline 1 & $10.31 \mathrm{psi}$ & $8.10 \mathrm{psi}$ & $7.86 \mathrm{psi}$ & $7.86 \mathrm{psi}$ \\
\hline 2 & $12.28 \mathrm{psi}$ & $11.79 \mathrm{psi}$ & $11.30 \mathrm{psi}$ & $11.30 \mathrm{psi}$ \\
\hline
\end{tabular}




\begin{tabular}{|r|r|r|r|r|}
\hline 3 & $10.31 \mathrm{psi}$ & $10.31 \mathrm{psi}$ & $9.82 \mathrm{psi}$ & $7.86 \mathrm{psi}$ \\
\hline 4 & $9.33 \mathrm{psi}$ & $8.84 \mathrm{psi}$ & $8.84 \mathrm{psi}$ & $7.37 \mathrm{psi}$ \\
\hline \multicolumn{5}{|c|}{ Whole Right Manifold } \\
\hline $\begin{array}{r}\text { Tested } \\
\text { Section }\end{array}$ & All Sections Closed & 1 Section Open & 2 Sections Open & 3 Sections Open \\
\hline 1 & $8.84 \mathrm{psi}$ & $3.93 \mathrm{psi}$ & $2.95 \mathrm{psi}$ & $5.65 \mathrm{psi}$ \\
\hline 2 & $12.28 \mathrm{psi}$ & $11.79 \mathrm{psi}$ & $10.80 \mathrm{psi}$ & $10.56 \mathrm{psi}$ \\
\hline 3 & $11.79 \mathrm{psi}$ & $11.79 \mathrm{psi}$ & $11.79 \mathrm{psi}$ & $10.56 \mathrm{psi}$ \\
\hline 4 & $12.28 \mathrm{psi}$ & $12.28 \mathrm{psi}$ & $12.28 \mathrm{psi}$ & $11.79 \mathrm{psi}$ \\
\hline
\end{tabular}

Table 58 shows the comparison between the results of the split right manifold vertical surface testing and the whole right manifold vertical surface test. The results of the no force versus applied force vertical surface testing for the split right manifold and the left manifold can be found in Table 59 and Table 60 respectively.

Table 58: Performance of the split right manifold versus the whole right manifold for the vertical surface test.

\begin{tabular}{|c|r|r|r|}
\hline Section & Right Manifold - Split & Right Manifold - Whole & \% Difference \\
\hline 1 & $0.00 \mathrm{psi}$ & $5.89 \mathrm{psi}$ & $100 \%$ \\
\hline 2 & $0.00 \mathrm{psi}$ & $11.30 \mathrm{psi}$ & $100 \%$ \\
\hline 3 & $9.82 \mathrm{psi}$ & $9.82 \mathrm{psi}$ & $0.0 \%$ \\
\hline 4 & $12.3 \mathrm{psi}$ & $7.37 \mathrm{psi}$ & $66 \%$ \\
\hline Average & $5.53 \mathrm{psi}$ & $8.60 \mathrm{psi}$ & $36 \%$ \\
\hline
\end{tabular}

Table 59: Results of the applied force test for the right manifold. Applied load was $14 \mathrm{lbf}$.

\begin{tabular}{|c|r|r|r|}
\hline Section & No Applied Load & Applied Load & \multicolumn{1}{|c|}{ \% Difference } \\
\hline 1 & $0.00 \mathrm{psi}$ & $10.80 \mathrm{psi}$ & $100 \%$ \\
\hline 2 & $0.00 \mathrm{psi}$ & $12.28 \mathrm{psi}$ & $100 \%$ \\
\hline 3 & $9.82 \mathrm{psi}$ & $9.82 \mathrm{psi}$ & 0.00 \\
\hline 4 & $10.80 \mathrm{psi}$ & $11.30 \mathrm{psi}$ & $4.4 \%$ \\
\hline Average & $5.16 \mathrm{psi}$ & $11.1 \mathrm{psi}$ & $53 \%$ \\
\hline
\end{tabular}

Table 60: Results of the applied force test for the left manifold. Applied load was $14 \mathrm{lbf}$.

\begin{tabular}{|c|r|r|r|}
\hline Section & No Applied Load & Applied Load & \multicolumn{1}{c|}{ Difference } \\
\hline 1 & $10.8 \mathrm{psi}$ & $9.82 \mathrm{psi}$ & $10 \%$ \\
\hline 2 & $12.3 \mathrm{psi}$ & $0.00 \mathrm{psi}$ & $100 \%$ \\
\hline 3 & $9.82 \mathrm{psi}$ & $0.00 \mathrm{psi}$ & $100 \%$ \\
\hline 4 & $11.3 \mathrm{psi}$ & $0.00 \mathrm{psi}$ & $350 \%$ \\
\hline Average & $11.1 \mathrm{psi}$ & $2.46 \mathrm{psi}$ & \\
\hline
\end{tabular}

\subsubsection{Discussion}

The section independence testing was completely successful. Each section was independent from its neighbor and each was fully sealed from the atmosphere. The off wheel testing was also successful with the 
average vacuum pressure in the split manifold increasing when compared to the whole manifold off wheel testing.

The fixed manifold testing had mixed results. The average vacuum pressure in the split manifold was slightly lower than the than pressure observed in the whole manifold. This could be the result of the misalignment in the tread or the manner in which the manifolds were fixed in place. The splitting of the manifolds did seem to reduce the effect that the front two and back two sections had on each other, especially at front section of the manifold.

The splitting of the right manifold drastically reduced the effectiveness of the manifold when it was adhered to vertical surfaces. The front half of the manifold did not engage at all, and the effectiveness of the first section on the back half was reduced. This was a direct result of the misalignment of the frame, which caused the front of the right tread to lift off the surface. When the manifold was whole and supported by two struts, this only caused the front section of the manifold to fail. Because the two halves of the manifold only had a single support system, the manifold halves were much easier to shift, allowing the tread to push the front manifold half out of position.

The misalignment in the frame was further highlighted when the modified vertical surface testing was performed. When a load was applied to the right front corner of the vehicle, forcing the right tread flat against the surface, an increase in the section pressure in the two right manifold halves was seen. The application of the force to the front right corner of the vehicle caused all but the first section of the left manifold to fail completely. This implies that as the front right corner of the vehicle was pushed down the back left corner was lifted when the force was applied.

\subsubsection{Vacuum Force Testing}

\subsubsection{Procedure}

This testing was done to determine the vacuum force developed between the wall and the vehicle. The information gathered during the test was used to validate the tread hole engagement calculation performed in Appendix A. The first step taken during this testing was the determination of the number of tread holes 
that were engaged during the testing. This was done by simply counting the tread holes which were exposed to the slots in the manifolds as shown in Figure 106.

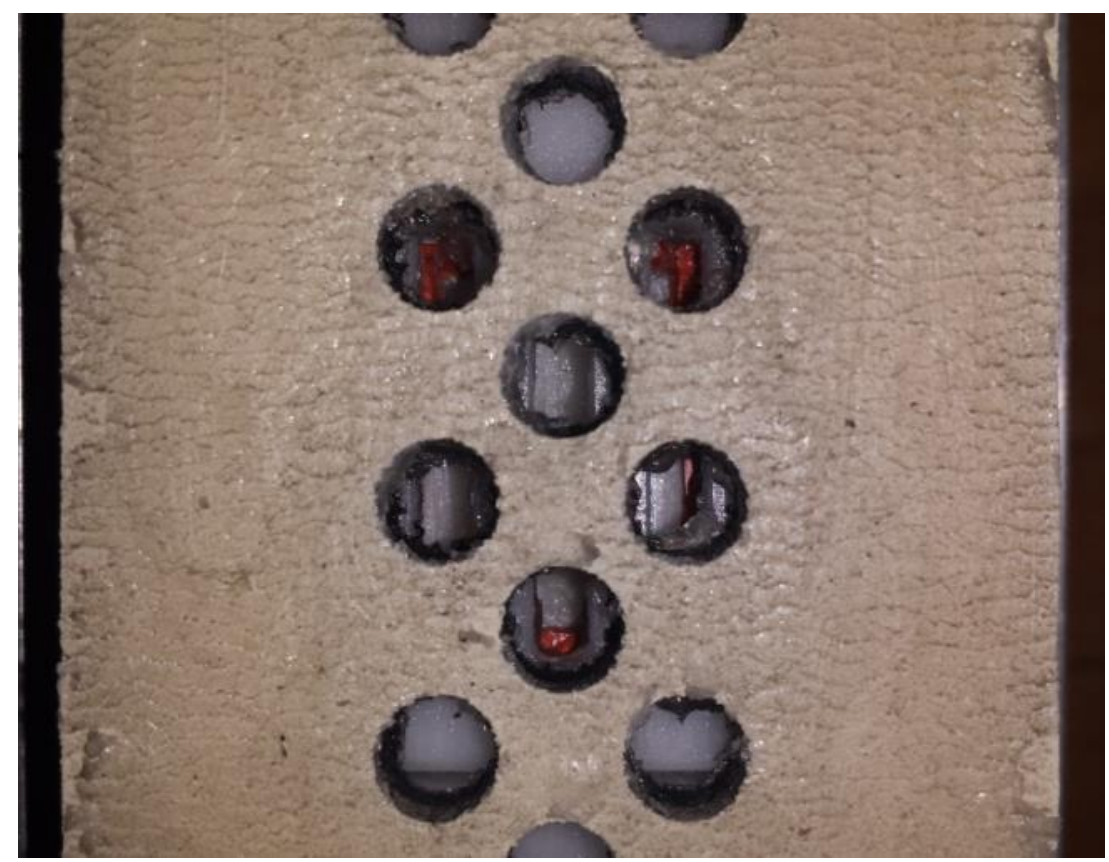

Figure 106: A section of the manifold and the tread holes which it engages.

A series of zip ties, show in Figure 107, were then used to create an attachment point over the center of gravity of the vehicle. This was done to minimize the effects that the mass of the vehicle would have on the testing.

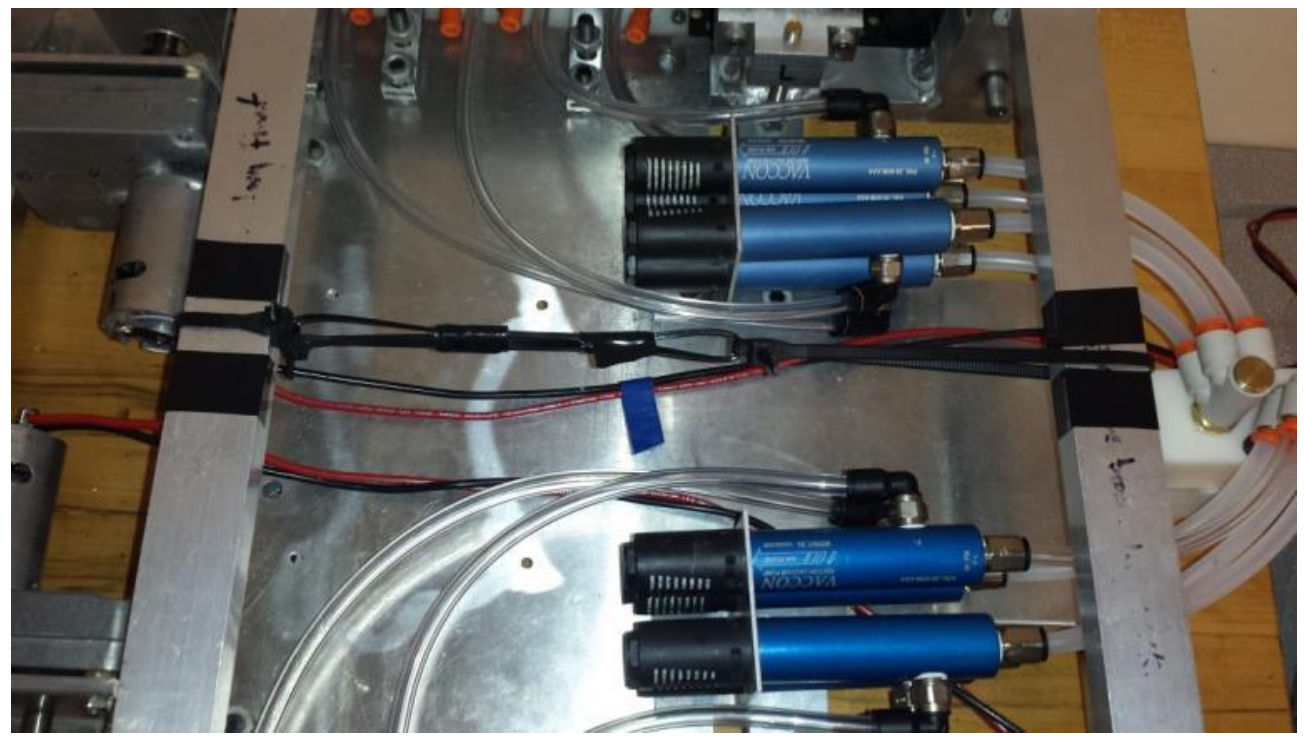

Figure 107: The zip tie suspension system used to perform the pull tests. 
The testing was conducted while the vehicle was both on both a horizontal and vertical surface. For both test cases the vehicle was adhered to the surface and the vacuum pressure in each of the manifold sections. A dial force gauge, shown in Figure 108, was then inserted into the attachment point and then pulled perpendicular to the surface as seen in Figure 109. The force when the vehicle began to leave the surface was recorded.

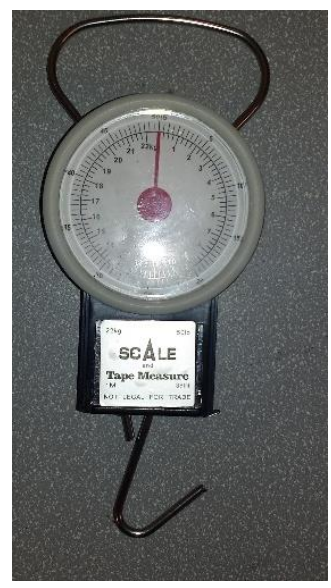

Figure 108: Force gauge used in the pull testing.

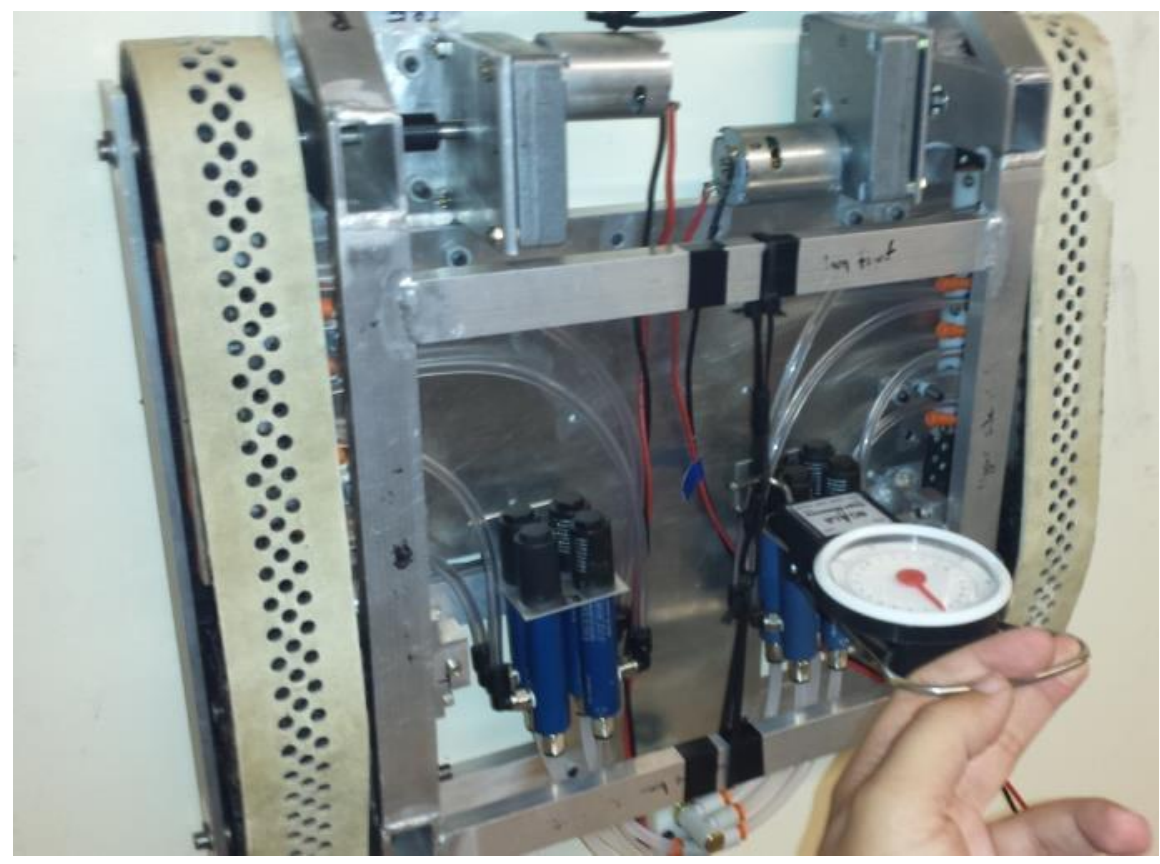

Figure 109: Vertical wall pull test example. 
The results of the initial horizontal testing prompted a modified version of the horizontal test to be conducted. Pull-off forces of known magnitude were applied to the vehicle. The vacuum pressure of each manifold section was measured as the force was applied until the vehicle left the surface.

\subsubsection{Results}

The complete data gathered during the vacuum force testing can be found in Appendix D. The number of holes engaged with each manifold section during the pull-off force testing can be found in Table 61. This was important information that was used in the calculation of the predicted pull-off force for the vehicle.

Table 62 gives the average values for the pull-off force of both tests and the average pressure in the manifolds during the tests.

Table 61: Holes engaged during the pull force testing.

\begin{tabular}{|c|r|r|}
\hline Section Number & Right Manifold & Left Manifold \\
\hline 1 & 8 & 6 \\
\hline 2 & 6 & 8 \\
\hline 3 & 7 & 6 \\
\hline 4 & 6 & 7 \\
\hline Total & 27 & 27 \\
\hline
\end{tabular}

Table 62: Average pull off force and manifold pressure for the horizontal and vertical tests.

\begin{tabular}{|c|c|c|}
\hline Test Case & $\begin{array}{c}\text { Average Pull -Off } \\
\text { Force }\end{array}$ & Average Manifold Pressure \\
\hline Vertical Surface & $17 \mathrm{lbf}$ & $7.27 \mathrm{psi}$ \\
\hline Horizontal Surface & $24.5 \mathrm{lbf}$ & $10.7 \mathrm{psi}$ \\
\hline
\end{tabular}

Table 63 and Table 64 contain the predicted and actual pull-off forces for the horizontal and vertical surface tests respectively. Note that the predicted values in Table 65 include the weight of the vehicle. The predicted values were obtained by multiply the number of holes engaged for a given section with the vacuum pressure measured in that section and the area of a single tread hole. Table 66 contains the results from the manifold pressure test and Figure 110 and Figure 111 show plots for the right and the left manifold respectively. Table 67 contains the predicted and actual pull-off forces for the horizontal surface test with the weight of the vehicle excluded from the predictions. 
Table 63: Predicted versus actual pull off force for the horizontal test with the mass included in the predicted value.

\begin{tabular}{|r|r|r|r|}
\hline Test Number & $\begin{array}{r}\text { Predicted Pull-Off } \\
\text { Force }\end{array}$ & Actual Pull-Off Force & \% Difference \\
\hline 1 & $39.7 \mathrm{lbf}$ & $25.0 \mathrm{lbf}$ & $37 \%$ \\
\hline 2 & $40.3 \mathrm{lbf}$ & $24.5 \mathrm{lbf}$ & $39 \%$ \\
\hline 3 & $42.0 \mathrm{lbf}$ & $23.0 \mathrm{lbf}$ & $45 \%$ \\
\hline 4 & $41.4 \mathrm{lbf}$ & $24.0 \mathrm{lbf}$ & $42 \%$ \\
\hline Average & $40.9 \mathrm{lbf}$ & $24.1 \mathrm{lbf}$ & $41 \%$ \\
\hline
\end{tabular}

Table 64: Predicted versus actual pull off force for the vertical test.

\begin{tabular}{|r|r|r|r|}
\hline Test Number & $\begin{array}{r}\text { Predicted Pull-Off } \\
\text { Force }\end{array}$ & Actual Pull-Off Force & \% Difference \\
\hline 1 & 18.8 & 19 & $1.1 \%$ \\
\hline 2 & 19.5 & 17 & $12 \%$ \\
\hline 3 & 18.5 & 15 & $18 \%$ \\
\hline 4 & 19.4 & 16 & $18 \%$ \\
\hline 5 & 19.3 & 20 & $3.6 \%$ \\
\hline Average & 19.1 & 17.4 & $8.9 \%$ \\
\hline
\end{tabular}

Table 65: Pressure in the right and left manifold sections at different pull off force values.

\begin{tabular}{|c|c|c|c|c|}
\hline \multicolumn{5}{|c|}{ Right Manifold } \\
\hline Pull Force & Section 1 & Section 2 & Section 3 & Section 4 \\
\hline $0 \mathrm{lbf}$ & $10.31 \mathrm{psi}$ & $12.28 \mathrm{psi}$ & $10.31 \mathrm{psi}$ & $10.31 \mathrm{psi}$ \\
\hline $10 \mathrm{lbf}$ & $8.84 \mathrm{psi}$ & $12.28 \mathrm{psi}$ & $9.82 \mathrm{psi}$ & $10.31 \mathrm{psi}$ \\
\hline $15 \mathrm{lbf}$ & $8.84 \mathrm{psi}$ & $11.79 \mathrm{psi}$ & $8.84 \mathrm{psi}$ & $10.31 \mathrm{psi}$ \\
\hline $20 \mathrm{lbf}$ & $7.86 \mathrm{psi}$ & $11.79 \mathrm{psi}$ & $8.84 \mathrm{psi}$ & $9.33 \mathrm{psi}$ \\
\hline \multicolumn{5}{|c|}{ Left Manifold } \\
\hline Pull Force & Section 1 & Section 2 & Section 3 & Section 4 \\
\hline $0 \mathrm{lbf}$ & $11.79 \mathrm{psi}$ & $10.80 \mathrm{psi}$ & $9.82 \mathrm{psi}$ & $10.31 \mathrm{psi}$ \\
\hline $10 \mathrm{lbf}$ & $11.79 \mathrm{psi}$ & $10.80 \mathrm{psi}$ & $8.84 \mathrm{psi}$ & $10.31 \mathrm{psi}$ \\
\hline $15 \mathrm{lbf}$ & $11.79 \mathrm{psi}$ & $10.31 \mathrm{psi}$ & $8.35 \mathrm{psi}$ & $9.82 \mathrm{psi}$ \\
\hline $20 \mathrm{lbf}$ & $11.79 \mathrm{psi}$ & $10.31 \mathrm{psi}$ & $8.35 \mathrm{psi}$ & $7.86 \mathrm{psi}$ \\
\hline
\end{tabular}




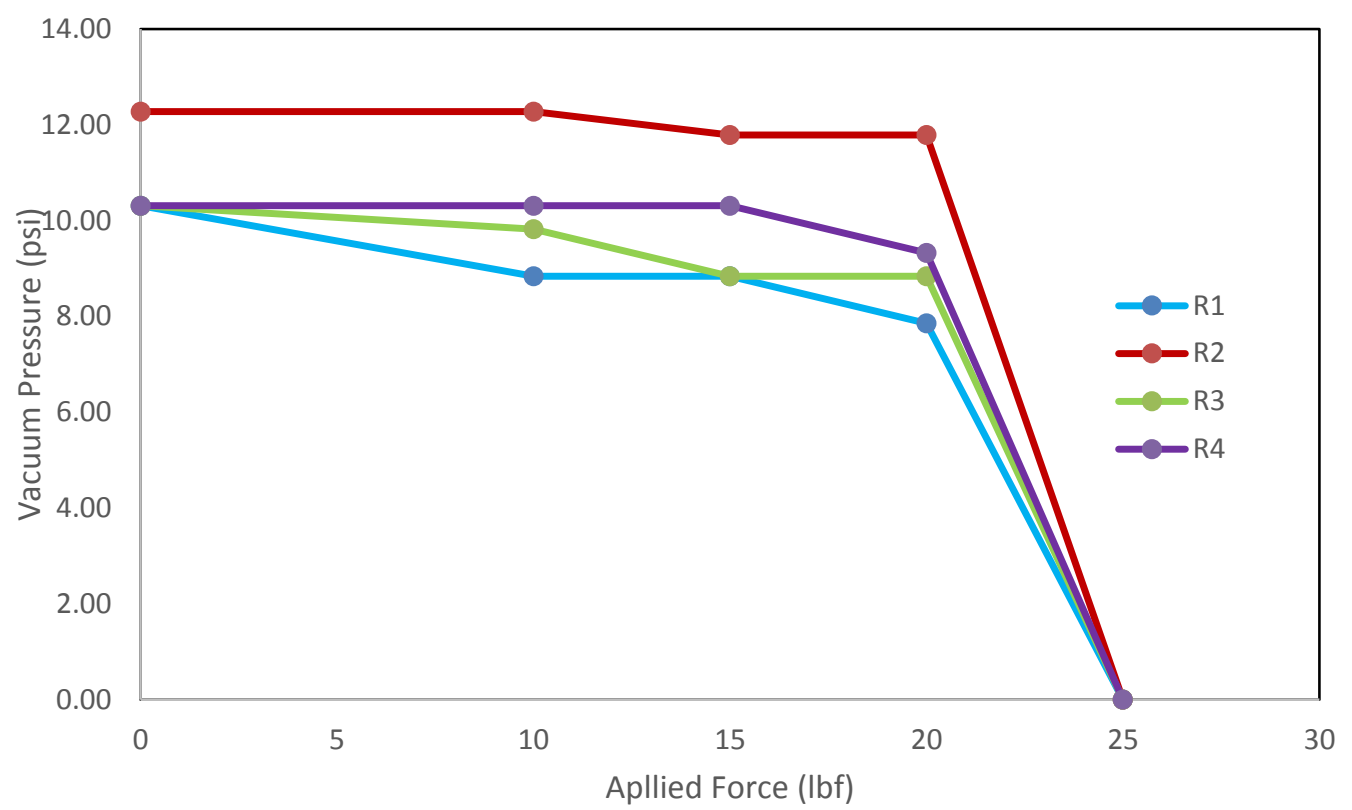

Figure 110: Right side manifold pressure as load was applied.

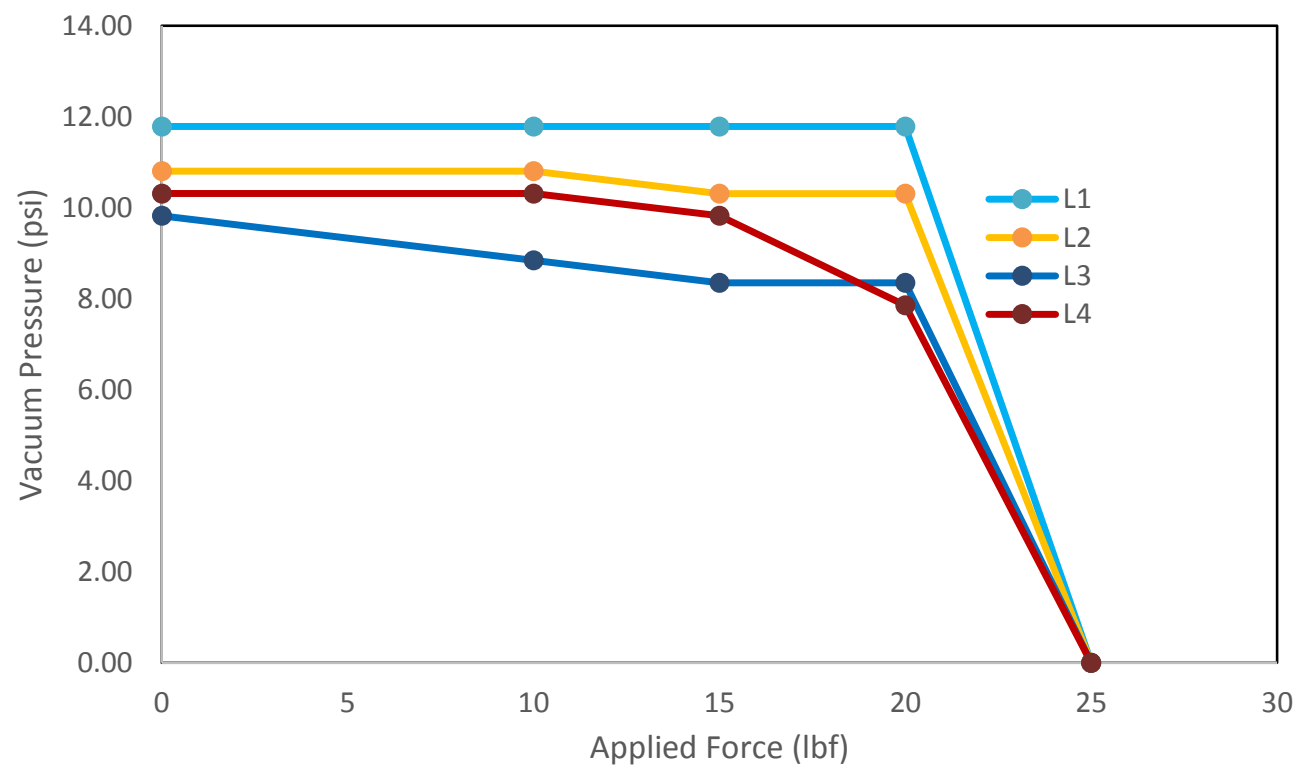

Figure 111: Left side manifold pressure as load was applied.

Table 66: Predicted versus actual pull off force for the horizontal test without the mass included in the predicted value.

\begin{tabular}{|c|r|r|r|}
\hline Test Number & $\begin{array}{c}\text { Predicted Pull-Off } \\
\text { Force }\end{array}$ & Actual Pull-Off Force & \% Difference \\
\hline 1 & $27.3 \mathrm{lbf}$ & $25.0 \mathrm{lbf}$ & $8.4 \%$ \\
\hline 2 & $27.8 \mathrm{lbf}$ & $24.5 \mathrm{lbf}$ & $12 \%$ \\
\hline 3 & $29.5 \mathrm{lbf}$ & $23.0 \mathrm{lbf}$ & $22 \%$ \\
\hline
\end{tabular}




\begin{tabular}{|c|r|r|r|}
\hline 4 & $28.9 \mathrm{lbf}$ & $24.0 \mathrm{lbf}$ & $17 \%$ \\
\hline Average & $28.4 \mathrm{lbf}$ & $24.1 \mathrm{lbf}$ & $15 \%$ \\
\hline
\end{tabular}

\subsubsection{Discussion}

The discrepancies between the actual and predicted pull-off forces for both of the test cases that were investigated could have several different possible explanations. If the pull-off forces were applied to the vehicle at an angle relative to the testing surface, the uneven loading could cause the development of moments. These moments could initiate premature separation between the treads and the testing surface and cause a loss of pressure. Another possible explanation could be that the pull-off force causes the manifolds to shift. This shifting could then result in the seal between the manifolds and the treads to begin to fail. Finally, the motion of the vehicle being lifted away from the surface could cause the portions of the tread not under the manifold to lift. This lifting of the tread around the manifold could have both opened gaps between the tread and the wall and caused the manifolds to lift at their ends, both of which would cause a premature loss of suction.

The results of the vertical surface testing were very encouraging. The discrepancies between the predicted and actual pull-off force values, while significant, do not preclude the prediction method used for being employed in future works. Further refinement of the method is necessary, but it could be used to estimate the expected force between a vertical surface and the vehicle.

The horizontal testing proved to be much more interesting than the vertical testing. Initially, the mass of the vehicle was included in the predicted pull-off force. When the conducted testing was performed, the discrepancies between the predicted values and the measured values were extremely high. When the weight of the vehicle was removed from the prediction, the discrepancy between the predicted and actual pull-off force dropped considerably.

This observation prompted two new sets of testing to be performed. The first test involved observing the pressure in each section at different applied pull-off forces. This was to determine if the pull-off forces was causing premature loss of seal at the manifolds or the treads. The second test was adding weight to vehicle to determine what effect the additional weight had on the pull-off force. 
While some loss in seal was seen as the pull-off force was applied in the pressure system testing, the drop off was not dramatic until the maximum pull-off force was approached. At this point each manifold section lost pressure rapidly and the vehicle left the surface. This seemed to indicate that either the

This result can be interpreted in two different ways to get two distinct scenarios. The first scenario is that the force gauge was measuring the weight of the vehicle and the pull-off force for the entire test. The lowered force between the surface and the vehicle could be the result of tread deformation or the manifolds being shifted. This implies that the predicted force between the surface and the vehicle is much lower than the actual force, but the results of the vertical surface testing seems to contradict this.

The other possible scenario is that the force gauge is measuring the vacuum force between the surface and the vehicle, and only after this force has been overcome does the gauge measure the weight of the vehicle. This is analogous to pre-tension in bolts, where the pre-load must be overcome before the bolt begins to carry any of the loading placed on the members it is attaching. This scenario seems to be supported by the results of the vertical surface testing and the manifold section testing, which indicates that the loss in pressure in the manifold sections is very gradual until a critical point is reached. Further testing should be conducted to confirm which scenario is correct, as the results will have serious implications about future vehicles abilities to adhere to ceilings.

The measured pull-off forces are far from the desire pull-off forces which the vehicle was designed for. This can be directly attributed to the inability of the manifolds to maintain a perfect seal across all sections when the vehicle is on a vertical surface. This will need to be addressed in future iterations of the project.

\subsection{Other Testing}

\subsubsection{Motor Torque Testing}

This testing was done in order to determine the torque required drive the vehicle under different conditions. The information gathered from this was used to validate the model used to determine the theoretical torque requirements of the motors. 


\subsubsection{Procedure}

The procedure used to determine the motor torque relatively simple. The motors were run during for several different test case, such as when the motors had no load applied to them and when the vehicle was run on a horizontal surface with no vacuum applied. The vehicle was allowed to reach steady state when possible and the current draw from the power supply of the test cases was recorded.

\subsubsection{Results}

The first step was to determine the no load current draw for the motors. This was done by disconnecting the motors from the drive wheels and running them. Because the stall torque was given as fifty inch pounds of torque, the current vs torque relationship could be established. Figure 112 shows the plot that was used to determine the relationship between torque and current for the motors.

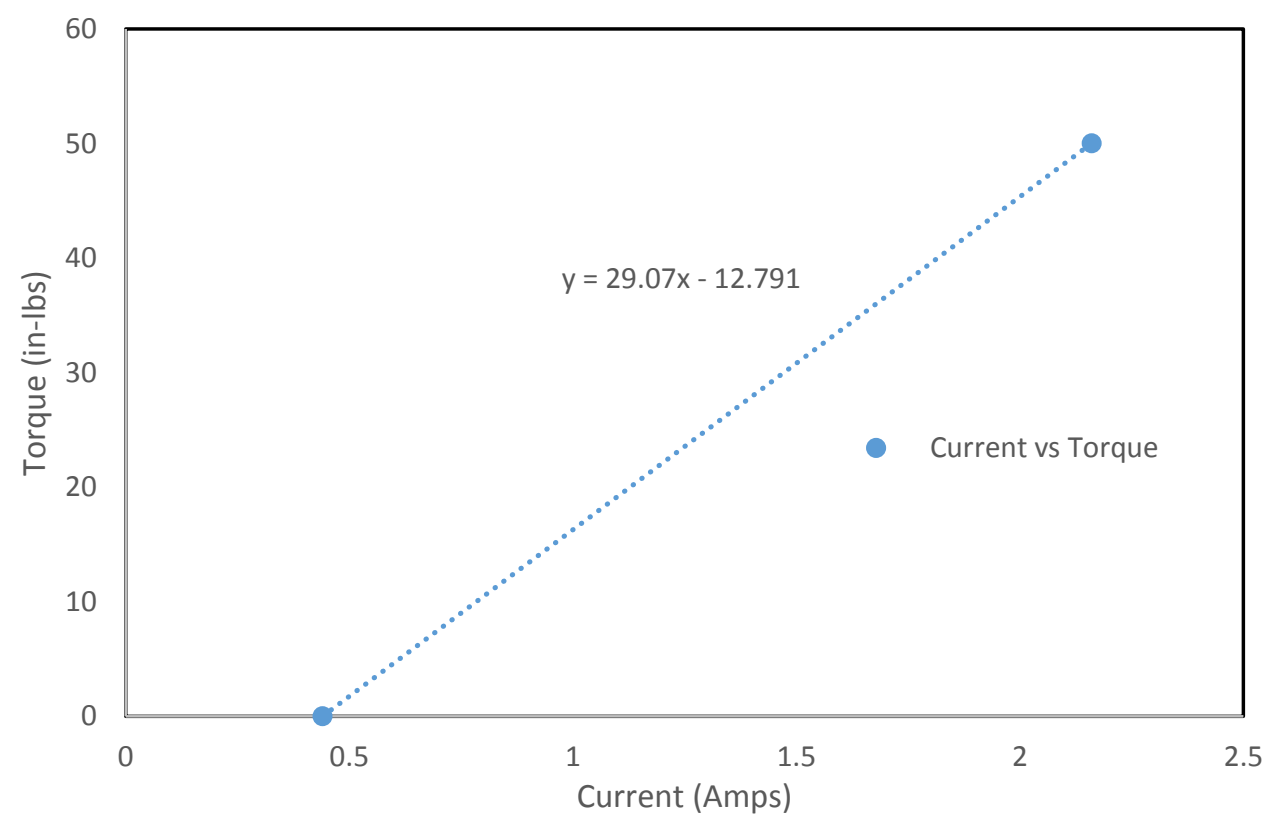

Figure 112: Current versus torque plot.

The current draw of the motors for several different test cases were then measured and recorded. The motor torque for each case was then calculated using the equation from Figure 112. Table 67 contains the current draw for each test case and the corresponding torque produced by the motors and Figure 113 shows a plot of the torque current relationship. 
Table 67: Current values measured during different test cases and the corresponding torque values.

\begin{tabular}{|l|l|r|r|}
\hline Test Case Number & \multicolumn{1}{|c|}{ Case Description } & \multicolumn{1}{c|}{ Current } & \multicolumn{1}{c|}{ Torque } \\
\hline 1 & Vertical Surface Before Failure & $1.55 \mathrm{~A}$ & $32.27 \mathrm{in}-1 \mathrm{bf}$ \\
\hline 2 & Vertical Surface after Failure & $1.22 \mathrm{~A}$ & $22.67 \mathrm{in}-1 \mathrm{bf}$ \\
\hline 3 & Table Top w/ Vacuum & $1.00 \mathrm{~A}$ & $16.28 \mathrm{in}-1 \mathrm{bf}$ \\
\hline 4 & Table Top w/o Vacuum & $0.83 \mathrm{~A}$ & $11.34 \mathrm{in}-1 \mathrm{bf}$ \\
\hline 5 & Table Top w/ Manifolds Removed & $0.65 \mathrm{~A}$ & $6.10 \mathrm{in}-1 \mathrm{bf}$ \\
\hline 6 & Not on Surface w/ Manifold & $0.60 \mathrm{~A}$ & $4.65 \mathrm{in}-1 \mathrm{bf}$ \\
\hline 7 & Not on Surface w/o Manifold & $0.60 \mathrm{~A}$ & $4.65 \mathrm{in}-1 \mathrm{bf}$ \\
\hline 8 & No Load & $0.44 \mathrm{~A}$ & $0.00 \mathrm{in}-1 \mathrm{bf}$ \\
\hline 9 & Stall Torque & $2.16 \mathrm{~A}$ & $50.00 \mathrm{in}-1 \mathrm{bf}$ \\
\hline
\end{tabular}

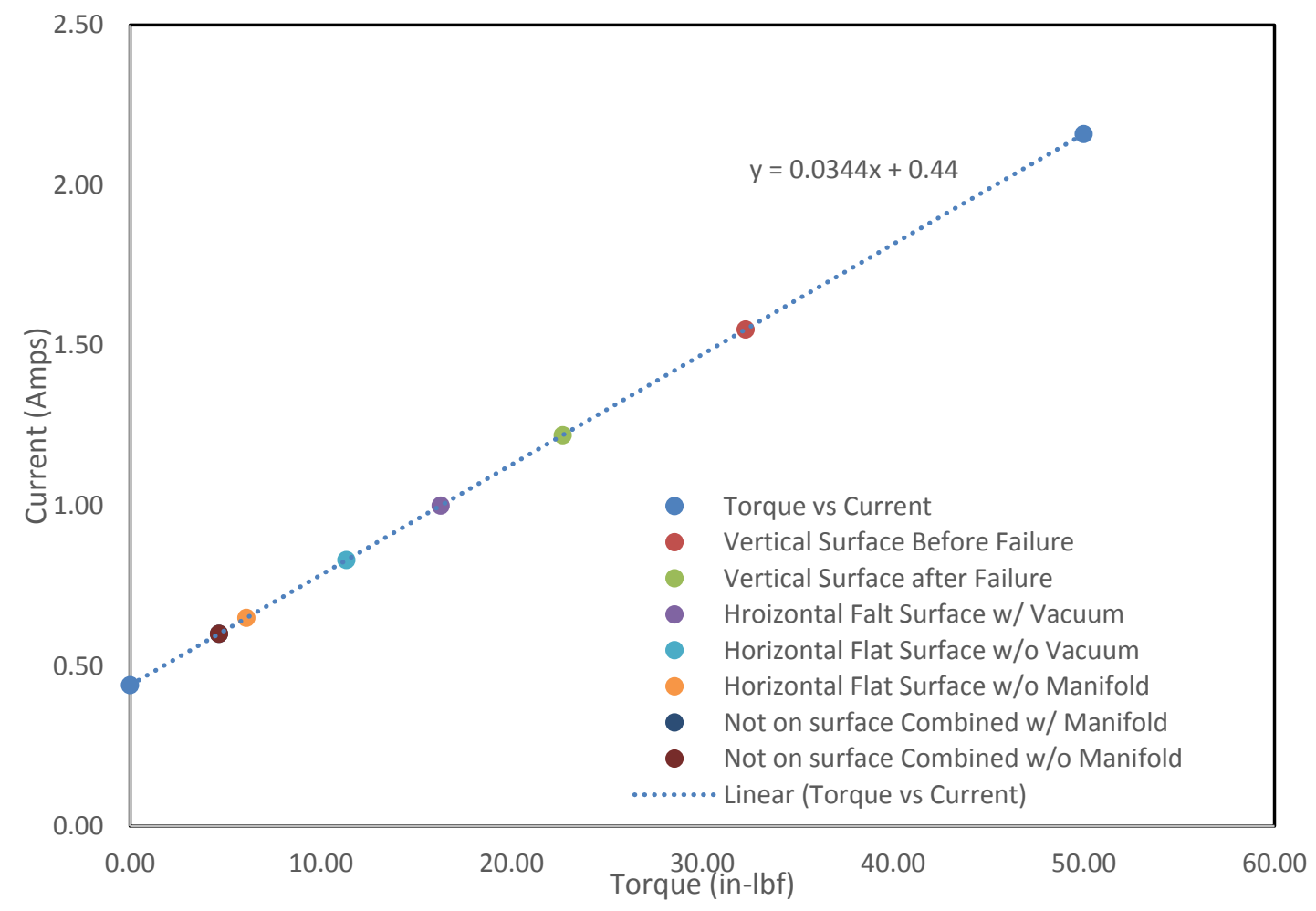

Figure 113: Torque versus current plot showing each test cases torque and current value.

Using the torque values for the different test cases, the contribution of different elements to the motor torque can be estimated. For instance the test case were the vehicle was run on a table top with the manifold removed gives an estimated of the losses in the wheel system when the vehicle is on a surface. Table 68 contains the estimates of the contribution of several different elements of the design to the motor torque and what test cases were used to calculate them. contains a comparison between the calculated and estimated torque contributions and the measured torque. Table 69 contributions of different elements of the vehicle. 
Table 68: Calculated values for different motor torque contributors.

\begin{tabular}{|l|c|r|}
\hline \multicolumn{1}{|r|}{ Motor Torque Contributor } & Test Cases Used & Torque Contribution \\
\hline Wheel Losses Off Surface & $(7)$ & 4.65 in-lbf \\
\hline Wheel Losses Due to Being on a Surface & $(6-7)$ & $1.45 \mathrm{in}-\mathrm{lbf}$ \\
\hline Friction Between Manifold/ Tread Due to Vacuum & $(3-4)$ & $4.94 \mathrm{in}-\mathrm{lbf}$ \\
\hline $\begin{array}{l}\text { Friction Between Manifold/Tread Due to Weight and } \\
\text { Spring Forces }\end{array}$ & $(4-5)$ & $5.23 \mathrm{in}-\mathrm{lbf}$ \\
\hline Total friction Loss Between Treads and Manifolds & $(3-5)$ & $10.17 \mathrm{in}$-lbf \\
\hline Weight & $(1-3)$ & $15.99 \mathrm{in}-\mathrm{lbf}$ \\
\hline
\end{tabular}

Table 69: Calculated versus measured values of the motor torque contribution of different elements of the design.

\begin{tabular}{|l|r|r|r|}
\hline \multicolumn{1}{|c|}{ Motor Torque Contributor } & $\begin{array}{r}\text { Calculated or } \\
\text { Estimated Value }\end{array}$ & Measured Value & \% Difference \\
\hline Losses in Wheel/Tread System & $5.00 \mathrm{in}-\mathrm{lbf}$ & $6.10 \mathrm{in}-\mathrm{lbf}$ & $18 \%$ \\
\hline Due to Manifold Friction & $10.64 \mathrm{in}-1 \mathrm{bf}$ & $10.17 \mathrm{in} .-\mathrm{lbf}$ & $4.6 \%$ \\
\hline Due to Acceleration/Weight & $11.13 \mathrm{in} .-\mathrm{lbf}$ & $15.99 \mathrm{in}-\mathrm{lbf}$ & $30 \%$ \\
\hline Total & $26.77 \mathrm{in}-1 \mathrm{bf}$ & $32.27 \mathrm{in}-\mathrm{lbf}$ & $17 \%$ \\
\hline
\end{tabular}

\subsubsection{Discussion}

The measured torque contributions of the different design elements were relatively close to the values which were calculated in Appendix A. Because of the complexity of the wheel system and its interaction with the tread, the value for the losses in the system was estimated. Since the value was estimated and not calculated, it was expected that there would some difference between the measured and predicted value.

Overall, the vehicle required about seventeen percent more torque than was initially calculated. The major contributor to this discrepancy was the torque contribution of the weight and the acceleration of the vehicle. The contribution of the acceleration and the weight of the vehicle were difficult to separate because it was unknown if the vehicle reached a constant velocity before it slipped from the wall. This meant that measured current during the vertical testing was a lumped value and so when it was compared to the calculated weight and acceleration contributions, these values had to be lumped as well. The discrepancies between measured and calculated values were relatively large. This is likely due to the idealized nature of the calculations used to determine the weight and accelerations values. Further investigation should be conducted to determine the root cause of this in future iterations of the design. 


\subsubsection{Wheel Alignment}

This test was used to determine how well the drive, idler and tensioner wheels were aligned. Misalignment of the wheels in the system could have significant effects on the performance of the belt system and the life time of the tread. The method used was relatively simple and was found in the SDP/SI timing belt design guide.

\subsubsection{Procedure}

To measure the relative misalignments between the wheels one end of a ruler was pressed flush against the face of each wheel. The other end of the straight edge was then positioned over one of the other wheels in the system, as seen in Figure 114, and the distance between the straight edge and the face of the second wheel was measured. This gave the parallel misalignment for each pair of wheels. If the straight edge was not parallel with the face of the wheel, then the angle between the straight edge and the second wheel was determined. An example of angular misalignment can be seen in Figure 115.

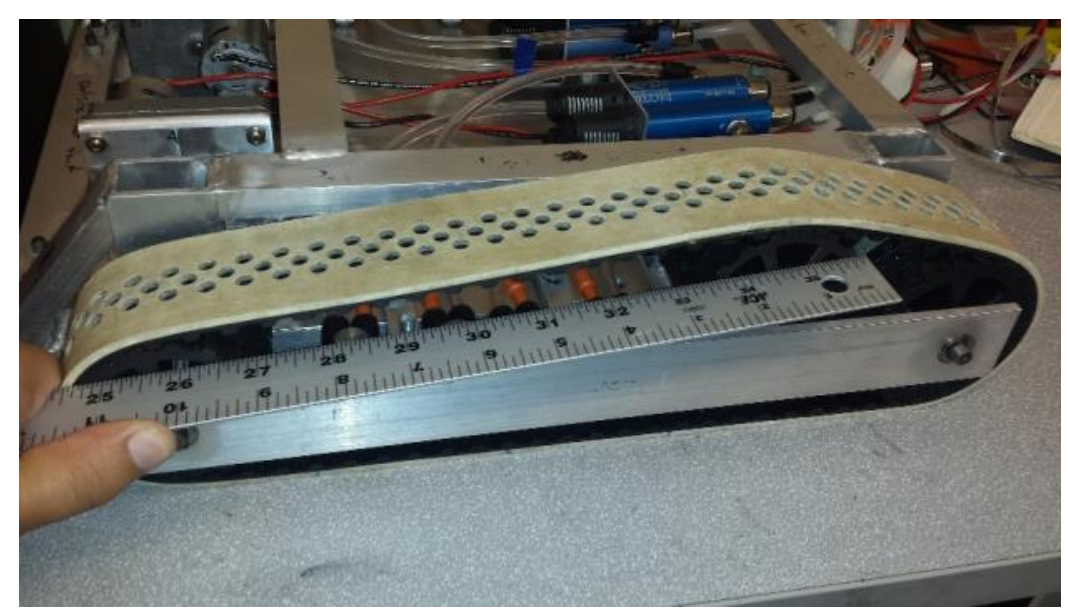

Figure 114: Straight edge going between the drive and tensioner wheels on the right side of the vehicle. 


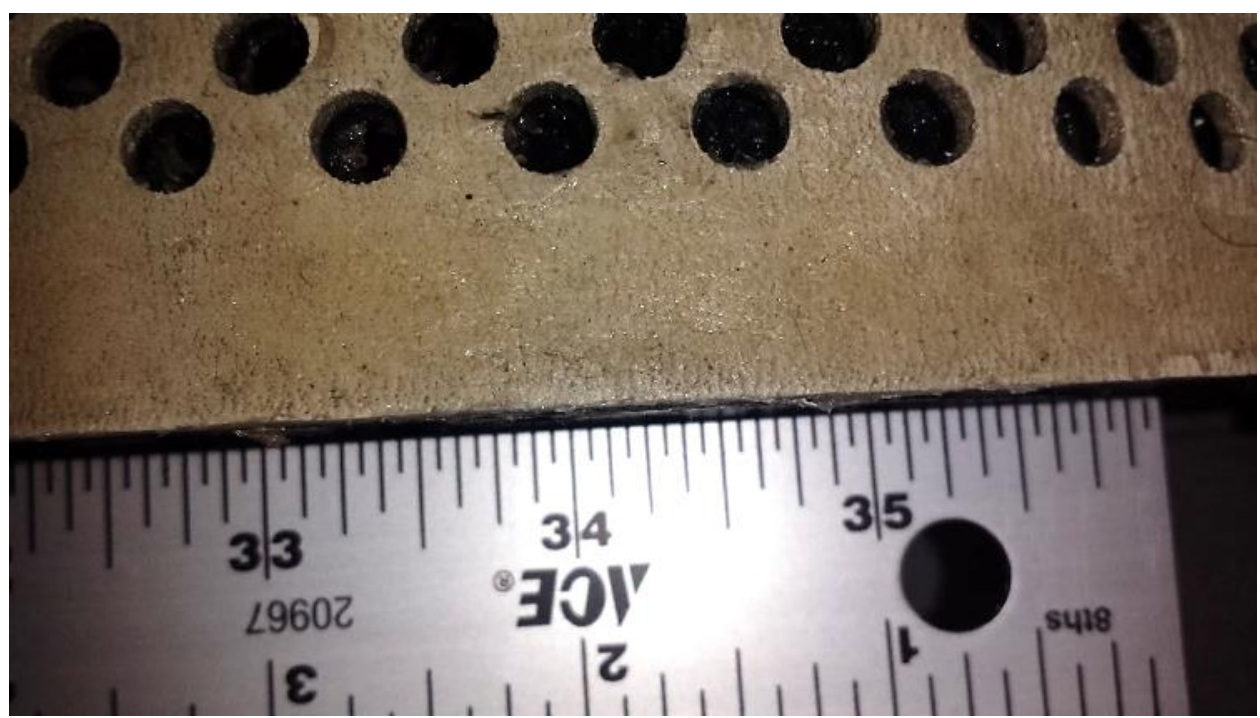

Figure 115: Angular misalignment of the tensioner wheel on the right side of the vehicle.

The angle between the wheels and the surface that the vehicle was resting on was also measured. The vehicle was place on a flat smooth surface and the straight edge was place flush against it. The straight edge was then moved into contact with the wheel and the angle between the face of the wheel and the straight edge was measured. To measure the angular misalignment of the tensioner wheel with ground, the braces on each side were check for their perpendicularity with the ground, as shown in Figure 116. The alignment between the top of the brace and the tensioners was then measured as shown in Figure 117.

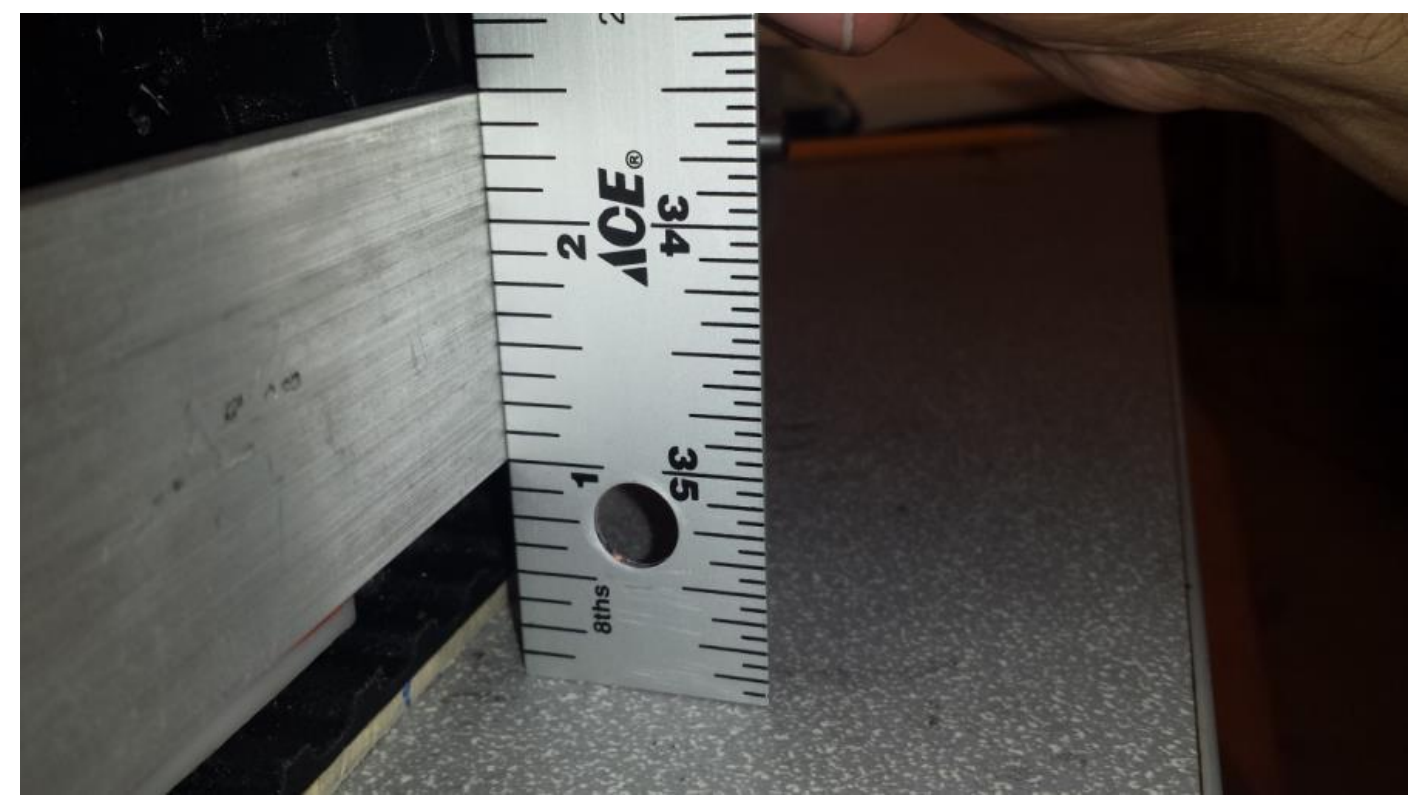

Figure 116: Straight edge against the brace. 


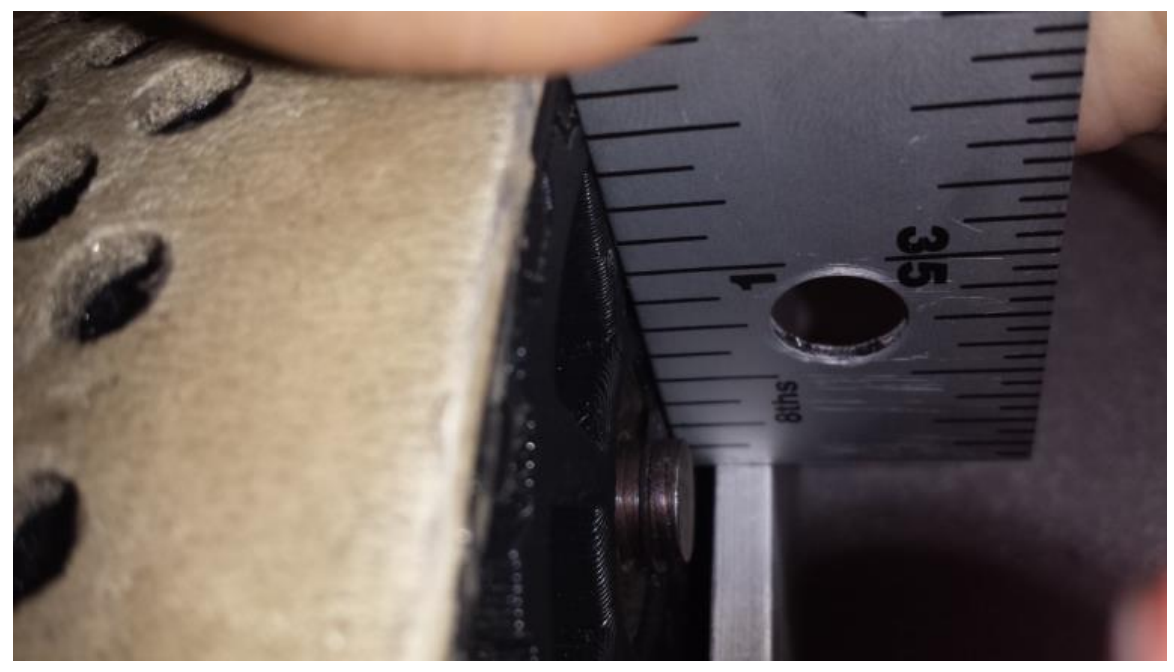

Figure 117: Angular misalignment between the brace and the tensioner wheel on the right side of the vehicle.

\subsubsection{Results}

The parallel and angular misalignments measured for the right and left sides of the vehicle can be seen in Table 70 and Table 71 respectively. The angular misalignment between the wheels on the right and left sides of the vehicle compared to a flat surface can be found in Table 72 and Table 73 respectively.

Table 70: Angular and parallel misalignment between wheels on the right side of the vehicle.

\begin{tabular}{|r|r|r|}
\hline Test Case & Parallel Misalignment & Angular Misalignment \\
\hline Drive - Tensioner & $0.0 \mathrm{in.}$ & $0.5^{\circ}$ \\
\hline Drive - Idler & $0.0 \mathrm{in.}$ & $0.0^{\circ}$ \\
\hline Idler - Tensioner & $0.0 \mathrm{in.}$ & $0.5^{\circ}$ \\
\hline
\end{tabular}

Table 71: Angular and parallel misalignment between wheels on the left side of the vehicle.

\begin{tabular}{|r|r|r|}
\hline Test Case & Parallel Misalignment & Angular Misalignment \\
\hline Drive - Tensioner & $0.0 \mathrm{in.}$ & $0.5^{\circ}$ \\
\hline Drive - Idler & $0.0 \mathrm{in.}$ & $0.41^{\circ}$ \\
\hline Idler - Tensioner & $0.0 \mathrm{in.}$ & $0.5^{\circ}$ \\
\hline
\end{tabular}

Table 72: Angular misalignment between wheels on the right side of the vehicle a flat surface.

\begin{tabular}{|r|r|}
\hline Test Case & Angular Misalignment \\
\hline Drive Wheel & $0.0^{\circ}$ \\
\hline Idler Wheel & $0.0^{\circ}$ \\
\hline Tensioner & $0.5^{\circ}$ \\
\hline
\end{tabular}


Table 73: Angular misalignment between wheels on the left side of the vehicle a flat surface.

\begin{tabular}{|r|r|}
\hline Test Case & Angular Misalignment \\
\hline Drive Wheel & $0.25^{\circ}$ \\
\hline Idler Wheel & $0.25^{\circ}$ \\
\hline Tensioner & $0.46^{\circ}$ \\
\hline
\end{tabular}

\subsubsection{Discussion}

The method used to determine wheel alignment was very difficult to perform. This was mostly due to the fact that the misalignments were extremely small, with the measurable misalignment being no greater than twenty thousandths of an inch, and occurred over a small distance. The geometries of the wheel system made taking some of the measurements awkward. Despite these difficulties, estimates of the angular and parallel misalignments in the wheels systems were taken.

No parallel misalignment was seen between the wheels on either side of the vehicle was observed. This indicates that the spacing system for the drive, idler and tensioner wheels was successful and the frames wheel support struts where very close to parallel.

The angular misalignment between the tensioner wheels and idler and drive wheels was expected. This is because the tensioner struts were not very well aligned with the rest of the frame. The braces were found to be parallel with the ground, and so the angular misalignment between the tensioner wheel and the ground could be estimated. The angular misalignment between the ground and the tensioner wheel was expected as the tensioner struts were not well aligned with the ground.

The right side of the vehicle was generally well aligned. The drive and idler wheels had no discernable misalignment with each other and with the ground. This was not the case with the left side of the vehicle, which had misalignment between the drive and idler wheel and the ground. This misalignment was likely due to the slop between the shafts of the wheels and the bearings, as well as angular misalignment between the frame struts.

Despite the angular misalignment seen the wheel systems of the robot the tread wheel system worked well during the other testing conducted. This indicates that the system can tolerate small misalignments and still operate properly, but may have reduced lifetime. Future iterations of the design should strive to eliminate 
measurable misalignment in the wheel system, as this will increase the life span of the treads and will likely reduce the losses in the wheel system.

\subsubsection{Belt Tension Determination}

This test was meant to determine the static tension which was developed in the treads after they had been tensioned. The following section covers the procedure used to determine the belt tension and the results of the testing.

\subsubsection{Procedure}

The procedure used to determine the belt tension was relatively straight forward and was found in SPD/SI's timing belt design guide manual. The method used to determine the belt tension is as follows. The belt was deflected by $1 / 64$ in. for each inch of the span where the belt was being tested and the force required to achieve the deflection was recorded. The deflection and force measurement were performed using a belt tension gauge and can be seen in Figure 118. Figure 119 shows the deflection force being measured using the belt tension gauge.

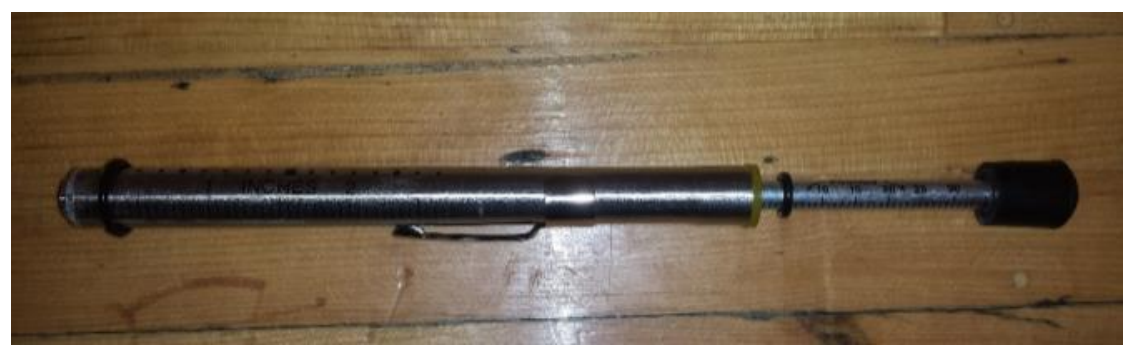

Figure 118: The force gauge used to determine the tread tension. 


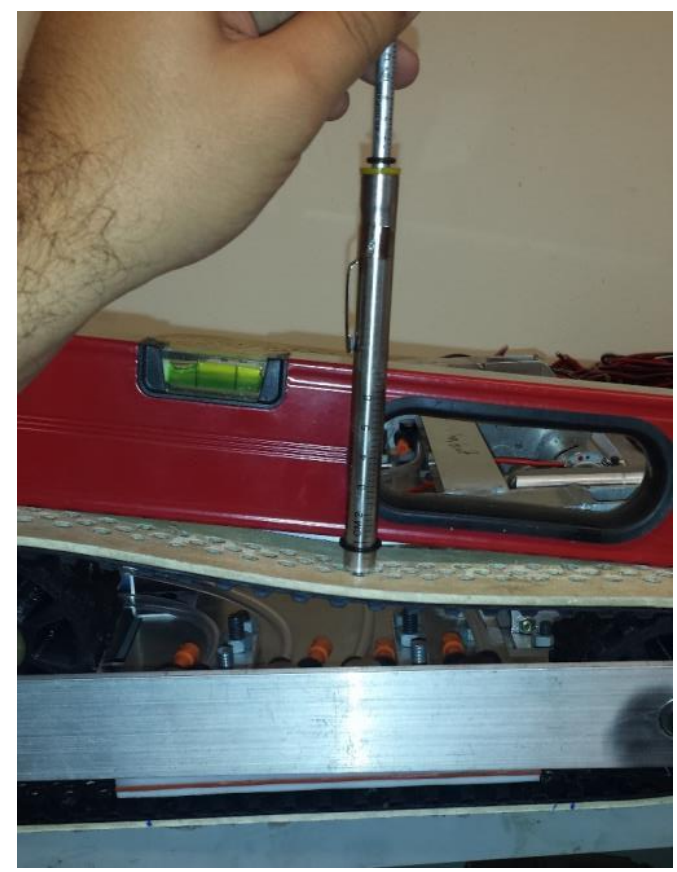

Figure 119: The tension in the left tread being measured.

\subsubsection{Results}

Equation 4.1 was used to calculate the static tension that was developed in the belt. Table 74 gives an explanation of the variables found in Equation 4.1. The constant $Y$ was calculated using a similar method to that used to determine the mass factor for the tread in Appendix A. The results for the right and left side treads can be found in Table 75 and Table 76 respectively. Table 77 compares the desired tread tension value with the estimated actual tread tension value.

$$
T_{s t}=\frac{16 * D F-\frac{t}{L} * Y}{1.1}
$$

Table 74: Variables used in Equation 4.1.

\begin{tabular}{|c|r|l|}
\hline Symbol & Units & \\
\hline$D F$ & lbf & Force required to deflect the tread 1/64 in. per in of span length. \\
\hline$t$ & in. & Length of span where the measurement was taken. \\
\hline$L$ & in. & Pitch length of the tread. \\
\hline$Y$ & lbf & Constant from SPD/SI. \\
\hline$T_{s t}$ & lbf & Static tension developed in the tread. \\
\hline
\end{tabular}


Table 75: Predicted tension in the left tread and the values used to calculate it.

\begin{tabular}{|rr|r|}
\hline & Input Variable & Value \\
\hline$D F$ & & $4 \mathrm{lbf}$ \\
\hline$t$ & & $9.0625 \mathrm{in}$. \\
\hline$L$ & & $34 \mathrm{in}$. \\
\hline$Y$ & & $27 \mathrm{lbf}$ \\
\hline & Output Variable & Value \\
\hline$T_{s t}$ & & $51.6 \mathrm{lbf}$ \\
\hline
\end{tabular}

Table 76: Predicted tension in the right tread and the values used to calculate it.

\begin{tabular}{|rr|r|}
\hline & Input Variable & Value \\
\hline$D F$ & & $4 \mathrm{lbf}$ \\
\hline$t$ & & $9.125 \mathrm{in}$. \\
\hline$L$ & & $34 \mathrm{in}$. \\
\hline$Y$ & & $27 \mathrm{lbf}$ \\
\hline & Output Variable & Value \\
\hline$T_{s t}$ & & $58.91 \mathrm{lbf}$ \\
\hline
\end{tabular}

Table 77: A comparison between the measured and desired tread tension values.

\begin{tabular}{|r|r|r|r|}
\hline & Measured Value & Desired Value & \% Difference \\
\hline Right & $58.9 \mathrm{lbf}$ & $46.3 \mathrm{lbf}$ & $27 \%$ \\
\hline Left & $51.6 \mathrm{lbf}$ & $46.3 \mathrm{lbf}$ & $11 \%$ \\
\hline
\end{tabular}

\subsubsection{Discussion}

The belt tension testing provided several important insights into not only the tension developed in the treads when installed on the vehicle, but also how belt tension effects the performance of the tread and the vehicle as a whole. The measured belt tension in both treads was higher than the minimum desired value calculated in Appendix A. This validates the design of the tensioning system. It also demonstrates that the losses in the wheel system are minimal if the treads are tensioned above the minimum desired belt tension. Because the tensioning system could not be raised further higher belt tensions and their effects on the vehicles performance could not be tested.

The testing procedure also gave insight into improvements which would be made to future iterations of the design. While the treads were tensioned to the minimum recommended belt tension, the span on which the manifolds rested was relatively loose and several instances of the treads slumping away from the manifolds when the vehicle was lifted were noted. Additionally, the fact that the span deflected as much as it did could likely have contributed to the reduction in the vacuum pressure in the manifolds. The test procedure used in calculating the belt tension seems to indicate that span length and belt tension directly affect the 
amount the treads can deflect. By reducing the effective span length around the manifolds in future designs and increasing the belt tension, the amount of deflection in the tread can be reduced.

\subsection{Vehicle Operation Observations}

This section gives an overview of performance of the vehicle as a whole and of the observations that were made during process of making vehicle operational which are salient to future iterations of the project. It also gives an overview of the different changes which were made to the vehicle as the operational testing was conducted. Videos of the vehicle operating can be found in the attached files that accompany this report.

\subsubsection{Initial Vertical Vehicle Adhesion and Testing}

\subsubsection{Creating the Manifold-Tread Seal}

The first step taken to get the vehicle operation was to statically adhere the vehicle to a vertical surface.

This was initially impossible as the low friction fabric on the treads was ridged which prevented the manifolds from sealing to the treads. To solve this issue, Dow Corning high-vacuum grease was purchased. The grease had very low volatility and would be stable when subjected to the vacuum. The purpose of the grease was to fill in the gaps in the low friction fabric and to help further reduce the friction between the treads and the manifolds. The grease was liberally applied to fabric and the manifolds were able to form a seal with treads. Figure 120 shows an image of one of the treads being adhered to wall with the manifolds.

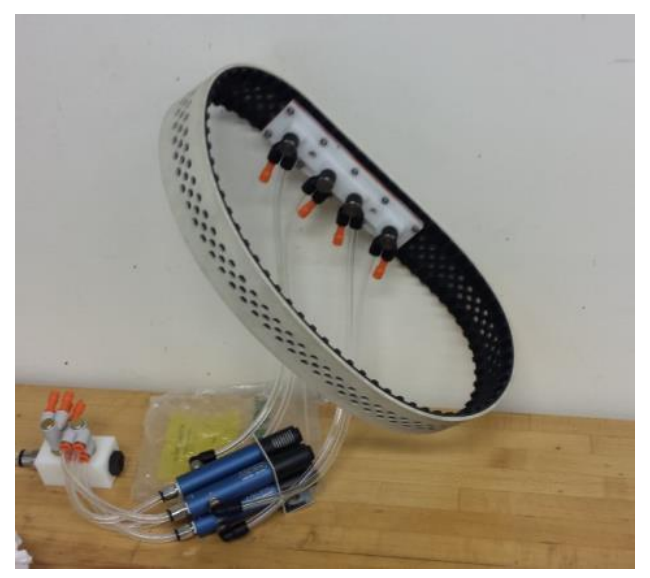

Figure 120: A tread being adhered to the wall with a manifold. 


\subsubsection{Vehicle Static Adhesion}

Once the manifold-tread seal was successfully created and both treads had been coated by the vacuum grease, the vehicle was fully assembled. The vehicle was then placed on a horizontal surface and the vacuum system was turned on and the vehicle was adhered to the surface. This was done to ensure that the assembly process had not adversely affected the vacuum system. Once it had been confirmed that the vehicle adhered to the horizontal surface, it was moved on to a vertical surface. Figure 121 shows the vehicle when it had been adhered to a vertical surface successfully.

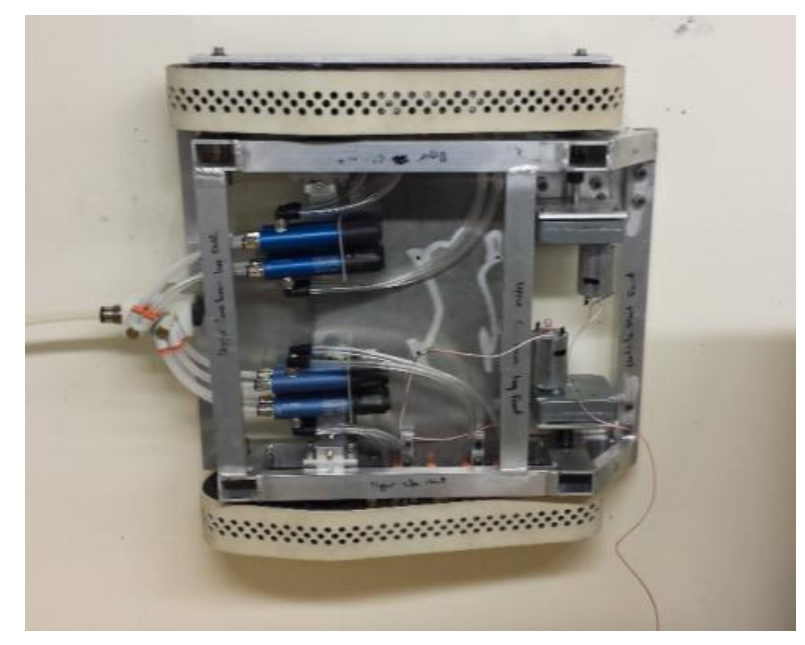

Figure 121: The vehicle being adhered to a vertical surface.

To determine if the vertical adhesion was repeatable the vehicle was removed and replaced on the vertical surface several different times. While the vehicle adhered to the wall successfully most of the time, it was noted that the process of removing the vehicle from the wall caused the manifolds to shift which prevented re-adhesion until the manifold was re adjusted.

Initially, the manifold springs had relatively low spring force, about four pounds at maximum compression, and it was found that they did not provide enough force to push the manifolds back into place. An attempt at fixing the manifolds completely, i.e. holding them in place by using a nut on either side of the support strut, was attempted, but was found to only exacerbate the problem. Stiffer springs which could provide much higher forces, approximately eleven pounds, were purchased and installed. These springs proved to be sufficient, and the adhesion and re-adhesion of the vehicle became much more reliable. 


\subsubsection{Powered Testing}

\subsubsection{Horizontal Surface Testing}

Before the vehicle was placed on the wall and driven under its own power, some horizontal operational testing was performed. This was done by setting the vehicle onto a flat horizontal surface and powering the motors. This was done with the vacuum system engaged and disengaged. The main purpose for this step was to determine if the vehicle would drive in a straight line without using the controller. This testing was successful and so the vertical surface powered testing began.

\subsubsection{Initial Vertical Surface Testing}

The initial vertical powered testing did not go well. Immediately after the motors were powered one of the vehicles treads would slip and the vehicle would begin to fall. Although both treads slipped, the right side slipped much more often than the left. To correct this problem, or to at least diagnosis its cause, several different adjustments to the vehicle were made. For instance, the vehicle was placed sideways on the wall so that its travel path was parallel with the ground rather than perpendicular to it. The manifold suspension system was both loosened and tightened in an attempt to ensure that the manifolds remained in contact with the treads. Finally, large amounts of the vacuum grease were added to the treads as it was noticed that the treads were becoming degreased as the vehicle operated. None of these attempts were successful and seemed to make little to no difference in the vehicles performance.

It was also noted that after the vehicle was run, it became very difficult to re-adhere it to the wall. The cause of this seemed to be the loss of the grease on the tread when the vehicle was run. This loss of grease was caused by two distinct phenomena. The first of these phenomena was the buildup of grease in the vacuum chambers of the manifolds. Figure 122 shows the inside of one of the manifolds after this problem was identified. The second phenomena was the grease being squeezed into the treads hole as the tread passed around the wheels. Interestingly, this did not cause a blockage of the tread holes as the grease remained on the wheels as seen in Figure 123. Channels were cut in the drive and idler wheels to prevent the grease from being squeezed and while this did improve the grease distribution on the tread, it did not solve the vehicles inability to maintain suction after repeated runs. 


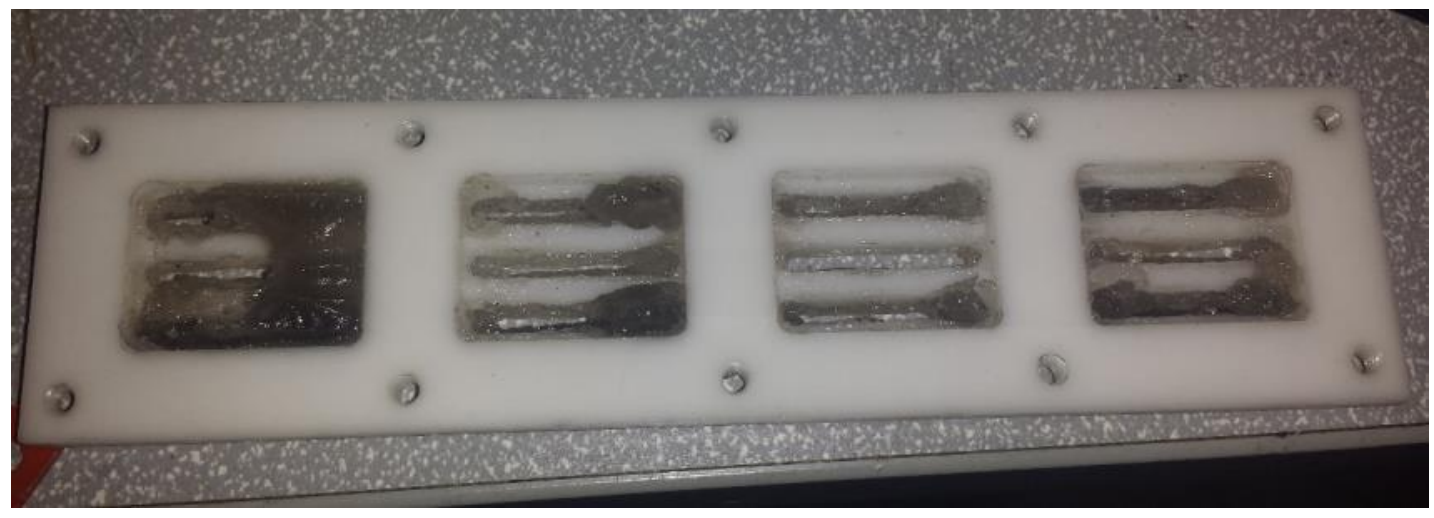

Figure 122: Interior of a manifold after it had become clogged.

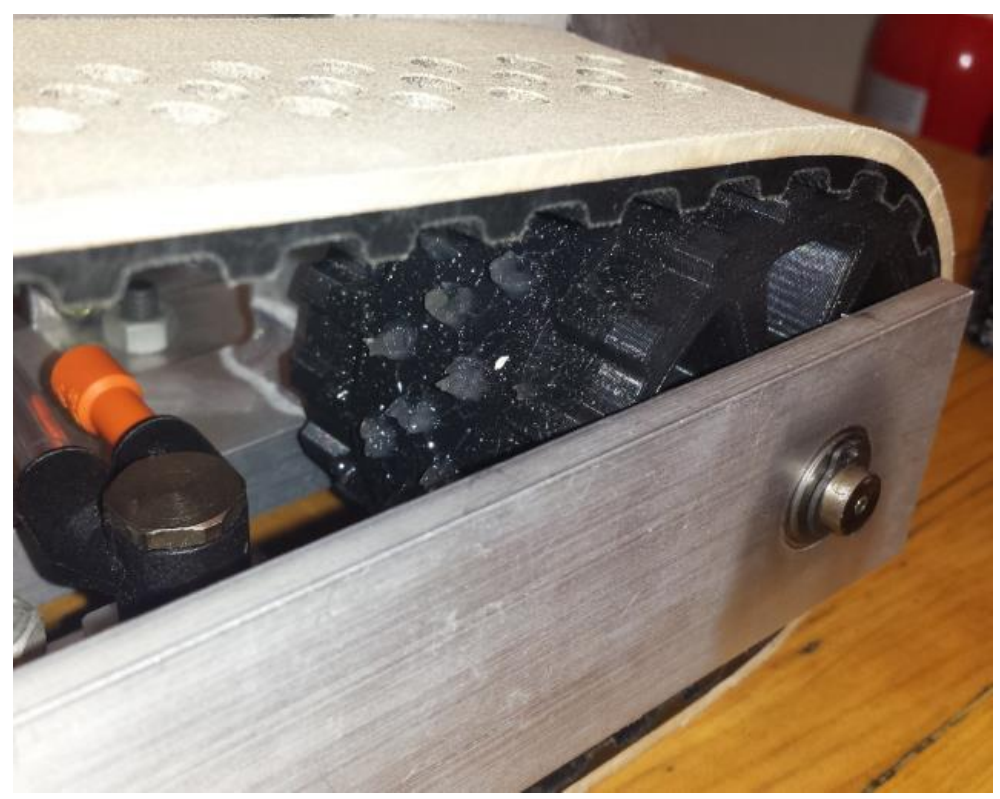

Figure 123: Grease spikes which were squeezed into the tread holes during operation.

\subsubsection{Final Vertical Surface Testing}

Because the treads were losing grease and therefore suction, a more permanent solution was sought out to create the seal between the manifolds and the treads. Ultimately, silicone sealant gel was chosen as the coating for the inside of the treads as it was readily available and easy to work with. The treads were cleaned and degreased and the silicone gel was applied to the low friction fabric and allowed to dry. Figure 124 shows the tread after the silicone sealant was applied and allowed to dry. A small amount of grease was then reapplied to the inside of the treads as the friction between the manifolds and the silicone gel was relatively high. The vehicle was then reassembled and tested. 


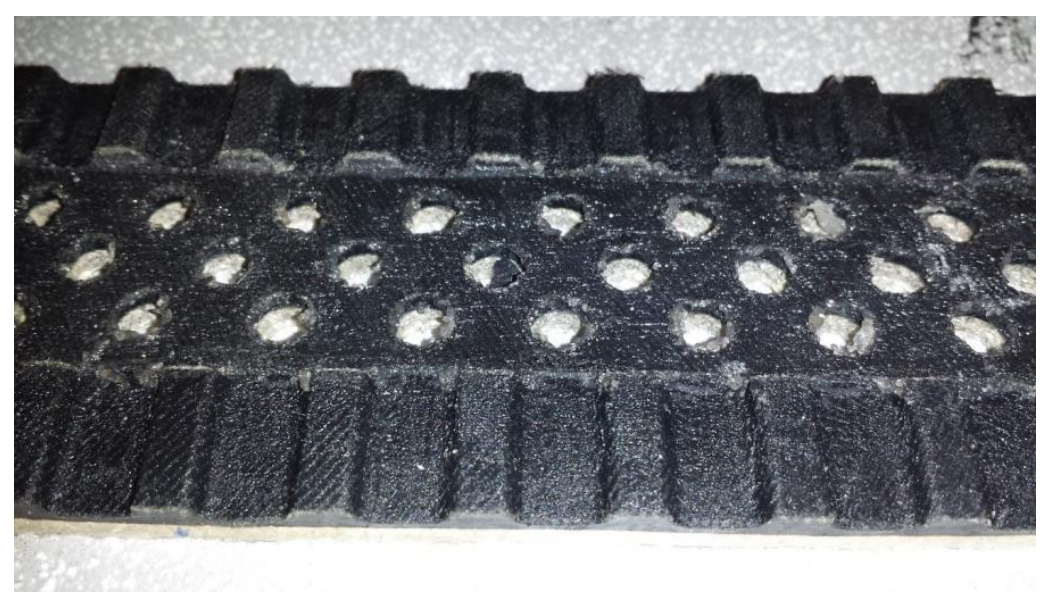

Figure 124: Low friction fabric after the silicone sealant had been applied.

The slipping problem seen in the earlier testing was still occurring, but the vehicle was able to travel further than it had during the grease only testing. While the grease only testing failed immediately upon motor startup, the vehicle was able to travel approximately six inches to a foot before slipping occurred. It should also be noted that the adhesion of the vehicle became much more reliable after the silicone was applied as the silicone remained intact and in place as the vehicle operated.

Because the silicone sealant had worked well for the inside of the treads, the outer surface of the tread, which was surprisingly slick, was coated in the sealant. This was done to increase the coefficient of friction between the tread and the wall. The sealant was applied in a thin coat and allowed to dry. The application of sealant to the outer portion of the tread had a marginal effect on the performance of the vehicle, though it still slipped after a short distance.

Up until this point in the attempts to get the vehicle fully operational, the current and voltage supplied to the motors was the maximum allowable values. This meant that the vehicle was accelerated very quickly which could have caused shifting in the frame and the release of the manifolds. To test this theory the voltage supplied to the motors was halved and the maximum current limit on the power supply was lowered slightly. This would prove to be the final operation test that was performed on the vehicle because during the test the drive shafts on both sides of the vehicle stripped the interior of their drive wheels, meaning the drive shafts spun but the wheels did not. This meant that the vehicle was no longer capable of climbing and so operational testing had to be ended. 


\section{Chapter 5: Recommendations for Future Work and Conclusion}

\subsection{Recommendations for Future Work}

There are several improvements which can be made to improve the design of this wall climbing robot. This section presents and discusses these improvements.

\subsubsection{Tread}

There are several modifications which can be made to the tread to improve vehicle performance. The most significant of these is simply to leave the bottoms of the manifold channels as rubber. This would ensure that the interface between the manifold and the tread has a consistent seal which will not degrade over time. Small amounts of the vacuum grease can be applied to the tread to reduce the friction between the manifolds and the tread, but a low-friction, dry interface is preferred.

The next modification would be the use of super high friction and highly compliant material as the backing of the tread. This would help to compensate for any losses in manifold pressure by increasing the coefficient of friction between the tread and the wall. The greater compliance would also allow the vehicle to operate on rougher surfaces. Natural rubber might be a good option for this material, but testing should be done to identify an ideal material.

\subsubsection{Wheel System}

A suspension system should be added to the wheel system. This would allow the wheels to push flat against the wall and help to compensate for the moment that the vehicle creates as it sits on the wall. This would also help prevent the vehicle from peeling off the wall when it is accelerated.

To prevent the drive wheels from being stripped by the drive shafts over time, the drive wheels should be constructed from aluminum and the shafts should be splined or keyed to assure torque transfer. This would also allow for the wheels to be easily removed from the vehicle if modifications were needed. While this would increase the price of the wheels significantly, it would also increase long term vehicle reliability. 


\subsubsection{Manifold}

The manifolds should be constructed from aluminum because the delrin used in this project, while easy to machine, was prone to warping after machining was complete. Having the manifolds constructed from aluminum would also help to ensure that the manifold suspension system did not strip the manifold threads over time.

The manifold should consist of physically independent floating sections, which have their own suspension system. This suspension system should allow the manifolds to shift with the tread, but should keep them in constant contact. This would help to eliminate the interactions between sections that were observed during this project.

The manifold should also extend over a larger section of the tread. This serves two purposes, it will help to reduce the effect that changes at one end of the vehicle have on the manifold sections at the back and will allow for greater vacuum forces to be generated.

\subsubsection{Vacuum Systems}

The vacuum system for the project performed well overall, so only minor modifications to the system need to be considered. If smaller venturis could be found then the weight contribution and foot print of the vacuum system in the vehicle could be reduced. An active or passive control system should also be considered for the vacuum system which would allow the vacuum force to be varied as the vehicle moves.

\subsubsection{Frame System}

The frame should be made generally smaller and lighter. It should also be very low profile relative to the wall to help reduce the distance between the vehicles center of mass and the wall. This would reduce the peel off force produced by the vehicle's center of mass. The frame should also be optimized for the expected loading to reduce its weight without sacrificing frame stiffness.

\subsubsection{Motor System}

The motors for future projects should be smaller both physically and dimensionally. This would help to reduce the overall weight of the vehicle and reduce the vehicles overall size. The selected motors should 
also include encoders to eliminate the need for an external motor speed tracking system. It would also be good to select several motors of similar dimensions, but different maximum torque ratings so that once the vehicle has been built and tested, its motor system can be optimized.

\subsubsection{Tensioner System}

The third wheel tensioner system should be abandoned. While this system worked, it prevented the manifold from extending further along the tread and increased the height of the frame. It also did not allow for high tread tension values to be attained and prevented the effect that belt tension had on the systems performance from being determined. As an alternative to the third wheel tensioner system, the idler wheel could double as the tensioner.

\subsubsection{General Vehicle Improvements}

The weight and size of future vehicles should be reduced as much as possible. This would make testing the vehicle much easier and would reduce the risk of injury if the vehicle losses adhesion. Components that are critical to the design of the future project should be professionally machined. This would serve a dual purpose: it would allow for much tighter tolerances to be specified for the vehicles design: and would help to eliminate the misalignments seen in this project.

In addition to reducing the weight of the vehicle, its center of gravity should also be moved closer to the wall to reduce the moment it creates on the vehicle. The center of gravitie's moment could also be compensated for through the addition of a tail to the vehicle. This tail would push on the wall and lift the vehicle rear end slightly, thereby preventing the front of the vehicle from rocking away from the wall. This tail would also help to eliminate any rocking that the vehicle would experience while it was accelerating. However, a fixed tail could make matters worse when traversing down the wall!

\subsection{Conclusion}

Despite the inability of the vehicle, as designed and manufactured in this project, to climb reliably for any great distance, a great deal of insight was gained into the vacuum tread concept. This information can be used to develop future embodiments of the design and more convincingly demonstrate the concepts viability. Ultimately, this project showed that the concept should be pursued further. 


\section{BIBLIOGRAPHY}

[1] G. La Rosa, M. Messina, G. Muscato and R. Sinatra, "A low-cost lightweight climbing robot for the inspection of vertical surfaces," Mechatronics, no. 12, pp. 71-96, 2002.

[2] K. Berns, C. Hillenbrand and T. Luksch, "Climbing Robots for Commercial Applications - a Survey," University of Kaiserslautern, Kaiserslautern, Germany, 2011.

[3] D. Schmidt and K. Berns, "Climbing robots for maintenance and inspections of vertical structures - A survey of design aspects and technologies," Robotics and Autonomous Systems, no. 61 , pp. 1288-1305, 2013.

[4] H. Kim, D. Kim and H. Yang, "A wall climbing robot with vacuum caterpillar wheel system operated by mechanical valve," in Proceedings of the 9th International Conference on Climbing and Walking Robots, Brussels, Belgium, 2006.

[5] B. Hu, L. Wang, Y. Zhao and Z. Fu, "A miniature wall climbing robot with biomechanical suction cups," Industrial Robot: An International Jounral, vol. 36, no. 6, pp. 551-561, 2009.

[6] S. Liu, X. Gao, K. Li, J. Li and X. Duan, "A Small-sized Wall-climbing Robot for Anti-terror Scout," in IEEE International Conference on Robotics and Biomimetics, Sanya, China, 2007.

[7] T. Akinfiev, M. Armada and S. Nabulsi, "Climbing cleaning robot for vertical surfaces," Industrial Robot: An International Journal, vol. 36, no. 4, pp. 352-357, 2009. Reinforced Concrete Structures," in 2nd International Conference on Applied Robotics for the Power Industry, Zurich, Switzerland, 2012.

[9] M. Taylor, X. Chen, M. Lang, T. McKee, J. Robertson and S. Aston, "TigBot - A Wall Climbing Robot for TIG Welding of Stainless Steel Tanks," in 15th International conference on 
Mechatronics and Machine Vision in Practice, Auckland,New-Zealand, 2008.

[10] J. C. Grieco, M. Prieto, M. Armada and P. Gonzalez de Santos, "A SIX-LEGGED CLIMBING ROBOT FOR HIGH PAYLOADS," in 1998 IEEE International Conference on Control Applications, Trieste, Italy, 1998.

[11] D. Longo and G. Muscato, "The Alicia Climbing Robot," IEEE Robotics \& Automation Magazine, pp. 42-50, 2006.

[12] H. X. Zhang, W. Wang and J. W. Zhang, "High Stiffness Pneumatic Actuating Scheme and Improved Position Control Strategy Realization of a Pneumatic Climbing Robot," in IEEE International Conference on Robotics and Biomimetics, Bangkok, Thailand, 2008.

F. XU, D. XIA and G. JIANG, "Initial Design and Analysis of a Helix Cable Detecting Robot," in 32nd Chinese Control Conference, Xi'an, China, 2013.

F. ROCHAT, P. SCHOENEICH, O. TRUONG-DAT NGUYEN and F. MONDADA, "TRIPILLAR: MINIATURE MAGNETIC CATERPILLAR CLIMBING ROBOT WITH PLANE TRANSITION ABILITY," Ecole polytechnique fédérale de Lausanne, Lausanne, Switzerland.

[15] W. Shen, J. Gu and Y. Shen, "Permanent Magnetic System Design for the Wall-climbing Robot," in IEEE International Conference on Mechatronics \& Automation, Niagara Falls, Canada, 2005.

[16] M. Spenko, G. Haynes, J. Sanders, M. Cutkosky and A. Rizzi, "Biologically Inspired Climbing with a Hexapedal Robot," Journal of Field Robotics, vol. 25, no. 4-5, pp. 223-242, 2008.

[17] T. Lun Lam and Y. Xu, "Tree Climbing Robot - Design, Kinematics and Motion PLanning," Springer Tracts in Advanced Robotics, vol. 78, 2012.

[18] G. L. K. Cui and J. Guo, "Design of a Climbing Robot Based on Electrically Controllable 
Adhesion Technology," in 2012 International Conference on Solid State and Materials, 2012.

[19] "A gecko-inspired electroadhesive wall-climbing robot," IEEE Potentials, pp. 15-19, 2015.

[20] Y. Yoshida and S. Ma, "Design of a Wall-Climbing Robot with Passive Suction Cups," in IEEE International Conference on Robotics and Biomimetics, Tianjin, China, 2010.

[21] M. Guimaraes and J. Lindberg, "REMOTE CONTROLLED VEHICLE FOR INSPECTION OF VERTICAL CONCRETE STRUCTURES," IEEE, vol. 14, 2014.

[22] A. Fallah, M. Rezasoltani and A. M. H. Riasi, "Numerical Analysis of a Non-Contact Surface Adhesion System based on Vortex Cup for Wall Climbing Robots," in RSIIISM International Conference on Robotics and Mechatronics, Tehran, Iran, 2013.

[23] R. Budynas and K. Nisbett, Shigley's Mechanical Engineering Design, New York: McGraw-Hill, 2011.

[24] NYU Tandon School of Engineering, "NYU Tandon School of Engineering," [Online]. Available: http://engineering.nyu.edu/gk12/Information/Vault_of_Labs/Physics_Labs/static\%20and\%20kineti c\%20friction.doc.. [Accessed 26 May 2016].

[25] SDP/SI, "SDP/SI Technical Section," [Online]. Available: http://www.sdpsi.com/D265/HTML/D265T050.html. [Accessed 275 2016].

[26] Smalley Steel Ring Company, "Load Capacity," 2016. [Online]. Available: http://www.smalley.com/ring-design/load-capacity. [Accessed 275 2016]. 


\section{APPENDICES}

\section{Appendix A - Simulations and Calculations}

\section{A.1 Symbolic Calculation of Motor Torque}

\section{A.1.1 Calculation Description}

The purpose of this calculation was to create a set of equations which could be used to calculate the required drive motor torque. Because the vehicle is symmetrical about its longitudinal axis, the torque of a single motor was calculated for half of the vehicle.

\section{A.1.2 Assumptions}

- Losses in the wheel system due to friction can be lumped at the drive wheel.

- The inertia of all the wheels can be lumped at the drive wheel.

- The Idler wheel does not support any dynamic load during forward motion.

- Tread is rigid along its longitudinal axis.

- Tread does not slip at the wall.

- Frame and Manifold move relative to Tread and Wall.

- Vehicle is symmetrical and the solution for the required motor torque can be found by looking at half of the vehicle. 


\section{A.1.3 Free Body and Mass Acceleration Diagrams}

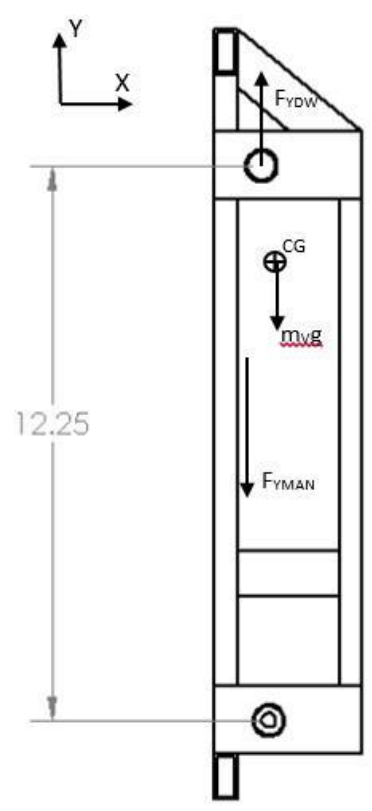

FBD - VEHICLE BODY

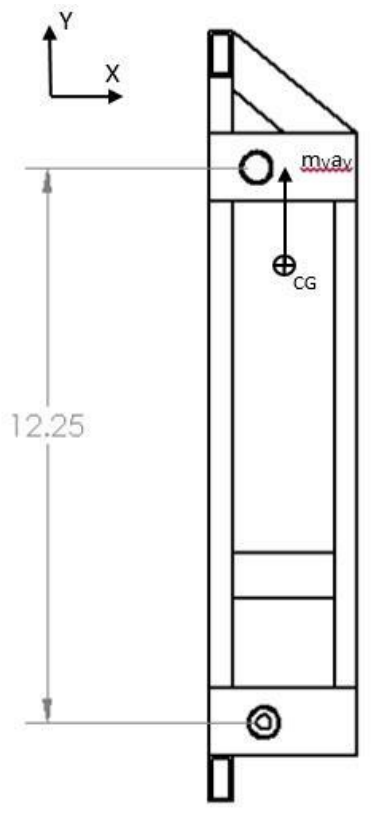

MAD - VEHICLE BODY

Figure 125: Free body and mass acceleration diagrams for the body of the vehicle.

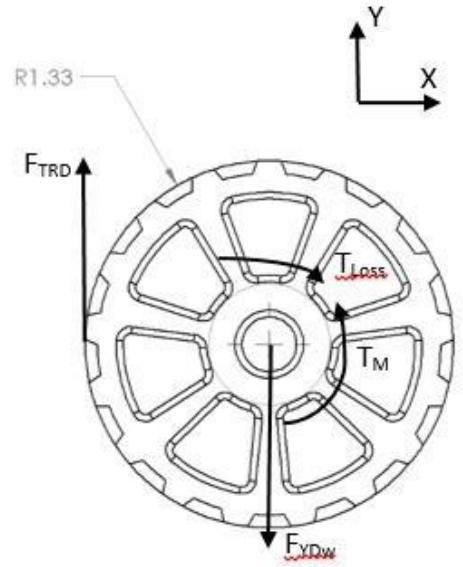

FBD - DRIVE WHEEL

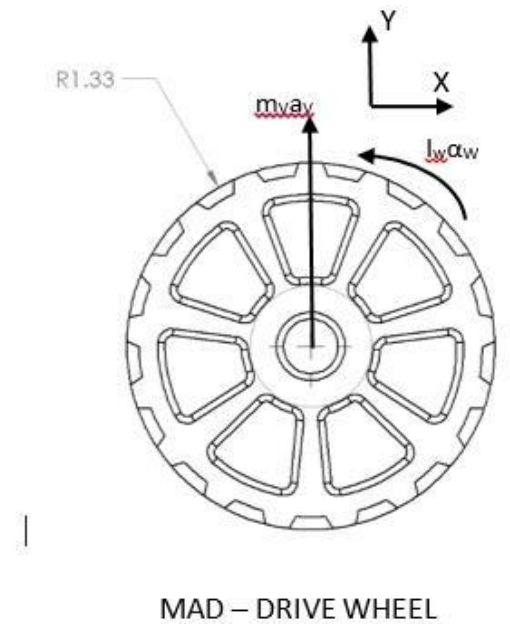

MAD - DRIVE WHEEL

Figure 126: Free body and mass acceleration diagrams for the drive wheel. 

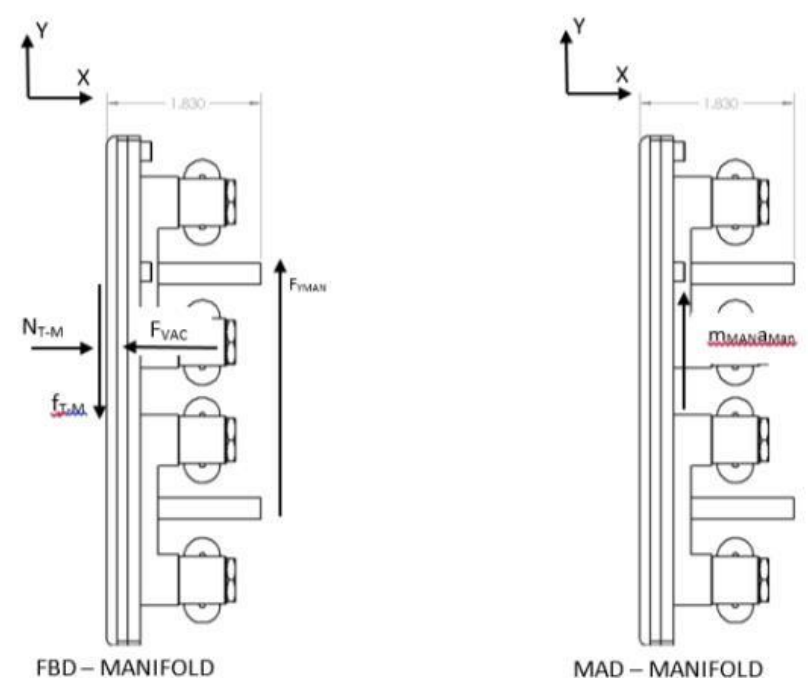

Figure 127: Free body and mass acceleration diagrams for the manifold.
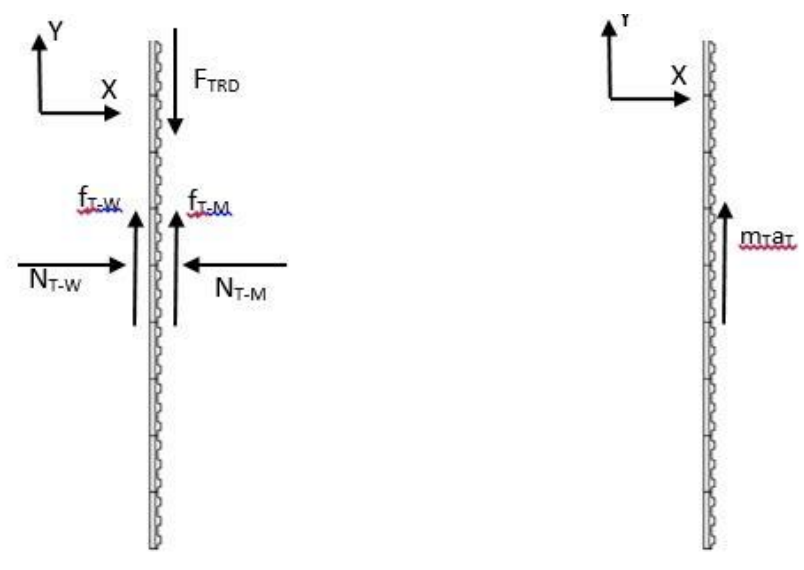

FBD - TREAD

MAD - TREAD

Figure 128: Free body and mass acceleration diagrams for the tread.

\section{A.1.4 Variable Definitions}

The variables used in the motor torque calculator can be found in Table 78 .

Table 78: Variable symbols, units and descriptions for the symbolic motor torque calculator.

\begin{tabular}{|c|c|l|}
\hline Symbol & Units & \multicolumn{1}{c|}{ Description } \\
\hline$m_{V}$ & SLUG & Mass of the Vehicle \\
\hline$F_{Y D W}$ & LBF & Force in the Y Direction on the Drive Wheel \\
\hline$F_{V M A N}$ & LBF & Force Produced by Manifold on Main Vehicle \\
\hline$a_{V}$ & FT/S^2 & Acceleration of the Vehicle \\
\hline$F_{T R D}$ & LBF & Force Between Drive Wheel and Tread \\
\hline$T_{M}$ & IN - LBF & Torque Produced by Motors \\
\hline$T_{L O S S}$ & IN - LBF & Lumped Losses in the Rotation of the Wheels \\
\hline
\end{tabular}




\begin{tabular}{|c|c|l|}
\hline$I_{W}$ & LBM $^{*} \mathrm{IN}^{\wedge} 2$ & Lumped Rotational Inertia of The Wheels and Shafts \\
\hline$\alpha_{W}$ & RAD/S^2 & Angular Acceleration of Drive Wheel \\
\hline$R_{D W}$ & IN & Radius of Drive Wheel. \\
\hline$N_{T-W}$ & LBF & Normal Force Between the Tread and the Wall \\
\hline$f_{T-W}$ & LBF & Friction Force Between tread and Wall \\
\hline$\mu_{T-W}$ & UNITLESS & Static Coefficient of Friction between Tread and Wall \\
\hline$N_{T-M}$ & LBF & Normal Force between the Tread and the Manifold \\
\hline$f_{T-M}$ & LBF & Friction Force between Tread and Manifold \\
\hline$\mu_{T-M}$ & UNITLESS & Static Coefficient of Friction between Tread and Manifold \\
\hline$F_{V A C}$ & LBF & Vacuum Force Produced by Manifold \\
\hline$m_{t}$ & SLUG & Mass of the Tread \\
\hline$a_{T}$ & FT/S^2 & Acceleration of Tread. Assumed equal to Zero. \\
\hline$m_{\text {Man }}$ & SLUG & Mass of Manifold. Assume accounted for in $m_{v}$. \\
\hline$a_{M a n}$ & FT/S^2 & Acceleration of Manifold. Assumed Equal to $a_{V}$. \\
\hline
\end{tabular}

\section{A.1.5 Symbolic Manipulation}

Begin by summing forces and accelerations for the vehicle body, given in Equation A.1.1 and solving for $F_{Y D W}$ which yields Equation A.1.2.

$$
\begin{gathered}
F_{Y D W}-m_{V} g-F_{V M A N}=m_{V} a_{V} \\
F_{Y D W}=m_{V} g+F_{V M A N}+m_{V} a_{V}
\end{gathered}
$$

Next, sum forces on the drive wheel, yielding Equation A.1.3. Also, sum moment about the center of the drive wheel to get Equation A.1.4.

$$
\begin{gathered}
F_{T R D}-F_{Y D W}=m_{V} a_{V} \\
T_{M}-T_{\text {Loss }}-R_{D W} F_{T R D}=I_{W} \alpha_{W}
\end{gathered}
$$

Use the relationship between angular acceleration and linear acceleration to find $\alpha_{W}$ in terms of $a_{V}$ as seen in Equation A.1.5. Solve for $F_{T R D}$ in Equation A.1.4 to yield Equation A.1.6.

$$
\begin{gathered}
\alpha_{W}=\frac{a_{V}}{R_{D W}} \\
F_{T R D}=\frac{T_{M}}{R_{D W}}-\frac{T_{L o s S}}{R_{D W}}-\frac{I_{W} \alpha_{W}}{R_{D W}}
\end{gathered}
$$

Substitute Equation A.1.5 and Equation A.1.6 into Equation A.1.3 and manipulate to solve for $T_{M}$ to yield Equation A.1.7. 


$$
T_{M}=T_{L o s s}+\frac{I_{W} a_{V}}{R_{D W}}+R_{D W} F_{Y D W}+R_{D W} m_{V} a_{V}
$$

Sum forces in the x-direction on the manifold to relate $F_{V A C}$ and $N_{T-M}$ in Equation A.1.8. Sum forces in the y-direction to find Equation A.1.9 and substitute in the relationship between normal force and frictional force to find Equation A.1.10.

$$
\begin{gathered}
N_{T-M}=F_{V A C} \\
F_{V M A N}=f_{T-M} \\
F_{V M A N}=\mu_{T-M} F_{V A C}
\end{gathered}
$$

Sum forces on the $\mathrm{x}$-direction on the tread to yield Equation A.1.11. The friction between the wall and the tread must be greater than both the friction between the manifold and the tread and the weight of the vehicle, this is expressed in Equation A.1.12 and Equation A.1.13 respectively.

$$
\begin{gathered}
N_{T-M}=N_{T-W} \\
f_{T-W}>f_{T-M} \\
f_{T-W}>m_{V} g
\end{gathered}
$$

Solve for $T_{M}$ by substituting Equation A.1.2 and Equation A.1.10 into Equation A.1.7 to find Equation A.1.14. Simplify to find Equation A.1.15.

$$
\begin{gathered}
T_{M}=T_{L o s S}+\frac{I_{W} a_{V}}{R_{D W}}+R_{D W}\left(m_{V} g+\mu_{T-M} F_{V A C}+m_{V} a_{V}\right)+R_{D W} m_{V} a_{V} \\
T_{M}=T_{\text {Loss }}+R_{D W} m_{V} g+R_{D W} \mu_{T-M} F_{V A C}+\left(\frac{I_{W}}{R_{D W}}+2 R_{D W} m_{V}\right) a_{V}
\end{gathered}
$$

Example of Use

This example represents the final settings used in the calculator to determine the motor torque required for the project. Table 79 contains the variable values used in the calculator. Table 80 contains the results of the calculation.

Table 79: Final input variables used in the motor torque calculator.

\begin{tabular}{|c|r|}
\hline Variable & Value \\
\hline$m_{V}$ & $.25 \mathrm{slugs}(8 \mathrm{lbs})$ \\
\hline$a_{V}$ & $.25 \mathrm{ft} / \mathrm{s}^{\wedge} 2$ \\
\hline$T_{\text {Loss }}$ & $5 \mathrm{in}-\mathrm{lbf}$ \\
\hline$I_{W}$ & $.0421 \mathrm{slug}$-in 2 \\
\hline$R_{D W}$ & $1.33 \mathrm{in}$ \\
\hline
\end{tabular}




\begin{tabular}{|l|r|}
\hline$\mu_{T-W}$ & 1 \\
\hline$\mu_{T-M}$ & .5 \\
\hline$F_{V A C}$ & $16 \mathrm{lbf}$ \\
\hline
\end{tabular}

Table 80: Final outputs from the motor torque calculator.

\begin{tabular}{|c|r|}
\hline Solution & Value \\
\hline$T_{M}$ & $26.77 \mathrm{in}-\mathrm{lbf}$ \\
\hline$\alpha_{W}$ & $2.26 \mathrm{Rad} / \mathrm{s}^{\wedge} 2$ \\
\hline$F_{Y D W}$ & $16.1 \mathrm{lbf}$ \\
\hline$f_{T-W}$ & $16 \mathrm{lbf}$ \\
\hline$f_{T-M}$ & $8 \mathrm{lbf}$ \\
\hline
\end{tabular}




\section{A.2 Motor RPM Requirement}

\section{Calculation Description}

This calculation determines the vehicles linear velocity based on a given motor output shaft angular velocity. This is simply the calculation to solve for the linear velocity of a rolling wheel based on the wheels angular velocity which is expressed in Equation A.2.1.

\section{A.2.1 Variables}

The variables used in the motor RPM requirements calculation can be found in Table 81 .

Table 81: Variable symbols, units and descriptions for the motor RPM requirement calculation.

\begin{tabular}{|c|r|l|}
\hline Symbol & Units & \multicolumn{1}{|c|}{ Description } \\
\hline$R_{D W}$ & in. & The radius of the drive wheel. \\
\hline$t_{T R D}$ & In. & Thickness of the tread. \\
\hline$\omega_{M}$ & RPM & Motor output shaft angular velocity \\
\hline$v_{V}$ & in/s & Vehicle velocity \\
\hline
\end{tabular}

\section{A.2.2 Equation}

$$
v_{V}=\left(.105 \frac{\mathrm{rad} * \min }{\mathrm{rev} * S}\right) R_{D W} \omega_{M}
$$

\section{A.2.3 Example}

This example uses the angular velocities of the two motors which were considered for the project. Two different drive wheel radiuses were used as well of sizes similar to the estimated drive wheel radius. Table 82 contains the results of this calculation.

Table 82: Results of the motor RPM requirements calculation for different motor and wheel combinations.

\begin{tabular}{|l|r|r|r|}
\hline \multicolumn{1}{|c|}{ Scenario } & $\boldsymbol{R}_{\boldsymbol{D} \boldsymbol{W}}$ & $\boldsymbol{\omega}_{\boldsymbol{M}}$ & \multicolumn{1}{c|}{$\boldsymbol{v}_{\boldsymbol{V}}$} \\
\hline 17 Tooth Wheel - Molon Motor & $1.33 \mathrm{in}$. & $25 \mathrm{RPM}$ & $3.5 \mathrm{in} / \mathrm{s}$ \\
\hline 17 Tooth Wheel - Maxon Motor & $1.33 \mathrm{in}$. & $50 \mathrm{RPM}$ & $7.0 \mathrm{in} / \mathrm{s}$ \\
\hline 18 Tooth Wheel - Molon Motor & $1.43 \mathrm{in}$. & $25 \mathrm{RPM}$ & $3.8 \mathrm{in} / \mathrm{s}$ \\
\hline 18 Tooth Wheel - Maxon Motor & $1.43 \mathrm{in}$. & $50 \mathrm{RPM}$ & $7.5 \mathrm{in} / \mathrm{s}$ \\
\hline
\end{tabular}




\section{A.3 Evacuation Rate Calculation}

\section{A.3.1 Calculation Description}

This calculation was used to determine the time it would take the venturis to evacuate the volume of vacuum system they were attached to. To begin, the volume of the different portions of the vacuum system are estimated and add to get an estimate of the total volume being evacuated. This value is then used in conjunction with the evacuation rates of the different venturis to determine the time it takes to achieve full evacuation.

\section{A.3.2 Volume Estimation}

There are four major volume sources that the venturi must evacuate. These are the manifold chamber, the tubing connecting the manifold and the venturi, and the volume of the tread perforations. Equation A.3.1 gives the combined volume calculations based on estimated tube lengths and tread thickness. Table 83 gives an explanation of the different variables found in Equation A.3.1.

$$
\left(l_{\text {cham }} * w_{\text {cham }} * d_{\text {cham }}\right)+\left(l_{\text {tube }} *\left(\pi * d_{\text {itube }}^{2}\right)\right)+\left(\frac{A_{\text {Vac }}}{8} * t_{\text {tread }}\right)=V_{\text {total }}
$$

Table 83: Variable symbols, units and descriptions for the evacuation rate calculation.

\begin{tabular}{|r|r|r|}
\hline Symbol & Value & Description \\
\hline$l_{\text {cham }}$ & $1 \mathrm{in}$ & Length of a single manifold chamber \\
\hline$w_{\text {cham }}$ & $0.75 \mathrm{in}$ & Width of a single manifold chamber \\
\hline$d_{\text {cham }}$ & $.1875 \mathrm{in}$ & Depth of a single manifold chamber \\
\hline$l_{\text {tube }}$ & $12 \mathrm{in}$ & Estimated length of tube between manifold and venturi \\
\hline$d_{\text {itube }}$ & $.2 \mathrm{in}$ & Inner diameter of tubing \\
\hline$A_{\text {Vac }}$ & $2.4 \mathrm{in}^{\wedge} 2$ & Total wall area exposed to vacuum \\
\hline$t_{\text {tread }}$ & $.2 \mathrm{in}$ & Estimated tread thickness \\
\hline$V_{\text {total }}$ & $9.4 \times 10^{-4} \mathrm{ft}^{\wedge} 3$ & Total area evacuated by a single venturi \\
\hline
\end{tabular}

\section{A.3.3 Evacuation Time}

Using the results from Equation A.3.1, the time to evacuate the estimated volume can found using the time to evacuate a cubic foot given by Vaccon. Equation A.3.2 is simply the rate given by Vaccon, Rate $_{\text {Evac }}$, multiplied by the estimated volume being evacuated, $V_{\text {total }}$. Table 84 gives evacuation times for each of the venturis considered for the project.

$$
V_{\text {total }} * \text { Rate }_{\text {Evac }}=t_{\text {evac }}
$$


Table 84: The evacuation rate and time to evacuate for each of the venturi models considered for the project.

\begin{tabular}{|l|r|r|}
\hline \multicolumn{1}{|c|}{ Venturi Model } & Evacuation Rate & \multicolumn{2}{c|}{ Time to Evacuation } \\
\hline JS-60M & $28.24 \mathrm{~s} / \mathrm{ft}^{\wedge} 3$ & $.027 \mathrm{~s}$ \\
\hline JS-90M & $10.05 \mathrm{~s} / \mathrm{ft}^{\wedge} 3$ & $.009 \mathrm{~s}$ \\
\hline JS-100M & $8.99 \mathrm{~s} / \mathrm{ft}^{\wedge} 3$ & $.008 \mathrm{~s}$ \\
\hline JS-150M & $4.46 \mathrm{~s} / \mathrm{ft}^{\wedge} 3$ & $.004 \mathrm{~s}$ \\
\hline
\end{tabular}




\section{A.4 Minimum Belt Tension Requirement Calculation}

\section{A.4.1 Calculation Description}

This calculation is used to determine the minimum tension which must be developed in treads for the vehicle to function properly. This was done by using a timing belt design guide produced by SDP/SI [25].

\section{A.4.2 Equation}

Equation A.4.1 was found in the SDP/SI design guide [25]. It solves for the minimum static belt tension per span required for maximum belt life and smooth belt operation. Table 85 contains a definition for each of the variables in Equation A.4.1.

$$
T_{s t}=\frac{.821 * D Q}{d}+m *\left(\frac{d * D R}{3820}\right)^{2}
$$

Table 85: Variable symbols, units and descriptions for the minimum belt tension requirement calculation.

\begin{tabular}{|c|r|l|}
\hline Symbol & Units & \multicolumn{1}{|c|}{ Description } \\
\hline$T_{s t}$ & lbf & Static Tension per Span \\
\hline$D Q$ & in-lbf & Driver Design Torque \\
\hline$d$ & in. & Driver Pitch Diameter \\
\hline$D R$ & RPM & Driver Angular Velocity \\
\hline $\mathrm{m}$ & Unit Less & Belt Mass Factor \\
\hline
\end{tabular}

\section{A.4.3 Calculating Mass Factor}

SDP/SI's design guide does not contain the mass factor for the H-type belt. To estimate it, two families of belt which SDP/SI did provide data for were analyzed. The belts within these families shared basic tooth geometries with each other. The two families were the GT2 type belts which had rounded teeth and the XL family which has trapezoidal teeth. The H-Type belts fall within the XL family. The following method was used to estimate the mass factor of the tread.

First, the linearity of the mass factor vs belt width was established by plotting the given mass factor vs belt width data for several different types of belts. Table 86 contains the data provided by SDP/SI for two different families of belts. The data for each family was plotted and shown to be linear as seen in Figure 129 and Figure 130. 
Table 86: Belt family data from SDP/SI.

\begin{tabular}{|r|r|r|r|}
\hline Belt Type & Belt Width & m & Pitch Length \\
\hline \multirow{4}{*}{$2 \mathrm{~mm}$ GT2 } & $4 \mathrm{~mm}$ & 0.026 & $2 \mathrm{~mm}$ \\
\cline { 2 - 4 } & $6 \mathrm{~mm}$ & 0.039 & $2 \mathrm{~mm}$ \\
\cline { 2 - 4 } & $9 \mathrm{~mm}$ & 0.058 & $2 \mathrm{~mm}$ \\
\cline { 2 - 4 } & $12 \mathrm{~mm}$ & 0.077 & $2 \mathrm{~mm}$ \\
\hline \multirow{4}{*}{$3 m m$ GT2 } & $6 \mathrm{~mm}$ & 0.077 & $3 \mathrm{~mm}$ \\
\cline { 2 - 4 } & $9 \mathrm{~mm}$ & 0.120 & $3 \mathrm{~mm}$ \\
\cline { 2 - 4 } & $12 \mathrm{~mm}$ & 0.150 & $3 \mathrm{~mm}$ \\
\cline { 2 - 4 } & $15 \mathrm{~mm}$ & 0.190 & $3 \mathrm{~mm}$ \\
\cline { 2 - 4 } & $9 \mathrm{~mm}$ & 0.170 & $5 \mathrm{~mm}$ \\
\hline \multirow{3}{*}{$5 \mathrm{~mm}$ GT2 } & $15 \mathrm{~mm}$ & 0.280 & $5 \mathrm{~mm}$ \\
\cline { 2 - 4 } & $20 \mathrm{~mm}$ & 0.380 & $5 \mathrm{~mm}$ \\
\cline { 2 - 4 } & $25 \mathrm{~mm}$ & 0.470 & $5 \mathrm{~mm}$ \\
\hline \multirow{3}{*}{ MXL } & $.125 \mathrm{in}$. & 0.003 & $0.08 \mathrm{in}$. \\
\cline { 2 - 4 } & $.1875 \mathrm{in}$. & 0.004 & $0.08 \mathrm{in}$. \\
\cline { 2 - 4 } & $.25 \mathrm{in}$. & 0.005 & $0.08 \mathrm{in}$. \\
\hline \multirow{3}{*}{ XL } & $.25 \mathrm{in}$ & 0.010 & $0.2 \mathrm{in}$ \\
\cline { 2 - 4 } & $.375 \mathrm{in}$. & 0.015 & $0.2 \mathrm{in}$ \\
\hline
\end{tabular}

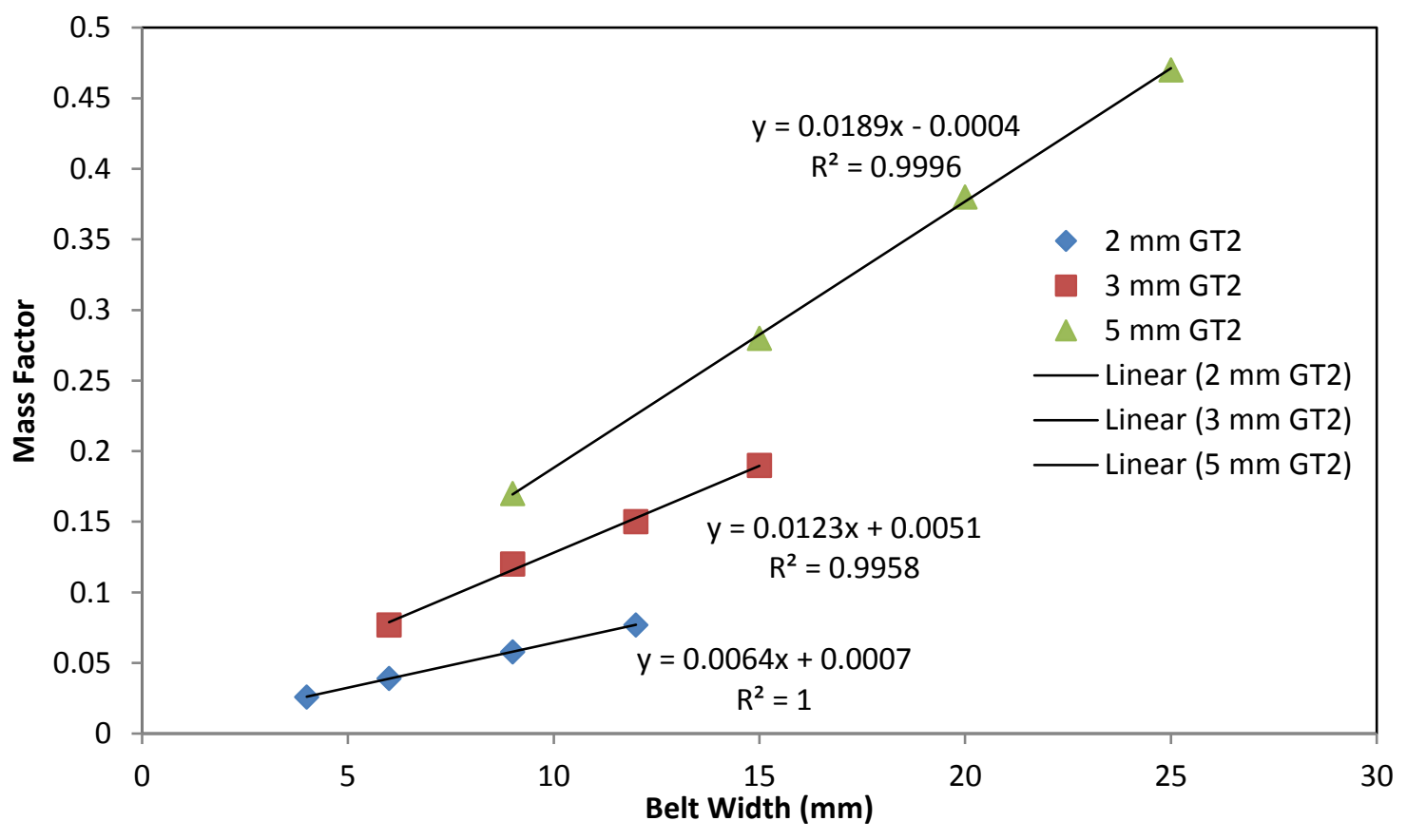

Figure 129: Mass factor versus belt width for the GT2 belt family. 


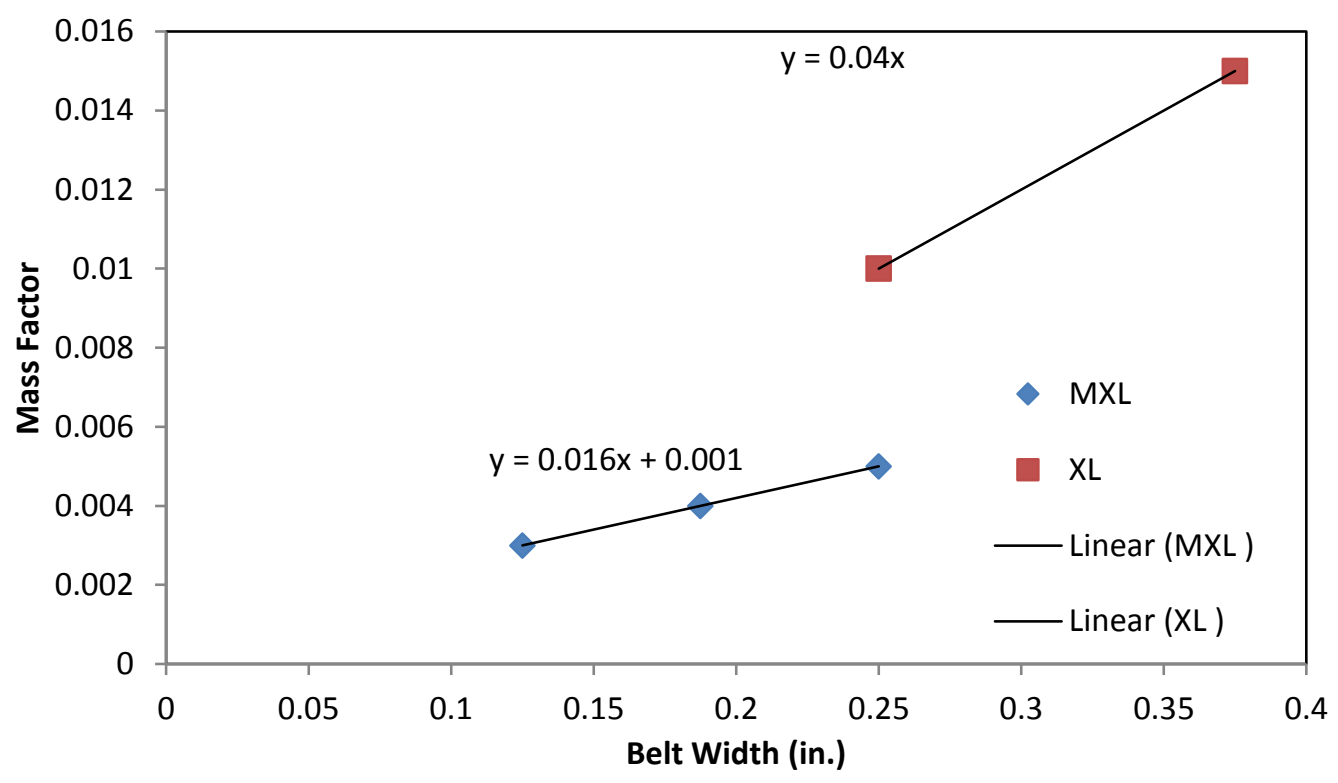

Figure 130: Mass Factor versus belt width for the trapezoidal tooth family.

The next step was to determine if there was a linear relationship between the pitch of the belts in each family and the ratio of mass factor vs belt width. To do this, the slopes from the GT2 family were plotted against their respective pitch lengths and this plot can be seen in Figure 131. The relationship was not perfectly linear, but was close enough that an estimate of the mass factor the tread could be found.

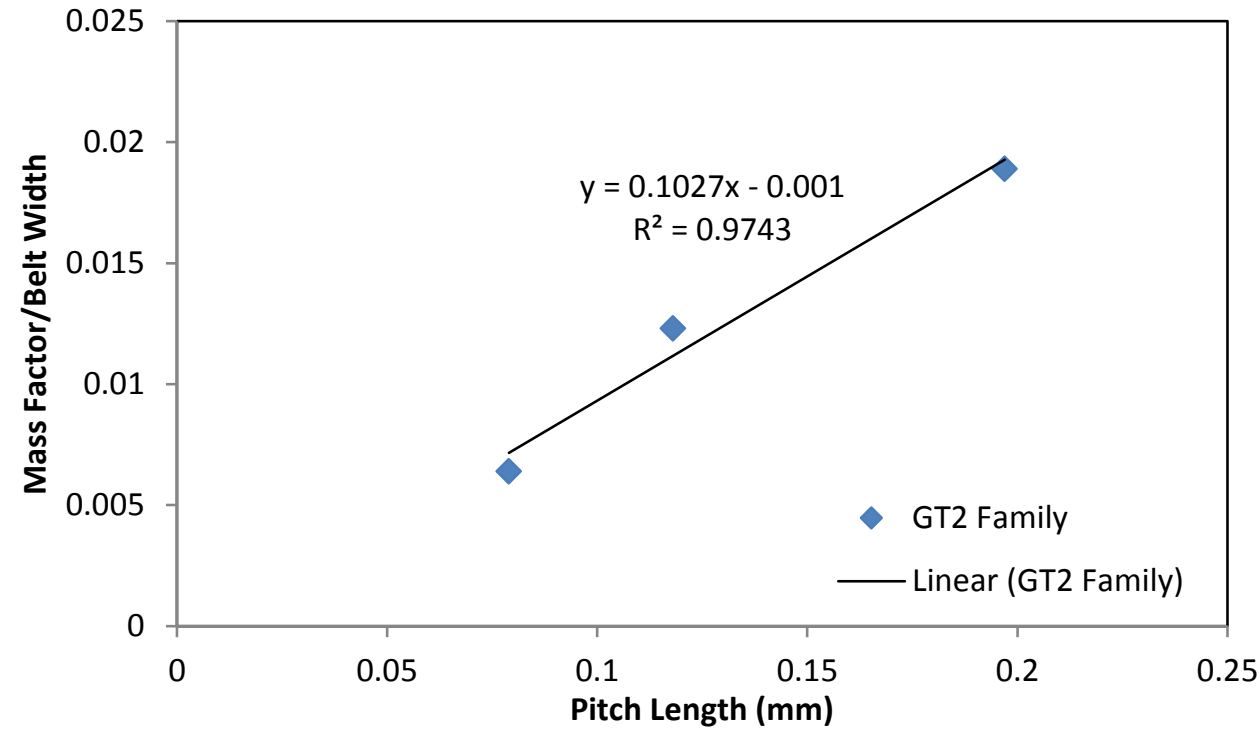

Figure 131: Mass factor per belt width versus pitch length for the GT2 belt family.

By plotting the trapezoidal family relationship of pitch length to the ratio of mass factor to belt width an equation can be found which will solve for the ratio of mass factor to belt width for the H-type belt. This 
ratio can then be multiplied by the width of the belt to get the mass factor estimate for the tread. Figure 132 shows the plot and equation generated from relationship of pitch length to the ratio of mass factor to belt width. Table 87 gives the mass factor to belt width ratio for the H-Type belt, as well as the mass factor for the tread.

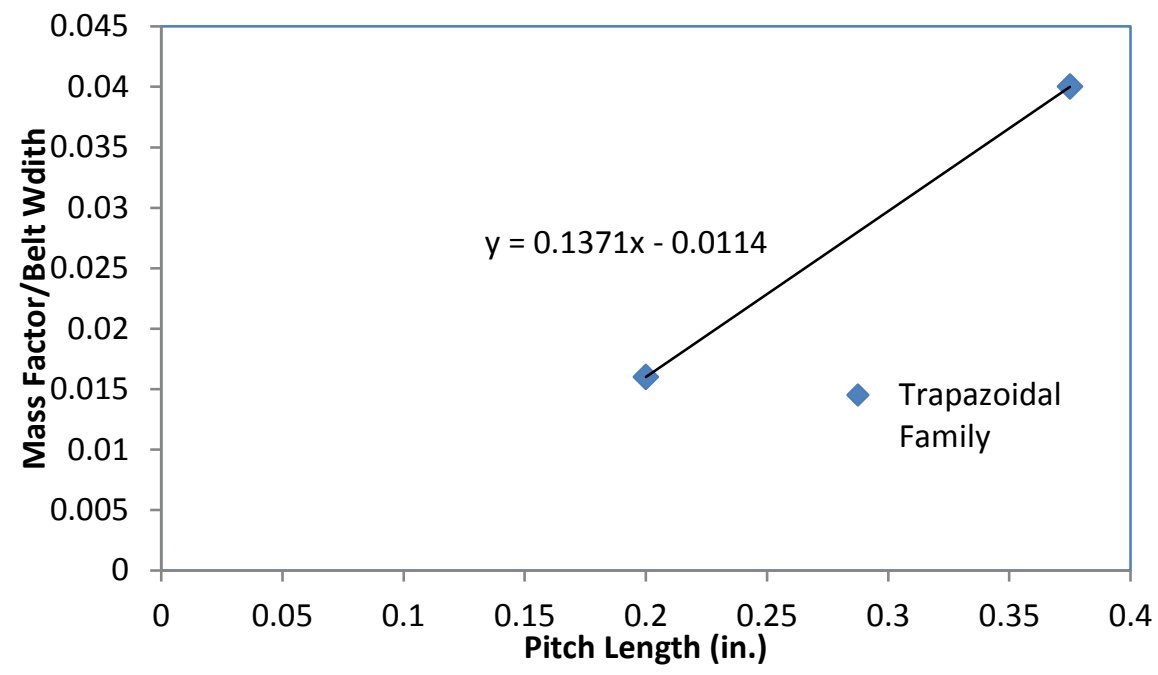

Figure 132: Mass factor per belt width versus pitch length for the trapezoidal tooth belt family.

Table 87: The estimated mass factor per belt width and estimated mass factor for the H-Type belt used for the tread.

\begin{tabular}{|l|r|}
\hline \multicolumn{1}{|c|}{ Parameter } & Value \\
\hline Mass Factor to Belt Width Ratio & 0.057 \\
\hline Estimated Mass Factor for H-Type Belt & 0.12 \\
\hline
\end{tabular}

\section{Solving for Minimum Tread Static Tension}

With an estimate for the mass factor, the minimum belt tension for single span can be solved. Table 88 contains the values input into Equation A.4.1 and the resulting tension per span and the total tension for all three spans.

Table 88: The input variables used to estimate the minimum belt tension required and the results of the calculation.

\begin{tabular}{|c|c|}
\hline Input Variable & Value \\
\hline$D Q$ & 50 in-lbf \\
\hline$d$ & $2.66 \mathrm{in.}$ \\
\hline$D R$ & $25 \mathrm{RPM}$ \\
\hline $\mathrm{m}$ & 0.12 \\
\hline Output Variable & Value \\
\hline$T_{s t}$ & $17.43 \mathrm{lbf}$ \\
\hline $3 * T_{s t}$ & $46.3 \mathrm{lbf}$ \\
\hline
\end{tabular}




\section{A.5 Tread Factor of Safety Estimation}

\section{A.5.1 Calculation Description}

This calculation was used to estimate the maximum tension force that the tread could be subjected to before failure. The results were then compared to the desired belt tension and a factor of safety was found.

\section{A.5.2 Solution}

Table 89 contains the variables used to calculate the maximum allowable tread tension. It was assumed that the steel cables imbedded in the tread would carry all of the tension loading and that tread failure occurred only when the cables failed

Table 89: Variables and their values used to calculate the maximum allowable tread tension.

\begin{tabular}{|r|r|r|}
\hline Symbol & Value & Description \\
\hline$Y_{S}$ & $36,000 \mathrm{psi}$ & Yield Strength of Steel \\
\hline$D_{C}$ & $0.0625 \mathrm{In}$. & Cable Diameter \\
\hline$N_{C}$ & 8 & Number of cables in the tread \\
\hline
\end{tabular}

Equation A.5.1 solves for the maximum tread tension allowable.

$$
T_{\max }=Y_{S} * N_{C} * \frac{\pi}{4} * D_{C}
$$

Table 90 gives the maximum tread tension, the desired tread tension and the factor of safety of the tread.

Table 90: Results of the tread factor of safety calculation.

\begin{tabular}{r|r|r|} 
Max Allowable Tension & Desired Tension & Factor of Safety \\
\hline
\end{tabular}




\section{A.6 Total Tread Perforation Area Calculation}

\section{A.6.1 Calculation Description}

This calculation was used to determine the total area of tread perforation required to generate the force required to hold the vehicle on the wall given a maximum vacuum pressure obtainable from the venturis selected. The force value used in this calculation was found in vacuum force requirements calculation.

\section{A.6.2 Solution}

Equation A.6.1 is simply the relationship between pressure and force solved for the area. Equation A.6.2 gives the numerical solution to the problem.

$$
\begin{gathered}
\frac{F_{V A C}}{P_{V e n}}=A_{P e r f} \\
\frac{33 l b f}{13.75 \frac{l b f}{m^{2}}}=2.4 \mathrm{in}^{2}
\end{gathered}
$$

\section{A.7 Vacuum Force Requirements}

\section{A.7.1 Calculation Description}

This calculation was used to determine the force which would be required to hold the vehicle to a vertical wall and the ceiling. This was done using the maximum desired system mass plus the desired payload mass and by estimating the friction coefficient between the wall and the tread.

\section{A.7.2 Equations and Variables}

Equation A.7.1 gives the minimum vacuum force which prevents slipping with a desired factor of safety. Equation A.7.2 gives the minimum vacuum force which will hold the vehicle to the ceiling with a desired factor of safety.

Table 91 contains a list of the variables used in Equation A.7.1 and Equation A.7.2.

$$
\begin{gathered}
\frac{w_{V} F S_{\text {wall }}}{\mu_{T-W}}=F_{\text {wall }} \\
F S_{\text {ceil }} w_{V}=F_{\text {ceil }}
\end{gathered}
$$


Table 91: Variable symbols, units and descriptions for the vacuum force requirements calculation.

\begin{tabular}{|c|r|r|}
\hline Symbol & \multicolumn{1}{|c|}{ Units } & \multicolumn{1}{c|}{ Description } \\
\hline$w_{V}$ & lbf & Weight of the vehicle plus payload \\
\hline$\mu_{T-W}$ & Unit less & Estimated coefficient of friction between the tread and the wall. \\
\hline$F S_{\text {wall }}$ & Unit less & Wall Factor of Safety \\
\hline$F S_{\text {ceil }}$ & Unit less & Ceiling Factor of Safety \\
\hline$F_{\text {wall }}$ & lbf & Required vacuum force on wall \\
\hline$F_{\text {ceil }}$ & lbf & Required vacuum force on ceiling \\
\hline
\end{tabular}

\section{A.7.3 Solution}

The input variables and the results of the vacuum force requirements can be found in Table 92 .

Table 92: Input values for the vacuum force requirements calculation and the results.

\begin{tabular}{|c|rr|}
\hline Input Variable & Value & \\
\hline$w_{V}$ & & $16.5 \mathrm{lbf}$ \\
\hline$\mu_{T-W}[24]$ & & 2 \\
\hline$F S_{\text {wall }}$ & Value \\
\hline$F S_{\text {ceil }}$ & & $33 \mathrm{lbf}$ \\
\hline Output Variable & $33 \mathrm{bf}$ \\
\hline$F_{\text {wall }}$ & & 2 \\
\hline$F_{\text {ceil }}$ & & \\
\hline
\end{tabular}




\section{A.8 Final Tread Hole Pattern and Manifold Slot Length Calculation}

\section{A.8.1 Calculation Description}

This calculation was used to determine if the tread hole pattern and manifold slot length chosen would provide sufficient wall area exposed to vacuum as the manifold moved along the tread. This was done by determining the number of tread holes which were exposed to vacuum for any given manifold position along the tread. This calculation only determines the wall area exposed for a single tread and the results of the calculation can simply be multiplied by two to obtain the total are for both treads.

\section{A.8.2 Process}

The process used to determine the number of tread holes exposed to each vacuum section of the manifold was relatively straight forward. An assembly was created in Solidworks which contained a length of tread and the lower half of the manifold. The manifold was positioned at the edge of the tread, as seen in Figure 133, using a distance mate. The number of holes exposed to each vacuum section of the manifold was then counted by looking at the bottom of the tread. The manifold was then incremented along the tread fifty thousandths of an inch and the process was repeated for a total displacement of one and a half inches. Figure 134 shows the tread holes which were exposed in the zero position and the Figure 135 shows the tread holes exposed after the manifold had been moved a tenth of an inch along the tread.

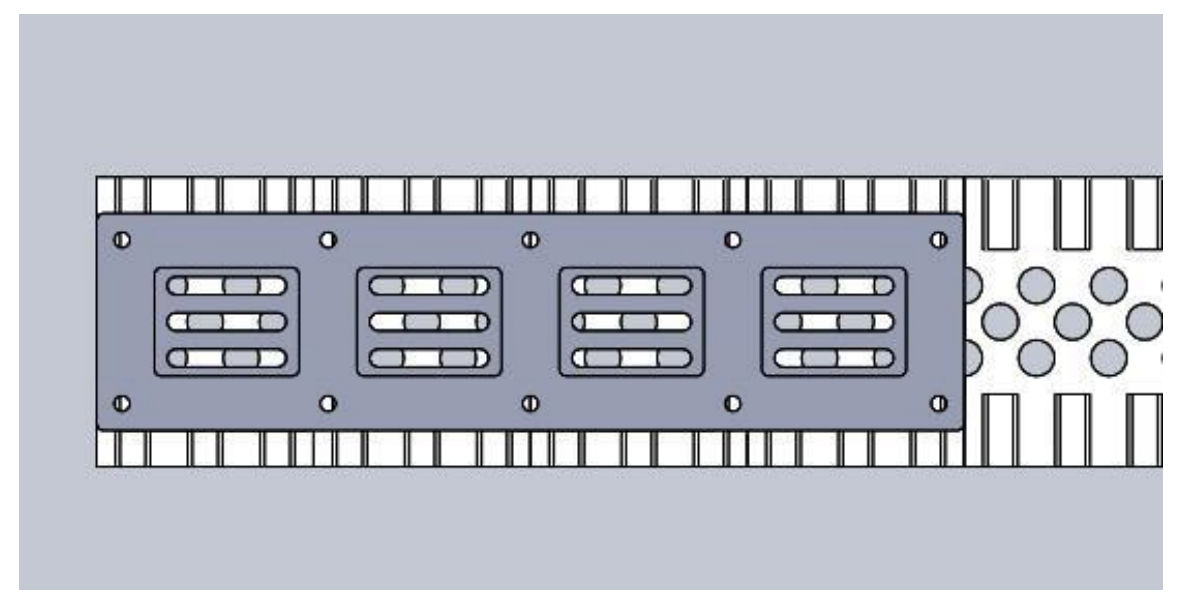

Figure 133: A top view of the model used to determine the tread hole engagement. 


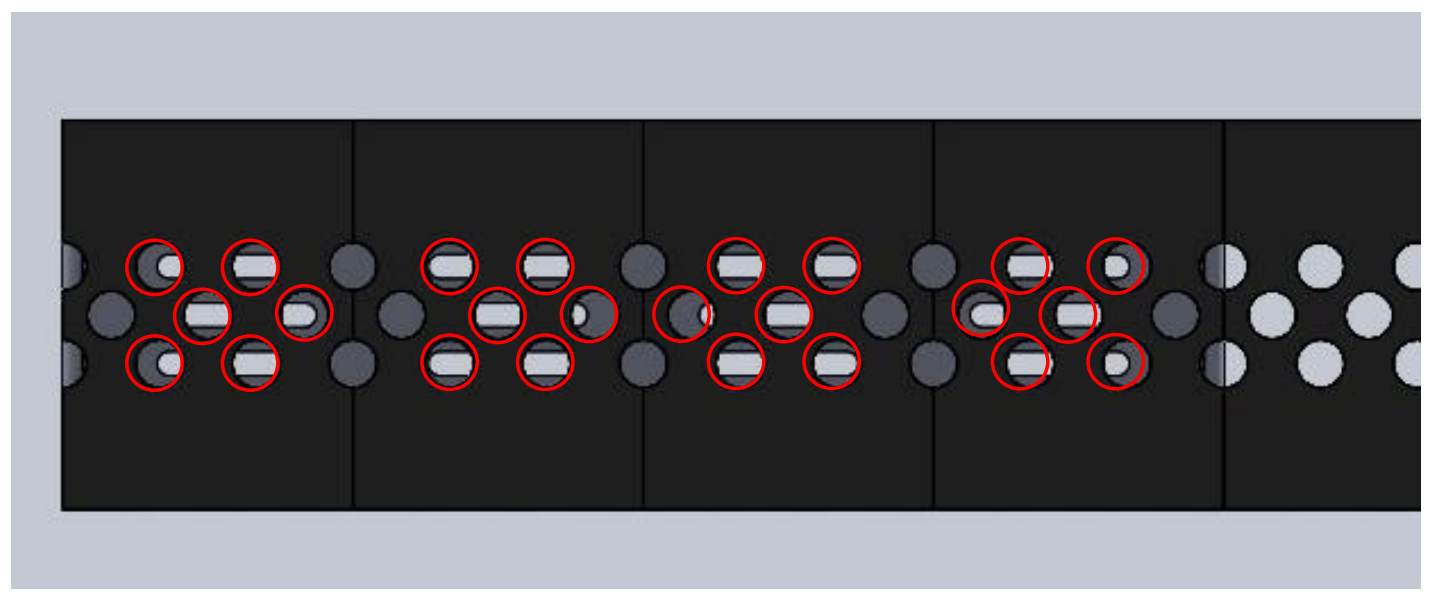

Figure 134: Tread holes engaged with the manifold sections with the manifold at zero displacement.

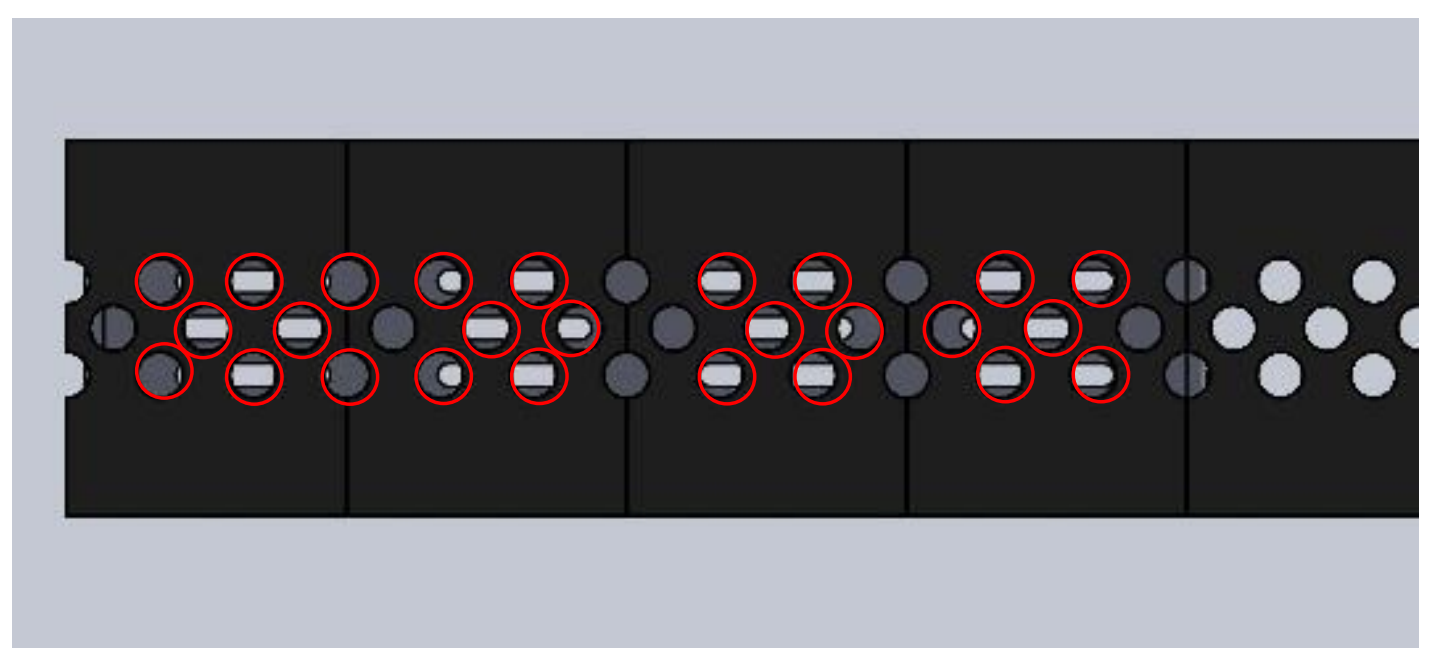

Figure 135: Tread holes engaged with the manifold sections with the manifold at a tenth of an inch of displacement.

\section{A.8.3 Results}

Two different conditions were placed on what would be considered engagement of a tread hole. These were the any engagement condition and the major engagement condition. The any engagement condition considered any tread hole exposed to be fully engaged. Table 93 contains the tabulated results of the any engagement condition interpretation of the data collected. The major engagement condition would only consider a tread hole engaged if the hole was exposed over approximately a quarter of its diameter. Figure 136 shows the hole exposure which was and was not considered in the major engagement condition. Table 94 contains the tabulated results of the major engagement condition interpretation of the data collected. 


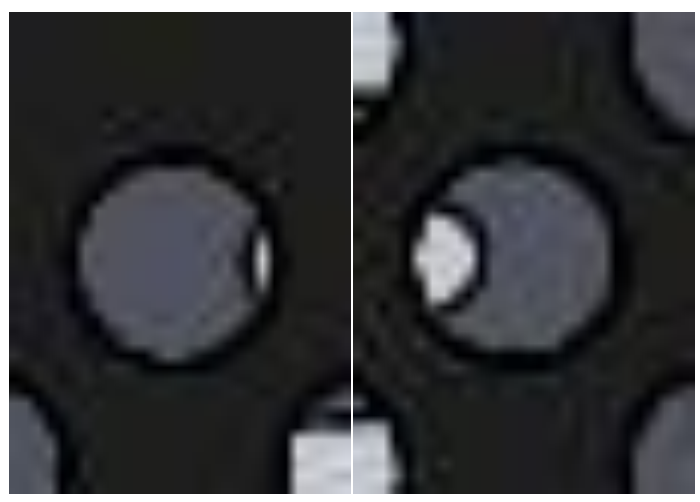

Figure 136: (Left) A hole which would be disregarded in the major engagement condition. (Right) A hole which would be considered engaged for the major engagement condition.

Table 93: Data for the any engagement condition.

\begin{tabular}{|c|c|c|c|c|c|}
\hline Displacement (in.) & First Section & $\begin{array}{l}\text { Second } \\
\text { Section } \\
\end{array}$ & $\begin{array}{r}\text { Third } \\
\text { Section } \\
\end{array}$ & $\begin{array}{l}\text { Fourth } \\
\text { Section } \\
\end{array}$ & $\begin{array}{r}\text { Total } \\
\text { Engaged }\end{array}$ \\
\hline 0 & 6 & 6 & 6 & 6 & 24 \\
\hline 0.05 & 6 & 6 & 7 & 6 & 25 \\
\hline 0.1 & 8 & 6 & 6 & 6 & 26 \\
\hline 0.15 & 6 & 6 & 6 & 7 & 25 \\
\hline 0.2 & 6 & 8 & 6 & 6 & 26 \\
\hline 0.25 & 6 & 6 & 6 & 6 & 24 \\
\hline 0.3 & 6 & 6 & 8 & 6 & 26 \\
\hline 0.35 & 7 & 6 & 6 & 6 & 25 \\
\hline 0.4 & 6 & 6 & 6 & 8 & 26 \\
\hline 0.45 & 6 & 7 & 6 & 6 & 25 \\
\hline 0.5 & 6 & 6 & 6 & 6 & 24 \\
\hline 0.55 & 6 & 6 & 7 & 6 & 25 \\
\hline 0.6 & 8 & 6 & 6 & 6 & 26 \\
\hline 0.65 & 6 & 6 & 6 & 7 & 25 \\
\hline 0.7 & 6 & 8 & 6 & 6 & 26 \\
\hline 0.75 & 6 & 6 & 6 & 6 & 24 \\
\hline 0.8 & 6 & 6 & 8 & 6 & 26 \\
\hline 0.85 & 7 & 6 & 6 & 6 & 25 \\
\hline 0.9 & 6 & 6 & 6 & 8 & 26 \\
\hline 0.95 & 6 & 7 & 6 & 6 & 25 \\
\hline 1 & 6 & 6 & 6 & 6 & 24 \\
\hline 1.05 & 6 & 6 & 7 & 6 & 25 \\
\hline 1.1 & 8 & 6 & 6 & 6 & 26 \\
\hline 1.15 & 6 & 6 & 6 & 7 & 25 \\
\hline 1.2 & 6 & 8 & 6 & 6 & 26 \\
\hline 1.25 & 6 & 6 & 6 & 6 & 24 \\
\hline 1.3 & 6 & 6 & 8 & 6 & 26 \\
\hline 1.35 & 7 & 6 & 6 & 6 & 25 \\
\hline 1.4 & 6 & 6 & 6 & 8 & 26 \\
\hline 1.45 & 6 & 7 & 6 & 6 & 25 \\
\hline 1.5 & 6 & 6 & 6 & 6 & 24 \\
\hline
\end{tabular}


Table 94: Data for the major engagement condition.

\begin{tabular}{|c|c|c|c|c|c|}
\hline Displacement (in.) & First Section & $\begin{array}{l}\text { Second } \\
\text { Section }\end{array}$ & Third Section & $\begin{array}{l}\text { Fourth } \\
\text { Section }\end{array}$ & $\begin{array}{c}\text { Total } \\
\text { Engaged } \\
\end{array}$ \\
\hline 0 & 6 & 6 & 6 & 6 & 24 \\
\hline 0.05 & 6 & 6 & 4 & 6 & 22 \\
\hline 0.1 & 4 & 6 & 6 & 6 & 22 \\
\hline 0.15 & 6 & 6 & 6 & 4 & 22 \\
\hline 0.2 & 6 & 4 & 6 & 6 & 22 \\
\hline 0.25 & 6 & 6 & 6 & 6 & 24 \\
\hline 0.3 & 6 & 6 & 4 & 6 & 22 \\
\hline 0.35 & 4 & 6 & 6 & 6 & 22 \\
\hline 0.4 & 6 & 6 & 6 & 4 & 22 \\
\hline 0.45 & 6 & 4 & 6 & 6 & 22 \\
\hline 0.5 & 6 & 6 & 6 & 6 & 24 \\
\hline 0.55 & 6 & 6 & 4 & 6 & 22 \\
\hline 0.6 & 4 & 6 & 6 & 6 & 22 \\
\hline 0.65 & 6 & 6 & 6 & 4 & 22 \\
\hline 0.7 & 6 & 4 & 6 & 6 & 22 \\
\hline 0.75 & 6 & 6 & 6 & 6 & 24 \\
\hline 0.8 & 6 & 6 & 4 & 6 & 22 \\
\hline 0.85 & 4 & 6 & 6 & 6 & 22 \\
\hline 0.9 & 6 & 6 & 6 & 4 & 22 \\
\hline 0.95 & 6 & 4 & 6 & 6 & 22 \\
\hline 1 & 6 & 6 & 6 & 6 & 24 \\
\hline 1.05 & 6 & 6 & 4 & 6 & 22 \\
\hline 1.1 & 4 & 6 & 6 & 6 & 22 \\
\hline 1.15 & 6 & 6 & 6 & 4 & 22 \\
\hline 1.2 & 6 & 4 & 6 & 6 & 22 \\
\hline 1.25 & 6 & 6 & 6 & 6 & 24 \\
\hline 1.3 & 6 & 6 & 4 & 6 & 22 \\
\hline 1.35 & 4 & 6 & 6 & 6 & 22 \\
\hline 1.4 & 6 & 6 & 6 & 4 & 22 \\
\hline 1.45 & 6 & 4 & 6 & 6 & 22 \\
\hline 1.5 & 6 & 6 & 6 & 6 & 24 \\
\hline
\end{tabular}

The tabulated data between the two cases were plotted get a visualization of the data. Figure 137shows the plot for the any engagement condition and Figure 138 shows the plot for the major engagement condition. 


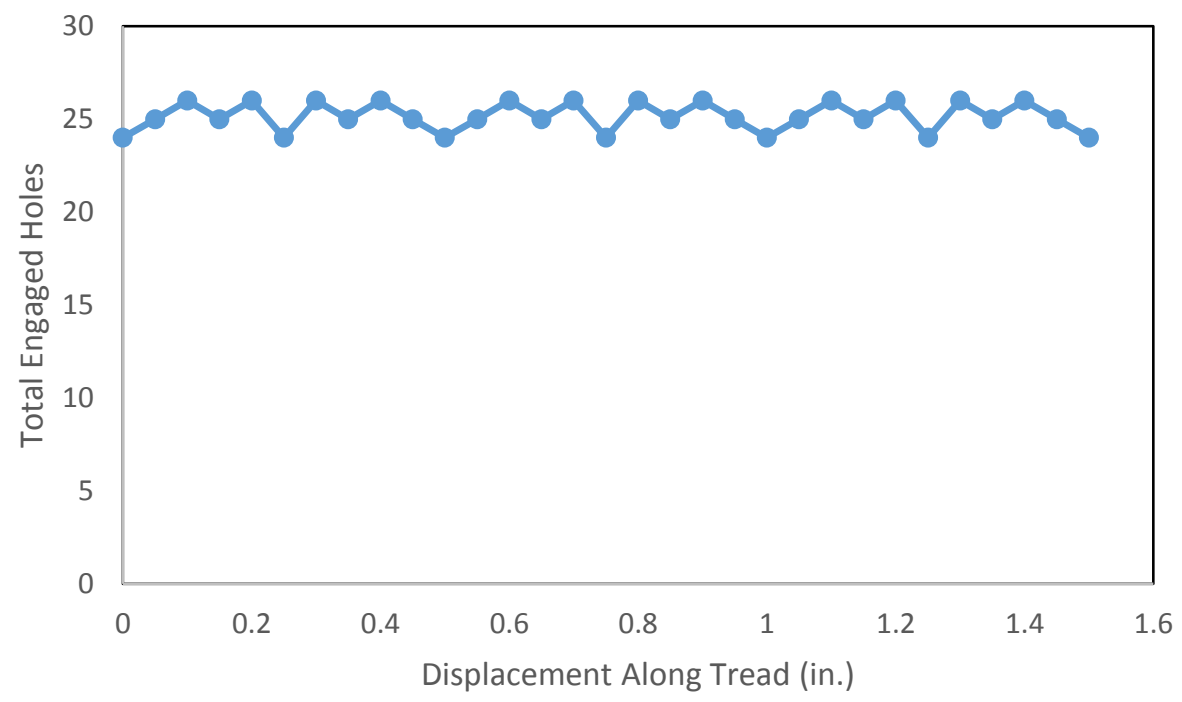

Figure 137: A plot of the total engaged holes versus the manifold displacement for the any engagement condition.

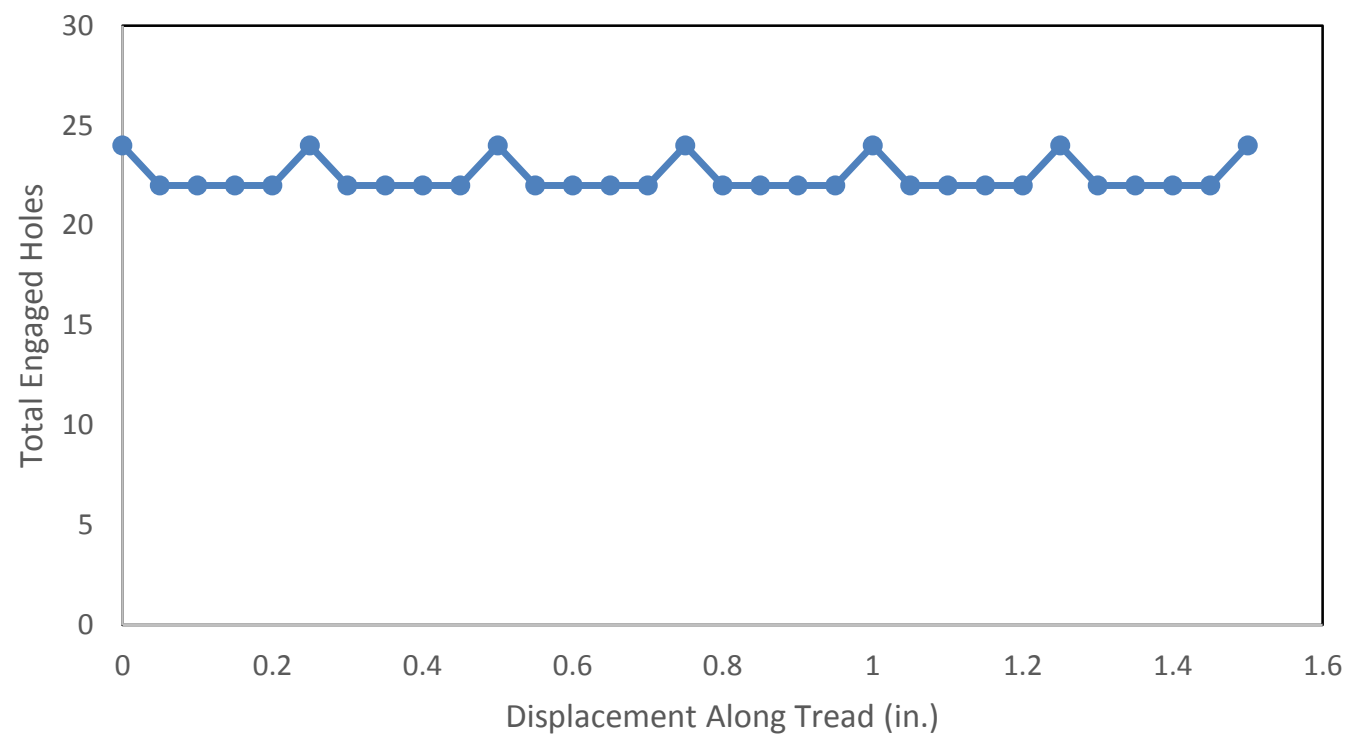

Figure 138: A plot of the total engaged holes versus the manifold displacement for the major engagement condition.

To determine the average vacuum force between the wall and the tread, the average number of holes engaged for each condition was determined. The area of a single tread hole was calculated and then multiplied by the average number of engaged holes. This gave the total area engaged. This value was then multiplied by the nominal vacuum pressure produced by the venturis to yield the average force between the wall and the tread for the given hole pattern. Table 95 contains the results of the any engagement condition 
and Table 96 gives the results for the major engagement condition. Because the major engagement condition is more conservative, the force value yielded was used in the design of the vehicle.

Table 95: The results of the any engagement condition calculation.

\begin{tabular}{|l|r|}
\hline \multicolumn{1}{|c|}{ Parameter } & Value \\
\hline Area of a Single Hole & $.05 \mathrm{in}^{\wedge} 2$ \\
\hline Average Number of Engaged Holes & 25.16 \\
\hline Average Area Engaged & $1.25 \mathrm{in}^{\wedge} 2$ \\
\hline Maximum Number of Engaged Holes & 26 \\
\hline Minimum Number of Engaged Holes & 24 \\
\hline Venturi Vacuum Pressure & $13.75 \mathrm{psi}$ \\
\hline Average Vacuum Force & $16.98 \mathrm{lbs}$ \\
\hline Maximum Force Value & $17.9 \mathrm{lbs}$ \\
\hline Minimum Force Value & $16.5 \mathrm{lbs}$ \\
\hline
\end{tabular}

Table 96: The results of the major engagement condition calculation.

\begin{tabular}{|l|r|}
\hline \multicolumn{1}{|c|}{ Parameter } & Value \\
\hline Area of a Single Hole & .05 in`2 $^{\wedge}$ \\
\hline Average Number of Engaged Holes & 22.45 \\
\hline Average Area Engaged & $1.12 \mathrm{in}^{\wedge} 2$ \\
\hline Maximum Number of Engaged Holes & 24 \\
\hline Minimum Number of Engaged Holes & 22 \\
\hline Venturi Vacuum Pressure & $13.75 \mathrm{psi}$ \\
\hline Average Vacuum Force & $15.15 \mathrm{lbs}$ \\
\hline Maximum Force Value & $16.5 \mathrm{lbs}$ \\
\hline Minimum Force Value & $15.125 \mathrm{lbs}$ \\
\hline
\end{tabular}




\section{A.9 Skid Steering Calculator}

\section{A.9.1 Calculation Description}

A symbolic analysis of the vehicle during skid steering was conducted and the resulting calculations were used to create a calculator. This calculator was then used in several later calculations such as the bearing loading calculation.

\section{A.9.2 Assumptions}

- The treads slip relative to the wall.

- The tread friction force which resists gravity acts at the geometric center of the vehicle.

- The distributed loading from the tread slipping can be split into three force.

- Assume that the drive force which causes rotation is equal to the dynamic friction between the wall and the tread

\section{A.9.3 Free Body Diagrams}

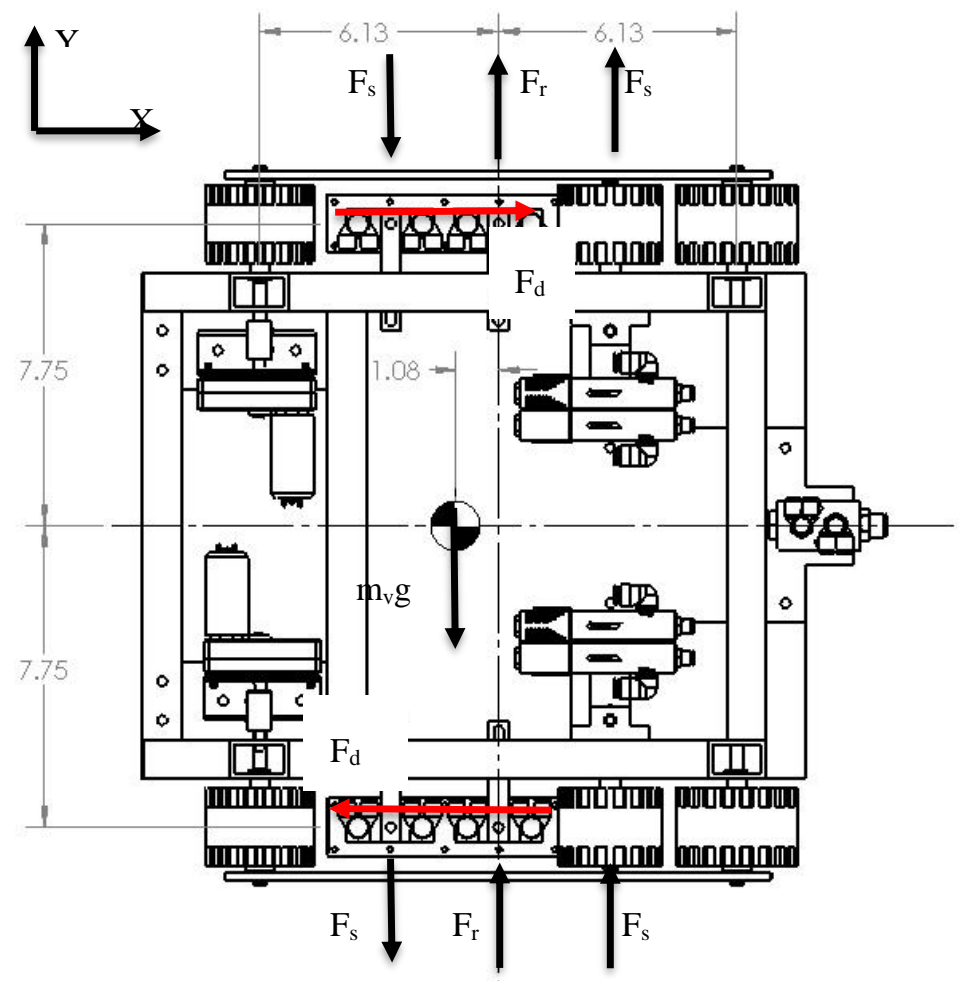

Figure 139: Vehicle Free Body Diagram. 


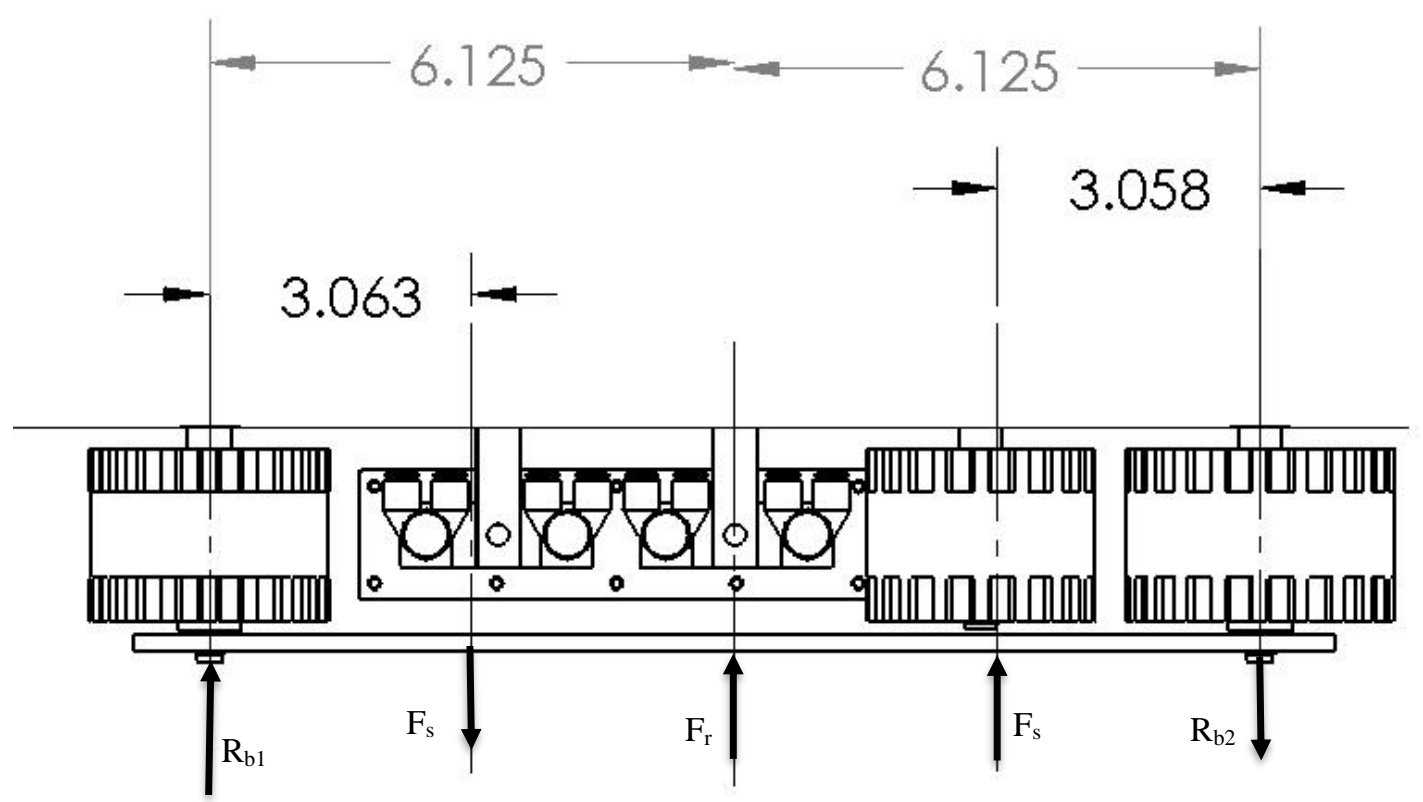

Figure 140: Wheel System Free Body Diagram.

\section{A.9.4 Variables}

Table 97: Variable symbols, units and description for the skid steering calculation.

\begin{tabular}{|c|c|c|}
\hline Symbol & Units & Description \\
\hline$m_{V}$ & SLUG & Mass of the Vehicle \\
\hline$F_{d}$ & LBF & The Tread Drive Force \\
\hline$F_{S}$ & LBF & Tread Resistance to Turning \\
\hline$F_{R}$ & FT/S^2 & The Tread resistance to vehicle weight \\
\hline$R_{b 1}-R_{b 2}$ & LBF & Bearing loads \\
\hline$F_{V A C}$ & LBF & Vacuum Force on Each Tread \\
\hline$\mu_{k}$ & N/A & Kinetic coefficient of friction between tread and wall \\
\hline
\end{tabular}

\section{A.9.5 Symbolic Manipulation}

Begin by setting the conditions for the friction forces as seen in Equation A.9.1 and Equation A.9.2.

$$
\begin{gathered}
\mu_{k} * F_{V A C}=F_{R}+F_{S} \\
\mu_{k} * F_{V A C}>F_{d}
\end{gathered}
$$

Sum moments about the geometric center of the vehicle, yielding Equation A.9.3.

$$
2 * F_{d} * 7.75 \mathrm{in} .=m_{V} g * 1.08 \mathrm{in} .+4 * F_{s} * 3.0625 \mathrm{in} .
$$

Next sum forces in the y-direction on the vehicle, yielding Equation A.9.4. This is then simplified to solve for $F_{R}$ which yields Equation A.9.5. 


$$
\begin{gathered}
2 * F_{S}+m_{V} g=2 * F_{R}+2 * F_{S} \\
F_{R}=0.5 * m_{V} g
\end{gathered}
$$

Sum moment about the center of the tread to find Equation A.9.6.

$$
R_{b 1} * 6.125 \text { in. }+R_{b 2} * 6.125 \text { in. }=2 * F_{s} * 3.0625 \text { in } .
$$

Sum forces in the y-direction on the wheel system to get Equation A.9.7. Simplify to get Equation A.9.8.

$$
\begin{gathered}
R_{b 1}+F_{s}+F_{R}=R_{b 1}+F_{s} \\
R_{b 2}=R_{b 1}+F_{R}
\end{gathered}
$$

\section{A.9.6 Solution}

The input variables and resulting outputs of the skid steering calculation can be found Table 98 .

Table 98: The input variables for the skid steering calculation and the results.

\begin{tabular}{|c|r|}
\hline Input Variables & \multicolumn{2}{|c|}{ Values } \\
\hline$F_{V A C}$ & $16 \mathrm{lbf}$ \\
\hline$\mu_{k}[24]$ & 0.8 \\
\hline$m_{V}$ & Values \\
\hline Output Variables & .5 Slugs \\
\hline$F_{d}$ & $4.9 \mathrm{lbf}$ \\
\hline$F_{S}$ & $4.75 \mathrm{lbf}$ \\
\hline$F_{R}$ & $8.05 \mathrm{lbf}$ \\
\hline$R_{b 1}$ & $-1.65 \mathrm{lbf}$ \\
\hline$R_{b 2}$ & $6.4 \mathrm{lbf}$ \\
\hline
\end{tabular}




\section{A.10 Allowable Friction Between The Tread and The Manifold}

\section{A.10.1 Calculation Description}

This calculation determines the maximum allowable friction force and coefficient of friction between the tread and the manifold. The criterion these values must satisfy is that the maximum friction force be twenty percent of the maximum motor torque.

\section{A.10.2 Solution}

The contribution that the friction contributes to the required motor torque is given by Equation A.10.1, which was determined in the symbolic motor torque calculation. Manipulating Equation A.10.1 yields Equation A.10.2, which solves for the maximum coefficient of friction between the tread and manifold. Table 99 gives a description of the variables found in Equation A.10.1 and Equation A.10.2.

$$
\begin{gathered}
R_{D W} * \mu_{T-M} * F_{V A C}=T_{\text {friction }} \\
\frac{T_{\text {friction }}}{R_{D W} * F_{V A C}}=\mu_{T-M}
\end{gathered}
$$

Table 99: The variable symbols, units and description for the allowable tread/manifold friction calculation.

\begin{tabular}{|c|r|l|}
\hline Symbol & \multicolumn{1}{|c|}{ Units } & \multicolumn{1}{|c|}{ Description } \\
\hline$R_{D W}$ & in & Drive wheel radius \\
\hline$\mu_{T-M}$ & Unit Less & Coefficient of Friction between the tread and the manifold \\
\hline$F_{V A C}$ & lbf & Vacuum Force between the manifold and the tread \\
\hline$T_{\text {friction }}$ & In-lbf & Torque contribution of the tread-manifold friction \\
\hline
\end{tabular}

Since the maximum torque of the motor is $50 \mathrm{in}-\mathrm{lbf}$, the allowable torque contribution from friction is 10 in-lb. inserting this value, the drive wheel radius and the vacuum force into Equation A.10.2 yields the maximum allowable coefficient of friction. Table 100 contains this maximum value and the resulting maximum allowable friction between the tread and the manifold.

Table 100: The results of the allowable tread/manifold friction calculation.

\begin{tabular}{|l|rr|}
\hline \multicolumn{1}{|c|}{ Parameter } & Value & 0.5 \\
\hline Max $\mu_{\mathrm{T}-\mathrm{M}}$ & & $10.5 \mathrm{lbf}$ \\
\hline Max $\mathrm{f}_{\mathrm{T}-\mathrm{M}}$ & & \\
\hline
\end{tabular}




\section{A.11 Frame Simulation}

\section{A.11.1 Simulation Description}

This simulation was used to validate the design of the vehicles frame. The simulation was performed in Solidworks simulation package. It describes the boundary conditions and loading conditions that were applied to the frame and how those conditions were determined. It reports the factor of safety, maximum Von Mises stress and the deflection of the bracket. The material for the entire frame was chosen to account for the degradation in strength of the frame due to welding.

\section{A.11.2 Boundary Conditions}

The boundary conditions of the frame were difficult to determine as the frame is not fixed. To simulate the inside holes of the drive and idler uprights were fixed as shown in Figure 141.

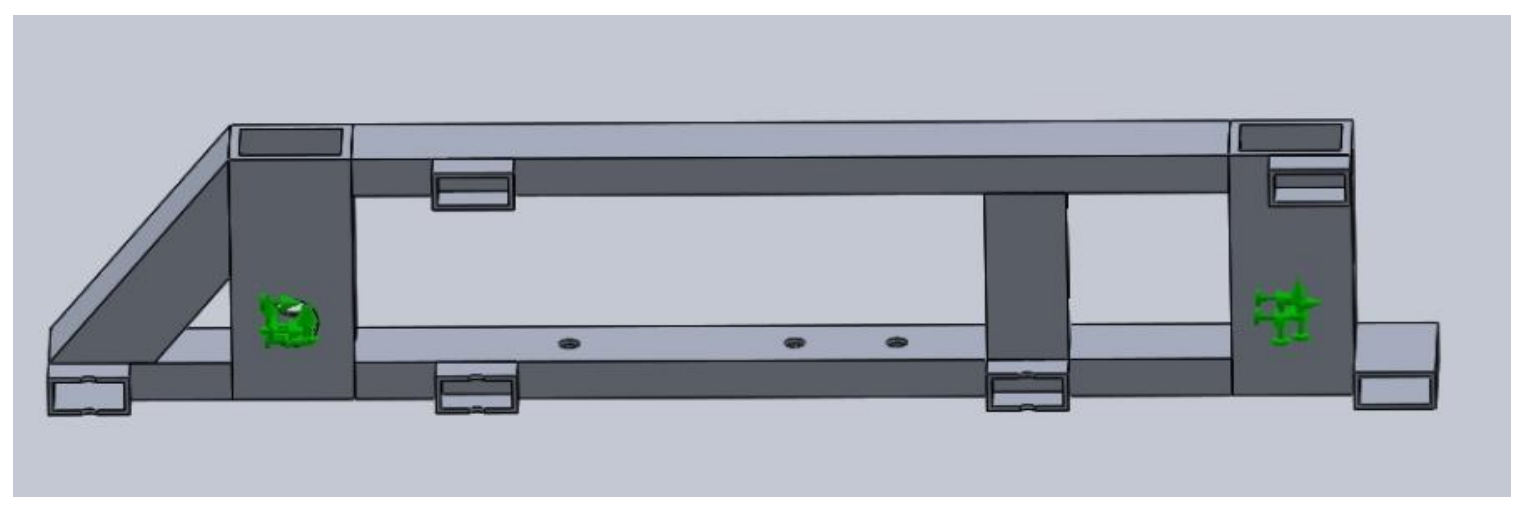

Figure 141: The fixed locations of the frame.

\section{A.11.3 Loading Conditions}

The loading conditions of the frame were assumed to be symmetrical about its midline. Three different sets of loads were applied to the vehicle. These were the bearing loading, the tensioner moment and the motor plate loading which can be seen in Figure 142, Figure 143 and Figure 144 respectively. Table 101 gives the values of the loading conditions applied to the frame. The loading was symmetrical about the center of the vehicle so only one side is shown.

Table 101: Loading conditions of the Frame.

\begin{tabular}{|l|r|r|}
\hline \multicolumn{1}{|c|}{ Load } & Magnitude & Direction \\
\hline Tread Tension - Idler & $55 \mathrm{lbf}$ & Negative X \\
\hline Bearing Load - Idler & $6.4 \mathrm{lbf}$ & Positive Z \\
\hline
\end{tabular}




\begin{tabular}{|l|r|r|}
\hline Tread Tension - Driver & $55 \mathrm{lbf}$ & Positive X \\
\hline Bearing Load - Driver & $6.4 \mathrm{lbf}$ & Negative Z \\
\hline Tensioner Moment & $90 \mathrm{lbf}$ & Positive About X Axis \\
\hline Forward Plate Force & $16 \mathrm{lbf}$ & Negative Y \\
\hline Rear Plate Force & $18 \mathrm{lbf}$ & Positive Y \\
\hline
\end{tabular}

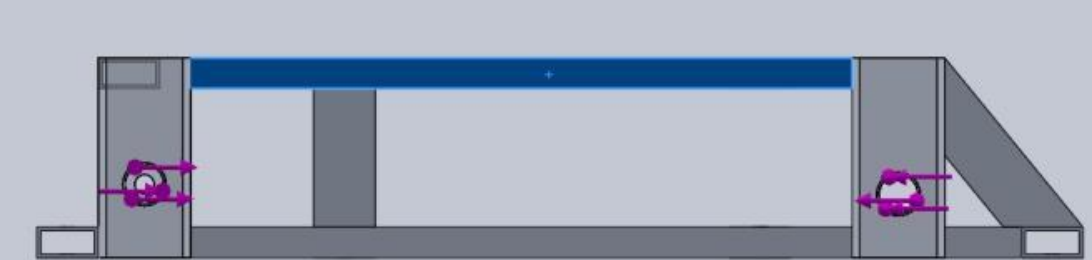

Figure 142: Tread tension and bearing loading conditions.

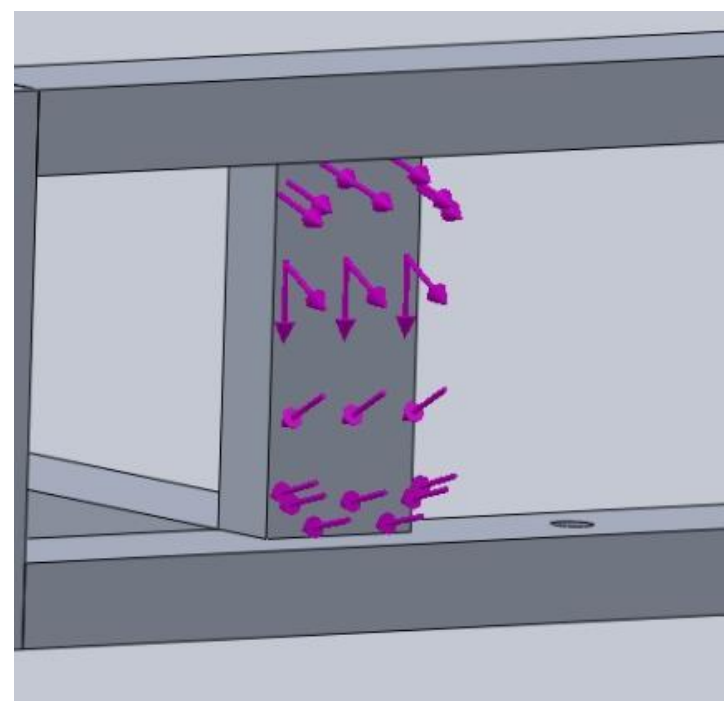

Figure 143: Torque produced by the tensioner assembly on the frame.

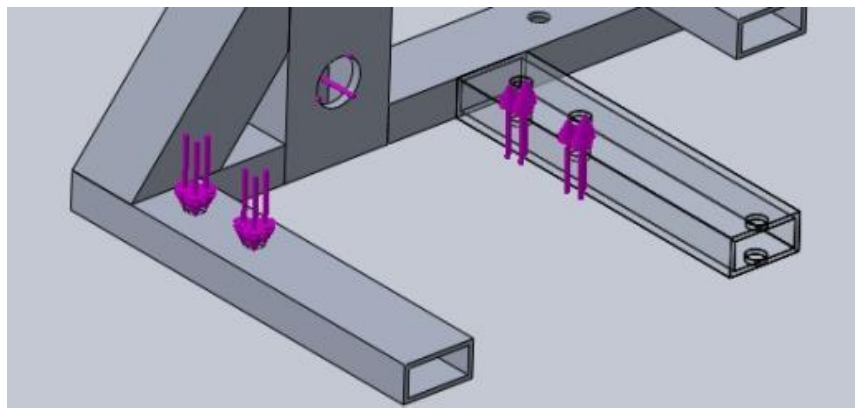

Figure 144: Forces produced by the motor plate. 


\section{A.11.4 Mesh}

The mesh of the frame was generated by Solidworks. Figure 145 shows the meshed frame.

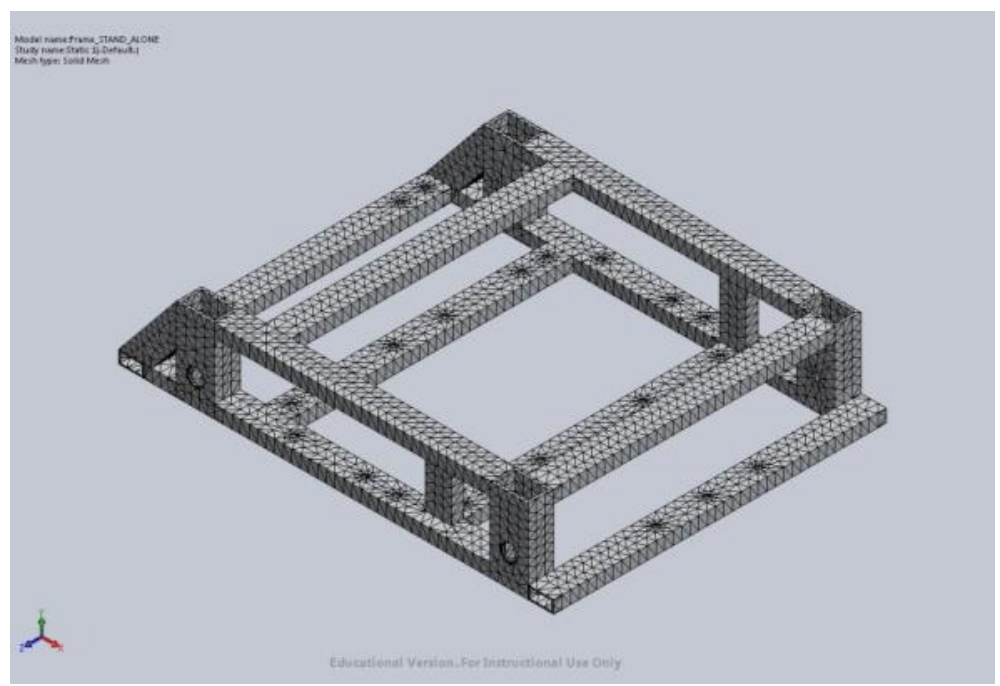

Figure 145: Meshed frame.

\section{A.11.5 Simulation Results}

The results of the simulation were promising. The resultant deflection, resultant stresses and factor of safety were well within acceptable bounds. Table 102 lists the results of the simulation. Figure 146, Figure 147 and Figure 148 show plots of the resultant deflection, resultant stresses and factor of safety respectively.

Table 102: Results of the motor bracket simulation.

\begin{tabular}{|l|r|}
\hline \multicolumn{1}{|c|}{ Parameter } & \multicolumn{1}{|c|}{ Value } \\
\hline Maximum Von Mises Stress & $4436.5 \mathrm{psi}$ \\
\hline Maximum Displacement & $0.002 \mathrm{in}$. \\
\hline Minimum Factor of Safety & 7.4 \\
\hline
\end{tabular}




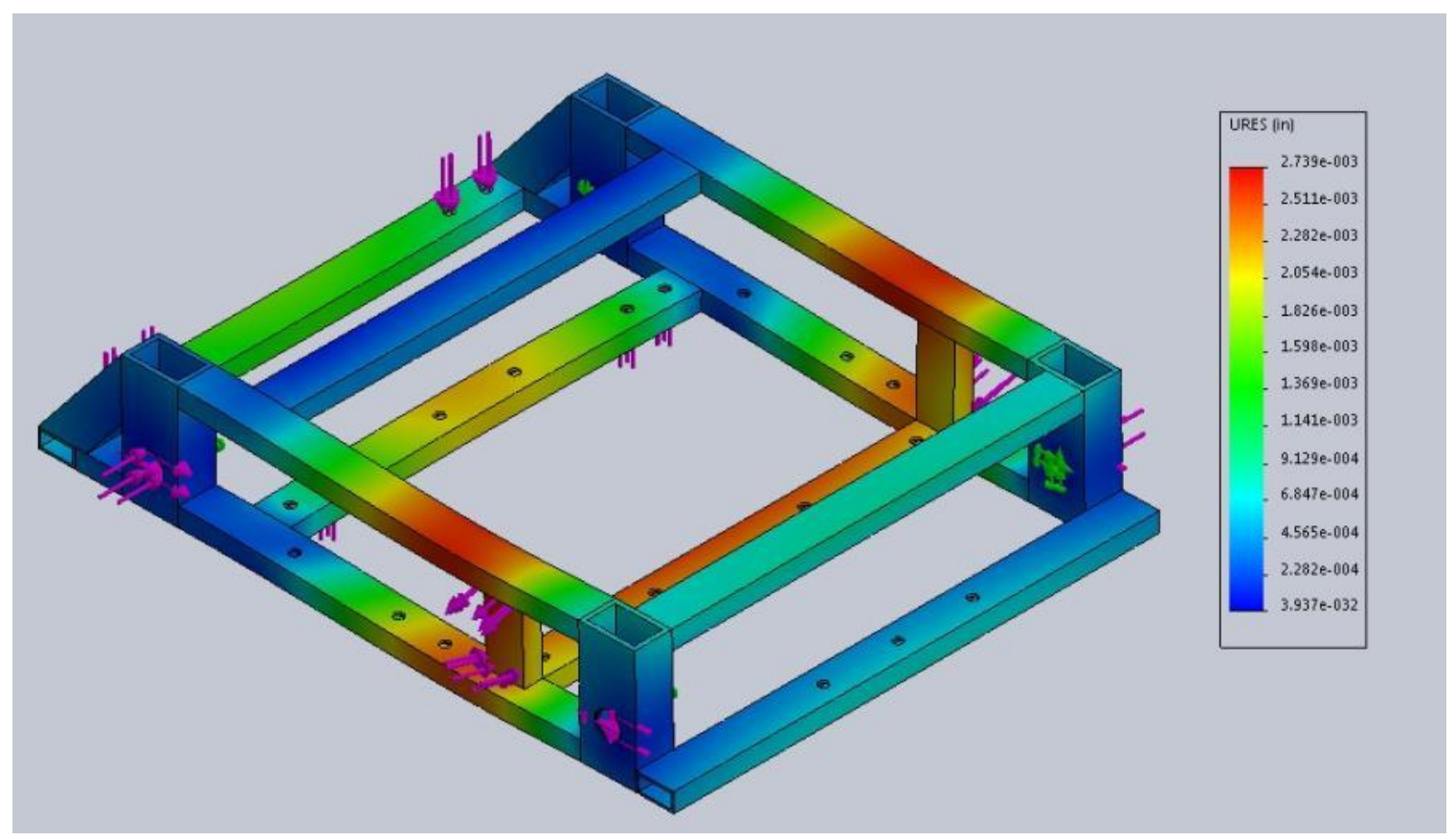

Figure 146: Displacement plot of the frame.

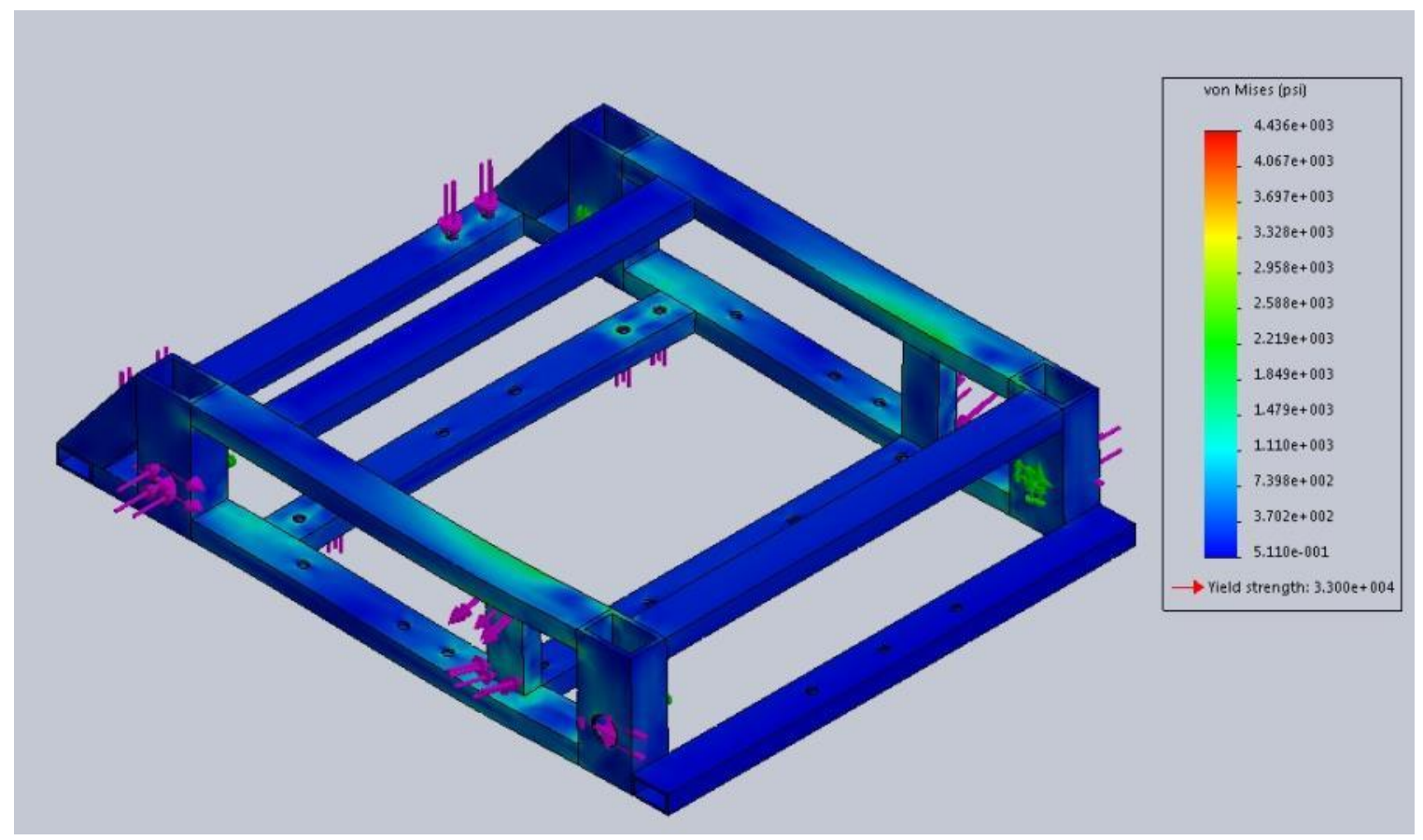

Figure 147: Von Mises stress plot of the frame. 


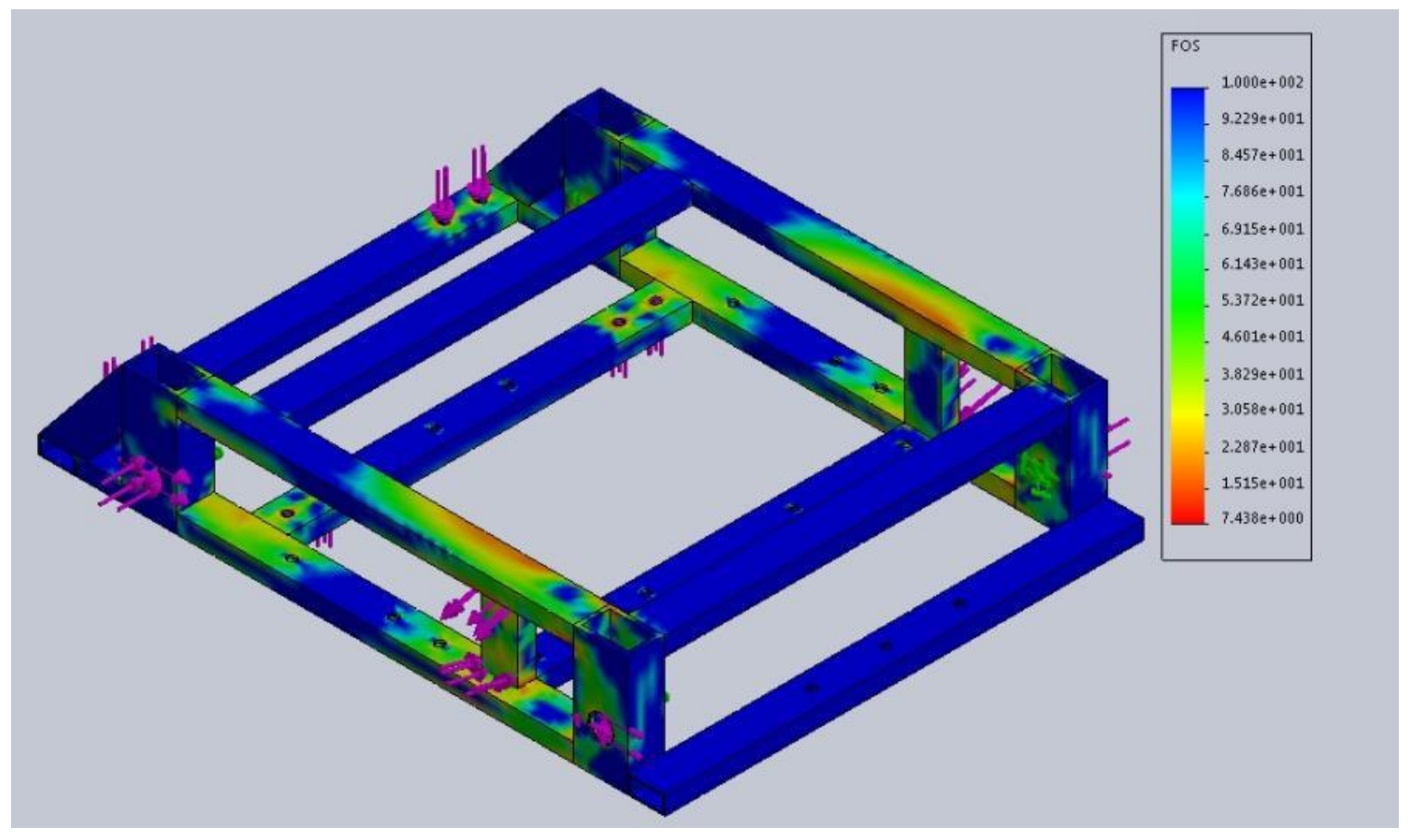

Figure 148: Factor of safety plot of the frame. 


\section{A.12 Motor Bracket Simulation}

\section{A.12.1 Simulation Description}

This simulation was used to validate the design of the motor mounting brackets. The simulation was performed in Solidworks simulation package. It describes the boundary conditions and loading conditions that were applied to the bracket and how those conditions were determined. It reports the factor of safety, maximum Von Mises stress and the deflection of the bracket.

\section{A.12.2 Boundary Conditions}

To simulate the mounting bracket being rigidly attached to the motor mounting plate the faces of the bolt holes in the lower leg of the bracket were fixed in all directions. Figure 149 shows the fixture locations used in the simulation.

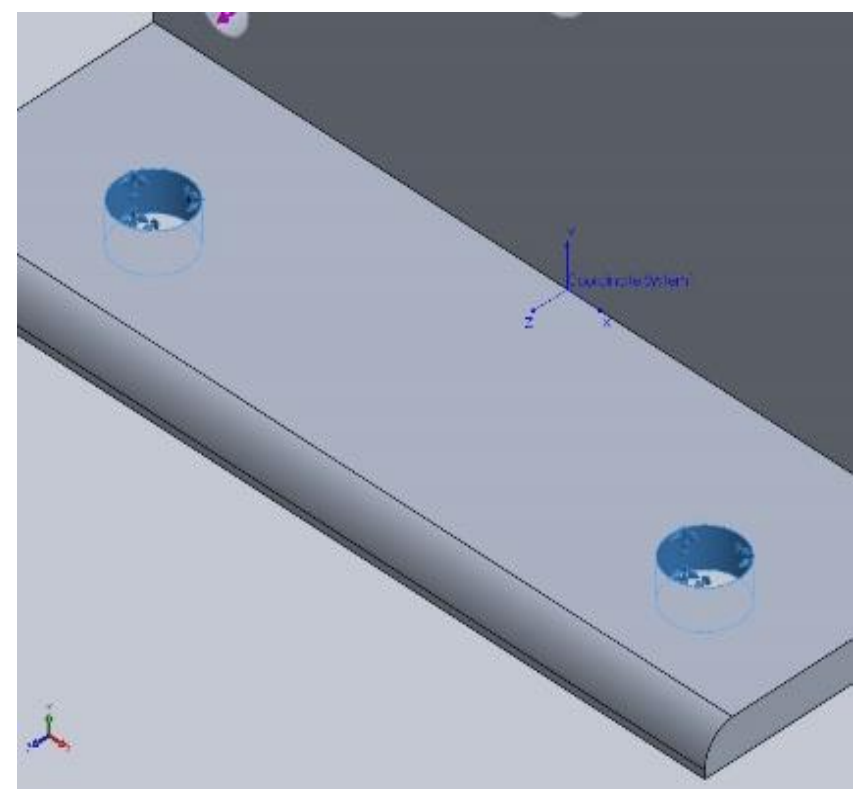

Figure 149: Fixtures used in the motor bracket simulation.

\section{A.12.3 Loading Conditions}

Two different sets of loads were applied to the bracket. One was meant to simulate the moment created by the weight of the motor hanging on bracket. The other was the bracket's resistance to the torque produce by the motor during operation. 
A simple hand calculation was used to determine the magnitudes and directions of reaction forces at the motor mounting holes due to the motor weights. The magnitude of the individual forces was found to be $0.51 \mathrm{lbf}$. The forces at the top of the bracket pointed in the negative $\mathrm{z}$-direction and those at the bottom pointed in the positive z-direction. These forces were inserted into the simulation as force loads on the inside faces of the motor mounting holes in the bracket. Figure 150 shows the loading condition due to the motor mass.

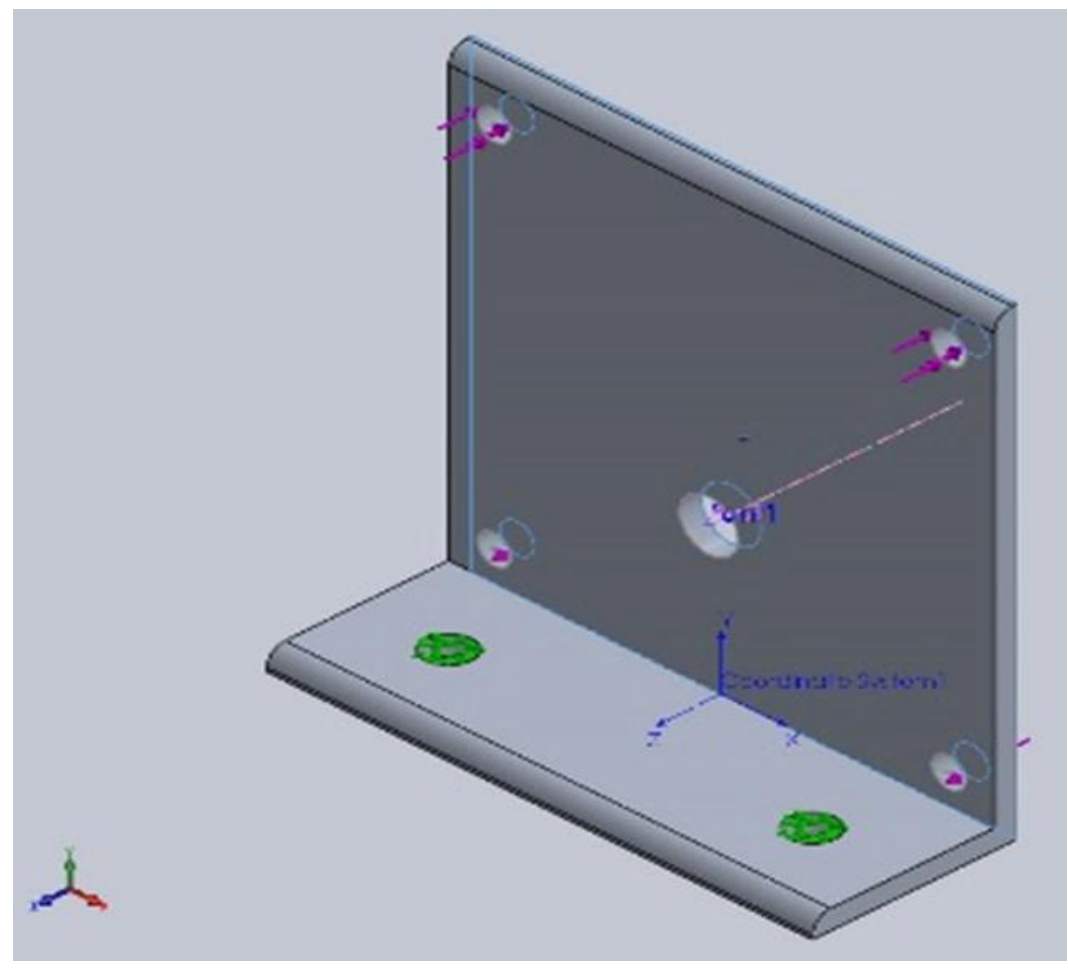

Figure 150: The loading on the motor bracket due to the mass of the motor.

It was assumed for the purposes of the determining the torque that the motor was operating at its full load capacity. This gave the torque applied to the bracket a magnitude of 50 in-lbf. It was also assumed that the vehicle was moving forwards which gave the torque applied to the bracket a counter-clockwise direction. The torque was centered on the axis of the trough hole for the motor shaft. The torque was applied to the back face of the bracket. Figure 151 shows the torque load applied to the motor bracket during the simulation. 


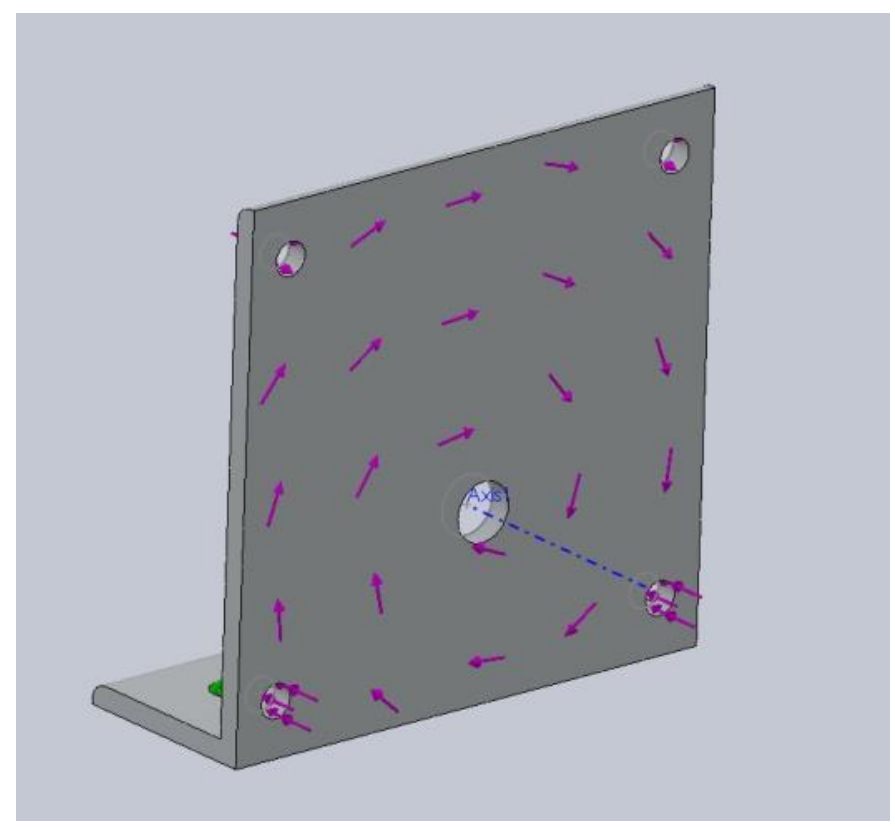

Figure 151: Torque load simulation the motor.

\section{A.12.4 Simulation Results}

The results of the simulation were promising. The resultant deflection, resultant stresses and factor of safety were well within acceptable bounds. Table 103 lists the results of the simulation. Figure 152, Figure 153 and Figure 154 show plots of the resultant deflection, resultant stresses and factor of safety respectively. Figure 155 shows the reaction forces at the fixed locations on the bracket. These reactions would be used in the motor plate validation simulation.

Table 103: Results of the motor bracket simulation.

\begin{tabular}{|l|r|}
\hline \multicolumn{1}{|c|}{ Parameter } & Value \\
\hline Maximum Von Mises Stress & $6390 \mathrm{psi}$ \\
\hline Maximum Displacement & $0.0035 \mathrm{in}$. \\
\hline Minimum Factor of Safety & 6.2 \\
\hline
\end{tabular}




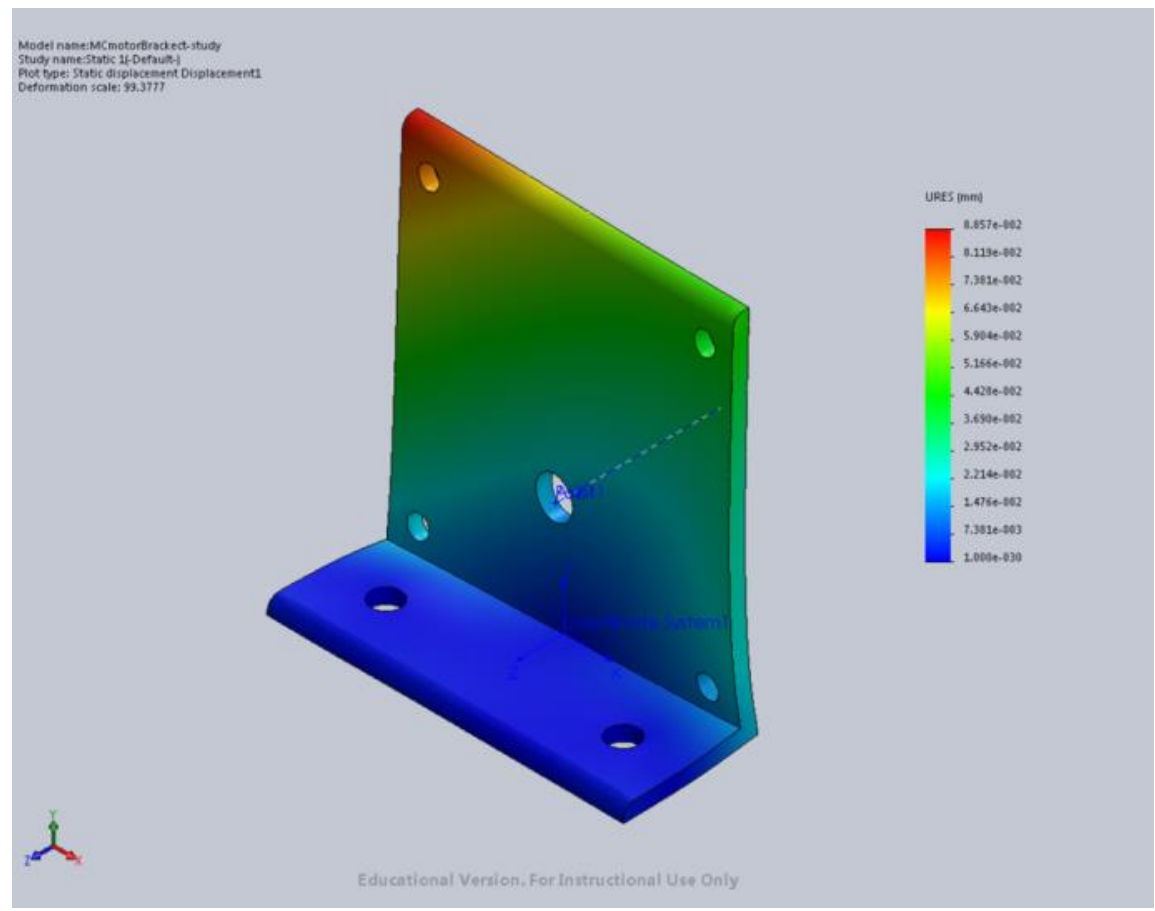

Figure 152: Deflection plot of the motor bracket.

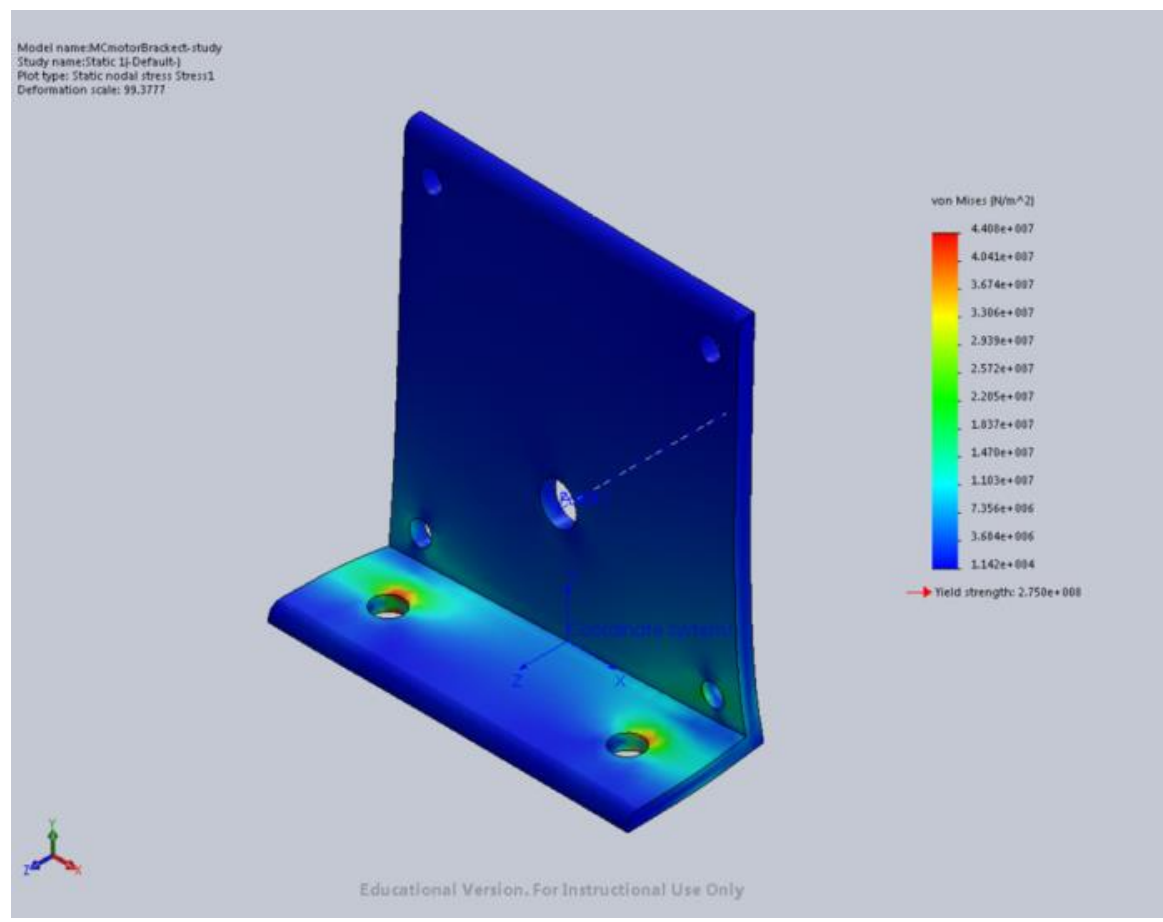

Figure 153: Von Mises stress plot of the motor bracket. 


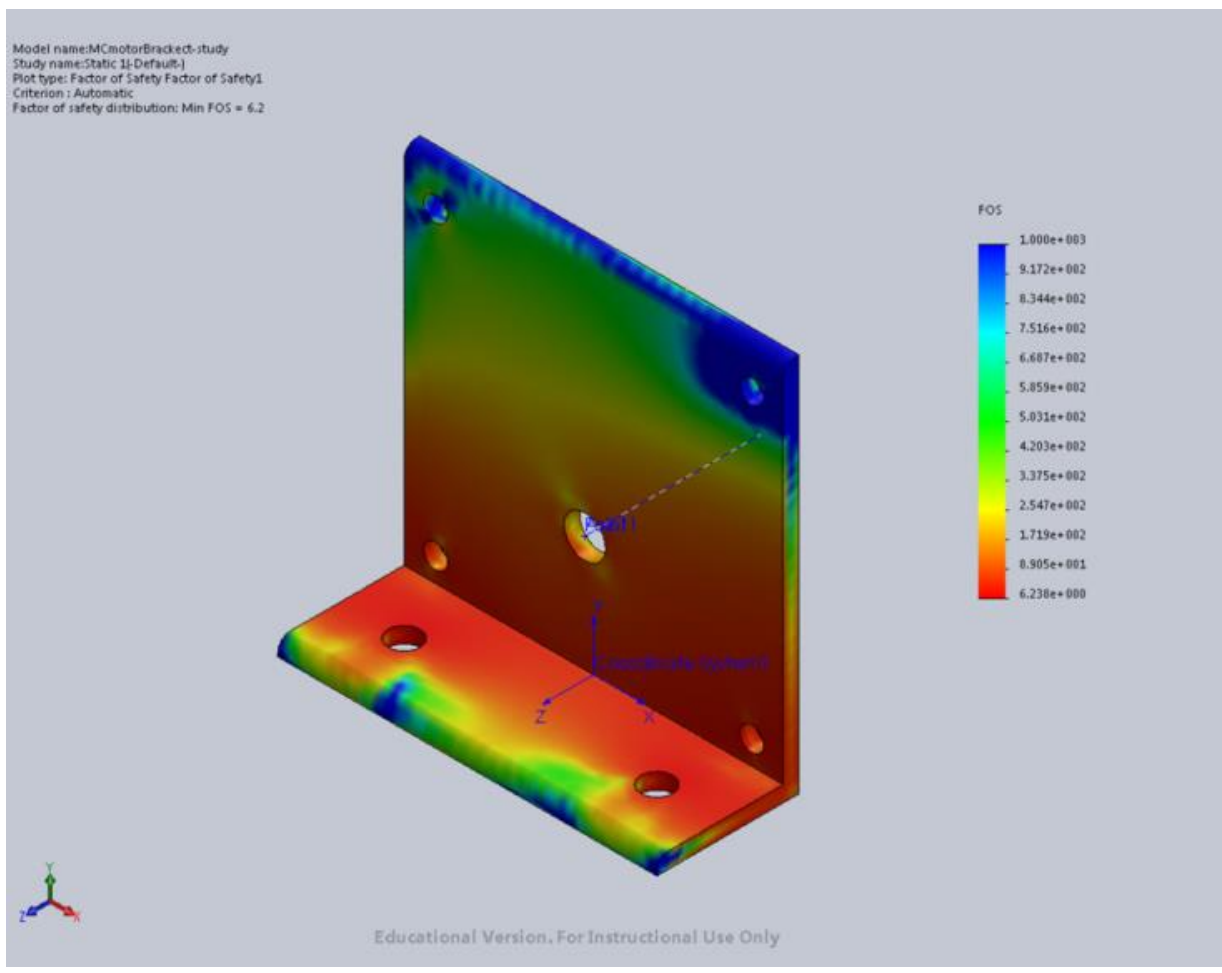

Figure 154: Factor of safety plot of the motor bracket.

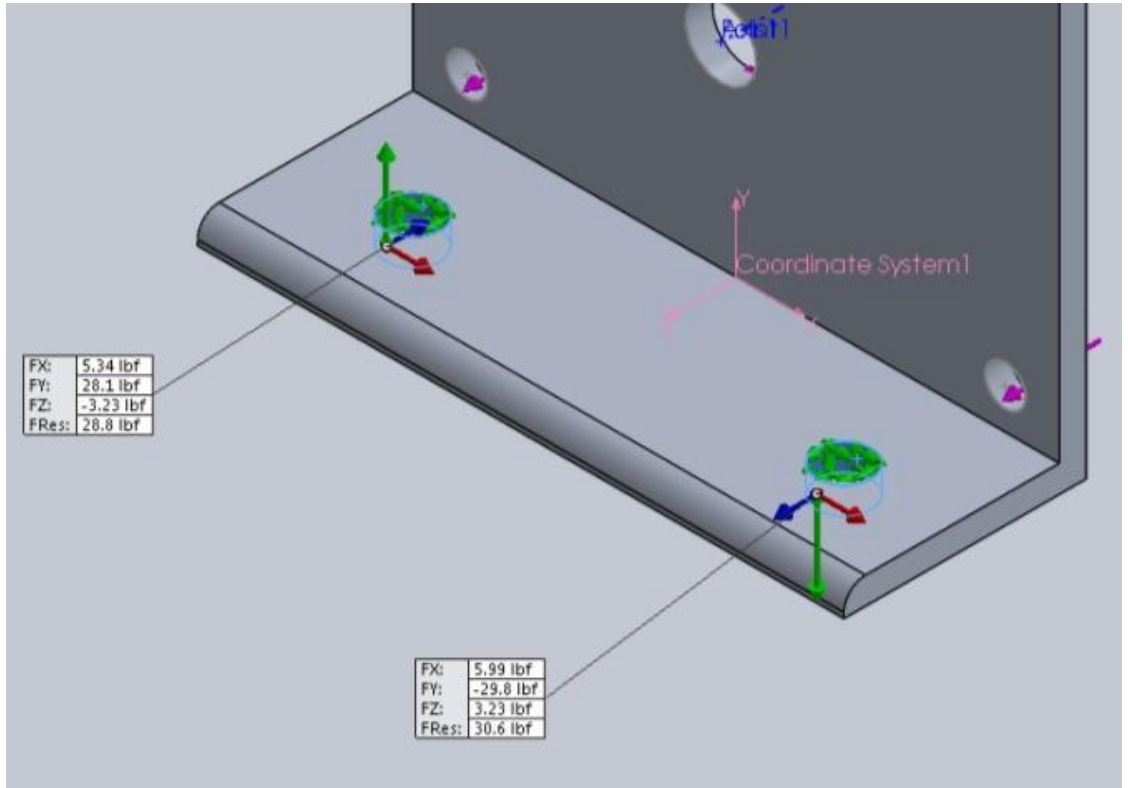

Figure 155: Reactions at the fixed holes of the motor bracket. 


\section{A.13 Motor Plate Validation}

\section{A.13.1 Simulation Description}

This simulation was used to validate the design of the motor base plates. The simulation was performed in Solidworks simulation package. It describes the boundary conditions and loading conditions that were applied to the plates and how those conditions were determined. It reports the factor of safety, maximum Von Mises stress and the deflection of the bracket.

\section{A.13.2 Boundary Conditions}

The plate was fixed in all directions at the four outer holes on the plate. This was done to simulate the rigid attachment of the plates to the fame. Figure 156 shows the fixture location used in the simulation.

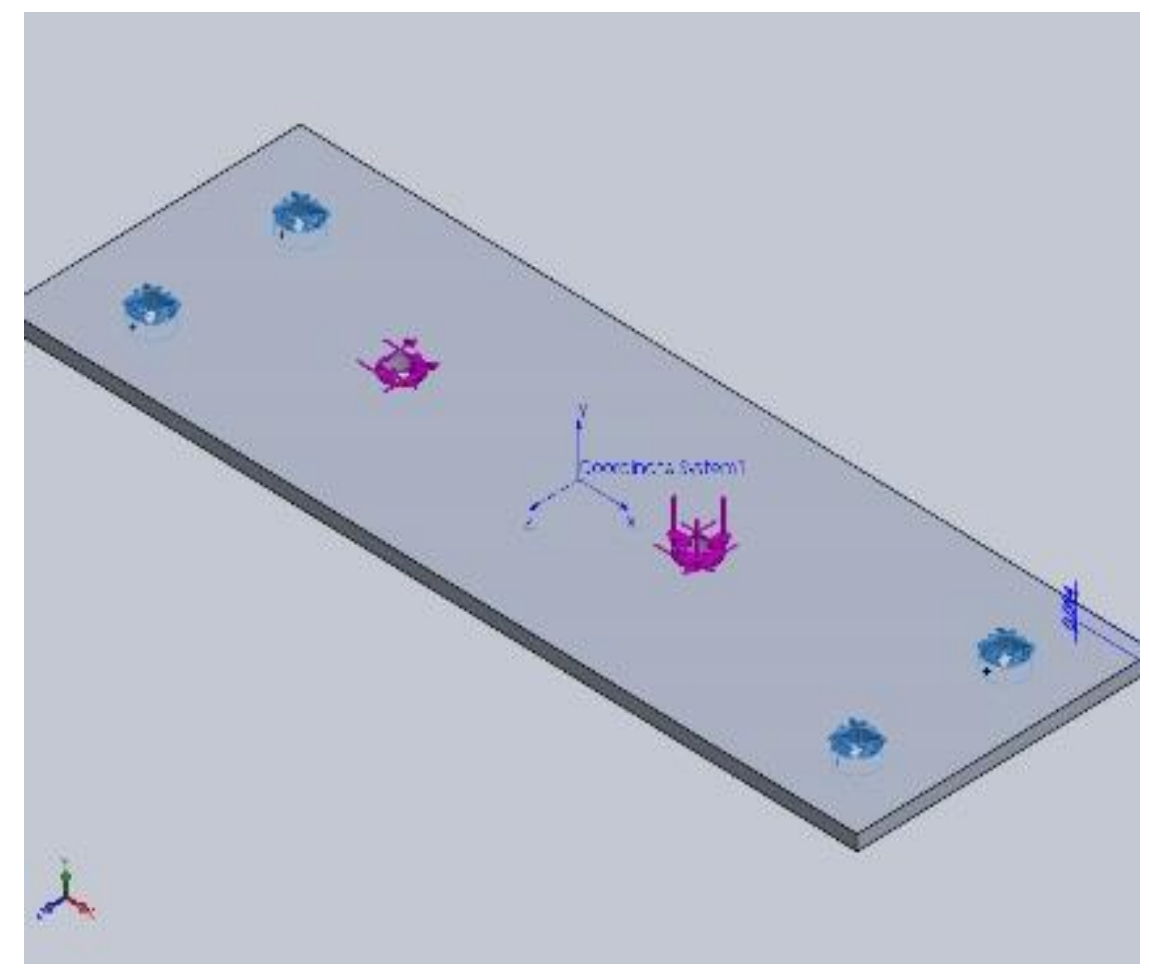

Figure 156: Fixtures used in the motor plate simulation, Highlighted in blue.

\section{A.13.3 Loading Conditions}

The loading conditions for the plates were taken directly from the reaction forces found during the simulation of the motor bracket. They were taken to act on the on the bolt holes which attached the motor plates to the motor brackets. Table 104 contains the magnitudes and directions of the loading conditions 
used in the plate simulation. Note that the direction of the forces is flipped relative to their counter parts on the motor bracket. Figure 157 shows the loading conditions applied to the plate.

Table 104: Loading conditions applied to the motor plate.

\begin{tabular}{|l|r|}
\hline \multicolumn{1}{|c|}{ Forward Hole } & Value \\
\hline$x$ - direction & $-5.34 \mathrm{lbf}$ \\
\hline y-direction & $-28.1 \mathrm{lbf}$ \\
\hline z-direction & $3.23 \mathrm{lbf}$ \\
\hline Rear Hole & Value \\
\hline$x$ - direction & $-5.99 \mathrm{lbf}$ \\
\hline y-direction & $29.8 \mathrm{lbf}$ \\
\hline z-direction & $-3.23 \mathrm{lbf}$ \\
\hline
\end{tabular}

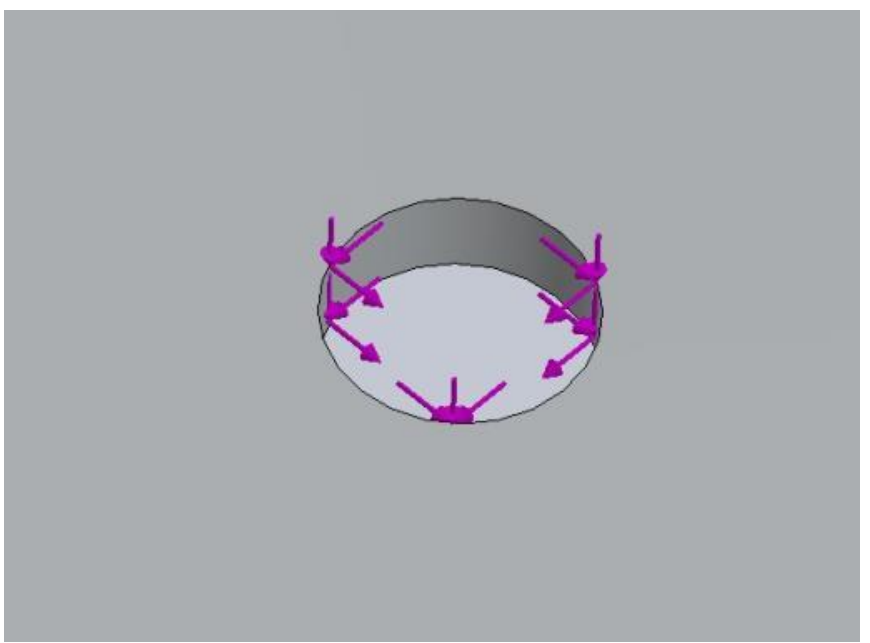

Figure 157: An image of the rear hole showing the loading conditions. 


\section{A.13.4 Mesh}

The mesh for the motor plate was auto generated by Solidworks. A fine mesh was chosen to increase the accuracy of the results. Figure 158 shows the meshed motor plate.

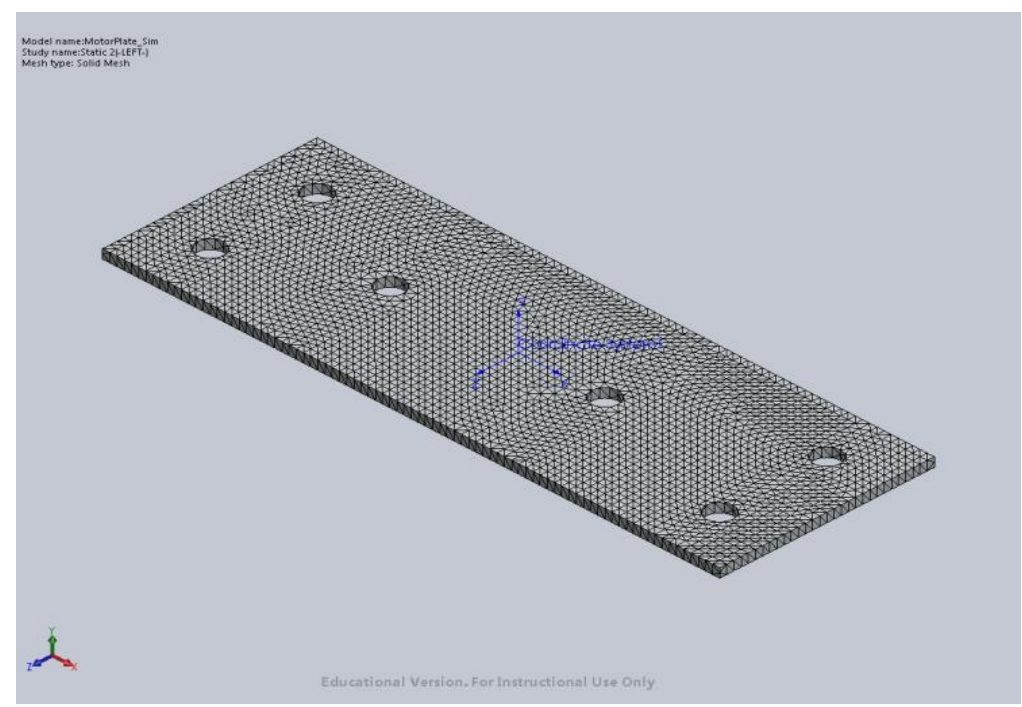

Figure 158: Meshed motor plate.

\section{A.13.5 Simulation Results}

The resultant deflection, resultant stresses and factor of safety were well within acceptable bounds. Table 105 lists the results of the simulation. Figure 159, Figure 160 and Figure 161 show plots of the resultant deflection, resultant stresses and factor of safety respectively. Figure 162 shows the reaction forces at the fixed locations on the motor plate. These reactions were used in the simulation of the frame.

Table 105: Results from the motor plate simulation.

\begin{tabular}{|l|r|}
\hline \multicolumn{1}{|c|}{ Simulation Result } & Value \\
\hline Maximum Von Mises Stress & $11,525 \mathrm{psi}$ \\
\hline Maximum Displacement & $0.005 \mathrm{in}$. \\
\hline Minimum Factor of Safety & 3.5 \\
\hline
\end{tabular}




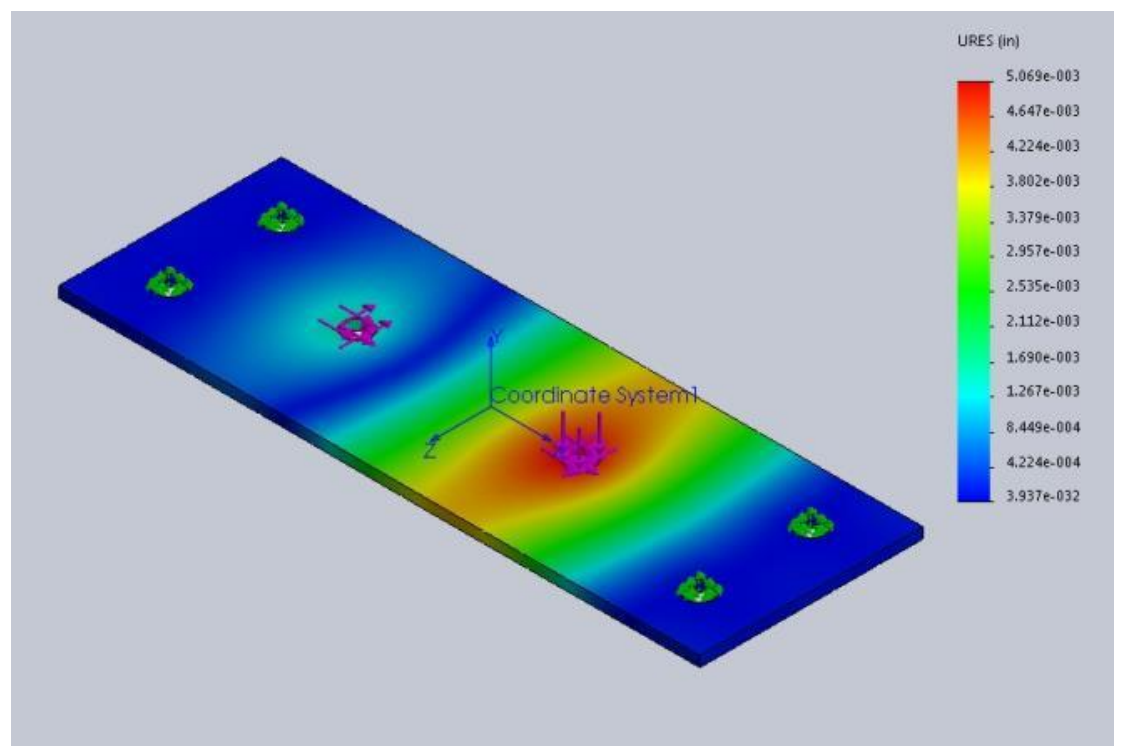

Figure 159: Deflection plot of the motor plate.

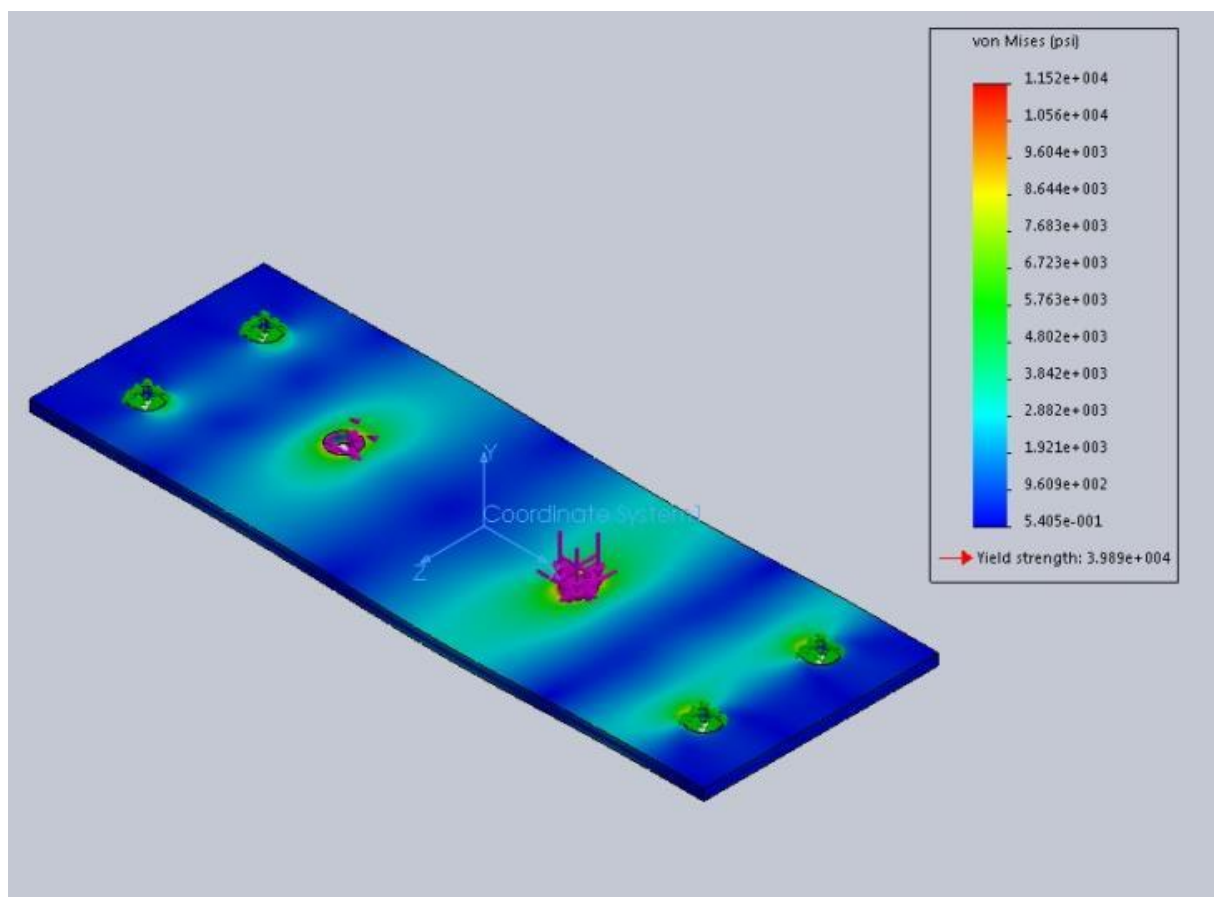

Figure 160: Von Mises Stress plot of the motor plate. 


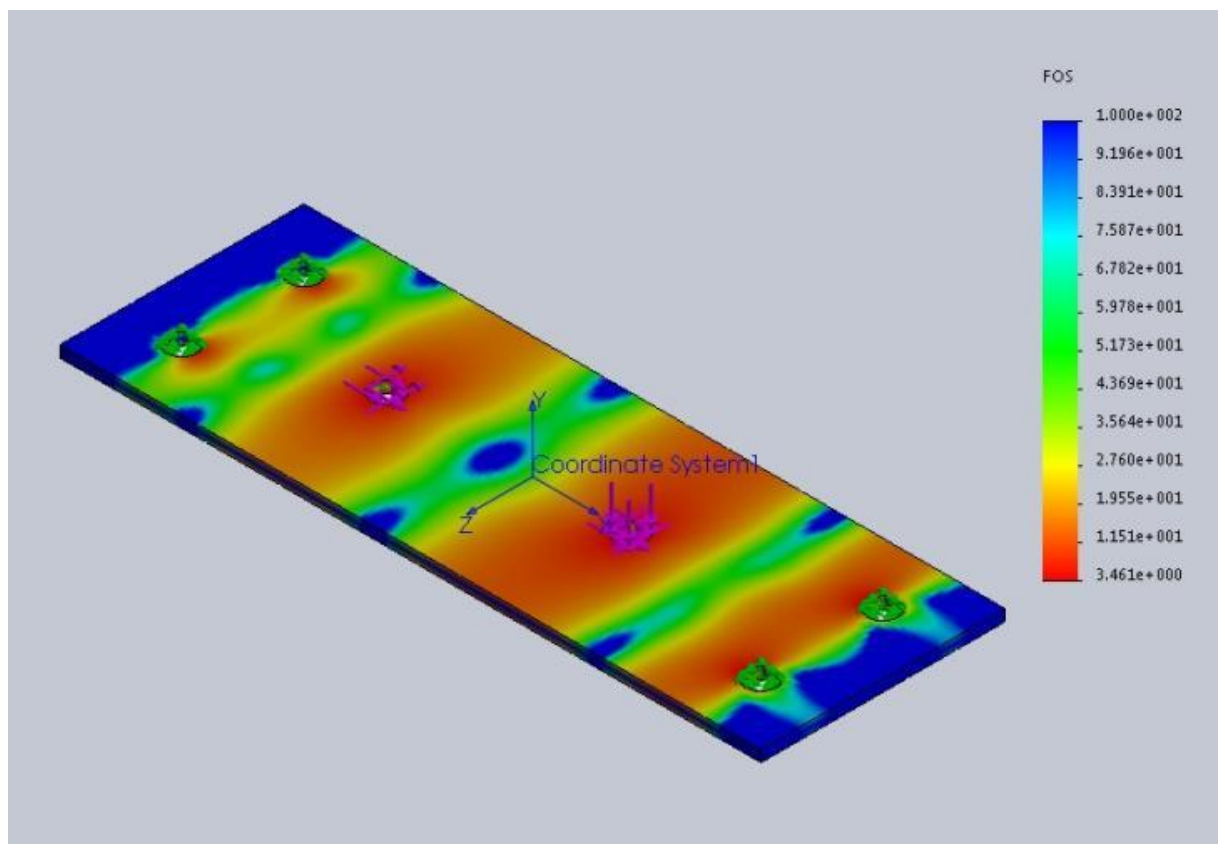

Figure 161: Factor of safety plot of the motor plate.

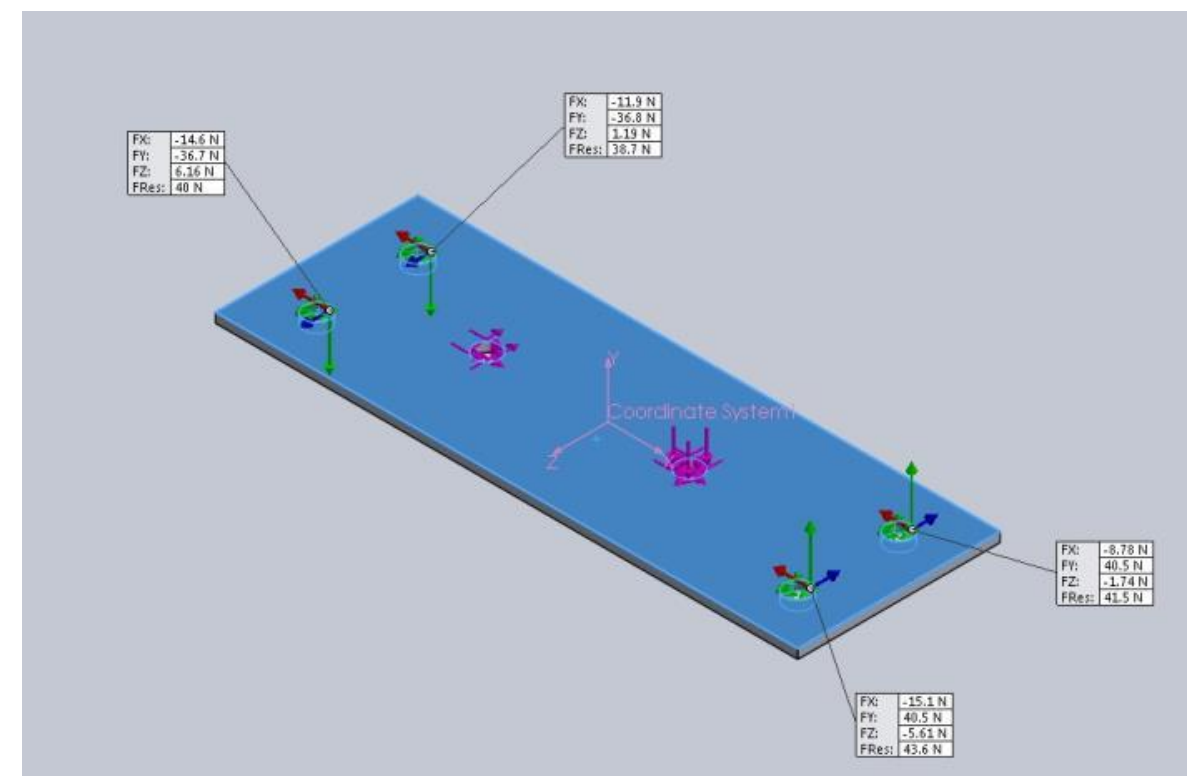

Figure 162: Reactions of the motor plate at the fixed holes. 


\section{A.14 Manifold Mounting Strut Validation}

\section{A.14.1 Simulation Description}

This simulation was used to validate the design of the manifold mounting struts. The simulation was performed in Solidworks simulation package. It describes the boundary conditions and loading conditions that were applied to the struts and how those conditions were determined. It reports the factor of safety, maximum Von Mises stress and the deflection of the bracket.

\section{A.14.2 Boundary Conditions}

The mounting strut was fixed in place at the frame mounting holes. Figure 163 shows the fixed locations of the mounting struts.

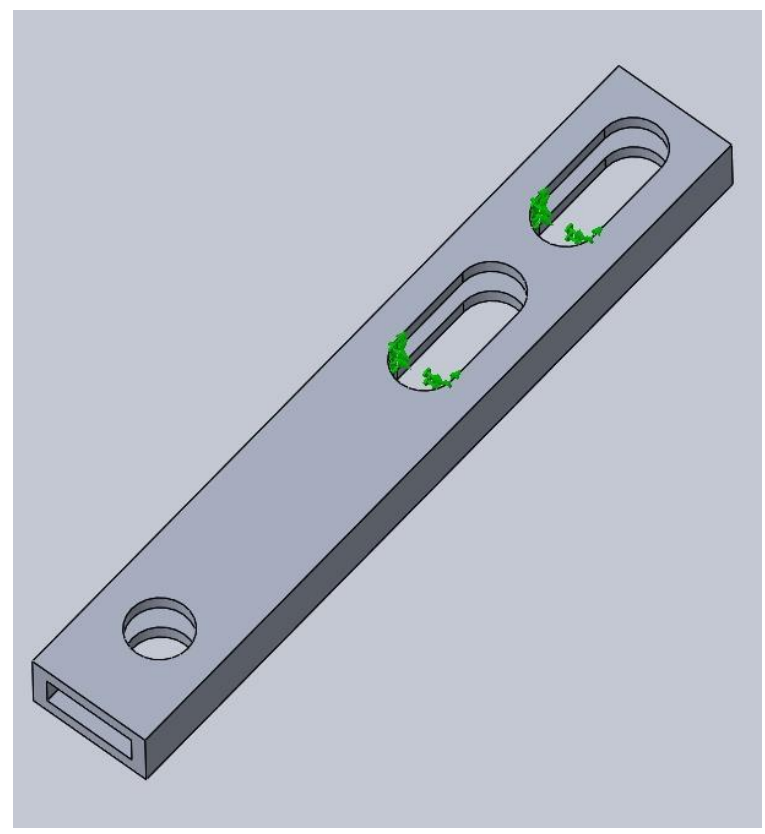

Figure 163: Fixed locations on the mounting strut.

\section{A.14.3 Loading Conditions}

The loading conditions of the strut were simple to determine. A load perpendicular to the bottom face of the strut was applied at the manifold mounting hole to simulate the spring force from the suspension. A 
horizontal load simulating the manifold friction was also applied. Table 106 contains the magnitudes of the applied loads and Figure 164 shows their location and direction.

Table 106: Magnitudes of the loads applied to the strut.

\begin{tabular}{|l|c|}
\hline \multicolumn{1}{|c|}{ Load } & Magnitude \\
\hline Spring Force & $11 \mathrm{lbf}$ \\
\hline Manifold Friction & $10 \mathrm{lbf}$ \\
\hline
\end{tabular}

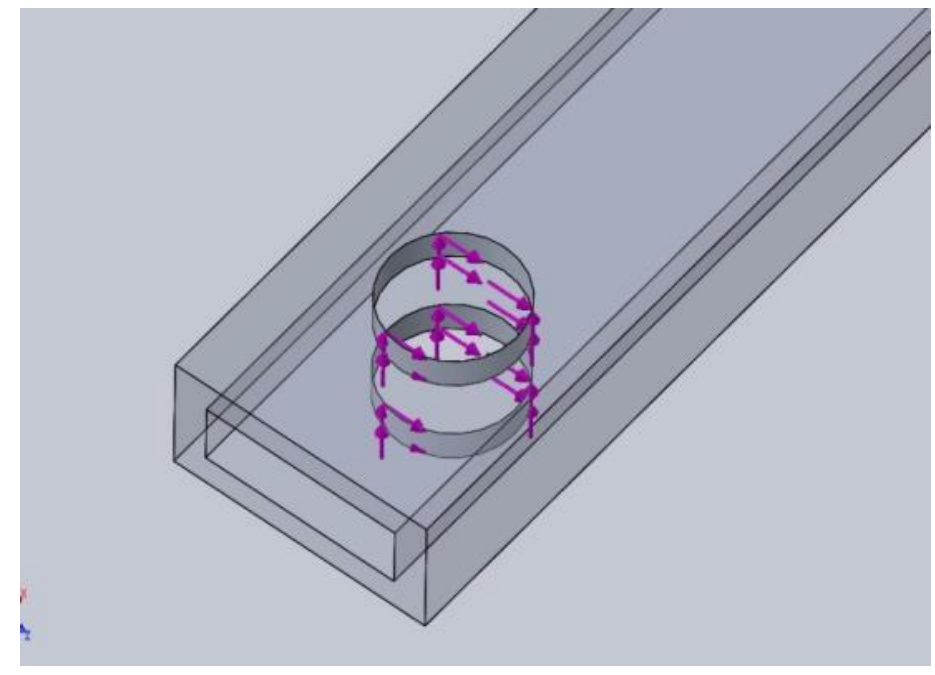

Figure 164: Loading conditions for the strut simulation.

\section{A.14.4 Mesh}

The mesh for the motor plate was auto generated by Solidworks. A fine mesh was chosen to increase the accuracy of the results. Figure 165 shows the strut.

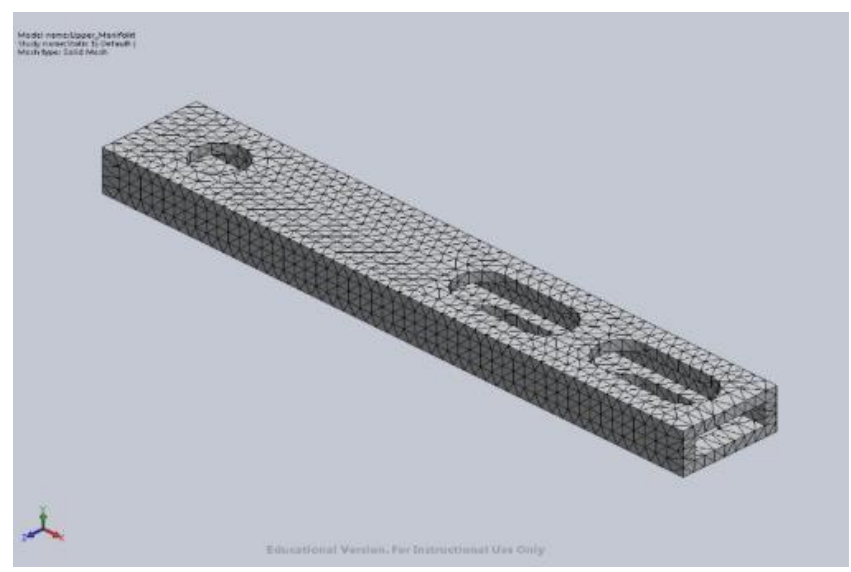

Figure 165: Meshed support strut. 


\section{A.14.5 Simulation Results}

Table 107 lists the results of the simulation. Figure 166, Figure 167 and Figure 168 show plots of the resultant deflection, resultant stresses and factor of safety respectively.

Table 107: Results from the motor plate simulation.

\begin{tabular}{|l|r|}
\hline \multicolumn{1}{|c|}{ Simulation Result } & \multicolumn{1}{|c|}{ Value } \\
\hline Maximum Von Mises Stress & $11,550 \mathrm{psi}$ \\
\hline Maximum Displacement & $0.004 \mathrm{in}$. \\
\hline Minimum Factor of Safety & 3.5 \\
\hline
\end{tabular}

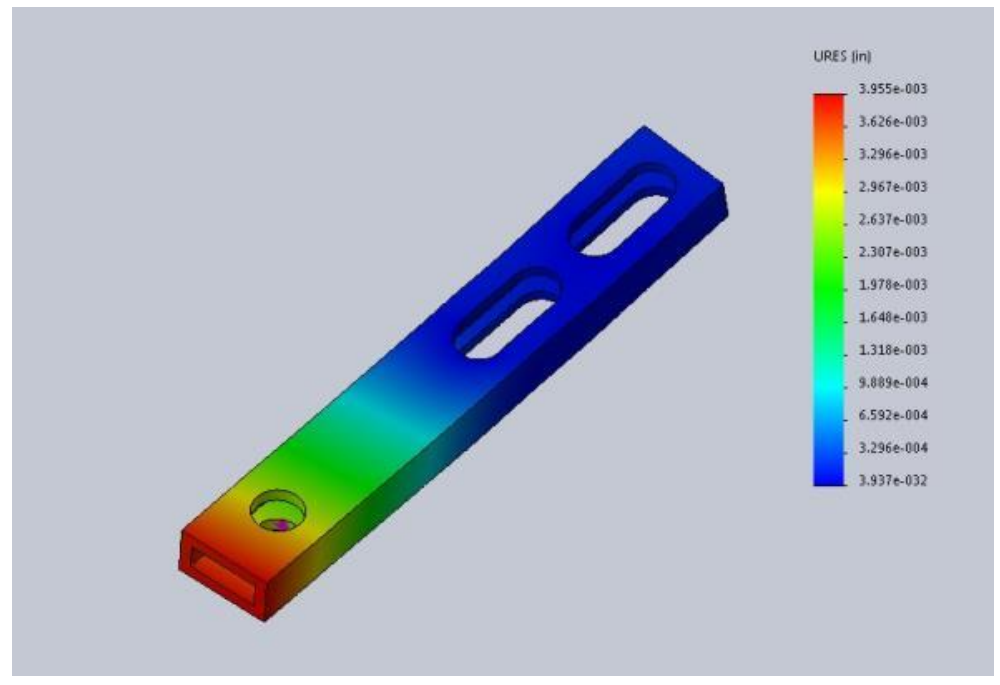

Figure 166: Deflection plot of the strut.

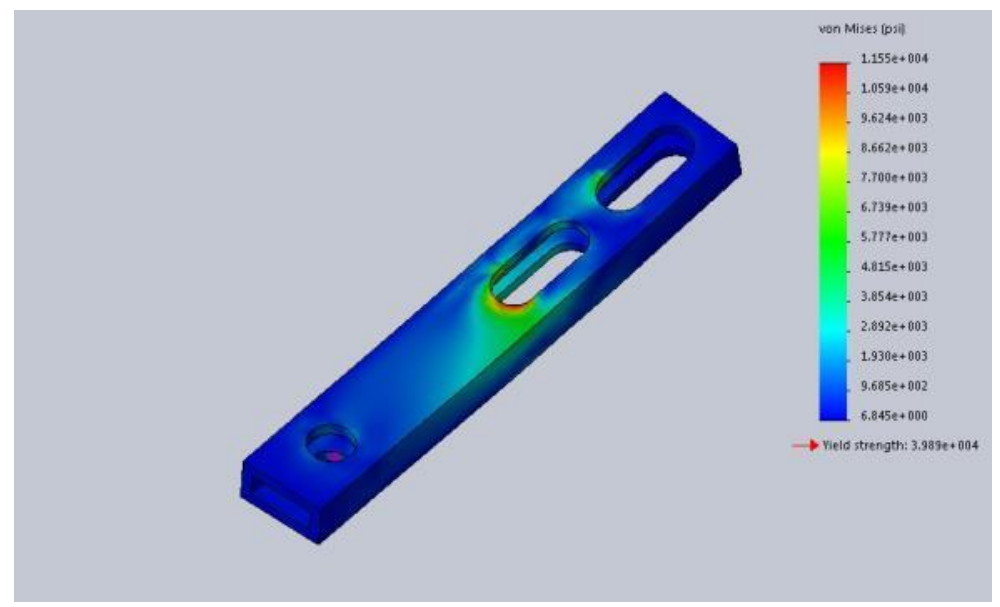

Figure 167: The Von Mises Stress plot of the strut. 


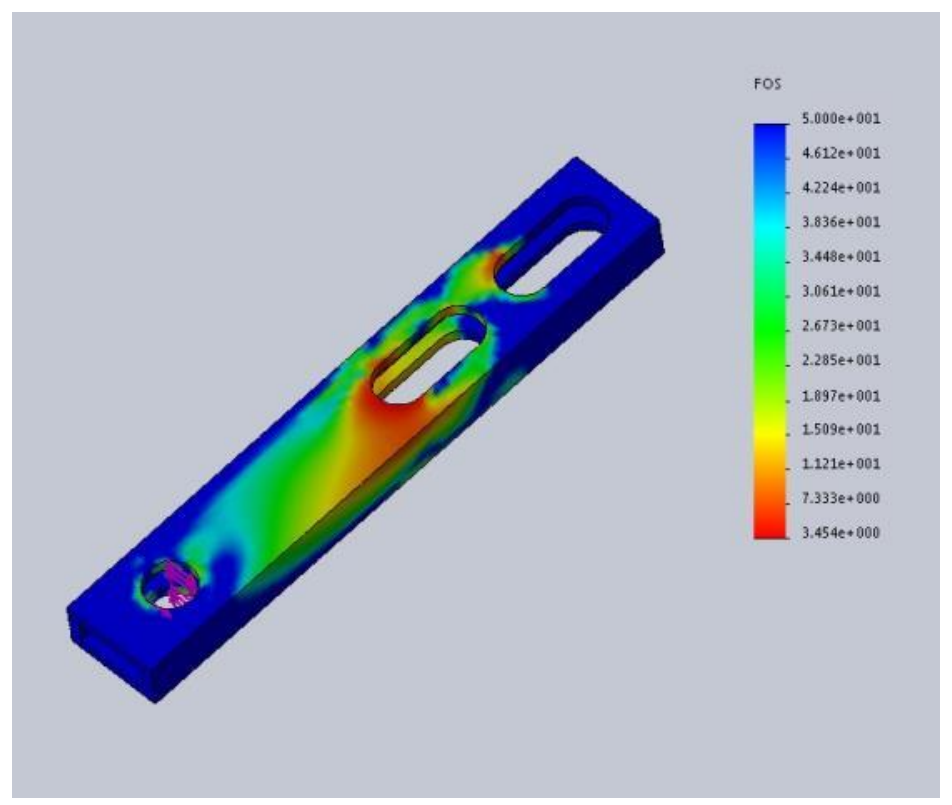

Figure 168: Factor of safety plot of the strut. 


\section{A.15 Bearing Loading Calculation}

\section{A.15.1 Calculation Description}

This calculation was used to determine maximum loading which the bearings in the vehicle would carry during operation.

\section{A.15.2 Assumptions}

- The tread tension load is distributed equally between the frame and brace bearings.

- The vehicle is parallel the ground.

- The weight of the vehicle is carried by only two of the bearings

\section{A.15.3 Solution}

Table 108 gives the variables and their values used to calculate the bearing loading.

Table 108: Force values which are applied across two bearings.

\begin{tabular}{|c|l|r|}
\hline Symbol & \multicolumn{1}{|c|}{ Description } & \multicolumn{1}{|c|}{ Value } \\
\hline$T_{T}$ & Tread Tension & $110 \mathrm{lbf}$ \\
\hline$w_{V}$ & Weight of the vehicle & $16 \mathrm{lbf}$ \\
\hline
\end{tabular}

The load on a single bearing is equal to half of the tension in the tread plus half of the weight of the vehicle, as seen in Equation A.15.1.

$$
F_{B}=.5 * T_{T}+.5 * w_{V}
$$

The results of this calculation can be found in Table 109.

Table 109: Results of the bearing loading calculation.

\begin{tabular}{|r|r|r|}
\hline Predicted Bearing Load & Max Bearing Load & Safety Factor \\
\hline $63 \mathrm{lbf}$ & $280 \mathrm{lbf}$ & 4.44 \\
\hline
\end{tabular}




\section{A.16 Drive Shaft Simulation}

\section{A.16.1 Simulation Description}

This simulation was used to validate the design of the drive shaft wheel and to determine the diameter and the material used to construct it. The simulation was performed in Solidworks simulation package. It describes the boundary conditions and loading conditions that were applied to the shaft and how those conditions were determined. It reports the factor of safety, maximum Von Mises stress and the deflection of the bracket.

\section{A.16.2 Boundary Conditions}

The end of the shaft which couples to the motor was fixed to simulate the stalling of the motor. The locations where the bearings contact the shafts was fixed radially and axially to prevent the shaft from shifting at these locations but still allowing for rotation. Figure 169 shows the locations of the shaft fixtures.

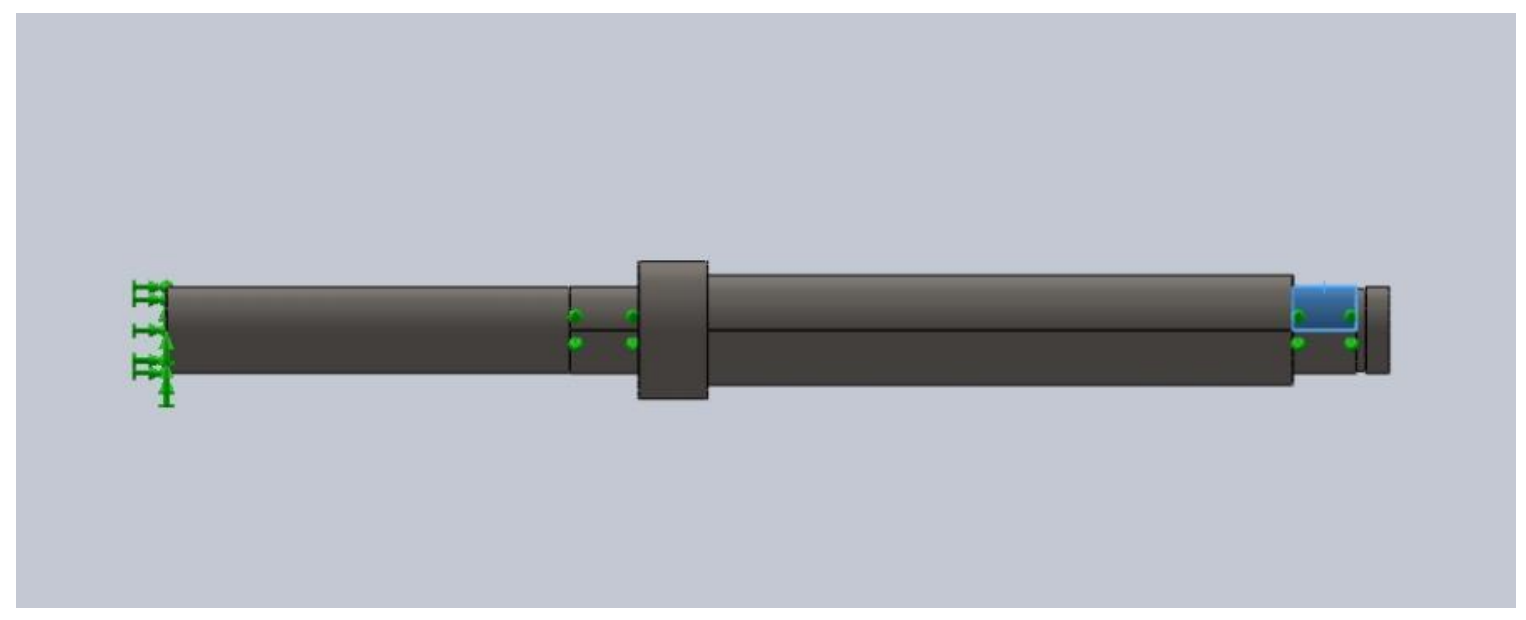

Figure 169: Fixed locations on the shaft.

\section{A.16.3 Loading Conditions}

Two loads were applied to the brace at the shaft. The first was the motor torque load and the second was the tread tension load. Table 110 gives the magnitudes of these loads and their location and directions can be seen in Figure 170. 
Table 110: Magnitudes of the loads applied to the brace.

\begin{tabular}{|l|rr|}
\hline \multicolumn{1}{|c|}{ Load } & \multicolumn{1}{c|}{ Magnitude } \\
\hline Tread Tension & $110 \mathrm{lbf}$ \\
\hline Motor Torque & $50 \mathrm{in}-\mathrm{lbf}$ \\
\hline
\end{tabular}

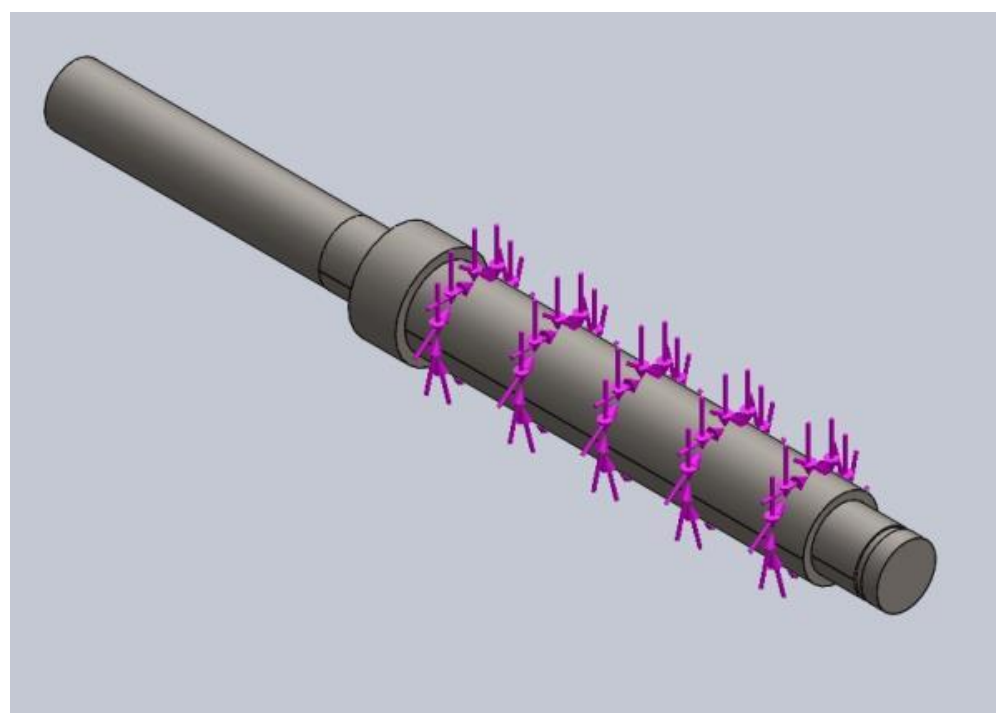

Figure 170: Locations of the loads applied to the brace.

\section{A.16.4 Mesh}

The mesh for the brace was auto generated by Solidworks. A fine mesh was chosen to increase the accuracy of the results. Figure 171 shows the meshed brace.

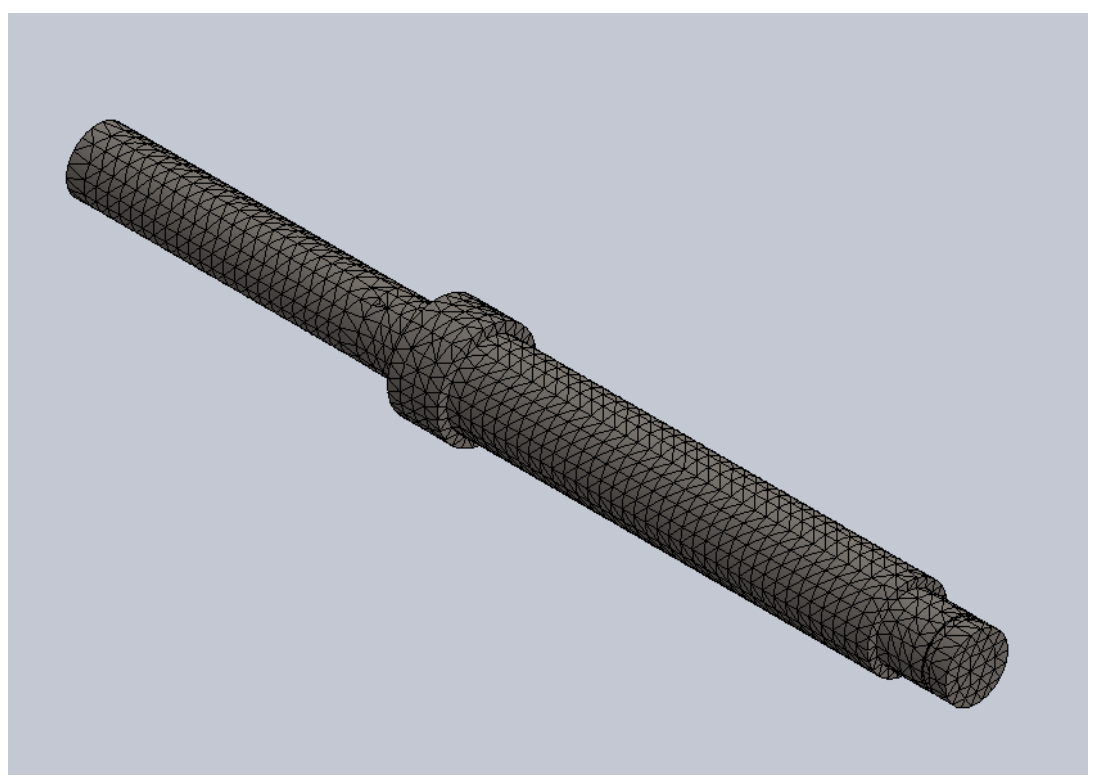

Figure 171: Meshed drive shaft. 


\section{A.16.5 Simulation Results}

The 0.4 in. diameter steel shaft was chosen. Table 111 lists the results of the simulation for each material type and diameter that was simulated. Figure 172, Figure 173 and Figure 174 show plots of the resultant deflection, resultant stresses and factor of safety respectively for the chosen shaft diameter and material.

Table 111: Results from the different shaft simulations.

\begin{tabular}{|l|c|c|r|}
\hline $\begin{array}{c}\text { Simulation Diameter and } \\
\text { Material }\end{array}$ & $\begin{array}{c}\text { Maximum } \\
\text { Displacement }\end{array}$ & $\begin{array}{c}\text { Maximum Von } \\
\text { Mises Stress }\end{array}$ & $\begin{array}{c}\text { Minimum Factor of } \\
\text { Safety }\end{array}$ \\
\hline 0.45 in. Steel & 0.002 in. & $17,440 \mathrm{psi}$ & 4.4 \\
\hline 0.45 in. Aluminum & 0.007 in. & $17,610 \mathrm{psi}$ & 2.3 \\
\hline 0.4 in. Steel & 0.002 in. & $17,960 \mathrm{psi}$ & 2.3 \\
\hline 0.4 in. Aluminum & $0.006 \mathrm{in}$. & $17,615 \mathrm{psi}$ & 4.0 \\
\hline 0.35 in. Steel & $0.002 \mathrm{in}$. & $19,130 \mathrm{psi}$ & 2.0 \\
\hline 0.35 in. Aluminum & $0.007 \mathrm{in}$. & $19,050 \mathrm{psi}$ & \\
\hline
\end{tabular}

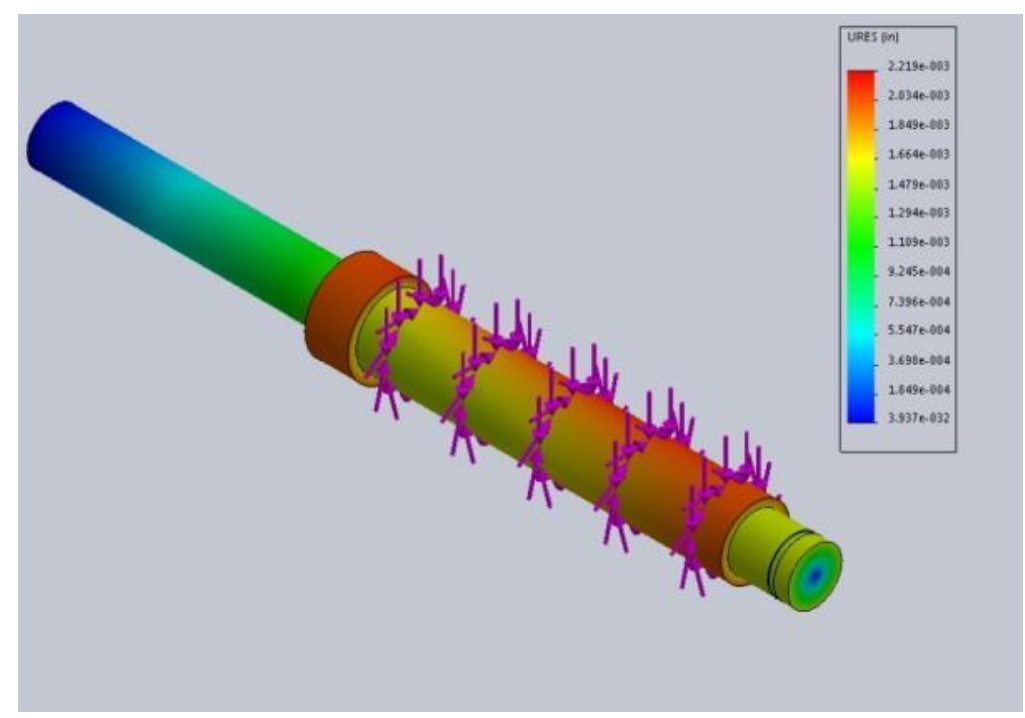

Figure 172: Deflection plot of the drive shaft. 


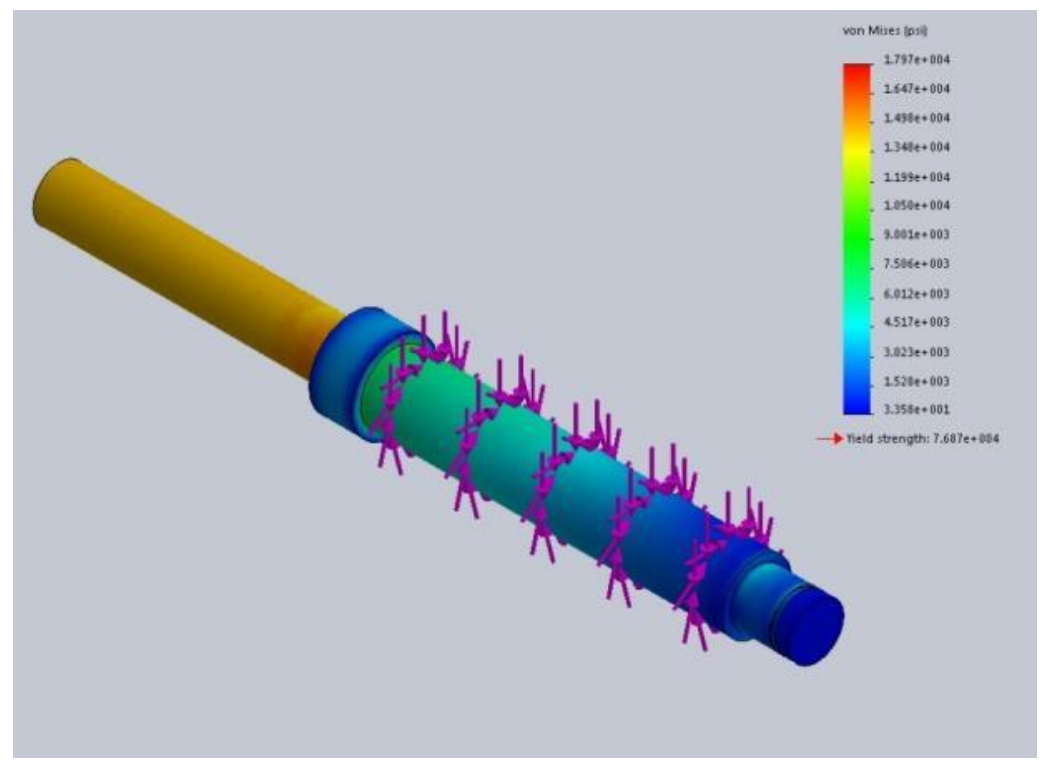

Figure 173: The Von Mises Stress plot of the drive shaft.

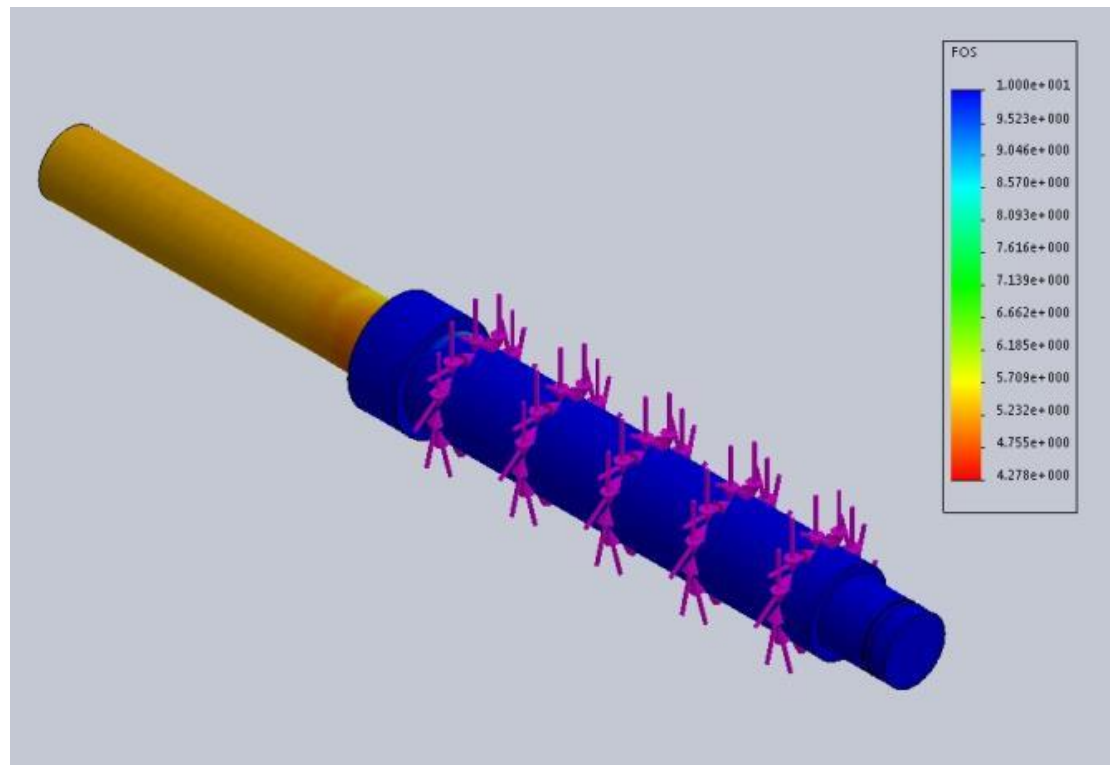

Figure 174: The Factor of safety plot of the drive shaft. 


\section{A.17 Retaining Ring Calculation}

\section{A.17.1 Calculation Description}

This calculation serves as the verification that the snap rings chosen for the project would be capable of withstanding the operating loads they were subjected to.

\section{A.17.2 Equations and Variable Descriptions}

Two equations are used. Equation A.17.1 calculates the maximum allowable thrust load which can be applied to the retaining rings before the rings shear. Equation A.17.2 calculates the maximum allowable thrust load which can be applied to the retaining rings before the groove in the shaft yields. Both equations used in this section were found on Smalley's website [26].

$$
\begin{gathered}
P_{R}=\frac{D T S_{S} \pi}{K} \\
P_{G}=\frac{D d S_{Y} \pi}{K}
\end{gathered}
$$

Table 112: Ring shear variable symbols and descriptions.

\begin{tabular}{|c|l|}
\hline Symbol & \multicolumn{1}{c|}{ Description } \\
\hline$P_{R}$ & Allowable Thrust Load Based on Ring Shear (lb) \\
\hline $\mathrm{D}$ & Shaft Diameter (in) \\
\hline $\mathrm{T}$ & Ring Thickness (in) \\
\hline$S_{S}$ & Ring Material Shear Strength (psi) \\
\hline $\mathrm{K}$ & Factor of Safety \\
\hline
\end{tabular}

Table 113: Slot yielding variable symbols and descriptions.

\begin{tabular}{|c|l|}
\hline & Symbol \\
\hline$P_{G}$ & Allowable Thrust Load Before Groove Deformation (lb) \\
\hline $\mathrm{D}$ & Shaft Diameter (in) \\
\hline $\mathrm{d}$ & Groove Depth (in) \\
\hline$S_{Y}$ & Groove Material Yield Strength (psi) \\
\hline $\mathrm{K}$ & Factor of Safety \\
\hline
\end{tabular}




\section{A.17.3 Values and Results}

The results of the drive and idler retaining ring calculations can be found in Table 114. The results of the tensioner shaft retaining ring calculation can be found in Table 115 .

Table 114: Input variable values for the drive and idler shaft retaining rings and the results of the calculation.

\begin{tabular}{|c|r|}
\hline Input Variable & Value \\
\hline $\mathrm{D}$ & \\
\hline $\mathrm{T}$ & $0.315 \mathrm{in}$ \\
\hline$S_{S}$ & $0.032 \mathrm{in}$ \\
\hline $\mathrm{d}$ & $45 \mathrm{kpsi}$ \\
\hline$S_{Y}$ & $0.008 \mathrm{in}$ \\
\hline $\mathrm{K}$ & $45 \mathrm{kpsi}$ \\
\hline Output Variable & 3 \\
\hline$P_{R}$ & Value \\
\hline$P_{G}$ & \\
\hline
\end{tabular}

Table 115: Input variable values for the tensioner shaft retaining rings and the results of the calculation.

\begin{tabular}{|c|c|}
\hline Variable & Value \\
\hline $\mathrm{D}$ & 0.375 in \\
\hline $\mathrm{T}$ & 0.025 in \\
\hline$S_{S}$ & $45 \mathrm{kpsi}$ \\
\hline $\mathrm{d}$ & 0.012 in \\
\hline$S_{Y}$ & $45 \mathrm{kpsi}$ \\
\hline $\mathrm{K}$ & 3 \\
\hline Output Variable & Value \\
\hline$P_{R}$ & $441 \mathrm{lbf}$ \\
\hline$P_{G}$ & $211 \mathrm{lbf}$ \\
\hline
\end{tabular}




\section{A.18 Drive Wheel Press Fit Calculation}

\section{A.18.1 Calculation Description}

This calculation was used to determine the required press fit to fix the drive wheel onto the drive shaft. It begins by determining the force required to hold the drive wheel in place. That force is then converted into a pressure and that is used to determine the required interference between the drive wheel and the drive shaft. It was assumed that these values would also be applicable to the idler wheel. All equations used for this calculator were obtained from [23].

\section{A.18.2 Required Pressure Calculation}

The first step was determining the friction force required to hold the drive wheel in place. Equation A.18.1 shows the calculation used.

$$
F_{r}=\frac{2}{D_{D S}} * T_{m} * F S
$$

This force value was then used to determine the required normal force in Equation A.18.2.

$$
N_{D W}=\frac{F_{r}}{\mu_{W-S}}
$$

This normal force was then converted to a pressure force using the area of the shaft as seen in Equation

A.18.3.

$$
P_{I}=\frac{N_{D W}}{\left(\pi * D_{D S} * w_{d w}\right)}
$$

Table 116 contains the variables and their values used to calculate the require interference pressure. Table 117 contains the results of this calculation.

Table 116: The input variable symbols, descriptions and values used to calculate the required press fit pressure.

\begin{tabular}{|c|l|r|}
\hline Symbol & \multicolumn{1}{|c|}{ Description } & \multicolumn{1}{|c|}{ Value } \\
\hline$D_{D S}$ & Drive Shaft Diameter & $0.4 \mathrm{in}$. \\
\hline$T_{m}$ & Motor Torque & 50 in-lbf \\
\hline$F S$ & Desired Safety Factor & 2 \\
\hline$\mu_{W-S}$ & Friction between drive shaft and drive wheel & 0.35 \\
\hline$w_{d w}$ & The Width of the Drive wheel & $2 \mathrm{in}$ \\
\hline
\end{tabular}


Table 117: The output variable symbols, descriptions and values from the required press fit pressure calculation.

\begin{tabular}{|c|r|r|}
\hline Symbol & Description & Value \\
\hline$F_{r}$ & Force required to resist the motor torque & $500 \mathrm{lbf}$ \\
\hline$N_{D W}$ & Normal Force Between the Drive wheel and the drive shaft & $1430 \mathrm{lbf}$ \\
\hline$P_{I}$ & The Required Interference Pressure & $570 \mathrm{psi}$ \\
\hline
\end{tabular}

\section{A.18.3 Required Interference Calculation}

Using Equation A.18.4, the required interference fit between the drive wheel and the drive shaft can be found.

$$
\delta=P_{I} * R_{D S} *\left[\frac{1}{E_{D W}} *\left(\frac{r_{d w}^{2}+R_{D S}^{2}}{r_{d w}^{2}-R_{D S}^{2}}+v_{D W}\right)+\frac{1}{E_{D S}} *\left(\frac{R_{D S}^{2}}{R_{D S}^{2}}-v_{D S}\right)\right.
$$

Table 118 contains the variables used in the calculation and the resulting interference required.

Table 118: Input variable symbols, descriptions and values used to calculate the required interference and the resulting interference.

\begin{tabular}{|c|l|r|}
\hline Variable & \multicolumn{1}{|c|}{ Description } & Value \\
\hline$P_{I}$ & The Required Interference Pressure & $570 \mathrm{psi}$ \\
\hline$R_{D S}$ & Radius of the Drive Shaft & $0.2 \mathrm{in}$. \\
\hline$E_{D W}$ & The Youngs modulus of ABS & $320 \mathrm{ksi}$ \\
\hline$r_{d w}$ & The outer radius of the drive wheel & $1.33 \mathrm{in}$. \\
\hline$v_{D W}$ & Poisons Ratio for ABS & 0.35 \\
\hline$E_{D S}$ & The Youngs modulus of steel & $29.9 \mathrm{Msi}$ \\
\hline$v_{D S}$ & Poisons ratio for Steel & 0.29 \\
\hline$\delta$ & The Required Interference & $0.001 \mathrm{in}$. \\
\hline
\end{tabular}




\section{A.19 Drive Wheel Validation}

\section{A.19.1 Simulation Description}

This simulation was used to validate the design of the drive wheel. The simulation was performed in Solidworks simulation package. It describes the boundary conditions and loading conditions that were applied to the wheel and how those conditions were determined. It reports the factor of safety, maximum Von Mises stress and the deflection of the bracket.

\section{A.19.2 Boundary Conditions}

The wheel was fixed at the tooth locations to simulate the engagement between the tread and the drive wheel. 8 sets of teeth were fixed as this was the estimated tooth engagement between the tread and the wheel. Figure 175 shows the location of the fixtures.

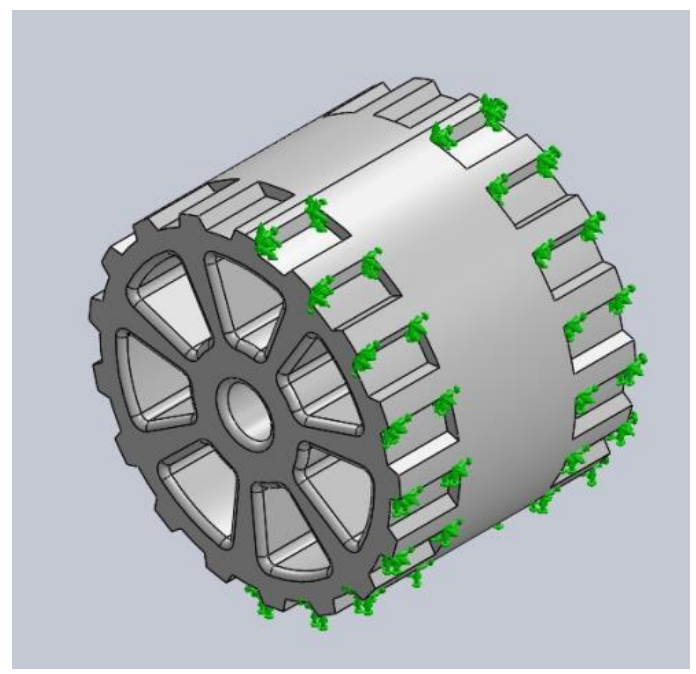

Figure 175: Fixed locations of drive wheel.

\section{A.19.3 Loading Conditions}

Three loads were applied to the wheel in the simulation. They were the pressure load due to press fitting the wheel onto the shaft, the motor torque and the belt tension. Table 119 gives the magnitudes of these loads and their location and directions can be seen in Figure 176. 
Table 119: Magnitudes of the loads applied to the drive wheel.

\begin{tabular}{|r|r|}
\hline Load & Magnitude \\
\hline Tread Tension & $110 \mathrm{lbf}$ \\
\hline Press Fit Pressure & $500 \mathrm{psi}$ \\
\hline Motor Torque & 50 in-lbf \\
\hline
\end{tabular}

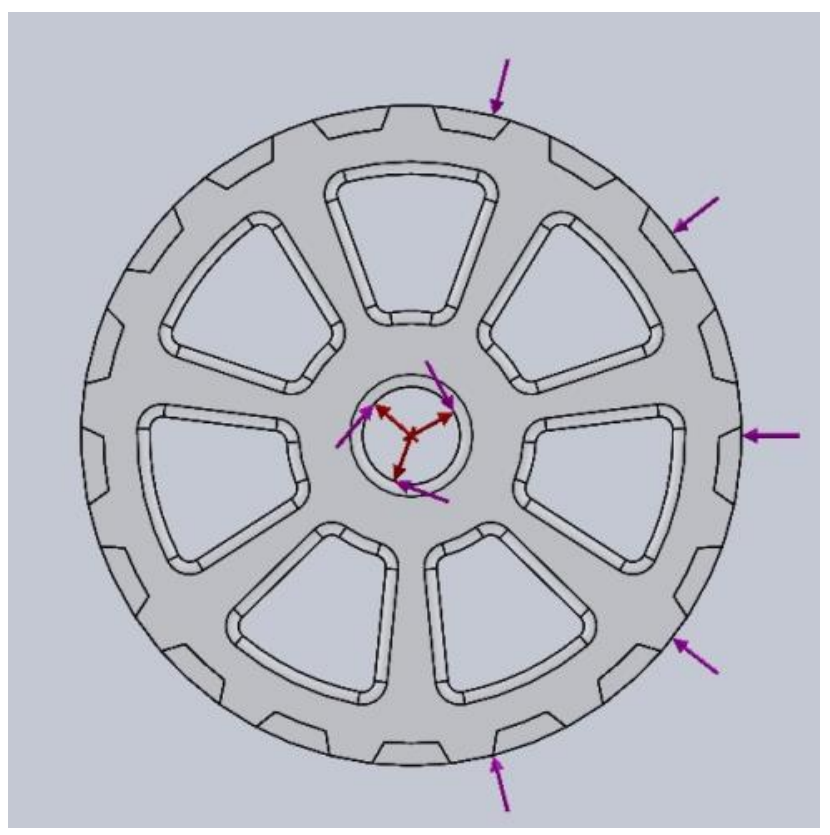

Figure 176: Locations of the loads applied to the drive wheel.

\section{A.19.4 Mesh}

The mesh for the wheel was auto generated by Solidworks. A fine mesh was chosen to increase the accuracy of the results. Figure 177 shows the meshed wheel.

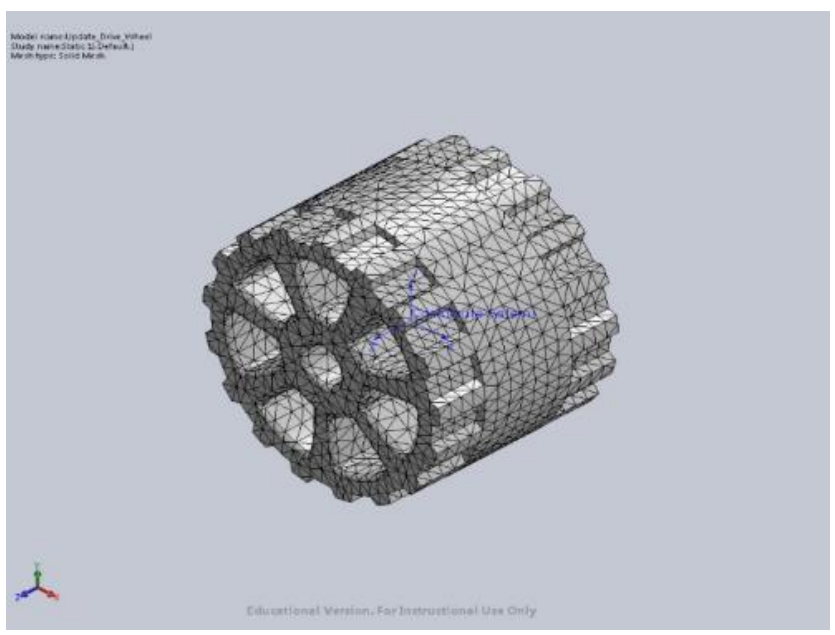

Figure 177: Meshed drive wheel. 


\section{A.19.5 Simulation Results}

Table 120 lists the results of the simulation. Figure 178, Figure 179 and Figure 180 show plots of the resultant deflection, resultant stresses and factor of safety respectively.

Table 120: Results from the drive wheel simulation.

\begin{tabular}{|r|r|}
\hline Simulation Result & Value \\
\hline Maximum Von Mises Stress & $1030 \mathrm{psi}$ \\
\hline Maximum Displacement & $0.001 \mathrm{in}$. \\
\hline Minimum Factor of Safety & 4.5 \\
\hline
\end{tabular}

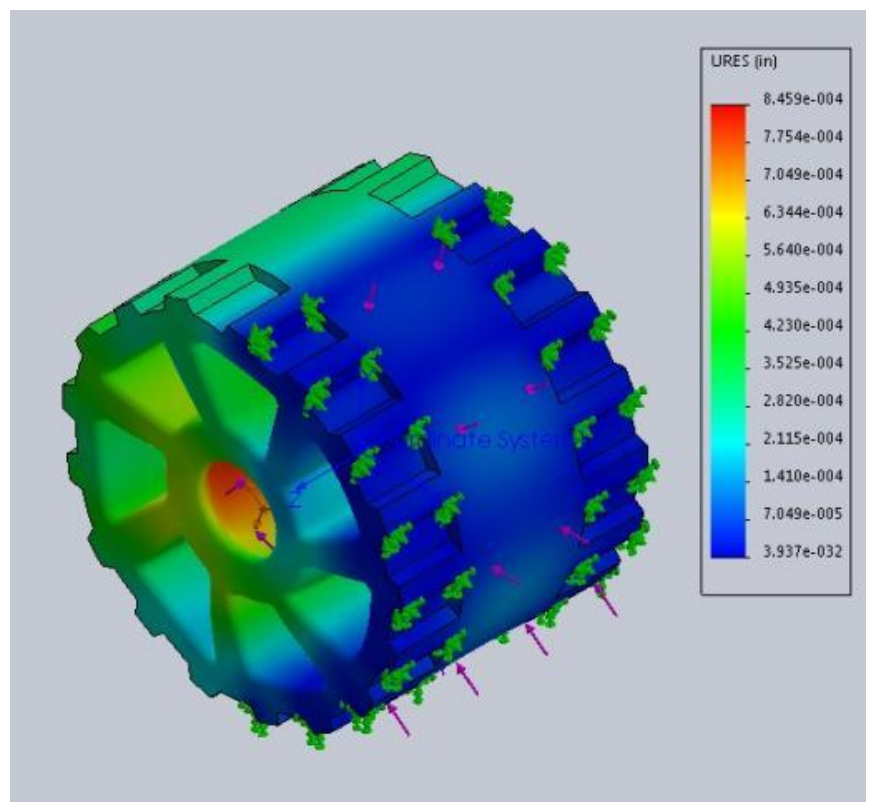

Figure 178: Deflection plot of the drive wheel. 


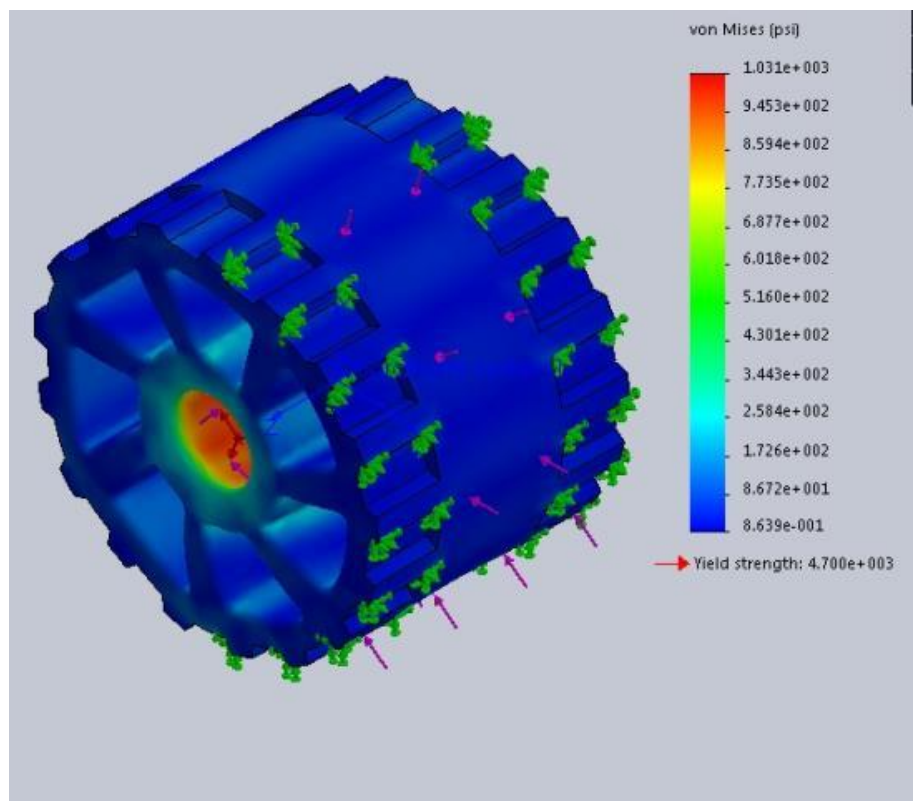

Figure 179: The Von Mises Stress plot of the drive wheel.

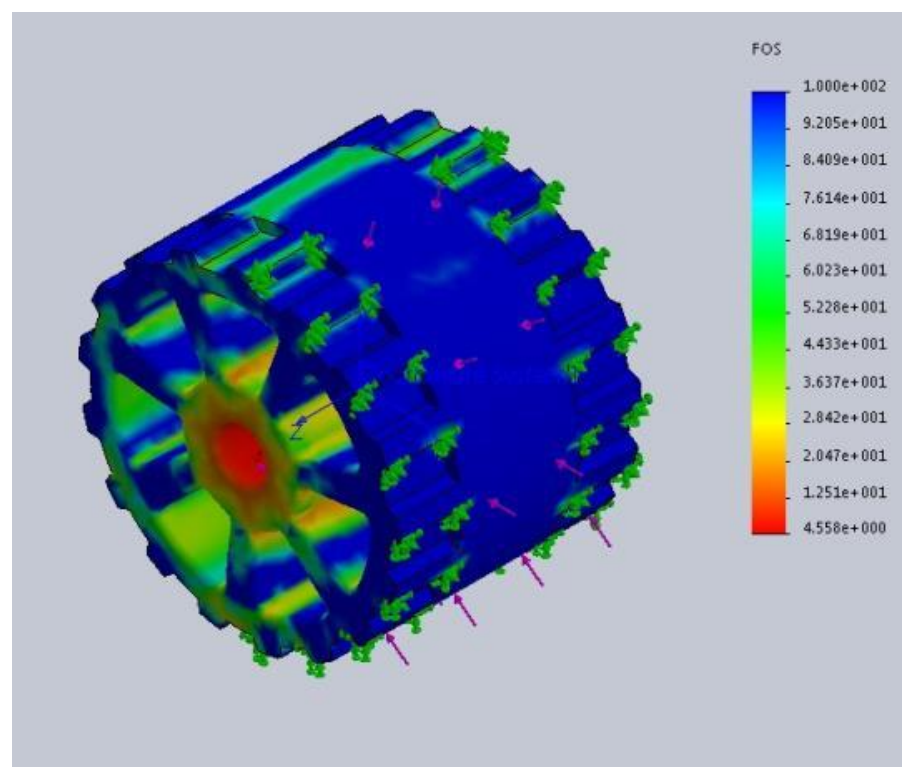

Figure 180: The Factor of safety plot of the drive wheel. 


\section{A.20 Brace Validation Simulation}

\section{A.20.1 Simulation Description}

This simulation was used to validate the design of the wheel stiffening brace. The simulation was performed in Solidworks simulation package. It describes the boundary conditions and loading conditions that were applied to the brace and how those conditions were determined. It reports the factor of safety, maximum Von Mises stress and the deflection of the bracket.

\section{A.20.2 Boundary Conditions}

The brace was fixed at the edges of bearing mounting holes. Figure 181 shows the fixed locations on the frame.

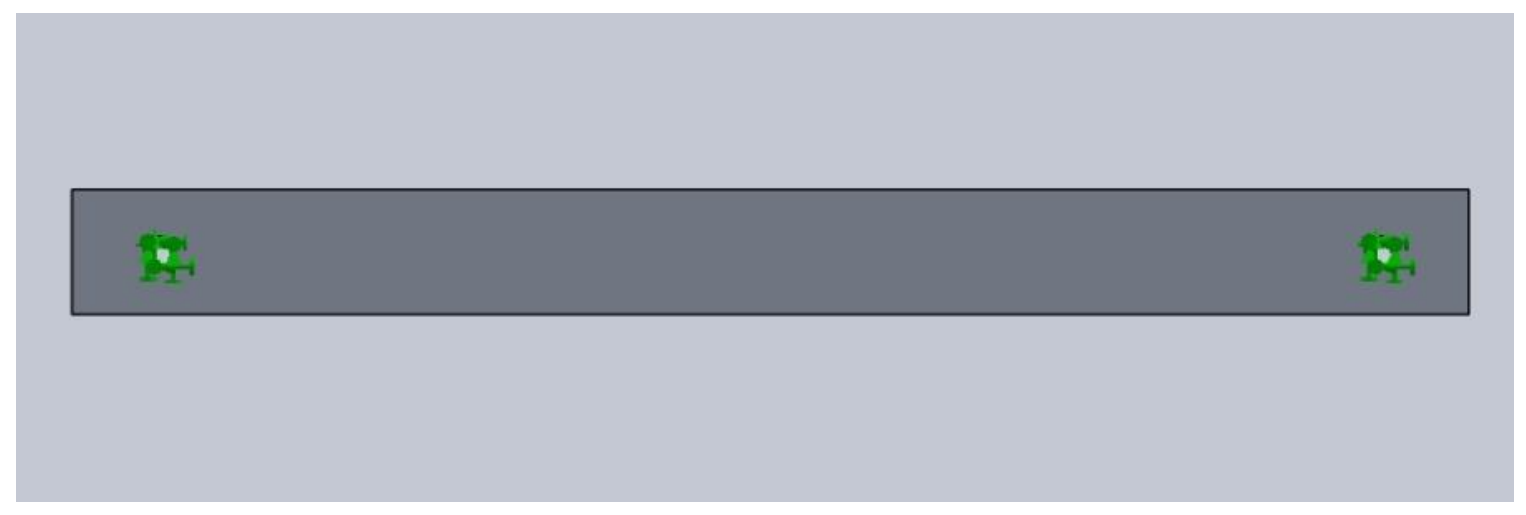

Figure 181: Fixed locations of the brace.

\section{A.20.3 Loading Conditions}

Two loads were applied to the brace at the bearing mounting holes. The first was the tread tension force which pointed at the center of the brace. The second were the bearing thrust loads which pointed towards the brace. Table 121 gives the magnitudes of these loads and their location and directions can be seen in Figure 182.

Table 121: Magnitudes of the loads applied to the brace.

\begin{tabular}{|r|r|}
\hline Load & Magnitude \\
\hline Tread Tension & $55 \mathrm{lbf}$ \\
\hline Bearing Thrust load & $6.4 \mathrm{lbf}$ \\
\hline
\end{tabular}




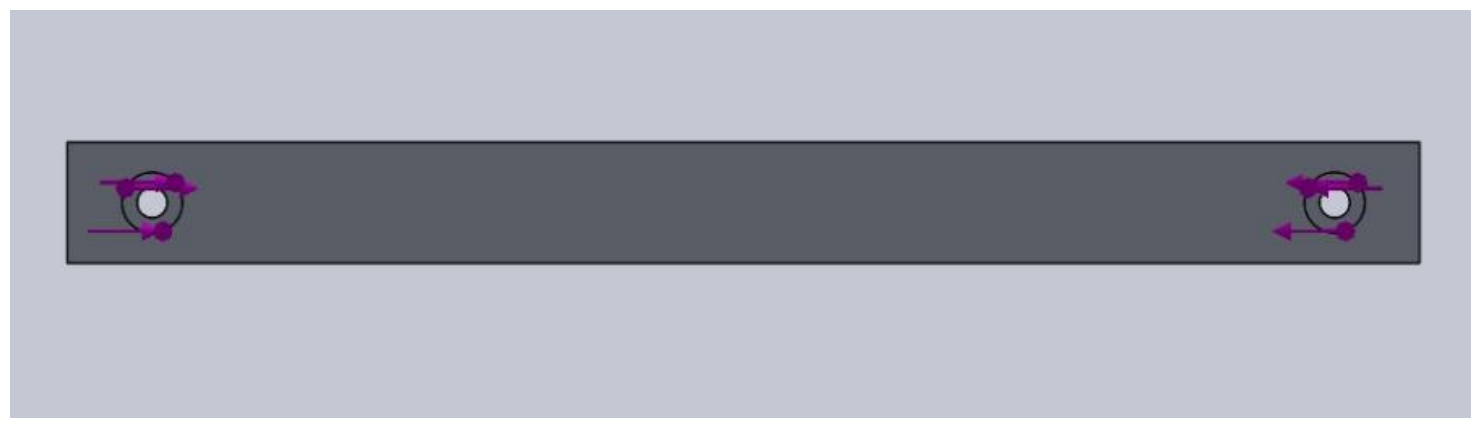

Figure 182: Locations of the loads applied to the brace.

\section{A.20.4 Mesh}

The mesh for the brace was auto generated by Solidworks. A fine mesh was chosen to increase the accuracy of the results. Figure 183 shows the meshed brace.

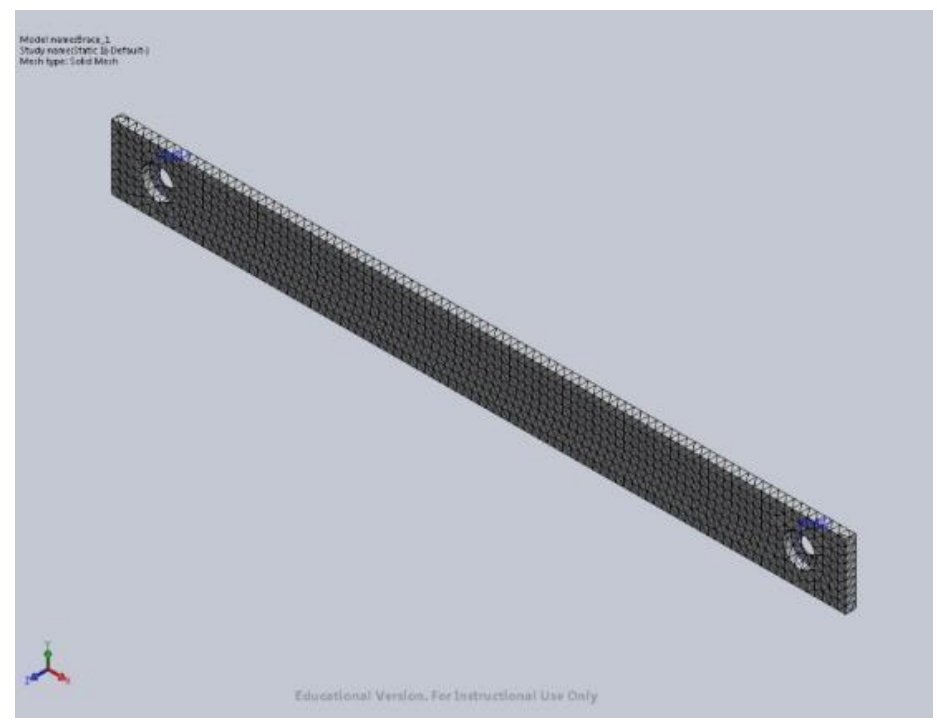

Figure 183: The meshed brace.

\section{A.20.5 Simulation Results}

Table 122 lists the results of the simulation. Figure 184, Figure 185 and Figure 186 show plots of the resultant deflection, resultant stresses and factor of safety respectively.

Table 122: Results from the motor plate simulation.

\begin{tabular}{|r|r|}
\hline Simulation Result & Value \\
\hline Maximum Von Mises Stress & $4,319 \mathrm{psi}$ \\
\hline Maximum Displacement & $0.002 \mathrm{in}$. \\
\hline Minimum Factor of Safety & 9.2 \\
\hline
\end{tabular}




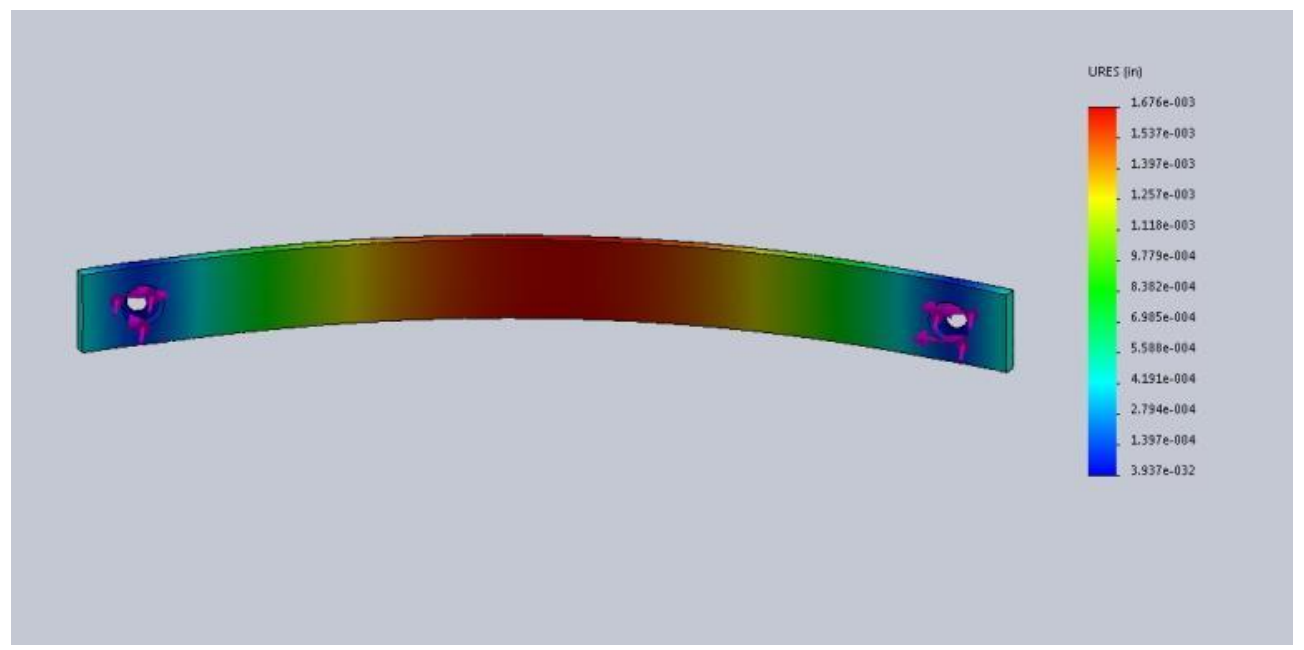

Figure 184: Deflection plot of the brace.

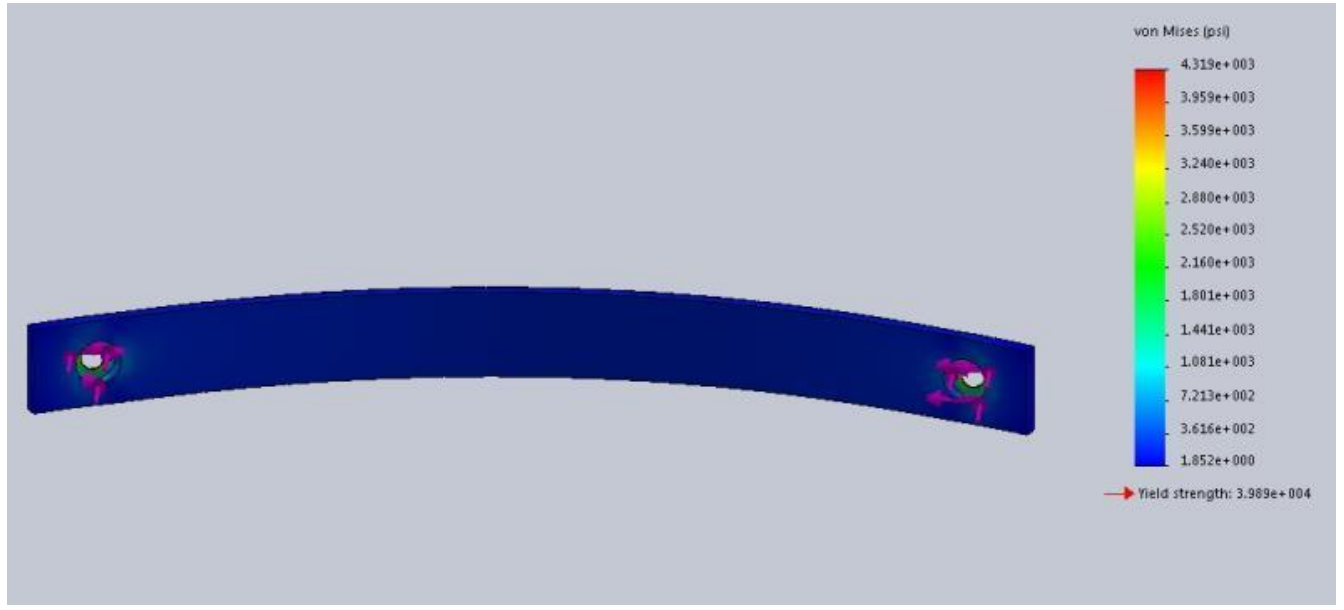

Figure 185: The Von Mises Stress plot of the brace.

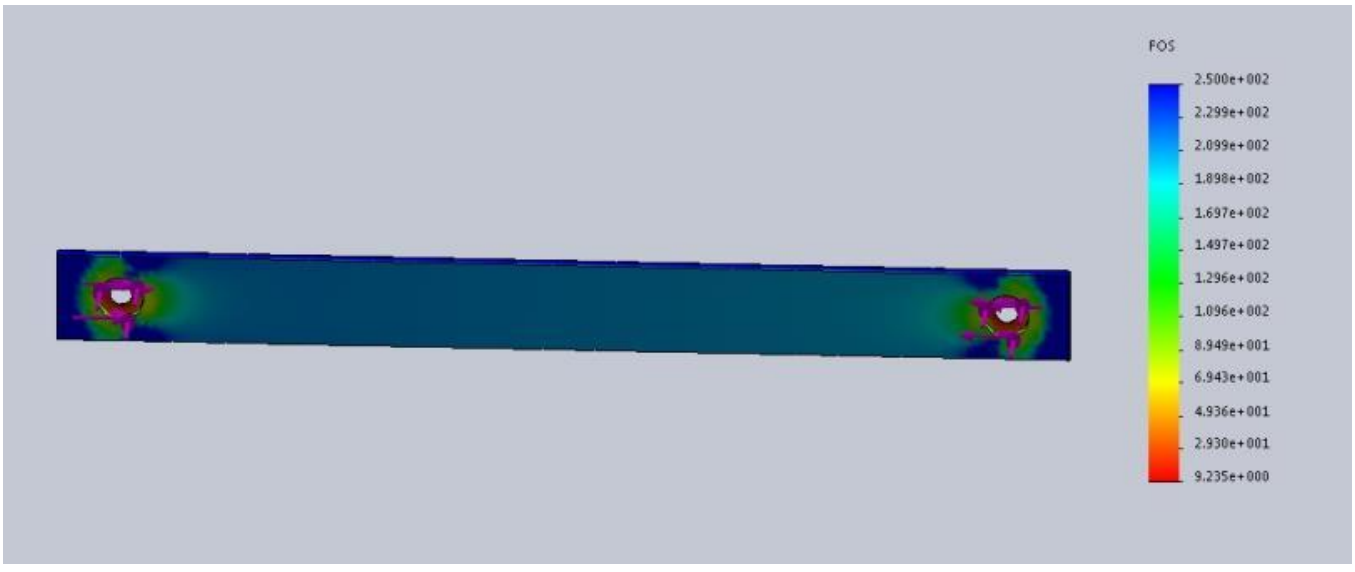

Figure 186: The Factor of safety plot of the brace. 


\section{A.21 Brace Buckling}

\section{A.21.1 Calculation Description}

This calculation checks the wheel brace for buckling. The results are used to justify the design of the brace.

\section{A.21.2 Solution}

Table 123 gives the known variables used in this calculation. The brace is assumed to have the both ends pivoted.

Table 123: Values used to calculate the buckling condition and factor of safety for the brace.

\begin{tabular}{|c|r|r|}
\hline Symbol & Description & Value \\
\hline$b$ & The base dimension of the brace & $0.1875 \mathrm{in}$. \\
\hline$h$ & The height dimension of the brace & $1.5 \mathrm{in}$. \\
\hline$l$ & The length of the beam & $12.25 \mathrm{in}$. \\
\hline$C$ & The constant used for the end conditions of the brace & 1 \\
\hline$E$ & The elastic modulus of aluminum 6061-t6 & $10 \mathrm{Msi}$ \\
\hline$S_{y}$ & The yield strength of aluminum 6061-T6 & $45 \mathrm{ksi}$ \\
\hline$P_{a}$ & The applied loading on the brace & $110 \mathrm{lbf}$ \\
\hline
\end{tabular}

The first thing that must be calculated is the radius of gyration of the brace, this is given by Equation

A.21.1 were $I_{b}$ is the area moment of inertia of the brace and $A_{b}$ is the area of the brace.

$$
k=\sqrt{\frac{I_{b}}{A_{b}}}=0.433 \mathrm{in} .
$$

The next step is to determine the slenderness ratio of the brace, which is given by Equation A.21.2.

$$
\frac{l}{k}=28.3
$$

The next step is to determine if the brace can be treated as an Euler column. This is done by calculating a minimum slenderness ratio and comparing that to the actual slenderness ratio. Equation A.21.3 gives the calculated minimum slenderness ratio.

$$
\left(\frac{l}{k}\right)_{1}=\sqrt{\frac{2 * \pi^{2} * C * E}{S_{y}}}=66.2
$$


Since the actual slenderness ratio is less than the minimum slenderness ratio, the brace must be treated as an intermediate length column. Equation A.21.4 solves for the critical load for the brace.

$$
P_{c r}=\left(S_{y}-\left(\frac{S_{y}}{2 * \pi} * \frac{l}{k}\right)^{2} * \frac{1}{C * E}\right) * A_{b}=12,600 \mathrm{lbf}
$$

The safety factor of the brace for buckling can be found by comparing the expected brace load with the critical load as seen in Equation A.21.5.

$$
\text { F.S. }=\frac{P_{c r}}{P_{a}}=114
$$




\section{A.22 Tensioner Shaft and Exterior Clamp Half Simulation}

\section{A.22.1 Simulation Description}

This simulation was used to validate the design of the tensioner shaft and exterior clamp half. The simulation was performed in Solidworks simulation package. It describes the boundary conditions and loading conditions that were applied to the shaft and clamp half and how those conditions were determined. It reports the factor of safety, maximum Von Mises stress and the deflection of the bracket.

\section{A.22.2 Boundary Conditions}

The model was fixed in two locations. Each of the threaded holes which attach the exterior and interior clamp halves were fixed. The back plate of the clamp half was fixed perpendicular to its surface to simulate the tensioner upright. Figure 187 shows the fixed locations of the model.



Figure 187: Fixed locations of the model. 


\section{A.22.3 Loading Conditions}

The only load applied to the model was at the shaft location. This was to simulate the portion of the tension load applied down on the shaft. Its magnitude was determined to be $55 \mathrm{lbf}$. Figure 188 shows the tension load on the shaft.

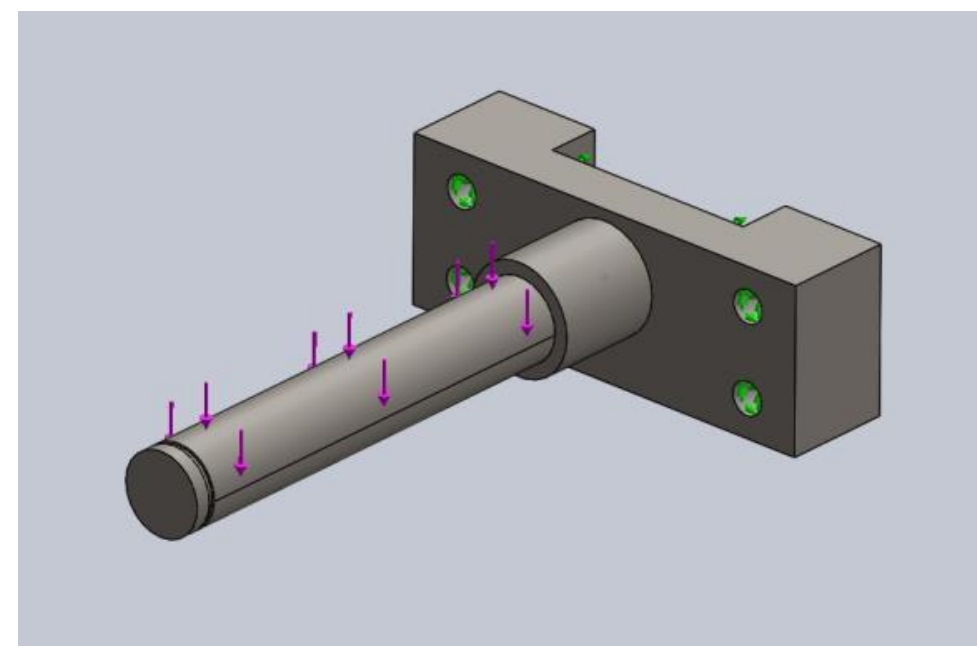

Figure 188: Tensioner shaft and exterior clamp loading.

\section{A.22.4 Mesh}

The mesh for the wheel was auto generated by Solidworks. A fine mesh was chosen to increase the accuracy of the results. Figure 189 shows the meshed wheel.

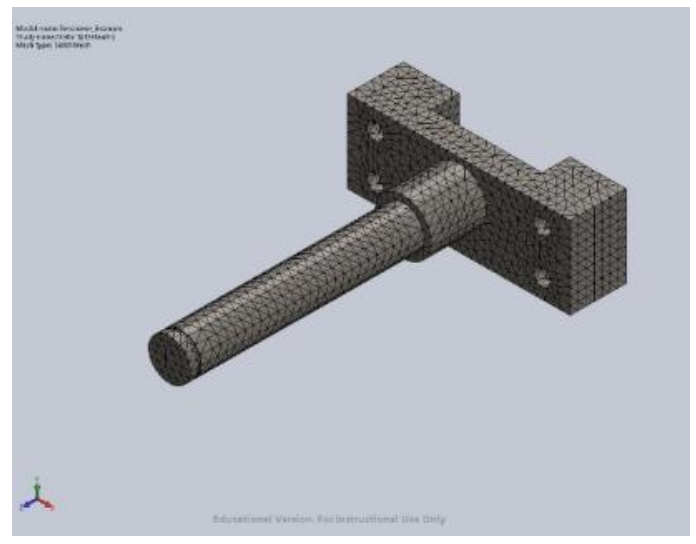

Figure 189: Meshed tensioner shaft and exterior clamp half. 


\section{A.22.5 Simulation Results}

Table 124 lists the results of the simulation. Figure 190, Figure 191 and Figure 192 show plots of the resultant deflection, resultant stresses and factor of safety respectively.

Table 124: Results from the tensioner shaft and exterior clamp half simulation.

\begin{tabular}{|r|r|}
\hline Simulation Result & Value \\
\hline Maximum Von Mises Stress & $11,850 \mathrm{psi}$ \\
\hline Maximum Displacement & 0.003 in. \\
\hline Minimum Factor of Safety & 6.5 \\
\hline
\end{tabular}

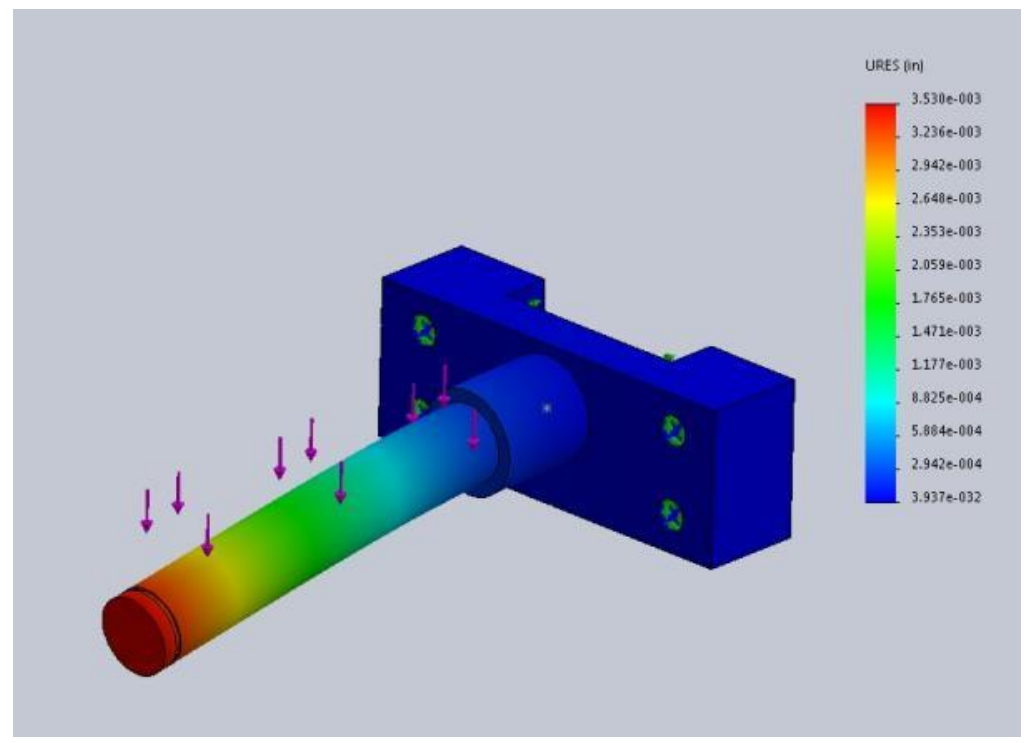

Figure 190: Deflection plot of the tensioner shaft and exterior clamp half.

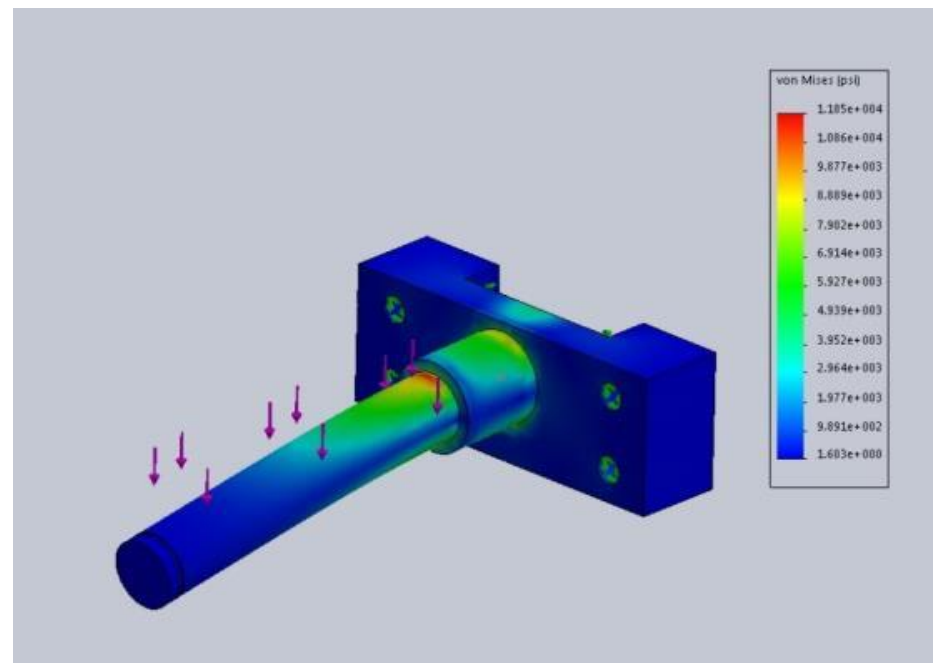

Figure 191: The Von Mises Stress plot of the tensioner shaft and exterior clamp half. 


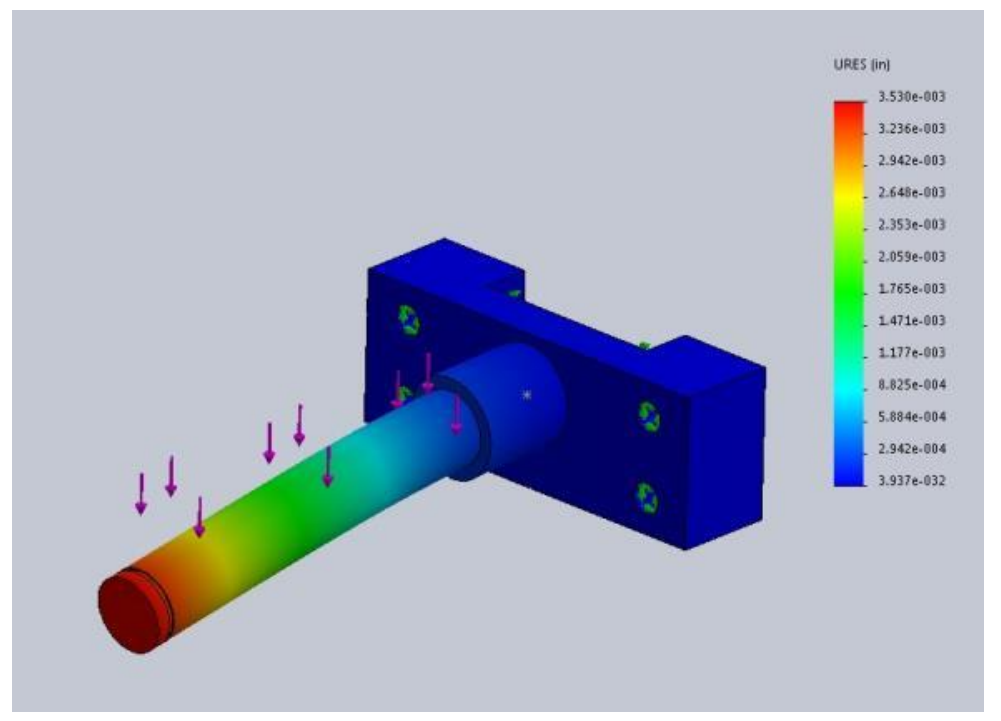

Figure 192: The Factor of safety plot of the tensioner shaft and exterior clamp half. 


\section{A.23 Tensioner Design Validation}

\section{A.23.1 Calculation Description}

This calculation was intended to validate the design of the tensioner system. This was done using Solidworks belt tool. It describes the process used to determine the tension developed in the belt as the tensioner is raised. It reports the tension in the belt.

\section{A.23.2 Procedure}

The procedure used to determine the tension in the treads as the tensioner is raised is relatively simple. Since the pitch length and the material composition of the treads is known, the tension in the belt can be estimated by checking the extension of the belt as the tensioner is raised. To do this, a model of the wheel system was created. The Solidworks belt tool was then used to wrap a belt around the drive, idler and tensioner wheels, as shown in Figure 193.

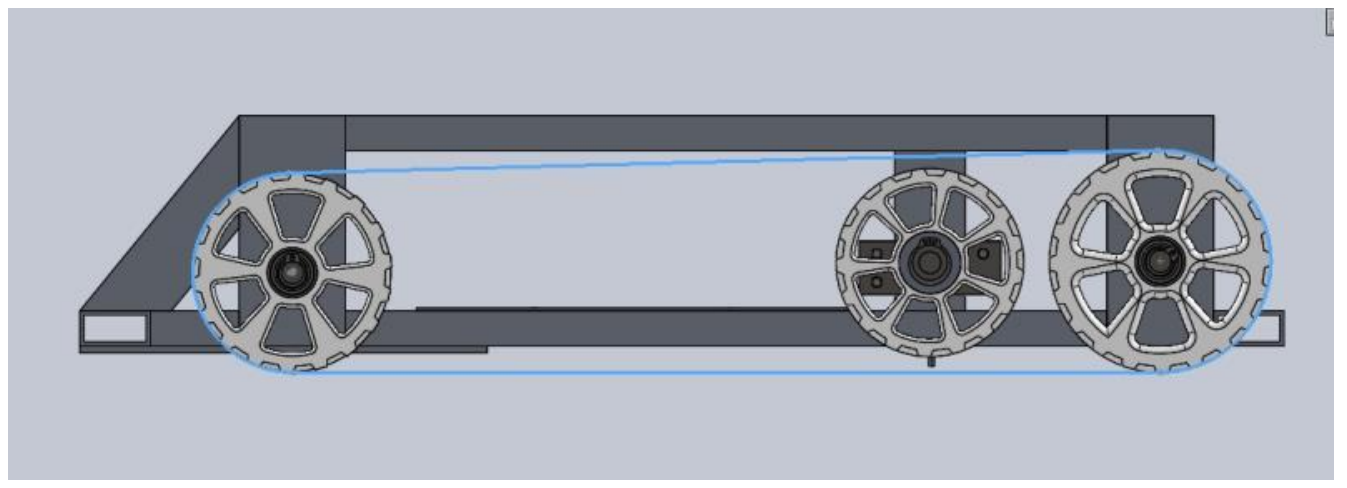

Figure 193: The slack condition of the belt. Belt length 33.83 in.

The tensioner assembly was then raised and the length of the belt was recorded. The actual tread length was then subtracted from this value to determine the strain in the belt. This strain value was then multiplied by the elastic modulus of the belt, which was assumed to be dominated by the modulus of the steel reinforcement cables, to determine the stress in the tread. This stress was then converted into a tension force. 


\section{A.23.3 Results}

Figure 194 shows the simulation when the tensioner has reached its assumed minimum distance to the top of the vehicle frame. This distance was determined based on the assumed weld bead dimensions, which limits how close the tensioner can get to the top of the vehicle. Table 125 gives the parameters used in the calculation of the developed tension at this height.

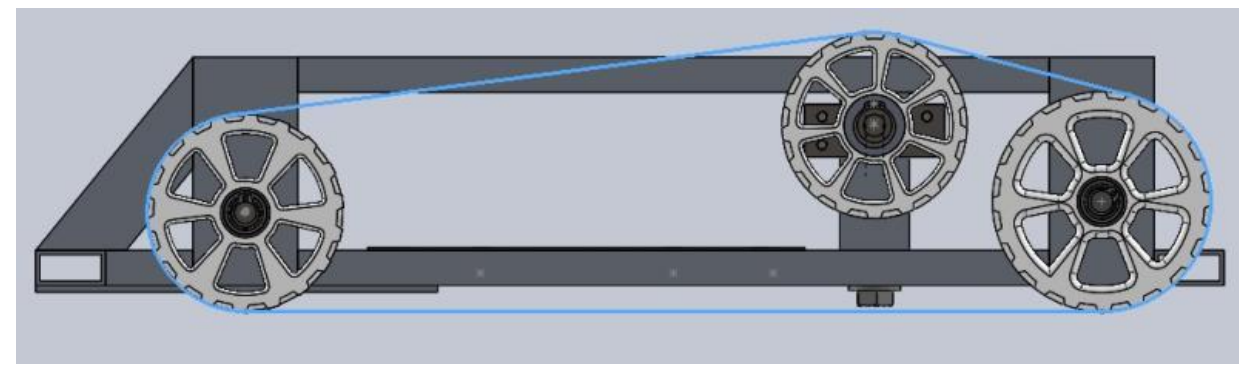

Figure 194: The assumed maximum tensioner height. Belt length 34.01 in.

Table 125: Parameters used to calculate belt tension.

\begin{tabular}{|c|l|r|}
\hline Symbol & \multicolumn{1}{|c|}{ Variable Description } & \multicolumn{1}{|c|}{ Value } \\
\hline$d_{T}$ & Tensioner distance from Top of frame & $0.171 \mathrm{in}$. \\
\hline$L_{T C}$ & Calculated Tread Length & $34.01 \mathrm{in}$. \\
\hline$L_{T A}$ & Actual belt Length & $34.00 \mathrm{in}$. \\
\hline$E_{S}$ & Young's Modulus of Steel cables & $29 \mathrm{Mpsi}$ \\
\hline$N_{C}$ & Number of Cables across width of the belt & 8 \\
\hline$d_{C}$ & Cable Diameter & $0.0625 \mathrm{in}$. \\
\hline
\end{tabular}

The strain developed in the tread is given by Equation B.22.1.

$$
\varepsilon_{T}=\frac{L_{T C}-L_{T A}}{L_{T A}}
$$

This strain was then used to calculate the stress in the tread using Equation B.22.2.

$$
\sigma_{T}=E_{S} * \varepsilon_{T}
$$

The tension in the tread was then calculated using Equation B.22.3.

$$
T_{T}=\sigma_{T} * N_{C} * \frac{\pi}{4} * d_{C}{ }^{2}
$$

Table 126 contains the results of the calculation.

Table 126: Results of the tensioner design validation calculation.

\begin{tabular}{|c|r|r|}
\hline Symbol & Description & \multicolumn{1}{|c|}{ Value } \\
\hline$\varepsilon_{T}$ & Belt Strain & $2.9 \times 10^{\wedge}-4 \mathrm{in} . / \mathrm{in}$. \\
\hline$\sigma_{T}$ & Belt Stress & $8529 \mathrm{psi}$ \\
\hline$T_{T}$ & Belt Tension & $209 \mathrm{lbf}$ \\
\hline
\end{tabular}




\section{Appendix B - Bill of Materials}

This section gives a breakdown of the parts and raw materials used in the project. This includes the cost, supplier and quantity of items purchased for the project. The bill of materials has been broken down by subsystem's which correspond to those found in Chapter 2.

Table 127: Frame raw material supplier, quantity and cost breakdown.

\begin{tabular}{|c|c|c|c|c|}
\hline Part Description & Supplier & SKU & Quantity & Cost \\
\hline 1/4"-20 5/8" Low-Profile Alloy Steel SHCS & McMaster & 92220A184 & $(1$ Box of 50) & $\$ 9.14$ \\
\hline $\begin{array}{l}\text { 1/4"-20 Zinc Aluminum Coated Steel Hex } \\
\text { Nut }\end{array}$ & McMaster & 93827A211 & (1 Box of 100) & $\$ 6.74$ \\
\hline 18 "x18"x0.04" Aluminum 6061 Sheet & McMaster & $89015 \mathrm{~K} 169$ & 1 & $\$ 28.80$ \\
\hline $\begin{array}{l}.5 " x 1 " x 3 \text { ' } 0.0625 " \text { Thick } 6061 \text { Aluminum } \\
\text { Tubing }\end{array}$ & McMaster & 6546K51 & 4 & $\$ 35.44$ \\
\hline $\begin{array}{l}\text { 1"x1.5"x3' 1/8"Thick } 6061 \text { Aluminum } \\
\text { Tubing }\end{array}$ & McMaster & 6546K38 & 1 & $\$ 21.15$ \\
\hline 1/4"-20 1/2" Low-Profile Alloy Steel SHCS & McMaster & 92220A183 & $8(1$ Box of 50$)$ & $\$ 9.15$ \\
\hline 1/4"-20 1" Low-Profile Alloy Steel SHCS & McMaster & 92220A186 & $8(1$ Box of 50$)$ & $\$ 11.48$ \\
\hline 3/16"x1.5"x3' 6061 Aluminum Bar & McMaster & $8975 \mathrm{~K} 588$ & 1 & $\$ 8.32$ \\
\hline \multicolumn{4}{|c|}{ Total } & $\$ 130.22$ \\
\hline
\end{tabular}

Table 128: Motor raw material supplier, quantity and cost breakdown.

\begin{tabular}{|c|c|c|c|c|}
\hline Part Description & Supplier & SKU & Quantity & Cost \\
\hline 2"x2'x3/32" 6061 Aluminum Plate & McMaster & $8975 \mathrm{~K} 342$ & 1 & $\$ 4.83$ \\
\hline Compact Square-Face DC Gearmotor & McMaster & $6409 \mathrm{~K} 26$ & 2 & $\$ 106.32$ \\
\hline $\begin{array}{l}3 " x 2 " X 2^{\prime} 3 / 16 \text { Thick } 6061 \text { Aluminum } 90^{\circ} \\
\text { Angle }\end{array}$ & $\begin{array}{r}\text { Metals } \\
\text { Depot }\end{array}$ & A332316 & 1 & $\$ 17.40$ \\
\hline Type 18-8 Stainless Steel Narrow Hex Nut & McMaster & 90730A411 & $8(1$ Box of 100$)$ & $\$ 5.92$ \\
\hline 5/16" ShaftDia. Straight Shaft Coupler & McMaster & $6412 \mathrm{~K} 12$ & 2 & $\$ 15.56$ \\
\hline \multicolumn{4}{|c|}{ Total } & $\$ 150.03$ \\
\hline
\end{tabular}

Table 129: Vacuum raw material supplier, quantity and cost breakdown.

\begin{tabular}{|l|r|r|r|r|}
\hline \multicolumn{1}{|r|}{ Part Description } & Supplier & SKU & Quantity & Cost \\
\hline Miniature J-Series Fixed Venturi Vacuum Pump & Vaccon & JS-90M-AA4 & 8 & $\$ 572$ \\
\hline Push-to-Connect Tube Fitting for Air - Straight & McMaster & $5779 \mathrm{~K} 108$ & 8 & $\$ 24.64$ \\
\hline $\begin{array}{l}\text { Push-to-Connect Tube Fitting for Air - Swivel 90 } \\
\text { Elbow }\end{array}$ & McMaster & $5779 \mathrm{~K} 151$ & 8 & $\$ 24.96$ \\
\hline 2"x24" 1/16" Thick 6061 Aluminum Sheet & McMaster & $89015 \mathrm{~K} 181$ & 1 & $\$ 7.08$ \\
\hline Low-Profile Alloy Steel Socket Head Cap Screw & McMaster & $92220 \mathrm{~A} 178$ & 2 & $\$ 6.07$ \\
\hline Nylon Manifold & McMaster & $5253 \mathrm{~K} 302$ & 1 & $\$ 29.75$ \\
\hline Hex-Head Polypropylene Plug & McMaster & $4515 \mathrm{~K} 211$ & 1 & $\$ 1.03$ \\
\hline $\begin{array}{l}\text { Push-to-Connect Tube Fitting for Air Straight } \\
\text { Adapter }\end{array}$ & McMaster & $5779 \mathrm{~K} 117$ & & $\$ 5.30$ \\
\hline \multicolumn{2}{|c|}{ Total } & & $\$ 670.83$ \\
\hline
\end{tabular}


Table 130: Manifold raw material supplier, quantity and cost breakdown.

\begin{tabular}{|c|c|c|c|c|}
\hline Part Description & Supplier & $\begin{array}{r}\text { Supplier } \\
\text { SKU }\end{array}$ & Quantity & Cost \\
\hline White Delrin ${ }^{\circledR}$ Acetal Resin Rectangular Bar & McMaster & $8739 \mathrm{~K} 74$ & 1 & $\$ 70.44$ \\
\hline $\begin{array}{r}\text { Multipurpose } 6061 \text { Aluminum Rectangular } \\
\text { Tube }\end{array}$ & McMaster & $6546 \mathrm{~K} 47$ & 1 & $\$ 4.29$ \\
\hline Multipurpose 6061 Aluminum Rod & McMaster & $8974 \mathrm{~K} 22$ & 1 & $\$ 2.00$ \\
\hline Steel Compression Spring $0.375^{\prime \prime} \mathrm{L}$ & McMaster & $9657 \mathrm{~K} 265$ & $4(1$ Box of 12$)$ & $\$ 10.35$ \\
\hline Steel Compression Spring 0.75" & McMaster & $9657 \mathrm{~K} 288$ & $4(1$ Box of 12$)$ & $\$ 10.35$ \\
\hline $\begin{array}{r}\text { Push-to-Connect Tube Fitting for Air Double } \\
90 \text { Degree Elbow }\end{array}$ & McMaster & $5779 \mathrm{~K} 254$ & $8(1$ Box of 100$)$ & $\$ 102.24$ \\
\hline $\begin{array}{r}\text { Black-Oxide Alloy Steel Socket Head Cap } \\
\text { Screw }\end{array}$ & McMaster & 91251A144 & 16 & $\$ 8.46$ \\
\hline Fiberglass Fabric-Reinforced Silicone Rubber & McMaster & $8612 \mathrm{~K} 51$ & 1 & $\$ 18.35$ \\
\hline \multicolumn{4}{|c|}{ Total } & $\$ 226.48$ \\
\hline
\end{tabular}

Table 131: Wheel raw material supplier, quantity and cost breakdown.

\begin{tabular}{|c|c|c|c|c|}
\hline Part Description & Supplier & $\begin{array}{r}\text { Supplier } \\
\text { SKU }\end{array}$ & Quantity & Cost \\
\hline .5 " Dia. 3' 1045 Medium Carbon Steel Rod & McMaster & $8279 \mathrm{~T} 27$ & 1 & $\$ 20.22$ \\
\hline $\begin{array}{r}\text { 8mm Black-Finish Steel External Retaining } \\
\text { Ring }\end{array}$ & McMaster & 98541A116 & 6 & $\$ 8.00$ \\
\hline 3/8" Shaft Brass Thrust Bearing & McMaster & $7447 \mathrm{~K} 3$ & 4 & $\$ 5.32$ \\
\hline $\begin{array}{r}\text { Flanged Stainless Steel Ball Bearing - } \\
\text { ABEC-5 }\end{array}$ & McMaster & 7804K147 & 8 & $\$ 57.20$ \\
\hline Drive Wheel & Cal Poly & N/A & 2 & $\$ 140.00$ \\
\hline Idler Wheel & Cal Poly & N/A & 2 & $\$ 160.00$ \\
\hline \multicolumn{4}{|c|}{ Total } & $\$ 390.74$ \\
\hline
\end{tabular}

Table 132: Tensioner raw material supplier, quantity and cost breakdown.

\begin{tabular}{|c|c|c|c|c|}
\hline Part Description & Supplier & $\begin{array}{r}\text { Supplier } \\
\text { SKU }\end{array}$ & Quantity & Cost \\
\hline 1/2"x3/4"x6"Low-Carbon Steel Rectangular & McMaster & $8910 \mathrm{~K} 693$ & 1 & $\$ 4.34$ \\
\hline $\begin{array}{r}\text { 1/2"x3/4"x 6" 6061 Aluminum Rectangular } \\
\text { Bar }\end{array}$ & McMaster & $8975 \mathrm{~K} 618$ & 1 & $\$ 2.41$ \\
\hline 1/4"-20 3"High-Strength Steel Cap Screw & McMaster & 92620A553 & $2(1$ Box of 5$)$ & $\$ 9.47$ \\
\hline 3/8" Dia. Steel External Retaining Ring & McMaster & 97633A170 & $2(1$ Box of 100$)$ & $\$ 8.74$ \\
\hline 3/8" Dia. Bronze Flange Bearing & McMaster & 9440T54 & 4 & $\$ 10.72$ \\
\hline Tensioner Wheel & Cal Poly & N/A & 2 & $\$ 120.00$ \\
\hline \multicolumn{4}{|c|}{ Total } & $\$ 155.68$ \\
\hline
\end{tabular}

Table 133: Tread raw material supplier, quantity and cost breakdown.

\begin{tabular}{|r|r|r|r|r|}
\hline Part Description & Supplier & $\begin{array}{r}\text { Supplier } \\
\text { SKU }\end{array}$ & Quantity & Cost \\
\hline Custom Timing Belt & F.N. Sheppard & N/A & 2 & $\$ 574.98$ \\
\hline
\end{tabular}




\begin{tabular}{|r|r|r|r|r|}
\hline 5.3-oz. High-Vacuum Grease & Mcmaster & 2966K52 & 1 & $\$ 29.19$ \\
\hline Silicone Sealant & ACE & 1012228 & 1 & $\$ 6.99$ \\
\hline \multicolumn{3}{|c|}{ Total } & & $\$ 611.16$ \\
\hline
\end{tabular}




\section{Appendix C - Vehicle Weight Break Down}

Table 134: Weight breakdown for left side components.

\begin{tabular}{|l|r|r|}
\hline \multicolumn{1}{|c|}{ Component Name } & Weight (oz.) & Weight (lbf) \\
\hline Tread & 9 & 0.56 \\
\hline Wheel Assembly & 15.8 & 0.99 \\
\hline Idler Wheel and Shaft & 4.6 & 0.29 \\
\hline Drive Wheel and Shaft & 4.9 & 0.31 \\
\hline Brace & 6.4 & 0.40 \\
\hline Manifold With Fasteners & 8.8 & 0.55 \\
\hline Venturi Bank & 10.5 & 0.66 \\
\hline Venturi Bracket & 0.5 & 0.03 \\
\hline Tensioner Assembly & 9.2 & 0.58 \\
\hline Interior Clamp Half & 1.3 & 0.08 \\
\hline Screw Assembly & 0.65 & 0.04 \\
\hline Tensioner Shaft and Exterior Clamp Half & 3.7 & 0.23 \\
\hline Tensioner Wheel & 3.4 & 0.21 \\
\hline Motor Assembly & 26.6 & 1.66 \\
\hline Mounting Plate & 1.4 & 0.09 \\
\hline Coupler & 0.9 & 0.06 \\
\hline Mounting Bracket & 2.8 & 0.18 \\
\hline Motor & 18.6 & 1.16 \\
\hline
\end{tabular}

Table 135: Weight breakdown for right side components.

\begin{tabular}{|l|r|r|}
\hline Tread & Weight (oz.) & Weight (lbf) \\
\hline Wheel Assembly & 9.1 & 0.57 \\
\hline Idler Wheel and Shaft & 15.5 & 0.97 \\
\hline Drive Wheel and Shaft & 4.7 & 0.29 \\
\hline Brace & 4.4 & 0.28 \\
\hline Manifold With Fasteners & 6.5 & 0.41 \\
\hline Venturi Bank & 8.8 & 0.55 \\
\hline Venturi Bracket & 10.2 & 0.64 \\
\hline Tensioner Assembly & 0.4 & 0.03 \\
\hline Interior Clamp Half & 9 & 0.56 \\
\hline Screw Assembly & 1.3 & 0.08 \\
\hline Tensioner Shaft and Exterior Clamp Half & 0.7 & 0.04 \\
\hline Tensioner Wheel & 3.7 & 0.23 \\
\hline Motor Assembly & 3.4 & 0.21 \\
\hline Mounting Plate & 26.6 & 1.66 \\
\hline Coupler & 1.4 & 0.09 \\
\hline
\end{tabular}




\begin{tabular}{|l|r|r|}
\hline Mounting Bracket & 0.9 & 0.06 \\
\hline Motor & 2.9 & 0.18 \\
\hline Tread & 18.6 & 1.16 \\
\hline
\end{tabular}

Table 136: Weight breakdown for other vehicle components.

\begin{tabular}{|l|r|r|}
\hline \multicolumn{1}{|c|}{ Component Name } & Weight (oz.) & \multicolumn{1}{c|}{ Weight (lbf) } \\
\hline Inlet Manifold & 6.1 & 0.38 \\
\hline Frame Plate Fastener & 52.6 & 3.29 \\
\hline Plate & 6.5 & 0.41 \\
\hline Frame & 45.4 & 2.84 \\
\hline
\end{tabular}

Table 137: The final weight of the vehicle. 


\section{Appendix D - Testing Data}

Table 138: Data for Fixed Right Manifold Unclogged Test.

\begin{tabular}{|c|c|c|c|c|}
\hline Section & 1 & 2 & 3 & 4 \\
\hline Venturi Power & 26.5 & 27 & 27 & 27 \\
\hline Section & 1 & 2 & 3 & 4 \\
\hline All Sections Closed - Initial & 19 & 25 & 25 & 25 \\
\hline Section & 1 & 2 & 3 & 4 \\
\hline All Sections Closed - Final & 19 & 25 & 25 & 25 \\
\hline Section & 1 & 2 & 3 & 4 \\
\hline \multirow{4}{*}{ Pressure in Section 1 (inHg) } & 18 & closed & closed & closed \\
\hline & 8 & open & closed & closed \\
\hline & 6 & open & open & closed \\
\hline & 11.5 & open & open & open \\
\hline Section & 1 & 2 & 3 & 4 \\
\hline \multirow{4}{*}{ Pressure in Section 2 (inHg) } & closed & 25 & closed & closed \\
\hline & open & 24 & closed & closed \\
\hline & open & 22 & open & closed \\
\hline & open & 21.5 & open & open \\
\hline Section & 1 & 2 & 3 & 4 \\
\hline \multirow{4}{*}{ Pressure in Section 3 (inHg) } & closed & closed & 24 & closed \\
\hline & open & closed & 24 & closed \\
\hline & open & open & 24 & closed \\
\hline & open & open & 21.5 & open \\
\hline Section & 1 & 2 & 3 & 4 \\
\hline \multirow{4}{*}{ Pressure in Section 4 (inHg) } & closed & closed & closed & 25 \\
\hline & open & closed & closed & 25 \\
\hline & open & open & closed & 25 \\
\hline & open & open & open & 24 \\
\hline
\end{tabular}

Table 139: Data for Unfixed Right Manifold Unclogged Test.

\begin{tabular}{|c|c|c|c|c|}
\hline Section & 1 & 2 & 3 & 4 \\
\hline Venturi Pressure (inHg) & 27 & 27.5 & 26 & 26 \\
\hline Section & 1 & 2 & 3 & 4 \\
\hline All Sections Closed - Initial (inHg) & 20 & 25 & 25 & 24 \\
\hline Section & 1 & 2 & 3 & 4 \\
\hline All Sections Closed - Final (inHg) & 20 & 25 & 24 & 25 \\
\hline Section & 1 & 2 & 3 & 4 \\
\hline \multirow{4}{*}{ Pressure in Section 1 (inHg) } & 20 & closed & closed & closed \\
\hline & 15 & open & closed & closed \\
\hline & 13 & open & open & closed \\
\hline & 20 & open & open & open \\
\hline Section & 1 & 2 & 3 & 4 \\
\hline \multirow{4}{*}{ Pressure in Section 2 (inHg) } & closed & 25 & closed & closed \\
\hline & open & 24 & closed & closed \\
\hline & open & 22 & open & closed \\
\hline & open & 21.5 & open & open \\
\hline Section & 1 & 2 & 3 & 4 \\
\hline \multirow{3}{*}{ Pressure in Section 3 (inHg) } & closed & closed & 24 & closed \\
\hline & open & closed & 24 & closed \\
\hline & open & open & 22.5 & closed \\
\hline
\end{tabular}




\begin{tabular}{|c|r|r|r|r|}
\hline & open & open & 19 & \multicolumn{2}{|c|}{ open } \\
\hline \multirow{2}{*}{ Section } & $\mathbf{1}$ & $\mathbf{2}$ & $\mathbf{3}$ & $\mathbf{4}$ \\
\hline \multirow{3}{*}{ Pressure in Section 4 (inHg) } & closed & closed & closed & 25 \\
\cline { 2 - 5 } & open & closed & closed & 25 \\
\cline { 2 - 5 } & open & open & closed & 25 \\
\cline { 2 - 5 } & open & open & open & 22 \\
\hline
\end{tabular}

Table 140: Data for the right manifold independence unclogged test.

\begin{tabular}{|c|c|c|c|c|}
\hline Section & 1 & 2 & 3 & 4 \\
\hline Venturi Pressure (inHg) & 27.5 & 28 & 27.5 & 27.5 \\
\hline Section & 1 & 2 & 3 & 4 \\
\hline All Sections Closed - Initial (inHg) & 26 & 25 & 26 & 26 \\
\hline Section & 1 & 2 & 3 & 4 \\
\hline All Sections Closed - Final (inHg) & 26.5 & 25 & 25 & 26 \\
\hline Section & 1 & 2 & 3 & 4 \\
\hline \multirow{4}{*}{ Pressure in Section 1 (inHg) } & 26 & closed & closed & closed \\
\hline & 26 & open & closed & closed \\
\hline & 26 & open & open & closed \\
\hline & 26 & open & open & open \\
\hline Section & 1 & 2 & 3 & 4 \\
\hline \multirow{4}{*}{ Pressure in Section 2 (inHg) } & closed & 26 & closed & closed \\
\hline & open & 26 & closed & closed \\
\hline & open & 26 & open & closed \\
\hline & open & 26 & open & open \\
\hline Section & 1 & 2 & 3 & 4 \\
\hline \multirow{4}{*}{ Pressure in Section 3 (inHg) } & closed & closed & 26 & closed \\
\hline & open & closed & 26 & closed \\
\hline & open & open & 26 & closed \\
\hline & open & open & 26 & open \\
\hline Section & 1 & 2 & 3 & 4 \\
\hline \multirow{4}{*}{ Pressure in Section $4(\mathrm{inHg})$} & closed & closed & closed & 26 \\
\hline & open & closed & closed & 26 \\
\hline & open & open & closed & 26 \\
\hline & open & open & open & 26 \\
\hline
\end{tabular}

Table 141: Data for the right manifold off vehicle unclogged test.

\begin{tabular}{|c|c|c|c|c|}
\hline Section & 1 & 2 & 3 & 4 \\
\hline Venturi Pressure (inHg) & 27 & 27 & 26.5 & 27 \\
\hline Section & 1 & 2 & 3 & 4 \\
\hline All Sections Closed - Initial (inHg) & 16 & 25 & 24 & 24 \\
\hline Section & 1 & 2 & 3 & 4 \\
\hline All Sections Closed - Final (inHg) & 15 & 25 & 24 & 25 \\
\hline Section & 1 & 2 & 3 & 4 \\
\hline \multirow{4}{*}{ Pressure in Section 1 (inHg) } & 15 & closed & closed & closed \\
\hline & 7 & open & closed & closed \\
\hline & 3 & open & open & closed \\
\hline & 16 & open & open & open \\
\hline Section & 1 & 2 & 3 & 4 \\
\hline \multirow{2}{*}{ Pressure in Section $2(\mathrm{inHg})$} & closed & 25 & closed & closed \\
\hline & open & 25 & closed & closed \\
\hline
\end{tabular}




\begin{tabular}{|c|r|r|r|r|}
\hline & open & 24 & open & closed \\
\cline { 2 - 5 } & open & 20 & open & open \\
\hline Section & $\mathbf{1}$ & $\mathbf{2}$ & $\mathbf{3}$ & $\mathbf{4}$ \\
\hline \multirow{3}{*}{ Pressure in Section 3 (inHg) } & closed & closed & 25 & closed \\
\cline { 2 - 5 } & open & closed & 24 & closed \\
\cline { 2 - 5 } & open & open & 22.5 & closed \\
\cline { 2 - 5 } & open & open & 15 & open \\
\hline Section & \multicolumn{1}{|c}{} & $\mathbf{2}$ & $\mathbf{3}$ & $\mathbf{4}$ \\
\hline \multirow{3}{*}{ Pressure in Section 4 (inHg) } & closed & closed & closed & 25 \\
\cline { 2 - 5 } & open & closed & closed & 25 \\
\cline { 2 - 5 } & open & open & closed & 25 \\
\cline { 2 - 5 } & open & open & open & 21 \\
\hline
\end{tabular}

Table 142: Data for Fixed Right Manifold Clogged Test.

\begin{tabular}{|c|c|c|c|c|}
\hline Section & $\mathbf{1}$ & 2 & 3 & 4 \\
\hline Venturi Pressure (inHg) & 26.5 & 26 & 26.5 & 26.5 \\
\hline Section & $\mathbf{1}$ & 2 & 3 & 4 \\
\hline All Sections Closed - Initial (inHg) & 16 & 25 & 25 & 21 \\
\hline Section & $\mathbf{1}$ & 2 & 3 & 4 \\
\hline All Sections Closed - Final (inHg) & 16.5 & 26.5 & 26 & 23 \\
\hline Section & 1 & 2 & 3 & 4 \\
\hline \multirow{4}{*}{ Pressure in Section 1 (inHg) } & 17 & closed & closed & closed \\
\hline & 12 & open & closed & closed \\
\hline & 9 & open & open & closed \\
\hline & 11 & open & open & open \\
\hline Section & 1 & 2 & 3 & 4 \\
\hline \multirow{4}{*}{ Pressure in Section 2 (inHg) } & closed & 25 & closed & closed \\
\hline & open & 25 & closed & closed \\
\hline & open & 23 & open & closed \\
\hline & open & 21 & open & open \\
\hline Section & 1 & 2 & 3 & 4 \\
\hline \multirow{4}{*}{ Pressure in Section 3 (inHg) } & closed & closed & 25 & closed \\
\hline & open & closed & 25 & closed \\
\hline & open & open & 23 & closed \\
\hline & open & open & 18 & open \\
\hline Section & 1 & 2 & 3 & 4 \\
\hline \multirow{4}{*}{ Pressure in Section 4 (inHg) } & closed & closed & closed & 23 \\
\hline & open & closed & closed & 23 \\
\hline & open & open & closed & 23 \\
\hline & open & open & open & 21 \\
\hline
\end{tabular}

Table 143: Data for Unfixed Right Manifold Clogged Test.

\begin{tabular}{|c|c|c|c|c|}
\hline Section & 1 & 2 & 3 & 4 \\
\hline Venturi Pressure (inHg) & 27 & 26.5 & 26 & 26.5 \\
\hline Section & 1 & 2 & 3 & 4 \\
\hline All Sections Closed - Initial (inHg) & 19 & 24 & 23 & 23 \\
\hline Section & 1 & 2 & 3 & 4 \\
\hline All Sections Closed - Final (inHg) & 18 & 24 & 23 & 23 \\
\hline Section & 1 & 2 & 3 & 4 \\
\hline Pressure in Section $1(\mathrm{inHg})$ & 20 & closed & closed & closed \\
\hline
\end{tabular}




\begin{tabular}{|c|c|c|c|c|}
\hline & 16 & open & closed & closed \\
\hline & 15 & open & open & closed \\
\hline & 17.5 & open & open & open \\
\hline Section & 1 & 2 & 3 & 4 \\
\hline \multirow{4}{*}{ Pressure in Section 2 (inHg) } & closed & 24 & closed & closed \\
\hline & open & 24 & closed & closed \\
\hline & open & 21 & open & closed \\
\hline & open & 16 & open & open \\
\hline Section & 1 & 2 & 3 & 4 \\
\hline \multirow{4}{*}{ Pressure in Section $3(\mathrm{inHg})$} & closed & closed & 24 & closed \\
\hline & open & closed & 24 & closed \\
\hline & open & open & 20 & closed \\
\hline & open & open & 2 & open \\
\hline Section & 1 & 2 & 3 & 4 \\
\hline \multirow{4}{*}{ Pressure in Section 4 (inHg) } & closed & closed & closed & 22 \\
\hline & open & closed & closed & 23 \\
\hline & open & open & closed & 23 \\
\hline & open & open & open & 20 \\
\hline
\end{tabular}

Table 144: Data for the right manifold independence clogged test.

\begin{tabular}{|c|c|c|c|c|}
\hline Section & 1 & 2 & 3 & 4 \\
\hline Venturi Pressure (inHg) & 26 & 27 & 27 & 27 \\
\hline Section & 1 & 2 & 3 & 4 \\
\hline All Sections Closed - Initial (inHg) & 26 & 27 & 27 & 26.5 \\
\hline Section & 1 & 2 & 3 & 4 \\
\hline All Sections Closed - Final (inHg) & 26.5 & 26.5 & 26 & 26.5 \\
\hline Section & 1 & 2 & 3 & 4 \\
\hline \multirow{4}{*}{ Pressure in Section 1 (inHg) } & 26.5 & closed & closed & closed \\
\hline & 26 & open & closed & closed \\
\hline & 26 & open & open & closed \\
\hline & 26 & open & open & open \\
\hline Section & 1 & 2 & 3 & 4 \\
\hline \multirow{4}{*}{ Pressure in Section 2 (inHg) } & closed & 27 & closed & closed \\
\hline & open & 27 & closed & closed \\
\hline & open & 27 & open & closed \\
\hline & open & 27 & open & open \\
\hline Section & 1 & 2 & 3 & 4 \\
\hline \multirow{4}{*}{ Pressure in Section 3 (inHg) } & closed & closed & 26.5 & closed \\
\hline & open & closed & 26.5 & closed \\
\hline & open & open & 26.5 & closed \\
\hline & open & open & 26.5 & open \\
\hline Section & 1 & 2 & 3 & 4 \\
\hline \multirow{4}{*}{ Pressure in Section 4 (inHg) } & closed & closed & closed & 26.5 \\
\hline & open & closed & closed & 26.5 \\
\hline & open & open & closed & 26.5 \\
\hline & open & open & open & 26.5 \\
\hline
\end{tabular}

Table 145: Data for the right manifold off vehicle clogged test.

\begin{tabular}{|c|c|r|r|rr|}
\hline Section & $\mathbf{1}$ & $\mathbf{2}$ & $\mathbf{3}$ & $\mathbf{4}$ \\
\hline Venturi Pressure (inHg) & 26.5 & 27 & 26.5 & 27 \\
\hline
\end{tabular}




\begin{tabular}{|c|c|c|c|c|}
\hline Section & 1 & 2 & 3 & 4 \\
\hline All Sections Closed - Initial (inHg) & 20 & 25.5 & 24 & 25 \\
\hline Section & $\mathbf{1}$ & 2 & 3 & 4 \\
\hline All Sections Closed - Final (inHg) & 19 & 24 & 24 & 25 \\
\hline Section & 1 & 2 & 3 & 4 \\
\hline \multirow{4}{*}{ Pressure in Section 1 (inHg) } & 20 & closed & closed & closed \\
\hline & 15 & open & closed & closed \\
\hline & 14 & open & open & closed \\
\hline & 20 & open & open & open \\
\hline Section & 1 & 2 & 3 & 4 \\
\hline \multirow{4}{*}{ Pressure in Section 2 (inHg) } & closed & 25 & closed & closed \\
\hline & open & 24 & closed & closed \\
\hline & open & 23 & open & closed \\
\hline & open & 21 & open & open \\
\hline Section & 1 & 2 & 3 & 4 \\
\hline \multirow{4}{*}{ Pressure in Section 3 (inHg) } & closed & closed & 23 & closed \\
\hline & open & closed & 23 & closed \\
\hline & open & open & 20 & closed \\
\hline & open & open & 11 & open \\
\hline Section & 1 & 2 & 3 & 4 \\
\hline \multirow{4}{*}{ Pressure in Section 4 (inHg) } & closed & closed & closed & 25 \\
\hline & open & closed & closed & 24 \\
\hline & open & open & closed & 24 \\
\hline & open & open & open & 24 \\
\hline
\end{tabular}

Table 146: Data for Fixed Left Manifold Unclogged Test.

\begin{tabular}{|c|c|c|c|c|}
\hline Section & 1 & 2 & 3 & 4 \\
\hline Venturi Pressure (inHg) & 27 & 27 & 27 & 27 \\
\hline Section & $\mathbf{1}$ & 2 & 3 & 4 \\
\hline All Sections Closed - Initial (inHg) & 18 & 22 & 21 & 24 \\
\hline Section & 1 & 2 & 3 & 4 \\
\hline All Sections Closed - Final (inHg) & 17 & 23 & 21.5 & 25 \\
\hline Section & $\mathbf{1}$ & 2 & 3 & 4 \\
\hline \multirow{4}{*}{ Pressure in Section 1 (inHg) } & 17 & closed & closed & closed \\
\hline & 14 & open & closed & closed \\
\hline & 12 & open & open & closed \\
\hline & 21 & open & open & open \\
\hline Section & 1 & 2 & 3 & 4 \\
\hline \multirow{4}{*}{ Pressure in Section 2 (inHg) } & closed & 22 & closed & closed \\
\hline & open & 21 & closed & closed \\
\hline & open & 15 & open & closed \\
\hline & open & 7.5 & open & open \\
\hline Section & 1 & 2 & 3 & 4 \\
\hline \multirow{4}{*}{ Pressure in Section 3 (inHg) } & closed & closed & 21 & closed \\
\hline & open & closed & 20 & closed \\
\hline & open & open & 13 & closed \\
\hline & open & open & 4 & open \\
\hline Section & 1 & 2 & 3 & 4 \\
\hline \multirow{4}{*}{ Pressure in Section 4 (inHg) } & closed & closed & closed & 24 \\
\hline & open & closed & closed & 24 \\
\hline & open & open & closed & 23 \\
\hline & open & open & open & 20 \\
\hline
\end{tabular}


Table 147: Data for Unfixed Left Manifold Unclogged Test.

\begin{tabular}{|c|c|c|c|c|}
\hline Section & 1 & 2 & 3 & 4 \\
\hline Venturi Pressure (inHg) & 27.5 & 27 & 27 & 27 \\
\hline Section & 1 & 2 & 3 & 4 \\
\hline All Sections Closed - Initial (inHg) & 18 & 23.5 & 20 & 23 \\
\hline Section & 1 & 2 & 3 & 4 \\
\hline All Sections Closed - Final (inHg) & 17.5 & 20 & 19 & 23 \\
\hline Section & 1 & 2 & 3 & 4 \\
\hline \multirow{4}{*}{ Pressure in Section 1 (inHg) } & 17 & closed & closed & closed \\
\hline & 10 & open & closed & closed \\
\hline & 4 & open & open & closed \\
\hline & 14 & open & open & open \\
\hline Section & 1 & 2 & 3 & 4 \\
\hline \multirow{4}{*}{ Pressure in Section 2 (inHg) } & closed & 21 & closed & closed \\
\hline & open & 20 & closed & closed \\
\hline & open & 10 & open & closed \\
\hline & open & 2 & open & open \\
\hline Section & 1 & 2 & 3 & 4 \\
\hline \multirow{4}{*}{ Pressure in Section 3 (inHg) } & closed & closed & 19 & closed \\
\hline & open & closed & 19 & closed \\
\hline & open & open & 9 & closed \\
\hline & open & open & 1 & open \\
\hline Section & 1 & 2 & 3 & 4 \\
\hline \multirow{4}{*}{ Pressure in Section 4 (inHg) } & closed & closed & closed & 23 \\
\hline & open & closed & closed & 23 \\
\hline & open & open & closed & 22 \\
\hline & open & open & open & 20 \\
\hline
\end{tabular}

Table 148: Data for the left manifold independence unclogged test.

\begin{tabular}{|c|c|c|c|c|}
\hline Section & 1 & 2 & 3 & 4 \\
\hline Venturi Pressure (inHg) & 27 & 27 & 27 & 27.5 \\
\hline Section & 1 & 2 & 3 & 4 \\
\hline All Sections Closed - Initial (inHg) & 27 & 27 & 26.5 & 26 \\
\hline Section & 1 & 2 & 3 & 4 \\
\hline All Sections Closed - Final (inHg) & 27 & 27 & 27 & 26.5 \\
\hline Section & 1 & 2 & 3 & 4 \\
\hline \multirow{4}{*}{ Pressure in Section 1 (inHg) } & 27 & closed & closed & closed \\
\hline & 27 & open & closed & closed \\
\hline & 26.5 & open & open & closed \\
\hline & 27 & open & open & open \\
\hline Section & 1 & 2 & 3 & 4 \\
\hline \multirow{4}{*}{ Pressure in Section 2 (inHg) } & closed & 27 & closed & closed \\
\hline & open & 27 & closed & closed \\
\hline & open & 27 & open & closed \\
\hline & open & 27 & open & open \\
\hline Section & 1 & 2 & 3 & 4 \\
\hline \multirow{4}{*}{ Pressure in Section 3 (inHg) } & closed & closed & 26.5 & closed \\
\hline & open & closed & 26 & closed \\
\hline & open & open & 26.5 & closed \\
\hline & open & open & 26.5 & open \\
\hline Section & 1 & 2 & 3 & 4 \\
\hline
\end{tabular}


Pressure in Section 4 (inHg)

\begin{tabular}{|r|r|r|r|}
\hline closed & closed & closed & 26 \\
\hline open & closed & closed & 26 \\
\hline open & open & closed & 26 \\
\hline open & open & open & 26 \\
\hline
\end{tabular}

Table 149: Data for the left manifold off vehicle unclogged test.

\begin{tabular}{|c|c|c|c|c|}
\hline Section & 1 & 2 & 3 & 4 \\
\hline Venturi Pressure (inHg) & 27 & 27 & 27 & 27 \\
\hline Section & 1 & 2 & 3 & 4 \\
\hline All Sections Closed - Initial (inHg) & 21 & 21.5 & 21 & 25 \\
\hline Section & 1 & 2 & 3 & 4 \\
\hline All Sections Closed - Final (inHg) & 22 & 20 & 20 & 25 \\
\hline Section & 1 & 2 & 3 & 4 \\
\hline \multirow{4}{*}{ Pressure in Section 1 (inHg) } & 21 & closed & closed & closed \\
\hline & 19 & open & closed & closed \\
\hline & 18 & open & open & closed \\
\hline & 18 & open & open & open \\
\hline Section & 1 & 2 & 3 & 4 \\
\hline \multirow{4}{*}{ Pressure in Section 2 (inHg) } & closed & 20 & closed & closed \\
\hline & open & 17.5 & closed & closed \\
\hline & open & 10 & open & closed \\
\hline & open & 3 & open & open \\
\hline Section & 1 & 2 & 3 & 4 \\
\hline \multirow{4}{*}{ Pressure in Section 3 (inHg) } & closed & closed & 19 & closed \\
\hline & open & closed & 18 & closed \\
\hline & open & open & 10 & closed \\
\hline & open & open & 2 & open \\
\hline Section & 1 & 2 & 3 & 4 \\
\hline \multirow{4}{*}{ Pressure in Section 4 (inHg) } & closed & closed & closed & 25 \\
\hline & open & closed & closed & 25 \\
\hline & open & open & closed & 25 \\
\hline & open & open & open & 22 \\
\hline
\end{tabular}

Table 150: Data for Fixed Left Manifold clogged Test.

\begin{tabular}{|c|c|c|c|c|}
\hline Section & 1 & 2 & 3 & 4 \\
\hline Venturi Pressure (inHg) & 27.5 & 27 & 27 & 27.5 \\
\hline Section & 1 & 2 & 3 & 4 \\
\hline All Sections Closed - Initial (inHg) & 15 & 23.5 & 22.5 & 18 \\
\hline Section & 1 & 2 & 3 & 4 \\
\hline All Sections Closed - Final (inHg) & 16.5 & 23 & 22.5 & 17.5 \\
\hline Section & 1 & 2 & 3 & 4 \\
\hline \multirow{4}{*}{ Pressure in Section 1 (inHg) } & 16 & closed & closed & closed \\
\hline & 11 & open & closed & closed \\
\hline & 10 & open & open & closed \\
\hline & 16.5 & open & open & open \\
\hline Section & 1 & 2 & 3 & 4 \\
\hline \multirow{4}{*}{ Pressure in Section 2 (inHg) } & closed & 23 & closed & closed \\
\hline & open & 22.5 & closed & closed \\
\hline & open & 22 & open & closed \\
\hline & open & 22 & open & open \\
\hline
\end{tabular}




\begin{tabular}{|c|r|r|r|r|}
\hline Section & $\mathbf{1}$ & $\mathbf{2}$ & $\mathbf{3}$ & \multicolumn{1}{|c|}{$\mathbf{4}$} \\
\hline \multirow{3}{*}{ Pressure in Section 3 (inHg) } & closed & closed & 23 & closed \\
\cline { 2 - 5 } & open & closed & 22 & closed \\
\cline { 2 - 5 } & open & open & 21 & closed \\
\cline { 2 - 5 } & open & open & 18 & open \\
\hline Section & $\mathbf{1}$ & $\mathbf{2}$ & $\mathbf{3}$ & $\mathbf{4}$ \\
\hline \multirow{3}{*}{ Pressure in Section 4 (inHg) } & closed & closed & closed & 18 \\
\cline { 2 - 5 } & open & closed & closed & 17.5 \\
\cline { 2 - 5 } & open & open & closed & 18 \\
\cline { 2 - 5 } & open & open & open & 15 \\
\hline
\end{tabular}

Table 151: Data for Unfixed Left Manifold clogged Test.

\begin{tabular}{|c|c|c|c|c|}
\hline Section & 1 & 2 & 3 & 4 \\
\hline Venturi Pressure (inHg) & 27 & 27.5 & 27 & 27.5 \\
\hline Section & 1 & 2 & 3 & 4 \\
\hline All Sections Closed - Initial (inHg) & 16 & 24 & 22 & 19 \\
\hline Section & 1 & 2 & 3 & 4 \\
\hline All Sections Closed - Final (inHg) & 17 & 24 & 21 & 19 \\
\hline Section & 1 & 2 & 3 & 4 \\
\hline \multirow{4}{*}{ Pressure in Section 1 (inHg) } & 16 & closed & closed & closed \\
\hline & 13 & open & closed & closed \\
\hline & 11 & open & open & closed \\
\hline & 20 & open & open & open \\
\hline Section & 1 & 2 & 3 & 4 \\
\hline \multirow{4}{*}{ Pressure in Section 2 (inHg) } & closed & 24 & closed & closed \\
\hline & open & 23 & closed & closed \\
\hline & open & 23 & open & closed \\
\hline & open & 21 & open & open \\
\hline Section & 1 & 2 & 3 & 4 \\
\hline \multirow{4}{*}{ Pressure in Section 3 (inHg) } & closed & closed & 21 & closed \\
\hline & open & closed & 21 & closed \\
\hline & open & open & 20 & closed \\
\hline & open & open & 12 & open \\
\hline Section & 1 & 2 & 3 & 4 \\
\hline \multirow{4}{*}{ Pressure in Section 4 (inHg) } & closed & closed & closed & 19 \\
\hline & open & closed & closed & 21 \\
\hline & open & open & closed & 19 \\
\hline & open & open & open & 16 \\
\hline
\end{tabular}

Table 152: Data for the left manifold independence clogged test.

\begin{tabular}{|c|c|c|c|c|}
\hline Section & 1 & 2 & 3 & 4 \\
\hline Venturi Pressure (inHg) & 28 & 27 & 28 & 27 \\
\hline Section & 1 & 2 & 3 & 4 \\
\hline All Sections Closed - Initial (inHg) & 27 & 27 & 26.5 & 27 \\
\hline Section & 1 & 2 & 3 & 4 \\
\hline All Sections Closed - Final (inHg) & 27 & 27 & 26.5 & 27 \\
\hline Section & 1 & 2 & 3 & 4 \\
\hline \multirow{3}{*}{ Pressure in Section 1 (inHg) } & 27 & closed & closed & closed \\
\hline & 27 & open & closed & closed \\
\hline & 27 & open & open & closed \\
\hline
\end{tabular}




\begin{tabular}{|c|c|c|c|c|}
\hline & 27 & open & open & open \\
\hline Section & 1 & 2 & 3 & 4 \\
\hline \multirow{4}{*}{ Pressure in Section $2(\mathrm{inHg})$} & closed & 26.5 & closed & closed \\
\hline & open & 26.5 & closed & closed \\
\hline & open & 26.5 & open & closed \\
\hline & open & 26.5 & open & open \\
\hline Section & 1 & 2 & 3 & 4 \\
\hline \multirow{4}{*}{ Pressure in Section $3(\mathrm{inHg})$} & closed & closed & 26.5 & closed \\
\hline & open & closed & 26.5 & closed \\
\hline & open & open & 26.5 & closed \\
\hline & open & open & 26.5 & open \\
\hline Section & 1 & 2 & 3 & 4 \\
\hline \multirow{4}{*}{ Pressure in Section $4(\mathrm{inHg})$} & closed & closed & closed & 26.5 \\
\hline & open & closed & closed & 26.5 \\
\hline & open & open & closed & 26.5 \\
\hline & open & open & open & 26.5 \\
\hline
\end{tabular}

Table 153: Data for the left manifold off vehicle unclogged test.

\begin{tabular}{|c|c|c|c|c|}
\hline Section & 1 & 2 & 3 & 4 \\
\hline Venturi Pressure (inHg) & 27 & 27 & 27 & 27.5 \\
\hline Section & 1 & 2 & 3 & 4 \\
\hline All Sections Closed - Initial (inHg) & 13 & 20 & 20 & 23 \\
\hline Section & 1 & 2 & 3 & 4 \\
\hline All Sections Closed - Final (inHg) & 13 & 20 & 19 & 23 \\
\hline Section & 1 & 2 & 3 & 4 \\
\hline \multirow{4}{*}{ Pressure in Section 1 (inHg) } & 15 & closed & closed & closed \\
\hline & 10 & open & closed & closed \\
\hline & 7 & open & open & closed \\
\hline & 12.5 & open & open & open \\
\hline Section & 1 & 2 & 3 & 4 \\
\hline \multirow{4}{*}{ Pressure in Section 2 (inHg) } & closed & 20 & closed & closed \\
\hline & open & 20 & closed & closed \\
\hline & open & 12 & open & closed \\
\hline & open & 1 & open & open \\
\hline Section & 1 & 2 & 3 & 4 \\
\hline \multirow{4}{*}{ Pressure in Section 3 (inHg) } & closed & closed & 20 & closed \\
\hline & open & closed & 19 & closed \\
\hline & open & open & 13 & closed \\
\hline & open & open & 2 & open \\
\hline Section & 1 & 2 & 3 & 4 \\
\hline \multirow{4}{*}{ Pressure in Section 4 (inHg) } & closed & closed & closed & 23 \\
\hline & open & closed & closed & 23 \\
\hline & open & open & closed & 23 \\
\hline & open & open & open & 21 \\
\hline
\end{tabular}

Table 154: Vertical surface test and following table top test data test set 1.

\begin{tabular}{|c|rr|r|r|r|r|}
\hline \multicolumn{7}{|c|}{ Vertical Test } \\
\hline Section & $\mathbf{1}$ & $\mathbf{2}$ & $\mathbf{3}$ & $\mathbf{4}$ \\
\hline Right Side Pressure (inHg) & & 0 & & 15 & & 24 \\
\hline
\end{tabular}




\begin{tabular}{|c|c|c|c|c|}
\hline Section & 1 & 2 & 3 & 4 \\
\hline Left Side Pressure (inHg) & 15 & 22 & 20 & 2.5 \\
\hline \multicolumn{5}{|c|}{ Table Top Test } \\
\hline Section & 1 & 2 & 3 & 4 \\
\hline Right Side Pressure (inHg) & 20 & 25 & 25 & 18 \\
\hline Section & 1 & 2 & 3 & 4 \\
\hline Left Side Pressure (inHg) & 23 & 23 & 23 & 22 \\
\hline
\end{tabular}

Table 155: Vertical surface test and following table top test data test set 2.

\begin{tabular}{|c|c|c|c|c|}
\hline \multicolumn{5}{|c|}{ Vertical Test } \\
\hline Section & 1 & 2 & 3 & 4 \\
\hline Right Side Pressure (inHg) & 3 & 23 & 24 & 25 \\
\hline Section & 1 & 2 & 3 & 4 \\
\hline Left Side Pressure (inHg) & 22 & 20 & 18 & 13 \\
\hline \multicolumn{5}{|c|}{ Table Top Test } \\
\hline Section & 1 & 2 & 3 & 4 \\
\hline Right Side Pressure (inHg) & 3 & 25 & 25 & 26 \\
\hline Section & 1 & 2 & 3 & 4 \\
\hline Left Side Pressure (inHg) & 23.5 & 20 & 20 & 17.5 \\
\hline
\end{tabular}

Table 156: Vertical surface test and following table top test data test set 3.

\begin{tabular}{|c|c|c|c|c|}
\hline \multicolumn{5}{|c|}{ Vertical Test } \\
\hline Section & 1 & 2 & 3 & 4 \\
\hline Right Side Pressure (inHg) & 0 & 24 & 25 & 25 \\
\hline Section & 1 & 2 & 3 & 4 \\
\hline Left Side Pressure (inHg) & 10 & 23 & 20 & 7 \\
\hline \multicolumn{5}{|c|}{ Table Top Test } \\
\hline Section & 1 & 2 & 3 & 4 \\
\hline Right Side Pressure (inHg) & 23 & 26 & 25 & 24.5 \\
\hline Section & 1 & 2 & 3 & 4 \\
\hline Left Side Pressure (inHg) & 23 & 18 & 20 & 21 \\
\hline
\end{tabular}

Table 157: Vertical surface test and following table top test data test set 4.

\begin{tabular}{|c|c|c|c|c|}
\hline \multicolumn{5}{|c|}{ Vertical Test } \\
\hline Section & 1 & 2 & 3 & 4 \\
\hline Right Side Pressure (inHg) & 2 & 22 & 25 & 25 \\
\hline Section & 1 & 2 & 3 & 4 \\
\hline Left Side Pressure (inHg) & 18 & 24 & 23 & 0 \\
\hline \multicolumn{5}{|c|}{ Table Top Test } \\
\hline Section & 1 & 2 & 3 & 4 \\
\hline Right Side Pressure (inHg) & 22.5 & 26 & 25 & 16 \\
\hline Section & 1 & 2 & 3 & 4 \\
\hline Left Side Pressure (inHg) & 22 & 22 & 23.5 & 20 \\
\hline
\end{tabular}

Table 158: Vertical surface test and following table top test data test set 5.

\section{Vertical Test \\ Section \\ 1

Vertical Test

\begin{tabular}{l|l}
2 & 3
\end{tabular}

3

4 


\begin{tabular}{|c|c|c|c|c|}
\hline Right Side Pressure (inHg) & 0 & 24 & 25 & 25 \\
\hline Section & 1 & 2 & 3 & 4 \\
\hline Left Side Pressure (inHg) & 0 & 22 & 23 & 20 \\
\hline \multicolumn{5}{|c|}{ Table Top Test } \\
\hline Section & 1 & 2 & 3 & 4 \\
\hline Right Side Pressure (inHg) & 20 & 26 & 25 & 10 \\
\hline Section & 1 & 2 & 3 & 4 \\
\hline Left Side Pressure (inHg) & 22 & 21.5 & 22 & 24 \\
\hline
\end{tabular}

Table 159: Split Manifold Testing. All Pressure Value in inHg.

\begin{tabular}{|c|c|c|c|c|}
\hline \multicolumn{5}{|c|}{ Manifold Section Independence Test } \\
\hline \multirow{2}{*}{ Section } & \multicolumn{2}{|c|}{ Front Half Sections } & \multicolumn{2}{|c|}{ Back Half Sections } \\
\hline & $\mathbf{1}$ & 2 & 3 & 4 \\
\hline Right Side Pressure (inHg) & 26 & 26.5 & 26 & 26 \\
\hline \multirow{2}{*}{ Section } & \multicolumn{4}{|c|}{ Whole Manifold } \\
\hline & 1 & 2 & 3 & 4 \\
\hline Left Side Pressure (inHg) & 26 & 26 & 27.5 & 27 \\
\hline \multicolumn{5}{|c|}{ On Tread Off Wheels } \\
\hline \multirow{2}{*}{ Section } & \multicolumn{2}{|c|}{ Front Half Sections } & \multicolumn{2}{|c|}{ Back Half Sections } \\
\hline & $\mathbf{1}$ & 2 & 3 & 4 \\
\hline Right Side Pressure (inHg) & 23 & 24 & 24 & 21 \\
\hline \multirow{2}{*}{ Section } & \multicolumn{4}{|c|}{ Whole Manifold } \\
\hline & $\mathbf{1}$ & 2 & 3 & 4 \\
\hline Left Side Pressure (inHg) & 18 & 23 & 21 & 24 \\
\hline \multicolumn{5}{|c|}{ Fixed Manifold Test } \\
\hline \multirow{2}{*}{ Section } & \multicolumn{2}{|c|}{ Front Half Sections } & \multicolumn{2}{|c|}{ Back Half Sections } \\
\hline & 1 & 2 & 3 & 4 \\
\hline Right Side Pressure (inHg) & 17.5 & 26 & 23 & 22 \\
\hline \multirow{2}{*}{ Section } & \multicolumn{4}{|c|}{ Whole Manifold } \\
\hline & 1 & 2 & 3 & 4 \\
\hline Left Side Pressure (inHg) & 17 & 23 & 22 & 20 \\
\hline \multicolumn{5}{|c|}{ Vertical Surface Test } \\
\hline \multirow{2}{*}{ Section } & \multicolumn{2}{|c|}{ Front Half Sections } & \multicolumn{2}{|c|}{ Back Half Sections } \\
\hline & 1 & 2 & 3 & 4 \\
\hline Right Side Pressure (inHg) & 0 & 0 & 20 & 25 \\
\hline \multirow{2}{*}{ Section } & \multicolumn{4}{|c|}{ Whole Manifold } \\
\hline & 1 & 2 & 3 & 4 \\
\hline Left Side Pressure (inHg) & 12 & 23 & 20 & 15 \\
\hline
\end{tabular}

Table 160: The split manifold vertical surface test data with and without applied load.

\begin{tabular}{|c|c|c|c|c|}
\hline \multicolumn{5}{|c|}{ No Applied Force } \\
\hline \multirow{2}{*}{ Section } & \multicolumn{2}{|c|}{ Front Half Sections } & \multicolumn{2}{|c|}{ Back Half Sections } \\
\hline & 1 & 2 & 3 & 4 \\
\hline Right Side Pressure (inHg) & 0 & 0 & 20 & 22 \\
\hline \multirow{2}{*}{ Section } & \multicolumn{4}{|c|}{ Whole Manifold } \\
\hline & 1 & 2 & 3 & 4 \\
\hline Left Side Pressure (inHg) & 15 & 23 & 21 & 10 \\
\hline \multicolumn{5}{|c|}{ With Applied Force - 14 lbf } \\
\hline \multirow{2}{*}{ Section } & \multirow{2}{*}{\multicolumn{2}{|c|}{\begin{tabular}{|r|r}
\multicolumn{2}{|c}{ Front Half Sections } \\
1
\end{tabular}}} & \multicolumn{2}{|c|}{ Back Half Sections } \\
\hline & & & 3 & 4 \\
\hline
\end{tabular}




\begin{tabular}{|c|c|c|c|c|}
\hline Right Side Pressure (inHg) & 22 & 25 & 20 & 23 \\
\hline \multirow{2}{*}{ Section } & \multicolumn{4}{|c|}{ Whole Manifold } \\
\hline & 1 & 2 & 3 & 4 \\
\hline Left Side Pressure (inHg) & 20 & 0 & 0 & 0 \\
\hline
\end{tabular}

Table 161: Fixed Split Manifold Influence Testing. All Pressure Values in inHg.

\begin{tabular}{|c|c|c|c|c|}
\hline Section & 1 & 2 & 3 & 4 \\
\hline Venturi Pressure (inHg) & 27 & 26.5 & 27 & 26 \\
\hline Section & 1 & 2 & 3 & 4 \\
\hline All Sections Closed - Initial (inHg) & 21 & 20 & 20 & 25 \\
\hline Section & 1 & 2 & 3 & 4 \\
\hline All Sections Closed - Final (inHg) & 22 & 20 & 19 & 25 \\
\hline Section & 1 & 2 & 3 & 4 \\
\hline \multirow{4}{*}{ Pressure in Section 1 (inHg) } & 21 & closed & closed & closed \\
\hline & 19 & open & closed & closed \\
\hline & 18 & open & open & closed \\
\hline & 18 & open & open & open \\
\hline Section & 1 & 2 & 3 & 4 \\
\hline \multirow{4}{*}{ Pressure in Section 2 (inHg) } & closed & 20 & closed & closed \\
\hline & open & 17.5 & closed & closed \\
\hline & open & 10 & open & closed \\
\hline & open & 3 & open & open \\
\hline Section & 1 & 2 & 3 & 4 \\
\hline \multirow{4}{*}{ Pressure in Section 3 (inHg) } & closed & closed & 19 & closed \\
\hline & open & closed & 18 & closed \\
\hline & open & open & 10 & closed \\
\hline & open & open & 2 & open \\
\hline Section & 1 & 2 & 3 & 4 \\
\hline \multirow{4}{*}{ Pressure in Section 4 (inHg) } & closed & closed & closed & 25 \\
\hline & open & closed & closed & 25 \\
\hline & open & open & closed & 25 \\
\hline & open & open & open & 22 \\
\hline
\end{tabular}

Table 162: Table top pull force data.

\begin{tabular}{|c|c|c|c|c|c|}
\hline \multicolumn{6}{|c|}{ Test 1} \\
\hline Section & 1 & 2 & 3 & 4 & Pull-Off Force \\
\hline Right Side Pressure (inHg) & 20 & 25 & 24 & 20 & \multirow{3}{*}{$25 \mathrm{lbf}$} \\
\hline Section & 1 & 2 & 3 & 4 & \\
\hline Left Side Pressure (inHg) & 22 & 21 & 19 & 17 & \\
\hline \multicolumn{6}{|c|}{ Test 2} \\
\hline Section & 1 & 2 & 3 & 4 & Pull-Off Force \\
\hline Right Side Pressure (inHg) & 21 & 25 & 22.5 & 21.5 & \multirow{3}{*}{$24.5 \mathrm{lbf}$} \\
\hline Section & 1 & 2 & 3 & 4 & \\
\hline Left Side Pressure (inHg) & 25 & 22.5 & 19 & 15 & \\
\hline \multicolumn{6}{|c|}{ Test 3} \\
\hline Section & 1 & 2 & 3 & 4 & Pull-Off Force \\
\hline Right Side Pressure (inHg) & 20 & 25 & 22 & 20 & \multirow{3}{*}{$23 \mathrm{lbf}$} \\
\hline Section & 1 & 2 & 3 & 4 & \\
\hline Left Side Pressure (inHg) & 25 & 24 & 24.5 & 21.5 & \\
\hline
\end{tabular}




\begin{tabular}{|c|c|c|c|c|c|}
\hline Section & 1 & 2 & 3 & 4 & Pull-Off Force \\
\hline Right Side Pressure (inHg) & 20 & 25 & 23.5 & 22 & \multirow{3}{*}{$24 \mathrm{lbf}$} \\
\hline Section & 1 & 2 & 3 & 4 & \\
\hline Left Side Pressure (inHg) & 25 & 23.5 & 20 & 19 & \\
\hline
\end{tabular}

Table 163: Vertical surface pull off testing data.

\begin{tabular}{|c|c|c|c|c|c|}
\hline \multicolumn{6}{|c|}{ Test 1} \\
\hline Section & 1 & 2 & 3 & 4 & Pull-Off Force \\
\hline Right Side Pressure (inHg) & 0 & 0 & 23 & 24 & \multirow{3}{*}{$19 \mathrm{lbf}$} \\
\hline Section & 1 & 2 & 3 & 4 & \\
\hline Left Side Pressure (inHg) & 15 & 22 & 22 & 11 & \\
\hline \multicolumn{6}{|c|}{ Test 2} \\
\hline Section & 1 & 2 & 3 & 4 & Pull-Off Force \\
\hline Right Side Pressure (inHg) & 0 & 0 & 23 & 25 & \multirow{3}{*}{$17 \mathrm{lbf}$} \\
\hline Section & 1 & 2 & 3 & 4 & \\
\hline Left Side Pressure (inHg) & 16 & 23 & 20 & 14 & \\
\hline \multicolumn{6}{|c|}{ Test 3} \\
\hline Section & 1 & 2 & 3 & 4 & Pull-Off Force \\
\hline Right Side Pressure (inHg) & 0 & 0 & 23 & 22 & \multirow{3}{*}{$15 \mathrm{lbf}$} \\
\hline Section & 1 & 2 & 3 & 4 & \\
\hline Left Side Pressure (inHg) & 13 & 25 & 21 & 10 & \\
\hline \multicolumn{6}{|c|}{ Test 4} \\
\hline Section & 1 & 2 & 3 & 4 & Pull-Off Force \\
\hline Right Side Pressure (inHg) & 0 & 0 & 24 & 23 & \multirow{3}{*}{$16 \mathrm{lbf}$} \\
\hline Section & 1 & 2 & 3 & 4 & \\
\hline Left Side Pressure (inHg) & 15 & 22 & 25 & 12 & \\
\hline \multicolumn{6}{|c|}{ Test 5} \\
\hline Section & 1 & 2 & 3 & 4 & Pull-Off Force \\
\hline Right Side Pressure (inHg) & 0 & 0 & 24 & 22 & \multirow{3}{*}{$20 \mathrm{lbf}$} \\
\hline Section & 1 & 2 & 3 & 4 & \\
\hline Left Side Pressure (inHg) & 10 & 23 & 24 & 16 & \\
\hline
\end{tabular}

Table 164: Holes engaged with manifold sections during pull force testing.

\begin{tabular}{|c|r|r|r|r|rr|}
\hline Section & \multicolumn{1}{|c|}{$\mathbf{2}$} & \multicolumn{1}{|c|}{$\mathbf{3}$} & $\mathbf{4}$ & \multicolumn{2}{c|}{ Sum } \\
\hline Right Side Engaged Holes & & 8 & 6 & 7 & 6 & 27 \\
\hline Section & $\mathbf{1}$ & $\mathbf{2}$ & $\mathbf{3}$ & $\mathbf{4}$ & \multicolumn{2}{|c|}{ Sum } \\
\hline Left Side Engaged Holes & & 6 & 8 & 6 & 7 & \\
\hline
\end{tabular}

